\title{
Die Initialphase der Vegetationsentwicklung nach Windwurf in Buchen-Wäldern auf Zechstein- und Buntsandstein-Standorten des südwestlichen Harzvorlandes
}

\author{
Dissertation \\ zur Erlangung des Doktorgrades \\ der Mathematisch-Naturwissenschaftlichen Fakultäten \\ der Georg-August-Universität zu Göttingen
}

\author{
vorgelegt von \\ Thomas Kompa \\ aus Frankenberg (Sachsen)
}

Göttingen 2004 
D 7

Referent: Prof. Dr. Wolfgang Schmidt

Korreferent: Prof. Dr. Hartmut Dierschke

Tag der mündlichen Prüfung: 03. November 2004 


\section{DANKSAGUNG}

Meinem Doktorvater Prof. Dr. Wolfgang Schmidt danke ich für die Überlassung und Betreuung des Themas, für die Möglichkeit zum freien und selbständigen Arbeiten, für ein jederzeit offenes Ohr bei Rückfragen und fachlichen Diskussionen sowie für die ausgezeichnete Versorgung mit Literaturhinweisen.

Im Rahmen von Diplom- und Praktikumsarbeiten erhoben Dipl.-Biol. Stefan Michel, Dipl.Biol. Stefanie Wild, Dipl.-Forstw. Marcus Lemke und Dipl.-Umweltwiss. Arkadiusz Szurman einen Teil der hier verwendeten Daten. Bei den Geländearbeiten halfen Dipl.-Forstw. Tina Meier, Herr Heiko Rubbert und Herr Andreas Parth tatkräftig mit. Die Laboruntersuchungen haben Frau Andrea Bauer, Frau Martina Knaust und Herr Andreas Parth durchgeführt. Für die computertechnische Unterstützung danke ich Herrn Andreas Parth. Herr Thomas Bernd (Untere Naturschutzbehörde Osterode a.H.) hat mich von amtlicher Seite aus unterstützt. Weiterhin erteilte die Bezirksregierung Braunschweig die erforderlichen naturschutzrechtlichen Ausnahmegenehmigungen. Mündliche Informationen zur Bestandesgeschichte stellten Herr Haase (Forstgenossenschaft Schwiegershausen), Herr Becker (Revierförsterei Hörden) und Herr Herzberg (Revierförsterei Rotenberg) bereit. Für mündliche bzw. bislang unveröffentlichte Informationen zur Naturwald- und Windwurfforschung danke ich Herrn Dr. Eberhard Aldinger (Forstliche Versuchs- und Forschungsanstalt Baden-Württemberg) und Herrn FR Wilhelm Unkrig (Niedersächsische Forstliche Versuchsanstalt). Dr. Martin Weckesser unterstützte mich mit kritischkonstruktiven Gesprächen und Anmerkungen, ebenso hilfreich war der Spaß beim gemeinsamen Erlernen einer neuen Sprache. Den Kollegen vom Göttinger Waldbau-Institut danke ich für das angenehme Arbeitsklima und so manche lustige Begebenheit.

Meinen Eltern danke ich, dass sie mir in der lang andauernden Endphase dieser Arbeit den Rücken frei gehalten haben. 


\section{INHALTSVERZEICHNIS}

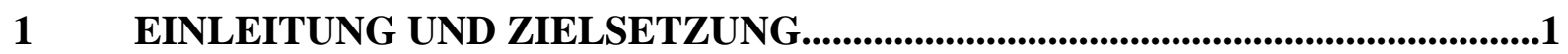

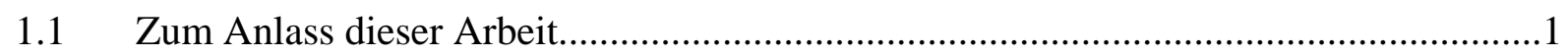

1.2 Windwurf unter meteorologischen, waldbaulichen und standörtlichen

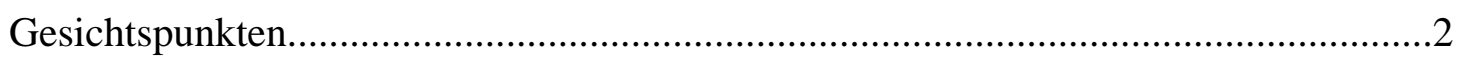

1.3 Bisherige Erkenntnisse der Windwurfsukzessionsforschung......................................4

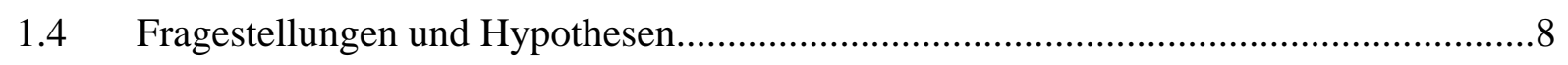

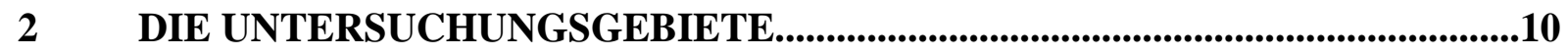

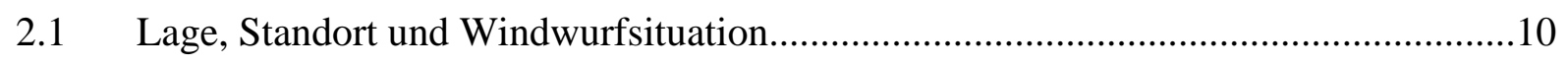

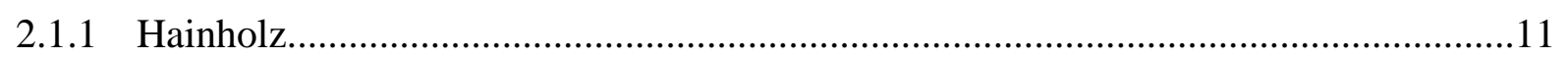

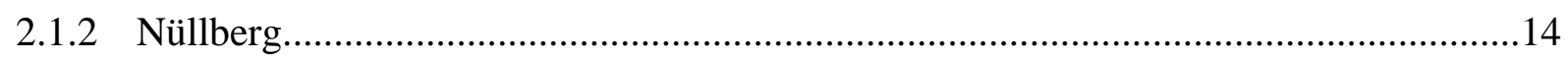

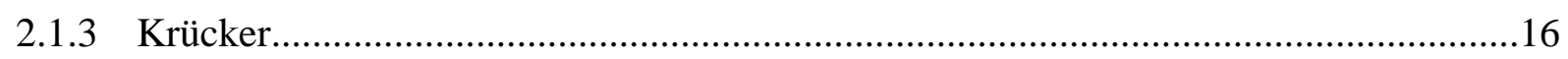

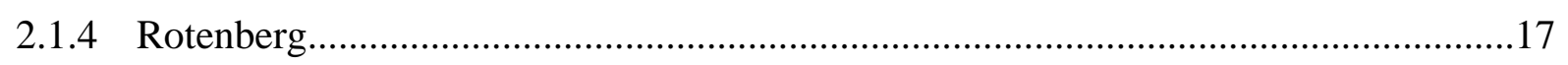

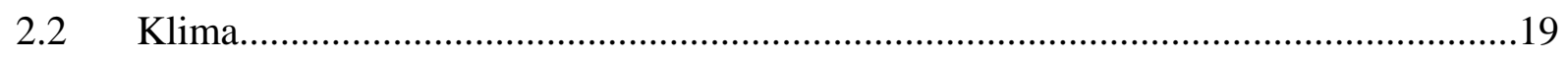

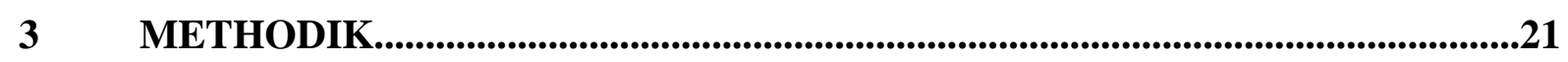

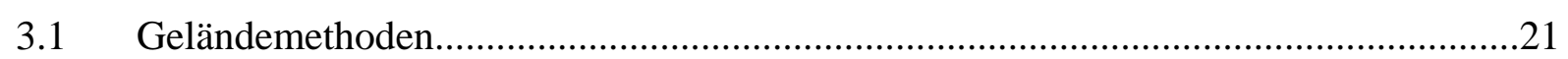

3.1.1 Stratifizierung der Untersuchungsflächen..........................................................21

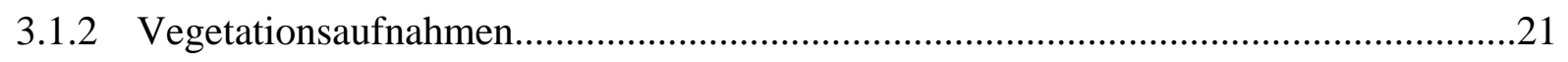

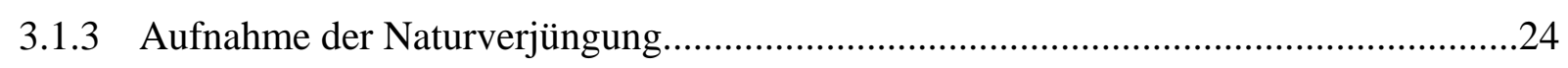

3.1.4 Populationsbiologische Untersuchungen zur Himbeere..........................................26

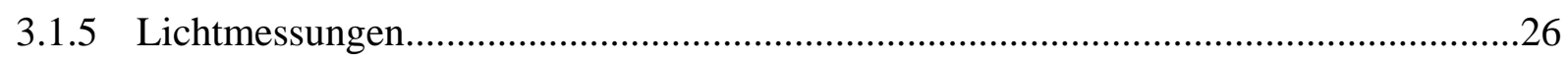

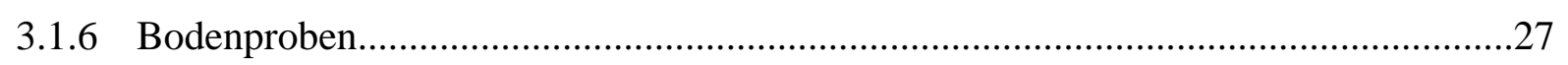

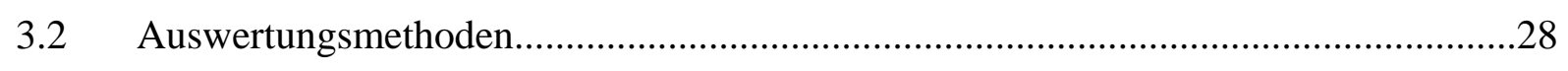

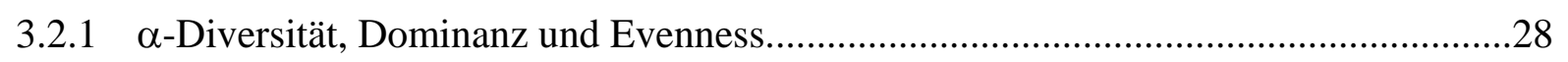

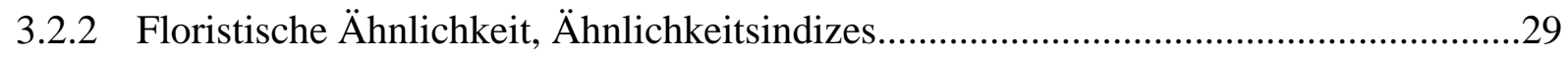

3.2.3 Qualitative (ungewichtete) und quantitative (gewichtete) Artengruppenspektren.......29

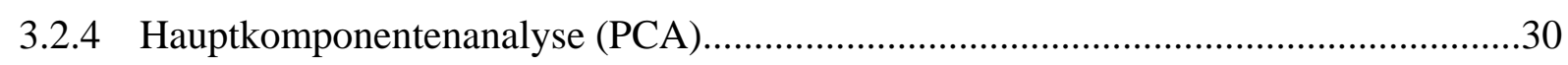

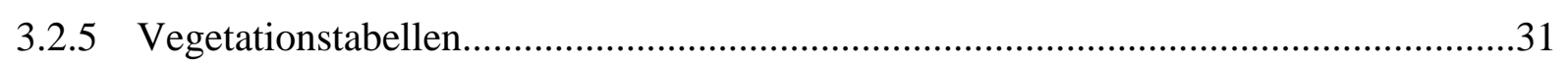

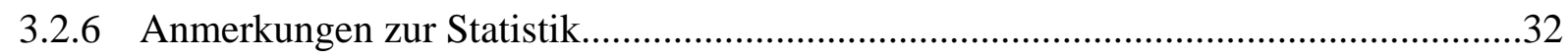

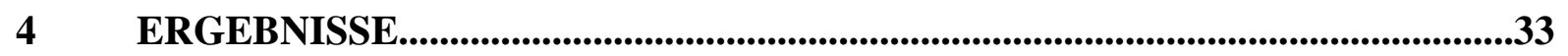

4.1 Standörtliche Charakterisierung der Untersuchungsflächen.......................................33

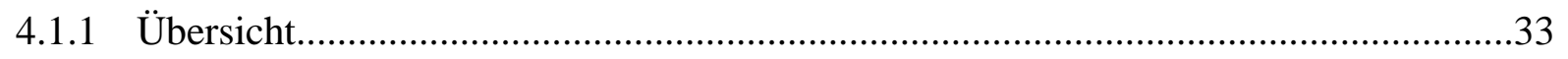

4.1.2 Pflanzensoziologische Zuordnung (DIERSCHKE 1985, 1989)....................................33

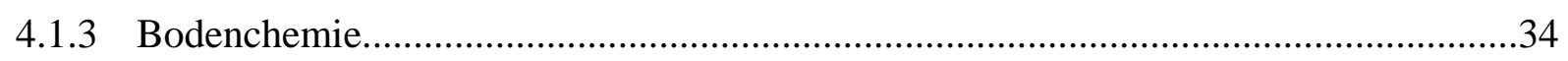




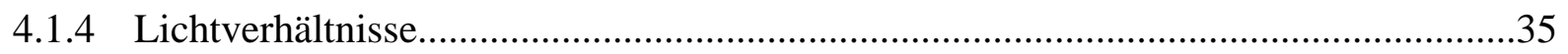

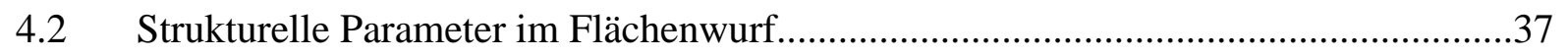

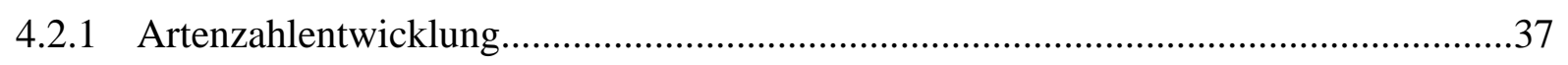

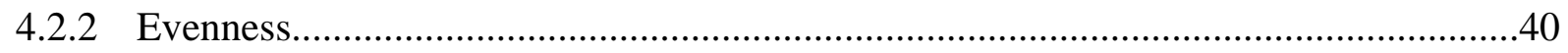

4.2.3 Deckungsgrade bzw. Deckungsgradsummen der Vegetationsschichten.....................41

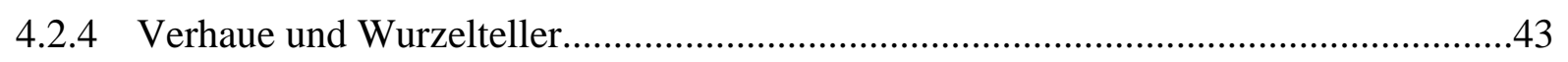

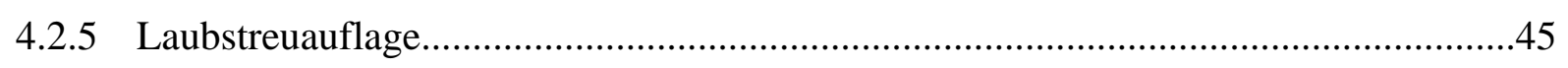

4.2.6 Vegetationsfreier Mineralboden oder Fels...........................................................45

4.2.7 Höhenentwicklung der Strauch- und Feldschicht (Maximalwerte)..............................46

4.2.8 Floristische Ähnlichkeit im Vergleich der Jahre 1999 und 2001.................................47

4.2.9 Hauptkomponentenanalyse (PCA) als Darstellungsmöglichkeit von

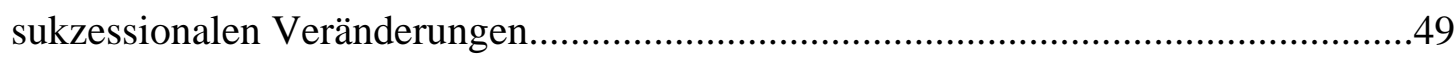

4.3 Qualitative (ungewichtete) und quantitative (gewichtete) Betrachtung ausgewählter vegetationsökologischer Parameter auf den Standorten mit Flächenwurf im Vergleich der Jahre 1999 und 2001................................................52

4.3.1 Ökologisches Verhalten nach Lichtzeigerwert (ELLENBERG et al. 1992)......................52

4.3.2 Ökologisches Verhalten nach Feuchtezeigerwert (ELLENBERG et al. 1992).................54

4.3.3 Ökologisches Verhalten nach Reaktionszeigerwert (ELLENBERG et al. 1992).............55

4.3.4 Ökologisches Verhalten nach Stickstoffzeigerwert (ELLENBERG et al. 1992)..............56

4.3.5 Pflanzensoziologische Klassen nach ELLENBERG et al. (1992)

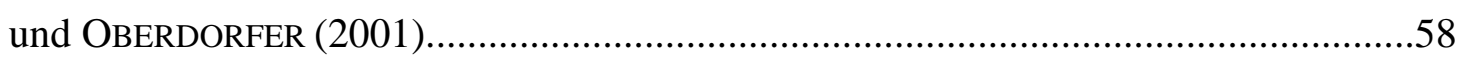

4.3.6 Wuchsformen (nach eigener Einteilung in Anlehnung an die forstliche

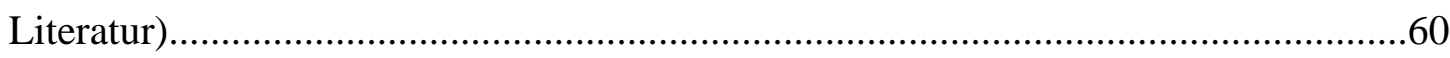

4.3.7 Lebensformtypen im Sinne RAUNKIAERs nach ELLENBERG et al. (1992).....................62

4.3.8 Samenbanktypen nach GRIME et al. (1989), KLEYER (1995), THOMPSON et al. (1997) und OBERDORFER (2001)....................................................64

4.3.9 Ausbreitungstypen nach FrANK et al. (1990).............................................................65

4.3.10 Vegetatives Ausbreitungsvermögen nach SCHMIDT (1983), GRIME et al. (1989), BARTELS (1993) und KLEYER (1995).......................................67

4.3.11 Hemerobietypen nach FRANK et al. (1990).............................................................68

4.4 Typische Sukzessionsmuster ausgewählter Arten..................................................69

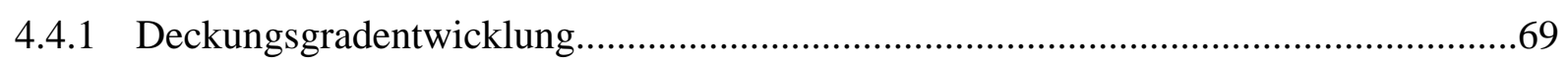

4.4.2 Populationsbiologischer Exkurs: Die Himbeere (Rubus idaeus) als dominantester Pionierstrauch der Windwurfgebiete im Harzvorland...........................73

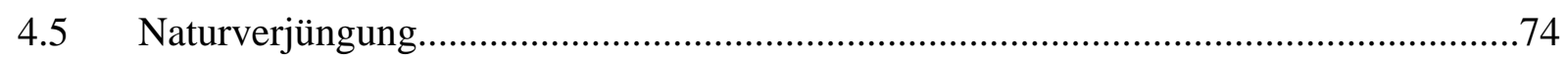

4.5.1 Naturverjüngung im belassenen Flächenwurf im vierten Jahr nach dem Sturm.

4.5.2 Naturverjüngung auf Buntsandstein-Standorten in Abhängigkeit vom Störungsgrad im vierten Jahr nach dem Sturm. 


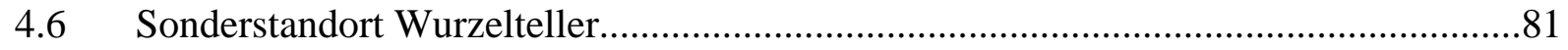

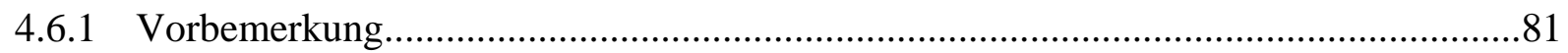

4.6.2 Strukturelle Parameter von Untersuchungsflächen und Wurzeltellern.........................82

4.6.3 Zunahme, Abnahme, Stagnation - das Verhalten der Arten im Vergleich von Untersuchungsfläche und Wurzelteller.

4.6.4 Zunahme, Abnahme, Stagnation - das Verhalten der Feldschichtarten bei isolierter Betrachtung nur der Wurzelteller.

4.6.5 Floristische Ähnlichkeit auf Untersuchungsflächen und Wurzeltellern anhand qualitativer und quantitativer Indizes...........................................................91

4.6.5.1 Floristische Ähnlichkeit zwischen den einzelnen Teilstandorten................................91

4.6.5.2 Intensität der floristischen Veränderung auf den einzelnen Teilstandorten..................93

4.6.6. Hauptkomponentenanalyse (PCA) zur Darstellung der Sukzession auf Untersuchungsflächen und Wurzeltellern.....

4.6.7 Vegetationsökologische Parameter im Vergleich von

Untersuchungsflächen und Wurzeltellern...............................................................96

4.6.7.1 Ökologische Zeigerwerte nach ELLENBERG et al. (1992)..........................................96

4.6.7.2 Pflanzensoziologische Klassen nach ELLENBERG et al. (1992).................................101

4.6.7.3 Lebensformtypen im Sinne RAUNKIAERs nach ELLENBERG et al. (1992)..................103

4.6.7.4 Vegetatives Ausbreitungsvermögen nach Schmidt (1983),

GRIME et al. (1989), BARTELS (1993) und KLEYER (1995)........................................105

4.6.8 Bedeutung der Wurzelteller als alleiniger Wuchsort für bestimmte Arten..................107

4.6.8.1 Häufigkeit des alleinigen Auftretens einzelner Arten auf Wurzeltellern....................107

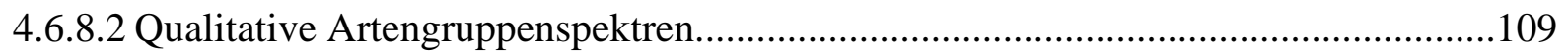

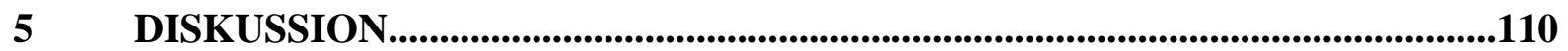

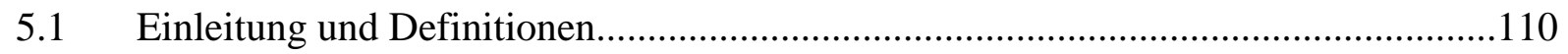

5.1.1 Grundsätzliches zur Herangehensweise...............................................................110

5.1.2 Fluktuation, Regeneration, Sukzession - Begrifflichkeiten der

Vegetationsdynamik.

5.2 Zur Soziologie und Phänologie von Schlagflur- und Ruderalgesellschaften...............112

5.3 Zum Einfluss des Standortes auf die Windwurfsukzession.......................................113

5.3.1 Übersicht über differenzierende Untersuchungsparameter.......................................113

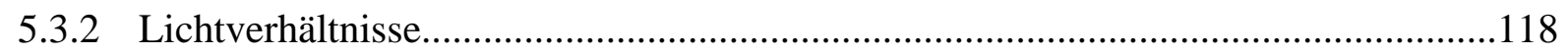

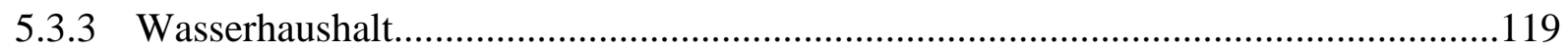

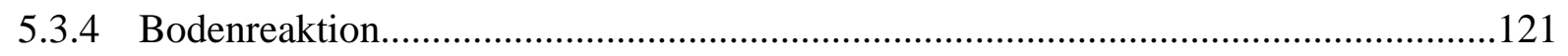

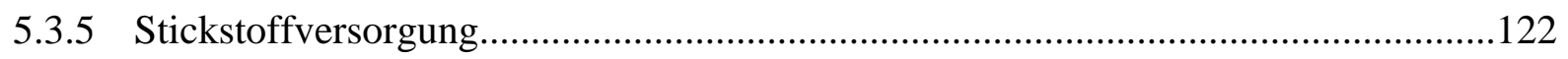

5.4 Zum Einfluss der forstlichen Bewirtschaftung auf die Windwurfsukzession.............124

5.4.1 Übersicht über differenzierende Untersuchungsparameter......................................124 
5.4.2 Einfluss forstlicher Maßnahmen nach dem Sturm: belassene vs. geräumte

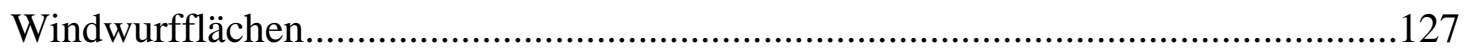

5.4.3 Einfluss der Nutzungsgeschichte: Naturwälder vs. Wirtschaftswälder......................130

5.5 Zum Einfluss der Störungsflächengröße auf die Windwurfsukzession.......................134

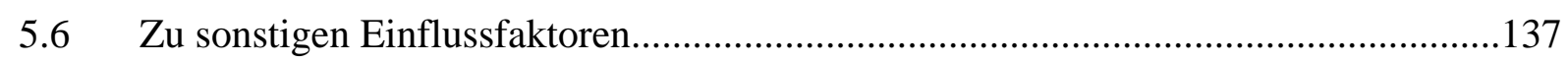

5.6.1 Vorteilsausnutzung am Sukzessionsbeginn: der Vorbestand an Bodenvegetation und Verjüngung.......................................................................138

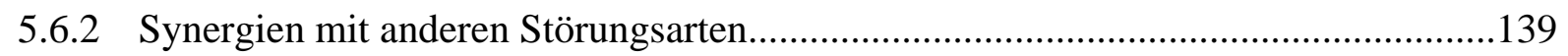

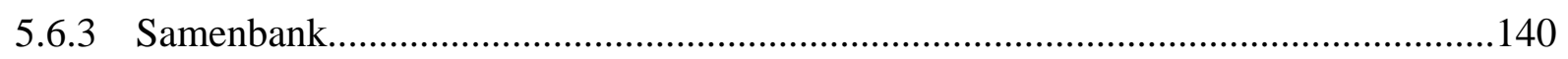

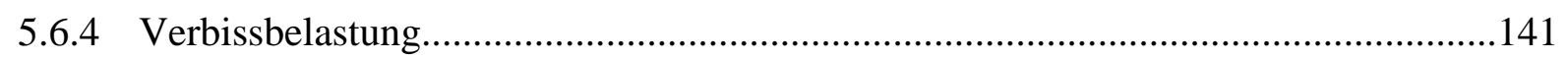

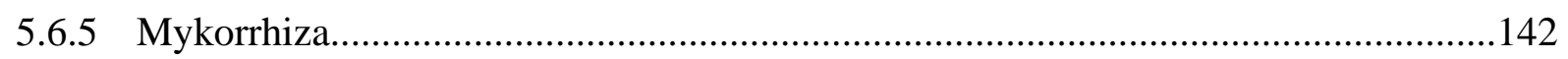

5.6.6 Alter des Waldstandortes und Mechanismen der Artenausbreitung

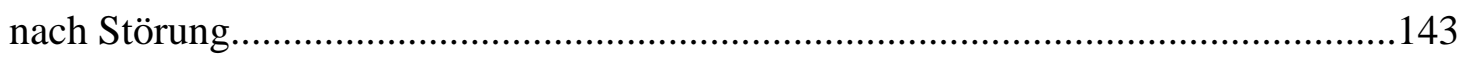

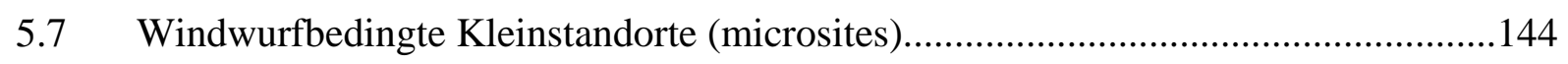

5.7.1 Kronen- und Stammverhaue geworfener Laubbäume..............................................144

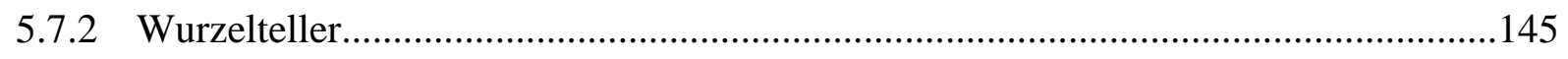

5.8 Zusammenfassende Diskussion: Windwurfdynamik in Wäldern...............................150

5.8.1 Typische Sukzessionsmuster im überregionalen Vergleich.......................................150

5.8.2 Konkurrenzfähigkeit der Buche (Fagus sylvatica) nach Windwurf..........................155

5.8.3 Sukzessionsmodelle in natürlich gestörten Wäldern unter besonderer

Berücksichtigung des Mosaik-Zyklus-Konzeptes.....................................................157

5.8.4 Eingangshypothesen: Standortsgradient und standörtliche Gunst.............................161

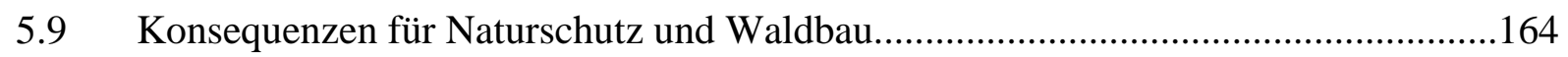

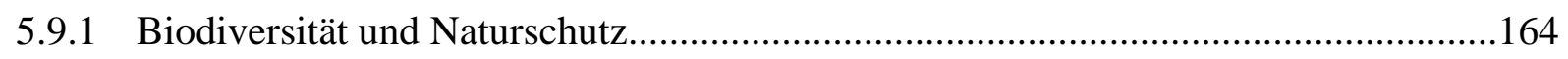

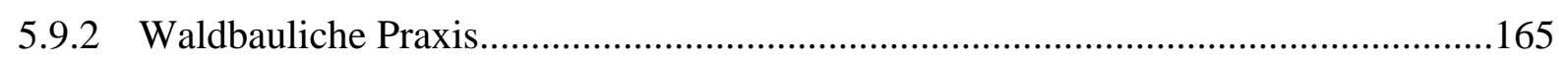

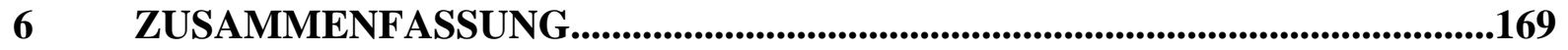

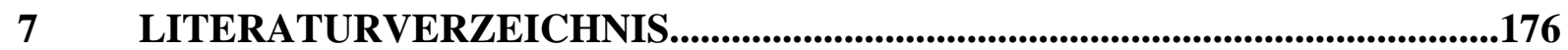

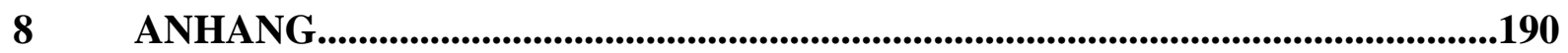




\section{ABKÜRZUNGSVERZEICHNIS}

Abb. Abbildung

Abt. Abteilung

DG Deckungsgrad

DGS Deckungsgradsumme (synonym Deckungssumme)

Kap. Kapitel

KF Kernfläche

NSG Naturschutzgebiet

NW Naturwald (synonym Naturwaldreservat, Bannwald)

Tab. Tabelle

Ufl Untersuchungsfläche (100 m²-Plot)

UG Untersuchungsgebiet

WT Wurzelteller 


\section{EINLEITUNG UND ZIELSETZUNG}

\subsection{Zum Anlass dieser Arbeit}

Ein am Abend des 29. Juni 1997 auftretender schwerer lokaler Sturm, der im Tiefdruckgebiet „Violetta“ entstand und u.a. in Form von horizontal rotierenden, springenden Böenwalzen mit Windspitzen von bis zu $180 \mathrm{~km} / \mathrm{h}$ von Leinefelde (thüringisches Eichsfeld) bis wenige Kilometer nördlich von Osterode (südwestlicher Harzrand/Niedersachsen) zog, verursachte innerhalb nur einer Stunde Windwurf und -bruch unterschiedlichen Ausmaßes (einzelstamm-, trupp-, horstweise, aber auch großflächig) auf verschiedensten Waldstandorten und in nahezu allen Altersklassen. In der 40 x $5 \mathrm{~km}$ langen Sturmschneise liegen Waldgebiete wie das Ohmgebirge, der Duderstädter Stadtwald, der Rotenberg, die „Aue“ südwestlich der Stadt Herzberg sowie der Nüllberg, das Hainholz und der Krücker zwischen Herzberg und Osterode. Während der größte Teil der geworfenen Wälder wie üblich bereits 1998 geräumt und aufgeforstet wurde, blieben in einigen wenigen, ausschließlich Buchenwald-bestockten Gebieten des südwestlichen Harzvorlandes geworfene Bestände sich selbst überlassen. Dazu gehören das Naturschutzgebiet „Gipskarstlandschaft Hainholz“ im Zechsteingürtel des Harzes und der Naturwald „Königsbuche“ im Rotenberg auf Buntsandstein. Die dort erfolgte Einrichtung von Dauerbeobachtungsflächen durch die Niedersächsische Forstliche Versuchsanstalt und die Universität Göttingen eröffnete vielfältige Perspektiven für eine waldökologische Sukzessionsforschung in der ansonsten stark vom menschlichen Handlungsund Gestaltungswillen beeinflussten Waldlandschaft Mitteleuropas. Die vorliegende Arbeit will einen Startpunkt für eine längerfristige vegetationsökologische Sukzessionsbeobachtung setzen, indem sie die verschiedenen Buchen-Waldgesellschaften in der Anfangsphase ihrer Entwicklung innerhalb der ersten vier Vegetationsperioden nach dem Sturmwurf in beschreibend-vergleichender Weise dokumentiert. Der Schwerpunkt liegt auf belassenem Windwurf, jedoch werden auch Vergleichsflächen mit Räumung/Bepflanzung einbezogen. Dabei wird an vorangegangene Untersuchungen im Windwurf des Harzvorlandes angeknüpft (CONRAD 1997; SEITZ 1998; Full 1999; UnKRIG et al. 1999; LUGINBÜHL 2000; WILD 2000; Kompa \& SCHMIDT 2001, 2002, 2003; LEMKE 2001; SCHMIDT 2002a, 2003). Die bisher überwiegend aus der süddeutschen und schweizerischen Windwurfforschung gewonnenen Erkenntnisse (FISCHER 1998; FISCHER \& MÖßMER 1999; HUSS \& HEHN 2001; SCHÖNENBERGER et al. 2002; WILLIG 2002a) sollen somit durch Ergebnisse aus einem weiter nördlich gelegenen Gebiet ergänzt und einem Vergleich zugänglich gemacht werden. Das Besondere daran ist, dass im Fall des Hainholzes deutschlandweit erstmals Windwurfflächen auf einem basenreichen Standort mit Gipskarst studiert werden können. 


\subsection{Windwurf unter meteorologischen, waldbaulichen und standörtlichen}

\section{Gesichtspunkten}

Die Klimaforschung der letzten Jahre geht u.a. von einer instabiler werdenden Atmosphäre infolge veränderter chemischer Zusammensetzung und steigender Jahrestemperaturen sowie von der Verlagerung der Zugbahnen des Island-Tiefs und des Azoren-Hochs aus, was zu heftigeren und häufigeren Stürmen führen müsste (FISCHER 1998; ANGST et al. 2000; AREVALO et al. 2000). Bislang ist aber nicht geklärt, ob im Zusammenhang damit die Sturmwurfhäufigkeit europaweit tatsächlich zugenommen hat. Vielfach ist die Dokumentation älterer Sturmwurfereignisse unterblieben oder lückenhaft, nur einige besonders schwere „Katastrophen“ sind schriftlich überliefert (z.B. die von 1870 im Bayerischen Wald, siehe FISCHER et al. 1990). Ein Vergleich ist auf Grund der Datenlage nicht immer möglich. Ausserdem müssen sich die veränderten klimatischen Bedingungen nicht überall in gleicher Weise auswirken. LÄssig \& Motschalow (1999, 2000a, 2002) fanden z.B. im Ural für die vergangenen 100 Jahre keine Zunahme der jährlichen Sturmereignisse und ihrer Durchschnittsgeschwindigkeiten. ANGST et al. (2000) argumentieren in Bezug auf die Schweiz, dass sich die (sturmreichen) winterlichen Westwindlagen eher nach Norden verlagern und das Alpenland zukünftig eher randlich treffen werden. Gestiegene finanzielle Schäden und Sturmholzmengen stehen in der Schweiz eher im Einklang mit zunehmender Siedlungsdichte, zunehmendem Versicherungsvolumen und steigendem Holzvorrat und nicht mit einer gestiegenen Sturmwurfhäufigkeit (LÄSSIG \& SCHÖNENBERGER 2000).

Nicht nur die klimatischen Rahmenbedingungen, sondern auch der Standort und der Baumbestand müssen in die Betrachtung einbezogen werden. In der Literatur werden verschiedene Gründe angegeben, die zur Prädispositionierung von Bäumen gegenüber Windwurf führen. Dies sind u.a. vorausgehende ergiebige Regenfälle (verminderte Bodenhaftung) bzw. dauerhaft nasse Böden mit Pseudovergleyung (flache Durchwurzelung infolge Staunässe), Erhöhung der aerodynamischen Rauhigkeit des Kronendaches durch überragende Einzelbäume und Bestandeslücken infolge Durchforstung oder vorangegangenen Windwurf, beschädigtes oder gelockertes Wurzelwerk infolge mechanischer Belastung (z.B. vorangegangene Stürme), geländemorphologische Exponierung, Flachgründigkeit des Bodens, Überalterung sowie schlechter Gesundheitszustand des Bestandes (HoFMANN 1960; MAYer 1985; Gravenhorst et al. 1991; Heupel \& BlOCK 1991; VAn den Berge et al. 1993; Peterson \& Rebertus 1997; Fischer 1998; Hubrig 1999; KulaKOWSKi \& Veblen 2002; SCHÖNENBERGER et al. 2003). Auch die Baumartenzusammensetzung kann von Bedeutung sein. Fichten-Reinbestände müssen vor allem auf Pseudogley-Standorten als besonders windwurfanfällig gelten, wohingegen das Risiko bei Mischbeständen, reinen Laubholzbeständen und auch bei der Kiefer deutlich niedriger liegt (FALINSKI 1976; JAHN 1979; Heupel \& Block 1991; Homann \& Engels 1991; Huss 1991; Angst et al. 2000). Beispielsweise wurden bei dem 1990er Sturmwurf in Bayern zwar 3,3\% des Gesamtvorrates an Fichte geworfen, aber nur 0,7\% an Buche und Tanne (FISCHER 1998). Anderen 
Untersuchungen zufolge lässt sich dies aber relativieren (z.B. HetzeL \& REIF 1997 für überproportional stark geworfene Buchen-Reinbestände der Schwäbischen Alb). Höhere Altersklassen sind nach übereinstimmenden überregionalen Angaben stärker windwurfgefährdet als jüngere (z.B. BREWER \& MERRITT 1978; HEUPEL \& BLOCK 1991; Hubrig 1999; UnKRig et al. 1999; AREVALO et al. 2000; SCHÖNENBERGER et al. 2003). Ebenso sind naturferne Wirtschaftskulturen anfälliger als naturnahe (Urwald-)Ökosysteme (MAYER et al. 1980). Entsprechend ausführlich ist der sinngemäße Themenkomplex „waldbauliche Maßnahmen zur Senkung des Sturmschadensrisikos“ in der forstlichen Literatur abgehandelt.

Alle Überlegungen zur waldbaulichen Minimierbarkeit von Sturmschäden werden jedoch hinfällig, wenn Orkane wie „Vivian/Wiebke“ (1990), „Violetta“ (1997) oder „Lothar“ (1999) auf Wälder einwirken. Je stärker der Sturm, umso weniger spielen dann noch Standort, Bestandesstruktur oder Baumart eine Rolle und umso größer ist die Wahrscheinlichkeit, dass der Baum geworfen wird. Während „Sturm“ definitionsgemäß ab Windstärke 9 der BeaufortSkala und damit ab 75 km/h Windgeschwindigkeit und „Orkan“ ab Windstärke 12 (ab 118 $\mathrm{km} / \mathrm{h}$ ) beginnt (HuBRIG 1999), gibt es unterschiedliche Angaben darüber, ab wann mit Windwurf bzw. -bruch zu rechnen ist. So argumentiert z.B. BRÜNIG (1973), dass weltweit keine Baumart existiert, die wirksamen Widerstand gegen Orkane mit Windgeschwindigkeiten ab $101 \mathrm{~km} / \mathrm{h}$ leisten kann (auf nassen Standorten noch niedrigerer Schwellenwert). Entscheidend sind dabei immer die erreichten Staudruckdosen und die Böigkeit des Sturmes in Verbindung mit geländebedingten Verstärkungseffekten, nicht nur mittlere Windgeschwindigkeiten (GRAVENHORST et al. 1991; HUBRIG 1999).

Bei dem Orkan im Harzvorland am 29.06.1997 bewegten sich die rotierenden Böenwalzen, die hauptsächlich für den Windwurf verantwortlich gewesen sein dürften, mit etwa 80-100 $\mathrm{km} / \mathrm{h}$ (Schätzwert) vorwärts. Als ein wesentliches Merkmal dieses Orkans fiel häufig eine flächenscharfe Begrenzung ins Auge (HuBRIG 1999), durch die sich eine punktuelle oder schneisenartige Verstärkung des Windwurfes ausdrückt und die oft zur Neuschaffung bizarr anmutender Waldkanten neben ausgedehnten Verhauflächen führte. Ein interessantes Wurfmuster lässt sich z.B. im Hainholz studieren (Abb. 4). Ein schmaler, galeriewaldartiger Saum am Rand der beiden großen Kahlflächen von Bollerkopf und Jettenhöhlenbreite wurde nicht geworfen und verdeckt zumindest im Sommer von außen den Blick auf diese Flächenwürfe. Als mögliche Erklärungen kommen die größere Stabilität der Traufbäume und das Entstehen einer relativ strömungsberuhigten Stauzone im Bereich des Waldmantels in Frage, aus der heraus sehr starke Böen über die Traufbäume hinweg in den dahinterliegenden Bestand gedrückt werden und dort den Windwurf verursachen (MAYER 1985). Bei Kuppen könnte weiterhin zutreffen, dass die Sturmwirkung aus aerodynamischen Gründen am mittleren Luvhang und am Leehang unmittelbar hinter dem Kuppenscheitel am stärksten ist, während sie am Kuppenfuß bereits wieder nachlässt und die äußersten Bestandesränder verschont hat. 
Eine genauere Klassifizierung der gefallenen Bäume nach Stamm-, Wurzel- und Stockbruch sowie Baumwurf (MAYER 1985) wurde im Rahmen der vorliegenden Arbeit nicht durchgeführt, jedoch sind in den Buchen-Wäldern im Harzvorland Stamm- und Stockbruch eher die Ausnahme. Die meisten umgeworfenen Bäume haben über den unteren Rand des Wurzeltellers und einige unverletzt gebliebene Wurzeln noch Bodenkontakt (Wurzelbruch), einige sind mit ihrem gesamten Wurzelteller herausgehebelt worden (Baumwurf i.e.S.). In der vorliegenden Arbeit wird verallgemeinernd von Wind- bzw. Sturmwurf die Rede sein.

\subsection{Bisherige Erkenntnisse der Windwurfsukzessionsforschung}

Sturmwurf ist die dominierende natürliche Störungsart in Wäldern nicht nur in Mitteluropa (FISCHER 1996a; SCHERZINGER 1996; FISCHER et al. 2002), sondern in der gesamten gemäßigten Zone (PALMER et al. 2000). Derartige natürliche Störungen sind von essentieller Bedeutung für die Regeneration und Stabilität vieler naturnaher und natürlicher Waldökosysteme. Sie sind häufiger als oftmals angenommen und gelten als Auslöser einer vielfältigen Sukzessionsdynamik, durch die geradlinige Entwicklungen hin zur Klimaxgesellschaft immer wieder unterbrochen werden (FALINSKI 1976; LEIBUNDGUT 1978; FISCHER et al. 1990; LÄSSIG \& SCHÖNENBERGER 1993; OtTO 1994; KORPEL 1995; UlANOVA 2000b). Neben dem von vielen Autoren bemängelten Fehlen von Langzeitstudien zur Waldsukzession auf Sturmwurf- oder (damit vergleichbaren) Kahlschlagsflächen lässt sich feststellen, dass insbesondere vegetationskundliche Untersuchungen im Vergleich zu waldbaulich-gehölzkundlichen bisher eher selten durchgeführt wurden (DIERSCHKE 1988; FISCHER et al. 1990, 2002; LÄSSIG \& SCHÖNENBERGER 1993; APFFELSTAEDT \& BERNHARDT 1996; Gregor \& SEIDLING 1997, 1998, 1999; Fischer 1998; AREVAlO et al. 2000; PALMER et al. 2000; KULAKOWSKI \& VEBLEN 2003). Vor allem auf belassenen Sturmwurfflächen ist die Forschung in Deutschland relativ jung, basenreiche Standorte sind dabei unterrepräsentiert. Kleinflächige Windwurfsukzession findet seit 1972/76 in einem Naturwaldreservat in den Stemwehder Bergen/Nordwestdeutschland statt (APFFELSTAEDT \& BERNHARDT 1996). Großflächig geworfenen Wald überließ man erstmals im Nationalpark Bayerischer Wald nach dem Stürmen von 1983/84 sich selbst und richtete Dauerflächen ein (FISCHER et al. 1990). Nach den 1990er Stürmen „Vivian/Wiebke“ wurden in BadenWürttemberg insgesamt fünf Sturmwurfbannwälder (synonym Naturwälder, Naturwaldreservate) sowie Vergleichsflächen in 10 Forstämtern eingerichtet (SAYER \& REIF 1998; HANKE \& PISOKE 1999). Ebenfalls seit dieser Zeit stehen das hessische Naturwaldreservat Weiherskopf im Vogelsberg (WILLIG 1994), eine Teilfläche im Nationalpark Berchtesgaden (ZIERL \& SIEGRIST 1999) und mehr als 50 Vergleichsflächen in 10 Bayerischen Forstämtern unter Dauerbeobachtung (FISCHER 1992). Nach einem lokalen Orkan im Jahr 1997 wurden das Naturschutzgebiet „Gipskarstlandschaft Hainholz“ sowie der Naturwald „Königsbuche“ im südniedersächsischen Harzvorland in diese spezielle Form der 
Wald-Sukzessionsforschung einbezogen (SCHMIDT 2002a, 2003; KOMPA \& SCHMIDT 2003). Nach dem 1999er Sturm „Lothar“ kamen 20 weitere Untersuchungsflächen in verschiedenen Landschaftsräumen Baden-Württembergs hinzu (ALDINGER et al. 2001). Veröffentlichte Ergebnisse von längeren, wenn auch nicht lückenlos dokumentierten Sukzessionszeiträumen im belassenen Windwurf existieren bisher von APFFELSTAEDT \& BERNHARDT (1996, nach 20 Jahren), Fischer et al. (2002, nach 15 Jahren) und JEHL (2001, nach 13 Jahren). Die Windwurfforschung in den Schweizer Bergwäldern wurde etwa 10 Jahre nach „Vivian/Wiebke“ von ScHÖNENBERGER et al. (2002, 2003) zusammengefasst. In den USA dagegen besteht schon wesentlich längere Zeit ein recht entspanntes Verhältnis zu „Wildnis“ und zur Belassung natürlicher Prozesse auf Katastrophenflächen. Aus einem Naturwald in den Rocky Mountains (Colorado) liegt beispielsweise eine 65 Jahre alte Windwurfstudie vor (KULAKOWSKi \& VEBLEN 2003).

Generelle Entwicklungen nach Windwurf gestalten sich nach bisherigen Erkenntnissen aus Deutschland, der Schweiz, Polen, Russland, den USA und Japan wie folgt:

\section{Windwurf führt durch die Öffnung des Kronendaches zu erhöhter struktureller} Diversität des Bestandes und zur Schaffung eines Mosaiks an Kleinstandorten (z.B. Wurfböden mit Wurzeltellerstrukturen, „mound-and-pit topography“, Stamm- und Kronenverhaue). Dadurch werden charakteristische Muster in Vegetation, Mikroklima und Boden mit entsprechenden ökologischen Nischen ausgebildet (FALINSKI 1976; KoOP 1982; ELLENBERG et al. 1986; NAKASHIZUKA 1989; FISCHER et al. 1990, 2002; JEHL 2001; ELLENBERG 1996; BATTAGLiA et al. 2000; MCALister et al. 2000; UlANOVA 2000b).

2. Windwurf führt zu heterogeneren Umweltbedingungen im Bestand. Davon sind Lichtklima, Temperatur sowie Wasser- und Nährstoffverfügbarkeit betroffen (FISCHER 1996a, 1998; SchönEnBerger et al. 2003). Das Klima der Freifläche ist „kontinentaler“ als im Bestand. Einstrahlung, Transpiration sowie Frost- und Dürrewahrscheinlichkeit sind erhöht. Durch den Wegfall der Transpiration des Kronendaches kommt es zu erhöhter Bodenwasserverfügbarkeit und Bodenvernässung (SCHÜLER 1999; POLOMSKI \& KUHN 2000). Verstärkte Bodendurchmischung durch aufgeklappte Wurzelteller sowie Licht- und Wärmeeinstrahlung bewirken einen regelrechten „Mineralisierungsschub“ mit entsprechendem Nährstoffaustrag. Dichter werdende Bodenvegetation und Verjüngung fängt diesen Wasser- und Nährstoffüberschuss zunehmend auf (KENK et al. 1991; BAUHUS 1994; BARTSCH et al. 1999; MELLERT et al. 1998; SCHMIDT 2002c). Im belassenen Windwurf wird langfristig der Nährstoffeintrag eine große Rolle spielen, wenn dort enorme Mengen an Stammholz durch holzzersetzende Pilze abgebaut werden (HONOLD \& OBERWINKLER 1998; REXER et al. 1999; SCHÄFER 2002; SCHLECHTE 2002).

3. Windwurf führt über Bodenverwundungen zur Aktivierung der Samenbank des Bodens. Da typische Waldarten kaum eine langfristige Samenbank aufbauen, sind waldfremde Störzeiger in der Samenbank besonders von Wirtschaftswäldern häufig 
überrepräsentiert (z.B. FISCHER 1987). Nach windwurfbedingter Auflichtung und Bodenverwundung (Wurzelteller, Räumung) laufen diese Arten massenhaft auf und nutzen ihre langjährige unterirdische Präsenz gewissermaßen als Konkurrenzvorteil (APFFELSTAEDT \& BernhardT 1996; Bonn \& PosCHLOD 1998; FIsCHER et al. 1998, 2002; ABs et al. 1999).

\section{Windwurf führt zu erhöhter floristischer, faunistischer und mykologischer Diversität} (KENK et al. 1991; FISCHER 1998; LÄSSIG 2000; PALMER et al. 2000; SMIRNOVA et al. 2000; Dorow 2002; FleChTNER 2002; KoMPa \& SCHMIDT 2002, 2003; SCHLECHTE 2002; SCHMIDT 2002a, 2003; SCHÖNENBERGER et al. 2003). Es treten Dominanzwechsel bei vielen Pflanzenartengruppen auf. So können Schlagflur- und Ruderalstadien sowie Vergrasung begünstigt werden. Besonders auf wasserstauenden, tonreichen Böden nehmen Vernässungs-, Wechselfeuchte- und Verdichtungszeiger zu (FISCHER 1992, 1996a; RUNGE 1993; REIF \& Przybilla 1995; Peterson \& Rebertus 1997; Fischer et al. 1998; MÄRKL \& EGgLSEer 2001). In der temperaten Nordhemisphäre wurden häufig Rubus-Dominanzen beschrieben (Fischer 1992; Wohlgemuth et al. 1995; Hetzel \& ReIF 1998; Hyatt \& CASPer 2000; PALMER et al. 2000; Ulanova 2000b; Kompa \& SCHMidT 2003). In vielen Fällen bleibt die alte Waldbodenflora nahezu unbehelligt und überdauert die Zeit bis zur Wiederherstellung von waldartigen Standortsverhältnissen auch unter zeitweise dominierenden Pionierstadien. Das Waldökosystem verändert sich in dieser Hinsicht relativ wenig (DIERSCHKE 1988; WILLIG 1994, 2002b; HETZEL \& REIF 1998; SAYER \& REIF 1998; KeITEL 1998, 1999; HARCOMBE et al. 2002; SCHMIDT 2002a, 2003; KOMPA \& SCHMIDT 2003).

5. Windwurf leitet die Waldregeneration aus der Vorverjüngung oder durch Neuansamung ein. Diese verläuft standortabhängig unterschiedlich schnell. Der Vorbestand an Bodenvegetation und Verjüngung, der Umfang und die Qualität der Samenbank, das Vorhandensein freier Mineralbodenkeimplätze, das Mykorrhizapotenzial, der Wildverbiss, die Störungsflächengröße und die Entfernung zur nächsten Samenquelle sind dabei entscheidend für die Qualität und die Geschwindigkeit der Wiederbewaldung (FISCHER 1998; SCHÖNENBERGER et al. 2002, 2003). Bei ausreichender Verjüngung der Schlusswaldbaumarten können frühsukzessionale Waldstadien gehindert oder „,übersprungen“ und spätsukzessionale Stadien in ihrem Wachstum beschleunigt werden (LORIMER 1980; KenK et al. 1991; SchÖlCh 1998; Wilmanns et al. 1998; Arevalo et al. 2000; HarcomBE et al. 2002; SCHMIDT 2002a, 2003; KompA \& SCHMidT 2003). Andererseits kann die Verjüngung der Schlusswaldbaumarten auch sehr gering ausfallen oder es können Artenwechsel auftreten, die eine schnelle Wiederbewaldung im Sinne der standortstypischen und potenziell natürlichen Waldgesellschaft verzögern (BARDEN 1979; KOOP 1982; REIF \& PrZYBiLla 1995; SCHMidT-SchÜtZ 1999; MÄRKL \& EGglSEER 2001; SCHÖNENBERGER 2002). Gelegentlich werden Baumarteninversionen in der Verjüngung beschrieben, bei denen im Altbestand subdominant vorhandene Klimaxbaumarten vorübergehend eine dominierende Pionierrolle übernehmen. Beschrieben wurden solche Dominanzwechsel z.B. von Fagus sylvatica zu Fraxinus excelsior (KeITEL 1998, 1999; SCHMIDT 2002a, 2003 für 
Mitteldeutschland), von Nothofagus menziesii zu N. fusca (STEWART et al. 1991 für Neuseeland) oder von Picea engelmannii zu Abies lasiocarpa (KULAKOWSKI \& VeBLEN 2003 für die Rocky Mountains/USA).

Es ist umstritten, ob und unter welchen Voraussetzungen die nahezu identische Wiederherstellung der Vor-Windwurf-Bedingungen nach sehr langen Sukzessionszeiträumen wahrscheinlich ist (PALMER et al. 2000). Insbesondere in Mitteleuropa waren und sind Aussagen zur natürlichen Walddynamik auf Grund des starken menschlichen Einflusses mit großen Unsicherheiten behaftet. Es zeichnet sich aber ab, dass eine menschlich unbeeinflusste Windwurfsukzession die Rückentwicklung forstlich überprägter zu naturnahen Beständen beschleunigen oder zumindest einleiten kann (FISCHER et al. 1990).

Die zitierten Beispiele mögen verdeutlichen, dass das Interesse an Langzeituntersuchungen zunehmend Eingang in die Forschungspraxis gefunden hat und Sturmwürfe offenbar ein angemessenes „Initialisierungsereignis“ dafür darstellen. Bei in den 1980er und 1990er Jahren durchgeführten Literaturauswertungen (TILMAN 1989; SCHMIDT 1998a) wurde noch festgestellt, dass nur minimale Prozentsätze (weit unter 5\%) von freilandökologischen Arbeiten Zeitreihen von mehr als 10 Jahren beinhalteten. Noch drastischer galt dies für Langzeituntersuchungen speziell zu den Fagetalia (FISCHER et al. 1990 zitieren Literaturrecherchen aus den 1970er Jahren). Als hervorragend geeignete Dauerbeobachtungsflächen bieten sich die derzeit 668 deutschen Naturwaldreservate (Gesamtfläche etwa $250 \mathrm{~km}^{2}$ ), die Kernzonen der Nationalparke und Biosphärenreservate sowie einige wenige waldbestockte Naturschutzgebiete mit Null-Nutzung an, sofern sie von Windwurf betroffen waren oder in Zukunft sein werden. In diesen Gebieten ist schon aus rechtlichen Gründen eine Belassung von Windwurfflächen unkomplizierter durchsetzbar als in Wirtschaftswäldern. In Deutschland ist zur Zeit mit einer unbewirtschafteten Waldfläche von maximal $450 \mathrm{~km}^{2}$ zu rechnen, was einem Anteil von rund 0,4\% an der Gesamtwaldfläche entspricht (unter Ausschluss z.B. der Pflege- und Entwicklungszonen in Großschutzgebieten; Angaben gerundet nach BÜCKING 1997 und KÖLBEL 1999). Für einen machbaren Forschungsansatz zur natürlichen Walddynamik mag das viel erscheinen, zumal sich der Anteil weiter erhöhen wird (z.B. durch neuausgewiesene Nationalparke wie Hainich und Kellerwald bzw. durch langfristige Überführung weiterer Nationalparkflächen in Kernzonenstatus). Gemessen an den Vorstellungen des behördlichen und verbandlichen Naturschutzes bezüglich ausreichend großer Prozessschutzflächen in Wäldern ist das aber eher zu wenig. Nach BÜCKING (1997) schwanken solche Zielvorstellungen innerhalb Deutschlands zwischen 1-3\% der Waldfläche des jeweiligen Bundeslandes, europa- und weltweit sogar zwischen 5-10\%. In Europa sind momentan nur 0,7\% der Waldfläche ohne Nutzung. Die meisten Länder bleiben unter 1\%, einige wenige liegen bei 3-5\% (Ex- 
Jugoslawien, Schweden, Island, Slowakei), nur Albanien erreicht bereits 10\% (BÜCKING 1997).

\subsection{Fragestellungen und Hypothesen}

Ziel der hier vorliegenden Untersuchungen ist eine Beschreibung und Erklärung von Prozessen der ungestörten Waldregeneration und -dynamik nach Windwurf unterschiedlichen Ausmaßes auf basenreichen und basenarmen Standorten im Vergleich $\mathrm{zu}$ forstlich aufgearbeiteten Windwurfflächen. Dadurch ergeben sich die drei Betrachtungsebenen Standort, Bewirtschaftung und Störungsflächengröße bezüglich ihres Einflusses auf die Vegetationsdynamik. Folgenden Fragen soll dabei auf den Grund gegangen werden:

- Wie regenerieren sich Buchen-dominierte Waldbestände nach Windwurf? Welche Arten profitieren unmittelbar von den neuen Bedingungen? Wodurch wird die Sukzessionsgeschwindigkeit gehemmt oder beschleunigt (Konkurrenz zwischen Schlagflur- und Ruderalarten sowie der Gehölzverjüngung; Reaktion typischer Waldarten)?

- Wie durchsetzungsfähig ist die Klimaxbaumart Buche (Fagus sylvatica) nach Windwurf?

- Welche Bedeutung haben windwurfbedingte Strukturveränderungen (Stamm- und Kronenverhaue) und Bodenstörungen (Wurzelteller, Wurfmulden) für die Sukzession?

- Welche Rolle spielen Störungsflächengröße und andere Standortfaktoren (Boden, Geologie, Lichtklima)?

- Gibt es grundsätzlich verschiedene Entwicklungen auf belassenen und geräumten Windwurfflächen?

- Welche strukturellen oder vegetationsökologischen Parameter (z.B. Artenzahlen, Abundanzen, Artengruppenspektren, Diversitätsmaße) eignen sich am besten zur Beschreibung unterschiedlicher Entwicklungen? Welchen Erklärungsbeitrag liefern sie? Welche Artengruppen eignen sich als Bioindikatoren für eine Beurteilung von Diversität und Dynamik in Wäldern?

- Welche verallgemeinerbaren Sukzessionsmodelle können abgeleitet werden? Wann und unter welchen Bedingungen sind Mosaikzyklen im Sinne von REMMERT (1985, 1987, 1991) mit längerfristigen Pionierstadien zu erwarten oder verläuft die Waldregeneration gänzlich ohne sie?

Aus diesen Überlegungen und aus den bisherigen Kenntnissen zur Windwurfsukzession heraus (siehe Kap. 1.3) sollen folgende Hypothesen vorangestellt werden:

1. Die Sukzession von Buchen-Wäldern nach Windwurf ist stark standortabhängig. Der Standortsgradient von „frisch-basenreich-nährstoffreich“ über mittlere Verhältnisse hin zu 
„(trocken?)-sauer-nährstoffarm“ wird durch die Reaktion des Bestandes auf die Störung deutlich nachgezeichnet. Je reicher der Standort, umso resilienter reagiert das Ökosystem, umso weniger wird die waldtypische Vegetation beeinträchtigt, überlagert oder verdrängt, umso schneller schreitet die Wiederbewaldung in Richtung der standorttypischen Ausgangsgesellschaft voran und umso unwichtiger ist die Störungsflächengröße.

2. Mit zunehmender standörtlicher Ungunst verlangsamt sich die Wiederbewaldungsgeschwindigkeit, wächst das Ausmaß schlusswaldferner Übergangsstadien und nimmt die Bedeutung der Störungsflächengröße zu. Unter solch suboptimalen Bedingungen kann sich der Wald nur noch bei kleinflächiger (lückiger) Störung schnell regenerieren, während großflächiger Windwurf zur Verzögerung dieses Prozesses führt sowie krautige und/oder verholzte Pioniervegetation in größerem Umfang begünstigt wird.

3. Ein kompletter Artenwechsel findet aber trotzdem nicht statt, nur eine unterschiedlich lang andauernde und unterschiedlich intensiv ausgeprägte Überlagerung der vor dem Sturm vorhandenen Vegetationsdecke im Sinne eines Dominanzwechsels zwischen verschiedenen Artengruppen.

4. Der Einfluss forstlicher Maßnahmen auf die Vegetationsdynamik in Buchen-Wäldern nach Windwurf ist dementsprechend auf reichen Standorten eher klein, auf ärmeren dagegen größer.

Die ausgewählten Untersuchungsgebiete und Waldgesellschaften eignen sich für die Überprüfung dieser Fragen und Hypothesen aus folgenden Gründen:

Die vom Windwurf betroffenen Buchen-Waldgesellschaften sind für den nordwestdeutschen Raum typisch (auf Zechstein: Carici-Fagetum, Hordelymo-Fagetum; auf Buntsandstein: Galio odorati-Fagetum, Luzulo-Fagetum). Ein Teil der Wälder weist eine große Naturnähe auf und ist schon seit längerem ohne forstliche Nutzung (Hainholz, Königsbuche). Ein anderer Teil steht als konventionell bewirtschaftete Vergleichsfläche räumlich nah zur Verfügung (Nüllberg, Rotenberg-Abt. 1228). Eine gewisse Großflächigkeit der Störung ist gegeben. Mit rund 60 ha ist z.B. die vom Sturm beeinflußte Waldfläche im Hainholz fast doppelt so groß wie die mittlere Flächengröße von Naturwaldreservaten in Deutschland (34 ha nach BÜCKING 1997). 


\section{DIE UNTERSUCHUNGSGEBIETE}

\subsection{Lage, Standort und Windwurfsituation:}

Alle Untersuchungsgebiete liegen im südlichen Niedersachsen im Landkreis Osterode und gehören zum forstlichen Wuchsbezirk Südwestliches Harzvorland innerhalb des Wuchsgebietes Südniedersächsisches Bergland (ARBEITSKREIS STANDORTSKARTIERUNG 1985). Eine grobe Abgrenzung des gesamten Untersuchungsraumes ist durch ein gedachtes Viereck möglich, welches von den Ortschaften Osterode, Herzberg, Rhumspringe und Hattorf gebildet wird (Abb. 1).

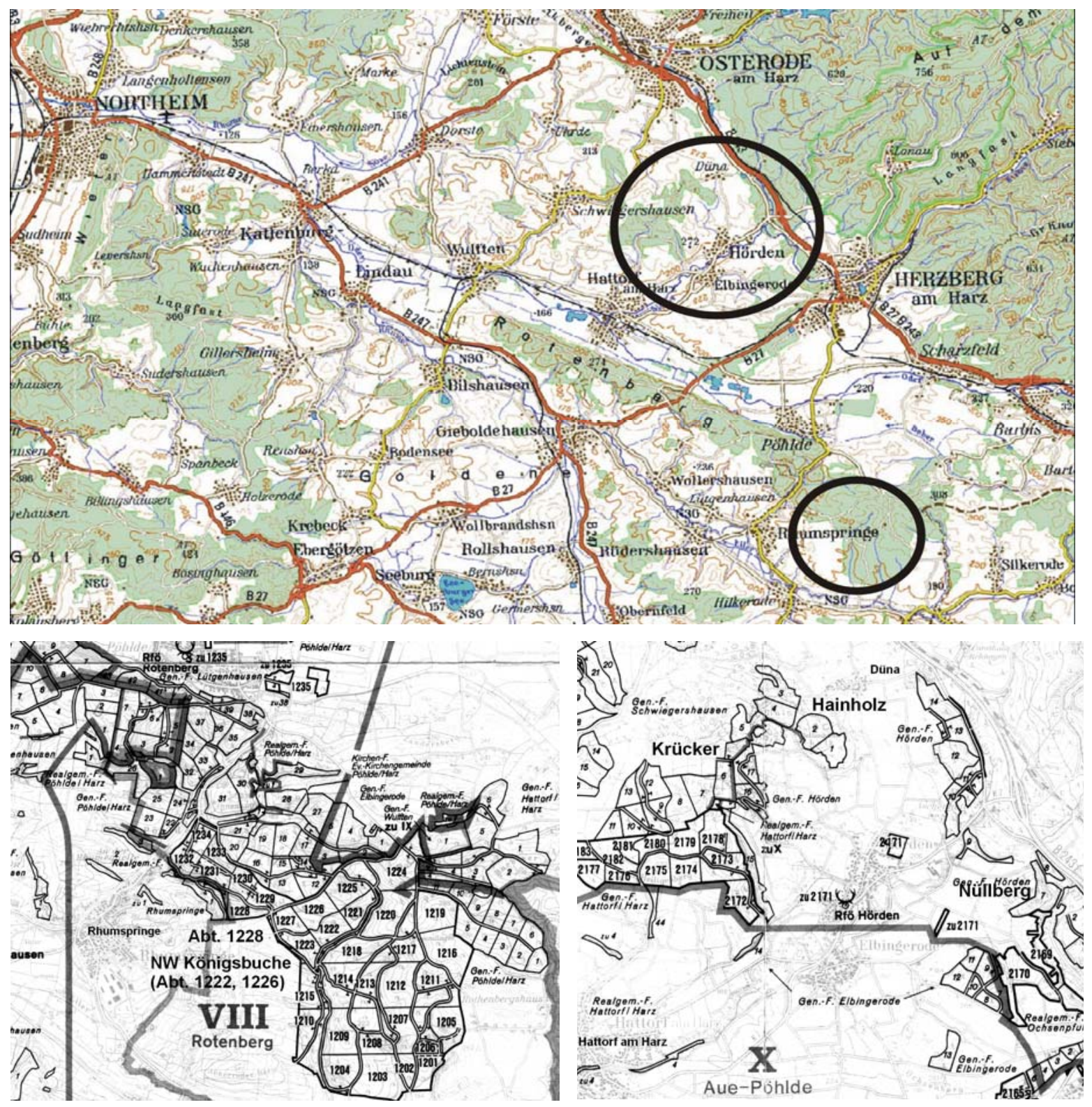

Abb. 1: Lage der Untersuchungsgebiete im südwestlichen Harzvorland; oben: Auszug aus der Topografischen Karte (1:200.000) - nördlicher Kreis: Hainholz, Krücker, Nüllberg - südlicher Kreis: Rotenberg; unten: Auszug aus der Forstkarte des Niedersächsischen Forstamtes Reinhausen (1:50.000) - einbezogene Forstabteilungen siehe Text (Kap. 2.1). 
Mit Höhenlagen von 220 bis 340 mNN gehören sie zur collinen Höhenstufe. In die Untersuchungen einbezogen wurden Buchen-Wälder frischer (im Fall des Bollerkopfes mäßig trockener) Standorte auf basenreichem Zechstein sowie auf basenarmem Buntsandstein, die sich bezüglich der Waldgesellschaft, des Störungsgrades sowie der forstlichen Nutzungsgeschichte vor und nach dem Sturmwurf vom Juni 1997 unterscheiden.

\subsubsection{Hainholz}

Das Hainholz ist ein etwa 60 ha großes Waldgebiet nahe der Ortschaft Düna, etwa $5 \mathrm{~km}$ südlich der Stadt Osterode. Es hat eine NW-SE-Ausdehnung von 1,5 km und eine mittlere Breite von $500 \mathrm{~m}$. Seine höchsten Erhebungen sind die im NW gelegenen Kuppen des Bollerkopfes (273 mNN) und des Sommersitzes (283 mNN). Nach Südosten fällt das Gelände auf etwa $240 \mathrm{mNN}$ ab. Das Hainholz erscheint als Laubwaldinsel in einer ansonsten durch Grünland (besonders im Norden) und Ackerflächen (besonders im Süden) geprägten Landschaft. Im Westen existiert über ein stark zerklüftetes, Buchen-bestocktes Teilareal („Mädchenlöcher“) sowie über einige Fichten-Aufforstungen ein Übergang zum südwestlich gelegenen Krücker.
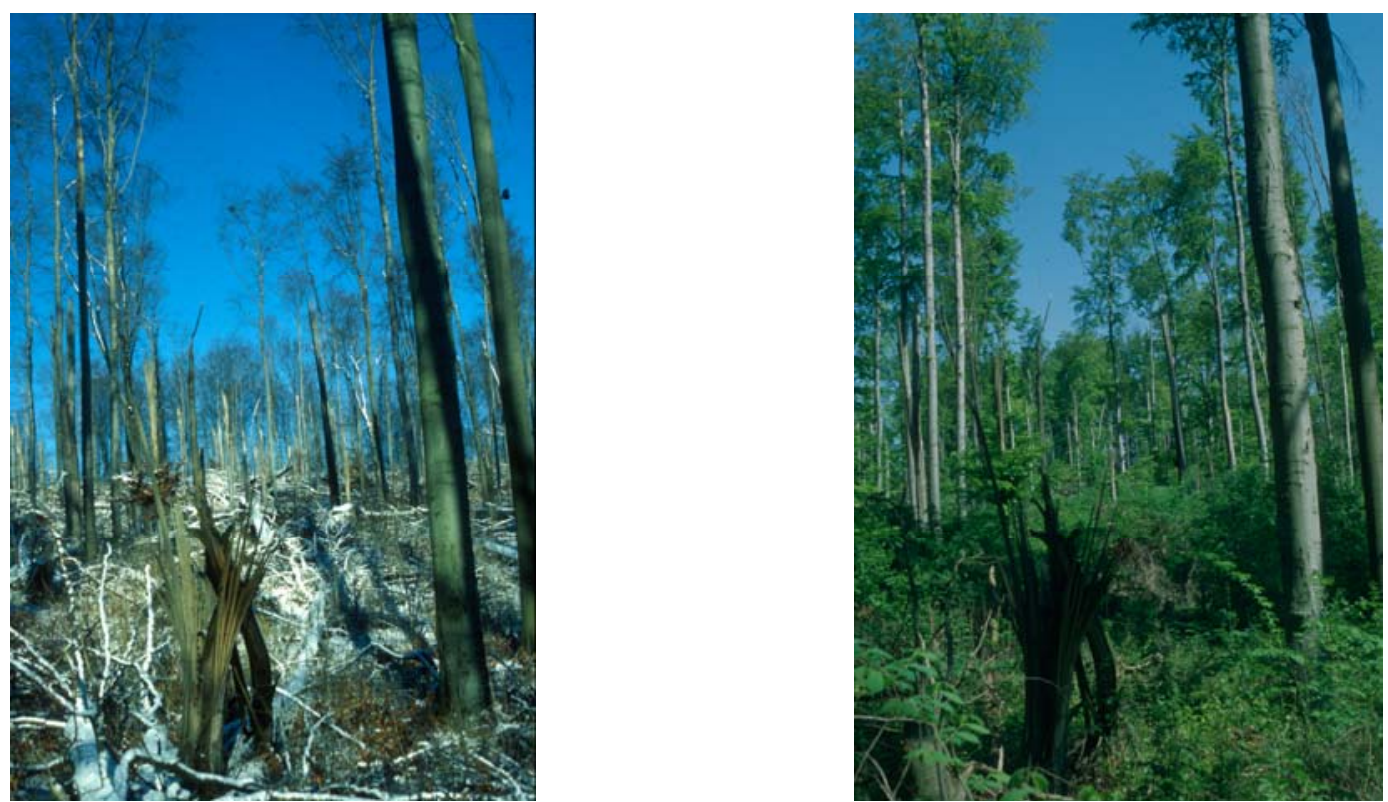

Abb. 2: Hainholz-KF1 („Jettenhöhlenbreite“) von Süden; links: Jan. 2000; rechts: Mai 2000.

Geologie und Böden: Das Hainholz liegt im Zechsteingürtel des Harzes und wird durch ausstreichende Anhydritschichten (Werra-, Basal- und Hauptanhydrit) geprägt, welche unterschiedlich stark vergipst sind und mit Lagerungen aus Salz und Dolomit wechseln (SCHÖNFELDER 1978; SCHMIDT 2002a). Nach der Substrattypenkarte von UNKRIG et al. (1999), die auf einer aktuellen Standortskartierung des Hainholzes basiert, sind ca. 70\% des Gebietes als Gipsstandorte anzusprechen. 

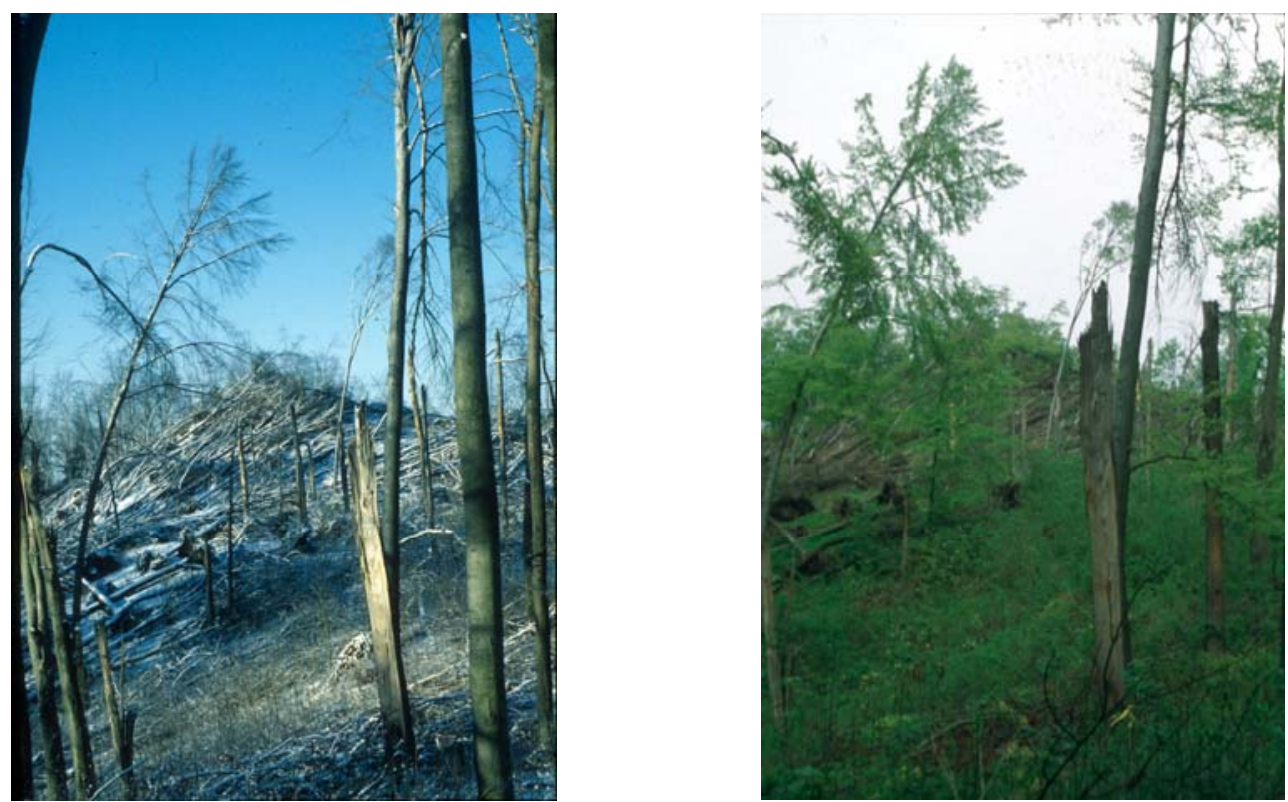

Abb. 3: Hainholz-KF2 („Bollerkopf“) von Südosten; links: Januar 2000; rechts: April 2000.

Im Südosten („Jettenhöhlenbreite“) liegt ein größeres Dolomit-Areal und im Westen („Mädchenlöcher“) besteht ein Übergang zum Unteren Buntsandstein des Krückers. Im Bereich des Zechsteins existieren weiterhin Gips/Dolomit-Mischstandorte. Das Hainholz ist von vielfältigen Karsterscheinungen geprägt, die in Form steiler Kuppen, weitläufiger Karrenfelder, tiefer und teilweise wassergefüllter Dolinen und auch in Gestalt zweier überregional bekannter Höhlen (Jetten- und Marthahöhle) auftreten (BERND et al. 1994; ELlWANGER 1999). Die Böden der Gipsstandorte sind meist sehr flachgründig und den GipsRendzinen zuzuordnen, welche kleinflächig und z.T. durch Lössbeimengungen (z.B. an Hangfüßen) in Lehm- oder Braunerde-Rendzinen übergehen können (SCHMIDT 2002a). Die Dolomit- und die Gips-/Dolomit-Mischstandorte sind meist (ausser in Kuppenlage) von mittel- bis tiefgründigen Braunerde-Rendzinen geprägt. Tiefgründige Lösslehmüberdeckungen sind auf den Buntsandstein-Standorten sowie in einem Teilareal auf Gips an der Nordflanke des südöstlichen Hainholzes zu finden. Somit ist das Hainholz durch eine kleinräumig sehr stark wechselnde Standortsvielfalt charakterisiert, die sich auch in der Vegetationsbedeckung niederschlägt (SCHMIDT 2002a).

Bestockung: Nach der Vegetationskarte von SCHÖNFELDER (1978) stocken im Hainholz verschiedene Ausprägungen des Seggen- und Waldgersten- (damals: Perlgras-) Buchenwaldes, die teilweise ineinander übergehen können (siehe Kap. 4.1). Im westlich gelegenen Buntsandstein-Bereich kommen Waldmeister- und Hainsimsen-Buchenwald hinzu. Buche ist die Hauptbaumart, standörtlich ergänzt durch Esche, Berg- und Spitz-Ahorn, Sommer-Linde, Fichte, Vogel-Kirsche und (selten) Berg-Ulme. Erwähnenswert ist auch das Carici-Fagetum luzuletosum (DIERSCHKE 1985), eine stellenweise auf oberflächlich versauerten Gipskuppen oder Karrenfeldern auftretende Untereinheit des SeggenBuchenwaldes mit vielen Säurezeigern. Azonale Vegetation zeigt sich im Bereich der Dolinen 
und feuchten Senken (Ahorn-Eschen-Schluchtwald, Bach-Eschenwald, Flutrasenvegetation). Menschliche Aufforstungsaktivität wird z.B. durch einen größeren Eschen/Berg-AhornJungbestand um den Sommersitz herum und durch mehrere, im Randbereich liegende und mittlerweile im Zuge von Naturschutzmaßnahmen bereits wieder abgetriebene Fichtenaufforstungen dokumentiert (BERND et al. 1994). Die meisten Bestände sind nach UNKRIG (1997a) etwa 120-150 Jahre alt, befanden sich vor dem Sturm auf dem größten Teil ihrer Fläche in der Optimal- oder Wachstumsphase und sind je nach Tiefgründigkeit des Standortes unterschiedlich verjüngungsreich. Die Waldgrenzen des Hainholzes sind mindestens seit 1784/85 weitestgehend konstant (Kurhannoversche Landesaufnahme zitiert in SCHÖNFELDER 1978).

Windwurfsituation: Eine sehr präzise Aussage zum 1997er Windwurf liefert die Bestandeslückenkarte von FUCHS \& KUNTH (1999), die auf einer Luftbildauswertung basiert (Abb. 4).

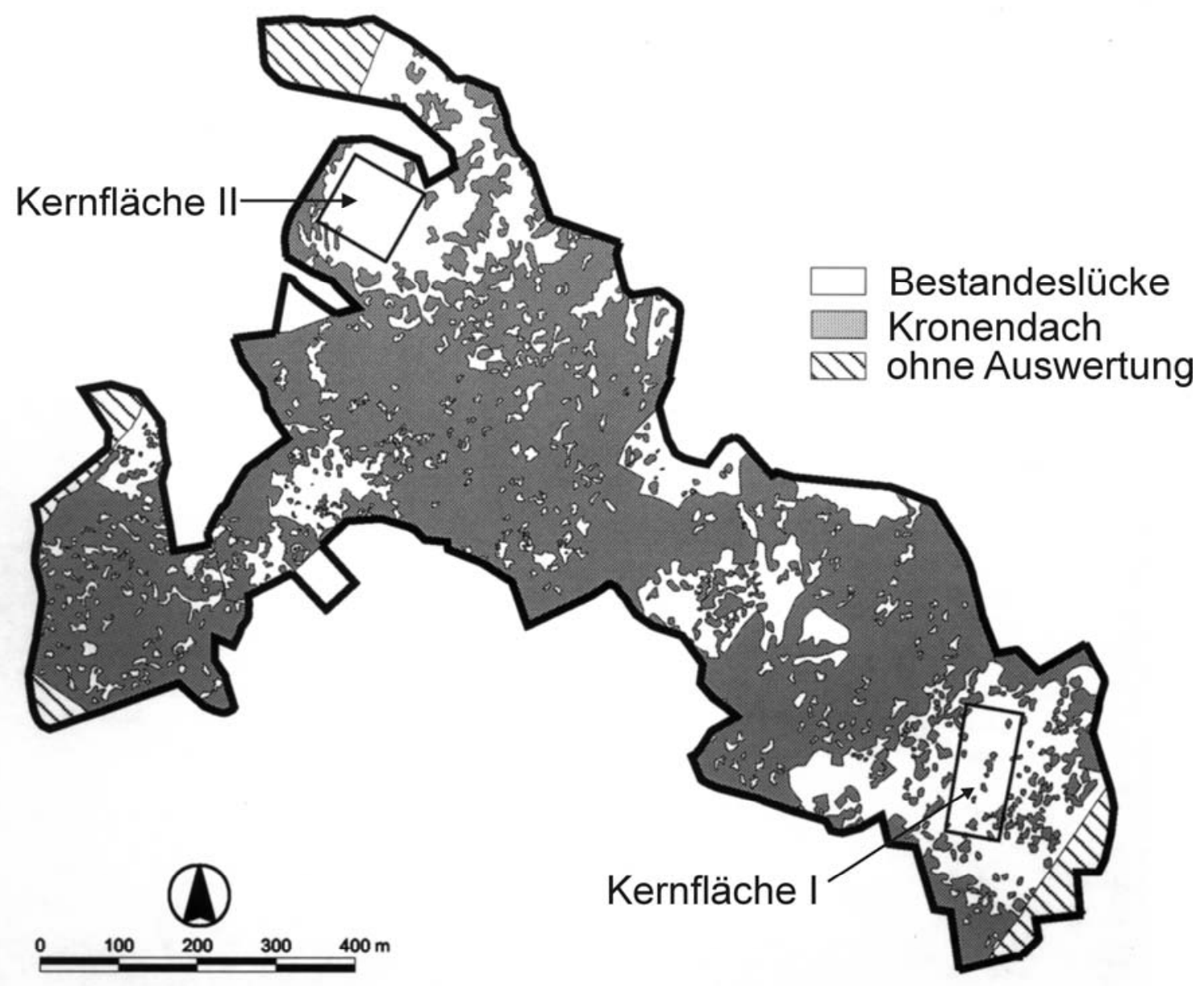

Abb. 4: Bestandeslückenkarte des Hainholzes nach dem Sturmwurf vom Juni 1997 (nach FUCHS \& KUNTH 1999). 
Etwa 66\% des Kronendaches im Hainholz blieben vom Sturm weitestgehend unbeeinflusst. Ein relativ großer Flächenanteil ist von lückigem Windwurf betroffen, wobei die allermeisten Lücken (etwa 86\%) unter $250 \mathrm{~m}^{2}$ groß sind und damit im Bereich von 1-2 Baumkronen liegen. Nur 3\% der Lücken sind über $2000 \mathrm{~m}^{2}$ groß, stellen damit aber immerhin $78 \%$ der geworfenen Fläche. Die größten Flächenwürfe (teilweise über 2 ha groß) befinden sich im Bereich der später eingerichteten Kernflächen („südöstliche Jettenhöhlenbreite“, „Bollerkopf“ - siehe Abb. 2, 3) sowie im zentralen Teil des Hainholzes und an seiner südwestlichen Flanke. Altbestände wurden eindeutig stärker vom Orkan getroffen als Jungbestände.

Verwaltung und Nutzung: Das Hainholz umfasst mehrere Abteilungen der Genossenschaftsforste Schwiegershausen und Hörden. Die in die Untersuchungen einbezogene Kernfläche 1 befindet sich in der Abt. 1 und die Kernfläche 2 in der Abt. 4 des Genossenschaftsforstes Schwiegershausen. Das UG gehört vollständig zum insgesamt 641 ha großen Naturschutzgebiet (NSG) „Gipskarstlandschaft Hainholz“, welches sich ansonsten durch eine wertvolle, meist halboffene Kulturlandschaft mit Xerotherm- und Feuchtvegetation auszeichnet (BERND et al. 1994; BEZIRKSREGIERUNG BRAUnSCHWEIG 2000). Desweiteren steht das Hainholz als Naturwald unter der wissenschaftlichen Betreuung durch die Niedersächsische Forstliche Versuchsanstalt. Da von Seiten des behördlichen Naturschutzes ohnehin geplant war, im Hainholz dem Prozessschutz Vorrang einzuräumen, beschränkte sich die forstlichen Aktivität vor dem Sturm etwa seit 1993 auf geringfügige Pflegeeingriffe. Nach dem Sturm von 1997 wurde beschlossen, der natürlichen Sukzession endgültig freien Lauf zu lassen. Die Durchsetzung dieser für Mitteleuropa immer noch wenig üblichen Null-Nutzung wird durch Anpachtung der naturschutzfachlich wertvollen Waldflächen durch den Landkreis Osterode „,auf 99 Jahre“ gewährleistet (SCHMIDT 2002a).

\subsubsection{Nüllberg}

Der Nüllberg ist ein von SW nach NE verlaufender, länglicher Bergrücken etwa 1,5 km nordwestlich der Stadt Herzberg. Er erreicht eine Maximalhöhe von 289 mNN und erhebt sich hoch über dem Tal der Sieber, welche hier etwa auf 220 mNN verläuft und - von SE kommend - durch diesen Höhenzug abgelenkt wird, bevor sie eine ausgedehnte Schleife beschreibt und weiter in südwestlicher Richtung fließt.

Geologie und Böden: Der Nüllberg liegt ebenso wie das Hainholz im Zechsteingürtel des Harzes. Das Hauptmassiv des Nüllberges besteht aus Dolomit. Der mittlere und untere Nordwesthang ist von pleistozänen hercynischen Schottern überlagert, welche aus unterschiedlichen Gesteinen des Harzes gebildet und von der Sieber abtransportiert wurden (GEOLOGISCHE SPECIALKARTE 1884). Eine relativ schmale Dolomitrippe bildet sehr markant den höchsten Bereich des Bergrückens. Gips steht nicht an, ist aber unter dem Dolomit am Fuß des Berges dokumentiert und führt dort im Bereich der Nordostflanke infolge Verkarstung zu einer geologischen Besonderheit, der Sieber-Schwinde (VLADI 1976, 1998). 

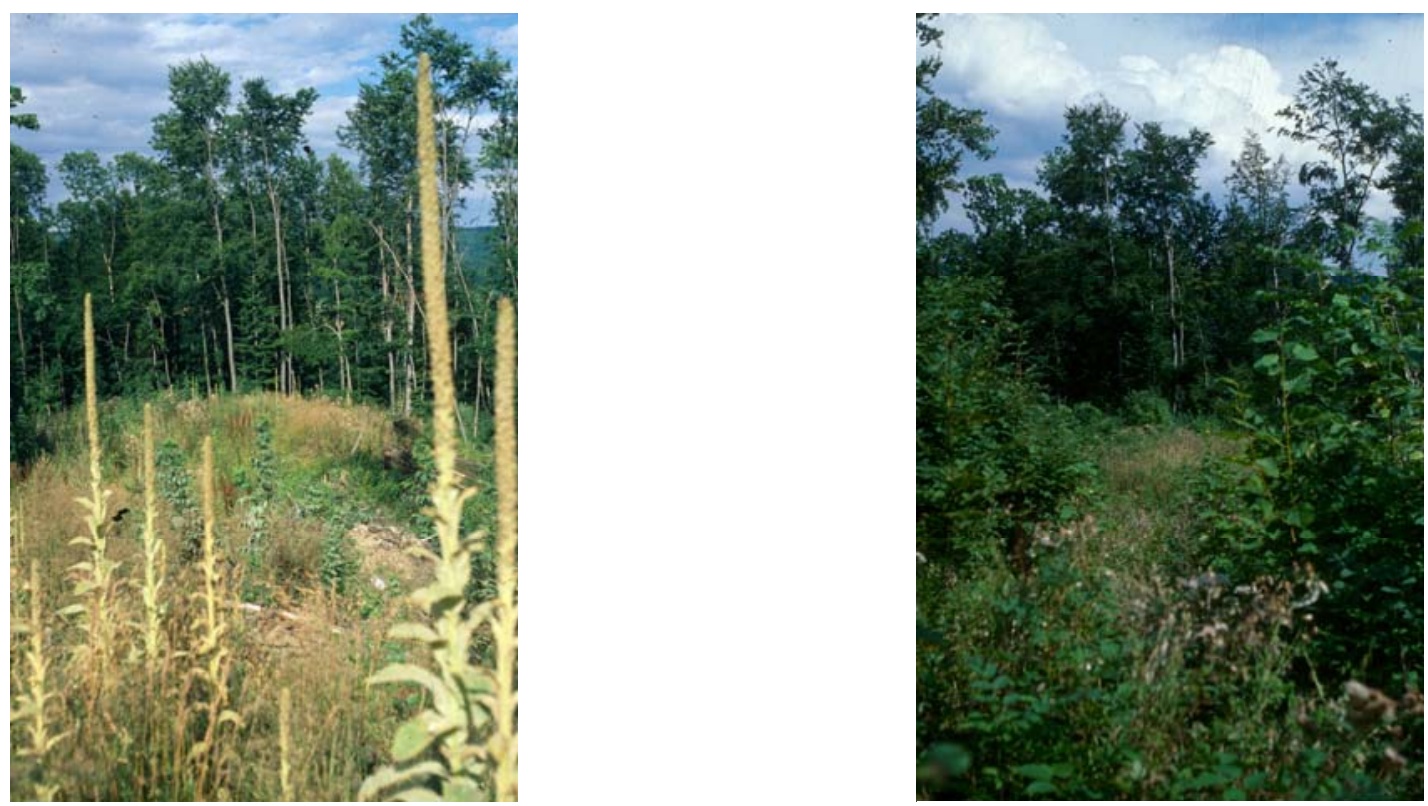

Abb. 5: Nüllberg; ehemaliger Rückeweg auf dem Grat der Dolomitrippe; links: August 1999; rechts: September 2002.

Im Bereich der Untersuchungsflächen überwiegen flach- bis mittelgründige BraunerdeRendzinen. Auf geringen Flächenanteilen am südöstlichen Oberhang steht der Dolomit als vegetationsfreier Fels an.

Bestockung: Der gesamte Zechstein des Nüllberges (höhergelegene Dolomitrippe, Nord- und Südosthang) wird überwiegend von einem Waldgersten-Buchenwald eingenommen (siehe Kap. 4.1). Die Baumschicht wird von Buche, ferner auch von Esche, Berg-Ahorn, SommerLinde und Hainbuche bestimmt. Auf den pleistozänen hercynischen Schottern am NWexponierten Mittel- und Unterhang dominieren Fichten-Forste. Die geworfenen BuchenBestände hatten ein Alter von etwa 90-100 Jahren (Herr BECKER, mündlich).

Windwurfsituation: Der Sturm von 1997 hat hauptsächlich den Bereich der höhergelegenen Dolomitrippe geworfen und die tieferliegenden Wälder ausgespart. Der vorherrschende Flächenwurf hebt sich sehr markant vom stehenden Bestand ab, jedoch existieren im Randbereich einige kleinere Lücken und auf den Kahlflächen einzeln oder truppweise stehengebliebene Überhälter.

Verwaltung und Nutzung: Die Untersuchungsflächen (Tab. 1) befinden sich alle im oberen Teil des Bergrückens und gehören zur Abt. 7 des Genossenschaftsforstes Hörden. Der Nüllberg gehört im Bereich der höhergelegenen Dolomitrippe und des gesamten Nord- und Südosthanges zum NSG „Siebertal“. Nach dem Sturm wurde das geworfene Holz maschinell geräumt und der Standort 1998 mit Buche, Esche, Berg-Ahorn und Vogel-Kirsche (nach eigener Beobachtung) aufgeforstet. Der Grat des Nüllberges ist dabei deutlich sichtbar als Rückeweg benutzt worden (Abb. 5). Der Wald wurde vor dem Sturm naturnah bewirtschaftet, was nach den Aufforstungen auch in Zukunft der Fall sein soll (Herr BECKER mündlich). Schwer nachvollziehbar ist in diesem Zusammenhang die im Sommer 2000 vom Hubschrauber aus erfolgte Kalkung der ohnehin schon basenreichen Standorte. 


\subsubsection{Krücker}

Der Krücker ist ein kompakt wirkender, teilweise zertalter Höhenzug mit ausgeprägter Plateaulage, etwa $7 \mathrm{~km}$ südlich der Stadt Osterode. Er erreicht etwa $340 \mathrm{mNN}$ und fällt in den Randbereichen auf 240-250 mNN ab. Sein nordöstlicher Teil, in dem auch alle Untersuchungsflächen (Tab. 1) liegen, schließt an das Hainholz an.
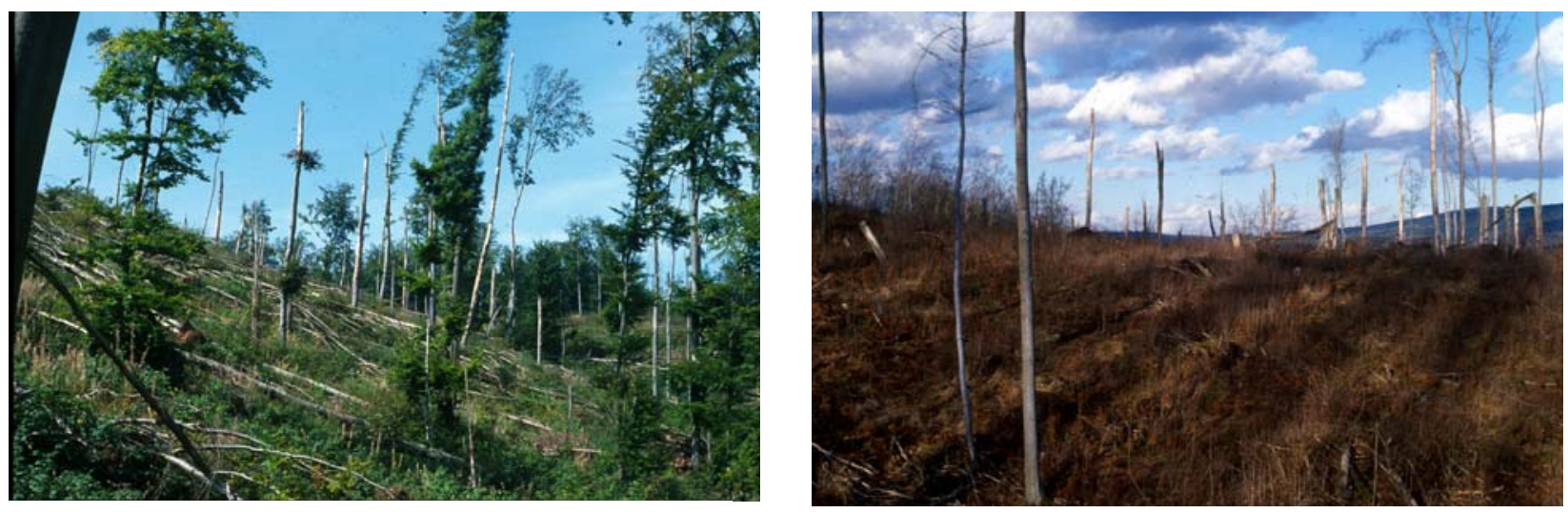

Abb. 6: Großflächiger Windwurf am Krücker; links: belassener Krücker-Osthang (August 2000); rechts: geräumtes Krücker-Plateau (Februar 2002).

Geologie und Böden: Der Krücker gehört zu einem mächtigen, vom Unteren Buntsandstein gebildeten Plateau, das sich zwischen den Ortschaften Wulften, Schwiegershausen, Hörden und Hattdorf erhebt (GeOlogische SPECialKarte 1884). Der Buntsandstein ist unterschiedlich stark mit Lößlehm überdeckt, es herrschen mittel- bis tiefgründige Braunerden vor.

Bestockung: Der Krücker wird in seinem südlichen Hauptareal von Nadelholzforsten dominiert. Besonders im Norden und Nordosten kommen Buchen- und Buchenmischwälder hinzu. Im Bereich der entsprechend Tab. 1 einbezogenen Untersuchungsflächen stocken mehrere Ausprägungen des Hainsimsen-Buchenwaldes (siehe Kap. 4.1). Die Buche ist hier häufig die allein dominierende Baumart, das Bestandesalter betrug vor dem Sturm etwa 100 Jahre (Herr HAASE, mündlich).

Windwurfsituation: Die Sturmwurffläche am nordöstlichen Krücker umfasst etwa 25-30 ha (eigene Schätzung nach Luftbildauswertung). Besonders beeindruckend ist die große Kahlfläche mit belassenem Flächenwurf am Osthang (Abb. 6). Im Randbereich dieser mächtigen Verhaue existiert ebenfalls in Osthanglage lückiger Windwurf im Übergang zum nicht geworfenen Bestand. Auf dem Plateau dagegen verläuft die Grenze zwischen Bestand und (geräumtem) Flächenwurf eher abrupt.

Verwaltung und Nutzung: Der Krücker umfasst mehrere Abteilungen des Niedersächsischen Forstamtes Reinhausen, der Genossenschaftsforste Schwiegershausen und Hörden sowie minimale Anteile des Realgemeindeforstes Hattorf. Die Untersuchungsflächen (Tab. 1) liegen in Abt. 17 des Genossenschaftsforstes Hörden („Krücker-Osthang“ sowie bei den Licht- und Verjüngungsaufnahmen das Stratum „Lücken“) und in Abt. 6 des Genossenschaftsforstes Schwiegershausen („Krücker-Plateau“ sowie bei den Licht- und Verjüngungsaufnahmen das 
Stratum „Bestand“). Der gesamte nordöstliche Teil des Krückers gehört - dem Namen nach nicht ganz korrekt - zum NSG „Gipskarstlandschaft Hainholz“. Vor dem Sturm wurden sowohl das Plateau als auch der Osthang des nordöstlichen Krückers konventionell bewirtschaftet. Der Windwurf am Osthang wurde nicht geräumt und soll in Zukunft der natürlichen Sukzession und Wiederbewaldung überlassen bleiben. Ähnlich wie im Hainholz wurden hier weite Flächen durch den Landkreis Osterode „auf 99 Jahre“ angepachtet. Die Plateaubereiche dagegen, die ebenfalls zum NSG gehören, wurden maschinell geräumt und anschließend mit Buche, Berg-Ahorn, Esche, Fichte und Vogel-Kirsche aufgeforstet. Desweiteren wurden Haselnuss, Schlehe und Gemeiner Schneeball im Zuge von Waldrandgestaltungsmaßnahmen ausgebracht (Herr HAASE, mündlich). Hier soll in Zukunft weiterhin naturnah gewirtschaftet werden.

\subsubsection{Rotenberg}

Der Rotenberg ist ein langgestreckter, bewaldeter Höhenzug zwischen den Auen der Flüsse Oder und Rhume und stellt gewissermaßen eine Schwelle zwischen Harzvorland und südniedersächsischem Eichsfeld dar. Seine höchste Erhebung ist der Rotenberg mit 317 mNN. In die Untersuchungen wurden zwei Teilgebiete im südlichen Rotenberg einbezogen. Es handelt sich dabei um den etwa 25 ha großen Naturwald Königsbuche (etwa 2,5 km östlich von Rhumspringe) und die bewirtschaftete Forstabteilung 1228 (etwa 1,5 km östlich von Rhumspringe).

Geologie und Böden: Bei allen Untersuchungsflächen bildet der Untere Buntsandstein den geologischen Untergrund, der unterschiedlich stark mit Lößlehm überdeckt ist. UNKRIG (1997a) verweist bei der Königsbuche auf die teilweise schlechte Durchwurzelbarkeit der Böden infolge von dichtlagerndem Ausgangsgestein und Pseudovergleyung der dortigen Parabraunerden. Damit ist eine Neigung dieser Buntsandstein-Böden zur Staunässebildung angezeigt. In der Abt. 1228 dagegen liegen mittel- bis tiefgründige Braunerden vor.

Bestockung: Der Rotenberg verfügt in seiner Gesamtheit über eine Vielzahl unterschiedlicher Waldtypen, die von naturnahen Buchen- und Buchen-Mischwäldern über Eichen-Bestände bis hin zu Fichten-Forsten reicht. Auf den Untersuchungsflächen stocken WaldmeisterBuchenwälder unterschiedlicher Ausprägung (s.a. Kap. 4.1) und Naturnähe. Der Buchendominierte Naturwald Königsbuche ist sehr strauchschicht- und verjüngungsarm, was nach UNKRIG (1997a) eine Folge des hohen Kronenschlussgrades und des intensiven Wildverbisses ist. Als Nebenbaumarten kommen Eiche, Birke und Fichte in geringem Umfang vor. Die dortigen Bestände sind etwa 120-150 Jahre alt und befanden sich vor dem Sturm auf dem größten Teil ihrer Fläche in der Optimal- oder Wachstumsphase. Im Gegensatz dazu verfügten die geworfenen Bestände der Abt. 1228 bereits vor dem Sturm infolge langjähriger forstlicher Eingriffe, entsprechender Kronenauflichtung und sicher auch wegen ihres hohen Eichen-Anteils über eine relativ stark ausgebildete Strauchschicht. 

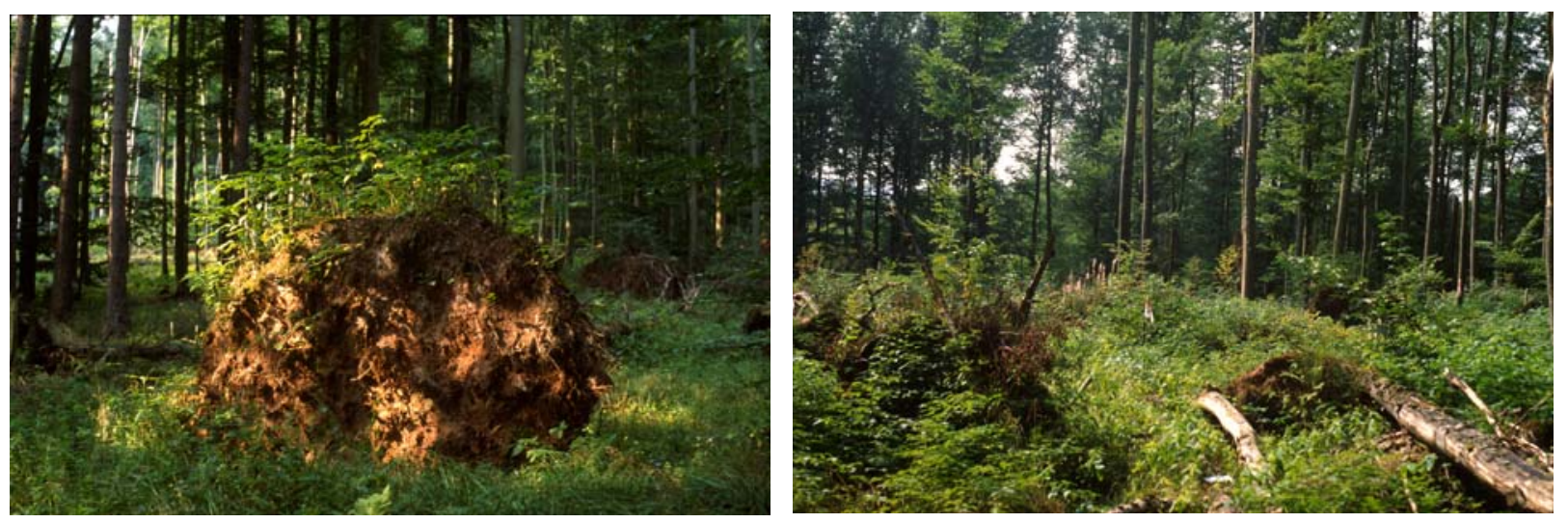

Abb. 7: Naturwald Königsbuche; links: Wurzelteller am Nordrand der KF2 (August 2001); rechts: belassener Flächenwurf im Zentrum der KF2 (August 2001).
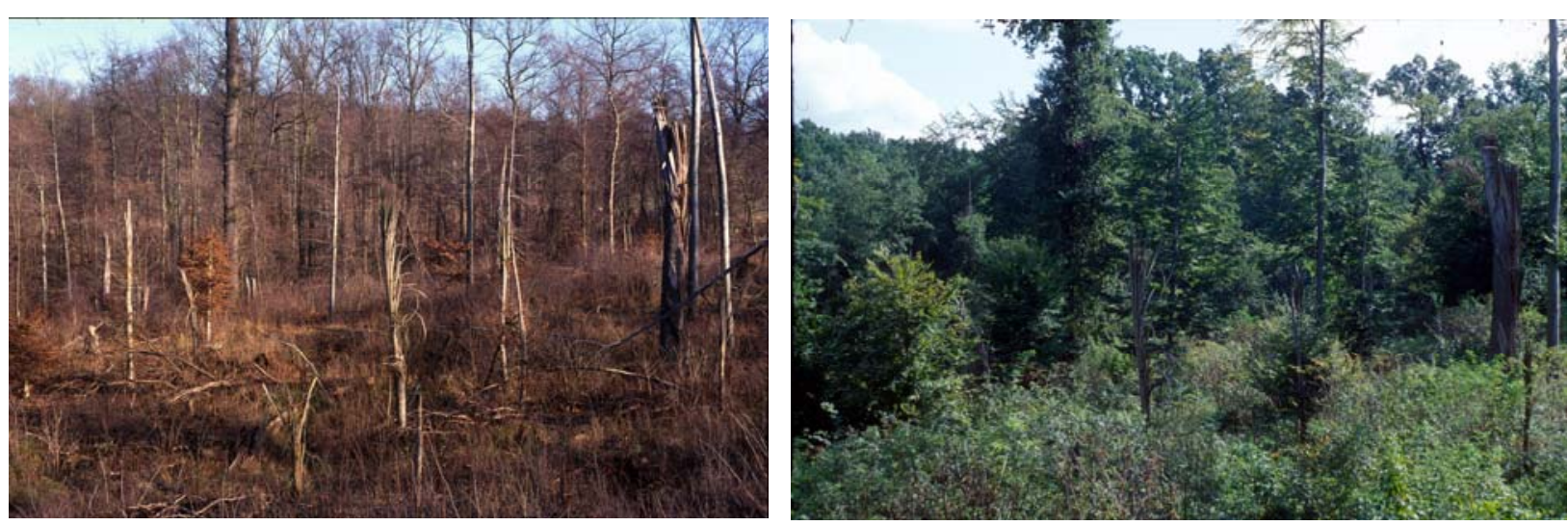

Abb. 8: Rotenberg-Abt. 1228; geräumter Flächenwurf im östlichen Drittel; links: Februar 2001; rechts: September 2002.

Im untersuchten östlichen Abschnitt der Abt. 1228 betrug das prozentuale Grundflächenverhältnis zwischen 156jähriger Stiel-Eiche und 116jähriger Buche vor dem Sturm annähernd 75:25, bei den Vorratsfestmetern etwa 60:40 (Angaben nach einer Inventur 1991, Herr HerzBerg mündlich). Diese Bestände weichen also bezüglich der Hauptbaumart von den übrigen UG ab. Sie wurden aber wegen der Artenzusammensetzung der Bodenvegetation trotzdem zum Galio odorati-Fagetum gestellt, zumal die Eichen-Dominanz auf diesen potentiellen Buchen-Waldstandorten forstlich bedingt ist. Weiterhin stockt im östlichen Abschnitt ein kleinerer Erlen-Bestand, in der nicht untersuchten westlichen Hälfte vorwiegend Trauben-Eiche und Fichte.

Windwurfsituation: Im Rotenberg lassen sich teilweise fünf parallel liegende, unterschiedlich breite Sturmschneisen ausmachen (HUBRIG 1999), wodurch dieses Gebiet als besonders stark vom Windwurf betroffen erscheint. Der Naturwald Königsbuche wurde jedoch nur randlich erfasst. Bei ihm stellt - neben einer Vielzahl von Windwurflücken im gesamten Reservat - das etwa 1 ha große Loch in Kernfläche 2 die großflächigste Störung dar (Abb. 7). An den Rändern dieses Loches setzt sich der Windwurf teilweise in Form von Lücken in den stehenden Bestand hinein fort, teilweise entstand aber auch ein neuer, abrupt wirkender 
Waldrand. Die Bestände in Abt. 1228 wurden besonders in ihrem östlichen Drittel teilweise lückig, teilweise flächig geworfen (Abb. 8). Etwa in der Mitte dieses östlichen Teilgebietes blieben ein kleiner Erlen- und ein Eichen-Buchen-Bestand weitestgehend unbeeinflusst. Im nicht untersuchten Westteil der Abt. 1228 dagegen trat kaum Windwurf auf.

Verwaltung und Nutzung: Der Rotenberg umfasst eine Vielzahl forstlich-administrativer Einheiten, wobei alle Untersuchungsflächen (Tab. 1) in den Abteilungen 1222 (also im südlichen Teil des NW Königsbuche) und 1228 des Niedersächsischen Forstamtes Reinhausen liegen. Die Königsbuche ist Naturwald in Verantwortung der Niedersächsischen Landesforstverwaltung und wurde 1972 (der nördliche Teil erst in den 1990er Jahren) aus der Nutzung genommen. Hier werden die Windwurfflächen entsprechend der Zweckbestimmung eines Naturwaldes sich selbst überlassen bleiben. Durch die seit 1998 existierende Zäunung der Kernfläche 2 soll ein zusätzlicher Schutz vor Wildverbiss gewährleistet werden. Die Abt. 1228 dagegen wurde konventionell bewirtschaftet, was auch in Zukunft der Fall sein wird. Nach dem Sturm wurde das geworfene Holz maschinell geräumt. Die Aufforstung erfolgte mit Buche, Berg-Ahorn, Trauben- und Stiel-Eiche, Vogel-Kirsche sowie Hainbuche (Herr HERZBERG, mündlich). Im Sommer 2001 musste ein erster Pflegeeingriff in Form von Freischneidearbeiten zugunsten der neu gepflanzten Bäume inmitten dicht schließender Pionierstrauchfluren erfolgen.

\subsection{Klima}

Das UG liegt im Klimakreis des Leineberglandes und befindet sich im Bereich verschiedener Klimagradienten. Die montanen Einflüsse des Harzes (höhere Niederschläge und Frostdauer, niedrigere Temperaturen) und die kontinentalen des südöstlichen Harzvorlandes (niedrigere Niederschläge, höhere Sommertemperaturen und Jahrestemperaturschwankungen) sind in unmittelbarer Nachbarschaft des südwestlichen Harzvorlandes wirksam (DEUTSCHER WETTERDIENST 1964 zitiert in ALAND 1994). Das UG selbst wird von subozeanischem Klima bestimmt. Die Temperaturen entsprechen der collinen Höhenstufe mit Höhen von 180-340 mNN. Bei den Niederschlägen wird dagegen der Einfluss des nahegelegenen Harzes deutlich, dessen Stauwirkung bei Hauptwindrichtungen aus West bis Südwest voll zum Tragen kommt (SCHÖNFELDER 1978).

Über die Jahresniederschlagssummen im UG gibt es verschiedene Angaben, die beim langjährigen Mittel übereinstimmend im Bereich 700-900 mm liegen. So befindet sich der Nüllberg nach der Klimakarte von HAASE (1954, zitiert in ScHÖNFELDER 1978) ziemlich genau auf der 900 mm-Isolinie des Jahresniederschlages, Hainholz und Krücker zwischen 800 und 900 mm und der Rotenberg im Bereich 700-800 mm. Für die Wetterstation Herzberg (240 mNN) wird das langjährige Mittel der Jahre 1913-1932 mit 783 mm angegeben, für die Jahre 1951-1980 dann aber mit 839 mm. Die weiter nordwestlich gelegene Wetterstation Osterode (285 mNN) hatte im langjährigen Mittel der Jahre 1913-1932 Niederschlagssummen 
von 913 mm zu verzeichnen, für die Jahre 1951-1980 aber nur noch 836 mm (jeweils frühere Jahresmittel: nach MEUSEL 1939 zitiert in SCHÖNFELDER 1978; jeweils spätere: nach GLÄSSER 1994 zitiert in LugINBÜHL 2000). Von BRANDT et al. (1976, zitiert in ALAND 1994) werden speziell für das Hainholz 820 mm angegeben. Innerhalb der Vegetationsperiode von Mai bis Juli fallen dort 220-240 mm Niederschlag, wobei der Juli mit 100-120 mm der regenreichste Monat ist. Die durchschnittliche Schneebedeckung beträgt etwa 50-60 Tage.

Das langjährige Mittel der Jahrestemperatur (1951-1980) wird für die Wetterstation Herzberg mit 7,6 $6^{\circ} \mathrm{C}$ angegeben (WALTER \& LIETH 1967 zitiert in LUGINBÜHL 2000). Übereinstimmend dazu liegt die mittlere Jahrestemperatur im nahen Hainholz nach BRANDT et al. (1976, zitiert in ALAND 1994) bei 7-8 ${ }^{\circ} \mathrm{C}$. Die mittlere Jahresschwankung von $17-17,5^{\circ} \mathrm{C}$ kennzeichnet einen Übergangsbereich zwischen atlantischem und kontinentalem Klima. Während der Vegetationsperiode werden im Mittel $14-15^{\circ} \mathrm{C}$ erreicht, der Juli ist mit $16-17^{\circ} \mathrm{C}$ der wärmste Monat. Die Frostperiode dauert etwa vom 5.-10. Oktober bis zum 20.-25. April, als Beginn der Apfelblüte wird der 10.-15. Mai genannt.

Kleinklimatische Besonderheiten, die insbesondere in Gipskarstgebieten wie dem Hainholz auftreten können, sind u.a. xerotherm ausgeprägte Südhänge (trotz hoher Niederschläge), Kaltluftsammelstellen in Dolinen und Erdfällen sowie Kaltluftabflussbahnen entlang stark eingeschnittener Kerbtälchen (ALAND 1994).

Für den weiter südlich gelegenen Rotenberg liegen solche detaillierten Angaben nicht vor. Die Klimabeschreibung für den Wuchsbezirk Südwestliches Harzvorland (ARBEITSKREIS STANDORTSKARTIERUNG 1985) nennt aber zumindest ähnliche Jahresdurchschnittstemperaturen wie im Hainholz, so dass diese auch für den Rotenberg angenommen werden können. 


\section{METHODIK}

\subsection{Geländemethoden}

\subsubsection{Stratifizierung der Untersuchungsflächen}

Eine erste Stratifizierungsebene der Untersuchungsflächen ist die in Tab. 1 vorgenommene Unterteilung in drei basenreiche Zechstein- und vier basenarme Buntsandstein-Standorte. Eine zweite Ebene unterteilt nach der Nutzung in vier belassene und drei geräumte/aufgeforstete Standorte. Auf einer dritten Ebene werden die Plots in Anlehnung an UNKRIG et al. (1999) folgendermaßen nach Störungsflächengröße und -struktur stratifiziert:

Bestand (randlich gestört): es sind keine aufgeklappten Wurzelteller, keine liegenden Stämme bzw. Kronenverhaue sowie keine auf den 1997er Windwurf zurückzuführende Kronenschäden vorzufinden; auf Grund der räumlichen Nähe zu den geworfenen Bestandesteilen muss aber von einer randlichen Beeinflussung ausgegangen werden.

Lücken: aufgeklappte Wurzelteller und/oder liegende Stämme bzw. Kronenverhaue, die aus dem 1997er Windwurf stammen, befinden sich in der Fläche; das Kronendach weist Lücken auf, die vom umliegenden Bestand langfristig wieder geschlossen werden können; Restüberschirmung bei mehr als 20\%.

Flächenwurf: wie Lücken, aber keine oder maximal 20\% Restüberschirmung; Kronenlücken werden auch langfristig vom umgebenden Bestand nicht geschlossen werden können (Freiflächensituation).

Die meisten Auswertungen beziehen sich auf den Flächenwurf der verschiedenen Standorte und Behandlungsvarianten. Bei den Untersuchungen zu Lichtverhältnissen, Naturverjüngung und Populationsbiologie der Himbeere wurden weitere Straten (Bestand, Lücke) einbezogen.

\subsubsection{Vegetationsaufnahmen}

Die Vegetationsaufnahmen erfolgten auf insgesamt 167 Untersuchungsflächen (100 m²-Plots) und 78 Wurzeltellern. Sie stellen somit den Großteil der Geländeerhebungen und eine wesentliche Basis der Datenauswertung dar.

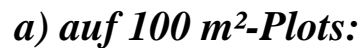

In Anlehnung an THOMAs et al. (1995) wurden Vegetationsaufnahmen mit abgestufter prozentgenauer Deckungsgradschätzung auf den in Tab. 1 aufgeführten $100 \mathrm{~m}^{2}$-Plots angefertigt, welche die Grundlage für die Vegetationsbeschreibung und die vegetationsökologische Auswertung bilden. Insgesamt wurden 167 Untersuchungsflächen mit einer Kantenlänge von jeweils 10 m einbezogen, für die eine unterschiedliche Anzahl von Untersuchungsjahren berücksichtigt wurde (Tab. 1). Da für die Jahre 1999 und 2001 aus allen UG Daten vorliegen, wird bei den meisten Auswertungsparametern auf diese beiden Jahre Bezug genommen. Die Flächen sind in den drei geräumten Varianten Nüllberg, Rotenberg- 
Abt. 1228 und Krücker-Plateau nach herkömmlichen Gesichtspunkten (d.h. unsystematisch verteilt) angeordnet, in den vier belassenen Varianten dagegen geklumpt in Form von lückenlos gerasterten Kernflächen (KF). Die Abb. 9 zeigt Form und Größe der vier Kernflächen sowie die tatsächlich einbezogenen Plots, da die Aufnahme von Streifen oder Kreuztransekten aus Praktikabilitätsgründen sinnvoller erschien als eine Komplettaufnahme.

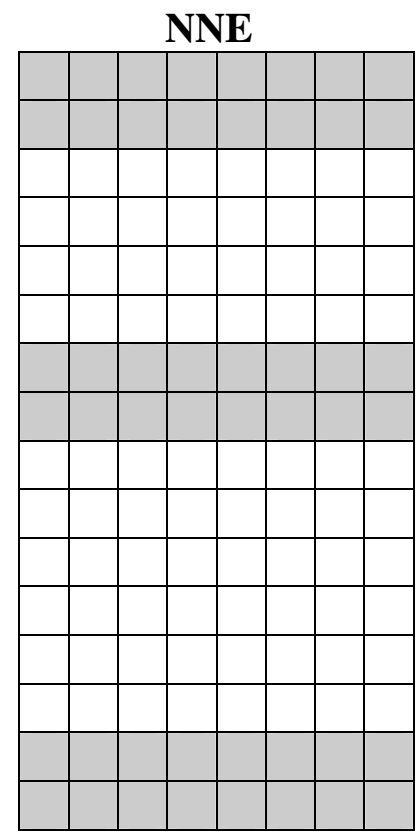

\section{Hainholz-KF1}

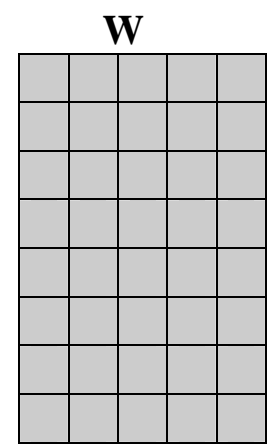

Krücker-Osthang

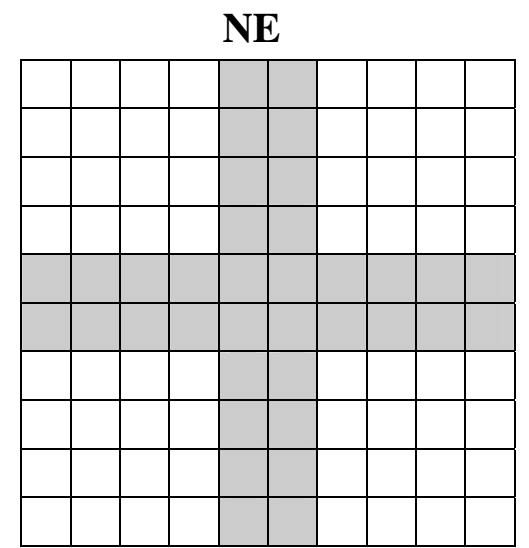

Hainholz-KF2

NW

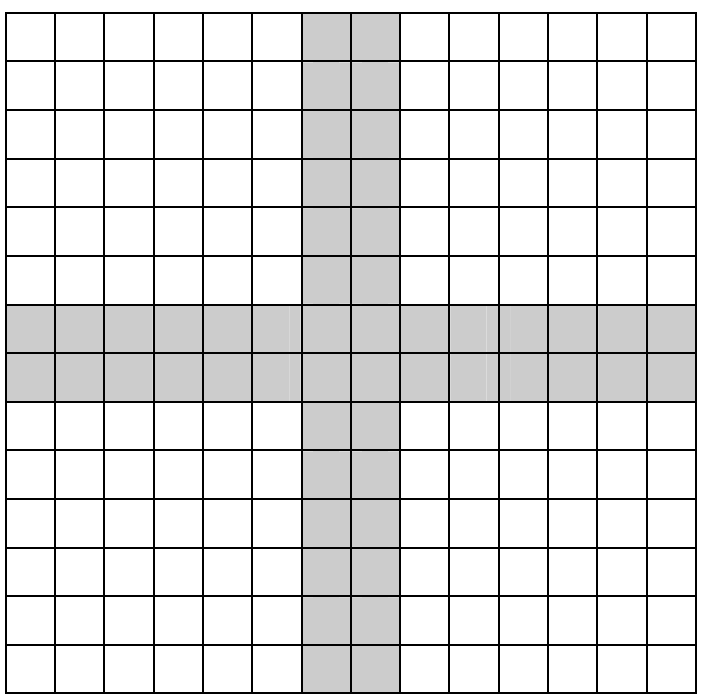

Königsbuche-KF2

Abb. 9: Lage der $100 \mathrm{~m}^{2}$-Plots mit Vegetationsaufnahmen innerhalb der vier belassenen Kernflächen von Hainholz, Königsbuche und Krücker.

Während die Kernflächen fest verpflockt sind und einer zukünftigen Sukzessionsforschung auf Dauerflächen zur Verfügung stehen, waren die geräumten und aufgeforsteten Plots nur im 
Untersuchungszeitraum markiert. Die basenarmen Standorte wurden einmal im Jahr (Juli/August) vegetationskundlich erfasst, die basenreichen unter Berücksichtigung des Frühjahrs- und des Sommeraspektes zweimal (April/Mai und Juli/August).

Folgende Schätzskala kam zur Anwendung:

r: nur 1 Exemplar, wenig deckend;

+: beliebige Anzahl Exemplare, $<1 \%$ deckend;

weiter $\mathbf{1}, \mathbf{2}, 3, \mathbf{4}, \mathbf{5}, \mathbf{8}, \mathbf{1 0}, \mathbf{1 2}, \mathbf{1 5}, \mathbf{2 0}, \mathbf{2 5 \%}$ deckend, anschließend in 5\%-Abschnitten bis zu $100 \%$.

Für die Mittelwertbildung und andere Berechnungen wurde die Klasse „r“ in 0,1\% und die Klasse „,“ in 0,5\% transformiert.

Die Vegetationsschichten wurden wie folgt eingeteilt:

Baumschicht 1 (BS1): Gehölze > 20 m Wuchshöhe;

Baumschicht 2 (BS2): Gehölze 5-20 m Wuchshöhe;

Strauchschicht (SS): Gehölze 0,5-5 m Wuchshöhe;

Feldschicht (FS): Krautige unabhängig von der Wuchshöhe sowie Gehölze bis 0,5 m;

Kryptogamenschicht (KryS): Moose, Flechten und Algen.

Zusätzlich erfolgte die Deckungsgradschätzung der lebenden Kronenteile geworfener Bäume, des Totholzes, der Laubstreu, des vegetationsfreien Bodens/Felses und der Wurzeltellerdeckung sowie die Bestimmung der Anzahl der Wurzelteller und liegenden Stämme und der Maximalhöhen von Strauch- und Feldschicht. Die Nomenklatur der Gefäßpflanzen folgt RoTHMALER (2002).

\section{b) auf Wurzeltellern:}

Bei der Deckungsgradschätzung auf Wurzeltellern kam dieselbe Methode zur Anwendung wie auf den $100 \mathrm{~m}^{2}$-Plots. Die aufgeklappten Wurzelteller lassen sich in die (hier meist) nordexponierte Oberseite sowie die (hier meist) südexponierte Unterseite und die Mulde unterteilen (Abb. 10). Diese drei Abschnitte wurden getrennt voneinander aufgenommen. Die Wurzeltelleruntersuchungen stellen einen Auszug aus der Datenmatrix der 100 m²-Plots dar. Die Arten der Wurzelteller gingen zunächst in die (Gesamt-)Vegetationsaufnahme der Untersuchungsfläche ein, bevor der Wurzelteller gesondert erfasst wurde. Insgesamt wurden 41 Untersuchungsflächen mit 78 Wurzeltellern in drei verschiedenen UG (Hainholz-KF1 und KF2, Königsbuche-KF2) in die vorliegenden Untersuchungen einbezogen und entsprechend Tab. 14 stratifiziert. Auch hier liegt der Schwerpunkt auf einem Vergleich der Jahre 1999 und 2001. 


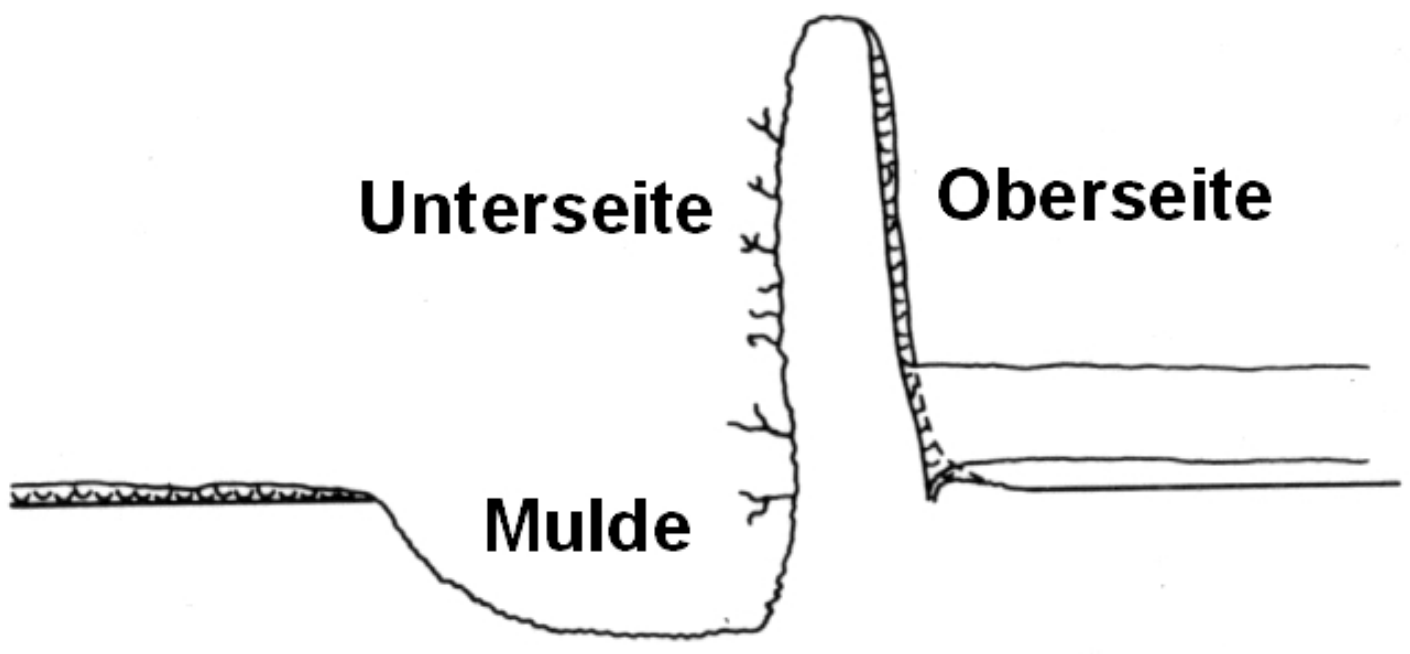

Abb. 10: Aufgeklappter Wurzelteller mit seinen drei Abschnitten Oberseite, Unterseite und Mulde (nach Skizzen von MuHLE 1975 in ELLENBERG et al. 1986).

\subsubsection{Aufnahme der Naturverjüngung}

Die Verjüngungsaufnahmen wurden zum Ende der vierten Vegetationsperiode nach dem Sturm im Herbst 2001 in den vier belassenen Untersuchungsvarianten (Hainholz-KF1 und KF2, Königsbuche-KF2, Krücker-Osthang) auf einem Teil der in Tab. 1 angegebenen Flächen durchgeführt (Abb. 12). Zusätzlich wurden im Krücker und in der Königsbuche die beiden Straten „Bestand“ und „,belassene Lücken“ einbezogen. In der Königsbuche sind diese beiden Zusatzvarianten innerhalb der KF2 teilweise in Plateau-, teilweise in Süd- und Osthanglage zu finden. Im Krücker befindet sich der (bewirtschaftete) Bestand auf dem Plateau, die (belassenen) Lücken dagegen in steiler Osthanglage.

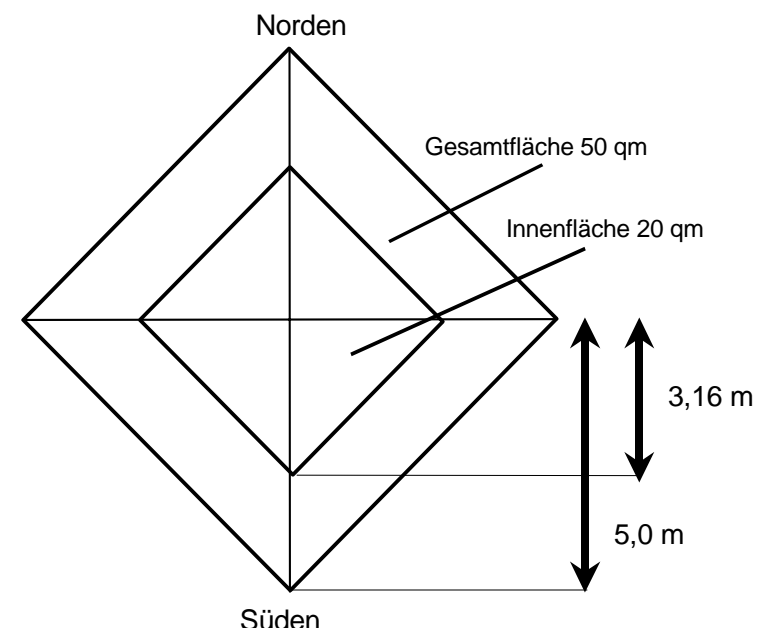

Abb. 11: Aufbau und Abmessungen der Probeflächen für die Verjüngungsaufnahmen auf Windwurfflächen im südwestlichen Harzvorland; nach MEYER (2000).

In den geräumten und aufgeforsteten Windwurfflächen erfolgte keine Verjüngungsaufnahme. Die genaue Anzahl einbezogener Plots ist den Abb. 43 bis $48 \mathrm{zu}$ entnehmen. Bei der Verjüngungsaufnahme wurde die Methode der Niedersächsischen Forstlichen Versuchsanstalt (MEYER 2000) angewendet, welche die Vollaufnahme und cm-genaue Höhenvermessung der 
gesamten Baumartenverjüngung (BHD $<7 \mathrm{~cm}$, ausser Keimlinge) auf einem $20 \mathrm{~m}^{2}$ großen Quadrat beinhaltet, dessen Ecken N-S- bzw. E-W-ausgerichtet sind und das sich genau in der Mitte des $100 \mathrm{~m}^{2}$-Plots befindet (Abb. 11). Die bei diesem Verfahren normalerweise übliche Auswertung, getrennt nach niedriger Verjüngung $(<150 \mathrm{~cm}$, vermessen im oben genannten 20 $\mathrm{m}^{2}$-Plot) und höherer Verjüngung ( $>150 \mathrm{~cm}$, vermessen in einer den $20 \mathrm{~m}^{2}$-Plot umschließenden $50 \mathrm{~m}^{2}$-Fläche) findet hier aus Gründen eines zweckmäßigen Vergleichs von vier Höhenklassen (<50, 51-100, 101-150 und >150 cm) keine Anwendung.

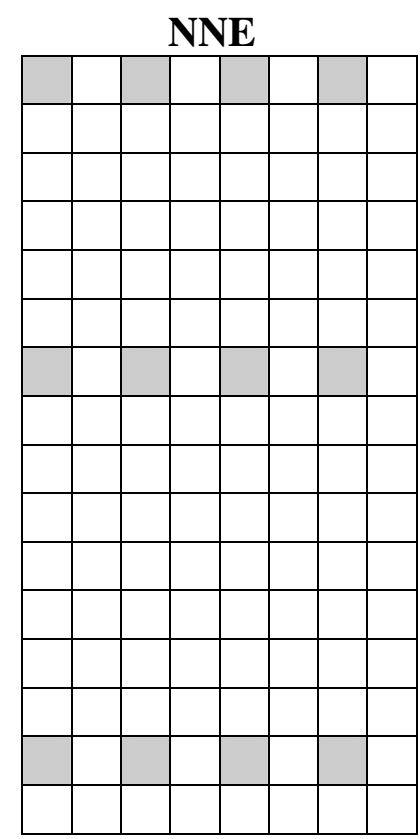

\section{Hainholz-KF1}

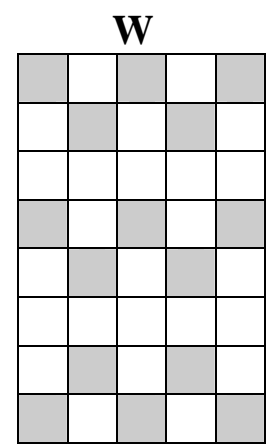

Krücker-Osthang
NE

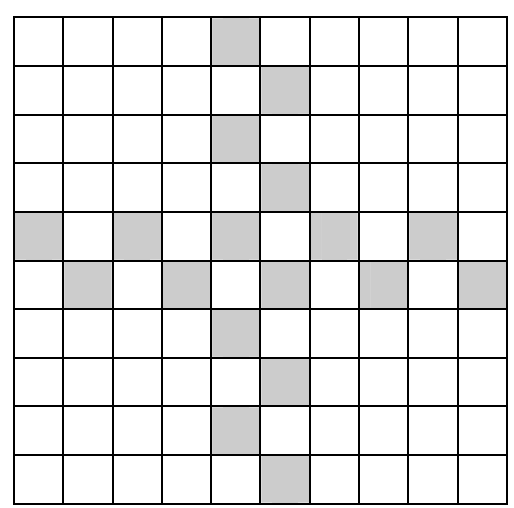

Hainholz-KF2

NW

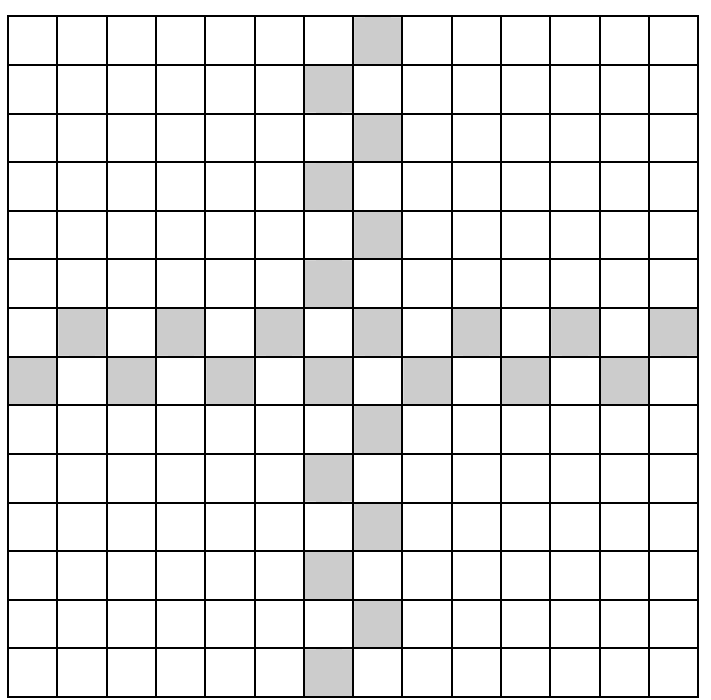

Königsbuche-KF2

Abb. 12: Lage der $100 \mathrm{~m}^{2}$-Plots mit Verjüngungsaufnahmen (Herbst 2001) in den vier belassenen Kernflächen von Hainholz, Königsbuche und Krücker; die zusätzlich aufgenommenen Flächen im Krücker (Bestand und Lücken) sind unsystematisch verteilt. 


\subsubsection{Populationsbiologische Untersuchungen zur Himbeere}

Zu Rubus idaeus als dem dominantesten Pionierstrauch der Untersuchungsgebiete wurden populationsbiologische Untersuchungen durchgeführt, von denen hier aber nur die Parameter „Anzahl Einzeltriebe bzw. Exemplare“ und „Prozentanteil fertiler Exemplare“ betrachtet werden (ein „Exemplar“ kennzeichnet jeweils eine Sprossgruppe gemeinsamen Wuchsursprungs). Diese Parameter ermöglichen einen ersten Überblick zu Fertilität und Wuchsverhalten der Himbeere bei unterschiedlicher Störungssituation. Die Pflanzen wurden auf gesondert eingerichteten 5 m²-Probeflächen in Lücken (6 Flächen) und im Flächenwurf (14 Flächen) ausgezählt und vermessen. Diese Probeflächen befanden sich überwiegend ausserhalb der in Tab. 1 aufgeführten $100 \mathrm{~m}^{2}$-Plots im westlichen Randbereich der HainholzKF1. Alle Angaben wurden auf $100 \mathrm{~m}^{2}$ extrapoliert.

\subsubsection{Lichtmessungen}

Die Lichtmessungen stammen aus dem Sommer 2001 und wurden zur Zeit der vollen Belaubung im August und im September bei homogener Wolkendecke durchgeführt. Dabei wurden alle in Tab. 1 aufgeführten $100 \mathrm{~m}^{2}$-Plots der drei belassenen UG Hainholz-KF1, Königsbuche-KF2 und Krücker-Osthang einbezogen, zusätzlich noch die Straten „Lücken“ und „Bestand“ im Krücker und in der Königsbuchen-KF2. Für die Hainholz-KF1 liegen zusätzliche Messungen aus dem Jahr 1998 vor, die von S. MichEL durchgeführt wurden. Die genaue Anzahl gemessener Plots ist aus den Abb. 15 und 16 sowie der Tab. 2 ersichtlich. Gemessen wurde die photosynthetisch aktive Strahlung (PAR in $\mu \mathrm{mol} / \mathrm{m}^{2 *}$ ) in einem starren 9-Punkte-Raster auf den beiden Diagonalen der $100 \mathrm{~m}^{2}$-Plots jeweils in Strauch- und in Feldschichthöhe (Abb. 13). Bei fehlender Strauchschicht wurde für die betreffende Fläche nur der Feldschichtwert verwendet.

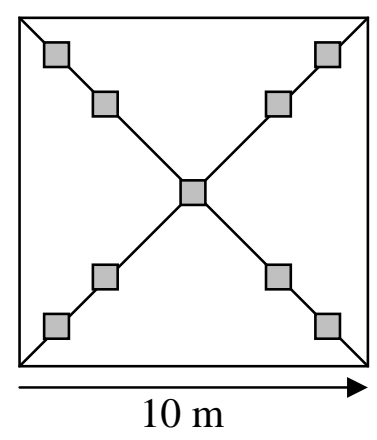

Abb. 13: Starres 9-Punkte-Raster der Messpunkte für die absolute Beleuchtungsstärke in

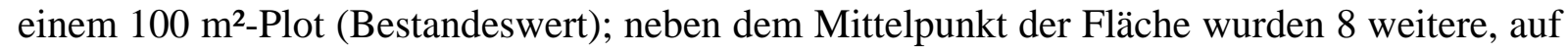
den Diagonalen liegende Punkte gewählt, die jeweils 2 bzw. $4 \mathrm{~m}$ vom Eckpunkt der Fläche entfernt lagen; der Freiflächen-Datalogger (Referenzwert) befand sich in maximal $1 \mathrm{~km}$ Entfernung. 
Die Werte wurden mit Hilfe von Lichtsensoren des Typs LICOR S190 und angeschlossenen Dataloggern des Typs LICOR LI-1400 durch Parallelmessungen jeweils auf der Windwurffläche (bzw. im Bestand) und auf einer nahegelegenen Freifläche mit voller Einstrahlung (Referenzwert) ermittelt. Der relative Lichtgenuss, der hier als Maß für die unterschiedlichen lichtökologischen Bedingungen herangezogen wird, ist das prozentuale Verhältnis aus absoluter Beleuchtungsstärke auf der Windwurffläche (bzw. im Bestand) und dem zeitlich entsprechenden Referenzwert der Freiflächenhelligkeit. Der absolute Bestandeswert wird aus dem arithmetischen Mittel der 9 Einzelmessungen gebildet, der Freiflächenwert dagegen aus jeweils 30 im Sekundentakt erfolgten Einzelmessungen. Vor Beginn der Messungen wurden beide Datalogger zeitlich synchronisiert, um später eine möglichst genaue Zuordnung der Bestandes- zu den Freiflächenwerten zu ermöglichen (Genauigkeit: max. 15 Sekunden Abweichung). Der zeitliche Aufwand für die 9 Einzelmessungen des Bestandeswertes schwankte sehr stark (ca. 1-5 min), da die $100 \mathrm{~m}^{2}$ Plots unter den speziellen Windwurfbedingungen (Stamm- und Kronenverhaue) unterschiedlich gut zugänglich waren. Lag ein Messpunkt in einem Kronenverhau, wurde der Wert auch dort in Feldschichthöhe aufgenommen, um das starre Raster einzuhalten.

\subsubsection{Bodenproben}

Die Bodenproben wurden im Herbst 1999 (Krücker) und im Herbst 2000 (übrige Gebiete) von einem Teil der in Tab. 1 aufgeführten $100 \mathrm{~m}^{2}$-Plots mit Hilfe eines Bohrstocks (Durchmesser $8 \mathrm{~cm}$ ) entnommen. Sie stellen Mischproben aus mehreren Einschlägen dar, welche getrennt nach den Tiefenstufen 0-10 und 10-20 cm eingesammelt wurden. Bei den Kernflächen im Hainholz und in der Königsbuche wurden jeweils 5 Proben um den gemeinsamen Eckpunkt von je 4 aneinandergrenzenden $100 \mathrm{~m}^{2}$-Plots entnommen und zu einer Mischprobe vereinigt. Bei den übrigen UG erfolgte die Entnahme von je 3 (Krücker) bzw. 5 Proben (Rotenberg, Nüllberg) von auf den Diagonalen der $100 \mathrm{~m}^{2}$-Plots angeordneten Einschlagpunkten (Abb. 14). Die genaue Anzahl verwendeter Mischproben ist Tab. 1 zu entnehmen.

Die Bodenproben wurden getrocknet und gesiebt und im Labor auf die drei Parameter $\mathrm{pH}$ Wert, C/N-Verhältnis und Carbonatgehalt untersucht, wiederum getrennt nach den Tiefenstufen 0-10 und 10-20 cm (keine Streuauflage). Der pH-Wert wurde elektrometrisch mittels einer Glaselektrode in $\mathrm{H}_{2} \mathrm{O}$ und in $\mathrm{KCl}$ gemessen (KöNIG \& ForTMANN 1996). Die Bestimmung des Carbonatgehaltes erfolgte gasvolumetrisch mit dem SCHEIBLER-Calcimeter (SCHLICHTiNG et al. 1995). Zur Emittlung des C/N-Verhältnisses wurden der $\mathrm{C}_{\text {ges- }}$ und der $\mathrm{N}_{\text {ges }}$-Gehalt gaschromatographisch mittels eines C/N-Analyzers vom Typ NA 1500 (Fa. Carlo Erba) bestimmt. 


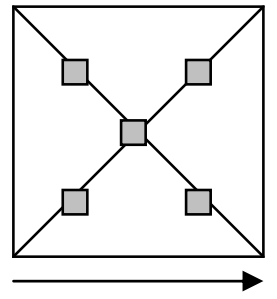

$10 \mathrm{~m}$

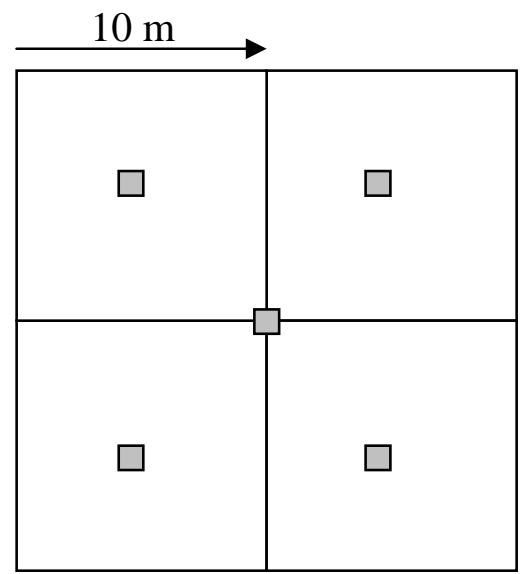

Abb. 14: Entnahmeschema der Bodenproben auf unsystematisch verteilten $100 \mathrm{~m}^{2}$-Plots im Nüllberg und Rotenberg bzw. im Krücker (links) sowie in den Kernflächen von Hainholz und Königsbuche (rechts).

\subsection{Auswertungsmethoden}

\subsection{1 $\alpha$-Diversität, Dominanz und Evenness}

Die $\boldsymbol{\alpha}$-Diversität kennzeichnet nach WHITTAKER (1973) den Artenreichtum z.B. eines Bestandes auf einer standardisierten Flächengröße. Als Maß der $\alpha$-Diversität wird in der vorliegenden Arbeit überwiegend die Artenzahl je $100 \mathrm{~m}^{2}$ verwendet, da dies die Einheitsgröße der vegetationskundlichen Aufnahmeflächen darstellt (Tab. 1). Ferner werden Artenzahl je UG (z.B. Tab. A22, A23) bzw. Artenzahl je Wurzelteller bzw. -abschnitt (Kap. 4.6) verwendet. Als grundlegendes Maß für die Dominanzverhältnisse wird der Deckungsgrad, bei Artengruppen und Vegetationsschichten auch die Deckungs(grad)summe angewendet. Die Deckungssummen ergeben sich aus der gruppenweisen Aufsummierung der im Feld geschätzten Einzeldeckungsrade der Arten. Bei den populationsbiologischen und den Verjüngungsaufnahmen findet weiterhin die Stückzahl je $100 \mathrm{~m}^{2}$ bzw. je Hektar Verwendung. Als Maß zum Vergleich der Dominanzstrukturen verschiedener Bestände, Pflanzengesellschaften oder Sukzessionsstadien eignet sich die Evenness („Gleichmäßigkeit“). Sie gibt in Prozent an, bis zu welchem Grad die maximal mögliche Gleichverteilung der Arten erreicht wird (HAEUPLER 1982). Die Evenness basiert auf dem SHANNON-Index, einem in der Vegetationsökologie weit verbreiteten Diversitätsmaß. Dieser ist aber artenzahlabhängig und eignet sich nur schlecht zum Vergleich unterschiedlich artenreicher Bestände. Deshalb wird bei der Berechnung der Evenness der SHANNON-Index auf seinen Maximalwert bezogen und somit normiert (HAEUPLER 1982).

Shannon-Index:

$\mathrm{H}^{\prime}=\Sigma \mathrm{p}_{\mathrm{i}} \ln \mathrm{p}_{\mathrm{i}}$ $\left(p_{i}=n_{i} / N ; H^{\prime}=\right.$ Diversitätsindex; $p_{i}=$ relative Häufigkeit einer Art; $n_{i}=$ Deckungsgrad einer Art i; N = Summe der Deckungsgradwerte aller Arten einer Vegetationsaufnahme) 
Evenness:

$\mathrm{E}=\mathrm{H}^{\prime} / \mathrm{H}_{\max } * 100$

$\left(\mathrm{H}_{\max }=\ln \mathrm{n} ; \mathrm{n}=\right.$ Artenzahl $)$

Häufig sind die hier beschriebenen Angaben als Boxplots dargestellt, um neben Mittelwert und Median auch die Streuung der Werte darzustellen (vier Perzentile sowie Ausreisser).

\subsubsection{Floristische Ähnlichkeit, Ähnlichkeitsindizes}

Für die Ermittlung der floristischen Ähnlichkeit zwischen Vegetationsbeständen oder Gesellschaften eignen sich Ähnlichkeitsindizes (Gemeinschaftskoeffizienten), die entweder auf qualitativen Daten (Präsenz) beruhen oder auch Mengenverhältnisse (z.B. Deckungsgrad) berücksichtigen. Damit können bei der Gegenüberstellung von Aufnahmekollektiven objektiv nachvollziehbare Ergebnisse erzielt werden, die allerdings auch schematischer erscheinen als der empirische Vergleich von Vegetationstabellen. In der vorliegenden Arbeit wurden der qualitative Jaccard-Index sowie der quantitative Index Percentage Similarity (synonym Czekanowski-Index) angewendet (PIELOU 1984). Die Indizes sind folgendermaßen definiert:

Jaccard-Index:

$\mathrm{CC}_{\text {Jaccard }}=\mathrm{a} /(\mathrm{a}+\mathrm{b}+\mathrm{c}) * 100$

( $\mathrm{a}=$ Anzahl der gemeinsamen Arten in Aufnahme A und B; $\mathrm{b}=$ Anzahl der Arten, die nur in Aufnahme A vorkommen; c = Anzahl der Arten, die nur in Aufnahme B vorkommen)

In Kap. 4.2.8 und 4.6.5 wird der Index nicht als Prozentwert dargestellt. Die höchstmögliche Ähnlichkeit ist dann also „1“.

Percentage Similarity:

PS $=200 * \sum_{i=1}^{n} \min \left(\mathrm{a}_{\mathrm{i}}, \mathrm{b}_{\mathrm{i}}\right) / \sum_{i=1}^{n}\left(\mathrm{a}_{\mathrm{i}}+\mathrm{b}_{\mathrm{i}}\right)$

( $n=$ Anzahl der in einem oder in beiden Aufnahmen vorkommenden Arten; $\Sigma \min \left(a_{i}, b_{i}\right)=$ Summe der jeweils kleineren Deckunsgrade derjenigen Arten, die in beiden Aufnahmen vorkommen; $\Sigma\left(a_{i}+b_{i}\right)=$ Summe der Einzeldeckungen in den verglichenen Aufnahmen)

\subsubsection{Qualitative (ungewichtete) und quantitative (gewichtete) Artengruppenspektren}

Bei allen errechneten Spektren (Kap. 4.3.) wurden Strauch- und Feldschicht zusammengefasst, da sich dies wegen des besonders engen Zusammenhanges der beiden Vegetationsschichten in der Windwurfsukzession anbietet. Bei quantitativer Betrachtung wurden die Abundanzanteile der entsprechenden Artengruppen am Gesamtspektrum (relativer Wert) als Grafik dargestellt. Ergänzend dazu erfolgt im Text und in den Tab. A22 und A23 die teilweise Angabe der Deckungssummen (absoluter Wert). Bei qualitativer Betrachtung werden die Anteile der entsprechenden Artengruppen an der Gesamtartenzahl (relativer Wert) tabellarisch dargestellt. Ergänzend dazu werden im Text teilweise die absoluten Artenzahlen genannt. 
Die Samenbanktypen sind nach Angaben von verschiedenen Autoren (GRIME et al. 1989; KLEYer 1995; THOMPSON et al. 1997; OBERDORFER 2001) zusammengestellt. Die Ausbreitungs- und Strategietypen richten sich nach FRANK et al. (1990). Die Lebensformspektren im Sinne RAUNKIAERS und die ökologischen Zeigerwertspektren basieren auf ELLENBERG et al. (1992). Die Zuordnung der Arten in den pflanzensoziologischen Spektren wurde teils nach ELLENBERG et al. (1992), teils nach OBERDORFER (2001) vorgenommen. Die Wuchsformen wurden zur Darstellung der Konkurrenzbeziehungen relativ grob gefasst und orientieren sich an der häufig in der forstlichen Literatur zu findenden Einteilung (Bäume, Sträucher, Farne und sonstige Krautige sowie Grasartige unter Einschluss der Poaceae, Cyperaceae und Juncaceae). Die Angaben zum vegetativen Ausbreitungsvermögen wurden nach mehrereren Autoren zusammengestellt (SCHMIDT 1983; GRIME et al. 1989; BARTELS 1993; KLEYER 1995). Zur Berechnung der Spektren diente das Programm SORT 4.0 (DURKA \& ACKERMANN 19911998).

\subsubsection{Hauptkomponentenanalyse (PCA)}

Die Hauptkomponentenanalyse (Principal Component Analysis - PCA) ist eine Methode der indirekten Ordination, bei der Aufnahmen und/oder Arten entsprechend ihrer floristischen Ähnlichkeitsstruktur graphisch angeordnet werden können. Geometrisch gesehen sind die Aufnahmen bzw. Arten als Punktwolken in einem mehrdimensionalen Raum vorstellbar, wobei die Positionierung dieser Punkte (z.B. Aufnahmen) durch ihre Attribute (z.B. die Bedeutungswerte der Pflanzenarten) bestimmt wird. Aus Gründen der Darstellbarkeit muss diese Mehrdimensionalität auf wenige Dimensionen reduziert und in Form von meist zwei, evtl. drei senkrecht aufeinander stehenden Achsen neu dargestellt werden (die übrigen Achsen werden nicht berücksichtigt). In dem von diesen „neuen“ Achsen aufgespannten Raum wird die Punktwolke dann neu angeordnet, wobei die Konfiguration der einzelnen Punkte weitestgehend unverändert bleibt (GLAVAC 1996). Die PCA wurde mit Hilfe des Programmes MVSP 3.1 (Kovach COMPUTing Systems 1986-1999) durchgeführt. Das Programm errechnet zunächst aus der Ausgangsmatrix (Vegetationstabelle) eine Ähnlichkeitsmatrix der Arten (Euklidische Distanz als verwendetes Ähnlichkeitsmaß), aus der anschließend Eigenwerte und Eigenvektoren sowie die Koordinatenwerte der einzelnen Punkte (Aufnahmen) für die Grafik abgeleitet werden. Verwendet wurden die Programmoptionen Zentrierung (der Arten) ohne weitere Transformierung (der Deckungsangaben), wobei sich letzteres aus der relativ geringen Deckungsspanne von 0,1 bis 100\% ergibt. Dadurch erfolgt eine Konzentrierung der Information auf die erste Ordinationsachse, welche den Hauptteil der Varianz erklärt. Die zweite Achse liefert den zweitgrößten Erklärungsbeitrag usw. (GLAVAc 1996). Als Darstellungsvariante wurde der Biplot gewählt, bei dem die Arten als vom Diagrammursprung ausgehende Pfeile (Vektoren) und die Aufnahmen als Punktwolke dargestellt sind. Die einzelnen Arten besitzen für die Achsen unterschiedlich große 
Bedeutung, je nach dem wie groß die Varianz ist, die sie im Datensatz verursachen. Geometrischer Ausdruck dieser Bedeutungsunterschiede sind der Winkel zwischen Artvektor und Achse sowie die Länge des Artvektors. Die Vektorpfeile weisen in Richtung der maximalen Varianz der Deckungsgrade bzw. -summen. Ihre Länge ist proportional zum Maximum der Variation, der Cosinus ihres Winkels gibt den korrelativen Zusammenhang der Größen an. Lange Pfeile kennzeichnen somit eine große quantitative Bedeutung der Art im Aufnahmekollektiv, kleine Winkel einen großen Erklärungsbeitrag der Art zur Achse. Dementsprechend sind die im Randbereich des Diagramms angezeigten Arten für die Interpretation am wichtigsten. Kurze Artvektoren im Diagrammzentrum sind dagegen relativ bedeutungslos und werden vom Programm nicht angezeigt. Den in Abb. 27, 28 und 53 verwendeten PCA-Grafiken liegen Vegetationstabellen mit mittleren Deckungsgraden zugrunde (Tab. A22, A23, A26, A28 und A30), da die Verwendung jeder einzelnen Aufnahme zur Erzeugung unübersichtlich großer Punktwolken geführt hätte. Der zurückgelegte Weg eines Standortsymbols entlang einer Achse kann als Ausdruck der Veränderungsintensität zwischen Ausgangs- (1999) und vorläufigem Endzustand (2001), bezogen auf die jeweils dominantesten Arten (Hauptkomponenten), gewertet werden. Vorsicht ist bei der ökologischen Interpretation der Grafiken geboten: Achsen, die mathematisch gesehen - hohe prozentuale Erklärungswerte erreichen, können ökologisch weitestgehend bedeutungslos sein (GLAVAC 1996).

\subsubsection{Vegetationstabellen}

Die hier beigefügten Vegetationstabellen sind jeweils nach basenreichen Zechstein- und basenarmen Buntsandstein-Gebieten unterteilt. Sie enthalten im Falle der Sukzessionstabellen (Tab. A22, A23) die mittleren Deckungsgrade bzw. im Falle der pflanzensoziologischen Tabellen (Tab. A24, A25) die Stetigkeitsklassen der einzelnen Arten aus n Aufnahmen.

Bei den Sukzessionstabellen, welche Daten aus jedem verfügbaren Untersuchungsjahr enthalten, erschien die Ausscheidung von Arten mit >1\% mittlerem Deckungsgrad sinnvoll, um eine gewisse quantitative Gewichtung zu erreichen. Zusätzlich sind diejenigen Arten grau unterlegt, bei denen sich der mittlere Deckungsgrad im Untersuchungszeitraum mindestens verdoppelt oder halbiert hat und die somit eine besonders hohe Sukzessionsdynamik aufweisen. Die Stetigkeitsklassen der pflanzensoziologischen Tabellen sind folgendermaßen definiert:

$\mathbf{r}=\max .5,0 \% ;+=5,1-10,0 \% ; \mathbf{I}=10,1-20,0 \% ; \mathbf{I I}=20,1-40,0 \% ; \mathbf{I I I}=40,1-60,0 \% ; \mathbf{I V}$ $=60,1-80 \% ; \mathbf{V}=80,1-100,0 \%$ Stetigkeit.

Die pflanzensoziologische Zuordnung erfolgte nach DiERSCHKE (1985, 1989), wobei das frühestmögliche Sukzessionsjahr (d.h. 1998 oder 1999) und das vierte Sukzessionsjahr (2001) gegenübergestellt wurden. Arten, die in den UG entgegen den Literaturangaben zusätzlich 
differenzierenden Charakter hatten, wurden in Klammern gesetzt und zu den entsprechenden Differentialarten der Syntaxa gestellt. Andere Arten, die der Literatur zufolge differenzierenden Charakter haben müssten, in den UG jedoch als Generalisten erscheinen, wurden ebenfalls mit Klammern gekennzeichnet und z.B. unter „sonstigen Arten“ bzw. bei den Verbands- oder Klassencharakterarten höherer Syntaxa (z.B. Fagion oder QuercoFagetea) einsortiert. Die Tabellenordnung fokussiert auf die Waldgesellschaften der Klasse Querco-Fagetea und lässt etwaige Sukzessionstadien mit Assoziationscharakter (z.B. aus der Klasse Epilobietea angustifolii) unberücksichtigt (siehe auch Kap. 5.2). Dadurch soll der Blick auf die Konstanz und Widerstandsfähigkeit der hier behandelten BuchenWaldgesellschaften in der frühen Windwurfsukzession gerichtet werden. Somit liegt eine Mischung aus induktiver und deduktiver Herangehensweise vor. Die pflanzensoziologischen Tabellen stellen insofern einen Kompromiss dar, als die Gruppierung der Aufnahmen entsprechend den in Tab. 1 angegebenen Untersuchungsvarianten erfolgte. Dadurch können bei Untersuchungsvarianten mit z.B. zwei vorhandenen Subassoziationen die Grenzen zwischen diesen künstlich unschärfer geworden sein.

\subsubsection{Anmerkungen zur Statistik}

Bei der Auswahl der Untersuchungsflächen, die dem Anliegen dieser Arbeit entsprechend ein möglichst breites Spektrum an Standorten und Behandlungsvarianten umfassen sollten, konnte kaum nach statistischen Gesichtspunkten vorgegangen werden. Die Subassoziationen der Waldgesellschaften wechseln - besonders im Gipskarst - kleinräumig. Die Windwurfflächen selbst variieren in Form und Ausdehnung. Plateaulagen wechseln mit in alle Himmelsrichtungen exponierten Ober-, Mittel- und Unterhängen. Windwurfbedingte Standortsmuster (Restüberschirmung, Wurzelteller, Verhaue) schwanken in ihrem Umfang innerhalb der bewusst grob gefassten Störungskategorien (Kap. 3.1.1). Zwei unterschiedliche Flächentypen (unsystematisch und geklumpt) wurden einbezogen. Nicht immer ließ sich ein geräumtes und ein belassenes Pendant finden (z.B. Hainholz-KF2). Die unterschiedliche Repräsentanz bestimmter Standorte und Störungssituationen spiegelt sich auch in der Untersuchungsflächenzahl wider, die zwischen 8 (Nüllberg) und 48 (Hainholz-KF1) liegt. Bei den vorliegenden Untersuchungen sollte man deshalb eher von Fallstudien sprechen. Vor diesem Hintergrund wurden hier relativ einfache Mittel der deskriptiven Statistik verwendet (Mittelwert, Median, Perzentile, Standardabweichung), die für die Beschreibung von Tendenzen und für Überblicksdarstellungen als ausreichend erachtet werden und die dem beschreibend-vergleichenden Charakter dieser Arbeit entsprechen. Ein komplexerer statistischer Ansatz liegt lediglich der PCA (Kap. 3.2.4) zugrunde, aber auch hier kommt die „Individualität“ und die teilweise geringe Anzahl der einzelnen Plots zum Tragen. Unter inhomogenen Bedingungen, wie sie für die Windwurfsukzession im Harzvorland typisch sind, bedarf jedes statistische Verfahren einer kritischen Hinterfragung hinsichtlich seiner Leistungsfähigkeit und seines Erklärungsbeitrages, um Fehlinterpretationen zu vermeiden. 


\section{ERGEBNISSE}

\subsection{Standörtliche Charakterisierung der Untersuchungsflächen}

\subsection{1 Übersicht}

In Tab. 1 erfolgt zunächst eine Kurzdarstellung aller in die Untersuchung einbezogenen Untersuchungsgebiete (UG) und ihrer Untersuchungsflächen (Ufl). Überblicksartig sind alle verfügbaren standörtlichen, bewirtschaftungsgeschichtlichen und naturschutzrechtlichen Angaben gegenübergestellt. Schwerpunktmäßig beschränken sich die Untersuchungsergebnisse auf die sieben Varianten mit Flächenwurf, jedoch wurden für einige Untersuchungen (Lichtverhältnisse, Naturverjüngung) die Vergleichsvarianten „Lücken“ und „Bestand“ hinzugezogen (Definition siehe Kap. 3.1). Die aufgeführten UG lassen sich grob den folgenden drei Standortseinheiten zuordnen:

- basenreich-flachgründiger Gips/Dolomit-Standort: Hainholz-KF2;

- basenreich-tiefgründige Dolomit-Standorte: Hainholz-KF1, Nüllberg;

- basenarme Buntsandtsandstein-Standorte: Königsbuche, Rotenberg-Abt. 1228, Krücker.

\subsubsection{Pflanzensoziologische Zuordnung (DIERSCHKE 1985, 1989)}

Auf der steilen Kuppe des Bollerkopfes (Hainholz-KF2) ist im Wesentlichen ein CariciFagetum zu finden, das aber in vielen Bereichen durch eine entsprechende gemeinsame Artengruppe zur Convallaria-Variante des Hordelymo-Fagetum lathyretosum vermittelt. Weiterhin ist in der Mehrzahl der Aufnahmen eine Frischezeigergruppe mit Circaea lutetiana, Stachys sylvatica, Urtica dioica und Impatiens noli-tangere vorhanden, wie sie für die Stachys-Variante dieser Gesellschaft bezeichnend wäre. Einige Aufnahmen am südöstlichen Hangfuß der Kuppe leiten zum frischeren Hordelymo-Fagetum circaeetosum über (Tab. 1, A24). Bereits SCHÖNFELDER (1978) weist darauf hin, dass im Hainholz Carici-Fagetum und Hordelymo-Fagetum lathyretosum schwer zu trennen sind.

Ausgehend von den Aufnahmen des Jahres 1998 lässt sich in der Hainholz-KF1 die AlliumVariante des Hordelymo-Fagetum typicum beschreiben, die im Unterhangbereich in die Allium-Variante eines Hordelymo-Fagetum circaeetosum übergeht (Tab. A24). Im Verlauf der Sukzession wird die Circaea-Differentialartengruppe steter (Hinweise auf Zunahme von Frischezeigern), so dass die Bestände der gesamten KF1 im Jahr 2001 dann überwiegend als Allium-Variante des Hordelymo-Fagetum circaeetosum anzusprechen sind (Tab. 1, A24).

Der Flächenwurf des Nüllberges wird überwiegend von einem Hordelymo-Fagetum circaeetosum eingenommen, teilweise in der zentralen Variante, teilweise (besonders auf dem Nordhang) in der Allium-Variante mit hochgradig deckenden Allium ursinum-Herden. In den nicht oder nur lückig geworfenen Randbereichen des Nüllberges sind Bestände eines Hordelymo-Fagetum lathyretosum und ebenso eines Hordelymo-Fagetum typicum zu finden, auch diese wiederum häufig in der Allium-Variante. Die Subassoziationen gehen stellenweise 
Tab. 1: Standörtliche Übersicht der Untersuchungsgebiete und -plots in großflächig geworfenen Buchen-Wäldern des südwestlichen Harzvorlandes; Angaben zu pH-Wert, Carbonatgehalt, C/N-Verhältnis und relativem Lichtgenuss mit Mittelwert und Standardabweichung.

\begin{tabular}{|c|c|c|c|c|c|c|c|}
\hline Standort & basenr.-flachgr. & basenreich & tiefgründig & & base & narm & \\
\hline Fläche & $\begin{array}{c}\text { Hainholz-KF2 } \\
\text { Bollerkopf }\end{array}$ & \begin{tabular}{|c|} 
Hainholz-KF1 \\
Jettenhöhlenbreite
\end{tabular} & Nüllberg & Königsbuche-KF2 & $\begin{array}{c}\text { Rotenberg } \\
\text { Abt. } 1228\end{array}$ & Krücker-Osthang & Krücker-Plateau \\
\hline $\begin{array}{l}\text { Pflanzen- } \\
\text { soziologische } \\
\text { Zuordnung }\end{array}$ & $\begin{array}{l}\text { Carici-Fagetum mit } \\
\text { Übergängen zum } \\
\text { Hordelymo-Fagetum } \\
\quad \text { lathyretosum }\end{array}$ & $\begin{array}{l}\text { Hordelymo-Fagetum } \\
\text { typicum, } H .-F . \\
\text { circaeetosum }\end{array}$ & $\begin{array}{c}\text { Hordelymo-Fagetum } \\
\text { circaeetosum mit } \\
\text { Übergängen zum H.-F. } \\
\text { lathyretosum }\end{array}$ & $\begin{array}{l}\text { Galio-Fagetum typicum, } \\
\text { G.-F. circaeetosum }\end{array}$ & $\begin{array}{c}\text { Galio-Fagetum typicum, } \\
\text { G.-F. circaeetosum, mit } \\
\text { starkem Eichen-Anteil } \\
\text { im Altbestand }\end{array}$ & $\begin{array}{c}\text { Luzulo-Fagetum } \\
\text { dryopteridetosum, L.-F. } \\
\text { galietosum, } L .-F . \\
\text { typicum }\end{array}$ & $\begin{array}{l}\text { Luzulo-Fagetum } \\
\text { dryopteridetosum, } L .-F \text {. } \\
\text { galietosum, } L .-F \text {. } \\
\text { typicum }\end{array}$ \\
\hline Geologie & Zechsteingips/-dolomit & Zechsteindolomit & Zechsteindolomit & Unterer Buntsandstein & Unterer Buntsandstein & Unterer Buntsandstein & Unterer Buntsandstein \\
\hline Bodentyp & $\begin{array}{l}\text { Gips-Rendzinen; } \\
\text { tw. lössüberdeckte } \\
\text { Rendzina-Braunerden }\end{array}$ & $\begin{array}{c}\text { tw. lössüberdeckte } \\
\text { Rendzina-Braunerden }\end{array}$ & $\begin{array}{c}\text { tw. lössüberdeckte } \\
\text { Rendzina-Braunerden }\end{array}$ & $\begin{array}{c}\text { lössüberdeckte } \\
\text { Parabraunerden, } \\
\text { tw. pseudovergleyt }\end{array}$ & $\begin{array}{l}\text { lössüberdeckte } \\
\text { Braunerden }\end{array}$ & $\begin{array}{l}\text { lössüberdeckte } \\
\text { Braunerden }\end{array}$ & $\begin{array}{l}\text { lössüberdeckte } \\
\text { Braunerden, } \\
\text { leicht podsolig }\end{array}$ \\
\hline $\begin{array}{l}\text { Exposition// } \\
\text { Geländeform }\end{array}$ & steile Kuppe & Südhang & Nord- und Südhang & Plateau & Nordhang & steiler Osthang & $\begin{array}{c}\text { Plateau } \\
\text { (tw. mit Taleinschnitt) }\end{array}$ \\
\hline Status & Naturwald \& NSG & Naturwald \& NSG & Wirtschaftswald \& NSG & Naturwald & Wirtschaftswald & unbewirtschaftetes NSG & Wirtschaftswald \& NSG \\
\hline Behandl. n. d. Sturm & belassen & belassen & geräumt \& aufgeforstet & belassen & geräumt \& aufgeforstet & belassen & geräumt \& aufgeforstet \\
\hline $\begin{array}{l}\text { aus der Nutzung } \\
\text { genommen }\end{array}$ & $\begin{array}{c}\text { seit } 1993 \\
\text { (mit geringen Eingriffen } \\
\text { bis zum Orkan) }\end{array}$ & $\begin{array}{c}\text { seit } 1993 \\
\text { (mit geringen Eingriffen } \\
\text { bis zum Orkan) }\end{array}$ & - & seit 1972 & - & $\begin{array}{c}\text { seit } 1997 \\
\text { (nach dem Orkan) }\end{array}$ & - \\
\hline Höhe & $250-280 \mathrm{mNN}$ & $240-260 \mathrm{mNN}$ & $260-288 \mathrm{mNN}$ & $230-240 \mathrm{mNN}$ & $220-230 \mathrm{mNN}$ & $280-320 \mathrm{mNN}$ & $330-340 \mathrm{mNN}$ \\
\hline $\begin{array}{l}\text { Jahresniederschlag } \\
\text { und -temperatur }\end{array}$ & $\begin{array}{l}\text { ca. } 820 \mathrm{~mm} \\
7-8^{\circ} \mathrm{C}\end{array}$ & $\begin{array}{l}\text { ca. } 820 \mathrm{~mm} \\
7-8^{\circ} \mathrm{C}\end{array}$ & $\begin{array}{c}839 \mathrm{~mm} \text { (Herzberg) } \\
7-8^{\circ} \mathrm{C}\end{array}$ & $\begin{array}{l}\text { ca. } 780 \mathrm{~mm} \\
8^{\circ} \mathrm{C}\end{array}$ & $\begin{array}{l}\text { ca. } 780 \mathrm{~mm} \\
8^{\circ} \mathrm{C}\end{array}$ & $\begin{array}{l}\text { ca. } 820 \mathrm{~mm} \\
7-8^{\circ} \mathrm{C}\end{array}$ & $\begin{array}{l}\text { ca. } 820 \mathrm{~mm} \\
7-8^{\circ} \mathrm{C}\end{array}$ \\
\hline Anzahl $100 \mathrm{~m}^{2}$-Plots & 36 & 48 & 8 & 15 & 9 & 40 & 11 \\
\hline Anordnung der Plots & $\begin{array}{l}\text { geklumpt, innerhalb } \\
\text { einer } 1 \text { ha großen } \\
\text { Kernfläche }\end{array}$ & $\begin{array}{c}\text { geklumpt, innerhalb } \\
\text { einer 1,26 ha großen } \\
\text { Kernfläche }\end{array}$ & unsystematisch verteilt & $\begin{array}{c}\text { geklumpt, innerhalb } \\
\text { einer 1,96 ha großen } \\
\text { Kernfläche }\end{array}$ & unsystematisch verteilt & $\begin{array}{l}\text { geklumpt, innerhalb } \\
\text { einer 0,4 ha großen } \\
\text { Kernfläche }\end{array}$ & unsystematisch verteilt \\
\hline $\begin{array}{l}\text { pH-Wert } \\
\text { in } \mathrm{H}_{2} \mathrm{O}\end{array}$ & $\begin{array}{c}\mathbf{7 , 3}( \pm 0,2) \\
\text { in } 0-10 \mathrm{~cm} \text { Tiefe }\end{array}$ & $\begin{array}{c}\mathbf{6 , 2}( \pm 0,6) \\
\text { in } 0-10 \mathrm{~cm} \text { Tiefe }\end{array}$ & $\begin{array}{c}\mathbf{6 , 8}( \pm 0,4) \\
\text { in } 0-10 \mathrm{~cm} \text { Tiefe }\end{array}$ & $\begin{array}{c}\mathbf{4 , 4}( \pm 0,1) \\
\text { in } 0-10 \mathrm{~cm} \text { Tiefe }\end{array}$ & $\begin{array}{c}\mathbf{4 , 6}( \pm 0,4) \\
\text { in } 0-10 \mathrm{~cm} \text { Tiefe }\end{array}$ & $\begin{array}{c}\mathbf{4 , 8}( \pm 0,4) \\
\text { in } 0-5 \mathrm{~cm} \text { Tiefe }\end{array}$ & $\begin{array}{c}\mathbf{4 , 6}( \pm 0,6) \\
\text { in } 0-5 \mathrm{~cm} \text { Tiefe }\end{array}$ \\
\hline $\begin{array}{l}\text { pH-Wert } \\
\text { in KCl }\end{array}$ & $\begin{array}{c}\mathbf{6 , 7}( \pm 0,3) \\
\text { in } 0-10 \mathrm{~cm} \text { Tiefe }\end{array}$ & $\begin{array}{c}\mathbf{5 , 6}( \pm 0,7) \\
\text { in } 0-10 \mathrm{~cm} \text { Tiefe }\end{array}$ & $\begin{array}{c}\mathbf{6 , 0}( \pm 1,0) \\
\text { in } 0-10 \mathrm{~cm} \text { Tiefe }\end{array}$ & $\begin{array}{c}3,3( \pm 0,1) \\
\text { in } 0-10 \mathrm{~cm} \text { Tiefe }\end{array}$ & $\begin{array}{c}3, \mathbf{8}( \pm 0,3) \\
\text { in } 0-10 \mathrm{~cm} \text { Tiefe }\end{array}$ & $\begin{array}{c}\mathbf{4 , 2}( \pm 0,2) \\
\text { in } 0-5 \mathrm{~cm} \text { Tiefe }\end{array}$ & $\begin{array}{c}3, \mathbf{6}( \pm 0,6) \\
\text { in } 0-5 \mathrm{~cm} \text { Tiefe }\end{array}$ \\
\hline $\begin{array}{l}\text { Carbonatgehalt } \\
\text { in } 0-10 \mathrm{~cm} \text { Tiefe }\end{array}$ & $42,4 \%( \pm 6,9)$ & $1,7 \%( \pm 1,9)$ & $17,6 \%( \pm 17,9)$ & k.A. & k.A. & k.A. & k.A. \\
\hline $\begin{array}{l}\text { C/N-Verhältnis } \\
\text { in } 0-10 \mathrm{~cm} \text { Tiefe }\end{array}$ & $22,7( \pm 2,7)$ & $\mathbf{1 5 , 9}( \pm 1,2)$ & $\mathbf{1 7 , 6}( \pm 2,0)$ & $21,7( \pm 1,2)$ & $\mathbf{1 8 , 8}( \pm 1,4)$ & k.A. & k.A. \\
\hline $\begin{array}{l}\text { relativer Lichtgenuss } \\
2001\end{array}$ & k.A. & $\begin{array}{c}\text { über Feldschicht: } \\
\text { 12,0 \% }( \pm 9,5) \\
\text { über Strauchschicht: } \\
\mathbf{6 3 , 8} \%( \pm 11,9) \\
\end{array}$ & k.A. & $\begin{array}{c}\text { über Feldschicht: } \\
\mathbf{2 4 , 0} \%( \pm 11,3) \\
\text { über Strauchschicht: } \\
\mathbf{9 1 , 0} \%( \pm 7,4) \\
\end{array}$ & k.A: & $\begin{array}{c}\text { über Feldschicht: } \\
\text { 15,1 \% }( \pm 7,0) \\
\text { über Strauchschicht: } \\
\mathbf{9 4 , 7} \%( \pm 6,4) \\
\end{array}$ & $\mathrm{k}: \mathrm{A}$. \\
\hline Bemerkung & - & - & Kalkung 2000 & Zäunung seit 1998 & Freischneidearbeit. 2001 & Kalkung 1991 & Kalkung 1991 \\
\hline Aufnahmejahre & $4(1998-2001)$ & $4(1998-2001)$ & $3(1999-2001)$ & $4(1998-2001)$ & $3(1999-2001)$ & $2(1999+2001)$ & $2(1999+2001)$ \\
\hline
\end{tabular}


ineinander über. Unklar ist, ob die Subassoziation lathyretosum vor dem Sturm eventuell weiter verbreitet war als im weiteren Verlauf der Sukzession und die Lathyrus-Artengruppe zugunsten der Frischezeiger der Subassoziation circaeetosum verdrängt wurde. Eine vorübergehende Besonderheit des Nüllberg-Windwurfes war die Ausbildung auffälliger Hochstaudenfluren von Euphorbia amygdaloides, die im zweiten und dritten Jahr nach dem Sturm noch hohe mittlere Deckungsgrade von 20 bzw. 16\% erreichten, im Jahr 2001 aber bereits zusammengebrochen waren (nur noch 3\%) und von der starken Gehölzentwicklung verdrängt wurden. Normalerweise wäre Euphorbia amygdaloides eher für die Subassoziation lathyretosum typisch, aber auf dem Nüllberg hat die Art keinen differenzierenden Charakter zwischen den eher trockeneren und den eher frischeren Ausbildungen des HordelymoFagetum (Tab. 1, A24).

Die Bestände im Buntsandstein lassen sich grob dem Galio odorati-Fagetum (Rotenberg) und dem Luzulo-Fagetum (Krücker) zuordnen (Tab. 1, A25). Auch hier sind Beispiele mehrerer Subassoziationen vertreten, die vielfältige Übergänge aufweisen. Vermutlich durch eine in der Vergangenheit durchgeführte Kalkung sind die Bestände im Krücker tendenziell weniger sauer (Tab. 1) und auch artenreicher (Abb. 17) als die im Rotenberg. Dennoch weist das Arteninventar der beiden Untersuchungsgebiete auf die genannte Einteilung in Waldmeister- und Hainsimsen-Buchenwald hin. Bei den Beständen im Rotenberg (beide Untersuchungsflächen) handelt es sich um ein Galio-Fagetum typicum bzw. circaeetosum, im Krücker um Bestände des Luzulo-Fagetum typicum bzw. galietosum und dryopteridetosum.

\subsubsection{Bodenchemie}

Die mittleren pH-Werte liegen für die beiden Dolomit-Standorte (Hainholz KF1, Nüllberg) mit 6,2-6,8 $\left(\mathrm{H}_{2} \mathrm{O}\right)$ bzw. 5,6-6,0 ( $\left.\mathrm{KCl}\right)$ im Silikat- bis Carbonat-Pufferbereich. Die HainholzKF2 (flachgründige Gips/Dolomit-Kuppe) hebt sich mit eindeutig im Carbonat-Pufferbereich liegenden mittleren pH-Werten von 7,3 $\left(\mathrm{H}_{2} \mathrm{O}\right)$ bzw. 6,7 $(\mathrm{KCl})$ noch einmal von den anderen Zechstein-Standorten ab. Die Buntsandstein-Varianten sind mit pH-Werten von 4,4-4,8 (in $\mathrm{H}_{2} \mathrm{O}$ ) bzw. 3,3-4,2 (KCl) im Austauscher- bis Eisen/Aluminium-Pufferbereich einzuordnen, wobei sich der Krücker gegenüber dem Rotenberg mit etwas höheren $\mathrm{pH}$-Werten als etwas besser basenversorgt abhebt (Tab. 1). Dies könnte ein Hinweis auf die 1991 im Krücker durchgeführte Kalkung sein. Trotz dieser günstigeren Bodenreaktion wurden die Bestände des Krückers als Luzulo-Fagetum eingestuft, die des Rotenberges dagegen als Galio odoratiFagetum (Kap. 4.1.2; Tab. A25).

Die Carbonatgehalte wurden nur für die Zechstein-Gebiete bestimmt (Tab. 1). Die Mittelwertangaben lassen eine deutliche Dreiteilung erkennen, die sich entsprechend der Forstlichen Standortaufnahme (AREBEITSKREIS STANDORTSKARTIERUNG 1996) von carbonatarm (Hainholz-KF1: Hordelymo-Fagetum typicum/circaeetosum) über carbonatreich (Nüllberg: Hordelymo-Fagetum circaeetosum/lathyretosum) bis hin $\mathrm{zu}$ sehr carbonatreich 
(Hainholz-KF2: Carici-Fagetum) erstreckt. Eine starke Unsicherheit in der Interpretation der Carbonatgehalte liegt in den hohen Standardabweichungen der beiden Dolomit-Standorte (Hainholz-KF1, Nüllberg). Die Werte auf den Nüllberg-Flächen dürften zudem künstlich erhöht sein, da hier im Sommer 2000 eine Kalkung durchgeführt wurde.

Die C/N-Verhältnisse (Tab. 1) der beiden Dolomit-Standorte (Hainholz KF1, Nüllberg) sind mit 16 - 18:1 als mäßig eng bis mittel einzustufen (ARBEITSKREIS STANDORTSKARTIERUNG 1996) und weisen auf eine gute Humusqualität hin (Übergangsbereich Mull und mullartiger Moder). Dagegen ist das C/N-Verhältnis in der Hainholz-KF2 mit 23:1 im Bereich mäßig weit einzuordnen. Es liegen also etwas ungünstigere Mineralisationsbedingungen vor und die Humusqualität (feinhumusarmer Moder) ist geringer als in den übrigen Zechstein-Gebieten. Im Rotenberg auf Buntsandstein liegen die C/N-Verhältnisse zwischen 19 und 22:1 (mittel bis mäßig weit) und weisen auf eine mittlere bis geringere Humusqualität hin. Sie kennzeichnen aber noch die Gruppe der biologisch günstigen Moder-Formen (AD-HOC ARBEITSGRUPPE Boden 1994; ArbeitSKReis StANDORTSKARTIERUnG 1996). Das lässt auf gute, aber nicht optimale Mineralisationsbedingungen schließen, wobei die belassene Königsbuchen-KF2 (Naturwald, pseudovergleyte Parabraunerden) etwas schlechtere Bedingungen aufweist als die geräumte Rotenberg-Abt. 1228 (Wirtschaftswald, Braunerden).

\subsubsection{Lichtverhältnisse}

Zur Beschreibung der Lichtverhältnisse wurden in den einbezogenen UG jeweils unterschiedliche Betrachtungsebenen gewählt (Hainholz-KF1: nur Flächenwurf im Vergleich der Jahre 1998 und 2001; Königsbuche/Krücker: Störungsgradient nur in 2001).

In der Hainholz-KF1 haben sich im besagten Zeitraum die Strahlungswerte über der jeweils höchsten Schicht (meist Strauch-, gelegentlich Feldschicht) auch noch nicht wesentlich verändert (Abb. 15 oben). Im Jahr 1998 waren im Mittel 68\% relativer Lichtgenuss vorhanden, im Jahr 2001 immer noch rund 64\%. Unklar bleiben die im Vergleich zu den Buntsandstein-Gebieten relativ geringen Werte (von 2001), die offenbar nicht oder nicht nur in der verbliebenen Restüberschirmung begründet scheinen (Hainholz-KF1: 8\%, Königsbuche-KF2: 6\%, Krücker-Osthang: 2\%). Über der Feldschicht dagegen (Abb. 15 unten) kam es zu einer beträchtlichen Ausdunkelung infolge der rasant aufwachsenden Naturverjüngung, besonders der jungen Eschen (Abb. 43-45). Der relative Lichtgenuss sank dort im Mittel von 54 auf 12\% und lag somit im Jahr 2001 ähnlich niedrig wie in den gestörten Flächen des Krückers oder wie im Bestand der Königsbuche (Tab. 2).

Im Naturwald Königsbuche zeigen die in Abb. 16 sowie in Tab. 2 angeführten Lichtmessungen des Jahres 2001 einen deutlich zunehmenden mittleren relativen Lichtgenuss über der Strauchschicht in der Reihenfolge Bestand (17\%) - Lücken (41\%) - Flächenwurf (91\%), im Krücker einen ähnlichen Gradienten mit 9\% - 44\% - 95\%. Dies bestätigt die auf Schätzwerten der Baumschicht-Deckungsgrade beruhende Grobstratifizierung. 
August/September 1998

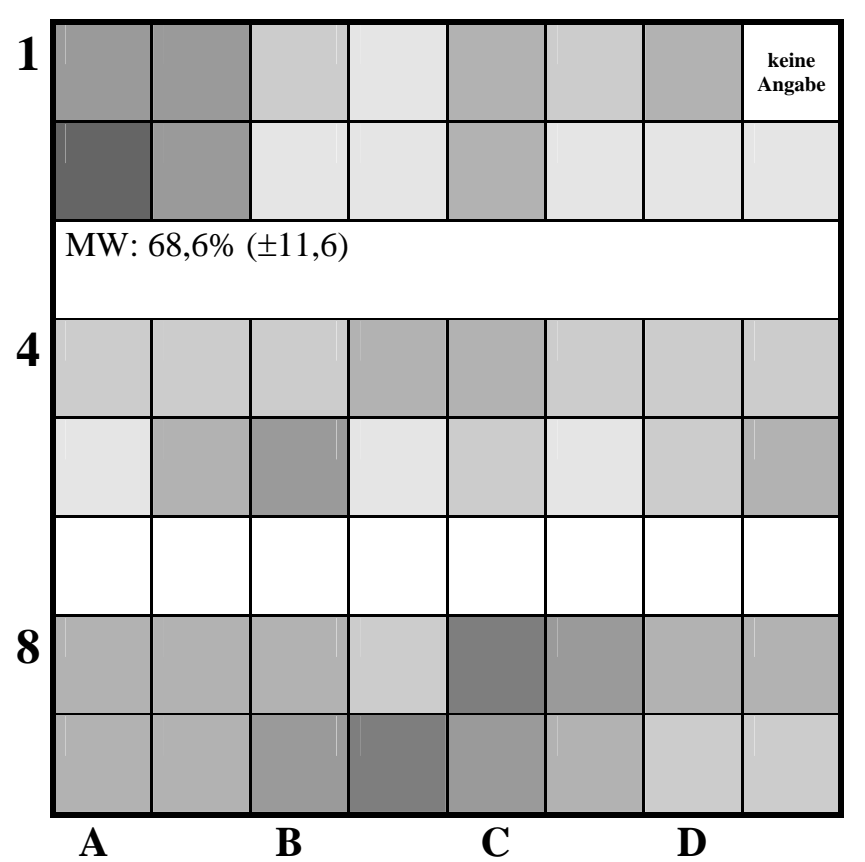

über der Strauchschicht

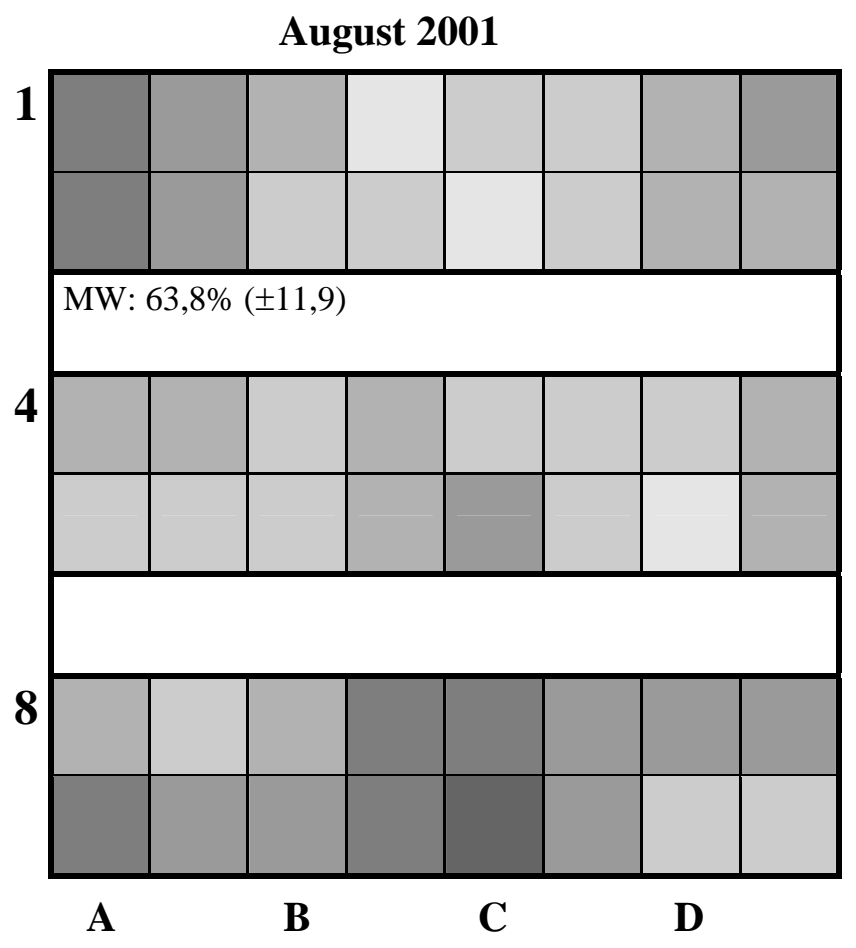

über der Feldschicht
August/September 1998

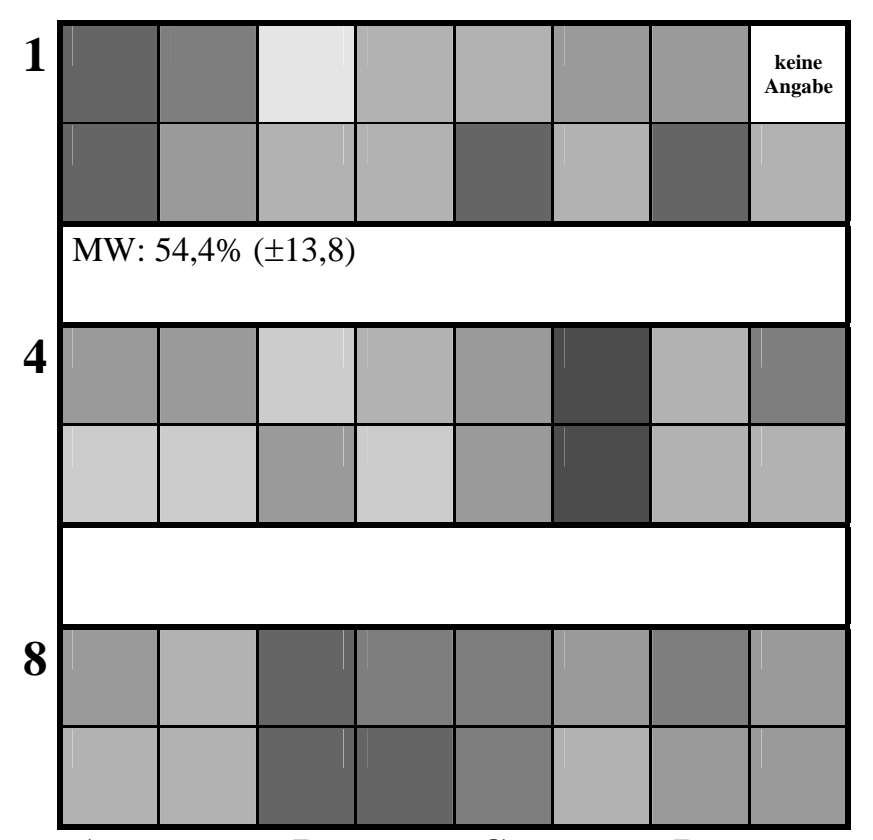

A

B

C

D

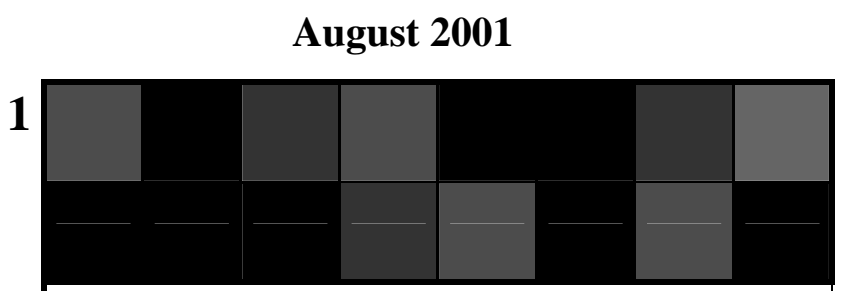

MW: $12,0 \%( \pm 9,5)$

4

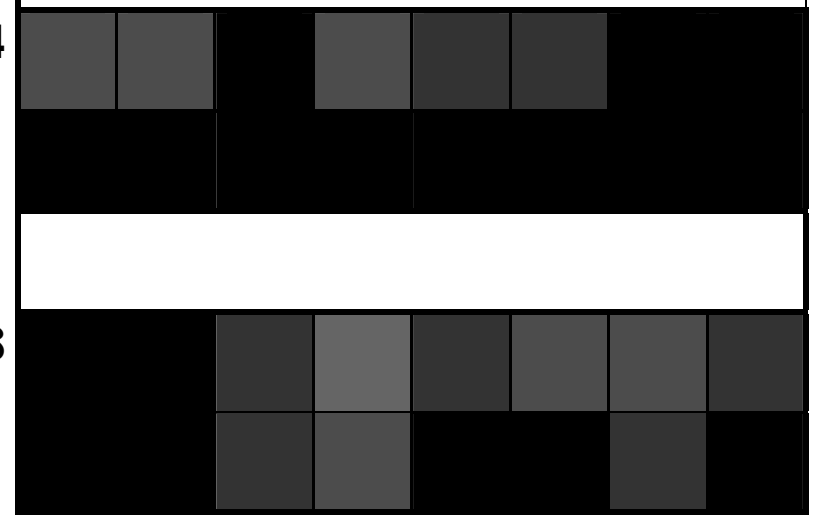

A

B

C

D

bis zu $\quad 10 \quad 20 \quad 30 \quad 40 \quad 50 \quad 60$

$\begin{array}{llll}70 & 80 & 90 & 100 \% \text { rel. Lichtgenuss }\end{array}$

Abb. 15: Relativer Lichtgenuss (PAR) in \% der Freilandhelligkeit über der Strauch- (oben) und der Feldschicht (unten) in den Sommern 1998 und 2001 in der großflächig geworfenen Hainholz-KF1 (Zechstein; Hordelymo-Fagetum); aufgenommen wurden 48 in drei hangparallelen Transekten angeordnete 100 m²-Plots (Kantenlänge $10 \mathrm{~m}$ ). 
Im vierten Jahr nach dem Sturm sorgt eine stark etablierte Strauchschicht in beiden UG dafür, dass in den Lücken, besonders aber im Flächenwurf in der darunterliegenden Feldschicht bereits wieder ähnlich niedrige Strahlungswerte vorherrschen wie im Bestand (Tab. 2).

Tab. 2: Mittlerer relativer Lichtgenuss (PAR) in \% der Freilandhelligkeit bei unterschiedlicher Störungssituation in zwei Buchen-Windwurfgebieten auf Buntsandstein im Sommer 2001 (mit Angabe der Standardabweichung und der einbezogenen $100 \mathrm{~m}^{2}$-Plots).

\begin{tabular}{|l|c|c|c|c|}
\hline & \multicolumn{2}{|c|}{ Königsbuche-KF2 } & \multicolumn{2}{c|}{ Krücker (Osthang und Plateau) } \\
\hline Messung über: & Feldschicht & Strauchschicht & Feldschicht & Strauchschicht \\
\hline Bestand & $\mathbf{1 5 , 6}( \pm 14,1) n=14$ & $\mathbf{1 7 , 1}( \pm 15,6) n=14$ & $\mathbf{7 , 4}( \pm 1,4) n=5$ & $\mathbf{9 , 1}( \pm 2,3) n=5$ \\
\hline Lücken & $\mathbf{2 9 , 3}( \pm 14,6) n=23$ & $\mathbf{4 1 , 2}( \pm 22,5) n=23$ & $\mathbf{1 6 , 8}( \pm 9,8) n=5$ & $\mathbf{4 4 , 2}( \pm 13,4) n=5$ \\
\hline Flächenwurf & $\mathbf{2 4 , 0}( \pm 11,3) n=15$ & $\mathbf{9 1 , 0}( \pm 7,4) n=15$ & $\mathbf{1 5 , 1}( \pm 7,0) n=32$ & $\mathbf{9 4 , 7}( \pm 6,4) n=32$ \\
\hline
\end{tabular}

Besonders in der Königsbuche erscheinen die Mittelwerte des stehenden Bestandes jedoch relativ hoch und werden durch die räumliche Nähe der geworfenen Bestandesteile und entsprechenden Seitenlichteinfall verursacht. Normalerweise liegt der relative Lichtgenuss in ungestörten Buchen-Wäldern unter 5\% (siehe EBER 1972; SCHMIDT 1997; WECKESSER 2003).

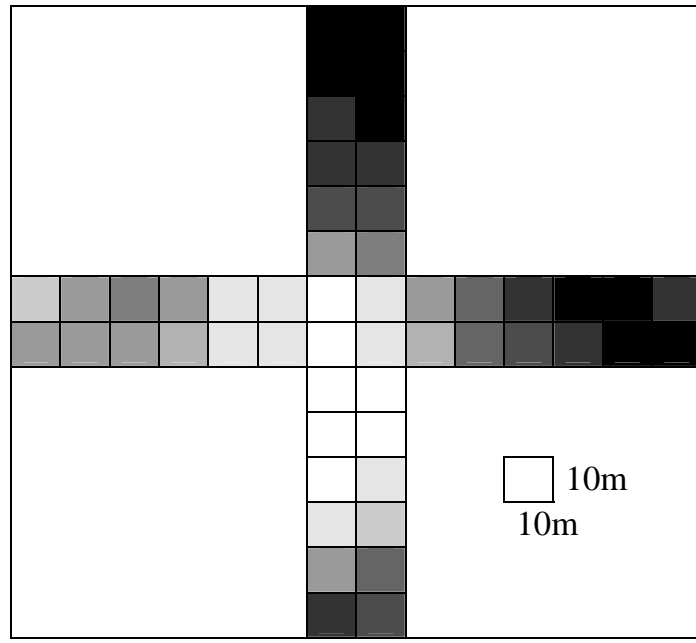

bis zu $\quad 10 \quad 20 \quad 30 \quad 40 \quad 50$

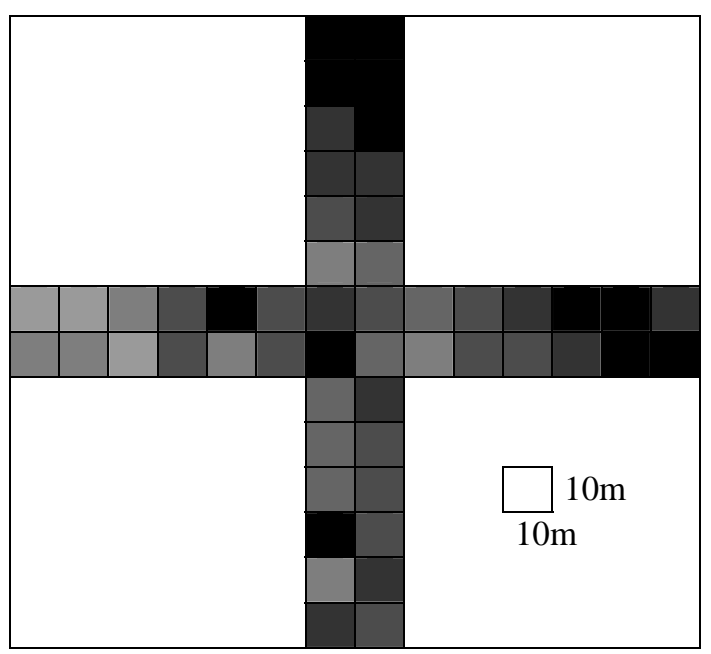

$60 \quad 70 \quad 80$

$90 \quad 100 \%$ rel. Lichtgenuss

Abb. 16: Relativer Lichtgenuss (PAR) in \% der Freilandhelligkeit über der Strauch- (links) und der Feldschicht (rechts) im Sommer 2001 in der Königsbuchen-KF2 (Buntsandstein; Galio odorati-Fagetum); aufgenommen wurden 52 in einem Kreuztransekt angeordnete 100 $\mathrm{m}^{2}$-Plots (Kantenlänge $10 \mathrm{~m}$ ).

\subsection{Strukturelle Parameter im Flächenwurf}

\subsubsection{Artenzahlentwicklung}

Im vierten Jahr nach dem Sturm (2001) kristallisieren sich bei den mittleren Artenzahlen je $100 \mathbf{~ m}^{2}$ zwei Niveaus heraus: Die beiden carbonatreicheren Zechstein-Standorte (Hainholz- 
KF2, Nüllberg - siehe Tab. 1) liegen deutlich vor allen anderen Untersuchungsgebieten (UG). Die seit 30 Jahren sich selbst überlassene Königsbuchen-KF2 hat das geringste Niveau (Abb. 17). Einheitlich ist aber festzustellen, dass sich die Artenzahl in allen UG gegenüber dem Ausgangszustand erhöht hat.

In der Feldschicht kam es dabei zu folgenden Entwicklungen: In der Hainholz-KF1 (Hordelymo-Fagetum) erreicht die Feldschichtartenzahl im dritten Jahr (2000) mit durchschnittlich 28/100 $\mathrm{m}^{2}$ ihr Maximum und geht 2001 bereits wieder auf 23/100 $\mathrm{m}^{2}$ zurück (Tab. A22). In der Königsbuchen-KF2 (Galio odorati-Fagetum) kommt es anfangs zu einer kurzen Stagnation zwischen 1998 und 1999, danach zu einem Anstieg (Tab. A23). In allen anderen Gebieten erhöht sich die mittlere Artenzahl in der Feldschicht kontinuierlich (Tab. A22, A23). Dieser Anstieg ist in den beiden artenreichsten Gebieten besonders steil (von 29 auf 49/100 m² in Hainholz-KF2; von 41 auf 56/100 m² im Nüllberg), während er ansonsten wesentlich moderater ausfällt. Auffällig ist, dass sowohl auf basenreichen als auch auf basenarmen Standorten die aufgeforsteten und geräumten Flächen (Nüllberg, Rotenberg-Abt. 1228, Krücker-Plateau) ausnahmslos artenreicher sind als die belassenen (Hainholz-KF1 und KF2, Königsbuche-KF2, Krücker-Osthang).

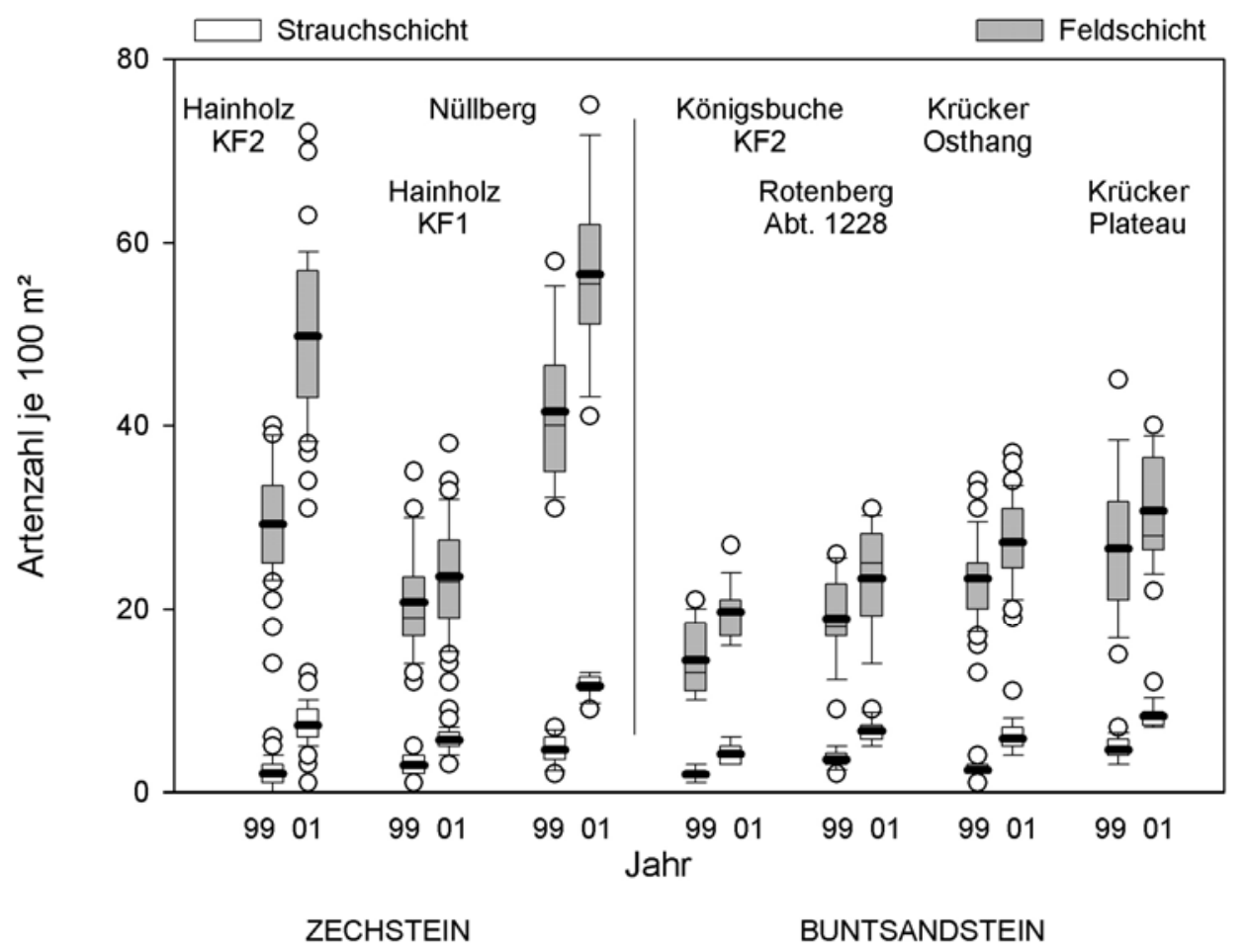

Abb. 17: Artenzahlentwicklung je $100 \mathrm{~m}^{2}$ in Buchen-Windwurfgebieten mit Flächenwurf im südwestlichen Harzvorland; getrennt nach Strauch- und Feldschicht; Anzahl einbezogener Plots siehe Tab. 1; Boxplot-Darstellung mit Mittelwert (dicker Querbalken), Median (dünner Querbalken), 25er und 75er Perzentil (grauer Kasten), 10er und 90er Perzentil (Fehlerbalken) sowie Ausreissern (Kreise). 
In der Strauchschicht (Gehölze über $50 \mathrm{~cm}$ ) steigt die mittlere Artenzahl in allen UG kontinuierlich an. Der Nüllberg ist im Jahr 2001 mit durchschnittlich 11,5 Strauchschichtarten je $100 \mathrm{~m}^{2}$ am artenreichsten, die Königsbuchen-KF2 am artenärmsten (4,1/100 m²). Auch hier gilt ohne Ausnahme, dass die geräumten und aufgeforsteten Plots im Mittel artenreicher sind als die belassenen (Abb. 17 und Tab. A22, A23).

Bei den Gesamtartenzahlen je Gebiet fallen wiederum Hainholz-KF2 bzw. Nüllberg mit besonders hohen (2001: 156 bzw. 118) und die Königsbuchen-KF2 mit besonders niedrigen Werten auf (2001: 48). Die Gesamtartenzahl je Gebiet und die mittlere Artenzahl je $100 \mathrm{~m}^{2}$ verhalten sich teilweise widersprüchlich (Tab. A22, A23). Kontinuierliche Anstiege der mittleren Artenzahlen je $100 \mathrm{~m}^{2}$ sind in einigen UG (Königsbuche-KF2, Rotenberg-Abt. 1228) ab dem vierten Sukzessionsjahr (2001) mit einem Rückgang der Gesamtartenzahlen verknüpft. Einem im gesamten UG bereits gesättigten Artenpool steht hier also die weitere Verbreitung vorhandener Arten in den einzelnen 100 m²-Plots gegenüber. In der HainholzKF1 erfolgt der Einbruch der Artenzahlentwicklung in beiden Fällen synchron ab 2001. In den beiden artenreichsten Gebieten (Hainholz-KF2, Nüllberg) dagegen nehmen die Artenzahlen absolut und relativ weiter zu. Für den Krücker ist dies aufgrund der unvollständigen Zeitreihe nicht nachprüfbar. Hier lässt sich nur allgemein feststellen, dass die Artenzahl auf den belassenen Flächen von 1999 zu 2001 absolut und relativ zunimmt, sich auf den geräumten Flächen aber gegenläufig verhält (Abb. 17; Tab. A23).

Insgesamt erreichen nur sehr wenige Arten einen Wert von über 1\% mittlerer Deckung (Abb. 18; Tab. A22, A23). Dabei erhöht sich deren Zahl (mit Ausnahme der Hainholz-KF2) in der Feldschicht bis zum vierten Jahr kaum, bei den geräumten Varianten Krücker und Nüllberg geht sie sogar wieder zurück. Dies unterstreicht die Dominanz einiger weniger Arten im Sukzessionsgeschehen. In der Strauchschicht erreichen in allen Gebieten ausser Königsbuche-KF2 und Rotenberg-Abt. 1228 (hier Stagnation) immer mehr Arten mittlere Deckungen von über 1\%, auch wenn es sich insgesamt um eine relativ geringe Anzahl handelt (Tab. A22, A23). Dabei sind auf dem Nüllberg und auf dem Krücker-Plateau (beide geräumt) mehr als 50\% aller Strauchschichtarten durch die Aufforstung in diese Kategorie gefallen. Weiterhin ist ersichtlich, dass die Mehrzahl der in den UG vorkommenden und der neu auftretenden Arten quantitativ eher bedeutungslos bleibt. Das betrifft vor allem die Arten der Feldschicht, weniger die der Strauchschicht (Abb. 18). Sowohl auf basenreichen als auch auf basenarmen Standorten weisen die geräumten Flächen einen höheren Prozentanteil an quantitativ bedeutsamen Feldschicht-Arten (d.h. Arten mit $>1 \%$ mittlerer Deckung) auf als die belassenen. Dabei zeichnete sich im bisherigen Sukzessionsverlauf im geräumten Flächenwurf eher eine gleichbleibend bis sinkende Tendenz ab, im belassenen dagegen eine gleichbleibend bis ansteigende. 


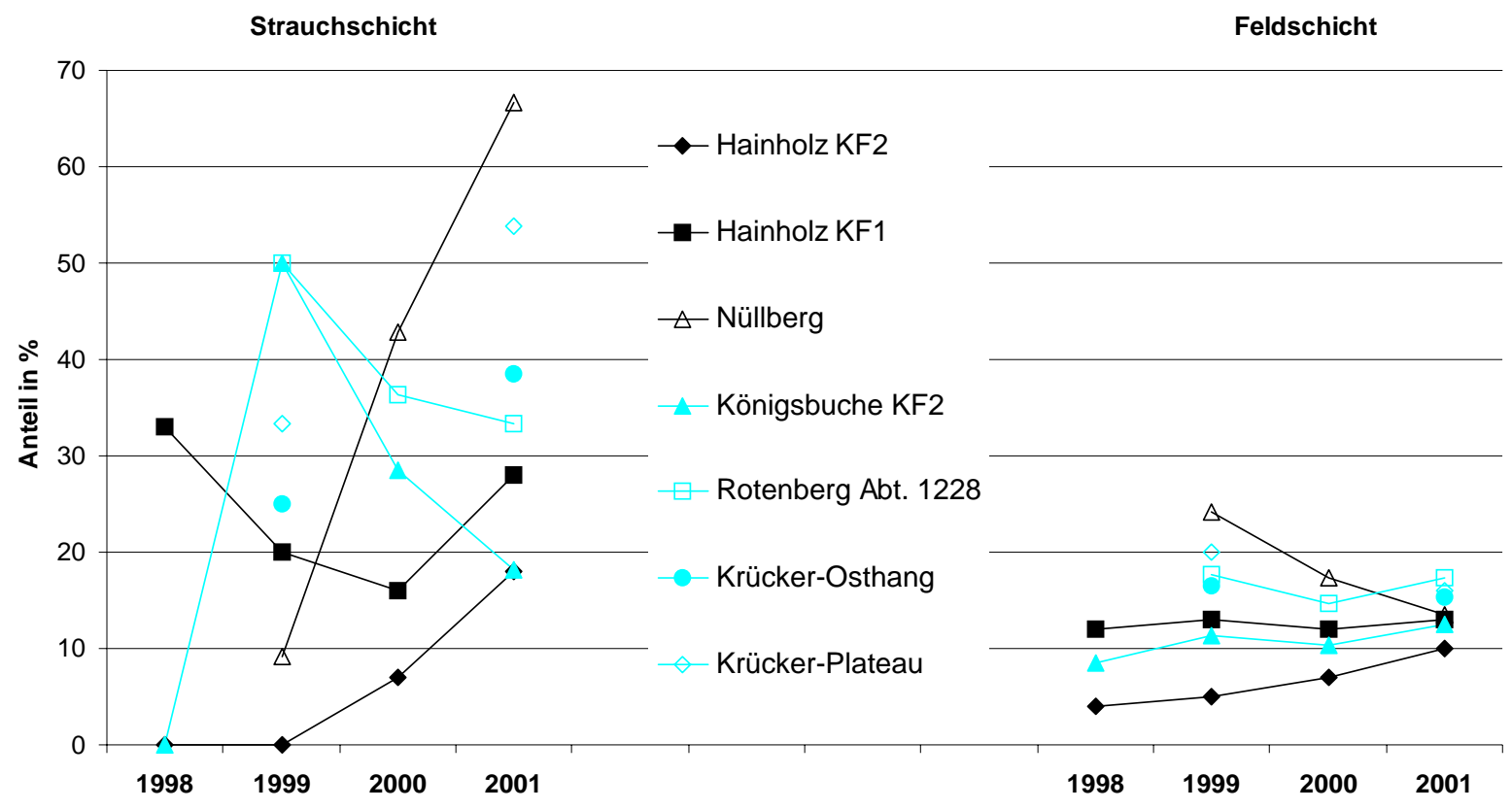

Abb. 18: Prozentanteil von Arten mit $>1 \%$ mittlerer Deckung an der Gesamtartenzahl von Strauch- (links) und Feldschicht (rechts) in Buchen-Windwurfgebieten mit Flächenwurf im südwestlichen Harzvorland in den Jahren (1998)1999 bis 2001; belassene Flächen mit vollen Symbolen, geräumte mit hohlen; Anzahl einbezogener 100 m²-Plots siehe Tab. 1.

\subsubsection{Evenness}

Im Vergleich der Jahre 1999 und 2001 ergeben sich hier sehr unterschiedliche Entwicklungen in der Feld- und in der Strauchschicht (Abb. 19). Während die mittleren Evenness-Werte der Feldschicht stagnieren (Hainholz-KF1, Nüllberg, Krücker-Plateau) oder leicht ansteigen (übrige UG) und sich dabei im Jahr 2001 in einem recht schmalen Rahmen von 50-70\% bewegen, nehmen die Werte der Strauchschicht kontinuierlich und teilweise rapide ab und liegen im Jahr 2001 standortsabhängig zwischen rund 20 und 80\%. Einzige Ausnahme ist die Strauchschicht in der geräumten Rotenberg-Abt. 1228, die sich mit 35-36\% in diesem Punkt kaum verändert. Sehr deutliche Abnahmen sind dagegen z.B. in der Strauchschicht von Königsbuche-KF2 (von 72 auf 19\%), Nüllberg (von 89 auf 54\%) und Krücker-Osthang (von 84 auf 52\%) zu verzeichnen, etwas moderatere Rückgänge in den übrigen Gebieten. Vier Jahre nach dem Sturm hat die Königsbuchen-KF2 somit die am einseitigsten dominierte Strauchschicht vorzuweisen (Evenness 19\%), die Hainholz-KF2 dagegen die am besten durchmischte (Evenness 80\%). Der Bollerkopf (Hainholz KF2) ist auch derjenige Standort, der mit seinen nach vier Jahren immer noch ansteigenden Artenzahlen (Tab. A22) und Feldschicht-Evenness-Werten (Zunahme von 60 auf 71\%) die dynamischste Sukzession und gleichmäßigste Durchmischung aufweist.

Zusammenfassend lässt sich also sagen, dass die Feldschichtarten in der bisherigen Zeitreihe im wesentlichen gleichverteilt bleiben und sich auch standörtlich bezüglich der Evenness nur 
wenig unterscheiden (Ausnahme: zunehmende Durchmischung in Hainholz-KF2), sich in der Strauchschicht aber standortsabhängig teilweise starke Dominanzen einzelner Arten herausbilden (am wenigsten in Hainholz-KF2, am stärksten in Königsbuche-KF2) oder auch schon länger bestehen (Rotenberg-Abt. 1228).

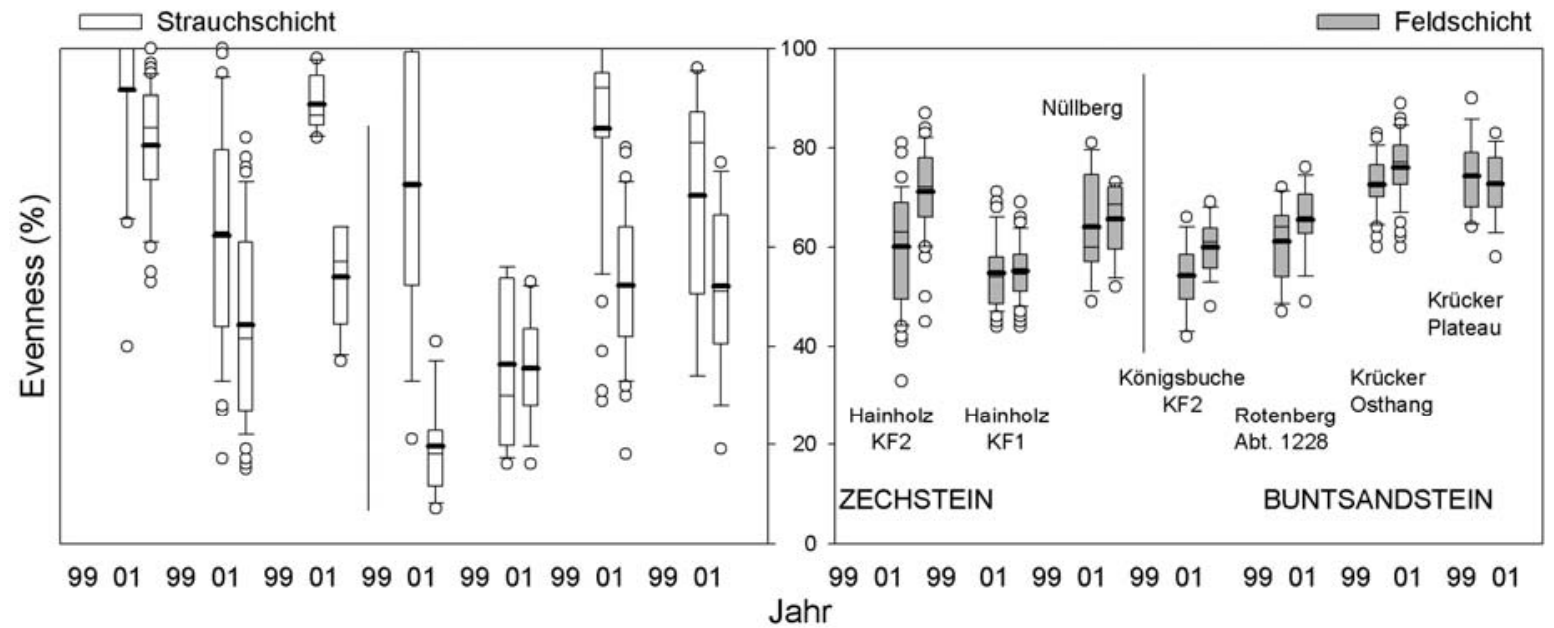

Abb. 19: Entwicklung der Evenness in der Strauch- (links) und in der Feldschicht (rechts) von Buchen-Windwurfgebieten mit Flächenwurf im südwestlichen Harzvorland in den Jahren 1999 und 2001; Anzahl einbezogener $100 \mathrm{~m}^{2}$-Plots siehe Tab. 1; Variantenanordnung der Strauchschicht (links) wie bei Feldschicht (rechts); Boxplot-Darstellung wie in Abb. 17.

\subsubsection{Deckungsgrade bzw. Deckungsgradsummen der Vegetationsschichten}

Im vierten Jahr nach dem Sturm (2001) lassen sich bei der Feldschicht zwei Niveaus erkennen: Die produktiveren Dolomit-Standorte mit Hordelymo-Fagetum (Hainholz KF1, Nüllberg) haben mit rund 150-160\% Deckungssumme eine relativ dichte Krautschicht ausgebildet, während alle übrigen Standorte nur bei 67-83\% liegen (Abb. 20; Tab. A22, A23). Die Feldschichtentwicklung ist in fast allen UG durch einen anfänglichen, in Hainholz-KF2 und Königsbuche-KF2 sogar sehr sprunghaften Anstieg und durch nachfolgende Stagnation bzw. Rückgang ab dem vierten Sukzessionsjahr (2001) gekennzeichnet. Dies ist vermutlich auf einen Ausdunkelungseffekt durch die sich parallel dazu rasant entwickelnde Strauchschicht zurückzuführen. Eine Ausnahme ist der flachgründige Gips/Dolomit-Standort mit Carici-Fagetum (Hainholz-KF2), wo mit nur 23\% mittlerer Deckungssumme zum Sukzessionsbeginn im Jahr 1998 eine äusserst lückige Feldschicht vorlag und die Ausbreitung der Feldschicht auch im Jahr 2001 noch nicht abgeschlossen zu sein scheint (Tab. A22). Für das Krücker-Plateau (geräumt) lässt sich ebenfalls ein leichter Anstieg der mittleren Feldschicht-Deckungssumme von 1999 zu 2001 feststellen (Abb. 20).

Die Strauchschicht (Gehölze ab $50 \mathrm{~cm}$ Höhe) ist nach vier Sukzessionsjahren recht unterschiedlich ausgebildet. Im Rotenberg (geräumte Variante) ist mit durchschnittlich 91\% Gehölzdeckungssumme im Jahr 2001 die dichteste Strauchschicht aller untersuchten Plots zu 
finden, gefolgt von den übrigen Standorten mit Werten zwischen 60 und 75\%. Die HainholzKF2 bleibt wegen ihrer flachgründigen Gips/Dolomit-Böden mit durchschnittlich 11\% (2001) auf dem niedrigsten Stand aller Untersuchungsflächen (Abb. 20; Tab. A22). Die Strauchschicht entwickelte sich bislang in ausnahmslos allen UG kontinuierlich, aber unterschiedlich dynamisch. Auf den geräumten Flächen im Rotenberg erfolgte dies wegen der hohen Ausgangswerte (1999 bereits 66\%) langsamer und weniger sprunghaft als in den übrigen Gebieten, in der Hainholz-KF2 kommt es standortbedingt zu einer verlangsamten Besiedlung mit Sträuchern und jungen Bäumen.

Ein weiterer Unterschied besteht darin, dass auf Zechstein auch nach vier Sukzessionsjahren die Feldschicht deutlich dichter ausgeprägt ist als die Strauchschicht, während im Buntsandstein die zunehmend dichtere Strauchschicht die Feldschichtwerte übertrifft (Rotenberg-Abt. 1228) oder einholt (übrige basenarme Standorte).

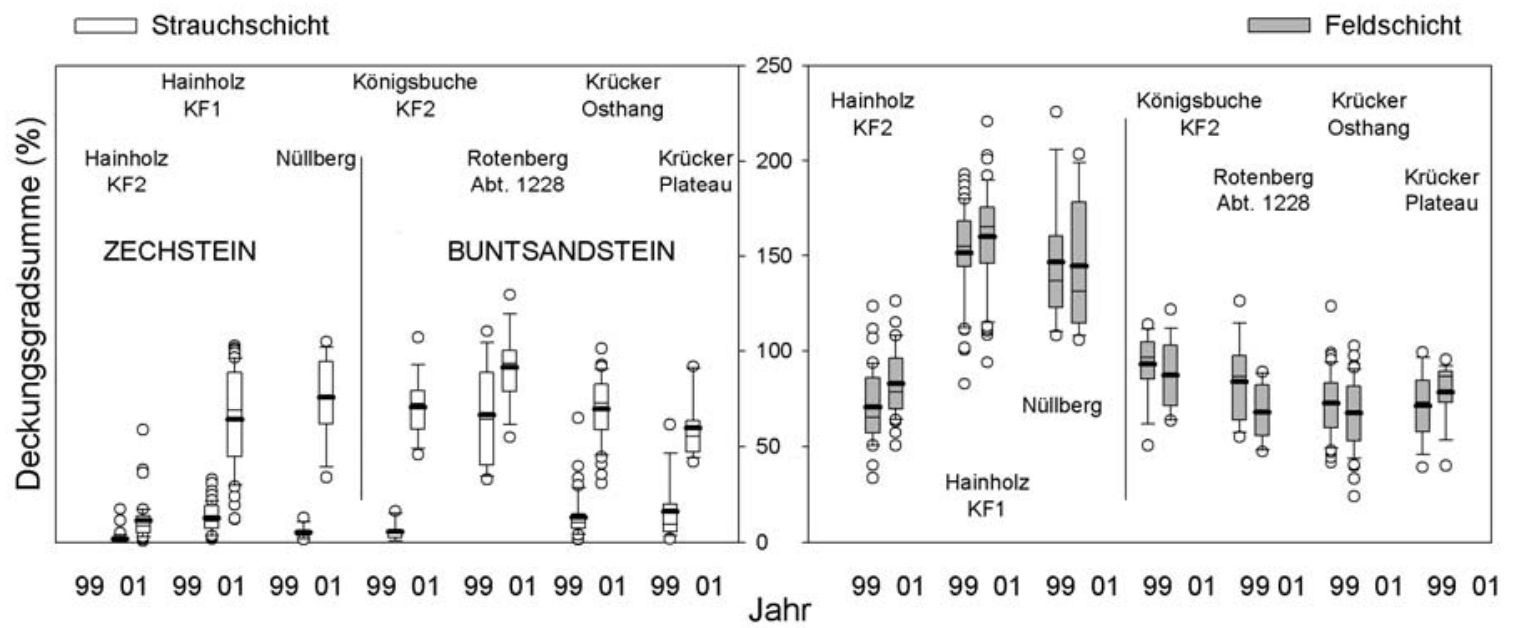

Abb. 20: Entwicklung der Deckungsgradsummen in der Strauch- (links) und in der Feldschicht (rechts) in Buchen-Windwurfgebieten mit Flächenwurf im südwestlichen

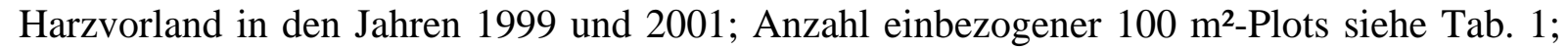
Boxplot-Darstellung wie in Abb. 17.

Für die Kryptogamenschicht liegen aus fünf UG für die Jahre 1999 bis 2001 Daten vor (nicht für den Krücker). Im Gegensatz zu Strauch- und Feldschicht ist hier die im Gelände geschätzte Deckung, nicht die Deckungssumme zugrunde gelegt. Mehrheitlich wird ein Anstieg der mittleren Kryptogamendeckungen auf unterschiedlichem Niveau angezeigt, lediglich in der Königsbuchen-KF2 stagniert die Entwicklung (Abb. 21; Tab. A22, A23). Vergleicht man die Werte im vierten Jahr nach dem Sturm, wofür Daten aus allen sieben Varianten vorhanden sind (Tab. A22, A23), so erweist sich der Krücker und damit das Luzulo-Fagetum mit 32\% (geräumt) bzw. 37\% (belassen) als das Kryptogamen-reichste Gebiet, gefolgt vom Galio odorati-Fagetum in der geräumten Rotenberg-Abt. 1228 (21\%) und den drei Zechstein-Varianten (8-16\%). Die Königsbuche (Galio odorati-Fagetum, belassen) liegt mit 3-4\% mittlerer Kryptogamendeckung an letzter Stelle. 


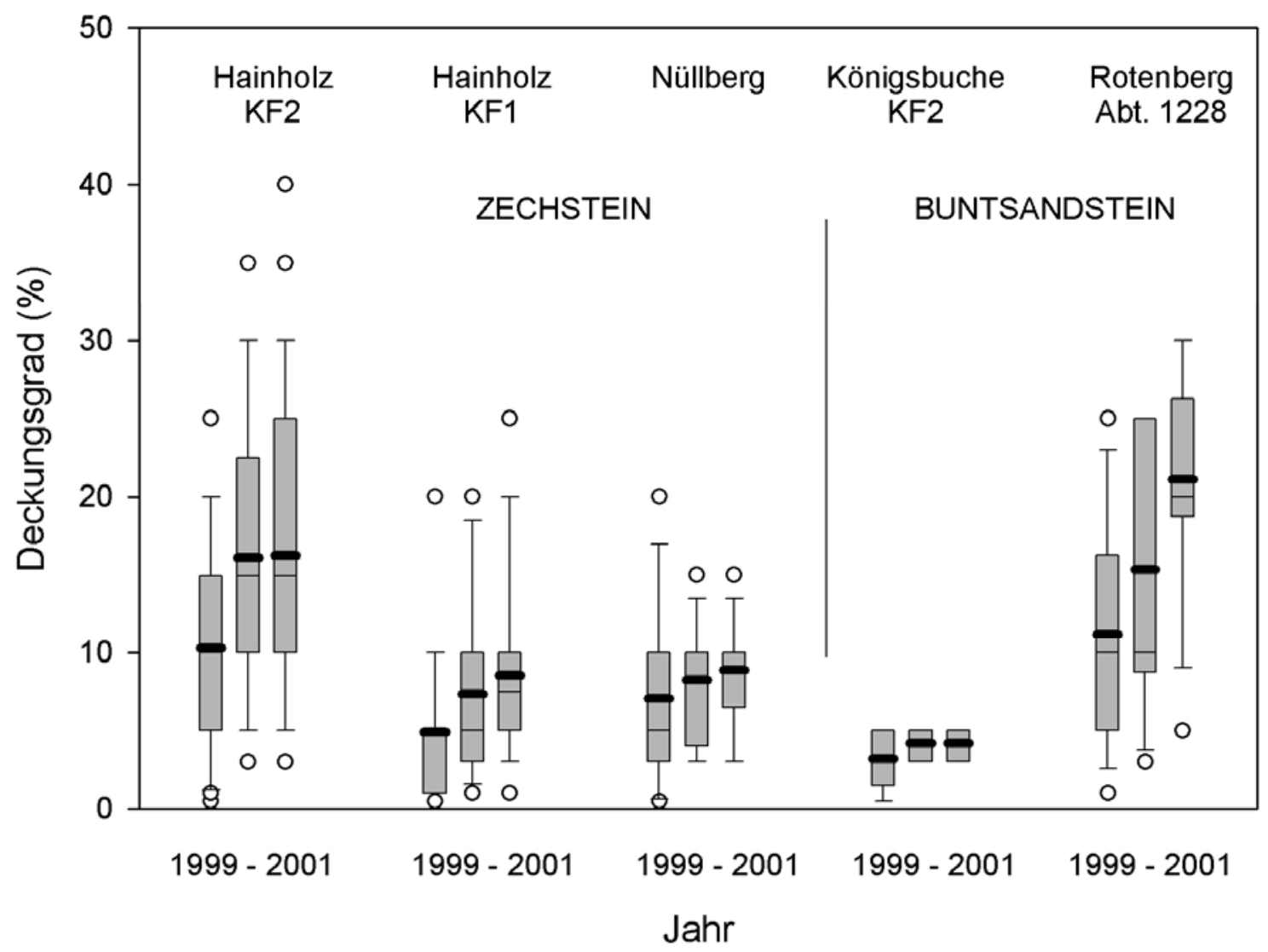

Abb. 21: Entwicklung der Kryptogamendeckungen in fünf Buchen-Windwurfgebieten mit Flächenwurf im südwestlichen Harzvorland in den Jahren 1999 bis 2001; Anzahl einbezogener 100 m²-Plots siehe Tab. 1; Boxplot-Darstellung wie in Abb. 17.

\subsubsection{Verhaue und Wurzelteller}

Für drei belassene UG (beide Hainholz-KF, Königsbuche-KF2) liegen Daten über die Deckungsgradentwicklung liegend-lebender Kronenteile von 1999 bis 2001 vor (Abb. 22). Erstaunlicherweise treiben diese Kronenteile Jahr für Jahr neu aus und fruktifizieren sogar, ihre mittleren Deckungsgrade nehmen aber erwartungsgemäß ab. Sie gehen im Flächenwurf der Königsbuche ab dem dritten Sukzessionsjahr gegen Null, dagegen sind sie in der Hainholz-KF2 vergleichsweise hoch (Abnahme von durchschnittlich 25 auf 9\%) und in der Hainholz-KF1 stagnieren sie fast (Abnahme von 6,4 auf 4,7\%). Am Krücker-Osthang (Tab. A23) liegen sie im vierten Sukzessionsjahr mit fast $14 \%$ am höchsten. Dies korreliert aber nur teilweise mit der recht unterschiedlichen mittleren Anzahl liegender Stämme, die sich am Krücker-Osthang auf 10 je $100 \mathrm{~m}^{2}$ beläuft, in der Hainholz-KF2 auf 9, in der KönigsbuchenKF2 auf 7 und in der Hainholz-KF1 auf 6 (Abb. 23). Wann die letzten Kronenteile absterben, bleibt abzuwarten (siehe Kap. 5.7.1). 


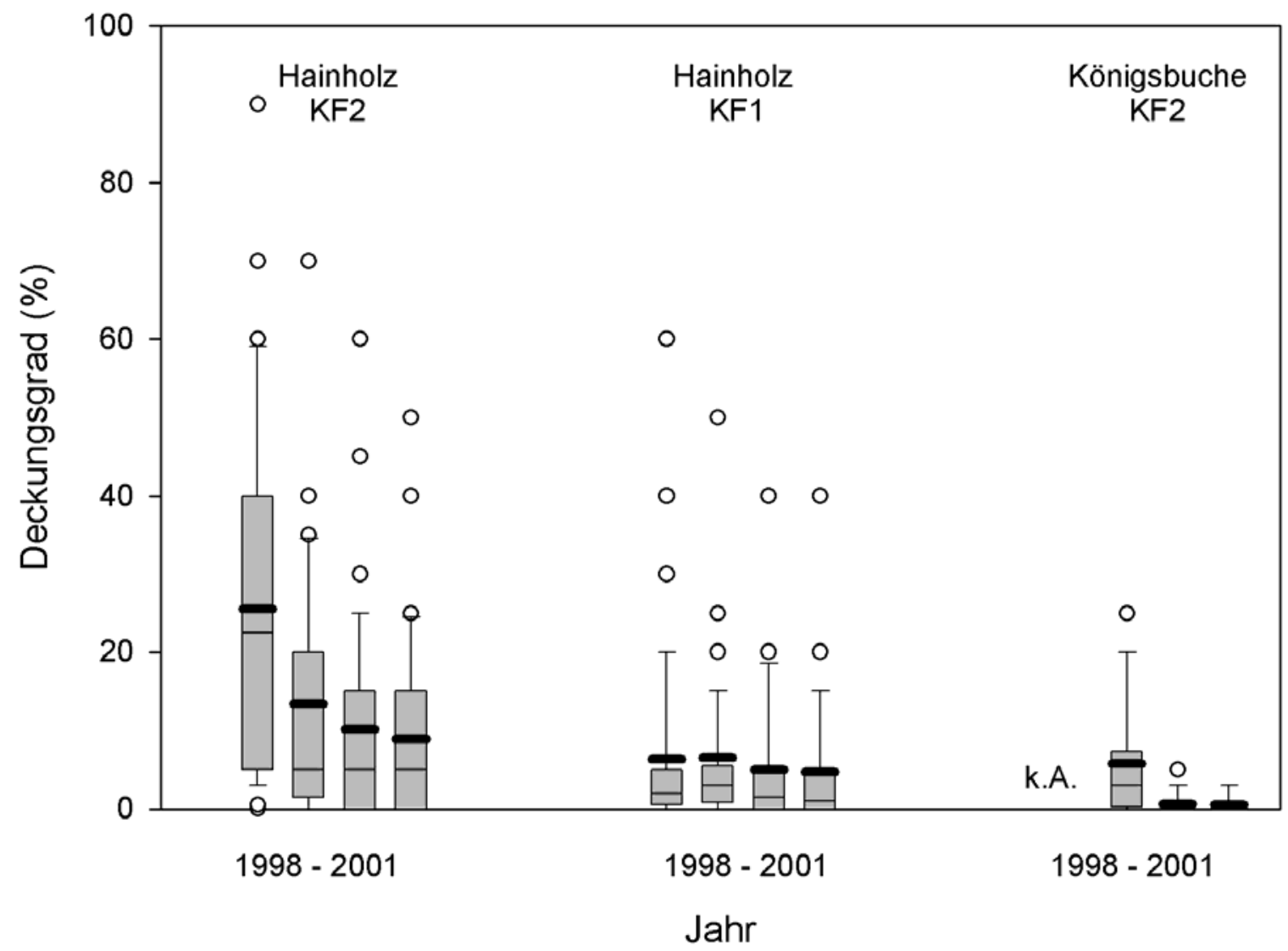

Abb. 22: Deckung lebender Kronenteile geworfener Laubbäume im belassenen Flächenwurf von Hainholz und Königsbuche im Verlauf der Jahre 1998-2001; Anzahl einbezogener 100 $\mathrm{m}^{2}$-Plots siehe Tab. 1; Boxplot-Darstellung wie in Abb. 17.

Die Zahl der Wurzelteller je $100 \mathrm{~m}^{2}$ variiert im Mittel zwischen rund 1 (Hainholz-KF1, Rotenberg-Abt. 1228) und rund 3 (Hainholz-KF2, Krücker-Osthang). In den übrigen Gebieten (Krücker-Plateau, Nüllberg, Königsbuche-KF2) liegen etwa anderthalb bis zweieinhalb Wurzelteller in jedem Plot (Abb. 23; Kap. 4.6). Da viele Stämme von ausserhalb in die Untersuchungsflächen hineingefallen sind, liegen in den belassenen Flächen im Durchschnitt drei- bis fünfmal so viele Stämme wie Wurzelteller vorhanden sind. Die mittleren Totholzdeckungsgrade (einschließlich tote Kronenteile - Abb. 23) liegen auf den geräumten Plots zwischen 5 und 13\%, im belassenen Windwurf zwischen 22\% (Hainholz-KF1) und 36\% (Krücker-Osthang). 


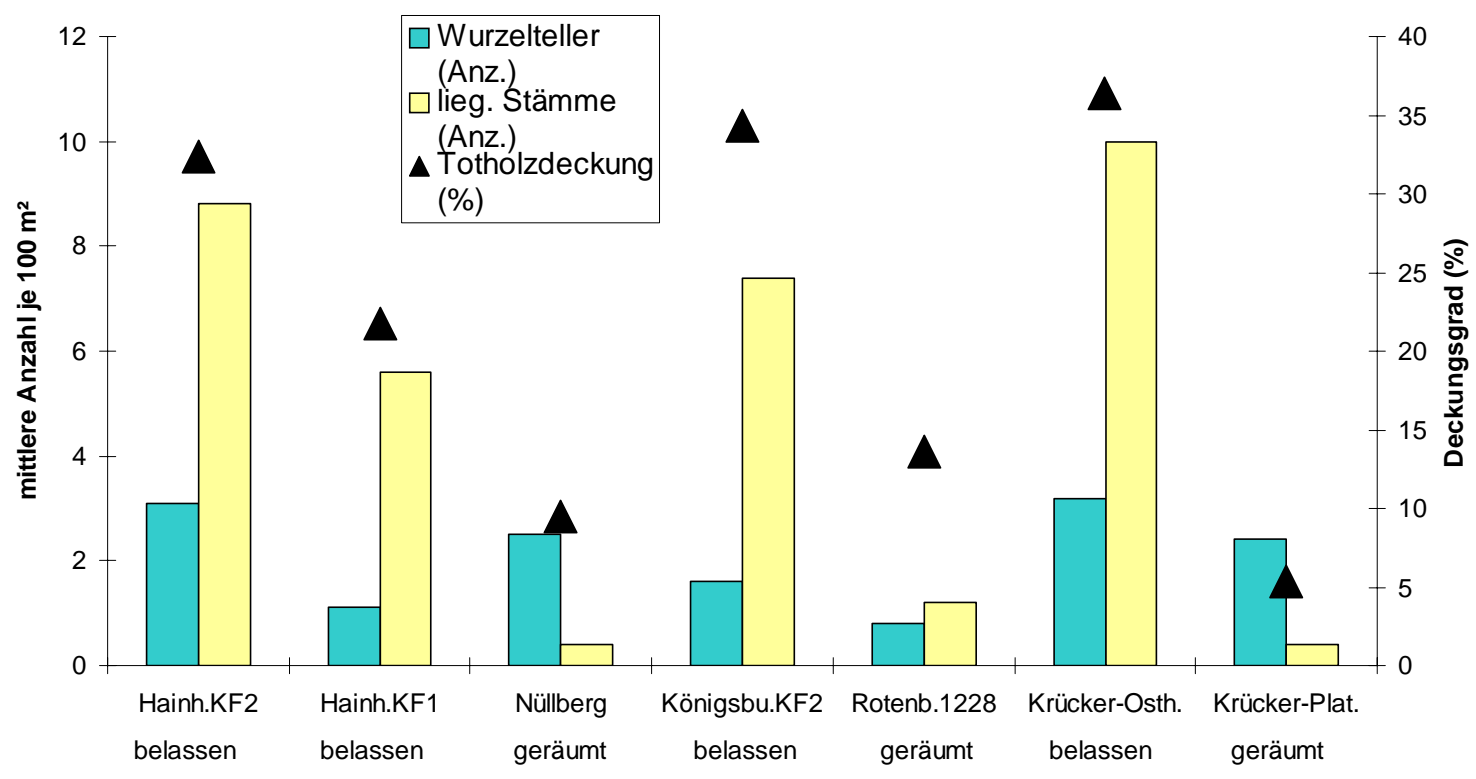

Abb. 23: Mittlere Anzahl liegender Stämme und aufgeklappter Wurzelteller je $100 \mathrm{~m}^{2}$ sowie mittlere Totholzdeckung (\%) in Buchen-Windwurfgebieten mit Flächenwurf im

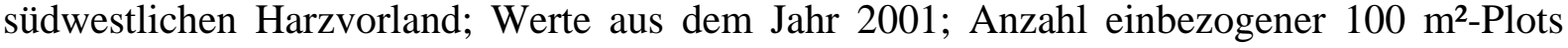
siehe Tab. 1.

\subsubsection{Laubstreuauflage}

Die Laubstreudeckung wurde für fünf UG im Zeitraum 1999 bis 2001 aufgenommen, für das vierte Sukzessionsjahr liegen Daten aus allen Varianten vor (Tab. A22, A23). Anfangs (d.h. 1999) waren die beiden Dolomit-Standorte (Hainholz-KF1, Nüllberg) mit rund 8\% mittlerer Deckung vergleichsweise laubstreuarm, während in den übrigen UG die Ausgangswerte im Mittel zwischen ca. 25\% (Buntsandstein) und ca. 30\% (Hainholz-KF2) lagen. In beiden Hainholz-KF nimmt die Laubstreudeckung bis 2001 kontinuierlich ab (auf ca. 2\% in KF1, auf ca. 8\% in KF2), auf den restlichen Standorten dagegen erfolgt nach einem Rückgang zwischen 1999 und 2000 ein Wiederanstieg der mittleren Deckungen im Jahr 2001. Der Krücker erscheint in 2001 mit 30\% Deckung sehr laubstreureich.

\subsubsection{Vegetationsfreier Mineralboden oder Fels}

Dieser Parameter wurde für fünf UG im Zeitraum 1999 bis 2001 erhoben (Abb. 24). Bezüglich der Ausgangswerte von 1999 lassen sich drei Niveaus erkennen. Die flachgründige Gips/Dolomit-Kuppe des Bollerkopfes (Hainholz-KF2) wirkte mit durchschnittlich 10\% vegetationsfreier Fläche (Maximalwerte bis 25\%) anfangs noch recht „kahl“. Dem folgten die beiden tiefgründigeren Dolomit-Standorte (Hainholz-KF1, Nüllberg) mit durchschnittlich ca. 4\% und die Buntsandstein-Flächen, welche auch schon 1999 fast vollständig mit Kryptogamen, Gefäßpflanzen oder Laubstreu bedeckt waren (nur 1-2\% vegetationsfreier Boden). In allen UG verringerte sich die vegetationsfreie Fläche bis zum vierten 
Sukzessionsjahr teilweise beträchtlich (besonders in der Hainholz-KF2) und ging im Buntsandstein schließlich gegen Null. Im Zechstein blieben im Mittel 1-3\% übrig. Trotzdem gab es im Jahr 2001 insbesondere auf dem Nüllberg und in der Hainholz-KF2 noch einzelne Plots, die bis zu 10\% bzw. 15\% vegetationsfrei blieben, wobei frische Bodenverwundungen (Wild, Spuren des Bearbeiters) eingeschlossen sein können.

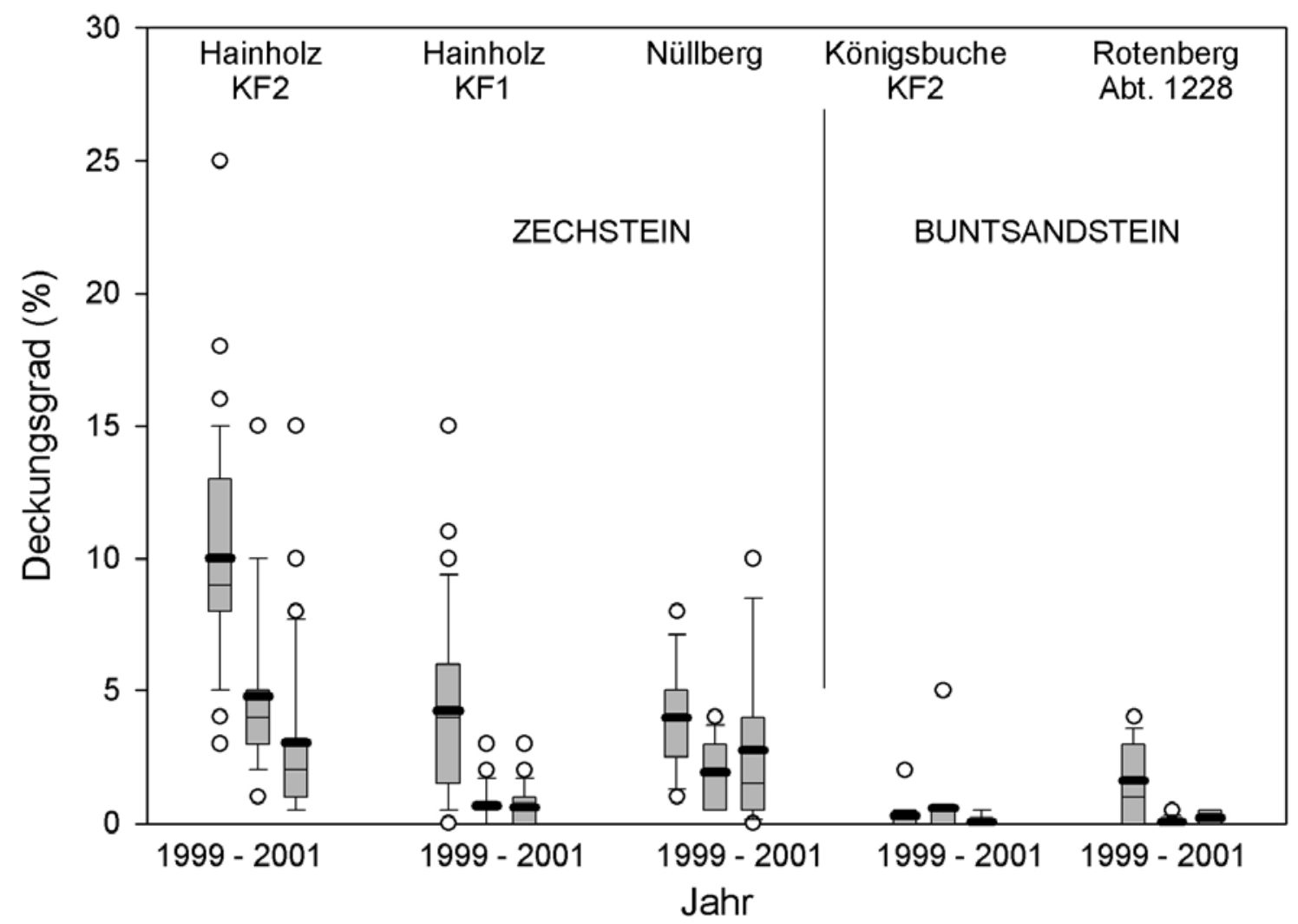

Abb. 24: Entwicklung der Deckungsgrade des vegetationsfreien Mineralbodens bzw. Felses in fünf Buchen-Windwurfgebieten mit Flächenwurf im südwestlichen Harzvorland in den Jahren 1999 bis 2001; Anzahl einbezogener 100 m²-Plots siehe Tab. 1; Boxplot-Darstellung wie in Abb. 17.

\subsubsection{Höhenentwicklung der Strauch- und Feldschicht (Maximalwerte)}

Die Maximalhöhen der Strauch- und Feldschicht wurden für fünf UG (Abb. 25) in den Sommern 1999 bis 2001 im Rahmen der Vegetationsaufnahmen erhoben. Mit Ausnahme von Rotenberg-Abt. 1228 (dort nur für Strauchschicht zutreffend) kommt es im genannten Zeitraum überall zu einem Anstieg der mittleren Maximalhöhen beider Schichten. Dabei liegt der Feldschicht-Mittelwert im Jahr 2001 in den Zechstein-Gebieten mit 1,80 m geringfügig über dem der Buntsandstein-Gebiete (1,50-1,60 m). Bei der Strauchschicht werden im vierten Sukzessionsjahr vor allem in der Hainholz-KF1 sehr hohe Maximalwerte erreicht (im Mittel 3,80 m, im Maximum $5 \mathrm{~m}$ ), was die Wüchsigkeit der Naturverjüngung dieses Standortes unterstreicht. Danach folgen - möglicherweise aufforstungsbedingt - die beiden geräumten 
Gebiete Nüllberg und Rotenberg-Abt. 1228 (beide mit 3,20 m mittlerer Maximalhöhe). Der Bollerkopf bzw. die Königsbuchen-KF2 erscheinen bei mittleren bzw. sehr geringen Naturverjüngungsdichten (Kap. 4.5.1) mit durchschnittlich 2,30 m bzw. 2,70 m vergleichsweise wenig wüchsig. Die jeweils beteiligten Baum- und/oder Straucharten spielen bei diesem Parameter natürlich eine wichtige Rolle (siehe Tab. A22, A23).

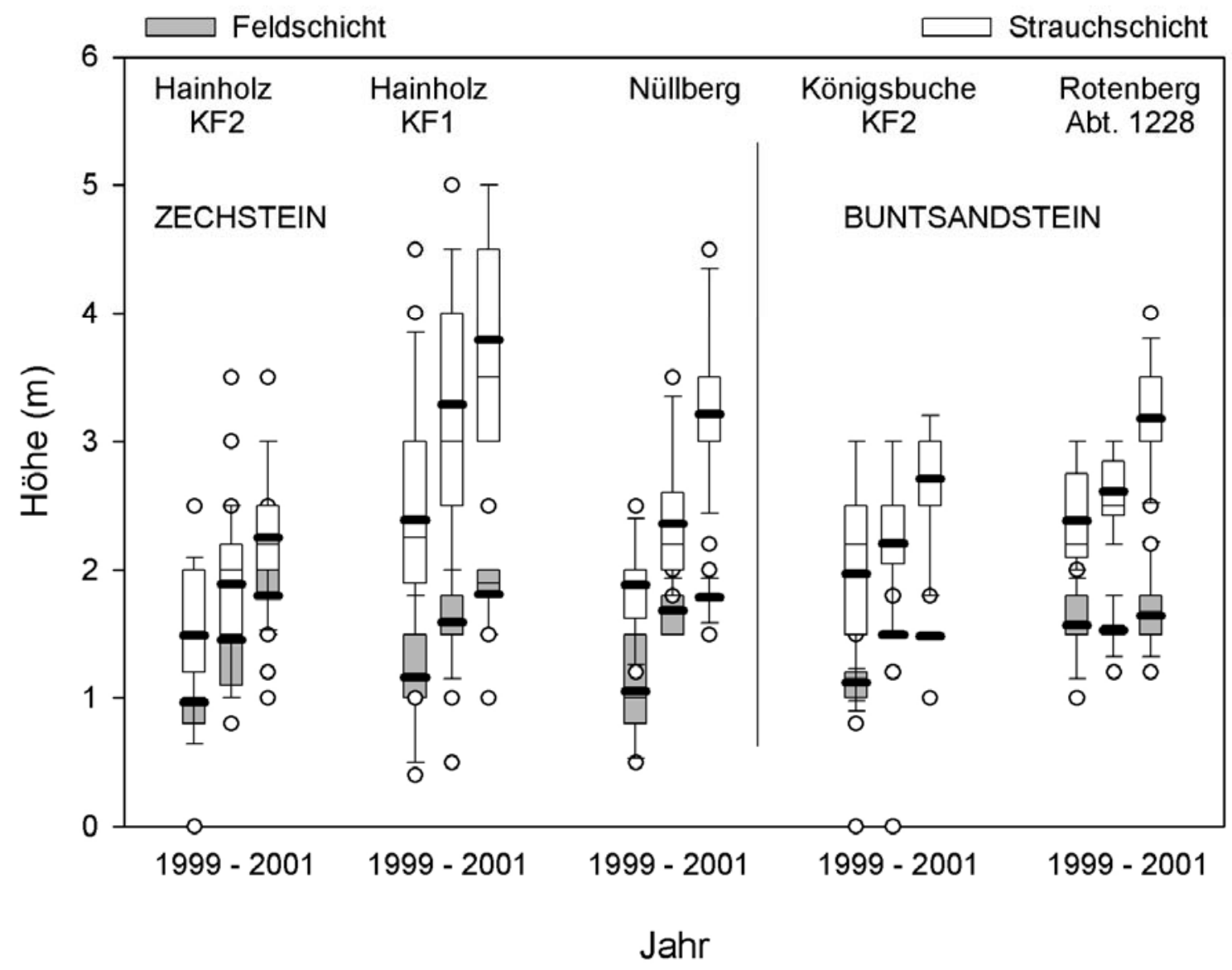

Abb. 25: Entwicklung der Maximalhöhen der Strauch- und Feldschicht in fünf BuchenWindwurfgebieten mit Flächenwurf im südwestlichen Harzvorland in den Jahren 1999 bis 2001; Anzahl einbezogener 100 m²-Plots siehe Tab. 1; Boxplot-Darstellung wie in Abb. 17.

\subsubsection{Floristische Ähnlichkeit im Vergleich der Jahre 1999 und 2001}

Für den qualitativen Vergleich der Untersuchungsflächen wurde der Jaccard-Index herangezogen, bei dem die Deckungsgrade der Arten unberücksichtigt bleiben (Abb. 26 oben; maximale Ähnlichkeit = 1). Zwischen den Zechstein- und den Buntsandstein-Standorten ergibt sich eine auffällige Differenzierung. Während sich die vier basenarmen Standorte mit einem mittleren Wert von rund 0,5 zwischen 1999 und 2001 alle etwa gleichstark verändert haben, erfährt unter den drei basenreichen Standorten die geräumte Variante (Nüllberg) mit einem Wert von rund 0,2 die stärkste Veränderung aller untersuchten Plots. Dem folgt die flachgründige Gipskuppe des Bollerkopfes (Hainholz KF2) mit 0,4 und der relativ gering veränderte Dolomit-Standort der Hainholz-KF1 mit 0,6. 
JACCARD

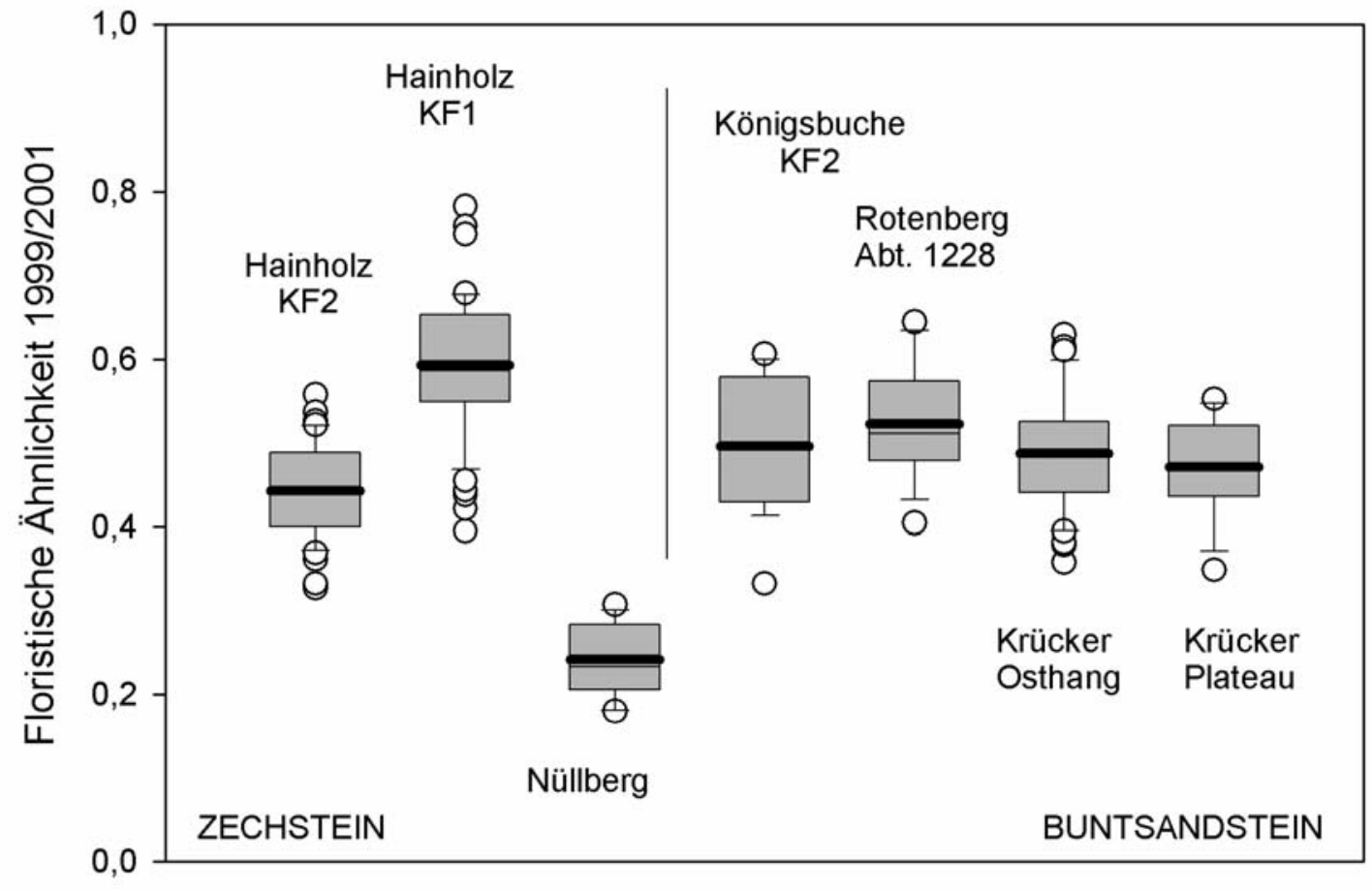

PERCENTAGE SIMILARITY

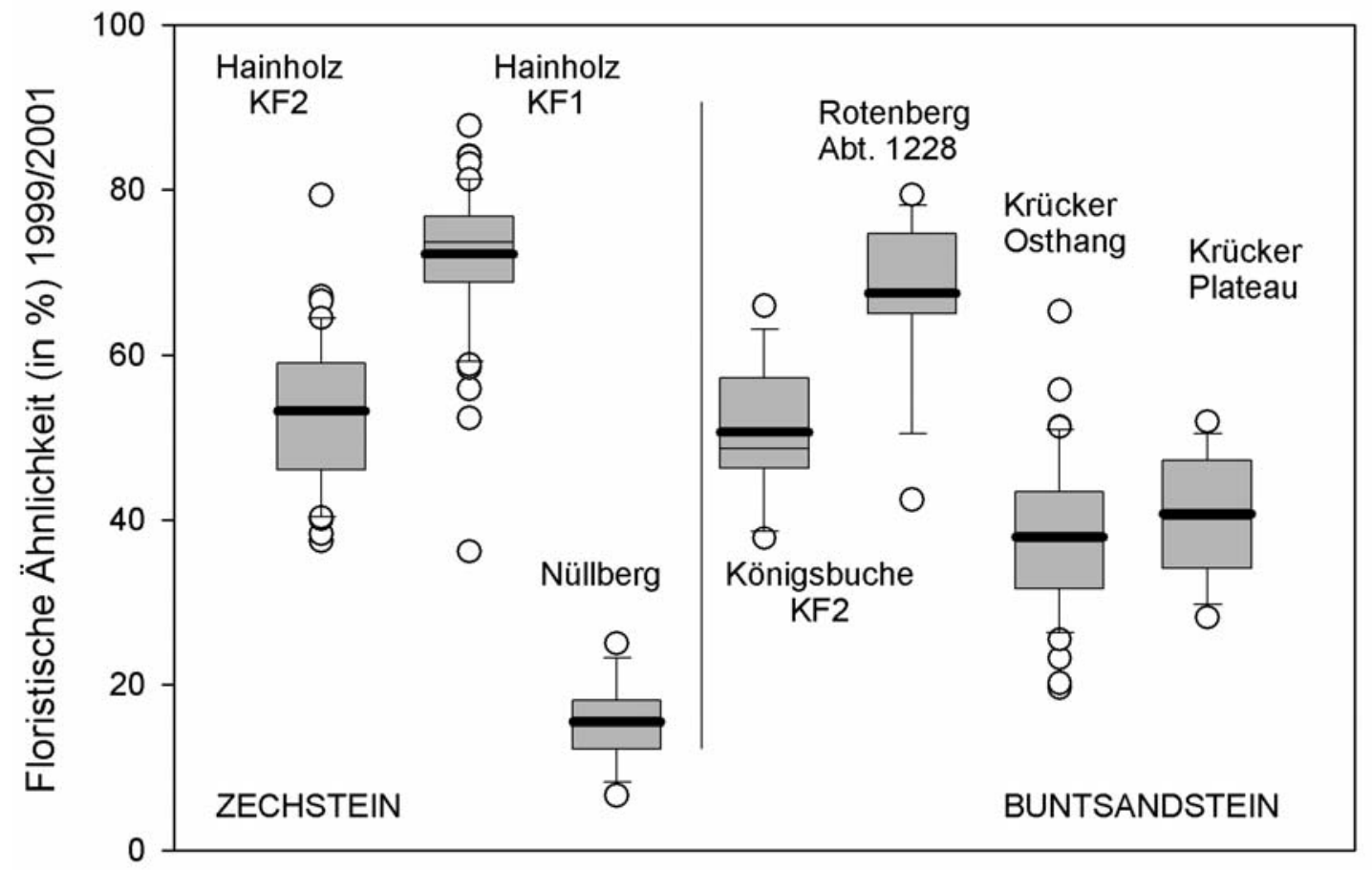

Abb. 26: Floristische Ähnlichkeit nach dem qualitativen Jaccard-Index (ohne Berücksichtigung der Deckungsgrade, oben) und dem quantitativen Index Percentage Similarity (unter Berücksichtigung der Deckungsgrade, unten) im Vergleich der Jahre 1999 und 2001 in Buchen-Windwurfgebieten mit Flächenwurf im südwestlichen Harzvorland; Anzahl einbezogener 100 m²-Plots siehe Tab. 1; Boxplot-Darstellung wie in Abb. 17. 
Für den quantitativen Vergleich unter Einbeziehung der Deckungsgrade wurde der Index Percentage Similarity (PILOU 1984) ausgewählt, der bei maximal möglicher Ähnlichkeit 100\% erreicht (Abb. 26 unten). Hierbei erscheinen die vier Buntsandstein-Standorte weniger einheitlich als bei qualitativer Betrachtung. Die beiden Krücker-Varianten erfuhren mit Werten um die 40\% eine relativ starke Veränderung zwischen 1999 und 2001, die Königsbuchen-KF2 liegt mit ca. 50\% im Mittelfeld und die geräumten Flächen im Rotenberg haben sich mit rund 70\% Ähnlichkeit recht wenig verändert. Bei den Zechstein-Standorten ergibt sich eine ähnliche Aussage wie bei qualitativer Betrachtung: Der Nüllberg besitzt mit Werten von rund 15\% eine sehr geringe Ähnlichkeit im Vergleich zum Ausgangszustand, die Hainholz-KF1 mit 70\% eine vergleichsweise große. Die Hainholz-KF2 liegt mit 50\% Ähnlichkeit dazwischen. So kann bei quantitativer Betrachtung variantenübergreifend folgende Einteilung getroffen werden:

- geringe floristische Veränderung (Hainholz-KF1, Rotenberg-Abt. 1228);

- mittelstarke floristische Veränderung (Hainholz-KF2, Königsbuche-KF2);

- starke floristische Veränderung (Krücker);

- sehr starke floristische Veränderung (Nüllberg).

\subsubsection{Hauptkomponentenanalyse (PCA) als Darstellungsmöglichkeit von sukzessionalen Veränderungen}

Entsprechend Abb. 27 bestimmen Allium ursinum, Galeobdolon luteum und die Esche (als Strauchschichtart) hauptsächlich die Achse 1 im Positivbereich und damit die beiden Dolomit-Standorte (Hainholz-KF1, Nüllberg). Juncus effusus, Oxalis acetosella und Rubus idaeus (als Strauchschichtart) bestimmen die Achse 1 im Negativbereich (die Himbeere auch den Positivbereich von Achse 2) und kennzeichnen den Zustand am Ende der Untersuchungen hauptsächlich im Buntsandstein. Impatiens parviflora ist eng mit dem Negativbereich von Achse 2 verknüpft und charakterisiert die Entwicklung auf dem Bollerkopf (Hainholz-KF2) und den Ausgangszustand der meisten Buntsandstein-Flächen. Damit ist die standörtliche Dreiteilung in flachgründige Gips/Dolomit-, tiefgründige Dolomit- und in BuntsandsteinStandorte gut sichtbar (Kap. 4.1.1). Allium ursinum bleibt unverändert die mit Abstand dominanteste Art im Sukzessionsgeschehen der Hainholz-KF1, was durch die relativ eng beieinander liegenden Symbole in der Nähe der Allium-Vektorpfeilspitze angezeigt wird. Die zunehmende Bedeutung der Esche wird hieraus weniger gut ersichtlich, welche im Jahr 2001 zusammen mit Galeobdolon luteum (beide jeweils mehr als 40\% Deckung) an zweiter Stelle liegt. Die Nüllberg-Flächen verändern sich etwas stärker (Bewegung entlang Achse 2 durch starke Deckungszunahme von Rubus idaeus) und werden in 2001 etwa gleichermaßen von Esche, Himbeere und Bärlauch dominiert (Tab. A22). Die nahezu unveränderte Positionierung der beiden (Jahres-)Symbole der Hainholz-KF2 um die ImpatiensVektorpfeilspitze herum deutet auf die mindestens vier Jahre andauernde SpringkrautDominanz auf dem Bollerkopf hin. Tatsächlich gab es dort trotz des in 2001 eintretenden 
Abundanzrückganges von Impatiens und der hohen Sukzessionsdynamik (siehe Artenzahlen, Evenness in Kap. 4.2.1 und 4.2.2; Ähnlichkeitsindizes in Kap. 4.2.8) keine einzige Art, die mehr als die Hälfte des Impatiens-Deckungsgrades erreichte. Am nächsten lagen noch Mycelis muralis und Atropa bella-donna im Jahr 1999 (Tab. A22), die aber programmbedingt keine Berücksichtigung in der Grafik finden. Ebenfalls zu dieser Impatiens-dominierten Punktwolke gehören die 1999er Sukzessionsstadien von Krücker und Königsbuche, die sich anschließend etwa gleichgerichtet zu unterschiedlich stark durch Himbeere dominierten Pionierstrauchfluren weiterentwickeln. Der Unterschied in den Rubus-Deckungen zwischen den beiden Rotenberg-Flächen (2001: über 60\%) und den beiden Krücker-Flächen (2001: über $40 \%$ ) drückt sich in einer entsprechend unterschiedlichen Positionierung entlang des Rubus-Vektors aus. Andere bedeutsame, aber niedriger deckende Arten wie Oxalis acetosella, Juncus effusus oder Calamagrostis epigejos werden in der Grafik nicht angezeigt, weil sie

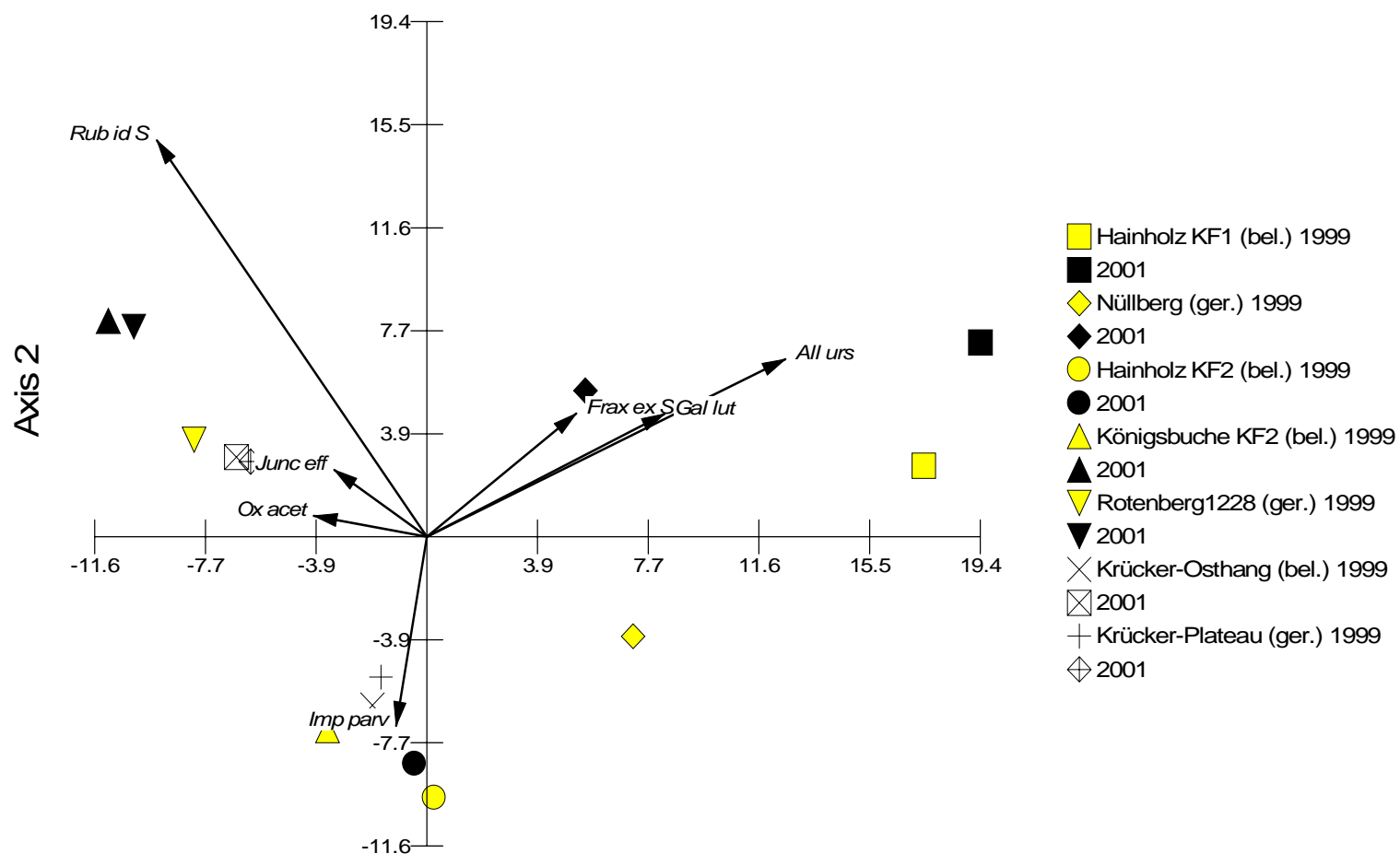

Axis 1

Vector scaling: 19,74

Abb. 27: Hauptkomponentenanalyse (PCA) von Buchen-Windwurfgebieten mit Flächenwurf im südwestlichen Harzvorland im Vergleich der Jahre 1999 und 2001; Euklidischer Biplot unter Verwendung mittlerer Deckungsgrade (entsprechend Tab. A22 und A23); Achse 1 und 2 erklären 79\% der Varianz; bel. = belassen, ger. = geräumt.

eine vergleichsweise geringe Rolle spielen. Gut sichtbar ist auch die geringe Veränderung der bewirtschafteten Rotenberg-Abt. 1228 (relativ kurzer Weg entlang des Rubus-Vektors) im Vergleich zur belassenen Königsbuche (relativ langer Weg). Eine erstaunlicherweise fast identische Entwicklung nahmen das geräumte Krücker-Plateau und der belassene KrückerOsthang, was auch anhand anderer Parameter (siehe Kap. 4.3) gezeigt werden konnte. 
Die in Abb. 28 dargestellte PCA-Grafik verdeutlicht diese Aussage noch einmal für drei UG mit belassenem Flächenwurf im lückenlosen Verlauf der ersten vier Sukzessionsjahre. Die Achsen sind im Prinzip durch die gleichen Arten bestimmt wie in Abb. 27. Dabei haben die Arten ähnliche Relevanz. Durch die abweichende Versuchsflächenauswahl kommt eine stärkere Korrelation von Rubus, Oxalis und Juncus zu Achse 2 (Positivbereich) und damit zur Entwicklung auf Buntsandstein zustande. Als neu angezeigte Art spielt Carex sylvatica für die Erklärung der Dominanzverhältnisse sowohl auf Dolomit als auch auf Buntsandstein eine Rolle. Die beiden Zechstein-Standorte lassen bei den hochdeckenden Arten keinen Wechsel erkennen, bleiben weiter von Allium ursinum (Hainholz-KF1) bzw. Impatiens parviflora (Hainholz-KF2) dominiert und bewegen sich auch kaum entlang der Vektoren oder Achsen. Die Flächen in der Königsbuche dagegen vollziehen eine deutliche „Wanderbewegung“ vom Impatiens-Dominanzstadium (1998) über Juncus- und Oxalis- bestimmte Muster (1999 und 2000) bis hin zu den auch physiognomisch markanten Himbeer-Fluren (2001). Ein Blick in Tab. A23 zeigt, dass Rubus idaeus (als Strauchschichtart) tatsächlich erst im vierten Sukzessionsjahr zur alles beherrschenden Spezies wird.

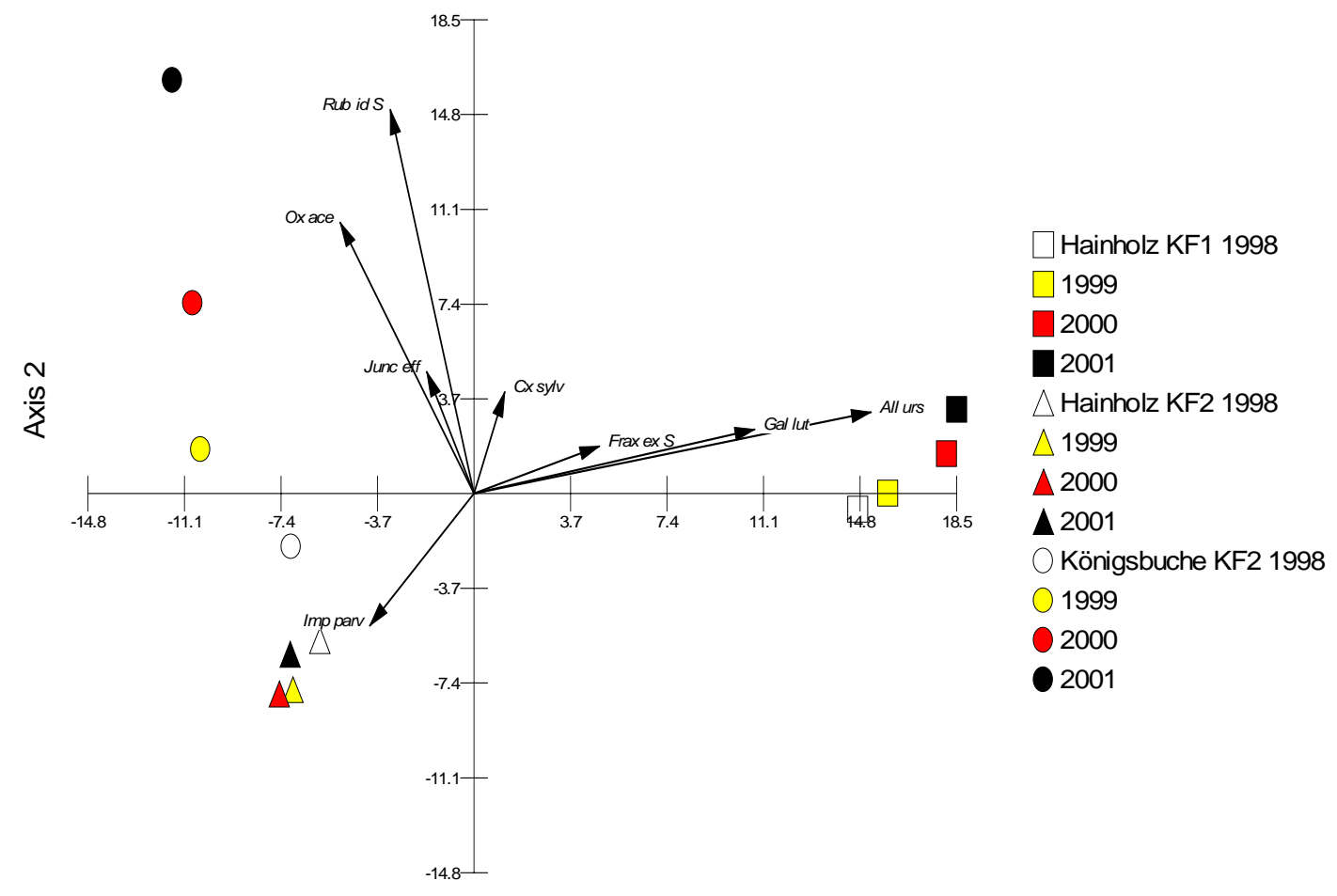

Axis 1

Vector scaling: 20,98

Abb. 28: Hauptkomponentenanalyse (PCA) von drei Buchen-Windwurfgebieten mit Flächenwurf im südwestlichen Harzvorland im Verlauf der Jahre 1998 bis 2001; Euklidischer Biplot unter Verwendung mittlerer Deckungsgrade (entsprechend Tab. A22 und A23); Achse 1 und 2 erklären $87 \%$ der Varianz. 
Anmerkung zu Ähnlichkeitsindizes und PCA: Die durch die PCA und die durch Ähnlichkeitsindizes gewonnenen Aussagen weichen oft voneinander ab, da sich die PCA besonders stark an den dominantesten Arten orientiert. So ist beispielsweise die „sehr starke floristische Veränderung“ auf dem Nüllberg (Kap. 4.2.8) hauptsächlich durch Arten im unteren Deckungsgradbereich bedingt, während viele der dominanten Arten im Sukzessionsverlauf konstant bleiben (relativ kurze Bewegung entlang Achse 2 in Abb. 27). Andererseits ist z.B. die Entwicklung in der Königsbuchen-KF2 durch eine enorme Deckungsgrad“explosion“ der Himbeere in der Strauchschicht gekennzeichnet (von 0 auf $65 \%$ in vier Jahren), was sich jedoch bei den Ähnlichkeitsindizes nicht so deutlich widerspiegelt (Kap. 4.2.8). Übereinstimmende Aussagen ergeben sich dagegen z.B. für die Hainholz-KF1 und die Rotenberg-Abt. 1228 (geringe floristische Veränderung).

\subsection{Qualitative (ungewichtete) und quantitative (gewichtete) Betrachtung ausgewählter vegetationsökologischer Parameter auf den Standorten mit Flächenwurf im Vergleich der Jahre 1999 und 2001}

Das Arteninventar der Untersuchungsflächen (Tab. A22, A23) wurde entsprechend der 11 hier verwendeten Untersuchungsparameter in ökologisch-funktionale Artengruppen unterteilt und mit Hilfe qualitativer und quantitativer Artengruppenspektren - bezogen auf 100\% (d.h. relativ) - dargestellt. Um Dominanz- und Artenwechsel voneinander unterscheiden zu können, empfiehlt sich zusätzlich die Betrachtung der absoluten Artenzahlen (indirekt aus Abb. 17 abzuleiten) und absoluten Deckungssummen dieser Gruppen (Tab. A22, A23).

\subsection{1 Ökologisches Verhalten nach Lichtzeigerwert (ELLENBERG et al. 1992)}

Trotz steigender Gesamtartenzahlen gibt es im qualitativen Artenspektrum der Lichtzeigerwerte kaum Veränderungen zwischen 1999 und 2001 (Tab. 3). In den meisten UG halten sich Licht- (L7-9) und Halbschattenzeiger (L4-6) mit Anteilen von jeweils rund einem Drittel in etwa die Waage. Schattenzeiger (L1-3) kommen mit rund einem Drittel Anteil besonders häufig in der Hainholz-KF1 und in der Königsbuchen-KF2 vor. In der HainholzKF2 ist ihr Anteil mit nur 8-9\% besonders gering, dafür dominieren dort die Lichtzeiger mit über 50\%. Aufgrund der geringen Wüchsigkeit dieses Standortes (flachgründige Gips/Dolomit-Böden in Kuppenlage) dürften auch schon vor dem Sturm viele Lichtzeiger im hier vorherrschenden Carici-Fagetum vertreten gewesen sein. Indifferente Arten kommen kaum vor.

Bei Betrachtung der Abundanzanteile fällt zunächst auf, dass die Buntsandstein-Standorte im vierten Jahr nach dem Sturm (2001) mit 57-64\% deutlich stärker von Lichtzeigern (L7-9) geprägt werden als die Zechstein-Standorte mit 11-40\% (Abb. 29). Mit Ausnahme der Rotenberg-Abt. 1228 (dort Stagnation) nehmen die Lichtzeiger überall relativ und absolut zu, wobei diese Artengruppe in zwei UG (Hainholz-KF2, Nüllberg) ihre Abundanzanteile 
verdoppeln, in einem (Königsbuche-KF2) sogar verdreifachen kann (Abb. 29 und Tab. A22, A23). Auffällig ist das im Jahr 1999 geringe Ausgangsniveau an Lichtzeigern auf den Zechstein-Standorten (7-19\%), aber auch in der seit langem unbewirtschafteten Königsbuche (21\%) im Gegensatz zu den langjährigen Wirtschaftswäldern im Buntsandstein (49-57\%).

Tab. 3: Qualitatives Licht-Zeigerwertspektrum der Arten in Buchen-Windwurfgebieten mit Flächenwurf im südwestlichen Harzvorland in den Jahren 1999 und 2001; Strauch- und Feldschicht zusammengefasst; Anzahl einbezogener $100 \mathrm{~m}^{2}$-Plots siehe Tab. 1; Angaben gerundet in \%.

\begin{tabular}{|l|c|c|c|c|c|c|c|c|c|c|c|c|c|c|}
\hline & \multicolumn{2}{|c|}{ Hhz KF2 } & \multicolumn{2}{c|}{ Hhz KF1 } & \multicolumn{2}{c|}{ Nüllberg } & \multicolumn{2}{c|}{ Köbu KF2 } & \multicolumn{2}{c|}{ Ro1228 } & \multicolumn{2}{c|}{ Krü-Osth. } & Krü-Plat. \\
\hline & $\mathbf{1 9 9 9}$ & $\mathbf{2 0 0 1}$ & $\mathbf{1 9 9 9}$ & $\mathbf{2 0 0 1}$ & $\mathbf{1 9 9 9}$ & $\mathbf{2 0 0 1}$ & $\mathbf{1 9 9 9}$ & $\mathbf{2 0 0 1}$ & $\mathbf{1 9 9 9}$ & $\mathbf{2 0 0 1}$ & $\mathbf{1 9 9 9}$ & $\mathbf{2 0 0 1}$ & $\mathbf{1 9 9 9}$ & $\mathbf{2 0 0 1}$ \\
\hline $\begin{array}{l}\text { Schattenzeiger } \\
\text { [L1-3] }\end{array}$ & 9 & 8 & 29 & 29 & 17 & 15 & 34 & 31 & 24 & 23 & 20 & 14 & 16 & 16 \\
\hline $\begin{array}{l}\text { Halbschattenzeiger } \\
\text { [L4-6] }\end{array}$ & 39 & 38 & 39 & 41 & 45 & 42 & 29 & 31 & 33 & 39 & 34 & 37 & 35 & 37 \\
\hline $\begin{array}{l}\text { Lichtzeiger } \\
\text { [L7-9] }\end{array}$ & 51 & 53 & 29 & 23 & 36 & 40 & 35 & 32 & 33 & 30 & 42 & 43 & 44 & 43 \\
\hline indifferente & 1 & 2 & 3 & 6 & 2 & 3 & 2 & 6 & 10 & 8 & 4 & 6 & 5 & 5 \\
\hline
\end{tabular}

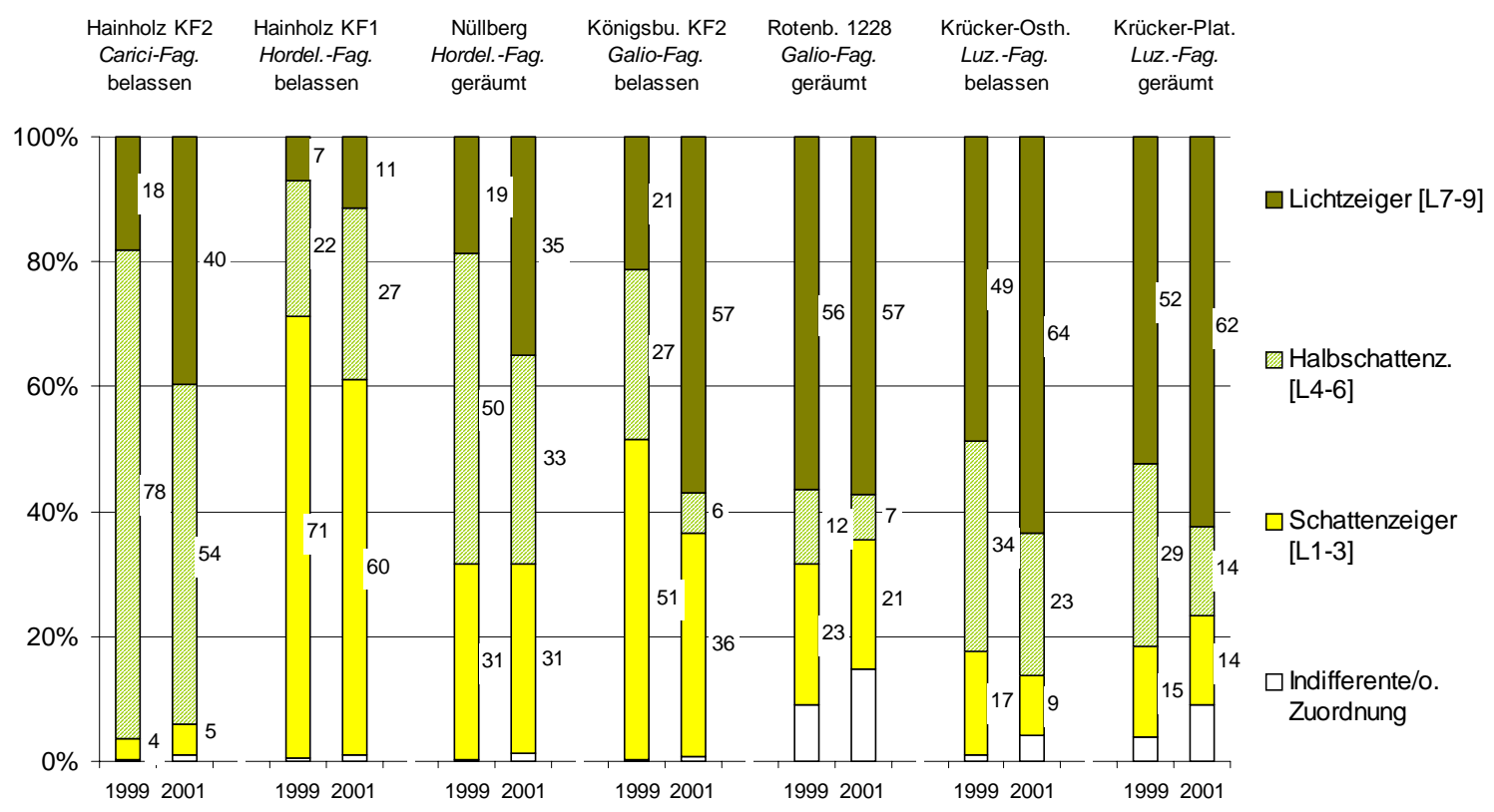

Abb. 29: Quantitatives Licht-Zeigerwertspektrum der Arten von Buchen-Windwurfgebieten mit Flächenwurf im südwestlichen Harzvorland in den Jahren 1999 und 2001; Strauch- und Feldschicht zusammengefasst; Anzahl einbezogener $100 \mathrm{~m}^{2}$-Plots siehe Tab. 1; Werte unter 3\% nicht angezeigt.

Parallel dazu stagnieren die Abundanzanteile der waldtypischen Schattenzeiger oder nehmen ab, während ihre absoluten Deckungssummen jedoch zunehmen oder ebenfalls stagnieren (Tab. A22, A23). Die Hainholz-KF2 und der Krücker-Osthang sind im Jahr 2001 durch besonders niedrige Anteile an Schattenzeigern charakterisiert (nur 5 bzw. 9\%), die HainholzKF1 durch besonders hohe (60\%). Der Nüllberg bzw. die Königsbuchen-KF2 liegen dazwischen (31 bzw. 36\%). Die Hainholz-KF2 stellt insofern einen Sonderfall dar, indem sie als einzige Untersuchungsfläche von Halbschattenpflanzen (L4-6) dominiert wird. 


\subsection{2 Ökologisches Verhalten nach Feuchtezeigerwert (ELLENBERG et al. 1992)}

Das qualitative Zeigerwertspektrum wird in allen sieben UG von Frischezeigern (F4-6) beherrscht, welche Anteile von zwei Dritteln bis drei Vierteln erreichen (Tab. 4). Ausgesprochene Feuchtezeiger (F7-9) sind im vierten Jahr nach dem Sturm im Buntsandstein mit Anteilen von 15-17\% häufiger vertreten als in den Zechstein-Gebieten mit 6-12\%. Ausgesprochene Trockenheitszeiger (F1-3) kommen mit minimalen Anteilen von 1-3\% nur in der Hainholz-KF2 und im Krücker vor. Daneben existiert eine Gruppe von indifferenten Arten, die überall rund 20\% Anteil erreicht. Im Vergleich der Jahre 1999 und 2001 verändern sich diese Verhältnisse trotz steigender Gesamtartenzahlen kaum.

Tab. 4: Qualitatives Feuchte-Zeigerwertspektrum der Arten in Buchen-Windwurfgebieten mit Flächenwurf im südwestlichen Harzvorland in den Jahren 1999 und 2001; Strauch- und Feldschicht zusammengefasst; Anzahl einbezogener $100 \mathrm{~m}^{2}$-Plots siehe Tab. 1; Angaben gerundet in \%.

\begin{tabular}{|l|c|c|c|c|c|c|c|c|c|c|c|c|c|c|c|}
\hline & Hhz KF2 & \multicolumn{2}{c|}{ Hhz KF1 } & \multicolumn{2}{c|}{ Nülberg } & \multicolumn{2}{|c|}{ Köbu KF2 } & \multicolumn{2}{|c|}{ Ro1228 } & \multicolumn{2}{|c|}{ Krü-Osth. } & \multicolumn{2}{|c|}{ Krü-Plat. } \\
\hline & $\mathbf{1 9 9 9}$ & $\mathbf{2 0 0 1}$ & $\mathbf{1 9 9 9}$ & $\mathbf{2 0 0 1}$ & $\mathbf{1 9 9 9}$ & $\mathbf{2 0 0 1}$ & $\mathbf{1 9 9 9}$ & $\mathbf{2 0 0 1}$ & $\mathbf{1 9 9 9}$ & $\mathbf{2 0 0 1}$ & $\mathbf{1 9 9 9}$ & $\mathbf{2 0 0 1}$ & $\mathbf{1 9 9 9}$ & $\mathbf{2 0 0 1}$ \\
\hline $\begin{array}{l}\text { Trockenheitszeiger } \\
\text { [F1-3] }\end{array}$ & 2 & 3 & 0 & 0 & 0 & 0 & 0 & 0 & 0 & 0 & 1 & 2 & 1 & 0 \\
\hline $\begin{array}{l}\text { Frischezeiger } \\
\text { [F4-6] }\end{array}$ & 74 & 72 & 72 & 67 & 77 & 75 & 67 & 62 & 62 & 58 & 64 & 60 & 65 & 61 \\
\hline $\begin{array}{l}\text { Feuchtezeiger } \\
\text { [F7-9] }\end{array}$ & 5 & 6 & 11 & 12 & 7 & 9 & 20 & 15 & 16 & 17 & 18 & 17 & 14 & 16 \\
\hline indifferente & 19 & 18 & 16 & 21 & 15 & 17 & 13 & 23 & 22 & 25 & 18 & 21 & 20 & 23 \\
\hline
\end{tabular}

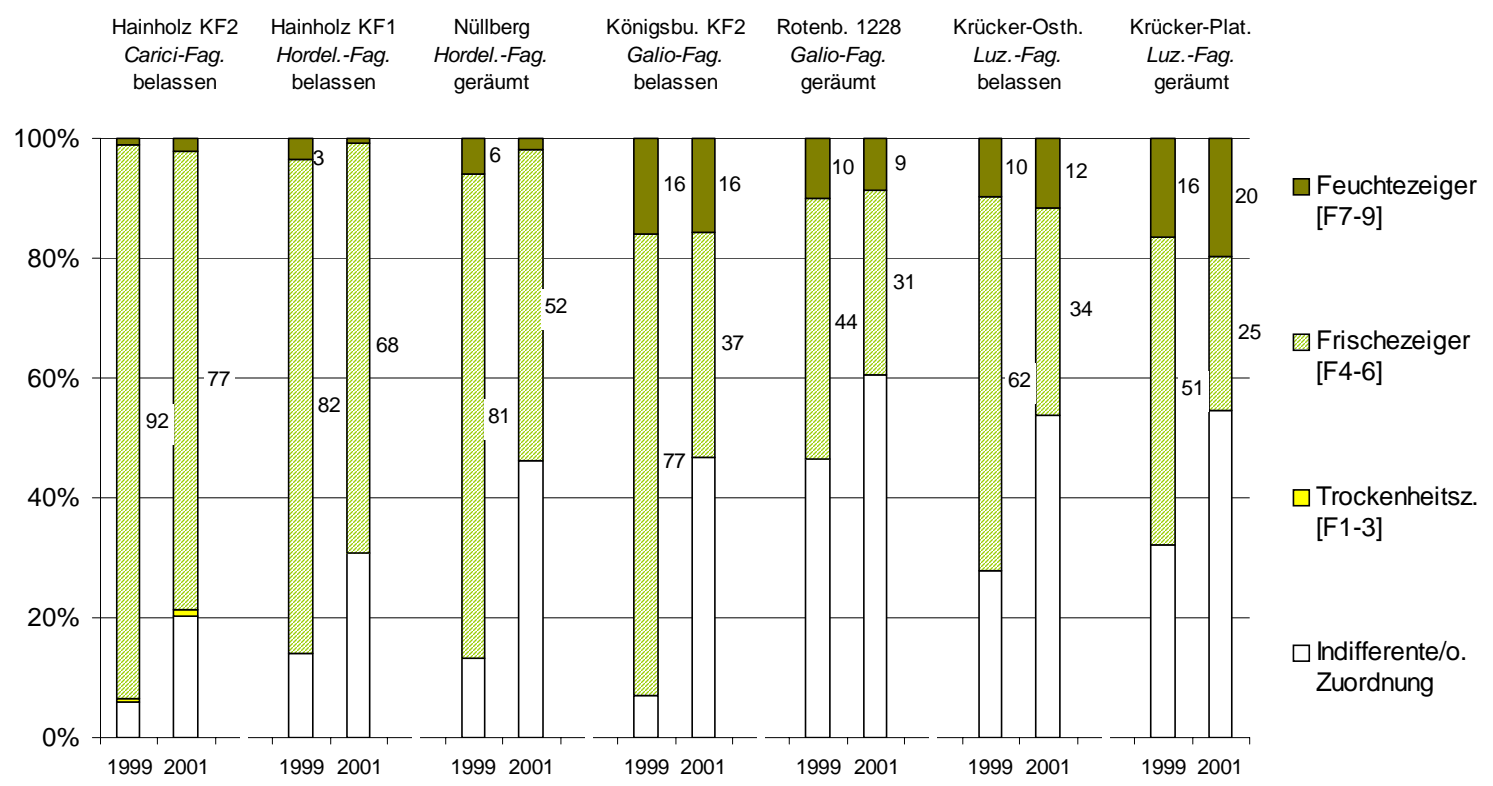

Abb. 30: Quantitatives Feuchte-Zeigerwertspektrum der Arten von BuchenWindwurfgebieten mit Flächenwurf im südwestlichen Harzvorland in den Jahren 1999 und 2001; Strauch- und Feldschicht zusammengefasst; Anzahl einbezogener $100 \mathrm{~m}^{2}$-Plots siehe Tab. 1; Werte unter 3\% nicht angezeigt.

Das quantitative Spektrum weist im Sukzessionsverlauf für alle UG eine starke absolute und relative Expansion indifferenter Arten aus, welche im Jahr 2001 - ausser im Hainholz - zur 
Dominanz gelangen (Abb. 30). Diese Entwicklung geht bei relativer Betrachtung zu Lasten der Frischezeiger, wobei diese im Hainholz ihre absoluten Deckungssummen sogar noch leicht erweitern können, während sie in den übrigen UG sowohl relativ als auch absolut zurückgedrängt werden (Tab. A22, A23). Die Feuchtezeiger verändern ihre Abundanzanteile nicht oder nur wenig (auf Dolomit eher Abnahme, im Buntsandstein eher Zunahme), wobei der Unterschied zwischen den Feuchtezeiger-reicheren Buntsandstein-Gebieten und den diesbezüglich ärmeren Zechstein-Gebieten noch deutlicher hervortritt als bei nur qualitativer Betrachtung. Trockenheitszeiger treten mengenmäßig fast gar nicht in Erscheinung.

\subsection{3 Ökologisches Verhalten nach Reaktionszeigerwert (ELLENBERG et al. 1992)}

Erwartungsgemäß sind die Zechstein-Standorte sowohl qualitativ als auch quantitativ relativ stark von Basenzeigern (R7-9) bestimmt (Tab. 5; Abb. 31). Mit 77 bzw. 49\% Abundanzanteil dominiert diese Artengruppe in den beiden UG mit Dolomit-Verwitterungsböden (HainholzKF1, Nüllberg), während die Hainholz-KF2 von indifferenten Arten beherrscht wird. Die Basenzeiger erreichen dort nur ein knappes Drittel Abundanzanteil. Stärkere Säurezeiger (R13) kommen dagegen fast nur im Buntsandstein vor (qualitative Anteile 4-13\%, quantitative Anteile 2-16\%). Charakteristisch für die basenarmen Standorte sind weiterhin die Dominanz indifferenter Arten und ein relativ starker Anteil von Mäßigsäurezeigern (R4-6), der in der Köngsbuchen-KF2 im Jahr 2001 mit 34\% den höchsten Wert dieser Artengruppe unter allen UG hält. In dem seit langem sich selbst überlassenen Naturwald Königsbuche fällt ausserdem der mit 1\% vergleichsweise minimale Abundanzanteil von Basenzeigern auf, während er in den bis zum Sturm bewirtschafteten Buntsandstein-Gebieten (Rotenberg-Abt. 1228, Krücker) zwischen 5 und 8\% erreicht. Möglicherweise sind dafür die im Wirtschaftswald erfolgten Kalkungsmaßnahmen verantwortlich.

Tab. 5: Qualitatives Reaktions-Zeigerwertspektrum der Arten in Buchen-Windwurfgebieten mit Flächenwurf im südwestlichen Harzvorland in den Jahren 1999 und 2001; Strauch- und Feldschicht zusammengefasst; Anzahl einbezogener $100 \mathrm{~m}^{2}$-Plots siehe Tab. 1; Angaben gerundet in \%.

\begin{tabular}{|l|c|c|c|c|c|c|c|c|c|c|c|c|c|c|c|}
\hline & Hhz KF2 & \multicolumn{2}{c|}{ Hhz KF1 } & \multicolumn{2}{c|}{ Nüllberg } & \multicolumn{2}{c|}{ Köbu KF2 } & \multicolumn{2}{|c|}{ Ro1228 } & \multicolumn{2}{|c|}{ Krü-Osth. } & Krü-Plat. \\
\hline & $\mathbf{1 9 9 9}$ & $\mathbf{2 0 0 1}$ & $\mathbf{1 9 9 9}$ & $\mathbf{2 0 0 1}$ & $\mathbf{1 9 9 9}$ & $\mathbf{2 0 0 1}$ & $\mathbf{1 9 9 9}$ & $\mathbf{2 0 0 1}$ & $\mathbf{1 9 9 9}$ & $\mathbf{2 0 0 1}$ & $\mathbf{1 9 9 9}$ & $\mathbf{2 0 0 1}$ & $\mathbf{1 9 9 9}$ & $\mathbf{2 0 0 1}$ \\
\hline $\begin{array}{l}\text { Säurezeiger } \\
\text { [R1-3] }\end{array}$ & 0 & 0 & 1 & 1 & 1 & 1 & 8 & 9 & 5 & 4 & 13 & 11 & 13 & 11 \\
\hline $\begin{array}{l}\text { Mäßigsäurezeiger } \\
\text { [R4-6] }\end{array}$ & 17 & 19 & 19 & 21 & 17 & 17 & 42 & 42 & 34 & 32 & 31 & 28 & 31 & 31 \\
\hline $\begin{array}{l}\text { Basenzeiger } \\
\text { [R7-9] }\end{array}$ & 40 & 42 & 50 & 46 & 44 & 41 & 11 & 10 & 9 & 13 & 14 & 16 & 15 & 17 \\
\hline indifferente & 44 & 39 & 31 & 32 & 39 & 41 & 38 & 39 & 51 & 51 & 41 & 44 & 41 & 42 \\
\hline
\end{tabular}

Was die Sukzessionsdynamik betrifft, so sind von 1999 zu 2001 im qualitativen Spektrum trotz steigender Artenzahlen keine wesentlichen Veränderungen auszumachen. Bei den Abundanzanteilen gibt es nur geringfügige Tendenzen. Mit Ausnahme der Hainholz-KF2 
breiten sich indifferente Arten überall aus, was auf den beiden Dolomit-Standorten zu Lasten der Basenzeiger (besonders im Nüllberg: Rückgang von 70 auf 49\% Anteil) und im Buntsandstein zu Lasten der Mäßigsäurezeiger (besonders in Königsbuche-KF2: Rückgang von 51 auf 34\%) geht. Diese Rückgänge sind nur relativ auszumachen, denn die betroffenen Artengruppen erweitern ihre absoluten Deckungssummen eher noch (Tab. A22, A23).

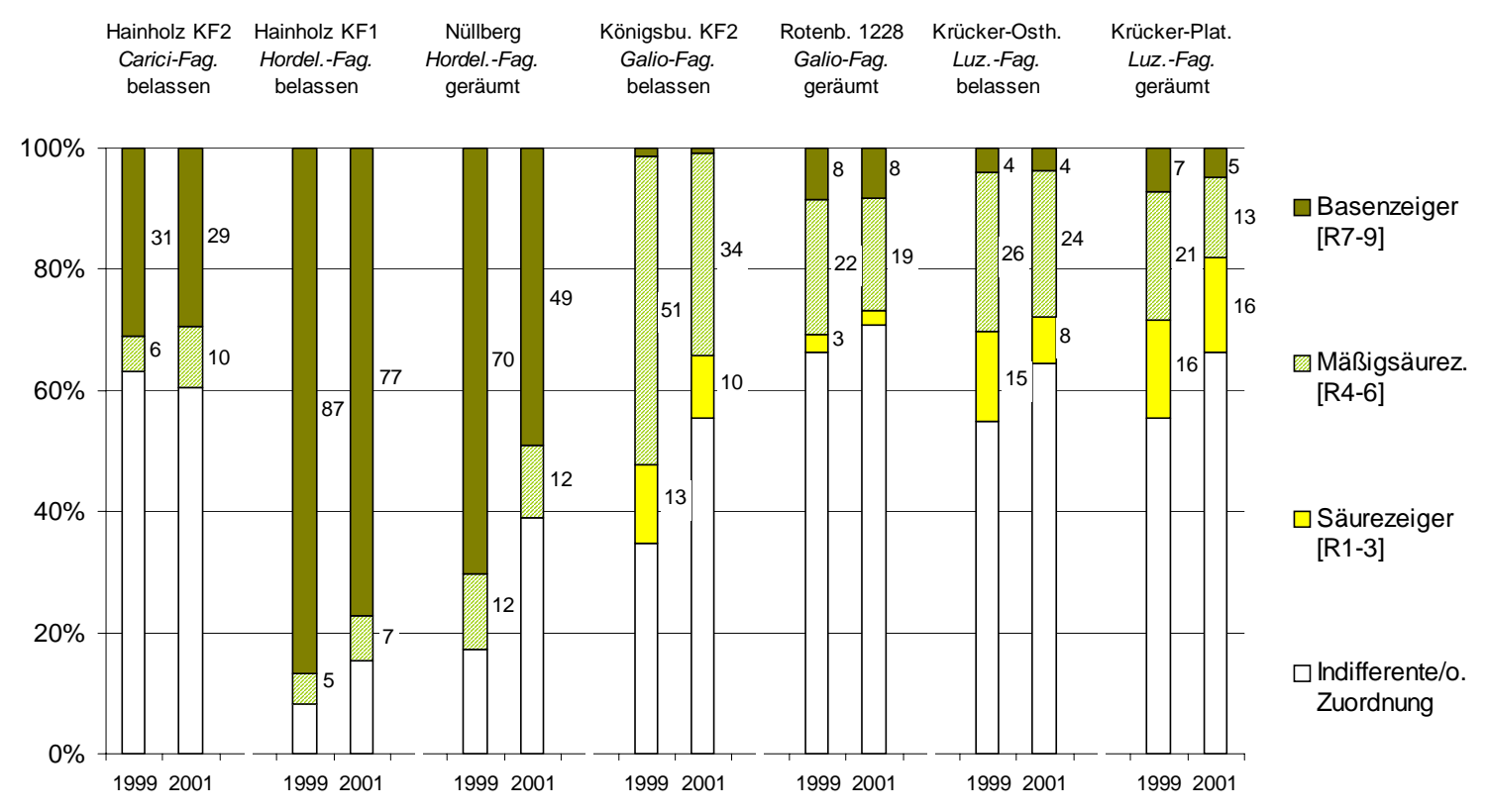

Abb. 31: Quantitatives Reaktions-Zeigerwertspektrum der Arten von BuchenWindwurfgebieten mit Flächenwurf im südwestlichen Harzvorland in den Jahren 1999 und 2001; Strauch- und Feldschicht zusammengefasst; Anzahl einbezogener $100 \mathrm{~m}^{2}$-Plots siehe Tab. 1; Werte unter 3\% nicht angezeigt.

\subsection{4 Ökologisches Verhalten nach Stickstoffzeigerwert (ELLENBERG et al. 1992)}

Sowohl bei qualitativer als auch bei quantitativer Betrachtung ergibt sich über den StickstoffFaktor eine Standortsdifferenzierung zwischen Zechstein- und Buntsandstein-Gebieten (Tab. 6 und Abb. 32). Während die basenreichen Standorte eher von Stickstoffzeigern (N7-9) dominiert werden, ist im Buntsandstein ein Vorherrschen von Mäßigstickstoffzeigern (N4-6) indiziert. In der Sukzessionsserie ergeben sich kaum nennenswerte Verschiebungen der hier beschriebenen Dominanzverhältnisse, d.h. sowohl die absoluten Artenzahlen als auch die Deckungssummen der einzelnen Artengruppen steigen ungefähr gleichmäßig an. Unter Einbeziehung der Deckungsgrade (quantitatives Spektrum, Abb. 32) sind die standörtlichen Unterschiede aber besonders deutlich sichtbar. Dabei fällt innerhalb der basenarmen Standorte auf, dass der seit 30 Jahren aus der Nutzung genommene Naturwald Königsbuche mit nur 3\% Anteil die mit Abstand geringste Beteiligung von Stickstoffzeigern (N7-9) aufweist, während diese Artengruppe z.B. im bis zum Sturm bewirtschafteten Krücker-Osthang immerhin 25\% Anteil (Jahr 2001) erreicht. Stickstoffmangelzeiger (N1-3) kommen im qualitativen 
Artenspektrum mit geringen und leicht steigenden Anteilen in allen UG vor, am deutlichsten etwa in der Königsbuchen-KF2 (Anstieg von 8 auf 15\%), in der Hainholz-KF2(von 5 auf 8\%) und in der Rotenberg-Abt. 1228 (von 6 auf 8\%).

Tab. 6: Qualitatives Stickstoff-Zeigerwertspektrum der Arten in Buchen-Windwurfgebieten mit Flächenwurf im südwestlichen Harzvorland in den Jahren 1999 und 2001; Strauch- und Feldschicht zusammengefasst; Anzahl einbezogener $100 \mathrm{~m}^{2}$-Plots siehe Tab. 1; Angaben gerundet in \%.

\begin{tabular}{|c|c|c|c|c|c|c|c|c|c|c|c|c|c|c|}
\hline & \multicolumn{2}{|c|}{ Hhz KF2 } & \multicolumn{2}{|c|}{ Hhz KF1 } & \multicolumn{2}{|c|}{ Nüllberg } & \multicolumn{2}{|c|}{ Köbu KF2 } & \multicolumn{2}{|c|}{ Ro1228 } & \multicolumn{2}{|c|}{ Krü-Osth. } & \multicolumn{2}{|c|}{ Krü-Plat. } \\
\hline & 1999 & 2001 & 1999 & 2001 & 1999 & 2001 & 1999 & 2001 & 1999 & 2001 & 1999 & 2001 & 1999 & 2001 \\
\hline $\begin{array}{l}\text { Stickstoffmangel- } \\
\text { zeiger [N1-3] }\end{array}$ & 5 & 8 & 2 & 4 & 0 & 3 & 8 & 15 & 6 & 8 & 2 & 3 & 4 & 5 \\
\hline $\begin{array}{l}\text { Mäßigstickstoff- } \\
\text { zeiger [N4-6] }\end{array}$ & 36 & 35 & 35 & 33 & 37 & 36 & 58 & 54 & 48 & 42 & 53 & 49 & 48 & 44 \\
\hline $\begin{array}{l}\text { Stickstoffzeiger } \\
\text { [N7-9] }\end{array}$ & 50 & 46 & 57 & 54 & 54 & 50 & 25 & 19 & 28 & 32 & 36 & 36 & 36 & 38 \\
\hline indifferente & 9 & 12 & 6 & 9 & 8 & 11 & 9 & 12 & 18 & 18 & 10 & 11 & 12 & 13 \\
\hline
\end{tabular}

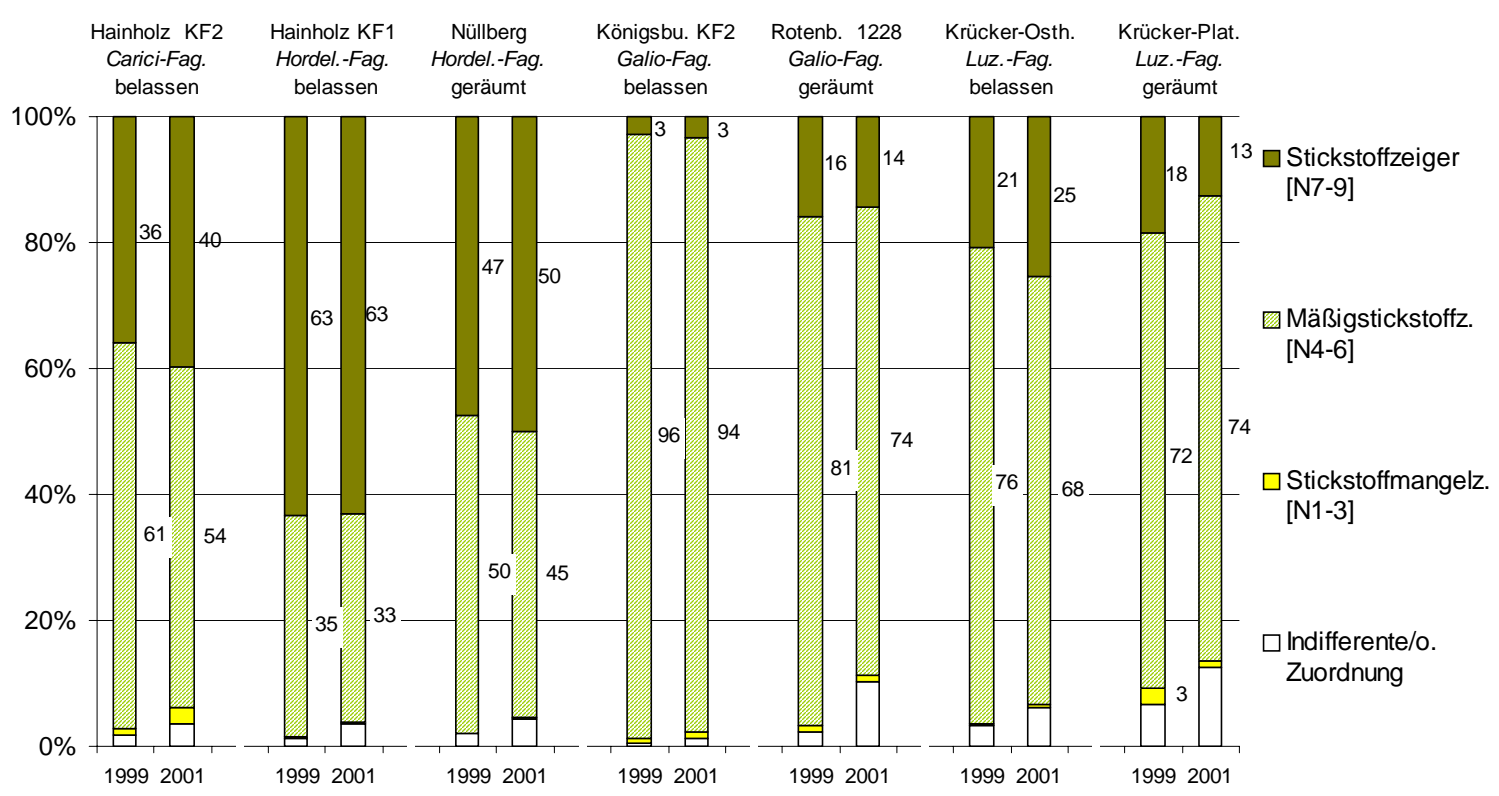

Abb. 32: Quantitatives Stickstoff-Zeigerwertspektrum der Arten von BuchenWindwurfgebieten mit Flächenwurf im südwestlichen Harzvorland in den Jahren 1999 und 2001; Strauch- und Feldschicht zusammengefasst; Anzahl einbezogener $100 \mathrm{~m}^{2}$-Plots siehe Tab. 1; Werte unter 3\% nicht angezeigt.

Quantitativ dagegen spielt diese Artengruppe keine Rolle. Selbst auf dem Bollerkopf (Hainholz-KF2), wo mit 2,4\% Abundanzanteil im Jahr 2001 der höchste Wert erreicht wird, fällt sie im Gesamtspektrum nicht auf. Eine bevorzugte Bindung der Mangelzeiger an Wurzelteller lässt sich nicht ausmachen, denn zwischen den qualitativen Spektren der Untersuchungsflächen und denen der Wurzelteller ergeben sich kaum Unterschiede (siehe Kap. 4.6.7). 


\subsubsection{Pflanzensoziologische Klassen nach ELLENBERG et al. (1992) und OBERDORFER (2001)}

Trotz steigender Artenzahlen verändert sich das qualitative pflanzensoziologische Spektrum vom zweiten zum vierten Sukzessionsjahr kaum und bleiben die sieben UG bezüglich der Zusammensetzung nach pflanzensoziologischen Klassen einander sehr ähnlich (Tab. 7). Die größte Gruppe ist auf allen Standorten die der Laubwald-typischen Querco-Fagetea-Arten. In der Hainholz-KF1 dominieren diese mit 57\% Anteil, in der Hainholz-KF2 liegt mit 33\% der geringste Anteil vor (in 2001). An zweiter Stelle folgen im vierten Sukzessionsjahr entweder die Epilobietea-Arten mit Anteilen zwischen 18 und 27\% (Buntsandstein-Gebiete und Hainholz-KF1) oder die Ruderalarten (Hainholz-KF2, Nüllberg) mit Anteilen zwischen 19 und 24\%. Grünlandarten erreichen meist um die 10\% Anteile, lediglich in der Hainholz-KF1 liegen sie mit 4\% darunter. Saumarten (Trifolio-Geranietea) kommen mit geringen Anteilen von 2-4\% nur in der Hainholz-KF2 (Carici-Fagetum) und auf dem Nüllberg (HordelymoFagetum circaeetosum/lathyretosum) vor.

Tab. 7: Qualitatives pflanzensoziologisches Spektrum der Arten in Buchen-Windwurfgebieten mit Flächenwurf im südwestlichen Harzvorland in den Jahren 1999 und 2001; Strauch- und Feldschicht zusammengefasst; Anzahl einbezogener $100 \mathrm{~m}^{2}$-Plots siehe Tab. 1; Angaben gerundet in \%.

\begin{tabular}{|l|c|c|c|c|c|c|c|c|c|c|c|c|c|c|}
\hline & \multicolumn{2}{|c|}{ Hhz KF2 } & \multicolumn{2}{c|}{ Hhz KF1 } & \multicolumn{2}{c|}{ Nüllberg } & \multicolumn{2}{c|}{ Köbu KF2 } & \multicolumn{2}{c|}{ Ro1228 } & \multicolumn{2}{c|}{ Krü-Osth. } & Krü-Plat. \\
\hline & $\mathbf{1 9 9 9}$ & $\mathbf{2 0 0 1}$ & $\mathbf{1 9 9 9}$ & $\mathbf{2 0 0 1}$ & $\mathbf{1 9 9 9}$ & $\mathbf{2 0 0 1}$ & $\mathbf{1 9 9 9}$ & $\mathbf{2 0 0 1}$ & $\mathbf{1 9 9 9}$ & $\mathbf{2 0 0 1}$ & $\mathbf{1 9 9 9}$ & $\mathbf{2 0 0 1}$ & $\mathbf{1 9 9 9}$ & $\mathbf{2 0 0 1}$ \\
\hline Laubwaldarten & 32 & 33 & 57 & 57 & 51 & 46 & 49 & 46 & 42 & 49 & 43 & 42 & 39 & 43 \\
\hline Schlagflurarten & 20 & 19 & 19 & 18 & 16 & 17 & 23 & 18 & 19 & 21 & 29 & 27 & 25 & 24 \\
\hline Ruderalarten & 29 & 24 & 16 & 14 & 23 & 19 & 10 & 11 & 19 & 13 & 14 & 14 & 15 & 13 \\
\hline Grünlandarten & 8 & 12 & 4 & 4 & 6 & 9 & 15 & 15 & 9 & 8 & 8 & 10 & 11 & 11 \\
\hline Saumarten & 4 & 4 & 0 & 0 & 2 & 2 & 0 & 0 & 0 & 0 & 0 & 0 & 0 & 0 \\
\hline ohne Zuordnung & 6 & 8 & 4 & 7 & 3 & 6 & 5 & 10 & 11 & 10 & 6 & 6 & 10 & 9 \\
\hline
\end{tabular}

Das quantitative pflanzensoziologische Spektrum zeigt eine differenziertere und dynamischere Entwicklung (Abb. 33). Auffällig ist die sich im Buntsandstein im vierten Jahr nach dem Orkan manifestierende Dominanz der Epilobietea-Arten (überwiegend Pioniersträucher wie Himbeere, Holunder und Brombeere, im Krücker auch Calamagrostis epigejos), die je nach Variante Abundanzanteile zwischen 48\% und 69\% erreichen. Die seit langem nicht mehr bewirtschaftete Königsbuche hatte bei dieser Artengruppe sowohl relativ als auch absolut im Jahr 1999 ein viel geringeres Ausgangsniveau als die übrigen Untersuchungsflächen (Tab. A22, A23), die auch schon vor bzw. kurz nach dem Sturm reich an Schlagflurarten waren (9\% Anteil gegenüber 42-52\%). Auch in den Zechstein-Gebieten nehmen die Schlagflurarten zu, erreichen aber mit nur 12-32\% im Jahr 2001 vergleichsweise geringe Anteile. Diese Entwicklung geht in allen UG einerseits zu Lasten der krautigen Ruderalarten (v.a. Artemisietea), die anfangs noch stark vertreten waren (ausser in der Hainholz-KF1) und im zweiten oder dritten Sukzessionsjahr ein vorübergehendes Ausbreitungsmaximum erreichten (hier nicht dargestellt; siehe Tab. A22, A23 und Kap. 4.4.1; 
ferner SCHMidT 2002a). Besonders der Bollerkopf (Hainholz-KF2) war mit 53\% Anteil in den Jahren 1999 und 2000 stark von Ruderalarten geprägt.

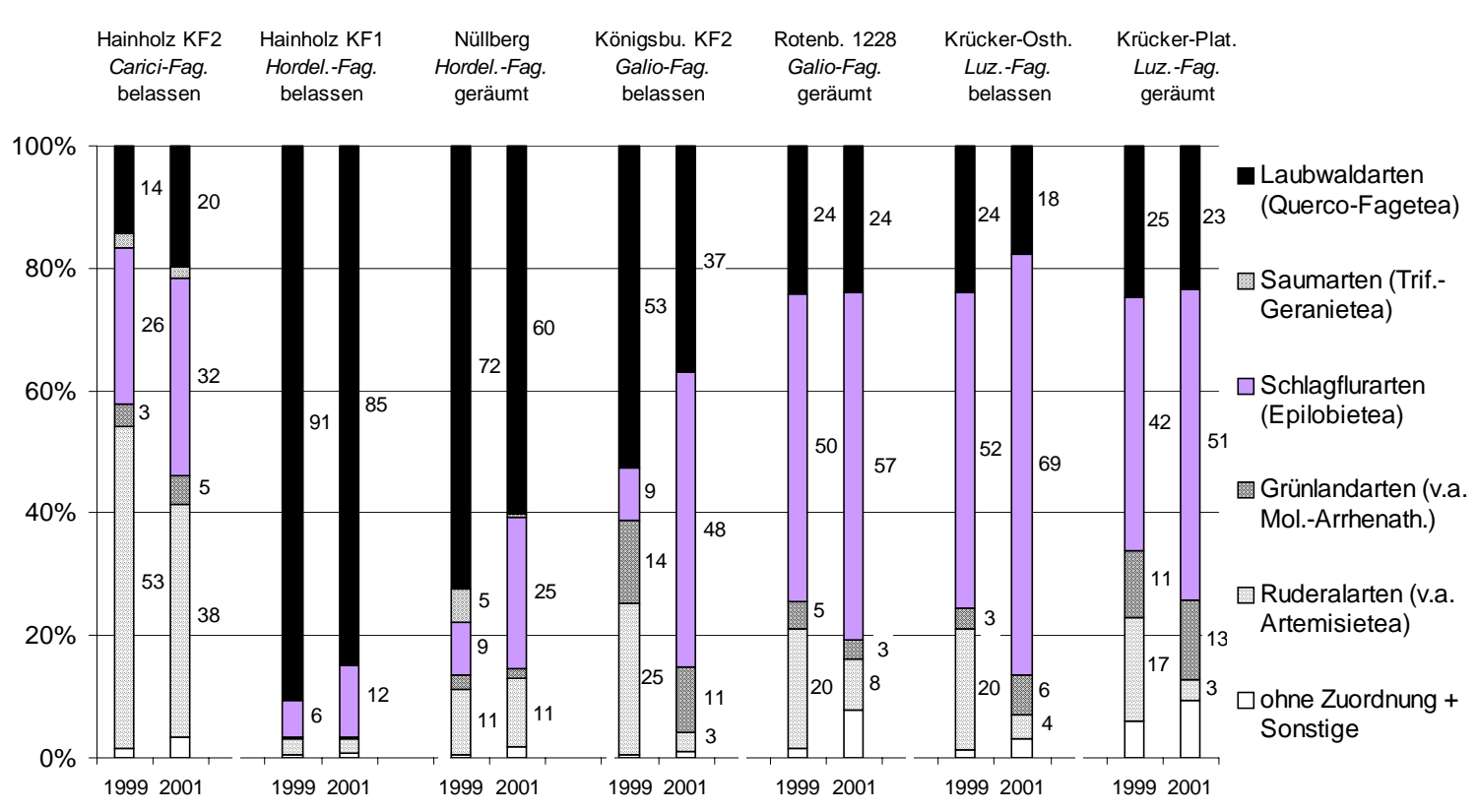

Abb. 33: Quantitatives pflanzensoziologisches (Klassen-)Spektrum der Arten in BuchenWindwurfgebieten mit Flächenwurf im südwestlichen Harzvorland in den Jahren 1999 und 2001; Strauch- und Feldschicht zusammengefasst; Anzahl einbezogener $100 \mathrm{~m}^{2}$-Plots siehe Tab. 1; Werte unter 3\% nicht angezeigt.

Die nachfolgenden Deckungsgradeinbrüche betrafen die in Wäldern nicht seltenen Störungszeiger wie z.B. Impatiens parviflora oder Mycelis muralis. Gleichzeitig verloren die typischen Querco-Fagetea-Arten im Vergleich zum Ausgangszustand überall Abundanzanteile, blieben in ihren absoluten Deckungssummen aber stabil bzw. nahmen vor allem im Zechstein sogar noch zu (Tab. A22, A23). Auf dem Bollerkopf (Hainholz-KF2) eroberten die Waldarten ab dem vierten Sukzessionsjahr bereits wieder Abundanzanteile zurück, nachdem sie von 1998 zu 1999 (hier nicht dargestellt, siehe SCHMIDT 2002) einen besonders drastischen Rückgang von 42 auf 14\% zu verzeichnen hatten. Im HordelymoFagetum (Hainholz-KF1, Nüllberg) behielten sie trotz rückläufiger Anteile weiterhin die Dominanz. Zwischen den standörtlich vergleichbaren Untersuchungsflächen von HainholzKF1 (belassen) und Nüllberg (geräumt \& aufgeforstet) fällt auf, dass die geräumten Plots stärker von Nicht-Waldarten geprägt sind und der relative Rückgang der Querco-FageteaArten dort deutlicher sichtbar ist als bei den belassenen. Einzelne Waldarten (z.B. Fraxinus excelsior, Carex sylvatica, Athyrium filix-femina) profitieren sehr stark von den WindwurfVeränderungen (Tab. A22, A23), andere verringern ihre Deckungsgrade (z.B. Oxalis acetosella, Stachys sylvatica, Circaea lutetiana, Hordelymus europaeus, Euphorbia amygdaloides). Die Grünlandarten (v.a. Molinio-Arrhenatheretea) spielen nur in den Plateaulagen von zwei Buntsandstein-Gebieten (Königsbuche, Krücker-Plateau) mit etwas 
mehr als 10\% Anteil eine größere Rolle, zeigen aber insgesamt kaum Veränderungen in der Sukzession. Die ohnehin seltenen Saumarten (Trifolio-Geranietea) bleiben mengenmäßig bedeutungslos und verschwinden auf dem Nüllberg fast vollständig (Abnahme von 5 auf $<1 \%)$.

Das Bild der hier dargestellten Windwurfgebiete ist im vierten Jahr nach dem Sturm folgendermaßen geprägt: auf den tiefgründigen Dolomit-Standorten (Hainholz-KF1, Nüllberg) vor allem durch krautige und verholzte Waldarten einschließlich einer sehr starken Baumartenverjüngung; auf der flachgründigen Gips/Dolomit-Kuppe (Hainholz-KF2) etwa gleichermaßen durch überwiegend krautige Schlagflur- und Ruderalarten; in den Buntsandstein-Gebieten durch ausgedehnte Pionierstrauchfluren der Epilobietea.

\subsubsection{Wuchsformen (nach eigener Einteilung in Anlehnung an die forstliche Literatur)}

Im qualitativen Spektrum (Tab. 8) fällt zunächst auf, dass die basenreichen Standorte im Gegensatz zu den basenarmen relativ reich an krautigen Arten sind (2001: 51-61\% Anteil gegenüber 29-45\%). Verholzte Lianen (meist nur Clematis vitalba als einzige Art) kommen ausschließlich auf den basenreichen Untersuchungsflächen vor. Dafür sind im Buntsandstein mehr Grasartige (2001: 20-32\% Anteil gegenüber 15-20\%) und Farne (2001: 7-16\% Anteil gegenüber 0-4\%) zu finden als im Zechstein. Sowohl bei den Baum- als auch bei den Straucharten liegt ungefähr ein Gleichstand zwischen allen Standorten vor, die Gehölze nehmen ungefähr ein Viertel am Artenspektrum ein. Trotz steigender Gesamtartenzahlen verschiebt sich dieses Verhältnis während der ersten vier Sukzessionsjahre kaum. Geringfügig erweitern die Grasartigen und teilweise auch die Farne ihre Anteile, die Krautigen gehen dafür tendenziell leicht zurück.

Tab. 8: Qualitatives Wuchsformenspektrum der Arten in Buchen-Windwurfgebieten mit Flächenwurf im südwestlichen Harzvorland in den Jahren 1999 und 2001; Strauch- und Feldschicht zusammengefasst; Anzahl einbezogener $100 \mathrm{~m}^{2}$-Plots siehe Tab. 1; Angaben gerundet in \%.

\begin{tabular}{|l|c|c|c|c|c|c|c|c|c|c|c|c|c|c|}
\hline & Hhz KF2 & \multicolumn{1}{c|}{ Hhz KF1 } & \multicolumn{2}{c|}{ Nüllberg } & Köbu KF2 & \multicolumn{2}{|c|}{ Ro1228 } & \multicolumn{2}{c|}{ Krü-Osth. } & \multicolumn{2}{|c|}{ Krü-Plat. } \\
\hline & $\mathbf{1 9 9 9}$ & $\mathbf{2 0 0 1}$ & $\mathbf{1 9 9 9}$ & $\mathbf{2 0 0 1}$ & $\mathbf{1 9 9 9}$ & $\mathbf{2 0 0 1}$ & $\mathbf{1 9 9 9}$ & $\mathbf{2 0 0 1}$ & $\mathbf{1 9 9 9}$ & $\mathbf{2 0 0 1}$ & $\mathbf{1 9 9 9}$ & $\mathbf{2 0 0 1}$ & $\mathbf{1 9 9 9}$ & $\mathbf{2 0 0 1}$ \\
\hline Bäume & 13 & 12 & 13 & 13 & 15 & 13 & 8 & 9 & 13 & 15 & 11 & 12 & 13 & 15 \\
\hline Sträucher & 9 & 9 & 9 & 11 & 8 & 9 & 17 & 15 & 15 & 14 & 11 & 11 & 12 & 11 \\
\hline holzige Lianen & 1 & 2 & 1 & 1 & 1 & 2 & 0 & 0 & 0 & 0 & 0 & 0 & 0 & 0 \\
\hline Grasartige & 12 & 14 & 16 & 20 & 12 & 15 & 29 & 32 & 17 & 20 & 22 & 25 & 20 & 23 \\
\hline Farne & 0 & 1 & 2 & 4 & 0 & 0 & 8 & 16 & 9 & 12 & 10 & 7 & 5 & 7 \\
\hline Sonsige Krautige & 65 & 61 & 60 & 51 & 64 & 61 & 37 & 29 & 45 & 38 & 45 & 45 & 51 & 44 \\
\hline
\end{tabular}

Ein wesentlich differenzierteres und dynamischeres Bild zeigt das quantitative Spektrum (Abb. 34). Überall gehen die Abundanzanteile der Krautigen zum Teil drastisch zurück, wobei in der Königsbuche und im Krücker bis zum Jahr 2001 eine besonders starke Reduzierung auf ein Drittel bis ein Viertel des Wertes von 1999 zu verzeichnen ist. Trotzdem 
erscheinen die Krautigen im Zechstein auch nach vier Sukzessionsjahren wesentlich stärker vertreten als im Buntsandstein (2001: 47-65\% Anteil gegenüber 9-26\%). Parallel dazu expandieren die Gehölze sowohl absolut als auch relativ in allen UG sehr stark.

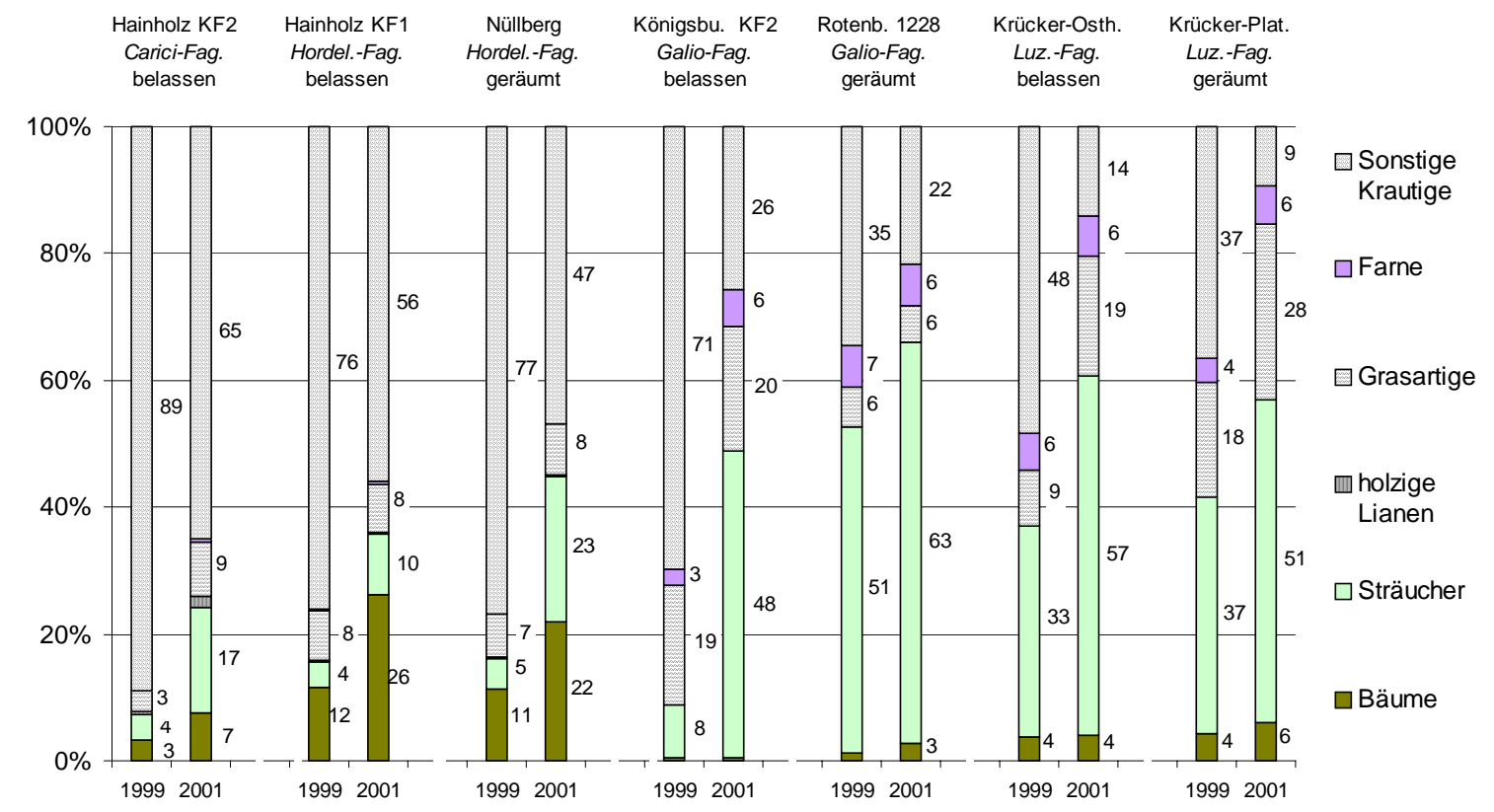

Abb. 34: Quantitatives Wuchsformenspektrum der Arten in Buchen-Windwurfgebieten mit Flächenwurf im südwestlichen Harzvorland in den Jahren 1999 und 2001; Strauch- und Feldschicht zusammengefasst; Anzahl einbezogener $100 \mathrm{~m}^{2}$-Plots siehe Tab. 1; Werte unter $3 \%$ nicht angezeigt.

Auf den beiden tiefgründigen Dolomit-Standorten (Hainholz-KF1, Nüllberg) setzt eine starke Baumarten-Naturverjüngung v.a. der Esche ein (Verdoppelung auf rund ein Viertel Anteil in 2001), die im Buntsandstein und auf der flachgründigen Gips/Dolomit-Kuppe (HainholzKF2) kaum eine Entsprechung findet. Dafür gelangen besonders auf den basenarmen Standorten die Sträucher (v.a. Himbeere und Holunder) mit Anteilen von 48-67\% zur Vorherrschaft. Eine besonders starke Strauch-Expansion ist in der Königsbuche zu verzeichnen (von 8 auf 48\%), eine relativ schwache in der Rotenberg-Abt. 1228 (von 51 auf 63\%). In den Zechstein-Gebieten entwickeln sich die Sträucher dagegen moderater und erlangen bei insgesamt steigender Tendenz im Jahr 2001 nur 10-23\% Abundanzanteil. Erwähnenswert sind weiterhin die in drei Buntsandstein-Gebieten (eine Ausnahme bildet die Rotenberg-Abt. 1228) recht hohen und teilweise steigenden Anteile an Grasartigen, die mit 20-28\% im Jahr 2001 deutlich über den Werten der basenreichen Standorte liegen (dort 89\%). Genauso wird der relative Farn-Reichtum der basenarmen UG deutlich. Vor dem Hintergrund steigender Gesamtdeckungssummen wird sichtbar, dass der Rückgang von waldtypischen krautigen Arten in den Zechstein-Gebieten nur relativ erfolgt, während die Absolutwerte eher stagnieren. Im Buntsandstein dagegen nehmen die Krautigen sowohl relativ als auch absolut ab (Abb. 34; Tab. A22, A23). 


\subsubsection{Lebensformtypen im Sinne RAUNKIAERS nach ELLENBERG et al. (1992)}

Bezüglich der RAUNKIAER'schen Lebensformtypen sind sich die sieben UG bei qualitativer Betrachtung ihres Arteninventars sehr ähnlich und innerhalb der ersten vier Sukzessionsjahre trotz steigender Gesamtartenzahlen auch recht konstant (Tab. 9). Die stärkste Gruppe ist überall die der Hemikryptophyten mit Anteilen zwischen 44\% (Hainholz-KF1) und 56\% (Krücker-Plateau), bezogen auf das Jahr 2001. Im Gegensatz zu den übrigen UG ist die Hainholz-KF1 mit 21\% Anteil relativ reich an Geophyten, dafür mit nur 3\% Anteil relativ arm an Therophyten. Letztere stellen die einzige Gruppe dar, die bei insgesamt geringer Beteiligung Anteile am Artenspektrum verlieren (z.B. Halbierung in Hainholz-KF1, Nüllberg, Krücker-Plateau) und die auch in einigen Fällen in der absoluten Artenzahl einbüßen (z.B. Nüllberg und Krücker-Plateau). Baum- und Straucharten halten mit jeweils rund $10 \%$ überall stabile Anteile. Chamaephyten kommen mit geringen Artenzahlen von 1-3 und Anteilen von 4-5\% hauptsächlich auf den beiden Dolomit-Standorten (Hainholz-KF1, Nüllberg) vor. Lianen kommen mit Anteilen von max. 3\% und Artenzahlen von höchstens 2 je Gebiet auf den meisten Plots ausser denen in der Königsbuche vor.

Tab. 9: Qualitatives Lebensformspektrum (im Sinne RAUNKIAERs) der Arten in BuchenWindwurfgebieten mit Flächenwurf im südwestlichen Harzvorland in den Jahren 1999 und 2001; Strauch- und Feldschicht zusammengefasst; Anzahl einbezogener $100 \mathrm{~m}^{2}$-Plots siehe Tab. 1; Angaben gerundet in \%.

\begin{tabular}{|lc|c|c|c|c|c|c|c|c|c|c|c|c|c|}
\hline & Hhz KF2 & \multicolumn{2}{c|}{ Hhz KF1 } & \multicolumn{2}{c|}{ Nüllberg } & \multicolumn{2}{c|}{ Köbu KF2 } & \multicolumn{2}{c|}{ Ro1228 } & \multicolumn{2}{c|}{ Krü-Osth. } & \multicolumn{2}{c|}{ Krü-Plat. } \\
\hline & $\mathbf{1 9 9 9}$ & $\mathbf{2 0 0 1}$ & $\mathbf{1 9 9 9}$ & $\mathbf{2 0 0 1}$ & $\mathbf{1 9 9 9}$ & $\mathbf{2 0 0 1}$ & $\mathbf{1 9 9 9}$ & $\mathbf{2 0 0 1}$ & $\mathbf{1 9 9 9}$ & $\mathbf{2 0 0 1}$ & $\mathbf{1 9 9 9}$ & $\mathbf{2 0 0 1}$ & $\mathbf{1 9 9 9}$ & $\mathbf{2 0 0 1}$ \\
\hline Chamaephyten & 0 & 1 & 5 & 4 & 5 & 4 & 1 & 2 & 1 & 0 & 1 & 1 & 2 & 2 \\
\hline Geophyten & 12 & 12 & 22 & 21 & 15 & 14 & 13 & 15 & 7 & 10 & 10 & 9 & 8 & 8 \\
\hline Hemikryptophyten & 45 & 51 & 43 & 44 & 44 & 51 & 53 & 53 & 51 & 48 & 53 & 54 & 49 & 56 \\
\hline Nanophaneroph. & 10 & 10 & 9 & 10 & 8 & 8 & 16 & 12 & 12 & 13 & 10 & 9 & 11 & 10 \\
\hline Phanerophyten & 12 & 11 & 13 & 13 & 16 & 13 & 8 & 8 & 12 & 13 & 11 & 11 & 12 & 13 \\
\hline Therophyten & 16 & 11 & 6 & 3 & 11 & 6 & 8 & 7 & 11 & 10 & 11 & 11 & 12 & 5 \\
\hline Lianen & 3 & 3 & 1 & 2 & 1 & 2 & 0 & 0 & 1 & 1 & 2 & 3 & 3 & 2 \\
\hline Sonstige & 0 & 1 & 1 & 3 & 1 & 2 & 1 & 3 & 5 & 4 & 2 & 3 & 3 & 3 \\
\hline
\end{tabular}

Die Abundanzspektren zeigen ein eher individuelles Bild der einzelnen UG (Abb. 35). Auf die standörtlich sehr unterschiedliche Entwicklung der Deckungssummen von Baum- und Straucharten (bzw. Phanerophyten und Nanophanerophyten) wurde bereits in den Kapiteln 4.3.5 und 4.3.6 hingewiesen. Die standörtliche Differenzierung beeinflusst offenbar auch die Entwicklung der Therophyten: 1. auf tiefgründigem Dolomit-Standort quantitative Bedeutungslosigkeit; 2. auf flachgründigem Gips/Dolomit-Standort relative und absolute Stagnation auf höchstem Niveau (ca. ein Drittel Abundanzanteil); 3. im Buntsandstein drastischer relativer und absoluter Rückgang. Auf basenarmen Standorten drückt sich dies vor allem im Deckungsgradeinbruch ausgedehnter Impatiens parviflora-Herden und teilweise auch von Galeopsis tetrahit ab dem dritten bzw. vierten Sukzessionsjahr aus (Tab. A22, A23). 


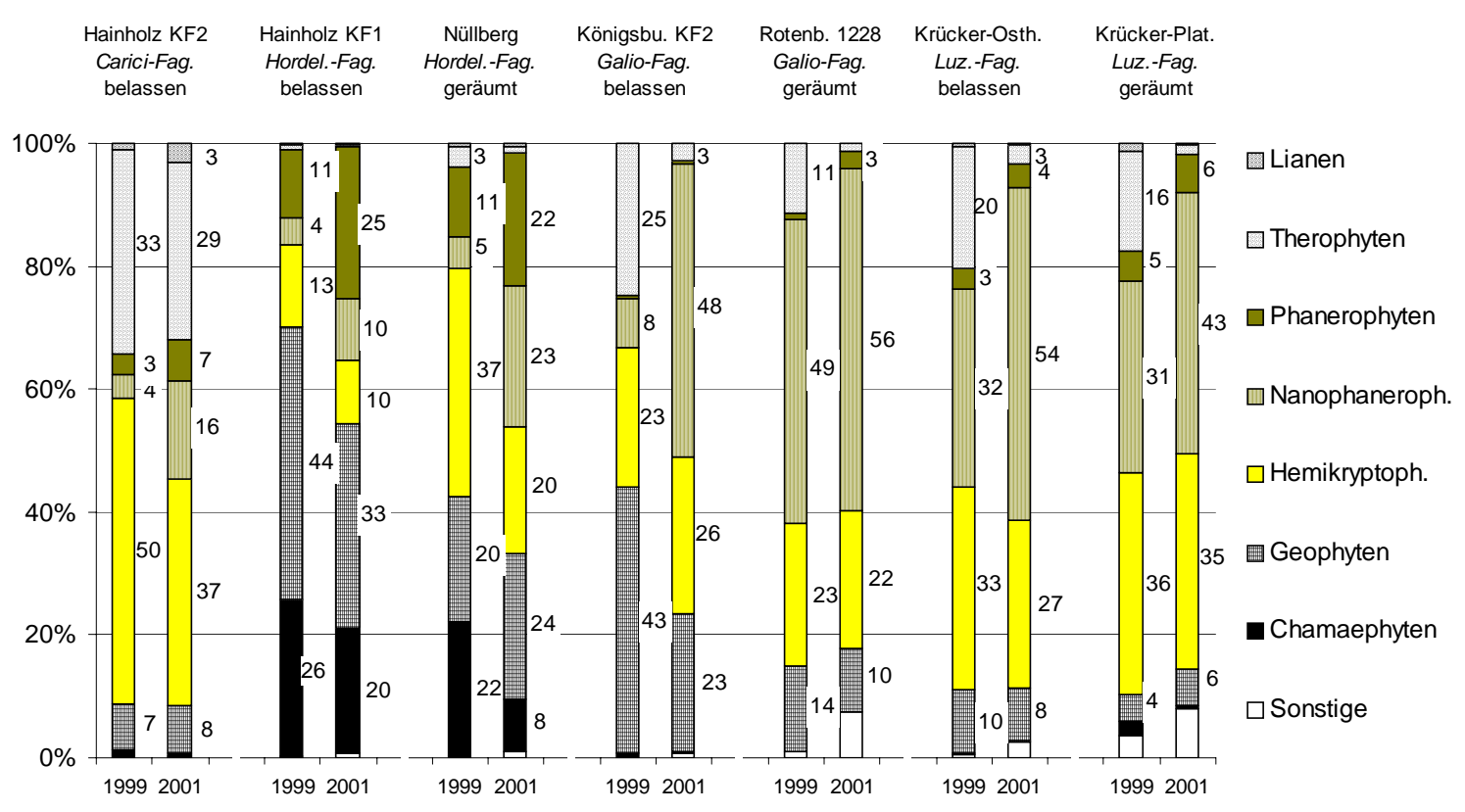

Abb. 35: Quantitatives Lebensformspektrum (im Sinne RAUNKIAERS) der Arten in BuchenWindwurfgebieten mit Flächenwurf im südwestlichen Harzvorland in den Jahren 1999 und 2001; Strauch- und Feldschicht zusammengefasst; Anzahl einbezogener $100 \mathrm{~m}^{2}$-Plots siehe Tab. 1; Werte unter 3\% nicht angezeigt.

Chamaephyten erreichen nur auf den beiden tiefgründigen Dolomit-Standorten beachtliche Anteile von anfangs etwa einem Viertel. Diese Artengruppe nimmt dort aber im Sukzessionsverlauf relativ ab, wobei sie in der Hainholz-KF1 v.a. durch die vom Windwurf unbeeinflussten Populationen von Galeobdolon luteum stabile Deckungssummen behält. Auf dem Nüllberg werden die krautigen Chamaephyten durch den Zusammenbruch der anfangs großflächigen Euphorbia amygdaloides-Staudenfluren auch absolut zurückgedrängt (Tab. A22). Die Geophyten zeigen je nach Standort ein unterschiedliches Verhalten. Die HainholzKF1, der Nüllberg und die Königsbuchen-KF2 sind mit Anteilen zwischen 20 und 40\% (1999) als relativ Geophyten-reich einzustufen. In den beiden Rotenberg-Flächen erfolgt ein relativer und absoluter Rückgang durch Deckungsverluste von Oxalis acetosella. Im Krücker waren stagnierende Anteile bei leicht steigenden Deckungssummen (Tab. A23) zu verzeichnen (z.B. durch die Zunahme von Calamagrostis epigejos). In der Hainholz-KF1 kam es zu einem relativen Rückgang der Geophyten-Anteile von 44 auf 33\% bei stabilen Deckungssummen, wobei die ausgedehnten Allium ursinum-Bestände im Deckungsgrad kaum auf den Windwurf reagierten (Tab. A22). In der Hainholz-KF2 stagnieren sowohl Abundanzanteile als auch absolute Deckungssummen auf niedrigem Niveau (5-10\%), während es auf dem Nüllberg zu einem leichten Anstieg der Geophyten-Anteile von 20 auf 24\% bei etwas deutlicher ansteigenden Deckungssummen von 35 auf 54\% kam. Dies wurde dort durch eine starke Zunahme des Rhizomgeophyten Cirsium arvense ausgelöst (Abb. 35; Tab. A22). 


\subsubsection{Samenbanktypen nach GRIME et al. (1989), KLEYER (1995), THOMPSON et al. (1997) und OBERDORFER (2001)}

Das qualitative Artenspektrum ist bezüglich der darin vertretenen Samenbanktypen in allen sieben UG relativ einheitlich und verändert sich in den ersten vier Sukzessionsjahren trotz steigender Gesamtartenzahlen kaum (Tab. 10). Rund ein Drittel der Arten baut eine langfristige Samenbank (>5 Jahre) auf, rund die Hälfte eine kürzerfristige unter 5 Jahren (Kategorien temporär, temporär-kurzfristig und kurzfristig). Der Rest verteilt sich auf nicht zuzuordnende Arten oder auf die Übergangskategorie „kurz- bis langfristig“.

Tab. 10: Qualitatives Samenbanktypenspektrum der Arten in Buchen-Windwurfgebieten mit Flächenwurf im südwestlichen Harzvorland in den Jahren 1999 und 2001; Strauch- und Feldschicht zusammengefasst; Anzahl einbezogener $100 \mathrm{~m}^{2}$-Plots siehe Tab. 1; Angaben gerundet in \%.

\begin{tabular}{|l|c|c|c|c|c|c|c|c|c|c|c|c|c|c|}
\hline & \multicolumn{2}{|c|}{ Hhz KF2 } & \multicolumn{2}{c|}{ Hhz KF1 } & \multicolumn{2}{c|}{ Nüllberg } & \multicolumn{2}{c|}{ Köbu KF2 } & \multicolumn{2}{c|}{ Ro1228 } & \multicolumn{2}{c|}{ Krü-Osth. } & Krü-Plat. \\
\hline & $\mathbf{1 9 9 9}$ & $\mathbf{2 0 0 1}$ & $\mathbf{1 9 9 9}$ & $\mathbf{2 0 0 1}$ & $\mathbf{1 9 9 9}$ & $\mathbf{2 0 0 1}$ & $\mathbf{1 9 9 9}$ & $\mathbf{2 0 0 1}$ & $\mathbf{1 9 9 9}$ & $\mathbf{2 0 0 1}$ & $\mathbf{1 9 9 9}$ & $\mathbf{2 0 0 1}$ & $\mathbf{1 9 9 9}$ & $\mathbf{2 0 0 1}$ \\
\hline lang & 31 & 29 & 31 & 30 & 29 & 29 & 36 & 35 & 40 & 31 & 31 & 36 & 36 & 33 \\
\hline kurz-lang & 6 & 6 & 4 & 2 & 5 & 5 & 8 & 6 & 5 & 5 & 11 & 9 & 10 & 9 \\
\hline kurz & 6 & 6 & 4 & 4 & 6 & 7 & 7 & 7 & 2 & 5 & 6 & 4 & 5 & 7 \\
\hline temporär-kurz & 22 & 25 & 13 & 14 & 13 & 17 & 17 & 17 & 21 & 17 & 22 & 21 & 22 & 24 \\
\hline temporär & 28 & 26 & 39 & 38 & 37 & 33 & 23 & 21 & 23 & 29 & 21 & 23 & 21 & 21 \\
\hline keine Angabe & 7 & 9 & 10 & 12 & 9 & 9 & 9 & 14 & 9 & 13 & 9 & 7 & 7 & 6 \\
\hline
\end{tabular}

Deutliche Differenzierungen ergeben sich jedoch beim quantitativen Spektrum (Abb. 36). Die Buntsandstein-Standorte werden deutlich stärker von Arten mit langfristiger Samenbank >5 Jahren beherrscht (67-75\% Anteil im Jahr 2001) als die Zechstein-Standorte (18-42\%). Gleiches gilt tendenziell für geräumte (42-75\% Anteil) gegenüber belassenen Flächen (1867\% Anteil). Arten mit langfristiger Samenbank expandieren aber in ausnahmslos allen UG. Relativ betrachtet führt das meistens zu einem Rückgang von Arten mit kürzerfristiger Samenbank, wobei der Nüllberg die einzige Ausnahme darstellt. Betrachtet man jedoch die absoluten Deckungssummen der einzelnen Artengruppen, so wird wiederum ein Standortsunterschied deutlich: im Zechstein nehmen die Arten mit kürzerfristiger Samenbank deutlich zu, im Buntsandstein stagnieren sie oder nehmen ab (Tab. A22, A23). Die stärkste relative Expansion von Arten mit langfristiger Samenbank findet in der seit langem unbewirtschafteten Königsbuchen-KF2 statt (Zunahme von 26 auf 67\% Abundanzanteil), die schwächste in der Hainholz-KF1 (von 13 auf 16\%) sowie in der Rotenberg-Abt. 1228 (von 69 auf $75 \%)$. 


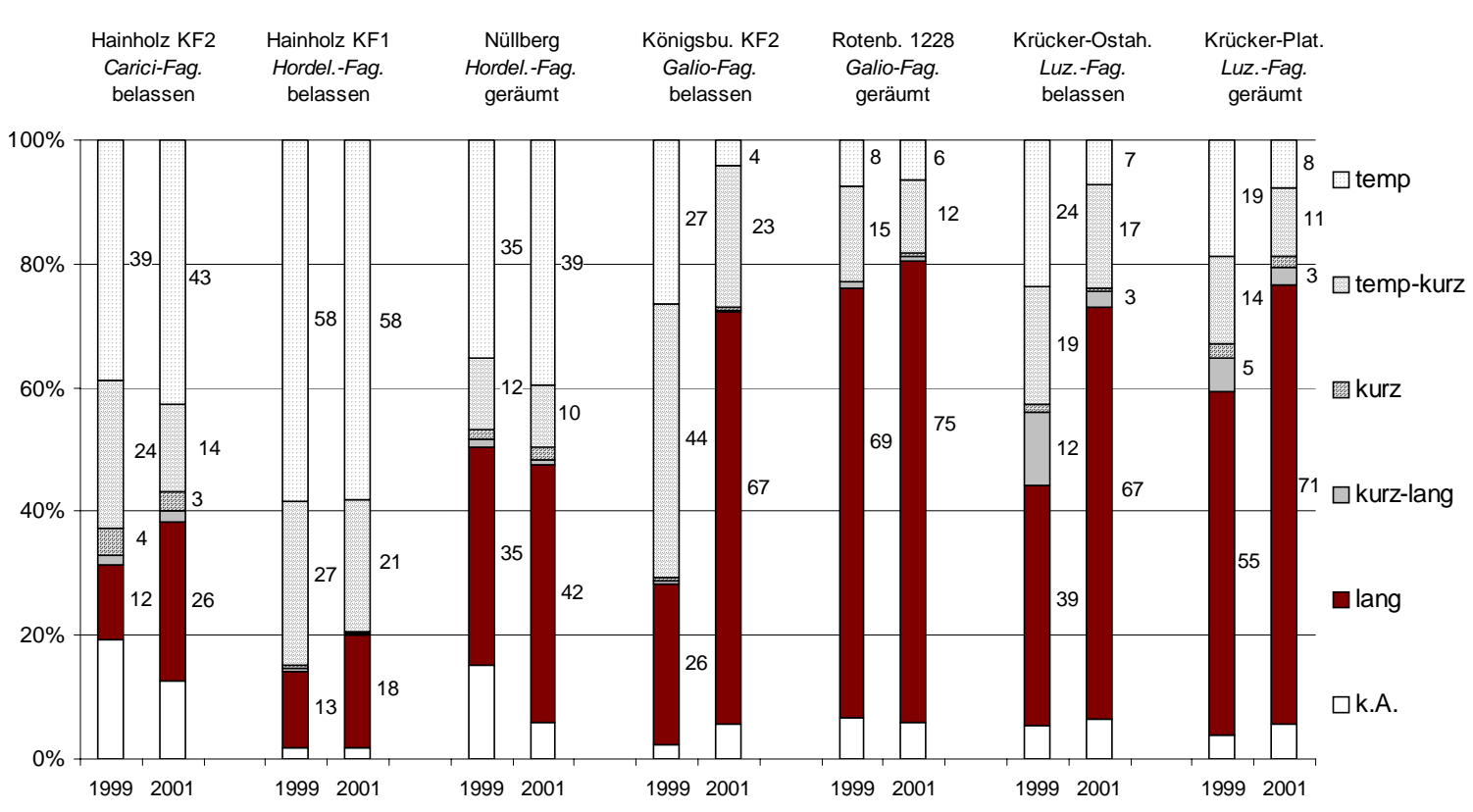

Abb. 36: Quantitatives Samenbanktypenspektrum der Arten in Buchen-Windwurfgebieten mit Flächenwurf im südwestlichen Harzvorland in den Jahren 1999 und 2001; Strauch- und Feldschicht zusammengefasst; Anzahl einbezogener $100 \mathrm{~m}^{2}$-Plots siehe Tab. 1; Werte unter 3\% nicht angezeigt.

\subsubsection{Ausbreitungstypen nach FrANK et al. (1990)}

Bezüglich der Ausbreitungstypen ist die Artenzusammensetzung aller sieben UG sehr ähnlich und verändert sich im Sukzessionsverlauf trotz steigender Gesamtartenzahlen kaum (Tab. 11). Die meisten Arten sind anemochor (Anteile zwischen 40 und 50\%), an zweiter bzw. dritter Stelle stehen epizoochore (ca. 20\%) bzw. endozoochore Arten (15-20\%). Weiterhin existiert eine Gruppe autochorer Arten mit Anteilen zwischen 10 und 15\%, in der Königsbuchen-KF2 bis zu 22\% (1999). Das geringste Gewicht haben myrmekochore Arten mit Anteilen bis zu 10\%, im Rotenberg und in der Hainholz-KF1 nur bis max. 4\%.

Tab. 11: Qualitatives Ausbreitungstypenspektrum der Arten in Buchen-Windwurfgebieten mit Flächenwurf im südwestlichen Harzvorland in den Jahren 1999 und 2001; Strauch- und Feldschicht zusammengefasst; Anzahl einbezogener $100 \mathrm{~m}^{2}$-Plots siehe Tab. 1; Angaben gerundet in \%.

\begin{tabular}{|l|c|c|c|c|c|c|c|c|c|c|c|c|c|c|}
\hline & \multicolumn{2}{|c|}{ Hhz KF2 } & \multicolumn{2}{c|}{ Hhz KF1 } & \multicolumn{2}{c|}{ Nüllberg } & \multicolumn{2}{c|}{ Köbu KF2 } & \multicolumn{2}{c|}{ Ro1228 } & \multicolumn{2}{c|}{ Krü-Osth. } & Krü-Plat. \\
\hline & $\mathbf{1 9 9 9}$ & $\mathbf{2 0 0 1}$ & $\mathbf{1 9 9 9}$ & $\mathbf{2 0 0 1}$ & $\mathbf{1 9 9 9}$ & $\mathbf{2 0 0 1}$ & $\mathbf{1 9 9 9}$ & $\mathbf{2 0 0 1}$ & $\mathbf{1 9 9 9}$ & $\mathbf{2 0 0 1}$ & $\mathbf{1 9 9 9}$ & $\mathbf{2 0 0 1}$ & $\mathbf{1 9 9 9}$ & $\mathbf{2 0 0 1}$ \\
\hline myrmekochor & 10 & 9 & 4 & 4 & 8 & 7 & 2 & 2 & 4 & 3 & 8 & 7 & 9 & 8 \\
\hline epizoochor & 18 & 21 & 21 & 21 & 20 & 22 & 19 & 22 & 20 & 21 & 15 & 19 & 17 & 17 \\
\hline endozoochor & 17 & 16 & 19 & 20 & 17 & 15 & 18 & 17 & 19 & 18 & 14 & 14 & 15 & 13 \\
\hline hydrochor & 0 & 0 & 0 & 0 & 0 & 0 & 0 & 0 & 0 & 0 & 0 & 0 & 1 & 1 \\
\hline anemochor & 40 & 40 & 39 & 40 & 44 & 46 & 37 & 41 & 40 & 41 & 47 & 44 & 42 & 48 \\
\hline autochor & 14 & 13 & 16 & 15 & 11 & 10 & 22 & 17 & 16 & 17 & 16 & 15 & 15 & 14 \\
\hline
\end{tabular}




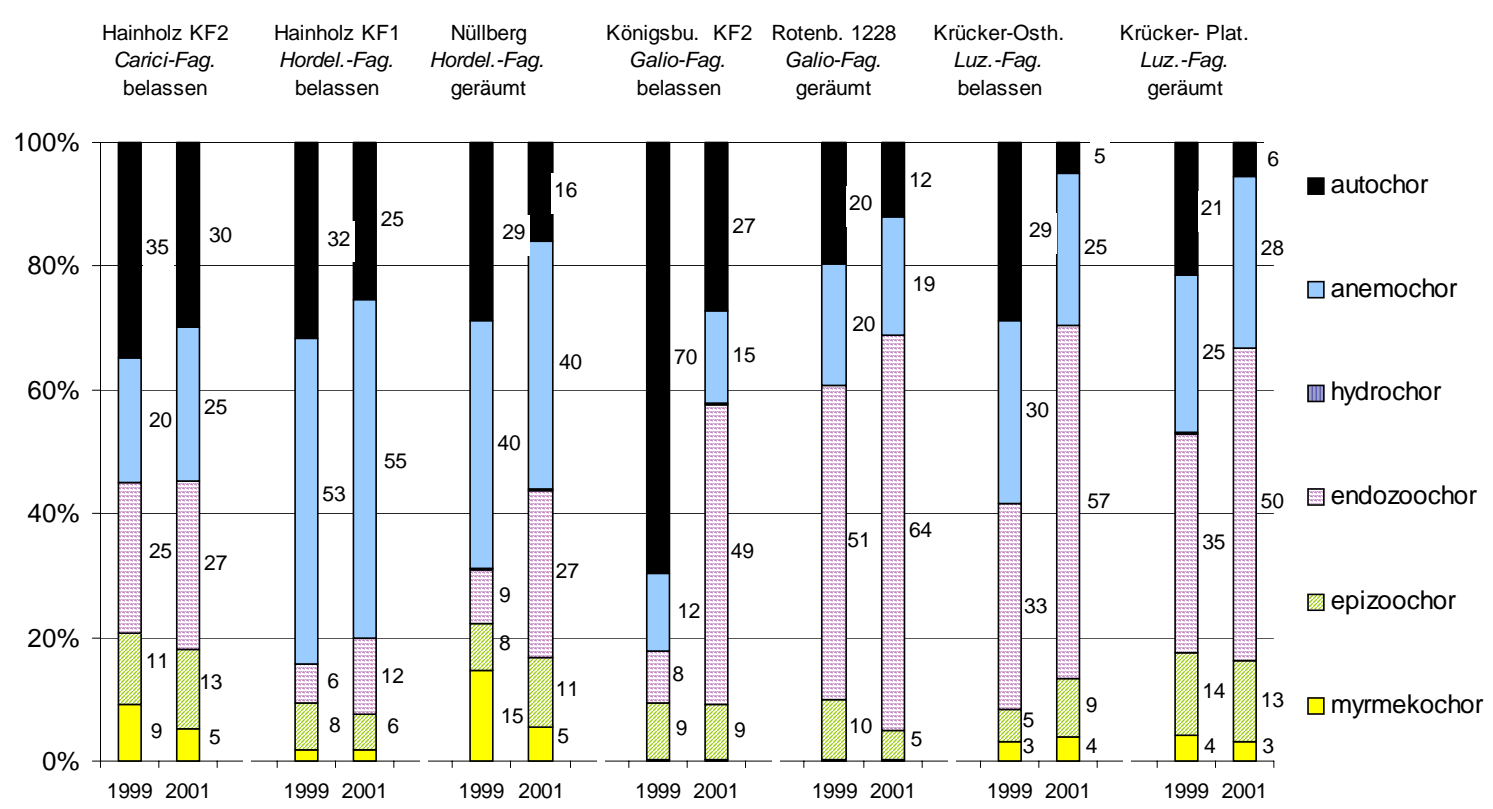

Abb. 37: Quantitatives Ausbreitungstypenspektrum der Arten in Buchen-Windwurfgebieten mit Flächenwurf im südwestlichen Harzvorland in den Jahren 1999 und 2001; Strauch- und Feldschicht zusammengefasst; Anzahl einbezogener $100 \mathrm{~m}^{2}$-Plots siehe Tab. 1; Werte unter 3\% nicht angezeigt.

Unter Einbeziehung der Deckungssummen (Abb. 37; Tab. A22, A23) fällt zunächst auf, dass in allen UG endozoochore Arten absolut und relativ zunehmen, wobei im vierten Sukzessionsjahr in den Buntsandstein-Gebieten generell höhere Anteile erreicht werden (4964\%) als auf den basenreichen Flächen (12-27\%). Besonders starke Zuwächse verzeichnet diese Artengruppe in der Königsbuchen-KF2 (von 8 auf 49\%). Parallel dazu verlieren die autochoren Arten überall Abundanzanteile, besonders stark in der seit 30 Jahren ungenutzten Königsbuche (von 70 auf 27\%) und auch am belassenen Krücker-Osthang (von 29 auf 5\%). Diesem relativen Rückgang liegen in den Buntsandstein-Gebieten auch absolute Verluste zugrunde, während in den Zechstein-Gebieten die Deckungssummen der autochoren Arten mehr oder weniger stabil bleiben (Tab. A22, A23). Für die anemochoren Arten ist charakteristisch, dass sie bei steigenden absoluten Deckungssummen in allen UG stagnierende Anteile am Abundanzspektrum halten. Dabei werden auf den beiden Dolomit-Standorten besonders hohe Werte erreicht (Hainholz-KF1: 55\%; Nüllberg: 40\%). Die epizoochoren Arten halten in allen UG geringe, aber stabile Anteile zwischen 5 und 13\%. Myrmekochorie spielt quantitativ vor allem in der Hainholz-KF2 und auf dem Nüllberg eine gewisse Rolle (Anteilsrückgang von 15 auf 5\% bzw. von 9 auf 5\%), in geringerem Maße auch im Krücker und in der Hainholz-KF1 (2-4\%), im Rotenberg dagegen überhaupt nicht. 


\subsubsection{Vegetatives Ausbreitungsvermögen nach SCHMIDT (1983), GRIME et al. (1989), BARTELS (1993) und KLEYER (1995)}

Das qualitative Artenspektrum der UG wird mehrheitlich von Arten mit vegetativem Ausbreitungsvermögen bestimmt, die meist um die 60\% Anteil erreichen (Tab. 12). Einzige Ausnahme ist die Hainholz-KF2, wo sich generativ vermehrende Arten mit max. 59\% Anteil (2001) leicht überwiegen. Zwischen dem zweiten (1999) und dem vierten Sukzessionsjahr (2001) ergeben sich trotz steigender Gesamtartenzahlen keine wesentlichen Veränderungen an diesem Verhältnis.

Tab. 12: Qualitatives Spektrum des vegetativen Ausbreitungsvermögens der Arten in BuchenWindwurfgebieten mit Flächenwurf im südwestlichen Harzvorland in den Jahren 1999 und 2001; Strauch- und Feldschicht zusammengefasst; Anzahl einbezogener $100 \mathrm{~m}^{2}$-Plots siehe Tab. 1; Angaben gerundet in \%.

\begin{tabular}{|l|c|c|c|c|c|c|c|c|c|c|c|c|c|c|}
\hline & \multicolumn{1}{c}{ Hhz KF2 } & \multicolumn{2}{c|}{ Hhz KF1 } & \multicolumn{2}{c|}{ Nüllberg } & \multicolumn{2}{c|}{ Köbu KF2 } & \multicolumn{2}{|c|}{ Ro1228 } & \multicolumn{2}{|c|}{ Krü-Osth. } & \multicolumn{2}{c|}{ Krü-Plat. } \\
\hline & $\mathbf{1 9 9 9}$ & $\mathbf{2 0 0 1}$ & $\mathbf{1 9 9 9}$ & $\mathbf{2 0 0 1}$ & $\mathbf{1 9 9 9}$ & $\mathbf{2 0 0 1}$ & $\mathbf{1 9 9 9}$ & $\mathbf{2 0 0 1}$ & $\mathbf{1 9 9 9}$ & $\mathbf{2 0 0 1}$ & $\mathbf{1 9 9 9}$ & $\mathbf{2 0 0 1}$ & $\mathbf{1 9 9 9}$ & $\mathbf{2 0 0 1}$ \\
\hline OHNE & 59 & 54 & 41 & 37 & 50 & 43 & 35 & 32 & 41 & 41 & 38 & 39 & 43 & 41 \\
\hline MIT & 41 & 46 & 59 & 63 & 50 & 57 & 65 & 68 & 59 & 59 & 62 & 61 & 57 & 59 \\
\hline
\end{tabular}

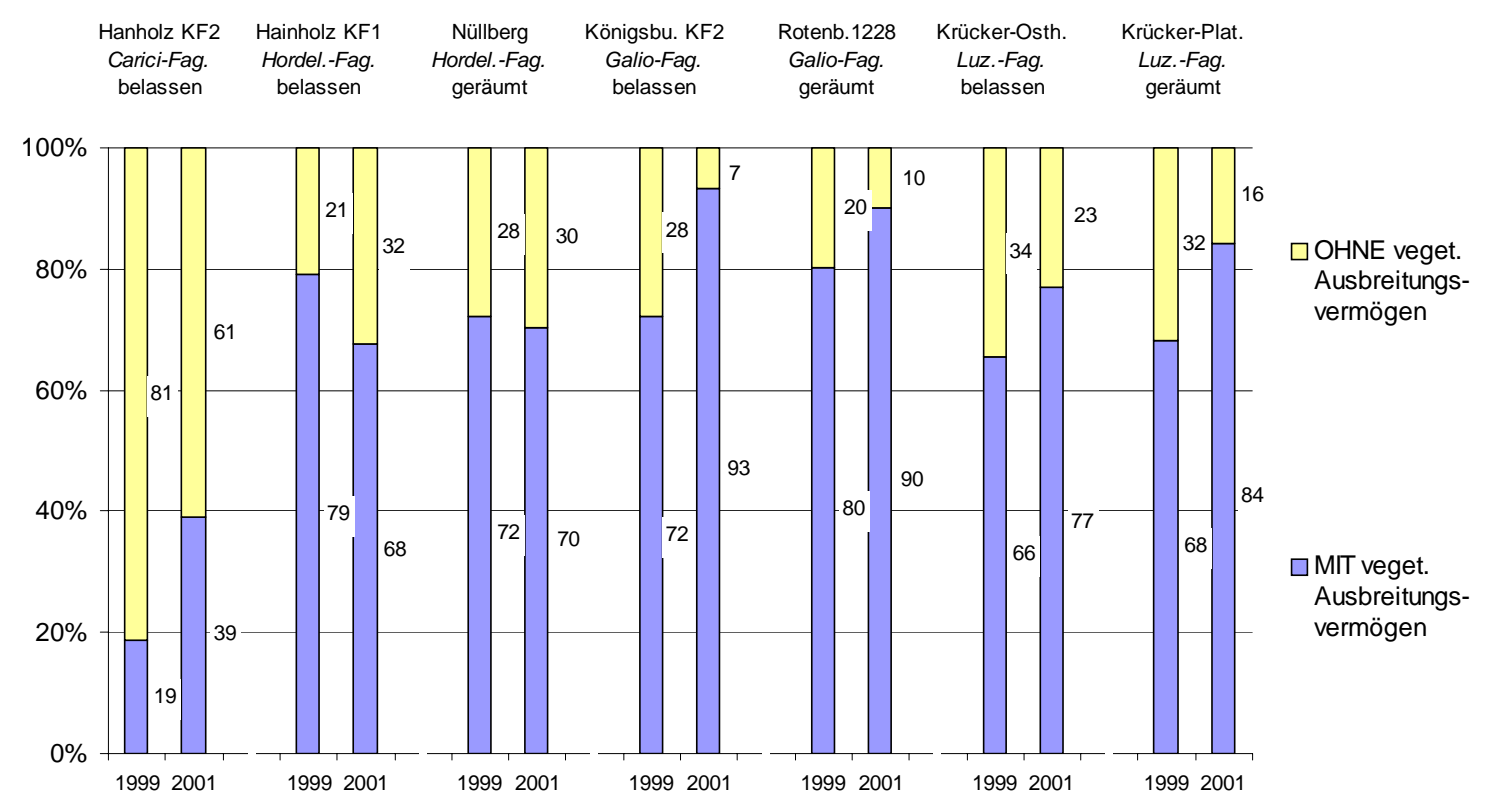

Abb. 38: Quantitatives Spektrum des vegetativen Ausbreitungsvermögens der Arten in Buchen-Windwurfgebieten mit Flächenwurf im südwestlichen Harzvorland in den Jahren 1999 und 2001; Strauch- und Feldschicht zusammengefasst; Anzahl einbezogener $100 \mathrm{~m}^{2}$ Plots siehe Tab. 1; Werte unter 3\% nicht angezeigt.

Deutliche Unterschiede lassen sich bei quantitativer Betrachtung erkennen (Abb. 38). Die Hainholz-KF2 wird sehr stark von sich generativ vermehrenden Arten dominiert (61\% Anteil in 2001), während vor allem die beiden Flächen im Rotenberg fast ausschließlich von Arten mit vegetativem Ausbreitungsvermögen beherrscht werden (mindestens 90\% Anteil im Jahr 2001). Die übrigen UG liegen dazwischen. Ausser auf den beiden Dolomit-Standorten 
(Hainholz-KF1, Nüllberg) nimmt der Anteil von Arten mit vegetativem Ausbreitungsvermögen im Untersuchungszeitraum um ca. 10-20\% zu.

\subsubsection{Hemerobietypen nach FrANK et al. (1990)}

Mit Ausnahme von Hainholz-KF2 dominieren in allen UG sowohl qualitativ als auch quantitativ mehr oder weniger stark die oligohemeroben Arten (Tab. 13; Abb. 39). Dabei fällt die Homogenität des qualitativen Artenspektrums aller sieben UG auf. Zwischen 64 und 75\% aller vorkommenden Arten sind oligohemerob und zwischen einem Viertel und einem Drittel sind mesohemerob.

Tab. 13: Qualitatives Hemerobietypenspektrum der Arten in Buchen-Windwurfgebieten mit Flächenwurf im südwestlichen Harzvorland in den Jahren 1999 und 2001; Strauch- und Feldschicht zusammengefasst; Anzahl einbezogener $100 \mathrm{~m}^{2}$-Plots siehe Tab. 1; Angaben gerundet in \%.

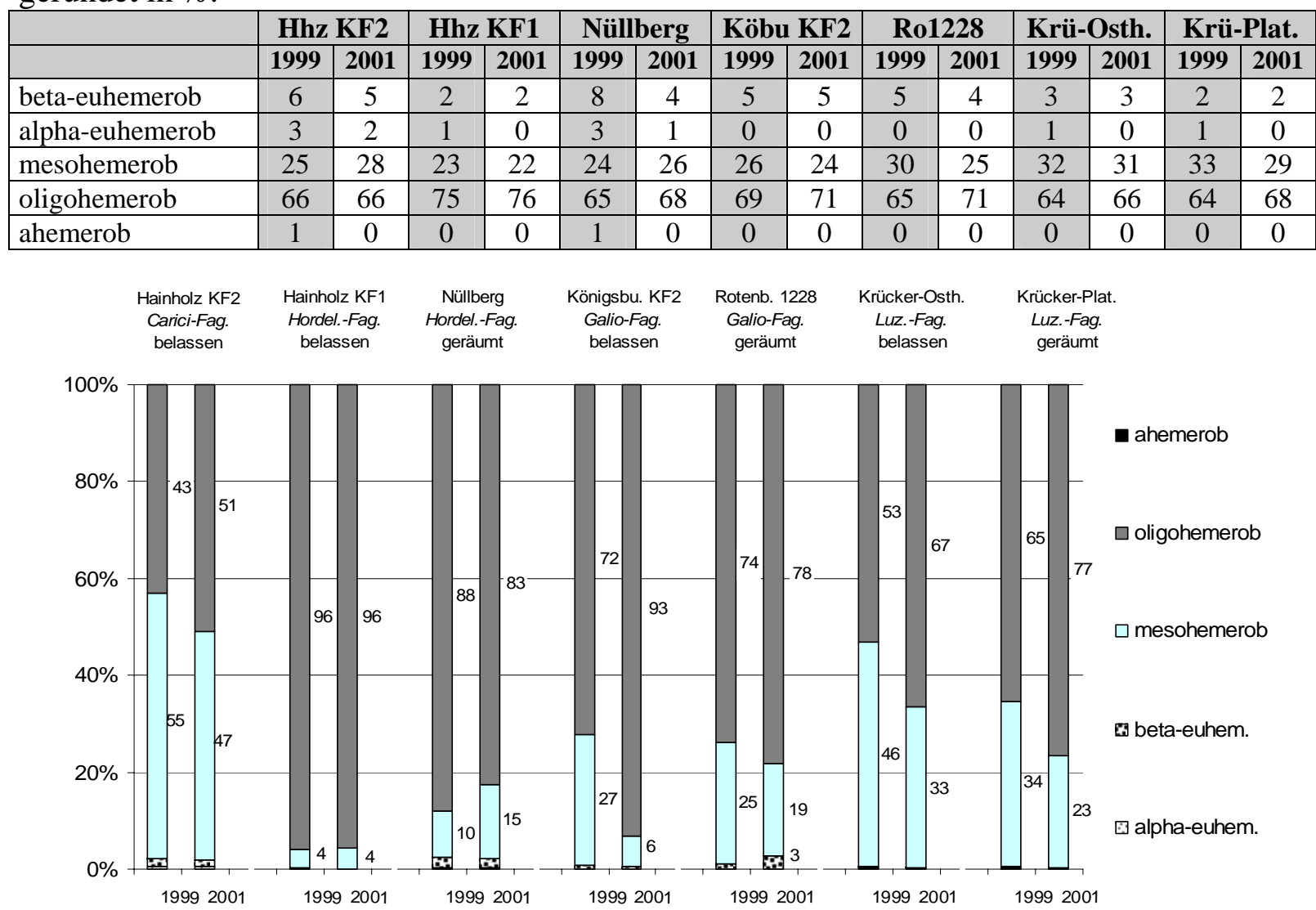

Abb. 39: Quantitatives Hemerobietypenspektrum der Arten in Buchen-Windwurfgebieten mit Flächenwurf im südwestlichen Harzvorland in den Jahren 1999 und 2001; Strauch- und Feldschicht zusammengefasst; Anzahl einbezogener $100 \mathrm{~m}^{2}$-Plots siehe Tab. 1; Werte unter $3 \%$ nicht angezeigt.

Deutlichere Unterschiede ergeben sich unter Einbeziehung der Deckungssummen (Abb. 39). Besonders die Hainholz-KF2, aber auch der Krücker erscheinen als relativ „naturfern“ (d.h. 
mesohemerobe Arten sind stark etabliert). Hainholz-KF1, Königsbuche-KF2 und Nüllberg erscheinen dagegen besonders „naturnah“ (d.h. besonders stark von oligohemeroben Arten dominiert). Eine leichte Zunahme in den Deckungsgraden der oligohemeroben Arten ist von 1999 zu 2001 in fünf UG zu verzeichnen (nicht auf den beiden Dolomit-Standorten), was zu Lasten der zweitstärksten Gruppe der mesohemeroben Arten geht. Ahemerobe Arten kommen mit Anteilen von $<1 \%$ am Gesamtartenspektrum einiger UG vor, spielen aber mengenmäßig keine Rolle. Polyhemerobe Arten existieren in den UG nicht. Euhemerobe Arten sind zwar überall mit geringen Anteilen von 3 bis maximal 11\% am Artenspektrum beteiligt (Tab. 13), erreichen aber bei quantitativer Betrachtung lediglich in drei UG (Hainholz-KF2, Nüllberg, Rotenberg-Abt. 1228) geringe Anteile von maximal 3\% an den Gesamtdeckungssummen (Abb. 39). Tendenziell sind sie in geräumten Flächen (Nüllberg, Rotenberg-Abt. 1228) etwas stärker verbreitet als in belassenen (Hainholz-KF1, Königsbuche-KF2).

\subsection{Typische Sukzessionsmuster ausgewählter Arten}

\subsubsection{Deckungsgradentwicklung}

Im Folgenden wird auf typische Sukzessionsmuster ausgewählter Arten anhand ihrer Deckungsgradentwickung innerhalb der ersten 4 Jahre eingegangen (Abb. 40). Dabei repräsentieren die in Abb. 41 gezeigten Arten eine bestimmte Anzahl weiterer Arten mit vergleichbarem Verhalten, die nur im Text erwähnt werden. Aufgeführt sind alle krautigen Arten sowie Gehölzarten der Strauchschicht $(>50 \mathrm{~cm})$, die in wenigstens einem Gebiet und einem Untersuchungsjahr eine mittlere Deckung von $>1 \%$ erreichen und somit eine gewisse quantitative Bedeutung im Sukzessionsgeschehen besitzen. Die genannten Tendenzen müssen - wenn standörtlich nicht anders zu erwarten - für mindestens zwei UG zutreffen, in denen die Art ihre Hauptvorkommen besitzt. Ansonsten wurde die Art unter Typ 5 (ambivalentes Verhalten) aufgeführt.

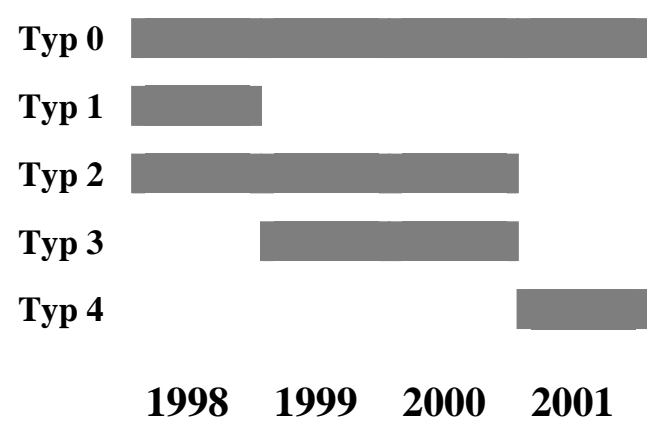

Abb. 40: Schematische Darstellung der Deckungsgradmaxima der in Kap. 4.4.1 beschriebenen Sukzessionsmuster in den ersten vier Jahren nach dem Sturm; Typendefinition siehe Text. 
Typ 0: keine erkennbare Deckungsgradänderung:

Allium ursinum (nur im Zechstein)

Arum maculatum (nur im Zechstein)

Viola reichenbachiana

Typ 1: Deckungsgradmaximum im ersten Jahr nach dem Sturm (1998), danach

\section{Abnahme}

Convallaria majalis (nur Hainholz-KF2)

Corydalis cava (nur Hainholz-KF1)

Stachys sylvatica (nur auf Dolomit)

Typ 2: nach anfänglich konstanter Phase Deckungsgradabnahme im vierten Jahr nach dem Sturm (2001):

Hordelymus europaeus (nur im Zechstein)

Typ 3: Deckungsgradmaximum im zweiten und/oder dritten Jahr nach dem Sturm (1999 bzw. 2000), danach Abnahme:

Alliaria petiolata (nur Nüllberg)

Atropa bella-donna (nur im Zechstein)

Carex muricata agg.

Digitalis purpurea (nur Krücker)

Euphorbia amygdaloides (nur Hainholz-KF2 und Nüllberg)

Galeopsis tetrahit

Hypericum perforatum

Impatiens parviflora

Galeobdolon luteum (sehr schwache Tendenz)

Mercurialis perennis (nur im Zechstein)

Mycelis muralis (nur Hainholz-KF2)

Oxalis acetosella (nur im Buntsandstein)

Rumex obtusifolius

Taraxacum officinale (nur im Zechstein)

Typ 4: konstante Zunahme und vorläufiges Deckungsgradmaximum im vierten Jahr nach dem Sturm (2001):

Athyrium filix-femina (nur im Buntsandstein)

Brachypodium sylvaticum

Calamagrostis epigejos (nur Krücker)

Carduus crispus (nur Hainholz-KF2)

Carex sylvatica

Carex flacca (nur im Zechstein)

Cirsum arvense (nur im Zechstein)

Clematis vitalba (nur im Zechstein)

Dactylis polygama (nur Hainholz-KF2)

Galium odoratum

Juncus effusus (nur im Buntsandstein)

Luzula luzuloides (nur Krücker)

Senecio ovatus

Gehölze $>50$ cm (Strauchschicht):

Acer pseudo-platanus 
Betula pendula (nur Krücker)

Fagus sylvatica

Fraxinus excelsior (nur auf Dolomit)

Prunus avium (nur Nüllberg)

Rubus fruticosus agg. (nur im Buntsandstein)

Rubus idaeus

Tilia platyphyllos (nur im Zechstein)

Ulmus glabra (nur im Zechstein)

Typ 5: ambivalentes Verhalten:

Agrostis capillaris

Carex remota (nur im Buntsandstein)

Circaea lutetiana (nur auf Dolomit)

Epilobium angustifolium (nur im Buntsandstein)

Galium aparine

Poa angustifolia

Urtica dioica

Gehölze $>50 \mathrm{~cm}$ (Strauchschicht):

Sambucus nigra

Sambucus racemosa

Aus dieser Typisierung wird ersichtlich, dass es nur relativ wenige Waldarten gibt (nämlich jene der Typen 1 und 2), die im bisherigen Sukzessionsverlauf quantitativ zurückgedrängt werden, wobei sie aber vermutlich auch in Zukunft an ihren Standorten bestehen bleiben. Das heisst nicht, dass nicht auch die eine oder andere quantitativ weniger bedeutsame Waldart von den Windwurfflächen verschwindet (Tab. A22, A23). Beobachtet wurde dies z.B. für Paris quadrifolia (Hainholz-KF1), Poa chaixii (Hainholz-KF2), Ranunculus auricomus (HainholzKF1, Krücker-Plateau), Calamagrostis arundinacea (Königsbuche-KF2), Hieracium murorum (Krücker-Plateau) oder Impatiens noli-tangere (Königsbuche-KF2, KrückerPlateau). Trotzdem sind diese Arten weiterhin in den umliegenden Beständen vorhanden.

Unter den Typen 3 und 4, also jenen, die zumindest zeitweise von der Windwurfsituation profitieren und ihre Deckungsgrade erweiterten, sind nicht nur neu einwandernde oder aus der Samenbank auflaufende Schlagflur-, Ruderal- und Grünlandarten, sondern auch viele Waldarten der Ausgangsgesellschaften vertreten. Standortübergreifend wären hier vor allem Carex sylvatica, Galeobdolon luteum, Galium odoratum und Brachypodium sylvaticum zu nennen. Standortsabhängig kommt noch eine Reihe weiterer Waldarten hinzu, z.B. Mercurialis perennis, Oxalis acetosella, Athyrium filix-femina, Clematis vitalba, Dactylis polygama und Luzula luzuloides.

Bei rein qualitativer Betrachtung des Arteninventars nach pflanzensoziologischen Klassen (Tab. 7) wurde bereits festgestellt, dass es zu keiner wesentlichen Verschiebung des Artenspektrums zugunsten irgendeiner Klasse kommt. Durch die Dominanzverschiebungen im Abundanzspektrum (Abb. 33) wird aber deutlich, dass unter den in der obigen Aufzählung 
vertretenen Arten die Schlagflur-, im zweiten und dritten Sukzessionsjahr auch noch die Ruderalarten eine überproportionale Rolle spielten.

Die meisten Gehölzarten mit mittleren Strauchschicht-Deckungen von $>1 \%$ sind dem Typ 4 (vorläufiges Deckungsgradmaximum im vierten Sukzessionsjahr) zuzuordnen. Ausnahmen sind Sambucus racemosa und Sambucus nigra, die im vierten Sukzessionsjahr vorzugsweise auf den geräumten Flächen bereits wieder zurückgehen oder zumindest stagnieren. Bedingt durch das Durchwachsen der Gehölze in die Strauchschicht ist parallel dazu etwa ab dem vierten Sukzessionsjahr ein Deckungsgradeinbruch der entsprechenden Gehölzarten in der Feldschicht zu verzeichnen. Bei genauerer Betrachtung der Strauchschicht-Deckungsgrade ergeben sich einige interessante Details. So fällt auf, dass bis zum vierten Sukzessionsjahr Fagus sylvatica und Acer pseudo-platanus die einzigen Arten sind, die über alle Standorte verteilt eine ähnliche Entwicklung nehmen, ansonsten liegen standorts- und/oder bewirtschaftungsbedingte Unterschiede vor.

Die beiden wichtigen Klimaxbaumarten Fagus sylvatica und Fraxinus excelsior erreichen in der belassenen Zechstein-Variante (Hainholz-KF1) höhere Deckungsgrade als in der geräumten und aufgeforsteten (Nüllberg). Für eine Reihe anderer Schlusswaldbaumarten ergibt sich jedoch eine dynamischere Entwicklung in den aufgeforsteten Flächen, so z.B. bei Acer pseudo-platanus, Fagus sylvatica (nur im Buntsandstein zutreffend), Carpinus betulus, Prunus avium und Ulmus glabra. Es kann hier nur vermutet werden, dass es sich um einen Aufforstungseffekt handelt. Aus der Rotenberg-Abt. 1228 liegen Informationen über die Aufforstung mit Buche, Berg-Ahorn, Trauben- und Stieleiche sowie Hainbuche vor. Der Nüllberg wurde mit Buche, Esche, Vogel-Kirsche und Berg-Ahorn aufgeforstet, das KrückerPlateau mit Buche, Berg-Ahorn, Esche, Fichte und Vogel-Kirsche (siehe Kap. 2.1.3).

Ein Teil der Pioniergehölze hat seinen Ausbreitungsschwerpunkt bevorzugt in den geräumten Flächen, was vor allem Rubus idaeus (nur im Hordelymo-Fagetum) und Rubus fruticosus agg. (im Buntsandstein) betrifft. Die Himbeere ist mit Abstand der dominanteste Pionierstrauch auf allen Buntsandstein-Flächen und auf dem Nüllberg. Im Hainholz hält sich Rubus idaeus mit den beiden Holunder-Arten in etwa die Waage. Im Rotenberg und im Krücker prägten ausgedehnte Himbeer-Fluren die Physiognomie der Windwurfflächen spätestens ab dem vierten Sukzessionsjahr (2001) maßgeblich. Allgemein sind die Pioniergehölze im Buntsandstein stärker als im Zechstein vertreten, was sich entweder in nahezu ausschließlichem Vorkommen (Betula pendula, Salix caprea, Rubus fruticosus agg.) oder in höheren Deckungsgraden (Rubus idaeus, Sambucus racemosa) auf den basenärmeren Standorten ausdrückt. Für Sambucus racemosa (stärker im Buntsandstein vertreten) und Sambucus nigra (stärker im Zechstein) gilt die oben bereits erwähnte Besonderheit des Deckungsgradeinbruches ab dem vierten Sukzessionsjahr bzw. der Stagnation vorrangig auf den geräumten Flächen, während die beiden Arten im belassenen Windwurf weiter im Deckungsgrad zunahmen. Eine Besonderheit hat die geräumte Rotenberg-Abt. 1228 aufzuweisen, in der eine große Zahl von Pioniergehölzen (Rubus idaeus, R. fruticosus agg., 
Salix caprea, Sambucus racemosa, S. nigra) von Anbeginn der Erhebungen im Jahr 1999 sehr stark etabliert war, während sich die übrigen, vor allem basenarmen Windwurfflächen erst im vierjährigen Sukzessionsverlauf auf dieses Niveau „zubewegten“. Das ist besonders deutlich am Beispiel der Königsbuche nachzuvollziehen (Abb. 34 und 35).

\subsubsection{Populationsbiologischer Exkurs: Die Himbeere (Rubus idaeus) als dominantester Pionierstrauch der Windwurfgebiete im Harzvorland}

Bei der Erfassung von Rubus idaeus wurden nur ein UG, dafür aber zwei Störungsvarianten berücksichtigt (Lücken und Flächenwurf im westlichen Randbereich der Hainholz-KF1). Allgemein entwickelte sich die Himbeere im lückigen Windwurf - wahrscheinlich konkurrenzbedingt - schneller, individuenreicher und fertiler als im Flächenwurf, wo wiederum eine sehr starke Eschen-Naturverjüngung vorherrschte (Abb. 42; Kap. 4.5)

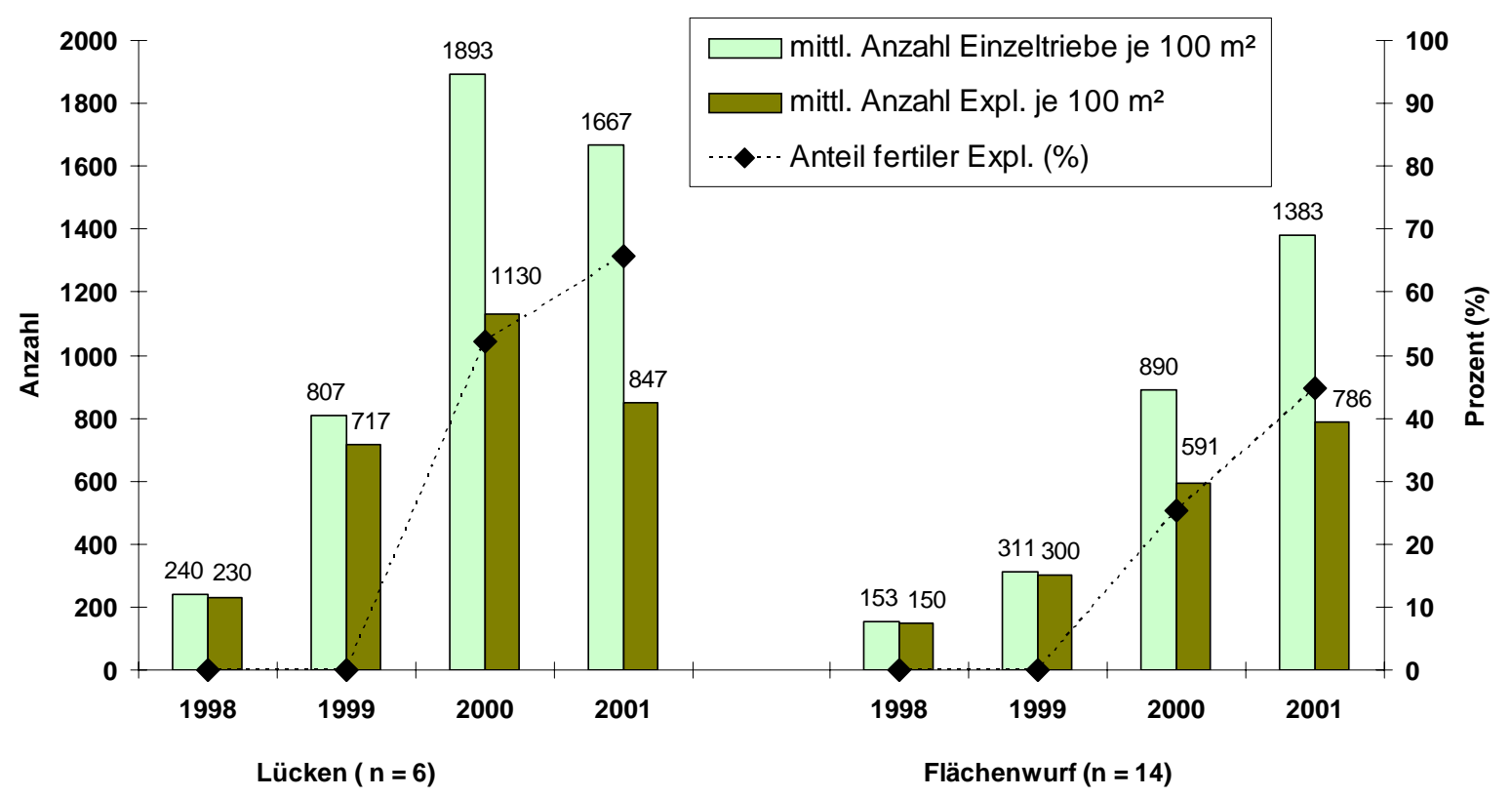

Abb. 42: Mittlere Anzahl Einzeltriebe und Exemplare (Sprossgruppen) je $100 \mathrm{~m}^{2}$ sowie Prozentanteil fertiler Exemplare von Rubus idaeus in belassenen Lücken bzw. im Flächenwurf des Hainholzes in den Jahren 1998-2001; Datengrundlage: Auszählung von 5 m²-Probeflächen im westlichen Randbereich der Hainholz-KF1.

Bis zum dritten Sukzessionsjahr kam es in beiden Straten bezüglich der Exemplare (d.h. je einer Sprossgruppe gemeinsamen Wuchsursprungs) zu einer kontinuierlichen Erhöhung der mittleren Anzahl/100 m², wobei die Werte in den Lücken von 230 auf 1.100, im Flächenwurf nur von 150 auf 590 anstiegen. Im Jahr 2001 erfolgte in den Lücken ein erster Rückgang auf ca. 850/100 $\mathrm{m}^{2}$, wohingegen im Flächenwurf ein weiterer Anstieg auf ca. 790/100 $\mathrm{m}^{2} \mathrm{zu}$ verzeichnen war. Bis zum zweiten Sukzessionsjahr (1999) bestand ein Exemplar meist nur aus dem lebenden Jahrestrieb, ab dem dritten Jahr (2000) dann aus durchschnittlich zwei 
lebenden Einzeltrieben. Darin eingeschlossen sind Exemplare mit maximal 4-6 lebenden Einzeltrieben. Die neu etablierten Himbeeren blühten und fruchteten erst ab dem dritten Sukzessionsjahr. Bei einheitlich ansteigender Tendenz bis 2001 waren in den Lücken höhere Prozentanteile fertiler Exemplare zu finden (Anstieg von 55 auf 65\%) als im Flächenwurf (Anstieg von 25 auf 45\%).

\subsection{Naturverjüngung}

\subsubsection{Naturverjüngung im belassenen Flächenwurf im vierten Jahr nach dem Sturm}

Die Ergebnisse der Verjüngungsinventur basieren auf den Aufnahmen von 20 m²-Flächen in den vier belassenen Varianten (beide Hainholz-KF, Königsbuche-KF2, Krücker-Osthang) im vierten Jahr nach dem Sturm (2001). Es bieten sich hier zwei Betrachtungsebenen an: Einerseits die gesamte Naturverjüngung unabhängig von der Pflanzenhöhe, andererseits die Unterteilung in vier Höhenklassen (0-50; 51-100; 101-150; >150 cm).

Die Hainholz-KF1 (Hordelymo-Fagetum auf tiefgründigem Dolomit-Standort) ist mit über 52.000 Sämlingen/ha (gesamte Verjüngung) mit Abstand die verjüngungsreichste, aber mit nur vier vorkommenden Arten auch die artenärmste Untersuchungsfläche (Abb. 43). Auffällig ist die Eschen-Dominanz mit 75\%, gefolgt von immerhin 23\% Buche und geringen Anteilen von Berg-Ahorn. Unterscheidet man bei der Verjüngung vier Höhenklassen (drei $50 \mathrm{~cm}$ Klassen sowie $>150 \mathrm{~cm}$ ), so verteilen sich die Sämlingszahlen mit jeweils 14-15.000/ha gleichmäßig über alle Höhenklassen. Eine Ausnahme ist die Klasse von 101-150 cm, wo nur etwa halb so viele Sämlinge vorzufinden sind (Abb. 44, 45). Die Buche hält in den beiden unteren Klassen zunächst Anteile von rund einem Drittel, die Esche etwa zwei Drittel und der Berg-Ahorn nur 3\%. Mit zunehmender Höhenklasse erringt die Esche mit bis zu 98\% (>150 cm) dann aber die absolute Dominanz und bestimmt auch physiognomisch diese Windwurfflächen. Die geringen Anteile an großwüchsigen Exemplaren von Buche und BergAhorn fallen kaum ins Gewicht (Klasse $>150$ cm: je 1\%).

Ein völlig anderes Bild ergibt sich in der Hainholz-KF2 (Carici-Fagetum auf flachgründigem Gips/Dolomit-Standort). Mit rund 9.000 Sämlingen/ha liegen hier nur etwa ein Sechstel der Verjüngungszahlen wie in der Hainholz-KF1 vor, aber mit 10 auftretenden Baumarten ist dieser Standort wesentlich artenreicher (Abb. 43). Insgesamt 5 Arten erreichen nennenswerte Anteile am Artenspektrum, wobei die beiden Ahorn-Arten (Berg-Ahorn 29\%, Spitz-Ahorn 13\%) knapp die Hälfte aller Sämlinge ausmachen und mit Sal-Weide (12\%) und Birke (2\%) auch Pionierbaumarten vertreten sind. Esche und Buche erreichen jeweils um die 20\% Anteil, fünf weitere Baumarten teilen sich die restlichen 7\%. Bei Unterteilung der Verjüngung in vier Höhenklassen (drei $50 \mathrm{~cm}$-Klassen und $>150 \mathrm{~cm}$ ) ergeben sich zunächst zwei Tendenzen (Abb. 44, 45): Mit steigender Höhenklasse sinken die Sämlingszahlen beständig von rund 5.300/ha (0-50 cm) auf rund 640/ha (>150 cm). Ebenso sinkt die Anzahl der daran beteiligten Arten von 10 auf 3 (Pflanzen >150 cm nur von Esche, Berg-Ahorn und Berg-Ulme). Berg- 
Ahorn bzw. Sal-Weide halten in den drei unteren Höhenklassen stabile Anteile von einem Drittel bzw. 10-15\%. Die Buche ist mit 30\% besonders stark in der Klasse zwischen 51 und $100 \mathrm{~cm}$ vorzufinden, noch nicht jedoch in der Klasse $>150 \mathrm{~cm}$. Bemerkenswert ist auch der mit 22\% große Anteil an Spitz-Ahorn in der untersten Höhenklasse (0-50 cm), denn diese Art spielt in keinem anderen UG eine Rolle. Die Esche erobert mit zunehmender Höhenklasse kontinuierlich Anteile (11 - 22 - 40 - 70\%) und dominiert somit unter den höchsten Bäumchen.
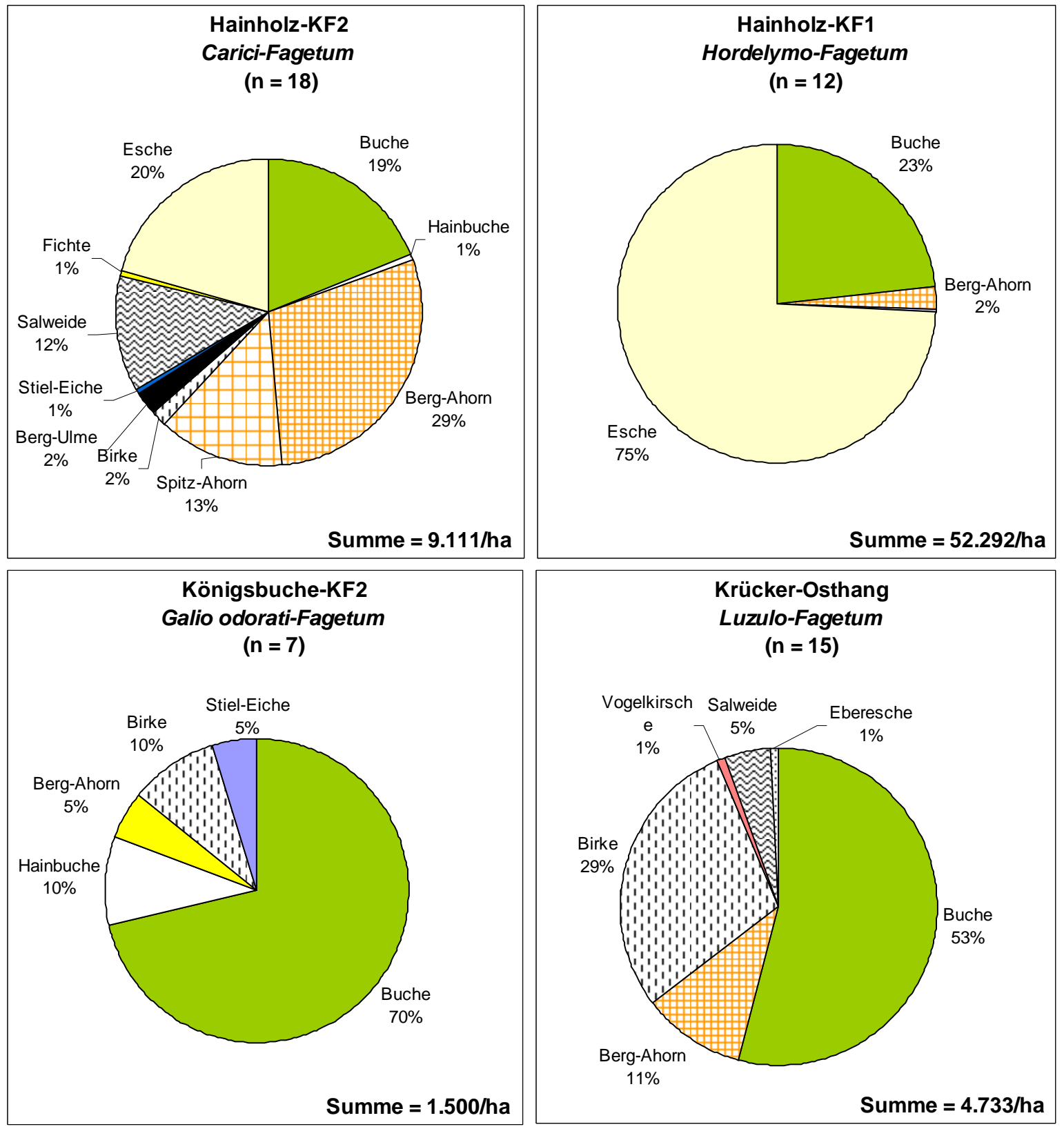

Abb. 43: Naturverjüngung in vier Buchen-Windwurfgebieten mit Flächenwurf im südwestlichen Harzvorland im vierten Jahr nach dem Sturm (2001); gesamte Naturverjüngung (ausser Keimlinge) ohne Berücksichtigung von Höhenklassen; Datengrundlage: Auszählung und Vermessung in $20 \mathrm{~m}^{2}$-Flächen, die in einem Teil der in Tab. 1 aufgeführten $100 \mathrm{~m}^{2}$-Plots liegen. 
Die ebenfalls schnellwüchsige Berg-Ulme fällt mit 13\% (>150 cm) ins Auge, der Berg-Ahorn erreicht dort mit 17\% etwas geringere Anteile als in den unteren Höhenklassen.

Die Königsbuchen-KF2 (Galio odorati-Fagetum auf Buntsandstein) ist die verjüngungsärmste aller einbezogenen Untersuchungsflächen, mit den fünf vorkommenden Baumarten liegt sie bezüglich der Artenzahl im mittleren Bereich. Hier dominiert die Buche mit 70\% Anteil an der gesamten Verjüngung in ähnlichem Maß wie die Esche in der Hainholz-KF1. Allerdings liegen die Gesamtverjüngungszahlen mit 1.500/ha nur bei einem Bruchteil derjenigen der Zechstein-Standorte (Abb. 43). Das restliche knappe Drittel am Spektrum teilen sich vier weitere Arten mit Anteilen zwischen 5 und 10\%, nämlich StielEiche, Berg-Ahorn, Hainbuche und Birke als einzige Pionierbaumart. Bezüglich der vier Höhenklassen (drei $50 \mathrm{~cm}$-Klassen und $>150 \mathrm{~cm}$ ) ergeben sich im Vergleich zu den übrigen UG deutliche Unterschiede. Die beiden oberen Höhenklassen sind mit je 71 Sämlingen/ha äußerst schwach vertreten, bei der Auszählung ließen sich nur Einzelexemplare von Buche und Stiel-Eiche finden (Abb. 44, 45). Die ohnehin schon geringen Gesamtverjüngungszahlen verteilen sich hauptsächlich auf die niedrigere Verjüngung $<50 \mathrm{~cm}$ (rund 780/ha) bzw. auf die Klasse 51-100 cm (rund 570/ha). Dabei hat die Buche in beiden Fällen mit 82 bzw. 62\% Anteil die Oberhand, gefolgt von Birke und Hainbuche (beide Klassen ) und Berg-Ahorn (nur Klasse 51-100 cm). Festzuhalten bleibt die Buchen-Dominanz bei sehr geringen Verjüngungszahlen und die bisher geringe Wuchsleistung der Naturverjüngung (kaum über $100 \mathrm{~cm}$ hoch), was vermutlich auch ein Konkurrenzeffekt der dichten Himbeerfluren oder der krautigen Vegetation (z.B. Juncus effusus, Carex sylvatica) bzw. die Folge der teilweisen Staunässe des Standortes sein könnte.

Der zweite basenarme Standort am Krücker-Osthang (Luzulo-Fagetum auf Buntsandstein) ist mit rund 4.700 Sämlingen/ha (gesamte Verjüngung) etwa dreimal so verjüngungsreich wie die Königsbuchen-KF2, liegt aber diesbezüglich auch weit hinter den Zechstein-Standorten zurück. Die Baumartenzahl liegt mit 6 eher im mittleren Bereich (Abb. 43). Auch hier dominiert die Buche mit immerhin 53\%. Auffällig ist am Krücker der unter allen UG höchste Pionierbaumarten-Anteil von rund einem Drittel, der von Birke (29\%), Sal-Weide (5\%) und Eberesche (1\%) gestellt wird. Dazu kommen Berg-Ahorn (11\%) und Vogel-Kirsche (1\%). Bei Unterteilung der Verjüngung in vier Höhenklassen (drei Klassen je $50 \mathrm{~cm}$, eine $>150 \mathrm{~cm}$ ) ergibt sich bezüglich der Sämlingszahlen/ha ein ähnliches Muster wie in Hainholz-KF1, wenn auch auf niedrigerem Niveau (Abb. 44, 45). Mit Ausnahme der Klasse 101-150 cm sind die Pflanzen mit rund 1.100-1.500/ha etwa gleichverteilt, bei der zweitgrößten Höhenklasse liegen sie dagegen mit rund 670/ha nur bei der Hälfte bis einem Drittel der übrigen Kategorien. Während die Buche mit zunehmender Höhenklasse kontinuierlich Anteile verliert (76 - 68 - 35 - 16\%), ist die Birke in der gleichen Reihenfolge immer stärker vertreten (9 - 16 - 45 - 66\%) und dominiert somit bei den jungen Bäumen >150 cm. Ebenfalls bemerkenswert ist bei der höheren Verjüngung (>150 cm) der mit Abstand höchste Buchen-Anteil aller UG von $16 \%$. Berg-Ahorn und Sal-Weide verfügen in allen vier Höhenklassen über mehr oder 
weniger konstante Anteile von 6-15 bzw. 4-6\%. Der Krücker ist das einzige UG, bei dem in der obersten Höhenklasse (>150 cm) mehr Arten (6) vertreten sind als in den niedrigeren (4), wozu hier einige hochgewachsene Exemplare von Eberesche und Vogel-Kirsche beitragen. Ausserdem ist der Krücker das einzige UG, in dem sich Pionierbäume anteilmäßig stärker etablieren konnten.

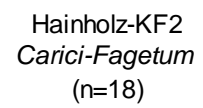
$(n=18)$

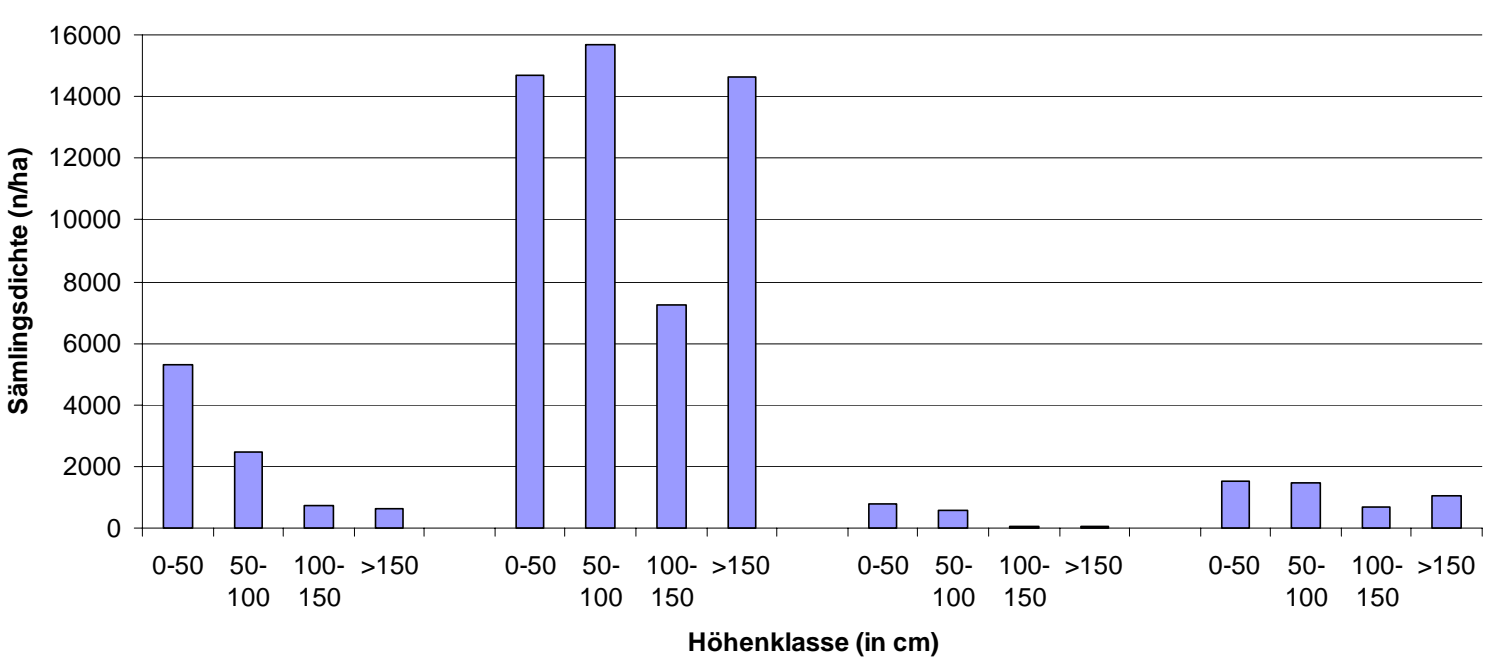

Abb. 44: Sämlingszahlen je ha in vier Buchen-Windwurfgebieten mit Flächenwurf im südwestlichen Harzvorland im vierten Jahr nach dem Sturm (2001); gesamte Verjüngung (ausser Keimlinge) unterteilt in vier Höhenklassen; Datengrundlage: Auszählung und Vermessung in $20 \mathrm{~m}^{2}$-Flächen, die in einem Teil der in Tab. 1 aufgeführten $100 \mathrm{~m}^{2}$-Plots liegen.

Vergleicht man alle vier Standorte bezüglich der Artenzahl (Abb. 43), so steht HainholzKF2 (10 Arten) vor Krücker-Osthang (6 Arten), Königsbuche-KF2 (5 Arten) und HainholzKF1 (3 Arten). Bezüglich der Sämlingszahlen je ha liegt Hainholz-KF1 (52.000) vor Hainholz-KF2 (9.000), Krücker-Osthang (4.700) und Königsbuche-KF2 (1.500). Vom Gesamtartenpool sind in der Hainholz-KF1 und am Krücker-Osthang bereits $100 \%$ in die Höhenklasse $>150 \mathrm{~cm}$ durchgewachsen, in der Hainholz-KF2 bzw. Königsbuche-KF2 dagegen nur 33\% bzw. 20\% (Abb. 45). Buche und Berg-Ahorn sind die einzigen beiden Arten, die in der Verjüngung aller vier untersuchten Standorte vorkommen. Für beide Zechstein-Standorte ist bis zum Jahr 2001 die auffällige Wuchsleistung der Esche bezeichnend (Abb. 43 bis 45), die aber standortsabhängig unterschiedliche Beimischungen anderer Laubhölzer enthält und sehr unterschiedliche Stückzahlen erreicht. Buche ist überall reichlich vorhanden, allerdings hält sie sich bezüglich der Wuchshöhe (noch) zurück.

Insgesamt kann die Hainholz-KF1 als Eschen-dominiert, sehr verjüngungsreich und relativ artenarm eingeschätzt werden. In der Hainholz-KF2 lässt sich dagegen keine ausgeprägte 


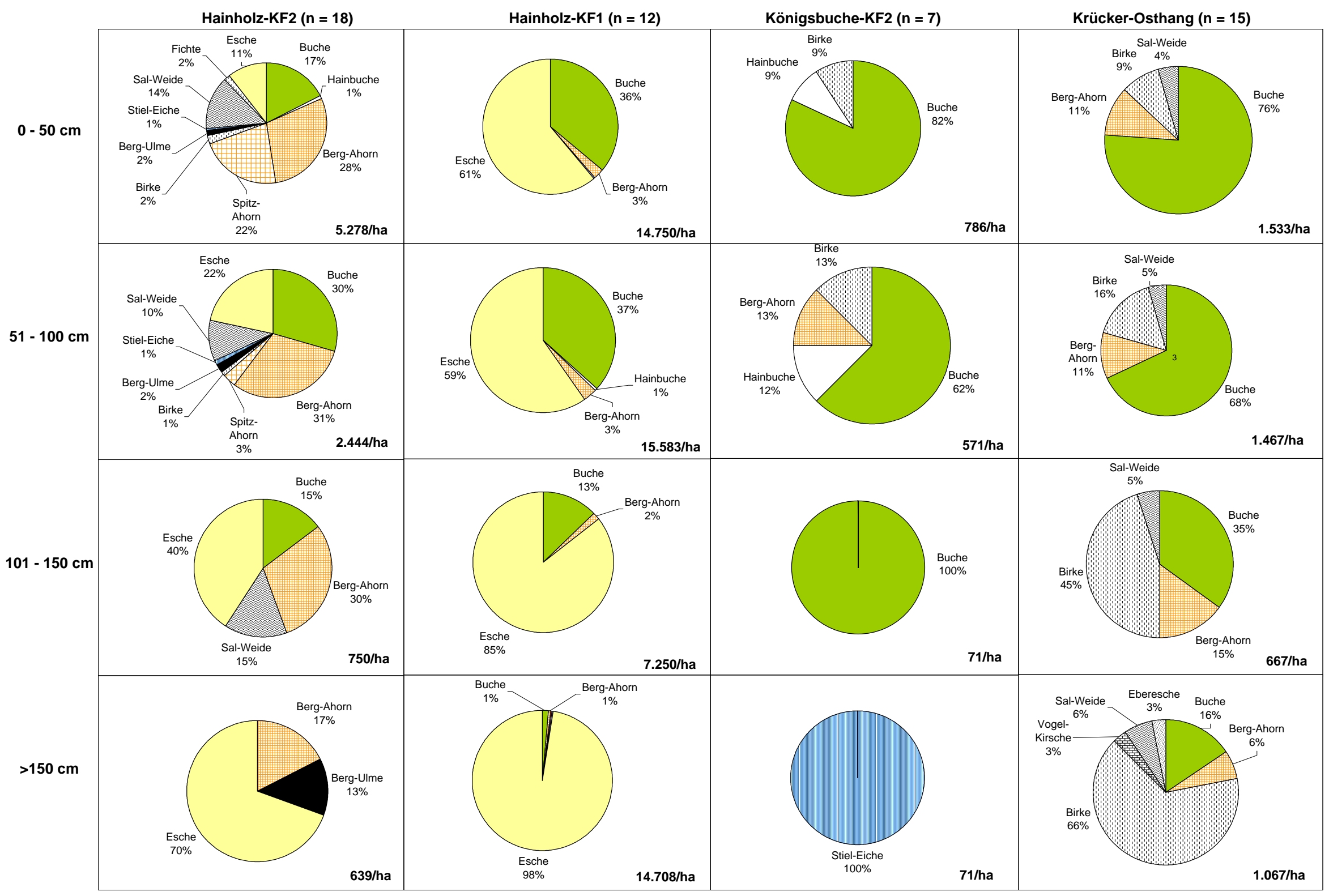

Abb. 45: Naturverjüngung in vier Buchen-Windwurfgebieten mit Flächenwurf im südwestlichen Harzvorland im vierten Jahr nach dem Sturm (2001); gesamte Verjüngung (ausser Keimlinge) unterteilt nach Arten und vier Höhenklassen; Datengrundlage: Auszählung und Vermessung in 20 m²-Flächen, die in einem Teil der in Tab. 1 aufgeführten 100 m²-Plots liegen. 
Dominanzart finden, die Fläche verfügt über mittlere Verjüngungszahlen und ist relativ artenreich. Die beiden Buntsandstein-Standorte wiederum sind Buchen-dominiert, relativ verjüngungsarm (besonders die Königsbuche) und haben ein mittleres Artenzahlniveau. In den einzelnen Höhenklassen ergeben sich Dominanzverhältnisse, die von der Gesamtzusammensetzung teilweise stark abweichen.

Focussiert man nur auf die Klimaxbaumart Buche, so fällt zunächst deren bereits erwähnte Dominanz in der Verjüngung der basenarmen Standorte auf (Königsbuche-KF2: 70\%, Krücker-Osthang: 53\%), wohingegen sie auf den basenreichen Standorten nur 23\% (Hainholz KF1) bzw. 19\% (Hainholz KF2) erreicht (Abb. 43). Allerdings ergibt sich ein anderes Bild, wenn man absolute Verjüngungszahlen einbezieht: die Hainholz-KF1 liegt mit rund 12.000 Buchen/ha weit vor den übrigen Standorten mit rund 2.500/ha (Krücker-Osthang), 1.700/ha (Hainholz-KF2) und 1.000/ha (Königsbuche-KF2).

Focussiert man nur auf die Pionierbaumarten, zu denen hier Birke, Sal-Weide und Eberesche gezählt werden, so ergeben sich die höchsten Werte am Krücker-Osthang (1.600/ha) und in der Hainholz-KF2 (1.300/ha), während in der Königsbuchen-KF2 nur 150 Pionierbäume/ha zu finden waren und in der Hainholz-KF1 gar keine. Eine ähnliche Abstufung ergibt sich bezüglich der Anteile dieser Artengruppe am Gesamtspektrum aller Sämlinge mit 34\% im Krücker, 14\% in der Hainholz-KF2, 10\% in der Königsbuchen-KF2 und $0 \%$ in der Hainholz-KF1 (Abb. 43).

\subsubsection{Naturverjüngung auf Buntsandstein-Standorten in Abhängigkeit vom Störungsgrad im vierten Jahr nach dem Sturm}

Auf beiden Buntsandstein-Standorten liegen die drei Straten „Bestand“, „Lücke“ und „Flächenwurf“ dicht beieinander. Es ist daher davon auszugehen, dass es vor dem Sturm keine Unterschiede in der waldbaulichen Behandlung und in der Entwicklung der Naturverjüngung gab. Das Artenspektrum umfasste im Jahr 2001 in beiden Gebieten hauptsächlich die überall vorherrschende Buche, die aber je nach Variante durch Berg-Ahorn, Hainbuche, Birke, Stiel-Eiche, Sal-Weide und Esche sowie durch minimale Anteile anderer Arten ergänzt wurde (Abb. 46). Neben der Buchen-Dominanz in der Verjüngung besteht eine weitere Gemeinsamkeit aller untersuchten Flächen in der sehr deutlichen Abnahme der Sämlingszahlen/ha vom Bestand (Königsbuche: 57.000/Krücker: 124.000) über die Lücken (11.000/54.000) zum Flächenwurf (1.500/4.700). Neben den höheren Verjüngungszahlen des Krückers gegenüber der Königsbuche besteht ein weiterer Unterschied zwischen den beiden Untersuchungsgebieten in der prozentualen Beteiligung anderer Baumarten am Artenspektrum. Generell verliert die Buche in der Reihenfolge Bestand - Lücken Flächenwurf immer mehr Anteile. Dies ist in der schon lange sich selbst überlassenen Königsbuche weniger ausgeprägt (Anteile wie 96 - 82 - 70\%) als in dem ehemaligen bzw. derzeitigen Wirtschaftswald des Krückers (Anteile wie 96 - 66 - 53\%). Weiterhin ist auffällig, 
dass die Birke als häufigste Pionierbaumart mit 1.300 Sämlingen/ha nur im Krücker nennenswerte, aber keine dominanten Anteile (29\%) an der Verjüngung des Flächenwurfes erreicht. Der Stückzahl-Unterschied der Baumarten spiegelt sich auf beiden flächig geworfenen Standorten - wenn auch nicht sehr prägnant - in den absoluten Deckungssummen bzw. Anteilen am Wuchsformenspektrum wider (Abb. 34, 35; Tab. A23).

Königsbuche-KF2

Krücker-Osthang

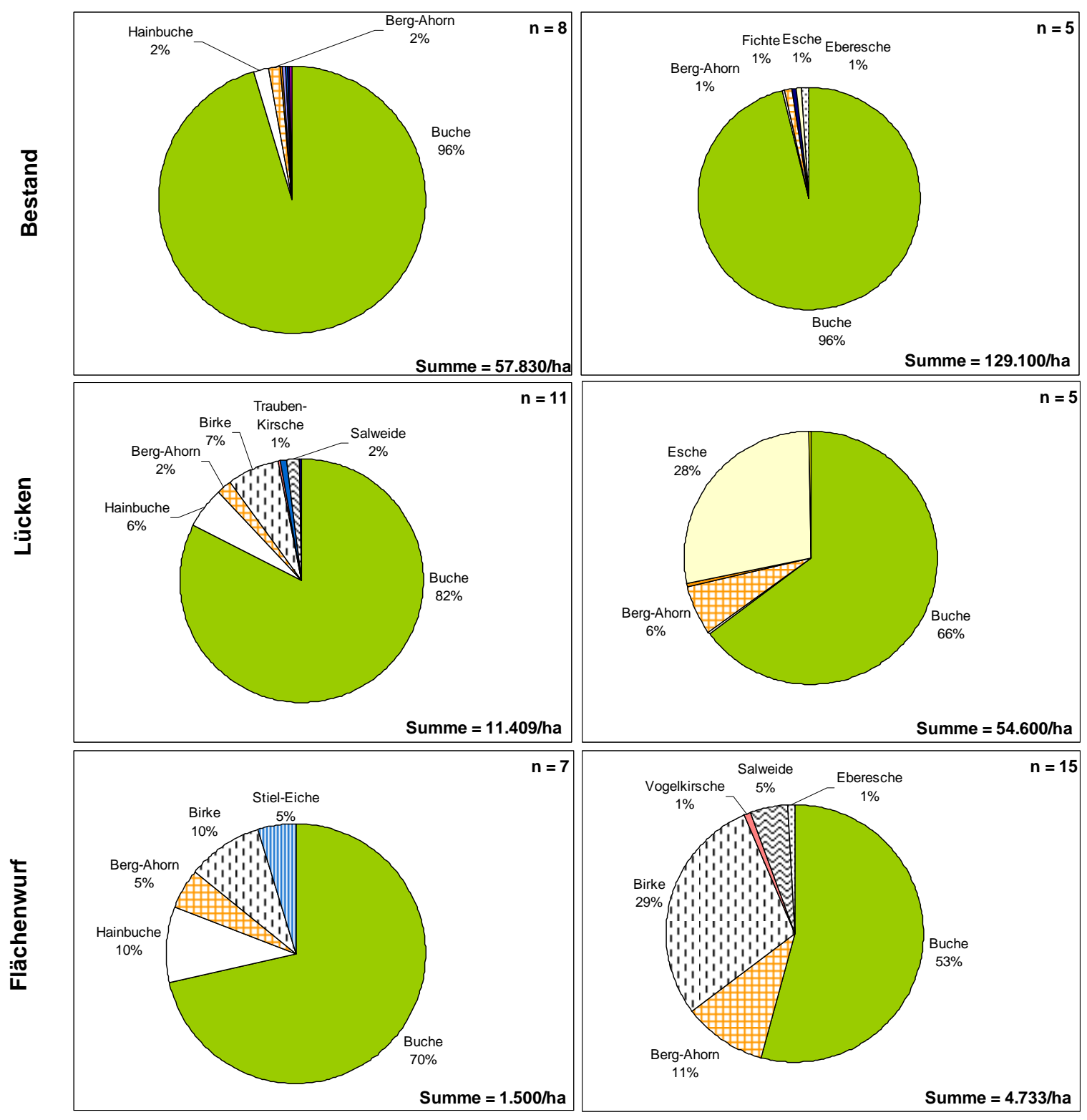

Abb. 46: Naturverjüngung in zwei Buchen-Windwurfgebieten auf Buntsandstein (Königsbuche-KF2: Galio odorati-Fagetum; Krücker-Osthang: Luzulo-Fagetum) unterteilt nach drei unterschiedlichen Störungsgraden im vierten Jahr nach dem Sturm (2001); gesamte Verjüngung (ausser Keimlinge) ohne Berücksichtigung von Höhenklassen; Datengrundlage: Auszählung und Vermessung in $20 \mathrm{~m}^{2}$-Flächen, die teilweise innerhalb, teilweise ausserhalb der in Tab. 1 aufgeführten $100 \mathrm{~m}^{2}$-Plots liegen. 
Unterteilt man die Verjüngung in die eingangs bereits erwähnten Höhenklassen (Abb. 47, 48), fällt zunächst auf, dass im stehenden Bestand beider UG bislang keine Verjüngung über 100 cm existiert. In den beiden niederen Klassen dominiert die Buche fast vollständig, lediglich im Krücker-Osthang ist in der Klasse 51-100 cm die Eberesche mit 22\% Anteil stärker vertreten. Die allermeisten Jungpflanzen sind unter $50 \mathrm{~cm}$ groß, wobei die Naturverjüngung im Bestand des Krückers in dieser Höhenklasse mit 128.000 Sämlingen/ha mehr als doppelt so zahlreich ist wie in der Königsbuchen-KF2. Bei den Lücken gibt es deutliche Unterschiede zwischen beiden Gebieten (Abb. 47, 48). In der Königsbuchen-KF2 konzentriert sich fast die gesamte Verjüngung (ca. 10.000/ha) auf die Klasse bis 50 cm, die nächsthöheren Klassen sind mit 909, 136 und 91 Sämlingen/ha nur sehr schwach vertreten. Mit zunehmender Höhenklasse verliert die Buche Anteile am Gesamtspektrum (86 - 65 - 34\%) und ist (noch) nicht mit Pflanzen $>150 \mathrm{~cm}$ vertreten. Stattdessen sind zunehmend Birke, Traubenkirsche, Sal-Weide und Hainbuche zu finden, wenn auch mit geringen Stückzahlen. Im Krücker dagegen sind die beiden unteren Höhenklassen mit 21-23.000/ha ungefähr gleichstark vertreten, danach kommt es mit zunehmender Höhenklasse zu einem Absinken der Verjüngungszahlen über 8.500/ha $(101-150 \mathrm{~cm})$ auf 1.600/ha (>150 cm). Die Buche ist maßgeblich an der Verjüngung beteiligt, wobei sie in den beiden mittleren Höhenklassen dominiert. Aber auch bei den Pflanzen >150 cm ist der Buchen-Anteil mit 38\% recht hoch. Standörtlich bedingt tritt hier die Esche hinzu, da im Bereich der lückig geworfenen Untersuchungsflächen eine Übergangszone zum benachbarten, basenreicheren Zechstein-Gebiet des Hainholzes verläuft. Der Berg-Ahorn ist mit 25\% Anteil ebenfalls sehr stark an der obersten Höhenklasse beteiligt. Die Artenzusammensetzung im Flächenwurf ist bereits in Kap. 4.5.1 beschrieben worden (Abb. 43-45).

Betrachtet man nun die einzelnen Höhenklassen in der Reihenfolge Bestand - Lücke Flächenwurf (Abb. 47, 48), so ist besonders für die Verjüngung bis $50 \mathrm{~cm}$ festzustellen, dass es zu einer drastischen Abnahme der Verjüngungszahlen kommt (Königsbuche: Reduktion auf ein Siebzigstel der im Bestand vorherrschenden Dichte, im Krücker sogar auf ein Hundertstel). Bei den darüberliegenden Höhenklassen ergeben sich in beiden UG unterschiedliche Entwicklungen. Als Gemeinsamkeit bleibt festzuhalten, dass die Lücken verjüngungsreicher sind als der Flächenwurf, wobei diese Aussage in der Königsbuche wegen der dortigen geringen Stückzahlen von Pflanzen >100 cm eingeschränkt gilt. Der oben konstatierte Rückgang des Buchen-Anteils vom Bestand über die Lücken zum Flächenwurf lässt sich nur in der Summe (Gesamtdarstellung) feststellen, nicht aber gleichermaßen in jeder der vier Höhenklassen. 


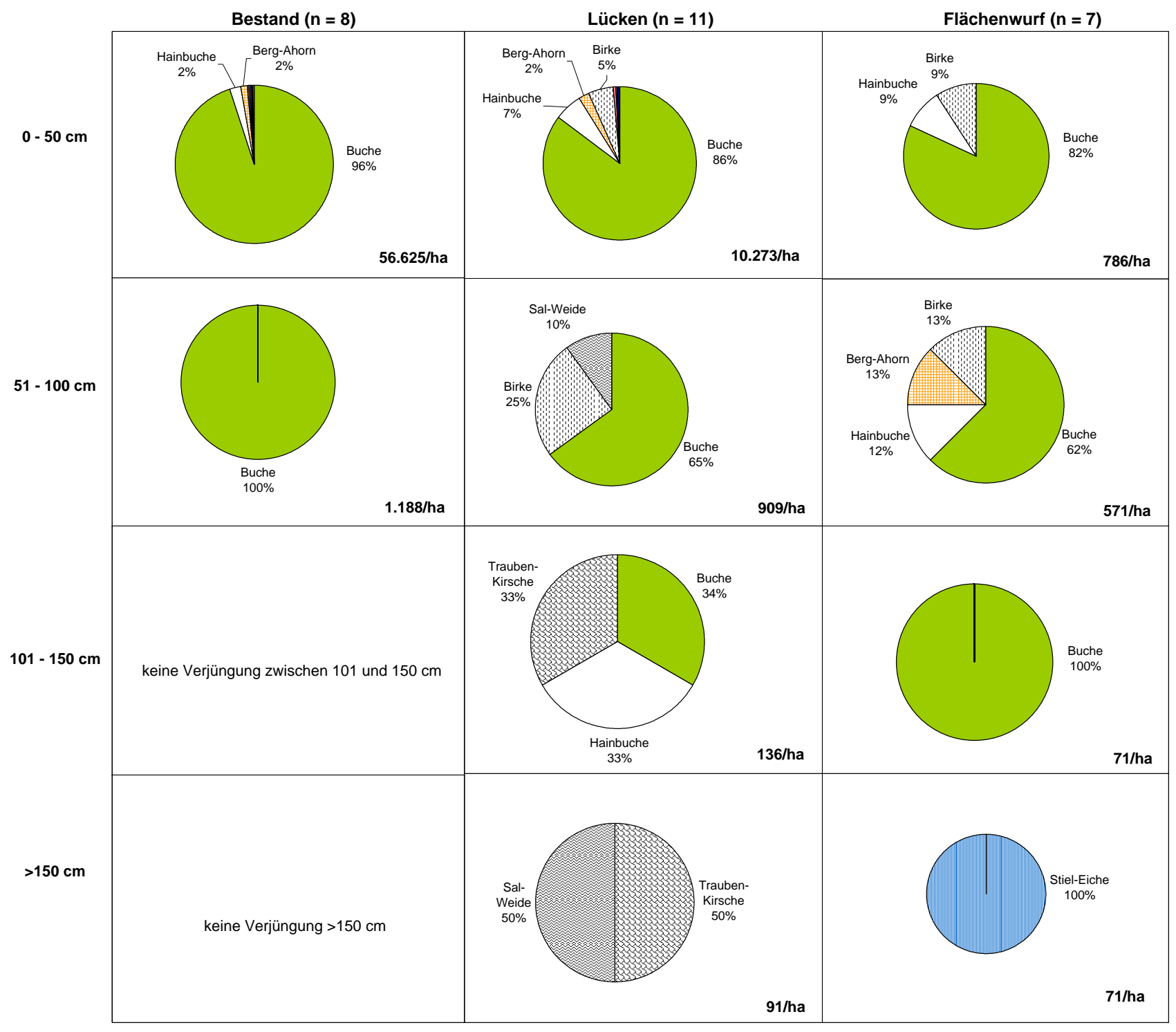

Abb. 47: Naturverjüngung in der Königsbuchen-KF2 (Galio odorati-Fagetum ), unterschieden nach drei Störungsgraden im vierten Jahr nach dem Windwurf (2001); gesamte Verjüngung (ausser Keimlinge) unterteilt in vier Höhenklassen; Datengrundlage: Auszählung und Vermessung in 20 m²-Flächen, die teilweise innerhalb (Flächenwurf), teilweise ausserhalb (Bestand, Lücken) der in Tab. 1 aufgeführten $100 \mathrm{~m}^{2}$-Plots liegen. 


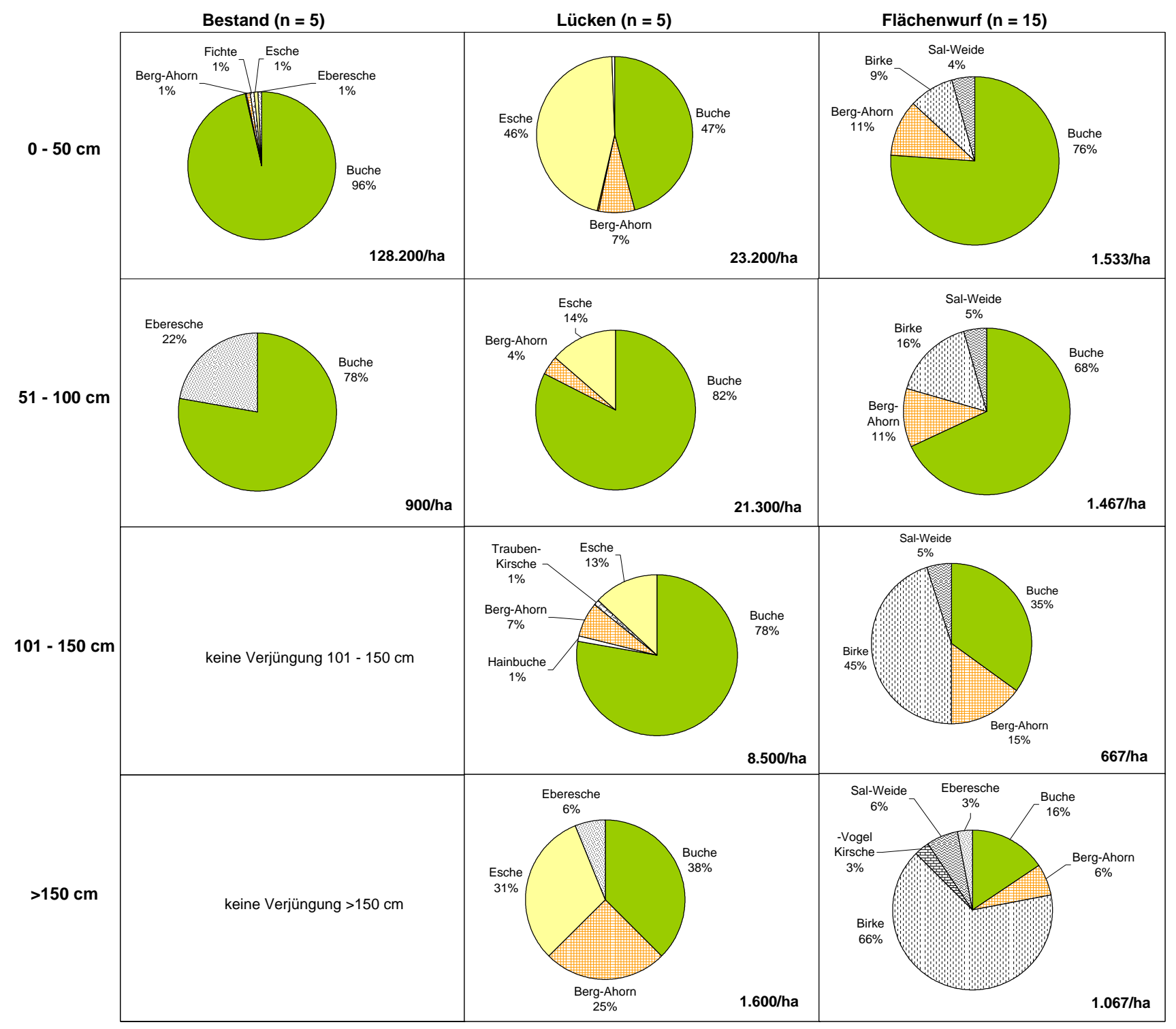

Abb. 48: Naturverjüngung auf dem Krücker-Plateau und am Krücker-Osthang (Luzulo-Fagetum ), unterschieden nach drei Störungsgraden im vierten Jahr nach dem Windwurf (2001); gesamte Verjüngung (ausser Keimlinge) unterteilt in vier Höhenklassen; Datengrundlage: Auszählung und Vermessung in $20 \mathrm{~m}^{2}$-Flächen, die teilweise innerhalb (Flächenwurf), teilweise ausserhalb (Bestand, Lücken) der in Tab. 1 aufgeführten 100 m²-Plots liegen. 


\subsection{Sonderstandort Wurzelteller}

\subsubsection{Vorbemerkung}

In den hier behandelten Buchen-Waldgebieten ist in der Regel Windwurf vorzufinden, d.h. liegende Stämme mit aufgeklappten Wurzeltellern herrschen vor. Windbruch, d.h. stehendes, sturmbedingt gebrochenes/gesplittertes Totholz ist dagegen wesentlich seltener (siehe Kap. 1.2). Die aufgeklappten Wurzelteller (WT) als Sonderstandorte der Vegetationsbesiedlung und -entwicklung sollen im Folgenden einerseits isoliert betrachtet und andererseits einem Vergleich mit der umgebenden Untersuchungsfläche (Ufl) unterzogen werden. Es erfolgt dabei eine Beschränkung auf drei belassene Windwurfgebiete mit Flächenwurf, nämlich die beiden Hainholz-KF sowie die Königsbuchen-KF2.

Resultierend aus der Hauptsturmrichtung liegen die Stämme überall nach Norden ausgerichtet, ebenso der alte Waldboden auf der Wurzeltelleroberseite (OS). Im Gegensatz dazu sind die Unterseite des Wurzeltellers (US) und die Wurfmulde (MU) südexponiert. Während die Oberseite des Wurzeltellers meist von Anfang an mit Vegetation bedeckt ist, stellen seine Unterseite und die Wurfmulde „Besiedlungs-Neuland“ dar (Abb. 10).

Es ist zu beachten, dass die Wurzeltellerdaten eine Auskoppelung aus der Datenmatrix der 100 m²$^{2}$-Flächen darstellen, d.h. Wurzelteller und umliegende Untersuchungsfläche wurden nicht vollständig getrennt aufgenommen. Deshalb werden in Tab. 14 Aussagen dazu getroffen, wie sich die untersuchten Parameter auf der Gesamtfläche (unter Einschluss der Wurzelteller) und bei isolierter Betrachtung nur der Wurzelteller verhalten. Die Angabe eines Zunahme- bzw. Abnahmefaktors (jeweils um das x-fache des Ausgangswertes) ermöglicht einen Vergleich unterschiedlicher Entwicklungsgeschwindigkeiten zwischen zweitem und viertem Sukzessionsjahr (1999...2001). Da aus Praktikabilitätsgründen nicht immer alle Flächen bzw. Wurzelteller einbezogen wurden (besonders in Hainholz-KF2), weichen die Angaben in den Tab. 14 sowie A26 bis A31 von denen in Tab. A22 und A23 sowie von Kap. 4.2 ab.

Der Vergleich von Untersuchungsfläche und Wurzelteller stellt insofern ein Problem dar, da einer einheitlichen Bezugsgröße bei den Untersuchungsflächen (100 $\mathrm{m}^{2}$ je Plot) eine sehr unterschiedliche Flächengröße der Wurzelteller bzw. ihrer einzelnen Abschnitte gegenübersteht, was sich besonders bei den mittleren und den Gesamtartenzahlen bemerkbar macht. Im Falle der Schichtdeckungsgrade resultieren die Angaben für den gesamten Wurzelteller (wie in Tab. 14 verwendet) aus einer arithmetischen Mittelwertbildung der drei Wurzeltellerabschnitte (Oberseite, Unterseite, Mulde), da diese Deckungen im Gelände nur für jeden der drei Abschnitte einzeln geschätzt wurden.

Die dargestellten Wurzeltelleruntersuchungen gleichen am ehesten einer „sukzessionalen Momentaufnahme“. Denn hier läuft ein sehr dynamischer Prozess ab, der durch immer wiederkehrenden Zerfall, Überschüttung und Neubesiedlung einzelner Wurzeltellerabschnitte charakterisiert ist, bis die Wurzelteller nach vielen Jahren nur noch flachen und dauerhaft bewachsenen Hügeln ähneln werden (siehe Kap. 5.7.2). 


\subsubsection{Strukturelle Parameter von Untersuchungsflächen und Wurzeltellern}

Anhand von Artenzahlen und Schichtdeckungsgraden sollen Untersuchungsflächen und darin befindliche Wurzelteller verglichen und unterschiedliche bzw. äquivalente Entwicklungen beschrieben werden (Tab. 14).

Tab. 14: Strukturelle Parameter auf den Untersuchungsflächen $\left(100 \mathrm{~m}^{2}\right.$-Plots unter Einbeziehung der Wurzelteller) und bei isolierter Betrachtung nur der Wurzelteller auf drei Buchen-Windwurfflächen mit Flächenwurf im südwestlichen Harzvorland im Vergleich der Sukzessionsjahre 1999 und 2001; WT = Wurzelteller, Ufl = Untersuchungsfläche; Darstellung: erster Wert (von 1999) ... zweiter Wert (von 2001); unter Angabe von Zunahme- (+) bzw. Abnahmefaktoren (-); z.B. „+1,5“ bedeutet „Zunahme um das 1,5-fache“ vom Ausgangswert.

\begin{tabular}{|c|c|c|c|}
\hline & $\begin{array}{l}\text { Hainholz-KF2 } \\
\text { Carici-Fagetum } \\
\quad(\mathrm{n}=15) \\
\text { 1999...2001 }\end{array}$ & $\begin{array}{c}\text { Hainholz-KF1 } \\
\begin{array}{c}\text { Hordelymo-Fagetum } \\
(\mathrm{n}=14)\end{array} \\
1999 . . .2001\end{array}$ & $\begin{array}{c}\text { Königsbuche-KF2 } \\
\begin{array}{c}\text { Galio-Fagetum } \\
(\mathrm{n}=12)\end{array} \\
1999 . .2001\end{array}$ \\
\hline vorhandene Wurzelteller & $\begin{array}{c}55 \\
\left(=3,7 / 100 \mathrm{~m}^{2}\right. \\
\text { bzw. } 370 / \mathrm{ha})\end{array}$ & $\begin{array}{c}\mathbf{2 6} \\
\left(=1,8 / 100 \mathrm{~m}^{2}\right. \\
\text { bzw. } 180 / \mathrm{ha})\end{array}$ & $\begin{array}{c}23 \\
\left(=1,9 / 100 \mathrm{~m}^{2}\right. \\
\text { bzw. } 190 / \mathrm{ha})\end{array}$ \\
\hline $\begin{array}{l}\text { mittlere Wurzelteller- } \\
\text { deckung/100 m² (2001) }\end{array}$ & $\mathbf{8 , 1 \%}$ & $4,4 \%$ & $4,5 \%$ \\
\hline einbezogene Wurzelteller & 31 & 24 & 23 \\
\hline \multicolumn{4}{|c|}{ Gesamtartenzahl Strauchschicht } \\
\hline auf WT + Ufl zusammen & $7 \ldots 18(+2,6)$ & $7 \ldots 13(+1,8)$ & $\mathbf{4 . . . 9}(+2,2)$ \\
\hline $\begin{array}{l}\text { nur auf WT } \\
\text { (entspricht.....\% vom } \\
\text { Gesamtartenpool der Ufl) }\end{array}$ & $\begin{array}{c}\mathbf{0 . . . 5}(+5,0) \\
(0 \%) \ldots(28 \%)\end{array}$ & $\begin{array}{c}1 . . .6(+6,0) \\
(14 \%) \ldots(46 \%)\end{array}$ & $\begin{array}{c}2 . . .5(+2,5) \\
(50 \%) \ldots(55 \%)\end{array}$ \\
\hline \multicolumn{4}{|c|}{ mittlere Artenzahl Strauchschicht } \\
\hline auf WT + Ufl zusammen & $2,0 \ldots .7,5(+3,7)$ & $2,8 \ldots .5,3(+1,9)$ & $\mathbf{2 , 5} \ldots \mathbf{4 , 1}(+1,6)$ \\
\hline nur auf WT & $\mathbf{0 , 0} \ldots \mathbf{0 , 7}(+0,7)$ & $\mathbf{0 , 1} \ldots \mathbf{1 , 5}(+15,0)$ & $0,7 \ldots 2,2(+3,1)$ \\
\hline \multicolumn{4}{|c|}{ Gesamtartenzahl Feldschicht } \\
\hline auf WT + Ufl zusammen & $112 . . .141(+1,3)$ & 61...70 (+1,2) & 53...44 $(-1,2)$ \\
\hline $\begin{array}{l}\text { nur auf WT } \\
\text { (entspricht.....\% vom } \\
\text { Gesamtartenpool der Ufl) }\end{array}$ & $\begin{array}{l}\mathbf{5 1 . . . 8 7}(+1,7) \\
(45 \%) \ldots(62 \%) \\
\end{array}$ & $\begin{array}{l}37 . . .43(+1,2) \\
(61 \%) \ldots(61 \%) \\
\end{array}$ & $\begin{array}{l}30 . . .29(-1,03) \\
(57 \%) \ldots(66 \%)\end{array}$ \\
\hline \multicolumn{4}{|c|}{ mittlere Artenzahl Feldschicht } \\
\hline auf WT + Ufl zusammen & $31,5 \ldots . .56,1(+1,8)$ & $23,3 . .28,4(+1,2)$ & $16,0 \ldots 19,3(+1,2)$ \\
\hline nur auf WT & $7,1 \ldots 16,5(+2,3)$ & $9,5 \ldots 9,5( \pm 1,0)$ & $9,0 \ldots 10,6(+1,2)$ \\
\hline \multicolumn{4}{|c|}{ mittlere Deckungssumme Strauchschicht (in \%) } \\
\hline auf WT + Ufl zusammen & $2,3 \ldots 14,0(+6,1)$ & $13,0 \ldots 63,4(+4,8)$ & 6,8...70,7 (+10,4) \\
\hline nur auf WT & $0,0 \ldots 4,3(+4,3)$ & 2,4...34,0 (+14,2) & $3,3 . . .45,5(+13,8)$ \\
\hline \multicolumn{4}{|c|}{ mittlere Deckungssumme Feldschicht (in \%) } \\
\hline auf WT + Ufl zusammen & $\mathbf{7 8 , 3 \ldots . . 8 5 , 8 ( + 1 , 1 )}$ & $146,9 \ldots 162,0(+1,1)$ & $91,4 \ldots 83,1(-1,1)$ \\
\hline nur auf WT & $24,7 \ldots .35,9(+1,4)$ & $42,0 . .46,2(+1,1)$ & $36,9 . .23,0(-1,6)$ \\
\hline \multicolumn{4}{|c|}{ mittlere Deckung Kryptogamen (in \%) } \\
\hline auf WT + Ufl zusammen & $11,1 \ldots 16,7(+1,5)$ & $5,0 . . .9,4(+1,9)$ & $3,8 . .4,3(+1,1)$ \\
\hline nur auf WT & $12,6 \ldots . .28,0(+2,2)$ & $11,4 \ldots 14,2(+1,2)$ & 20,6...16,7 (-1,2) \\
\hline \multicolumn{4}{|c|}{ mittlere Deckung Laubstreu + Totholz (in \%) } \\
\hline auf WT + Ufl zusammen & $50,0 \ldots . .34,9(-1,4)$ & 25,3...22,0 (-1,2) & $\mathbf{5 7 , 1 . . . 4 8 , 4}(-1,2)$ \\
\hline nur auf WT & $1,5 \ldots 3,3(+2,2)$ & $1,8 \ldots . .5,9(+3,3)$ & 0,8...18,5 (+23,1) \\
\hline \multicolumn{4}{|c|}{ mittlere Deckung vegetationsfreier Mineralboden/Fels (\%) } \\
\hline auf WT + Ufl zusammen & $17,8 \ldots .7,4(-2,4)$ & $5,6 \ldots 0,5(-11,2)$ & $\mathbf{0 , 3 \ldots . 0 , 1}(-3,0)$ \\
\hline nur auf WT & $60,8 \ldots . .42,5(-1,4)$ & $54,8 \ldots 33,8(-1,6)$ & $52,4 \ldots . .56,5(+1,1)$ \\
\hline
\end{tabular}


Wurzeltellerdichte und -deckung (Tab. 14): Die mittlere Anzahl Wurzelteller und die mittlere Wurzeltellerdeckung je $100 \mathrm{~m}^{2}$-Plot verhalten sich zueinander in etwa äquivalent. Die Hainholz-KF2 hebt sich mit 3,7 WT/100 $\mathrm{m}^{2}$ und etwa 8\% mittlerer WT-Deckung als besonders „wurzeltellerreich“ gegenüber den beiden anderen Kernflächen ab (dort rund 2 WT/100 $\mathrm{m}^{2}$ mit etwa 4,5\% mittlerer Deckung). Übereinstimmend zwischen allen einbezogenen UG ist somit jeder Wurzelteller mit rund 2,1 bis 2,4 $\mathrm{m}^{2}$ senkrechter Projektionsfläche zu veranschlagen. Die mittlere Fläche einzelner Wurzeltellerabschnitte schwankt zwischen 1,3 und 4,0 $\mathrm{m}^{2}$ und nimmt im Sukzessionsverlauf ab, da die Wurzelteller wenn auch unterschiedlich schnell - zerfallen. Die Unterseite des Wurzeltellers ist dabei normalerweise der größte der drei Abschnitte, die Oberseite und die Mulde folgen mit etwa derselben mittleren Größe an zweiter Stelle (in der Hainholz-KF2 sind Unterseite und Mulde im Mittel annähernd gleichgroß). Beispielsweise sind die Wurzeltellerunterseiten in der Königsbuche mit durchschnittlich 3,6 bis 4,1 $\mathrm{m}^{2}$ am größten, in der Hainholz-KF2 dagegen mit 2,0 bis 2,3 $\mathrm{m}^{2}$ am kleinsten, die der Hainholz-KF1 liegen mit 2,6 bis 3,0 $\mathrm{m}^{2}$ dazwischen (Tab. A26 bis A31).

Strauchschicht-Artenzahlen (Tab. 14): Bis zum vierten Sukzessionsjahr verdoppelt sich auf den $100 \mathrm{~m}^{2}$-Plots die Gesamtartenzahl standortsunabhängig. Dabei werden auf den beiden Zechstein-Standorten 13 (Hainholz-KF1) bzw. 18 (Hainholz-KF2) Strauchschichtarten erreicht, im Buntsandstein dagegen nur 9. Auf den Wurzeltellern wird dieser Trend nicht nachvollzogen, hier konnten bei unterschiedlichen Steigerungsraten bis zum vierten Sukzessionsjahr überall einheitlich 5-6 Strauchschichtarten gezählt werden. Im vierten Jahr nach dem Sturm wächst auf den Wurzeltellern des Gips/Dolomit-Standortes (Hainholz-KF2) nur ein gutes Viertel des Strauchschicht-Artenpools der Untersuchungsflächen, auf denen der tiefergründigen Buntsandstein- und Dolomit-Standorte (Königsbuche-KF2, Hainholz-KF1) dagegen bereits etwa die Hälfte. Die mittleren Artenzahlen liegen auf den Wurzeltellern bei sehr unterschiedlichen Steigerungsraten bis zum Jahr 2001 in der Hainholz-KF2 nur bei 0,7 auf den beiden anderen Standorten dagegen bei 1,5-2,2. Auf den umgebenden $100 \mathrm{~m}^{2}$-Plots zeigt sich ein umgekehrtes Bild. Die Hainholz-KF2 ist mit 7,5 Strauchschichtarten/100 m² relativ artenreich und hat mit einer knappen Vervierfachung auch die größte Steigerung aufzuweisen. Auf den übrigen Flächen kommt es zu einer knappen bzw. reichlichen Verdoppelung, wobei diese mit 3-5 Strauchschichtarten/100 $\mathrm{m}^{2}$ artenärmer sind als der flachgründige Gips/Dolomit-Standort. Von den drei Wurzeltellerabschnitten weisen Oberseite und Mulde - korrespondierend mit dem Gesamtniveau des Standortes - die höchsten mittleren Artenzahlen auf. Die Unterseite liegt meist beträchtlich darunter (Tab. A26 bis A31).

Feldschicht-Artenzahlen (Tab. 14): Für alle UG lässt sich einheitlich feststellen, dass bis zum vierten Sukzessionsjahr etwa zwei Drittel des gesamten Arteninventars der Feldschicht auch auf Wurzeltellern zu finden ist. Dieser Prozentsatz erhöht sich zwischen 1999 und 2001 
standortsabhängig unterschiedlich stark, wobei die Wurzelteller auf dem flachgründigen Gips/Dolomit-Standort eine relativ große Steigerung aufweisen (um 19\%), diejenigen auf den übrigen beiden Standorten jedoch eine relativ geringe (Königsbuche-KF2: um 9\% bzw. Hainholz-KF1: um 1\%). Dies zeigt sich auch bei der absoluten Artenzahl auf den Wurzeltellern, die sich in der Hainholz-KF2 auf 87 Arten knapp verdoppelt, während sie in der Hainholz-KF1 nur geringfügig von 37 auf 43 ansteigt und in der Königsbuchen-KF2 sogar von 30 auf 29 leicht zurückgeht. Ein teilweise ähnlicher Trend ist auf den $100 \mathrm{~m}^{2}$ Flächen zu verzeichnen: in der Königsbuche Artenrückgang, auf den übrigen Flächen relativ moderate Steigerung. Sowohl auf den Wurzeltellern als auch auf der Umgebungsfläche spiegelt sich über die Gesamtartenzahl die standörtliche Dreiteilung wider (flachgründiger Gips/Dolomit-Standort am artenreichsten, tiefgründiger Dolomit-Standort im Mittelfeld, Buntsandstein am artenärmsten). Betrachtet man die mittlere Artenzahl, so ist wiederum der flachgründige Gips/Dolomit-Standort sowohl auf den $100 \mathrm{~m}^{2}$-Flächen als auch auf den Wurzeltellern am artenreichsten (im Jahr 2001: Ufl: 56/100 m²; WT: 16). Die artenärmsten Wurzelteller befinden sich in Hainholz-KF1 (2001: 9,5/100 m²), die artenärmsten 100 m²- $^{2}$ Plots dagegen in der Königsbuche (2001: 19/100 m²). Als Besonderheit der KönigsbuchenKF2 verringert sich dort die mittlere Artenzahl im Gegensatz zur Gesamtartenzahl nicht (betrifft Ufl und WT), d.h. hier kommt es trotz absoluten Artenverlustes im gesamten Stratum zu einer weiteren Ausbreitung vorhandener Arten innerhalb der Flächenunterteilungen bzw. Wurzelteller. Während die Steigerungsfaktoren auf den $100 \mathrm{~m}^{2}$-Flächen zwischen $+1,2$ und $+1,8$ noch relativ eng beieinander liegen, gibt es auf den Wurzeltellern größere Standortsunterschiede. Die Wurzelteller in der Hainholz-KF2 verdoppeln ihre mittlere Artenzahl in etwa, während auf denen der Königsbuchen-KF2 nur ein moderater Anstieg erfolgt (um etwa 20\%) und in der Hainholz-KF1 Stagnation eintritt. Unter den einzelnen Wurzeltellerabschnitten sind Oberseite und Mulde jeweils artenreicher als die Unterseite (Tab. A26 bis A31).

Strauchschicht-Deckungssummen (Tab. 14): Sowohl auf den $100 \mathrm{~m}^{2}$-Plots als auch auf den Wurzeltellern hat sich in der Königsbuchen-KF2 (Ufl: 70\%/WT: 45\%) und der Hainholz-KF1 (Ufl: 63\%/WT: 34\%) bis zum vierten Sukzessionsjahr eine relativ dichte Strauchschicht ausgebildet, während in der Hainholz-KF2 (Ufl: 14\%/WT: 4\%) wesentlich geringere Deckungssummen erreicht werden. Im Buntsandstein entwickelt sich die Strauchschicht auf den $100 \mathrm{~m}^{2}$-Flächen etwa doppelt so schnell wie im Zechstein (Verzehnfachung der Deckungssumme gegenüber Verfünffachung), auf den Wurzeltellern dagegen liegen der Buntsandstein- bzw. Dolomit-Standort (jeweils Steigerung auf das 14-fache) vor dem Gips/Dolomit-Standort (Steigerung auf das Vierfache). Die Hainholz-KF2 und ihre Wurzelteller erscheinen bisher als relativ ungünstige Standorte für die Gehölzentwicklung. Dies zeigt sich auch auf den einzelnen Wurzeltellerabschnitten (Tab. A26 bis A31), wo in den Mulden max. 6\% mittlere Deckung erreicht werden, auf Ober- und Unterseite nur max. 3,5\%. 
Dagegen sind in den übrigen UG bei insgesamt höheren Deckungssummen die Oberseiten und Mulden wesentlich Strauchschicht-reicher als die Unterseiten.

Feldschicht-Deckungssummen (Tab. 14): Sowohl auf den Wurzeltellern als auch auf den umgebenden $100 \mathrm{~m}^{2}$-Plots nehmen die Feldschicht-Deckungssummen im Buntsandstein zwischen 1999 und 2001 um den Faktor 1,1-1,6 ab, wogegen sie im Zechstein um den Faktor 1,1-1,4 zunehmen. In der Königsbuche dunkelt die schnell und massiv entwickelte Strauchschicht die Feldschicht bereits aus. Während bei den $100 \mathrm{~m}^{2}$-Plots ein großer NiveauUnterschied zwischen der Hainholz-KF1 (in 2001: 162\% mittlere Deckungssumme) und den übrigen Varianten deutlich wird (in 2001: 83-86\%), sind auf den Wurzeltellern die standörtlichen Unterschiede wesentlich geringer. Hier ist im vierten Jahr nach dem Sturm mit 23-46\% mittlerer Deckungssumme überall nur eine recht lückige Feldschicht zu finden. Innerhalb der Zechstein-Standorte ist die Hainholz-KF1 einschließlich ihrer Wurzelteller dichter besiedelt als die Hainholz-KF2, wobei dies bei den $100 \mathrm{~m}^{2}$-Plots sehr deutlich, bei den Wurzeltellern dagegen weniger deutlich sichtbar wird (Ufl: 162\%/WT: 46\% gegenüber 86\%/36\%). Im Buntsandstein weisen die $100 \mathrm{~m}^{2}$-Plots bezüglich der Feldschicht eine ähnliche Besiedlungsdichte auf wie der Gips/Dolomit-Standort, die Wurzelteller dagegen wirken sehr „kahl“ (Ufl: 83\%/WT: 23\%). Von den einzelnen Wurzeltellerabschnitten (Tab. A26 bis A31) sind die Oberseiten immer am Feldschicht-reichsten, gefolgt von Mulde und Unterseite. Auf den Unterseiten nehmen die Deckungssummen in jedem Fall zu, während auf den Oberseiten und in den Mulden Zunahme (Hainholz-KF2) bzw. Stagnation oder Rückgang zu beobachten sind (übrige UG).

Kryptogamendeckungen (Tab. 14): Auf den $100 \mathrm{~m}^{2}$-Plots aller UG nehmen die mittleren Kryptogamendeckungen zu, wobei Steigerungsfaktoren von $+1,1$ (Königsbuche-KF2) bis zur knappen Verdoppelung (Hainholz-KF1) erreicht werden. Die basenreichen Standorte sind generell Kryptogamen-reicher als die basenarmen (in 2001: 9\% bzw. 17\% gegenüber 4\%). Auf den Wurzeltellern wird diese Entwicklung nur teilweise nachvollzogen. Im Buntsandstein kommt es hier zu einer leichten Abnahme der Kryptogamendeckung, im Zechstein dagegen zu einer leichten (Hainholz-KF1) bzw. deutlichen Zunahme (Hainholz-KF2). Die Kryptogamen-reichsten Wurzelteller sind in der Hainholz-KF2 mit 28\% mittlerer Deckung zu finden (2001), während in den beiden anderen Kernflächen nur 14-16\% erreicht werden. Unter den Wurzeltellerabschnitten (Tab. A26 bis A31) ist die Oberseite in allen UG die Kryptogamen-reichste. Auf den beiden Zechstein-Standorten weist die Unterseite fast ebensolche Deckungen auf wie die Oberseite, auf Buntsandstein liegen Unterseite und Mulde beträchtlich dahinter.

Laubstreu und Totholz (Tab. 14): Laubstreu- und Totholzdeckungen wurden in diesem Kapitel zu einer Deckungssumme addiert, wobei auf den $100 \mathrm{~m}^{2}$-Plots das Totholz 
anteilmäßig die größere Rolle spielt, auf den Wurzeltellern dagegen die Laubstreu. Während die Totholzdeckung auf den $100 \mathrm{~m}^{2}$-Flächen mehr oder weniger stabil blieb und auch durch vereinzelt nachbrechende Stämme oder Kronenteile im Mittel kaum beeinflusst wurde, verhält sich die Laubstreumenge variabel. Auf den $100 \mathrm{~m}^{2}$-Plots verringerte sich ihre Deckung im Sukzessionsverlauf variantenunabhängig um den Faktor 1,2-1,4. Dabei behielt der Buntsandstein-Standort im Jahr 2001 mit 48\% etwas höhere Deckungen als die ZechsteinStandorte mit 22 bzw. 35\%. Dagegen kommt es auf bzw. an den Wurzeltellern besonders im Buntsandstein $\mathrm{zu}$ einer starken Anhäufung vor allem von Laubstreu (etwa Verzwanzigfachung von 1999 zu 2001), wobei Deckungen von 18-19\% erreicht werden. Auf den Wurzeltellern der beiden Zechstein-Standorte liegen sowohl die Steigerungrate (Verdoppelung bis Verdreifachung) als auch die im vierten Sukzessionsjahr vorgefundenen Deckungen (3-6\%) wesentlich niedriger. Von den drei Wurzeltellerabschnitten fungiert hauptsächlich die Mulde als „Laubfänger“, während auf der Oberseite kaum und auf der Unterseite so gut wie gar kein Laub abgelagert wird (Tab. A26 bis A31).

Vegetationsfreier Mineralboden/Fels (Tab. 14): Während im Sukzessionsverlauf auf den $100 \mathrm{~m}^{2}$-Flächen immer weniger offener Boden oder Fels zu sehen ist (Verringerung um das Zweieinhalb- bis Elffache der Deckung von 1999), bleibt auf den Wurzeltellern (als Gesamtstandort betrachtet) auch im vierten Jahr nach dem Sturm überall ein Drittel bis die reichliche Hälfte der Fläche ohne jegliche Vegetationsbedeckung. Im Zechstein kommt es dabei zu einer moderaten Abnahme der vegetationsfreien Fläche (um den Faktor 1,4-1,6), im

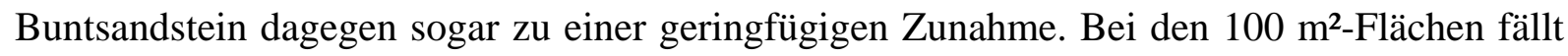
die flachgründige Gips/Dolomit-Kuppe des Bollerkopfes im Jahr 2001 mit immer noch rund $7 \%$ vegetationsfreiem Boden/Fels gegenüber den übrigen Varianten auf (dort $<1 \%$ ). Dagegen sind die Wurzelteller im Buntsandstein geringfügig „kahler“ (56\%) als im Zechstein (34 bzw. 42\%). Von den drei Wurzeltellerabschnitten ist die Unterseite in der Regel am stärksten durch vegetationsfreien Mineralboden/Fels charakterisiert (Maximum: Rückgang von 94 auf 93\% in Königsbuche-KF2; Minimum: Rückgang von 74 auf 57\% in Hainholz-KF2). Der schrittweise und schon heute sichtbare Zerfall der Wurzeltellerunterseiten ist in Zukunft wahrscheinlicher als deren zunehmende Vegetationsbesiedlung. Die Mulde ist dagegen in der Regel derjenige Abschnitt, auf dem der offene Mineralboden am schnellsten und mit hohen Deckungsgraden wiederbesiedelt wird. Nur in der Königsbuche sind Mulden und Oberseiten diesbezüglich gleichrangig. Im vierten Jahr nach dem Sturm verbleiben in den Mulden noch zwischen 11 (Hainholz-KF1) und 37\% (Königsbuche-KF2) vegetationsfreier Boden/Fels (Tab. A26 bis A31). 


\subsubsection{Zunahme, Abnahme, Stagnation - das Verhalten der Arten im Vergleich von Untersuchungsfläche und Wurzelteller}

Eine weitere Betrachtungsebene widmet sich nun der Entwicklung von Strauch- und Feldschichtarten nach Stetigkeit und mittlerem Deckungsgrad auf Wurzeltellern und deren Umgebungsflächen (100 m²-Plots) zwischen 1999 und 2001 (Tab. A26 bis A31). Die einzelnen Arten wurden dabei entsprechend ihres Verhaltens (Zunahme, Abnahme, Stagnation, ausschließliches Vorkommen auf Wurzelteller und/oder Untersuchungsfläche) in folgende 6 Kategorien eingeteilt:

Kategorie 1 = auf Ufl und auf WT zunehmend

Kategorie 2 = auf Ufl stagnierend oder abnehmend, aber auf WT zunehmend

Kategorie 3 = auf WT stagnierend

Kategorie 4 = auf Ufl stagnierend oder zunehmend, aber auf WT abnehmend

Kategorie 5 = auf Ufl und auf WT abnehmend

Kategorie 6 = auf WT nicht vorkommend

Speziell für die Feldschichtarten ist das Ergebnis in Abb. 49 zunächst überblicksartig dargestellt, bevor anschließend auf einzelne Arten bzw. Artengruppen eingegangen wird.

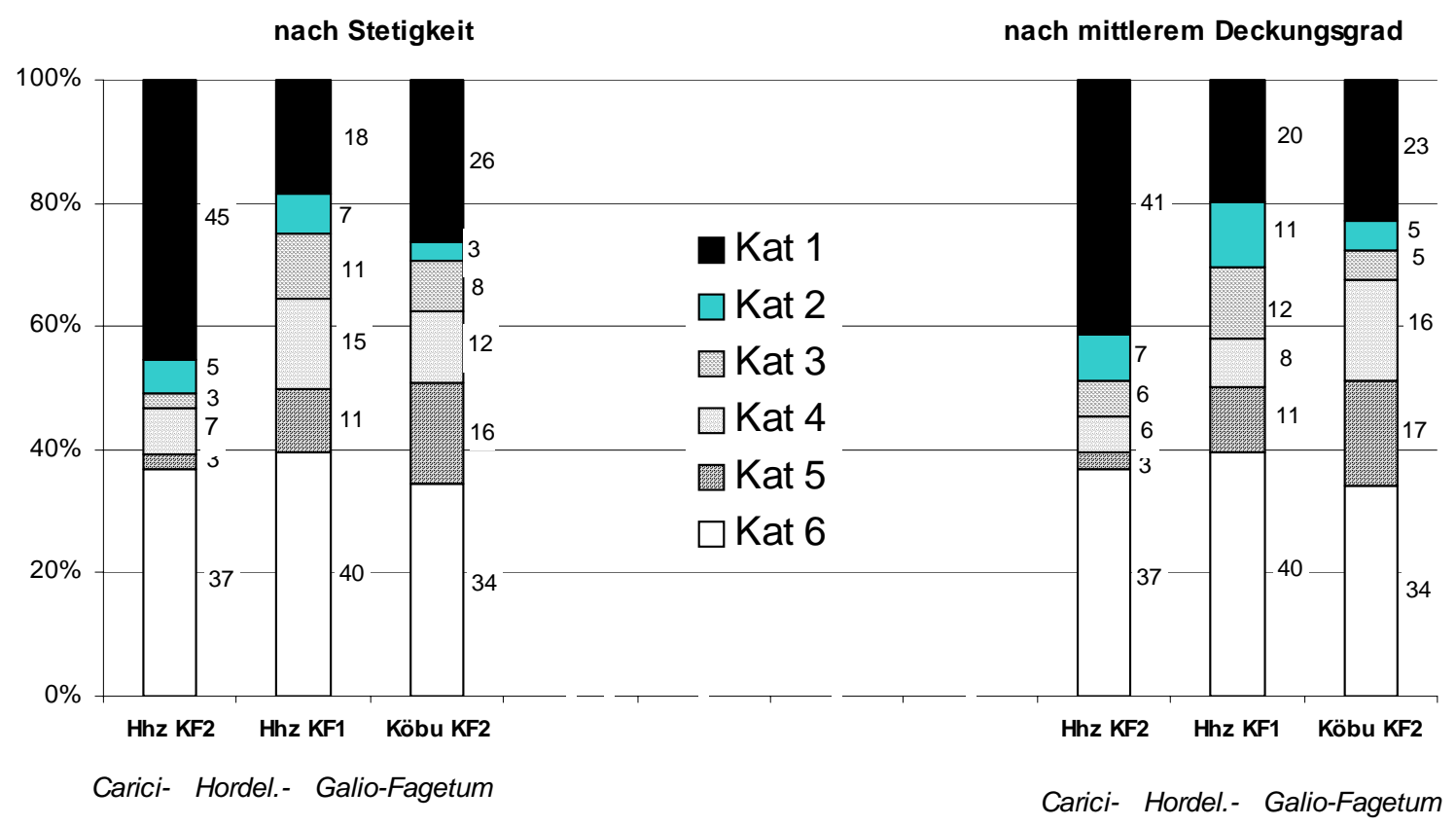

Abb. 49: Verhalten der Feldschichtarten nach Stetigkeit und mittlerem Deckungsgrad beim Vergleich von Wurzeltellern (WT) und Untersuchungsflächen (Ufl) auf drei BuchenWindwurfflächen mit Flächenwurf im südwestlichen Harzvorland im Sukzessionsverlauf der Jahre 1999 und 2001; Kat = Kategorie; Anzahl einbezogener WT und Ufl siehe Tab. 14; Einteilung der Kategorien siehe Text in Kap. 4.6.3.

Sinnvollerweise finden nur solche Arten Erwähnung, die standortsübergreifend in mindestens zwei UG die gleiche Entwicklung entsprechend Stetigkeit und mittlerer Deckung zeigen (Tab. 
A26 bis A31). Diese Kategorisierung ist bewusst sehr strikt gewählt worden. Bereits eine Abweichung bedingt die Zuordnung der Art zur Kategorie „Ambivalentes Verhalten“.

Strauchschichtarten (Tab. A26 bis A31): Zu den Gehölzen mit $>50 \mathrm{~cm}$ Wuchshöhe, die sowohl auf Untersuchungsflächen als auch auf Wurzeltellern zunehmen (Kategorie 1) gehören gebietsübergreifend Acer pseudo-platanus, Sambucus racemosa und Rubus idaeus (letztere nur nach mittlerer Deckung). Diese Gruppe umfasst in der Hainholz-KF2 etwa 28\% aller Strauchschichtarten (sowohl nach Stetigkeit als auch nach mittlerer Deckung), in der Hainholz-KF1 etwa 38\% (nach Stetigkeit) bzw. 46\% (nach mittlerer Deckung) und in der Königsbuchen-KF2 etwa 33\% (nach Stetigkeit) bzw. 44\% (nach mittlerer Deckung).

Dagegen kommt ein hoher Prozentsatz der Strauchschichtarten gar nicht auf Wurzeltellern vor und gehört somit zur Kategorie 6 (Hainholz-KF2: 72\%, Hainholz-KF1: 54\%, KönigsbucheKF2: 44\%). Das bedeutet aber in der Mehrzahl der Fälle, dass diese Gehölze die 50 cmHöhenmarke noch unterschreiten. Hierher gehören gebietsübergreifend Prunus avium, Salix caprea, Betula pendula, Cornus sanguinea, Corylus avellana und Ulmus glabra sowie weitere Arten, die aber nur in einem UG vorkommen.

Einige Strauchschichtarten zeigen ein gebietsweise ambivalentes Verhalten. Besonders hervorzuheben wäre hier die Buche, die auf den Wurzeltellern der Hainholz-KF2 noch nicht in Strauchschichthöhe vorkommt, in den übrigen UG aber bereits zur Kategorie 1 gehört. Dazu zählt weiterhin die Brombeere, die im Buntsandstein gleichermaßen auf Untersuchungsflächen und Wurzeltellern zunimmt (Kategorie 1), die jedoch in der HainholzKF1 auf Wurzeltellern noch nicht in die Strauchschichthöhe durchgewachsen ist und die in der Hainholz-KF2 auf Wurzeltellern sogar ganz fehlt. Junge Eschen mit $>50 \mathrm{~cm}$ Wuchshöhe

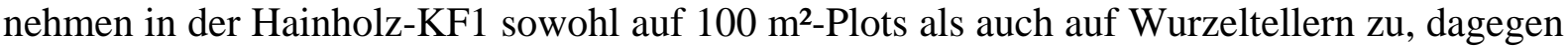
erreichen sie auf den Wurzeltellern der Hainholz-KF2 die Strauchschichthöhe (noch?) nicht und nehmen dort in der Feldschicht sogar ab. Im Buntsandstein hat die Art kein Vorkommen.

Feldschichtarten (Abb. 49; Tab. A26 bis A31): Bei der Einteilung der Arten in 6 Kategorien wird deutlich, dass sich den einzelnen Kategorien sowohl nach Stetigkeit als auch nach mittlerer Deckung annähernd gleichviele Arten zuordnen lassen, auch wenn diese häufig nicht identisch sind. Bezogen auf jeweils ein UG als Gesamtstratum war keine Feldschichtart ausschließlich auf Wurzeltellern zu finden. Jedoch werden in Kap. 4.6.8 noch bestimmte Arten näher untersucht werden, für die der Wurzelteller gelegentlich (d.h. in einzelnen Plots) der einzige Wuchsort war.

In jedem der drei UG kam gut ein Drittel der Gesamtartenzahl (zwischen 34 und 40\%) gar nicht auf Wurzeltellern vor (Kategorie 6), obwohl die entsprechenden Arten auf den Umgebungsflächen $\mathrm{zu}$ finden waren (Abb. 49). Das betrifft standortsübergreifend Deschampsia cespitosa, Poa annua, Agrostis canina, Poa nemoralis, Dactylis glomerata, Geum urbanum, Pimpinella major, Ranunculus repens und Rumex obtusifolius. Eine Reihe 
weiterer Arten der Kategorie 6 kommen nur in einem der drei UG vor, die meisten davon in der Hainholz-KF2 (Tab. A26 bis A31).

Eine quantitativ ebenfalls sehr bedeutende Gruppe ist die derjenigen Arten, die sowohl auf

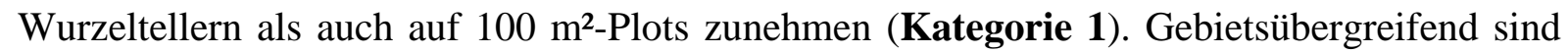
das Acer platanoides, Brachypodium sylvaticum, Bromus benekenii, Urtica dioica sowie Dryopteris carthusiana und Senecio viscosus (die beiden letzten nur nach Stetigkeit). Hinzu kommen noch weitere Arten, die jeweils nur in einem der drei UG vorkommen (meist in Hainholz-KF2). Dabei ist das gesamte Spektrum pflanzensoziologischer Klassen vertreten, wie es in Kap. 4.3.5 beschrieben wurde. Es ergibt sich dabei eine deutliche Zweiteilung zwischen dem Gips/Dolomit-Standort (Hainholz-KF2) einerseits sowie dem Buntsandsteinbzw. Dolomit-Standort (Königsbuche-KF2 bzw. Hainholz KF1) andererseits. Während in der erstgenannten Variante sehr hohe Anteile von Arten der Kategorie 1 erreicht werden (z.B. nach Stetigkeit 45\%), ist der Prozentsatz auf den beiden übrigen Standorten geringer (z.B. nach Stetigkeit 26 bzw. 18\%). Somit ist der Flächenwurf auf dem Bollerkopf sowohl im Falle

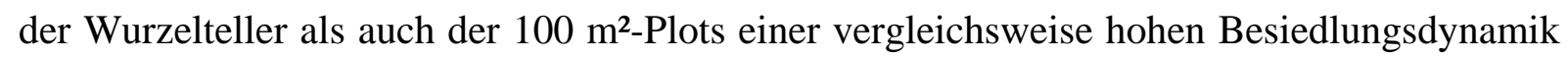
unterworfen.

Auf dem Bollerkopf drückt sich dieser Trend zusätzlich noch durch entsprechend geringe Prozentanteile von Arten aus, die auf den $100 \mathrm{~m}^{2}$-Plots und/oder den Wurzeltellern stagnieren oder abnehmen (Kategorien 2-5). Im Gegensatz dazu sind der produktivere Buntsandsteinund der Dolomit-Standort durch besonders viele Arten gekennzeichnet, die auf Wurzeltellern und/oder auf Untersuchungsflächen seltener werden und/oder an Deckung verlieren (Kategorien 4 und 5).

Zu den Arten, die auf Wurzeltellern standortsunabhängig immer noch zunehmen, während auf den umgebenden 100 m²-Plots Stagnation oder Rückgang eintritt (Kategorie 2), gehören z.B. Impatiens parviflora (nach Stetigkeit) und Mercurialis perennis (nach mittlerer Deckung). Weitere Arten verhalten sich ähnlich, kommen aber nur in der Hainholz-KF2 vor, so z.B. Campanula rapunculoides, Carduus nutans, Corylus avellana und Rumex acetosa.

Andere Arten nehmen auf den Wurzeltellern ab, während auf den umgebenden $100 \mathrm{~m}^{2}$ Flächen Stagnation oder Zunahme zu verzeichnen ist (Kategorie 4). Das trifft z.B. für Allium ursinum und Fraxinus excelsior zu (letztere standortsübergreifend nur nach Stetigkeit), wobei die Esche in der Hainholz-KF1 auf Wurzeltellern zunehmend in die Strauchschicht durchwächst (d.h. als Feldschichtart abnimmt), in der Hainholz-KF2 dagegen auf Wurzeltellern tatsächlich seltener wird und an Deckung verliert. Weitere Arten verhalten sich ebenfalls der Kategorie 4 entsprechend, kommen aber nur in der Hainholz-KF2 vor, so z.B. Chaenarrhinum minus, Vincetoxicum hirundinaria und Viola hirta.

Nur sehr wenige Arten gehören der Kategorie 5 (Abnahme auf Untersuchungsflächen und Wurzeltellern) an, darunter Verbascum thapsus als gebietsübergreifende Schlagflurart im Zechstein (nur nach Stetigkeit). Weitere Arten dieser Gruppe, die aber nur in einem der UG vorkommen, sind z.B. Rumex sanguineus und Epipactis helleborine. 
Unter den Arten der Kategorie 3, welche auf Wurzeltellern stagnieren, sich aber auf den 100 $\mathrm{m}^{2}$-Flächen ambivalent verhalten, sind Campanula trachelium und Sonchus asper erwähnenswert (letztere nur nach mittlerer Deckung). Ähnlich verhält sich z.B. Ajuga reptans, kommt aber nur in einem UG vor.

\subsubsection{Zunahme, Abnahme, Stagnation - das Verhalten der Feldschichtarten bei isolierter Betrachtung nur der Wurzelteller}

Zur isolierten Betrachtung nur der Wurzelteller wurden die auf den Wurzeltellern wachsenden Feldschichtarten bezüglich ihres Verhaltens nach Stetigkeit und mittlerem Deckungsrad im Zeitraum zwischen 1999 und 2001 in drei Kategorien eingeteilt (Zunahme, Abnahme, Stagnation - siehe Abb. 50 und Tab. A26 bis A31). Dabei bestätigt sich in weiten Teilen, was in Kap. 4.6.3 bereits gesagt wurde. Das Verhalten der Arten nach Stetigkeit und mittlerem Deckungsgrad zeigt innerhalb jeder der Untersuchungsvarianten ein ähnliches Muster. Besonders deutlich tritt in der Hainholz-KF2 wieder die Gruppe der zunehmenden Arten hervor, die etwa drei Viertel der Gesamtartenzahl repräsentiert. Auf Buntsandstein und auf Dolomit dagegen nimmt diese Gruppe nur die knappe Hälfte des Arteninventars ein.

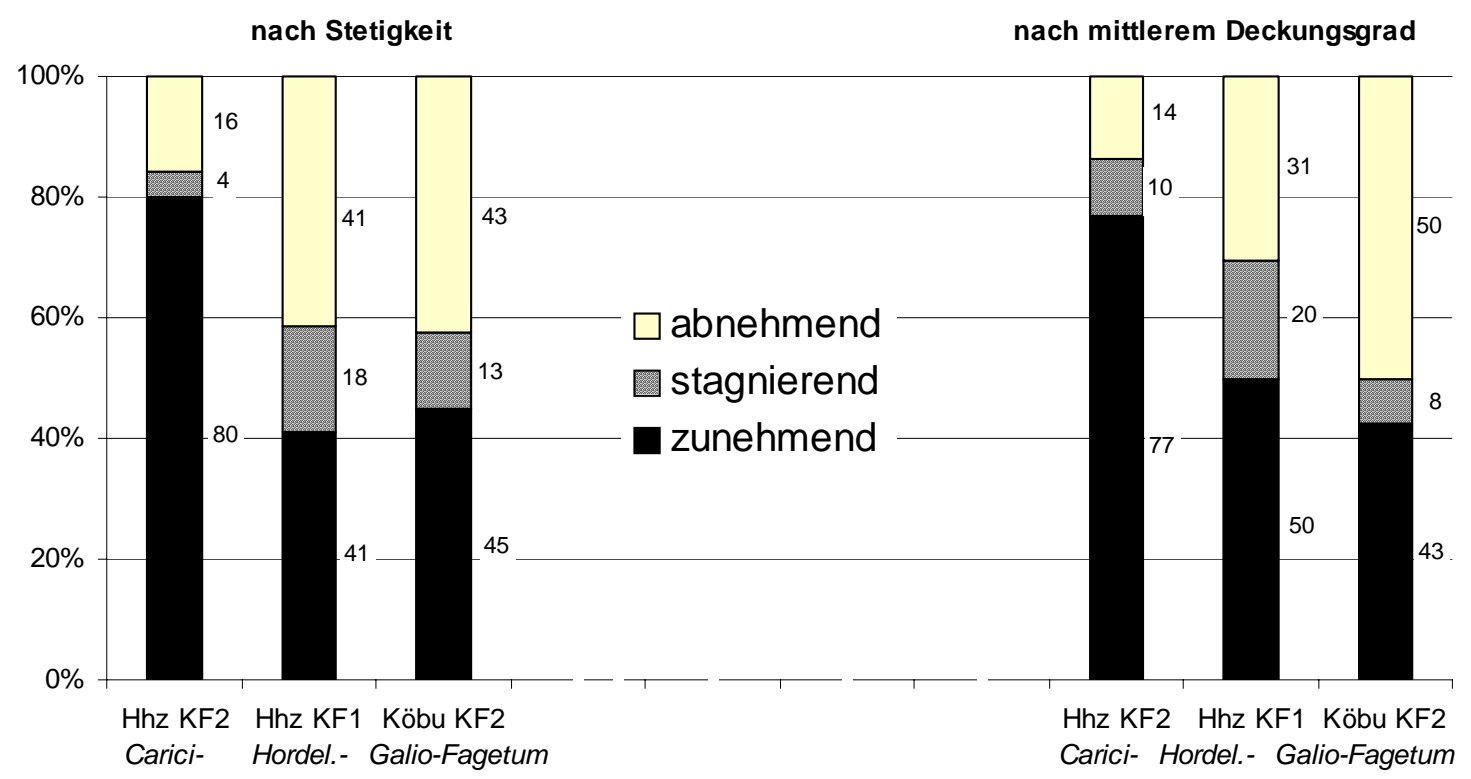

Abb. 50: Verhalten von Feldschichtarten auf Wurzeltellern nach Stetigkeit bzw. mittlerem Deckungsgrad im Vergleich der Jahre 1999 und 2001 auf drei Buchen-Windwurfflächen mit Flächenwurf im südwestlichen Harzvorland; Köbu = Königsbuche; Hhz = Hainholz; Anzahl einbezogener Wurzelteller siehe Tab. 14.

Dafür sind dort mehr Arten vorhanden, die bereits seltener werden bzw. an Deckung verlieren (betrifft ebenfalls die knappe Hälfte der Arten). Stagnierende Arten sind überall von randlicher Bedeutung (unter 20\% Anteil). Somit wird auch bei isolierter Betrachtung der Wurzelteller deutlich, dass der vor dem Sturm recht Krautschicht-arme Bollerkopf der 
dynamischsten Besiedlung unterworfen ist, während die Wurzelteller der übrigen Standorte vergleichsweise „gesättigt“ erscheinen.

\subsubsection{Floristische Ähnlichkeit auf Untersuchungsflächen und Wurzeltellern anhand qualitativer und quantitativer Indizes}

\subsubsection{Floristische Ähnlichkeit zwischen den einzelnen Teilstandorten}

Im Folgenden soll geprüft werden, inwiefern bereits verwendete Ähnlichkeitsindizes (Jaccard, Percentage Similarity) geeignet sind, die floristische Ähnlichkeit zwischen Untersuchungsfläche und Wurzelteller bzw. Wurzeltellerabschnitt im Vergleich des zweiten und vierten Sukzessionsjahres (1999 und 2001) zu beschreiben (Abb. 51).

Sowohl bei qualitativer (Jaccard) als auch bei quantitativer Auswertung (Percentage Similarity) wird deutlich, dass die Wurzelteller im Sukzessionsverlauf ihrer Umgebungsfläche immer ähnlicher werden. Diese Angleichung erfolgt unterschiedlich schnell und von jeweils unterschiedlichem Ausgangsniveau. Wieder heben sich HainholzKF2 einerseits sowie Hainholz-KF1 und Königsbuche-KF2 andererseits voneinander ab (siehe auch Kap. 4.6.3 und 4.6.4). Die Wurzelteller des flachgründigen Gips/DolomitStandortes haben anfangs eine vergleichsweise geringe floristische Ähnlichkeit (nach qualitativem Jaccard-Index) zur Umgebungsfläche. Sie gleichen sich aber schneller an ihre Umgebungsfläche an als die Wurzelteller auf den beiden tiefgründigeren Standorten (Anstieg von 0,27 auf 0,43 gegenüber Anstieg von 0,43 auf 0,45 bzw. von 0,52 auf 0,65). Unter Einbeziehung der Deckungsgrade (Percentage Similarity) ergibt sich ein davon abweichendes Bild: die Hainholz-KF2 hat das höhere Ausgangsniveau und die „Angleichungsgeschwindigkeit“ unter den Varianten ist ähnlich hoch (Anstieg von 48 auf 57\% gegenüber Anstieg von 35 auf 40\% bzw. von 38 auf 52\%).

Vom Gesamtniveau der floristischen Ähnlichkeit, das im vierten Sukzessionsjahr (2001) erreicht wird, lässt sich bei qualitativer Betrachtung dementsprechend die Reihenfolge Königsbuche-KF2 (große Ähnlichkeit) > Hainholz KF1 (mittlere Ähnlichkeit) > Hainholz KF2 (geringe Ähnlichkeit) aufstellen, bei quantitativer Betrachtung dagegen die Reihenfolge Hainholz-KF2 > Königsbuche-KF2 > Hainholz-KF1 (siehe auch Kap. 4.6.7).

Die von 1999 zu 2001 ansteigende floristische Ähnlichkeit zwischen Untersuchungsfläche und Wurzelteller wird von den einzelnen Wurzeltellerabschnitten unterschiedlich deutlich bestätigt. Sie zeigen aber mit geringen Ausnahmen generell dieselbe Entwicklung wie der Wurzelteller als Gesamtstandort. Dabei hat die Unterseite erwartungsgemäß die geringste Ähnlichkeit mit der Untersuchungsfläche, was bei quantitativer Betrachtung deutlicher hervortritt als bei qualitativer (Abb. 51). Die Oberseite weist ohne Berücksichtigung der Deckungsgrade (Jaccard-Index) in der Regel die höchste Ähnlichkeit zum 100 m²-Plot auf, unter Einbeziehung der Deckungsgrade (Percentage Similarity) ist es dagegen die Mulde. Das kann so interpretiert werden, dass die Mulden zwar am schnellsten zuwachsen, der alte 
Waldboden auf der Wurzeltelleroberseite dagegen das der Untersuchungsfläche ähnlichste Arteninventar beherbergt.
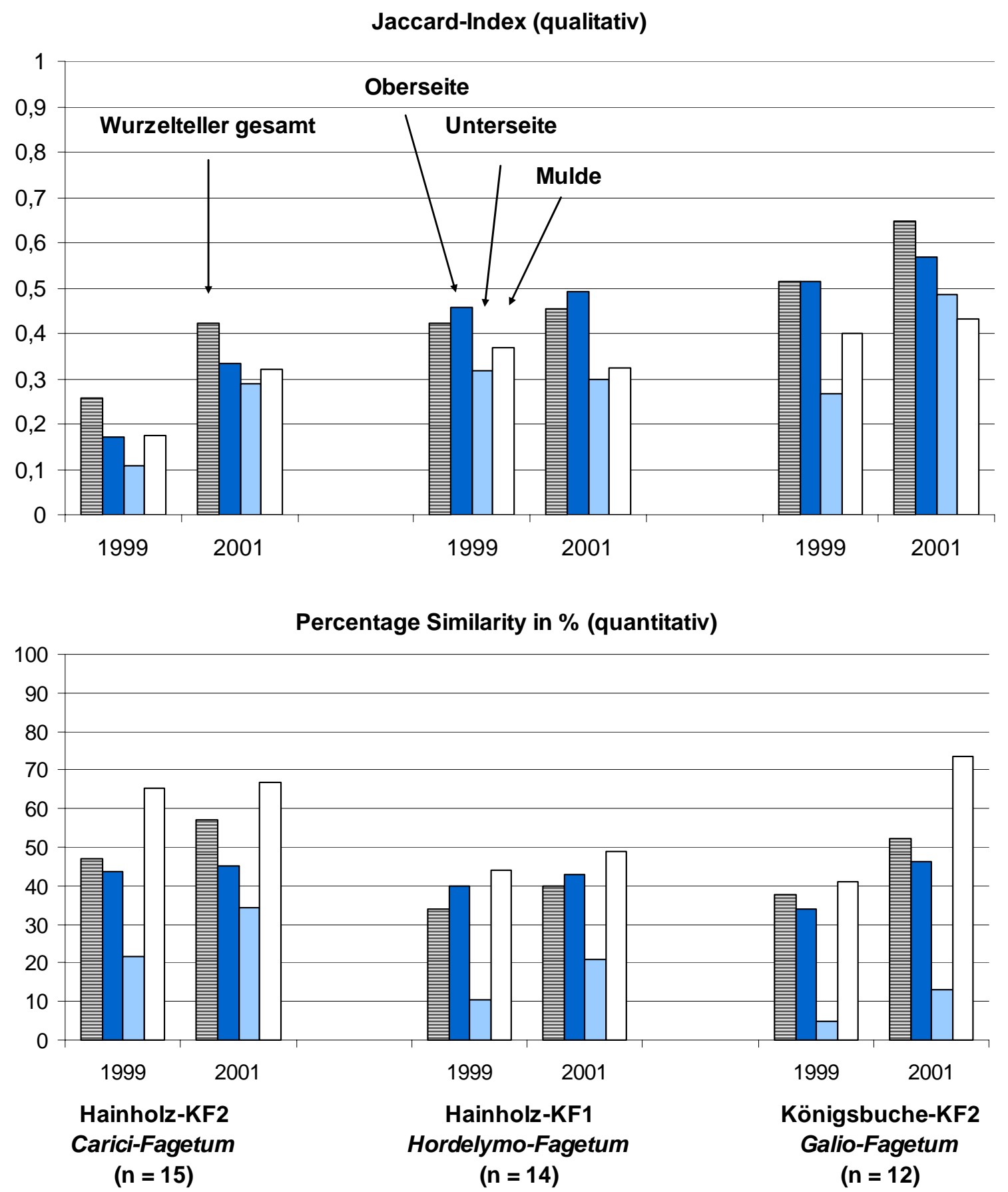

Abb. 51: Qualitative (oben) und quantitative (unten) floristische Ähnlichkeit zwischen dem $100 \mathrm{~m}^{2}$-Plot und dem Wurzelteller als Gesamtstandort bzw. einzelnen Wurzeltellerabschnitten auf drei Buchen-Windwurfflächen mit Flächenwurf im südwestlichen Harzvorland im Vergleich der Jahre 1999 und 2001; Datenbasis: Vegetationstabellen mit mittleren Deckungsgraden (Tab. A26, A28, A30). 


\subsubsection{Intensität der floristischen Veränderung auf den einzelnen Teilstandorten}

Mit den oben genannten Ähnlichkeitsindizes lässt sich prüfen, welcher Teilstandort (Untersuchungsfläche, Wurzelteller gesamt, Wurzeltellerabschnitte) sich zwischen zweitem und viertem Sukzessionsjahr am stärksten verändert hat. Sowohl mit als auch ohne Berücksichtigung der Deckungsgrade ergibt sich für den genannten Zeitraum, dass sich der Wurzelteller als Gesamtstandort in jedem Fall floristisch stärker verändert hat als seine Umgebungsfläche, d.h. die Indizes der Wurzelteller sind generell niedriger als die der $100 \mathrm{~m}^{2}$-Plots (Abb. 52).

\section{Jaccard-Index (qualitativ)}

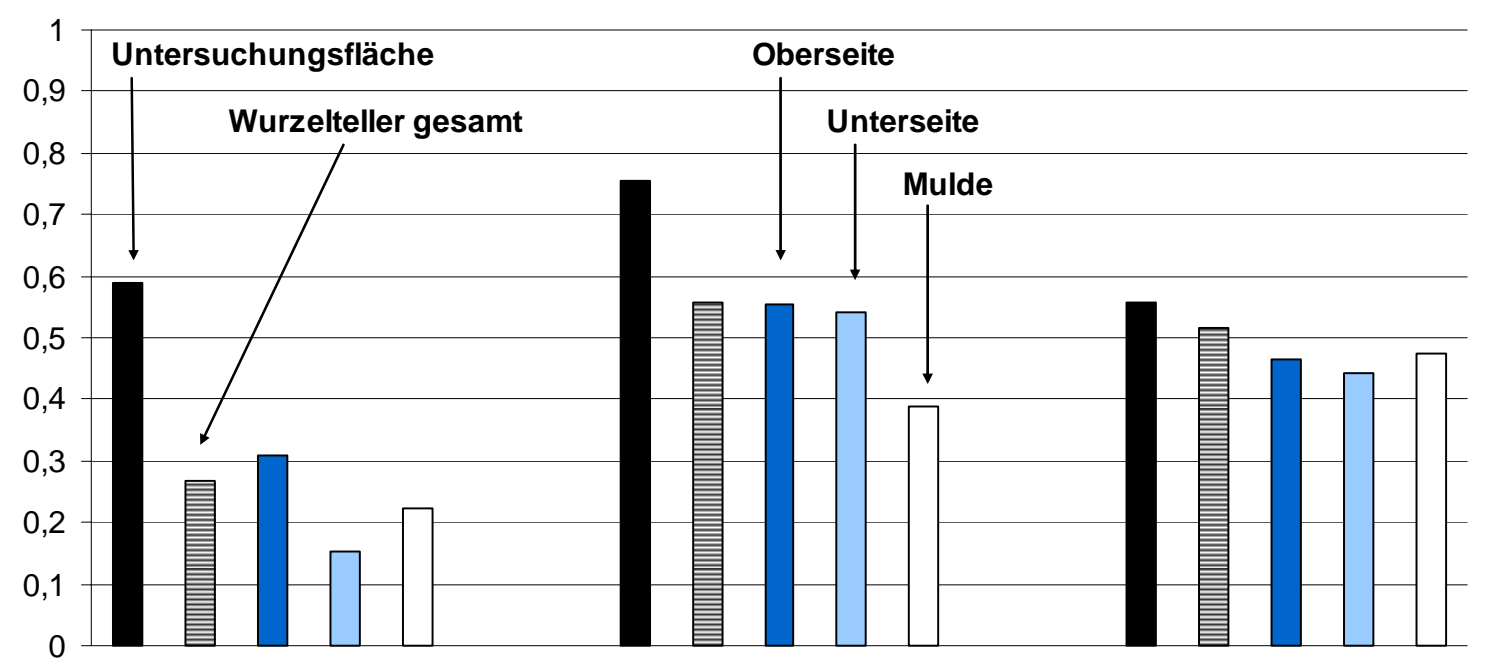

Percentage Similarity in \% (quantitativ)

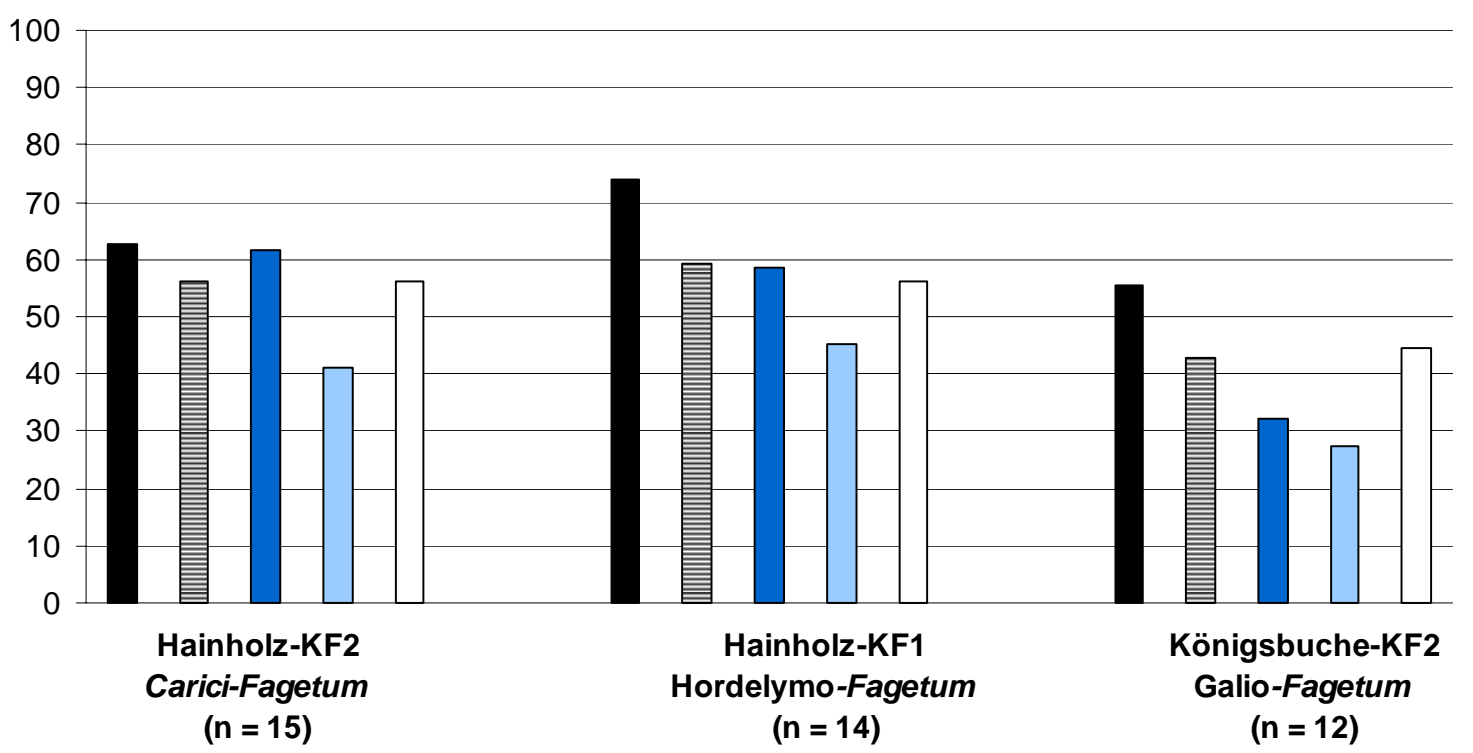

Abb. 52: Qualitative (oben) und quantitative (unten) floristische Ähnlichkeit von $100 \mathrm{~m}^{2}$ Plots, Wurzeltellern als Gesamtstandort und einzelnen Wurzeltellerabschnitten auf drei Buchen-Windwurflächen mit Flächenwurf im südwestlichen Harzvorland im Vergleich der Jahre 1999 und 2001; Datenbasis: Vegetationstabellen mit mittleren Deckungsgraden (Tab. A26, A28, A30). 
Bezüglich der einzelnen Wurzeltellerabschnitte gibt es wenig verallgemeinerbare Aussagen. Meistens ist sowohl der qualitative als auch der quantitative Index der Wurzeltellerunterseiten am niedrigsten, d.h. diese veränderten sich zwischen 1999 und 2001 am stärksten. Die Veränderung auf Oberseiten und in den Mulden ist im Vergleich dazu geringer und variiert zwischen den UG. Bezüglich der $100 \mathrm{~m}^{2}$-Plots ergibt sich eine relativ geringe floristische Veränderung in der Hainholz-KF1 (Index qualitativ: 0,75/quantitativ 75\%), eine relativ große dagegen in der Hainholz-KF2 (0,59/63\%) und in der Königsbuchen-KF2 (0,56/56\%). Das entspricht den in Kap. 4.2.8 dargestellten Ergebnissen.

Bei den Wurzeltellern dagegen muss zwischen qualitativer und quantitativer Bewertung unterschieden werden. Nach dem Jaccard-Index haben sich die Wurzelteller als Gesamtstandort in der Hainholz-KF2 $(0,28)$ relativ stark verändert, während diese Veränderung in den beiden anderen Varianten geringer ausfällt (Hainholz-KF1: 0,55/Königsbuche-KF2: 0,52). Damit bestätigt sich wiederum die auch in Kap. 4.6.3, 4.6.4 und 4.6.5 festgestellte Zweiteilung in die beiden Standortsgruppen „flachgründig“ (Gips/Dolomit) und „tiefgründig/produktiv“ (Dolomit bzw. Buntsandstein). Im Gegensatz dazu folgen die quantitativen Veränderungen (Percentage Similarity) eher dem Muster der umgebenden $100 \mathrm{~m}^{2}$-Plots. Dabei war die quantitative floristische Veränderung auf den Wurzeltellern im Buntsandstein (44\% Ähnlichkeit) etwas stärker als auf denen im Zechstein (Hainholz-KF2: 56\%/Hainholz-KF1: 59\%).

\subsubsection{Hauptkomponentenanalyse (PCA) zur Darstellung der Sukzession auf Untersuchungsflächen und Wurzeltellern}

Der hier verwendeten PCA-Grafik (Abb. 53) liegen mittlere Deckungsgrade (Tab. A26, A28, A30) zugrunde. Beim Vergleich der Wurzelteller (als Gesamtstandort) mit den umgebenden 100 m²-Plots im Verlauf der Jahre 1999 und 2001 ergibt sich folgendes Bild: Der Positivbereich von Achse 1 wird hauptsächlich von Allium ursinum, Galeobdolon luteum und Fraxinus excelsior (Strauchschicht), teilweise auch von Carex sylvatica definiert. Dies kennzeichnet sowohl den Ausgangs- als auch den vorläufigen Endzustand der $100 \mathrm{~m}^{2}$-Plots auf Dolomit. Den Positivbereich von Achse 2 bestimmen hauptsächlich Rubus idaeus (Strauchschicht), in geringerem Maße auch Carex sylvatica, Oxalis acetosella und Juncus effusus (Endzustand vor allem der 100 m²-Plots im Buntsandstein). Die beiden letztgenannten Arten erklären zusammen mit Impatiens parviflora auch den Negativbereich von Achse 1 (Ausgangszustand der Ufl und Endzustand der WT im Buntsandstein). Der Negativbereich von Achse 2 wird von Atropa bella-donna und Impatiens parviflora bestimmt (Ausgangs- und Endzustand der Ufl und WT in Hainholz-KF2, Ausgangszustand der WT von Königsbuche und Hainholz-KF1).

Alle Teilstandorte der Hainholz-KF2 bilden eine auffällige Punktwolke im Bereich der Impatiens-Vektorpfeilspitze. Sie bleiben demzufolge von dieser Art beherrscht (in geringerem 
Umfang auch von Atropa bella-donna), unterscheiden sich nur wenig voneinander bzw. verändern sich in der Sukzession kaum (Tab. A26). Demgegenüber heben sich in der Hainholz-KF1 die $100 \mathrm{~m}^{2}$-Plots deutlich von den Wurzeltellern ab, wobei auch

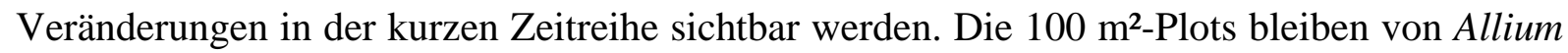
ursinum und Galeobdolon luteum beherrscht. Die kurze Bewegung entlang der Achse 1 ergibt sich aus der starken Deckungsgradzunahme der Esche als Strauchschichtart. Dagegen vollführen die Wurzelteller eine „Wanderbewegung“ entlang der Achse 2 von einem Atropaund Impatiens-beherrschten Stadium in Richtung Rubus- und Carex-Dominanz (Tab. A28).

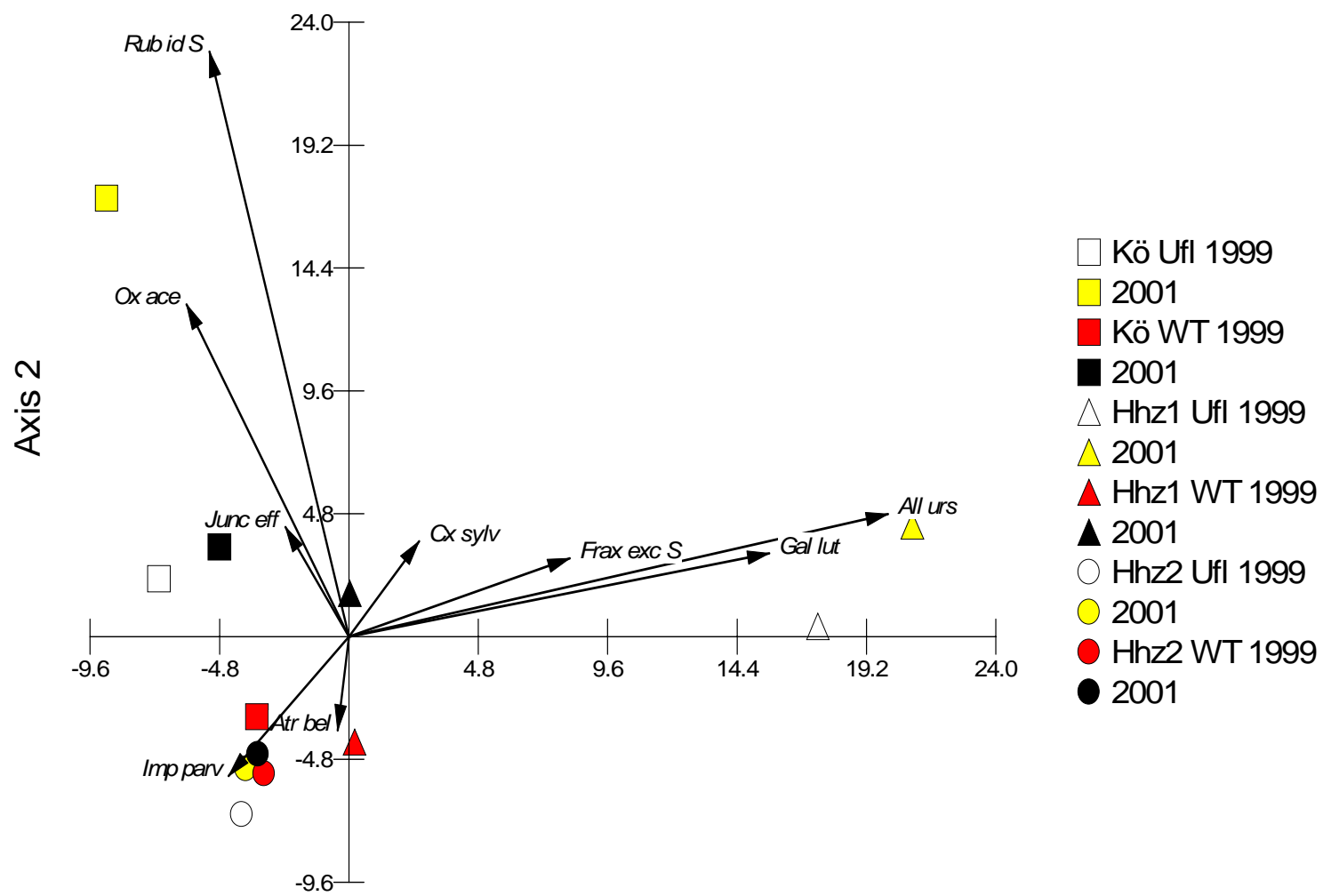

Axis 1

Vector scaling: 28,78

Abb. 53: Hauptkomponentenanalyse (PCA) für $100 \mathrm{~m}^{2}$-Plots und darin befindliche Wurzelteller auf drei Buchen-Windwurfflächen mit Flächenwurf im südwestlichen Harzvorland im Verlauf der Jahre 1999 und 2001; Köbu = Königsbuche-KF2; Hhz1 = Hainholz-KF1; Hhz2 = Hainholz-KF2; Ufl = Untersuchungsfläche (100 m²-Plot); WT = Wurzelteller; Euklidischer Biplot unter Verwendung mittlerer Deckungsgrade entsprechend Tab. A26, A28, A30; Achse 1 und 2 erklären 79\% der Varianz.

Die Vegetationsentwicklung in der Königsbuchen-KF2 zeigt gut sichtbar eine gleichgerichtete, aber unterschiedlich weite „Wanderung“ beider Teilstandorte von Impatiens(vor allem auf WT), Oxalis- und Juncus-dominiertem Ausgangszustand (1999) in Richtung Rubus-Strauchfluren (2001) entlang des entsprechenden Vektorpfeiles (Kap. 4.2.9). Diese quantitative Veränderung verläuft auf den Wurzeltellern etwas schwächer (kürzerer Weg) als

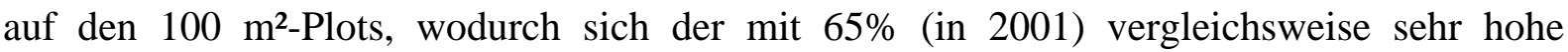


Deckungsgrad der Himbeere auf den Untersuchungsflächen ausdrückt. Die relativ große quantitative Bedeutung von Athyrium filfx-femina für die Wurzelteller wird in der Grafik programmbedingt nicht angezeigt (Tab. A30).

\subsubsection{Vegetationsökologische Parameter im Vergleich von Untersuchungsflächen und Wurzeltellern}

Zunächst werden im Zeitraum zwischen 1999 und 2001 für jedes einbezogene UG die qualitativen Artengruppenspektren der Untersuchungsflächen (100 m²-Plots) und die der darin befindlichen Wurzelteller verglichen. Damit soll geklärt werden, ob bzw. welche Artengruppen verstärkt auf Wurzeltellern vorkommen (Abb. 54 bis 59 jeweils oben). Danach erfolgt der Vergleich der quantitativen Abundanzspektren zur Prüfung der Dominanzverhältnisse (Abb. 54 bis 59 jeweils unten).

\subsubsection{1 Ökologische Zeigerwerte nach ELLENBERG et al. (1992)}

Licht-Zeigerwert: Im qualitativen Zeigerwertspektrum zeigen sich weder Unterschiede zwischen den untersuchten Teilstandorten noch Veränderungen im bisherigen Sukzessionsverlauf (Abb. 54 oben). In der Königsbuchen-KF2 und in der Hainholz-KF1 halten die Lichtzeiger auf Untersuchungsflächen und gleichermaßen auf Wurzeltellern etwa 40\% Anteil, die Halbschattenzeiger ebenfalls etwa 40\% und die Schattenzeiger die restlichen ca. 20\%. In der Hainholz-KF2 dagegen erreichen die dort stärker vertretenen Lichtzeiger etwa 50-60\% Anteil (auf Wurzeltellern sogar Zunahme um 13\%), die Halbschattenzeiger halten stabile 40\% und die Schattenzeiger nur max. 12\% (auf Wurzeltellern etwa um die Hälfte abnehmend).

Unter Berücksichtigung der Deckungsgrade (Abb. 54 unten) zeigt sich, dass mit Ausnahme der Hainholz-KF2 die Wurzelteller stärker von Lichtzeigern besiedelt sind als die umgebenden Untersuchungsflächen (in Hainholz-KF2 etwa Gleichstand). Generell kommt es auf allen Teilstandorten zwischen 1999 und 2001 zu einer Zunahme der Lichtzeigeranteile bei unterschiedlichem Ausgangsniveau. Lediglich die Wurzelteller der Königsbuchen-KF2 behalten einen konstanten Lichtzeigeranteil von rund 60\% und damit die höchsten Werte aller UG. Entsprechend gehen die Halbschatten- und Schattenzeiger zurück bzw. stagnieren. Trotz dieses Rückgangs ist besonders die Hainholz-KF1 im Jahr 2001 noch immer stark von Schattenzeigern geprägt (Ufl: 59\% Anteil/WT: 32\%), während diese in der Hainholz-KF2 mengenmäßig überhaupt keine Rolle spielen. Wiederum lassen sich auf dem Bollerkopf - im Gegensatz zu den übrigen Gebieten - kaum Unterschiede zwischen dem quantitativen Zeigerwertspektrum der Wurzelteller und dem ihrer Umgebungsfläche finden. 

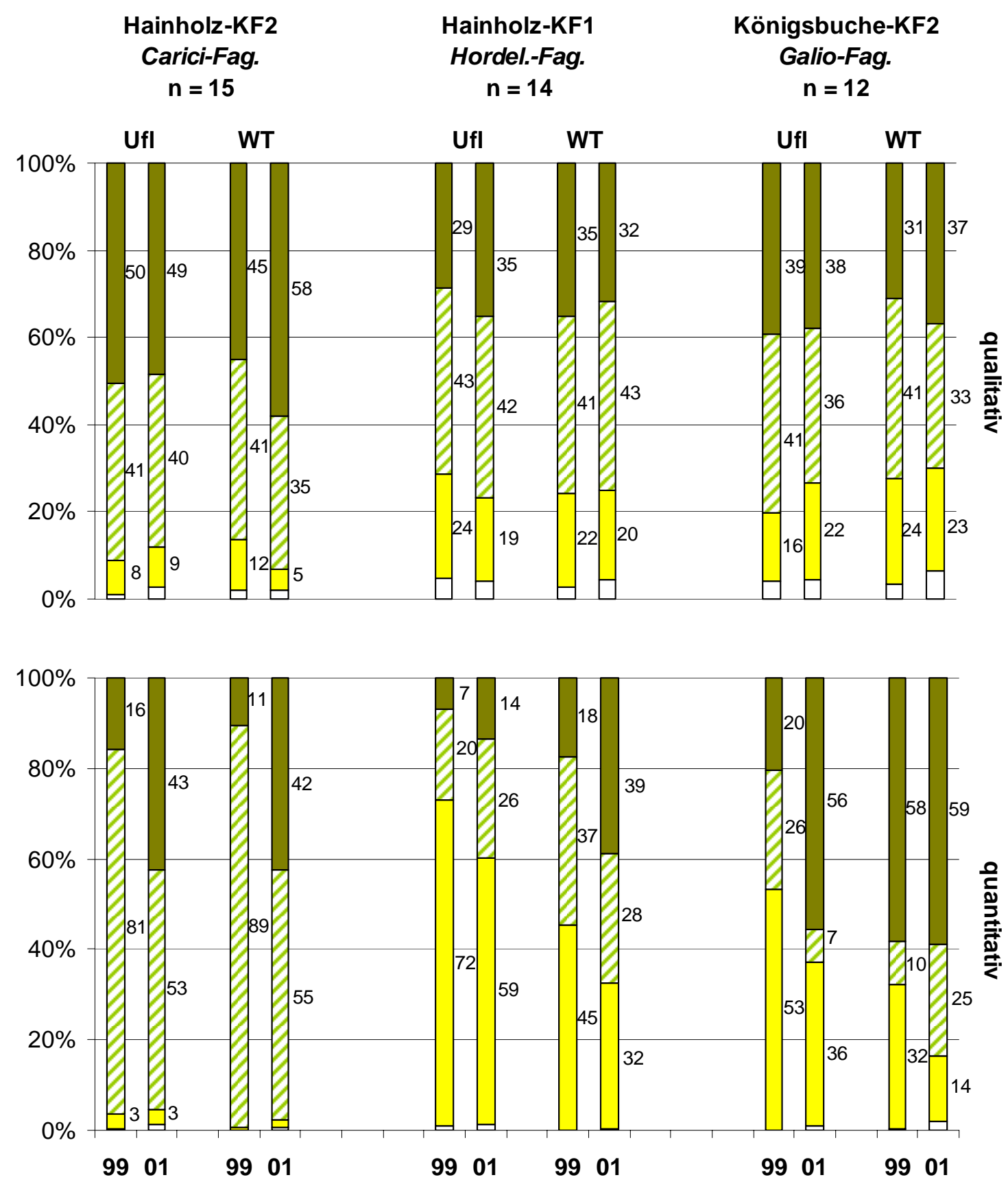

$\square$ Indifferente/O. Zuordnung $\square$ Schattenzeiger [L1-3]

$\square$ Halbschattenzeiger [L4-6] $\square$ Lichtzeiger [L7-9]

Abb. 54: Qualitatives (oben) und quantitatives (unten) Licht-Zeigerwertspektrum der Arten auf drei Buchen-Windwurfflächen mit Flächenwurf im südwestlichen Harzvorland in den Jahren 1999 und 2001; Anzahl einbezogener $100 \mathrm{~m}^{2}$-Plots und Wurzelteller siehe Tab. 14; Strauch- und Feldschicht zusammengefasst; Angaben unter 3\% nicht angezeigt.

Feuchte-Zeigerwert: Wurzelteller und $100 \mathrm{~m}^{2}$-Plots unterscheiden sich bezüglich ihrer qualitativen Zeigerwertspektren kaum und verändern sich in der bisherigen Sukzession auch nicht wesentlich (Abb. 55 oben). Das Bild wird in allen Fällen von Frischezeigern beherrscht, 

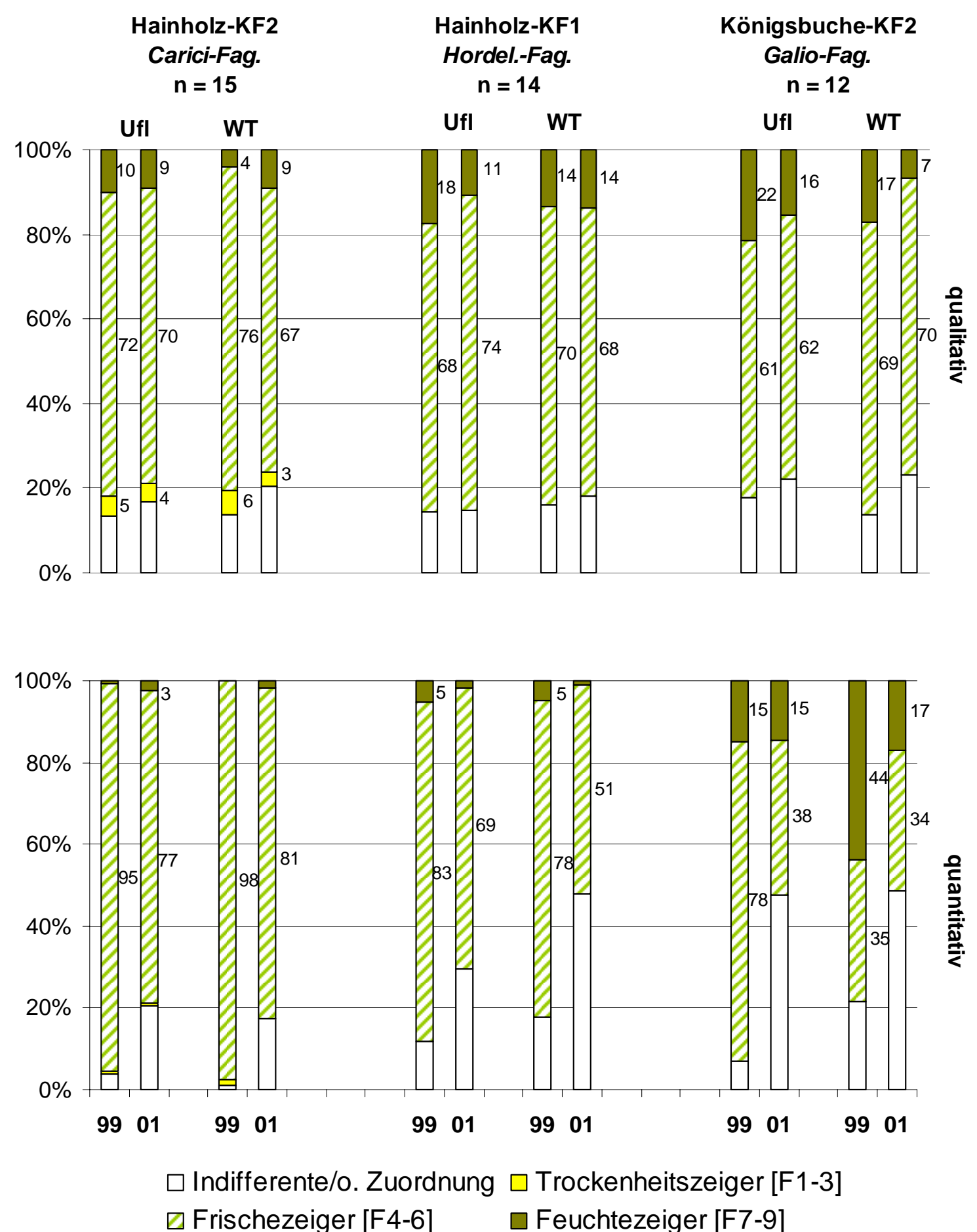

Abb. 55: Qualitatives (oben) und quantitatives (unten) Feuchte-Zeigerwertspektrum der Arten auf drei Buchen-Windwurfflächen mit Flächenwurf im südwestlichen Harzvorland in den Jahren 1999 und 2001; Anzahl einbezogener 100 m²-Plots und Wurzelteller siehe Tab. 14; Strauch- und Feldschicht zusammengefasst; Angaben unter 3\% nicht angezeigt.

die mit Anteilen von 60-70\% stabil dominieren. Feuchtezeiger halten teilweise konstante Anteile von etwa 10-20\%, teilweise ergeben sich geringfügige Veränderungen innerhalb der Zeitreihe (z.B. Königsbuche-KF2: auf WT Abnahme von 17 auf 7\%; Hainholz-KF1: in Ufl 
Abnahme von 18 auf 11\%; Hainholz-KF2: auf WT Zunahme von 4 auf 9\%). Die Trockenheitszeiger spielen nur in der Hainholz-KF2 eine minimale Rolle (rund 5\% Anteil).

Unter Berücksichtigung der Deckungsgrade (Abb. 55 unten) lässt sich bei allen Teilstandorten eine deutliche Zunahme von indifferenten Arten erkennen, welche bis zum vierten Sukzessionsjahr Abundanzanteile zwischen 20\% (Hainholz-KF2) und 50\% (KönigsbucheKF2) erobern. Zweitgrößte Artengruppe bleiben auch in 2001 überall die Frischezeiger. Feuchtezeiger sind in der Königsbuche deutlich stärker verbreitet als in den beiden ZechsteinGebieten. Dabei stellen die Wurzelteller der Königsbuche anfangs (d.h. 1999) gegenüber den $100 \mathrm{~m}^{2}$-Plots den deutlich Feuchtezeiger-reicheren Lebensraum dar (44\% Anteil gegenüber 15\%). Anschließend setzt dort aber ein Rückgang zugunsten indifferenter Arten ein, so dass sich die Feuchtezeiger-Anteile beider Teilstandorte angleichen. In der Hainholz-KF1 werden die Feuchtezeiger sowohl von den Untersuchungsflächen als auch von den Wurzeltellern verdrängt (Rückgang von 5 auf 1\% Anteil), in der Hainholz-KF2 spielen sie mengenmäßig gar keine Rolle. Letzteres gilt generell auch für die Trockenheitszeiger in allen UG. Insgesamt sind sich die untersuchten Teilstandorte bei quantitativer Betrachtung der FeuchteZeigerwerte in der Hainholz-KF2 am ähnlichsten.

Stickstoff-Zeigerwert: Beim qualitativen Spektrum (Abb. 56 oben) sind zwischen Wurzeltellern und Umgebungsflächen keine Unterschiede ersichtlich, ebensowenig ergeben sich Veränderungen innerhalb der Zeitreihe. Es dominieren Mäßigstickstoffzeiger mit 40-50\% Anteil (Königsbuche-KF2, Hainholz-KF2) oder Stickstoffzeiger mit 50-60\% Anteil (Hainholz-KF1). Auffällig ist die sehr geringe Präsenz von Stickstoffarmutszeigern in der Hainholz-KF1 (3-6\% Anteil), während auf den anderen Standorten mindestens doppelt so hohe Werte erreicht werden.

Unter Berücksichtigung der Deckungsgrade (quantitatives Spektrum, Abb. 56 unten) zeigt sich zunächst die deutlich stärkere Verbreitung der Stickstoffzeiger im Zechstein, was für alle Teilstandorte zutrifft. Indifferente Arten und Stickstoffarmutszeiger spielen quantitativ nirgendwo eine Rolle. Darüber hinaus lässt sich folgende Dreiteilung vornehmen: 1 . In der Hainholz-KF2 ähneln sich Wurzelteller und $100 \mathrm{~m}^{2}$-Plots sehr stark, wobei sich auf beiden Teilstandorten die Abundanzanteile der Stickstoffzeiger von ca. 30 auf 40\% erhöhen. 2. Auf den Wurzeltellern der Hainholz-KF1 gehen die Abundanzanteile der Stickstoffzeiger von 42 auf 34\% zurück, während sie auf den Untersuchungsflächen mit konstant 60\% dominieren. 3. Im Buntsandstein dominieren die Mäßigstickstoffzeiger am stärksten. Hier fungieren die Wurzelteller zunehmend als Ausbreitungsort für Stickstoffzeiger (Anstieg von 11 auf 21\% Abundanzanteil), während diese auf den umgebenden $100 \mathrm{~m}^{2}$-Plots bei $4 \%$ stagnieren.

Insgesamt sind sich die untersuchten Teilstandorte bei gewichteter Betrachtung der StickstoffZeigerwerte in der Hainholz-KF2 am ähnlichsten. 

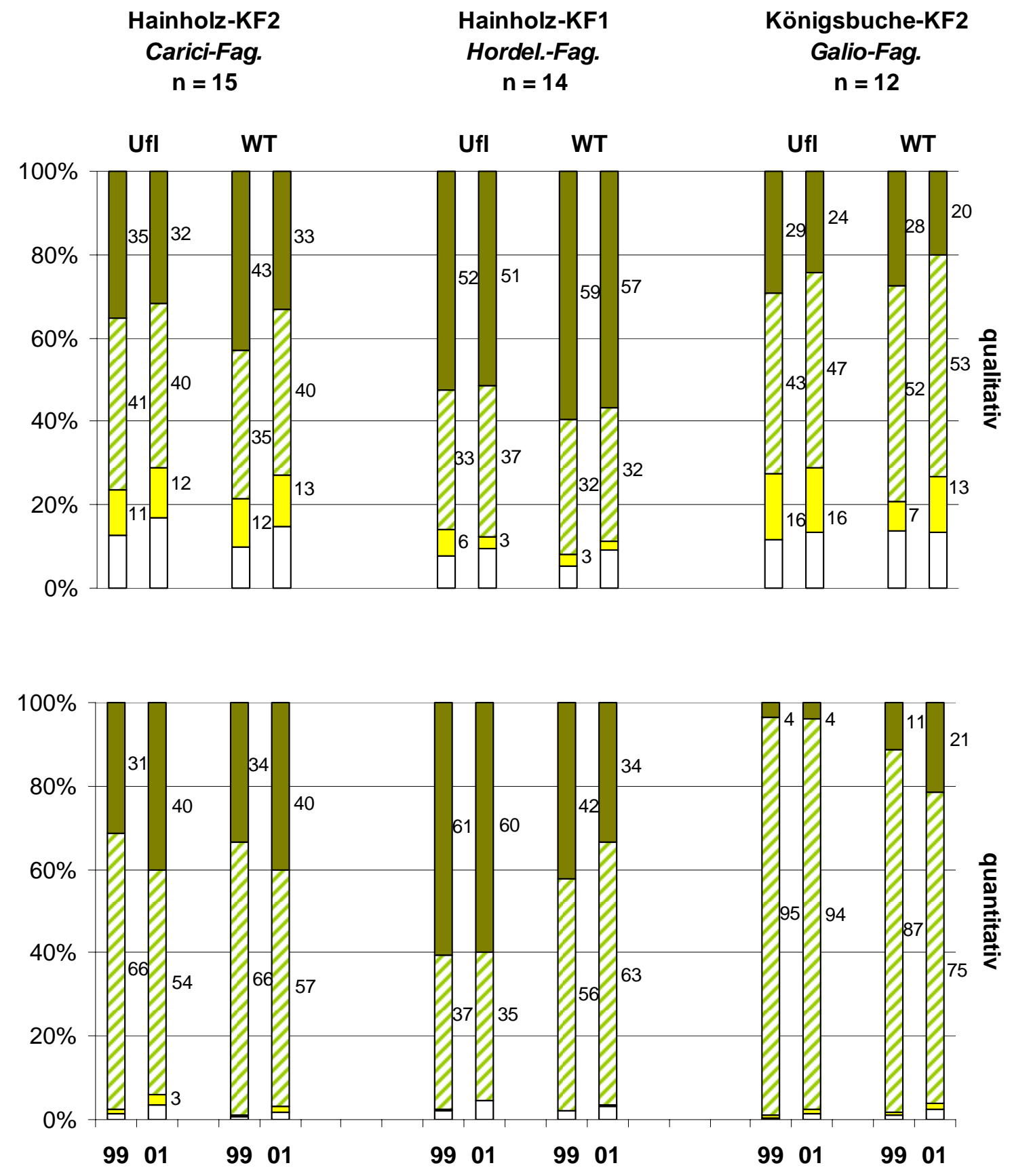

$\begin{array}{ll}\square \text { Indifferente/o. Zuordnung } & \square \text { Stickstoffmangelzeiger [N1-3] } \\ \square \text { Mäßigstickstoffzeiger [N4-6] } & \square \text { Stickstoffzeiger [N7-9] }\end{array}$

Abb. 56: Qualitatives (oben) und quantitatives (unten) Stickstoff-Zeigerwertspektrum der Arten auf drei Buchen-Windwurfflächen mit Flächenwurf im südwestlichen Harzvorland in den Jahren 1999 und 2001; Anzahl einbezogener 100 m²-Plots und Wurzelteller siehe Tab. 14; Strauch- und Feldschicht zusammengefasst; Angaben unter 3\% nicht angezeigt. 


\subsubsection{Pflanzensoziologische Klassen nach ELLENBERG et al. (1992)}

Die Laubwaldarten (Querco-Fagetea) stellen die qualitativ stärkste Artengruppe dar, wobei kaum Unterschiede zwischen $100 \mathrm{~m}^{2}$-Flächen und Wurzeltellern bzw. Veränderungen innerhalb der Zeitreihe feststellbar sind (Abb. 57 oben). Sie erreichen Anteile zwischen rund 35\% (Hainholz-KF2) und 50\% (übrige UG). Zwischen 1999 und 2001 verzeichnen sie auf den Wurzeltellern der Königsbuche Anteilsrückgänge um 15\%. Unter Berücksichtigung der Deckungsgrade (Abb. 57 unten) zeigt sich, dass die Laubwaldarten auf Wurzeltellern ausnahmslos schwächer verbreitet sind als auf den umgebenden $100 \mathrm{~m}^{2}$-Plots. Dabei gehen die Anteile dieser Artengruppe von unterschiedlichem Ausgangsniveau zurück. Lediglich in der Hainholz-KF2, die insgesamt am schwächsten von Querco-Fagetea-Arten geprägt wird, nehmen sie auf beiden Teilstandorten zu (Ufl: von 11 auf 17\%/WT: von 3 auf 9\% Anteil). Im vierten Sukzessionsjahr ist die Hainholz-KF1 sowohl auf Wurzeltellern als auch auf deren Umgebungsflächen immer noch am stärksten von Laubwaldarten besiedelt (Ufl: 83\%/WT: 49\% Abundanzanteil).

Die Schlagflurarten (Epilobietea) sind mit 10-20\% Anteil am qualitativen Artenspektrum beteiligt. Unterschiede zwischen den $100 \mathrm{~m}^{2}$-Plots und den darin befindlichen Wurzeltellern sowie innerhalb der Zeitreihe ergeben sich kaum (Abb. 57 oben). Unter Berücksichtigung der Deckungsgrade (Abb. 57 unten) sind die Schlagflurarten in der Königsbuche und in der Hainholz-KF1 auf den Wurzeltellern wesentlich stärker etabliert als auf den umliegenden 100 $\mathrm{m}^{2}$-Flächen, wohingegen sich auf dem Bollerkopf (Hainholz-KF2) diesbezüglich kaum Unterschiede ergeben. Die Epilobietea erweitern auf allen Teilstandorten ihre quantitativen Anteile. Sie erreichen im Jahr 2001 auf den Wurzeltellern der Königsbuche mit 67\% Anteil die höchsten Werte.

Die Ruderalarten (v.a. Artemisietea) sind im qualitativen Spektrum (Abb. 57 oben) der Buntsandstein-Flächen mit 7-12\% Anteil schwächer vertreten als im Zechstein mit 15-26\%. Zwischen $100 \mathrm{~m}^{2}$-Plots und Wurzeltellern sowie innerhalb der Zeitreihe ergeben sich keine Unterschiede. Unter Berücksichtigung der Deckungsgrade (Abb. 57 unten) lässt sich auf den meisten Teilstandorten ein Anteilsrückgang feststellen, wobei die Hainholz-KF2 sowohl auf

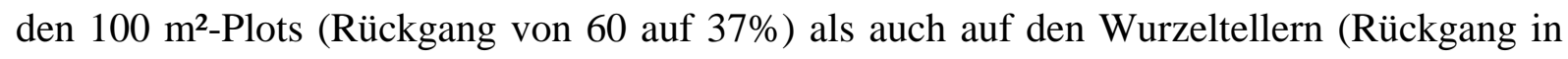
ähnlichem Umfang) besonders stark von Ruderalarten beherrscht bleibt. Bei den übrigen UG lässt sich erkennen, dass im Jahr 2001 die Wurzelteller im Vergleich zu ihrer Umgebungsfläche etwas stärker mit Ruderalarten besiedelt waren.

Grünlandarten (v.a. Molinio-Arrhenatheretea) sind in ähnlichem Umfang wie die Ruderalarten am qualitativen Artenspektrum beteiligt (Abb. 57 oben). Quantitativ spielen sie hauptsächlich im Buntsandstein eine Rolle (Abb. 57 unten), wobei sie vor allem im zweiten Sukzessionsjahr (1999) bei anschließend sinkender Tendenz auf den Wurzeltellern höhere Abundanzanteile erreichten als auf den 100 m²-Plots (Ufl: konstant rund 10\% /WT: Rückgang von ca. 26 auf 9\%). 

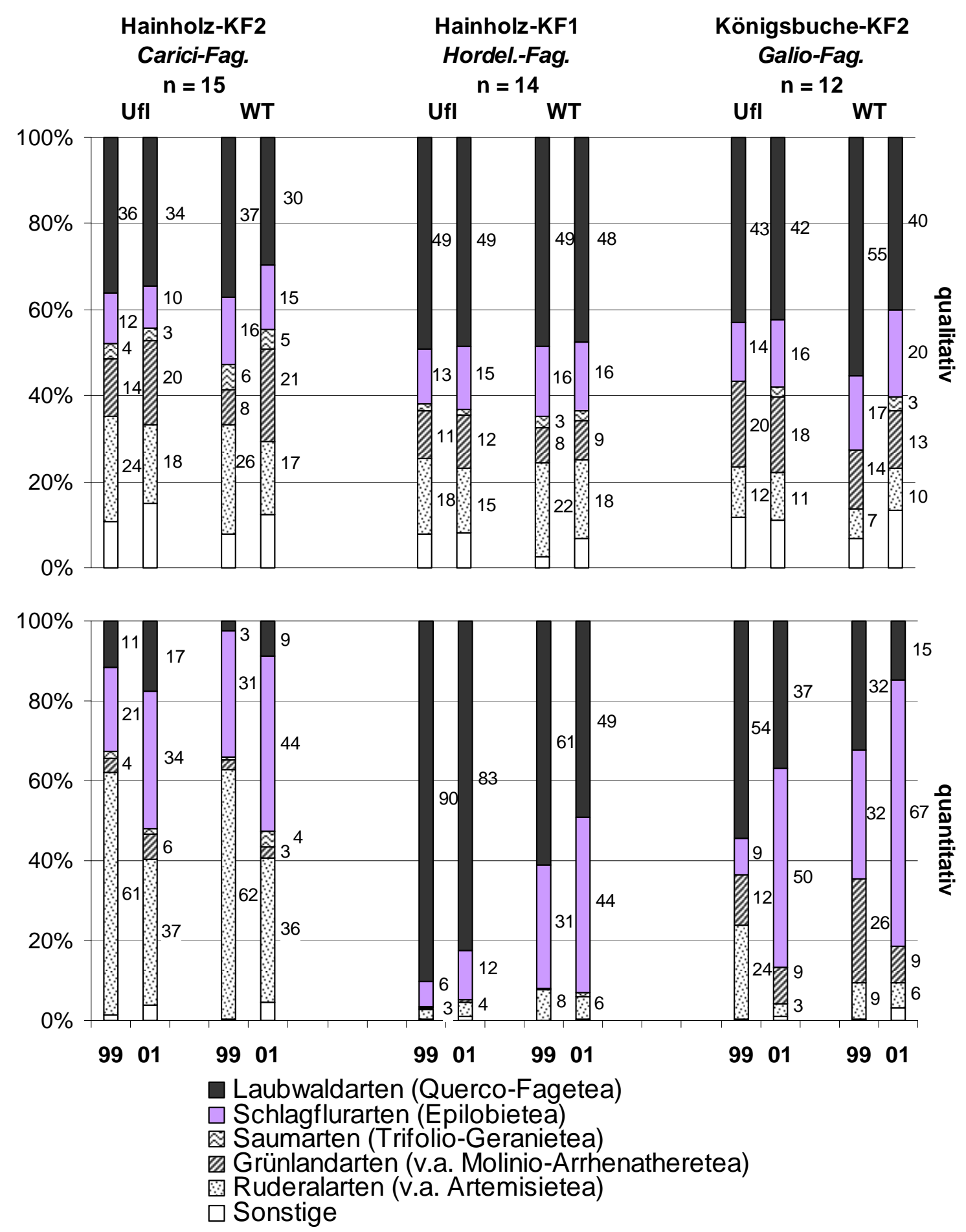

Abb. 57: Qualitatives (oben) und quantitatives (unten) Artengruppenspektrum nach pflanzensoziologischen Klassen der Arten auf drei Buchen-Windwurfflächen mit Flächenwurf im südwestlichen Harzvorland in den Jahren 1999 und 2001; Anzahl einbezogener $100 \mathrm{~m}^{2}$ Plots und Wurzelteller siehe Tab. 14; Strauch- und Feldschicht zusammengefasst; Angaben unter 3\% nicht angezeigt.

Saumarten (Trifolio-Geranietea) kommen zwar überall vor (Abb. 57 oben), erreichen aber nur geringe Anteile am qualitativen Artenspektrum (max. 3-6\%). Bemerkenswert erscheint, dass sie in der Königsbuche erst im vierten Sukzessionsjahr neu auftauchen. Quantitativ (Abb. 
57 unten) spielen sie nur auf den Wurzeltellern der Hainholz-KF2 eine geringe Rolle (2001: 4\% Abundanzanteil).

Bei quantitativer Betrachtung der in pflanzensoziologische Klassen eingeteilten Artengruppen unterscheiden sich Untersuchungsflächen und Wurzelteller auf dem Bollerkopf insgesamt am wenigsten.

\subsubsection{Lebensformtypen im Sinne RAUNKIAERS nach ELLENBERG et al. (1992)}

Bei qualitativer Betrachtung ergeben sich kaum Unterschiede zwischen $100 \mathrm{~m}^{2}$-Plots und Wurzeltellern bzw. innerhalb der Zeitreihe (Abb. 58 oben). Etwa 50-60\% der Arten sind Hemikryptophyten, jeweils ca. 5-10\% sind den Therophyten, Geophyten, Phanerophyten und Nanophanerophyten zuzuordnen. Chamaephyten sind mit max. 4\% Anteil auf den meisten Teilstandorten vertreten, lediglich auf den Wurzeltellern der Hainholz-KF2 fehlen sie.

Unter Berücksichtigung der Deckungsgrade (Abb. 58 unten) fällt zunächst auf, dass die Geophyten in den relativ Geophyten-reichen UG Königsbuche und Hainholz-KF1 (Ufl: anfangs 40-46\% Anteil) auf den Wurzeltellern nur Bruchteile der Abundanzanteile der Umgebungsflächen erreichen und diese Werte im Sukzessionsverlauf abnehmen oder stagnieren. Auf dem generell Geophyten-armen Bollerkopf dagegen (Ufl: konstant 6-7\% Anteil) gewinnen die Geophyten auf den Wurzeltellern sogar noch geringfügige Anteile hinzu (von ca. 1 auf 4\%). Chamaephyten spielen quantitativ nur in der Hainholz-KF1 eine Rolle und erreichen dort anfangs (d.h. 1999) bei überall sinkenden Werten auf den Wurzeltellern geringfügig höhere Abundanzanteile (Rückgang von 37 auf 23\%) als auf den umgebenden

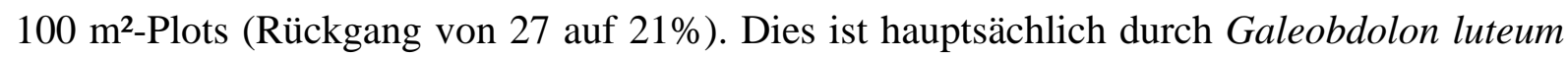
begründet, welche mit ihrem kriechenden Wuchs die Wurzeltellermulden besonders schnell erobert hat. Ebenfalls in der Hainholz-KF1 als dem einzigen UG mit auffällig starker Naturverjüngung kommen die Phanerophyten $\mathrm{zu}$ nennenswerten und steigenden Abundanzanteilen (Ufl: von 11 auf 24\%/WT: von 9 auf 15\%). In wesentlich geringerem Umfang wird das auch auf den $100 \mathrm{~m}^{2}$-Plots der Hainholz-KF2 deutlich (Anstieg von 3 auf 8\% Abundanzanteil), aber auf den Wurzeltellern treten die jungen Bäume dort kaum in Erscheinung. In der Königsbuche schließlich existiert weder auf den Wurzeltellern noch auf deren Umgebungsfläche nennenswerte Naturverjüngung. Nanophanerophyten erreichen bei unterschiedlich stark ansteigenden Werten auf den Wurzeltellern die deutlich höheren Abundanzanteile (mit Ausnahme des Bollerkopfes - hier etwa Gleichstand im Jahr 2001), wobei die Wurzelteller der Königsbuche im vierten Sukzessionsjahr mit 66\% Anteil am Strauch-reichsten sind. Hemikryptophyten waren anfangs auf Wurzeltellern generell stärker verbreitet als auf deren Umgebungsflächen (in 1999: auf WT der Hainholz-KF2 mit 58\% sogar dominierend, auf Ufl: mit 46\%). Sie verzeichnen aber im Sukzessionsverlauf überall sinkende Abundanzanteile, wodurch sich Wurzelteller und $100 \mathrm{~m}^{2}$-Plots diesbezüglich angleichen. Die Therophyten verzeichnen generell sinkende Abundanzanteile am 
Lebensformspektrum. Sie waren anfangs (d.h. 1999) besonders stark auf beiden Teilstandorten des Bollerkopfes vertreten (jeweils ca. 40\%), ferner auch auf den $100 \mathrm{~m}^{2}$-Plots der Königsbuche (24\%).

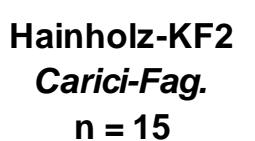

$\mathrm{n}=15$

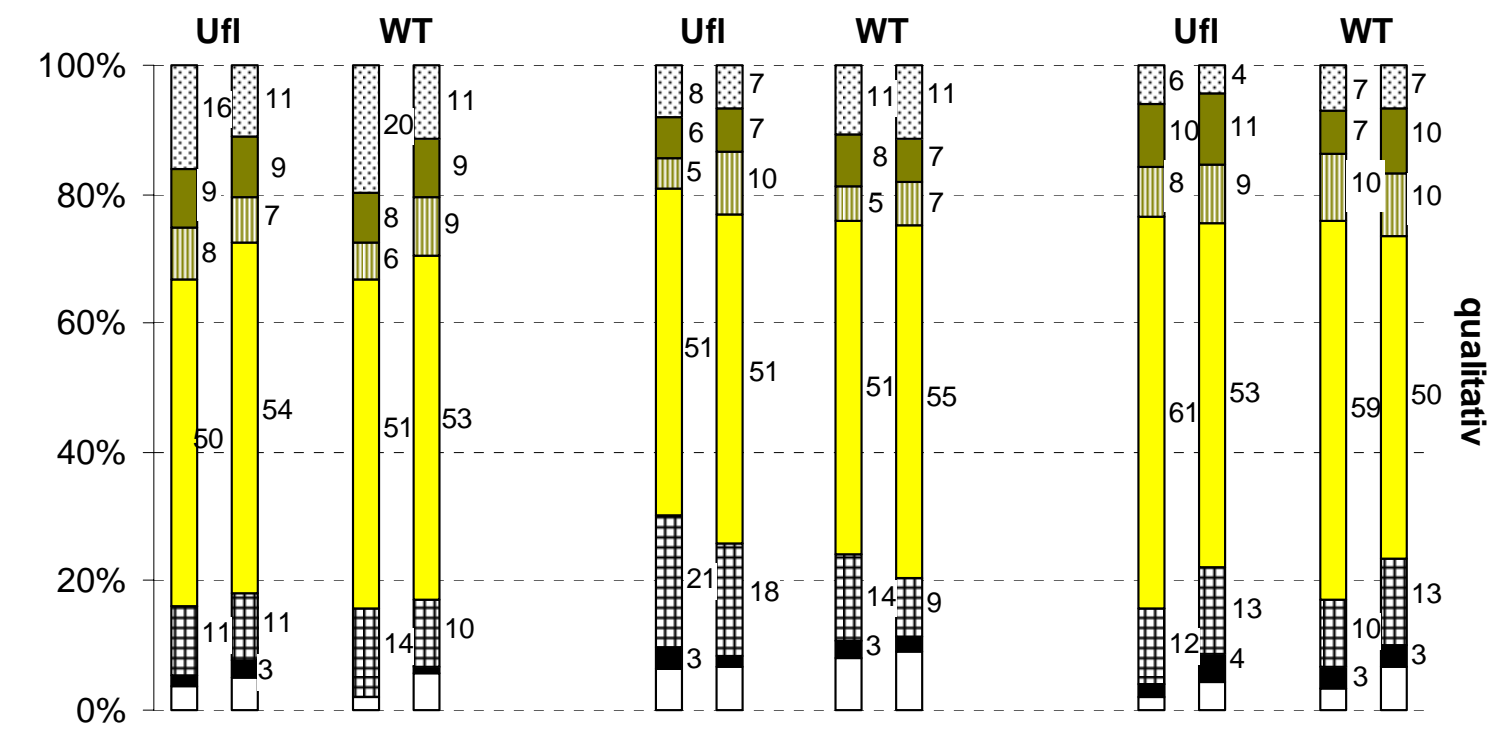

\section{Hainholz-KF1} Hordel.-Fag.

$\mathrm{n}=14$

\section{Königsbuche-KF2 \\ Galio-Fag. \\ $\mathrm{n}=12$}

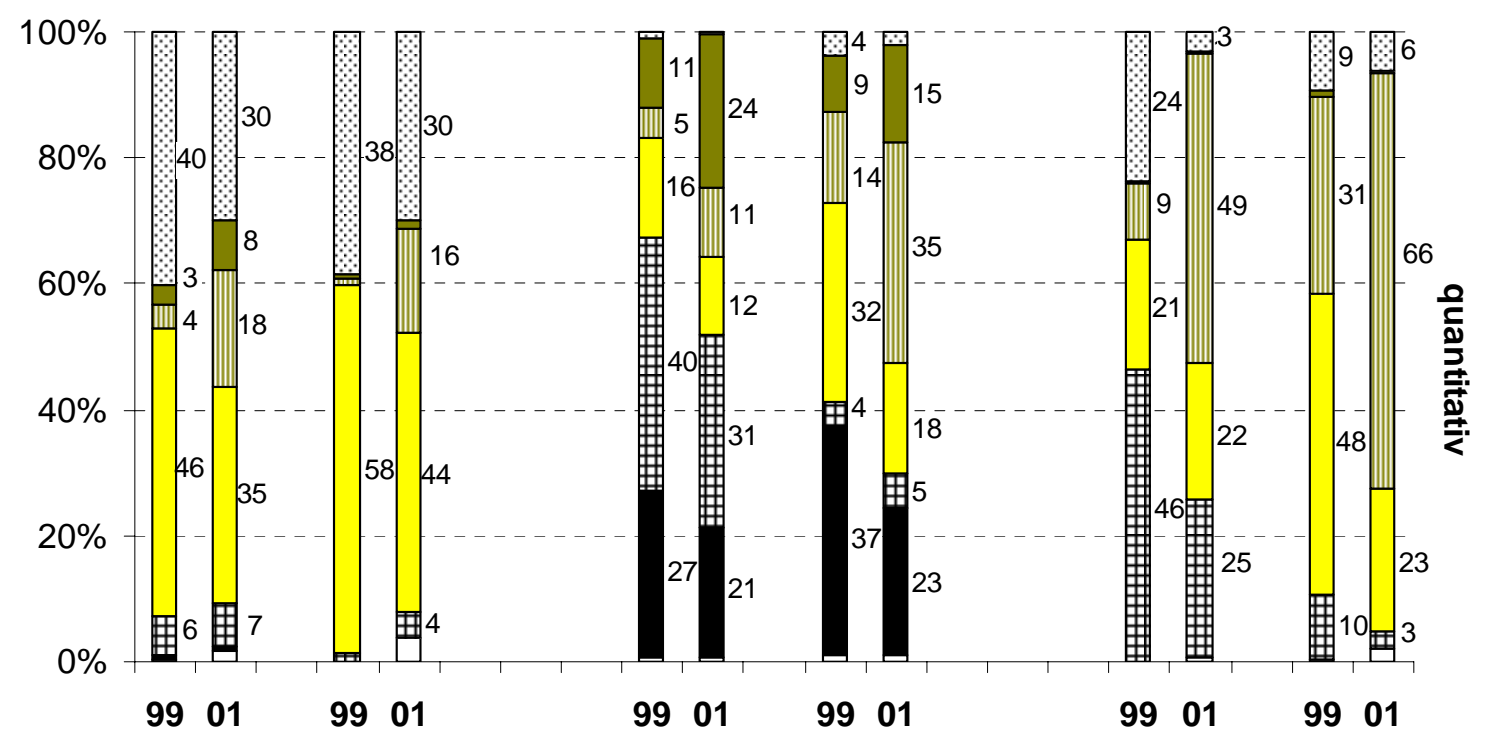

\section{$\square$ Sonstige \\ Chamaephyten $⿴$ Geophyten \\ $\square$ Hemikryptophyten $\quad \square$ Nanophanerophyten $\square$ Phanerophyten \\ 圆 Therophyten}

Abb. 58: Qualitatives (oben) und quantitatives (unten) Artengruppenspektrum nach Lebensformtypen der Arten auf drei Buchen-Windwurfflächen mit Flächenwurf im südwestlichen Harzvorland in den Jahren 1999 und 2001; Anzahl einbezogener 100 m²-Plots und Wurzelteller siehe Tab. 14; Strauch- und Feldschicht zusammengefasst; Angaben unter $3 \%$ nicht angezeigt. 
Während diese Gruppe auf dem flachgründigen Gips/Dolomit-Standort auch im vierten Sukzessionsjahr etwa gleichstark auf Wurzeltellern und $100 \mathrm{~m}^{2}$-Flächen vertreten bleibt, erscheinen die Wurzelteller in der Königsbuchen-KF2 bzw. in der Hainholz-KF1 als „Therophyten-Refugien“ inmitten dicht schließender Himbeer- bzw. Eschen-Fluren.

Insgesamt unterscheiden sich bei quantitativer Betrachtung der in Lebensformtypen eingeteilten Artengruppen Wurzelteller und umliegende $100 \mathrm{~m}^{2}$-Plots in der Hainholz-KF2 am wenigsten voneinander.

\subsubsection{Vegetatives Ausbreitungsvermögen nach SCHMIDT (1983), GRIME et al. (1989), BARTELS (1993) und KLEYER (1995)}

Bei qualitativer Betrachtung (Abb. 59 oben) zeigt sich sowohl auf den Wurzeltellern als auch

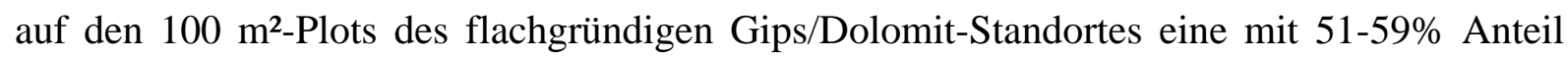
knappe Dominanz von Arten ohne vegetatives Ausbreitungsvermögen, während auf den produktiveren Dolomit- bzw. Buntsandstein-Standorten Arten mit vegetativem Ausbreitungsvermögen bei Anteilen um die 60\% im Artenzahlenspektrum vorherrschen. Innerhalb der Zeitreihe kommt es bezüglich der Arten ohne vegetatives Ausbreitungsvermögen auf den Wurzeltellern im Carici-Fagetum (Abnahme von 59 auf 51\% Anteil) und auf denen im Galio odorati-Fagetum (Abnahme von 38 auf 27\%) zu geringeren Veränderungen.

Unter Einbeziehung der Deckungsgrade (Abb. 59 unten) sind die bei qualitativer Betrachtung vorgefundenen Dominanzverhältnisse noch deutlich stärker ausgeprägt. Dabei kommt es auf beiden Teilstandorten der Hainholz-KF2 (Gips/Dolomit) zu einem relativ starken Rückgang von Arten ohne vegetatives Ausbreitungsvermögen bei ähnlichem Ausgangsniveau (Ufl: von 87 auf 63\%/WT: von 96 auf 65\% Anteil). Auf den beiden produktiveren Standorten (Hainholz-KF1, Königsbuche-KF2) laufen auf den Wurzeltellern und ihrer Umgebungsfläche gegenläufige Entwicklungen ab. Auf Dolomit nehmen die Arten ohne vegetatives

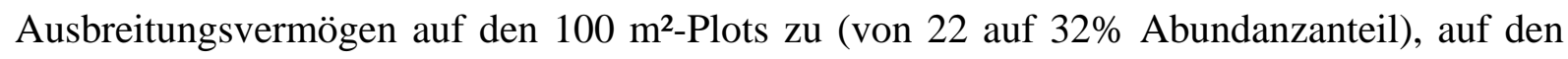
Wurzeltellern aber ab (von 41 auf 31\%). Im Buntsandstein ist es dagegen genau umgekehrt (Ufl: von 27 auf 7\%/WT: von 21 auf 27\%). Im vierten Sukzessionsjahr (2001) sind sich die Wurzelteller und die umgebenden $100 \mathrm{~m}^{2}$-Plots auf beiden Zechstein-Standorten sehr ähnlich, während im Buntsandstein die Wurzelteller als vergleichsweise bevorzugter Wuchsort für Arten ohne vegetatives Ausbreitungsvermögen erscheinen. 

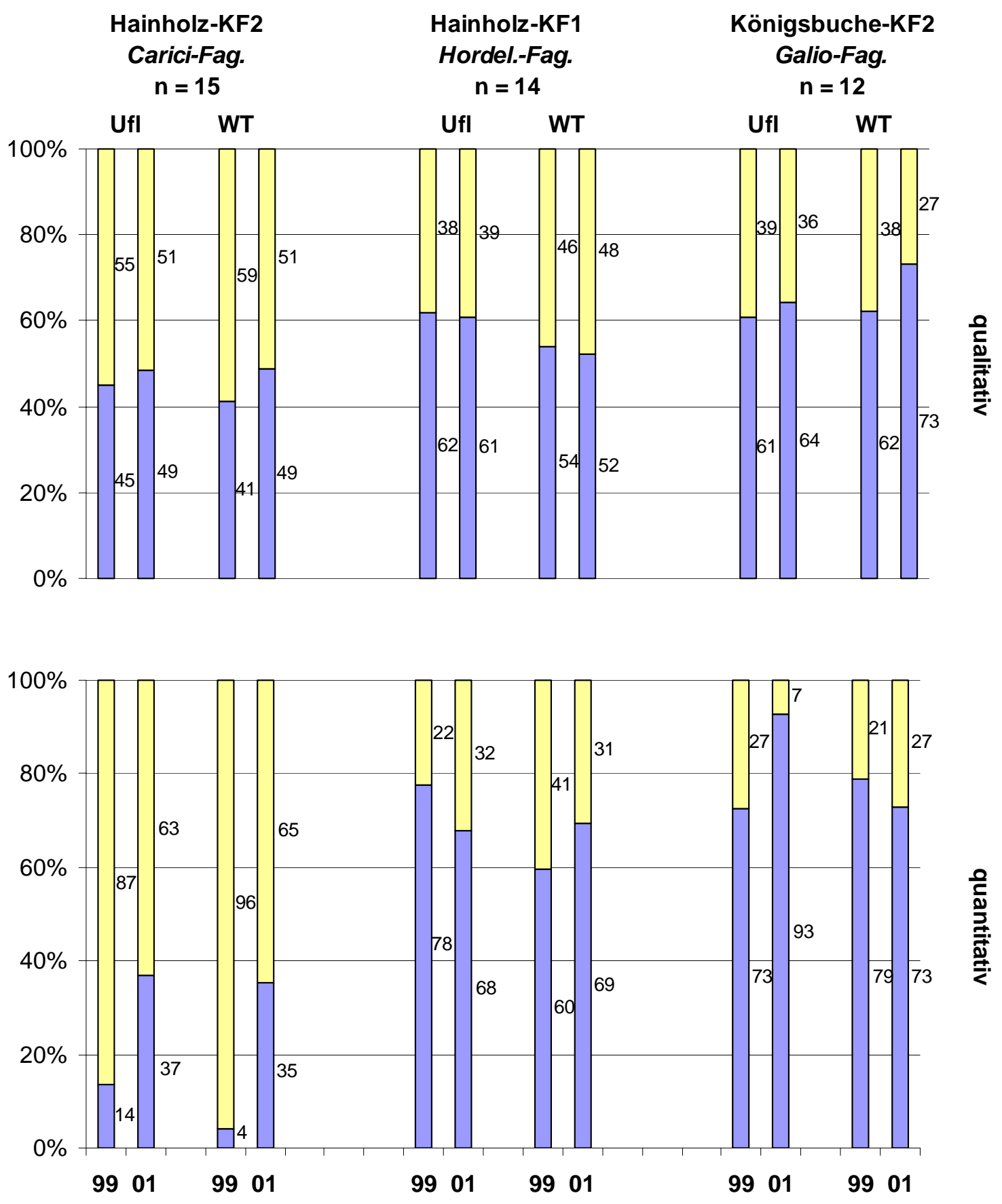

$\square$ MIT veget. Ausbreitungsvermögen $\square$ OHNE veget. Ausbreitungsvermögen

Abb. 59: Qualitatives (oben) und quantitatives (unten) Artengruppenspektrum nach vegetativem Ausbreitungsvermögen der Arten auf drei Buchen-Windwurfflächen mit Flächenwurf im südwestlichen Harzvorland in den Jahren 1999 und 2001; Anzahl einbezogener $100 \mathrm{~m}^{2}$-Plots und Wurzelteller siehe Tab. 14; Strauch- und Feldschicht zusammengefasst; Angaben unter 3\% nicht angezeigt.

Zusammenfassend lässt sich also bis auf wenige Ausnahmen feststellen, dass die qualitative Artenzusammensetzung auf Untersuchungsflächen und Wurzeltellern erstaunlich ähnlich und im Sukzessionsverlauf der ersten vier Jahre auch sehr stabil ist. Dagegen ergibt sich bei 
quantitativer Betrachtung, dass sich beide Teilstandorte im Sukzessionsverlauf sehr dynamisch entwickeln. Dabei zeigen besonders auf den produktiveren Buntsandstein- bzw. Dolomit-Standorte bestimmte Artengruppen auf den Wurzeltellern ein deutlich anderes Wuchsverhalten (im Sinne eines stärkeren quantitativen Vorkommens) als auf den umgebenden $100 \mathrm{~m}^{2}$-Plots. Dies betrifft Lichtzeiger, Epilobietea-Arten, Sträucher, Hemikryptophyten sowie speziell auf Buntsandstein auch Feuchte- und Stickstoffzeiger sowie Arten ohne vegetatives Ausbreitungsvermögen. Auf dem flachgründigen Gips/DolomitStandort unterscheiden sich Untersuchungsflächen und Wurzelteller auch bei quantitativer Betrachtung fast gar nicht.

\subsubsection{Bedeutung der Wurzelteller als alleiniger Wuchsort für bestimmte Arten}

\subsubsection{Häufigkeit des alleinigen Auftretens einzelner Arten auf Wurzeltellern}

Um die Bedeutung der Wurzelteller als alleiniger Wuchsort für bestimmte Arten ermitteln zu können, wurde die prozentuale Häufigkeit bestimmt, mit der manche Arten ausschließlich auf Wurzeltellern vorkamen (Tab. A32). Die Datenbasis dazu ist ein einfacher Vermerk im

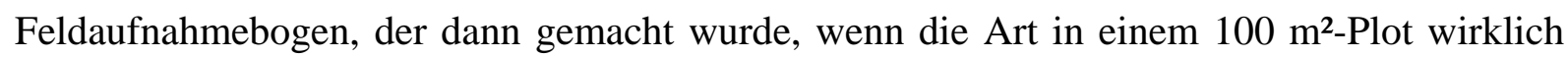
nur auf Wurzeltellern zu finden war, ohne deren Anzahl zu berücksichtigen. Damit soll geklärt werden, ob bestimmte Arten im Sukzessionsverlauf zunehmend auf die Wurzelteller „abgedrängt“ werden. Wiederum erfolgte für die drei bisher behandelten Untersuchungsvarianten (beide Hainholz-KF, Königsbuche-KF2) ein Vergleich zwischen zweitem und viertem Sukzessionsjahr (1999 und 2001).

Im Untersuchungszeitraum konnte bei insgesamt 5 Strauchschicht- und 90 Feldschichtarten ein alleiniges Vorkommen auf Wurzeltellern registriert werden, ohne dass Gefäßpflanzenarten ermittelt wurden, die - bezogen auf jeweils ein UG als Gesamtstratum - ausschließlich auf Wurzeltellern vorkamen (Tab. A32). Unterteilt nach den einzelnen Untersuchungsgebieten und -jahren ergeben sich für diese Unterfraktion die in Tab. 15 aufgeführten Prozentanteile an der Gesamtartenzahl.

Tab. 15: Anteil derjenigen Arten, die in manchen $100 \mathrm{~m}^{2}$-Plots nur auf Wurzeltellern vorkamen (entsprechend Tab. A32) an der Gesamtartenzahl (entsprechend Tab. A26 bis A31).

\begin{tabular}{|l|c|c|c|c|c|c|}
\hline & \multicolumn{2}{|c|}{ Hainholz-KF2 (n = 15) } & \multicolumn{2}{c|}{ Hainholz-KF1 (n = 14) } & \multicolumn{2}{c|}{ Königsbuche-KF2 (n = 12) } \\
\hline & $\mathbf{1 9 9 9}$ & $\mathbf{2 0 0 1}$ & $\mathbf{1 9 9 9}$ & $\mathbf{2 0 0 1}$ & $\mathbf{1 9 9 9}$ & $\mathbf{2 0 0 1}$ \\
\hline Strauchsch. & $0 \%$ & $11 \%$ & $0 \%$ & $8 \%$ & $50 \%$ & $22 \%$ \\
\hline Feldschicht & $18 \%$ & $32 \%$ & $29 \%$ & $40 \%$ & $40 \%$ & $41 \%$ \\
\hline
\end{tabular}

Die relativ hohen und teilweise noch steigenden Prozentanteile insbesondere bei den Feldschichtarten machen deutlich, dass die Wurzelteller für eine ganze Reihe von Arten zunehmend zu einem „Ausweichwuchsort“ werden oder im Falle der Königsbuchen-KF2 auch schon im zweiten Sukzessionsjahr waren. Dieser Trend ist auf den produktiveren Dolomit- bzw. Buntsandstein-Standorten (Hainholz-KF1, Königsbuche-KF2) mit ihrer immer 
dichter schließenden Strauchschicht (Esche, Himbeere) vorerst noch stärker ausgeprägt als auf der flachgründigen und anfangs nur dünn besiedelten Gips/Dolomit-Kuppe des Bollerkopfes, wo die Krautschichtvegetation weder auf Wurzeltellern noch auf $100 \mathrm{~m}^{2}$-Plots eine Sättigung bezüglich Artenzahlen und Deckungsgraden erkennen lässt (Kap. 4.6.3, 4.6.5, 4.6.7). In welchem Umfang auch höherwüchsige Gehölze $(>50 \mathrm{~cm})$ zukünftig auf Wurzelteller „abgedrängt“ werden, bleibt abzuwarten. Lediglich in der Königsbuche zeigte anfangs (1999) jede zweite Strauchschichtart ein gelegentlich alleiniges Vorkommen auf Wurzeltellern, danach (2001) aber nur noch jede fünfte.

Die Arten in Tab. A32 wurden ferner dahingehend unterteilt, ob für sie der Wurzelteller als alleiniger Keim-, Etablierungs- und Wuchsort an Bedeutung gewinnt (steigende Prozentzahl oder Neuvorkommen zwischen 1999 und 2001) oder verliert (sinkende Prozentzahl oder Verschwinden). Dabei muss die genannte Entwicklung für die Mehrzahl der UG zutreffen, ansonsten wurde die Art der Kategorie „ohne eindeutige Tendenz“ zugeordnet.

Für insgesamt 4 Strauch- bzw. 54 Feldschichtarten (entspricht 80 bzw. 60\% der Artenzahl der Tab. A32) war zwischen 1999 und 2001 eine Bedeutungszunahme der Wurzelteller als alleiniger Wuchsort zu verzeichnen. Gebietsübergreifend sind das hauptsächlich Bromus benekenii, Carex muricata, C. remota, Carpinus betulus (Feldschicht), Dryopteris carthusiana, Epilobium angustifolium, E. montanum, E. hirsutum, Galeopsis tetrahit, Gymnocarpium dryopteris, Hypericum perforatum, Poa trivialis, Quercus robur (Feldschicht), Scrophularia nodosa, Senecio viscosus und Urtica dioica sowie weitere Arten, für die ein solches Verhalten nur in einem der UG registriert wurde. Es handelt sich meist um Licht-, Halbschatten- und Mäßigstickstoff-Zeiger unterschiedlicher soziologischer Zuordnung. Für eine Strauch- bzw. 20 Feldschichtarten (entspricht 20 bzw. 22\% der Artenzahl der Tab. A32) nimmt die Bedeutung der Wurzelteller als alleiniger Wuchsort ab. Das sind gebietsübergreifend Fagus sylvatica (Feldschicht), Impatiens parviflora, Quercus petraea (Feldschicht) und Sambucus nigra (Feldschicht) sowie weitere Arten, für die ein derartiges Verhalten nur in einem der drei UG zutrifft. Weitere 16 Feldschichtarten (entspricht 18\% der Artenzahl der Tab. 32) zeigen zwischen 1999 und 2001 widersprüchliche Trends in den einzelnen UG.

Insgesamt ist die prozentuale Häufigkeit, mit der manche Arten allein auf Wurzeltellern vorkamen, eher gering: etwa 6-14\% sind die Regel (Tab A32). Ausnahmen, die in wenigstens einem Untersuchungsjahr und/oder -gebiet Häufigkeiten von über einem Drittel der Fälle (>33\%) erreichen, betreffen eine Strauch- und neun Feldschichtarten. Das sind Sambucus nigra (max. 58\%) als Strauch- und Feldschichtart sowie Carex pallescens (max. 75\%), C. pilulifera (max. 42\%), Dryopteris filix-mas (max. 33\%), Epilobium angustifolium (max. 58\%), E. ciliatum (max. 36\%), Gymnocarpium dryopteris (max. 42\%), Luzula luzuloides (max. 33\%) und Sonchus asper (max. 36\%). Den „Rekord“ hält also Carex pallescens, die im Jahr 2001 im Flächenwurf der Königsbuchen-KF2 in 75\% der 100 m²-Plots nur auf 
Wurzeltellern vorkam und dort ein zwischenzeitliches Refugium in der ansonsten vorherrschenden Himbeer-Flur fand.

\subsubsection{Qualitative Artengruppenspektren}

Dieses Kapitel bezieht sich wiederum nur auf diejenigen 5 Strauchschicht- und 90

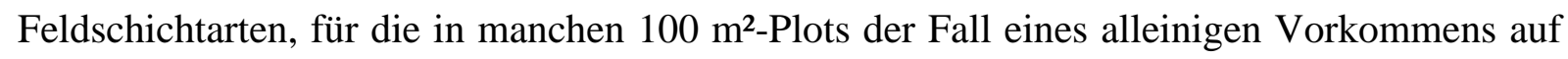
Wurzeltellern registriert wurde (vergleiche Kap. 4.6.8.1; Tab. A32). Die zusätzliche Einengung des Arteninventars der Wurzelteller auf diese Teilfraktion dient hier der Ausscheidung ökologisch-funktionaler Artengruppen, die als „Wurzeltellerspezialisten“ gelten können.

Bei qualitativer Betrachtung zeigen sich nur geringe Unterschiede zwischen den Spektren der hier behandelten Untergruppe (Abb. 60), denen der Wurzelteller (als Gesamtstandort) und auch denen der $100 \mathrm{~m}^{2}$-Plots (siehe Kap. 4.6.7). Besonderes Augenmerk kann man jedoch auf bestimmte Artengruppen legen, die zwischen 1999 und 2001 aus dieser Teilfraktion verschwanden, neu in ihr auftauchten, ihre qualitativen Anteile ganz besonders stark veränderten oder im Vergleich zum Spektrum der Umgebungsfläche besonders große oder geringe Bedeutung besaßen (Abb. 60). Erwähnenswert sind in diesem Zusammenhang:

1. die zunehmende, teilweise erstmalige Bedeutung der Wurzelteller als alleiniger Wuchsort für:

- Stickstoff- und auch Stickstoffmangelzeiger (nur auf Buntsandstein);

- Saum- und Ruderalarten (nur auf Buntsandstein);

- Trockenheitszeiger (nur auf Gips/Dolomit);

- Grünlandarten (nur auf Gips/Dolomit);

- Nanophanerophytenarten (nur auf Gips/Dolomit);

2. die abnehmende, teilweise verschwindende Bedeutung der Wurzelteller als alleiniger Wuchsort für:

- Feuchtezeiger (nur auf Buntsandstein);

- Schattenzeiger (auf allen Standorten);

- Saum- und Ruderalarten (nur auf Gips/Dolomit);

- Phanerophytenarten (nur auf tiefgründigem Dolomit);

- Arten ohne vegetatives Verbreitungsvermögen (nur im Zechstein - dort in 1999 noch mit relativ großer Bedeutung im Vergleich zum qualitativen Spektrum der Umgebungsfläche);

3. die im Vergleich zum Spektrum der Umgebungsfläche relativ große, aber gleichbleibende Bedeutung der Wurzelteller als alleiniger Wuchsort für:

- Schlagflurarten (nur auf tiefgründigem Dolomit);

4. die im Vergleich zum Spektrum zur Umgebungsfläche relativ geringe, aber gleichbleibende Bedeutung der Wurzelteller als alleiniger Wuchsort für:

- Geophytenarten (nur auf Buntsandstein und tiefgründigem Dolomit);

- Laubwaldarten (nur auf tiefgründigem Dolomit). 


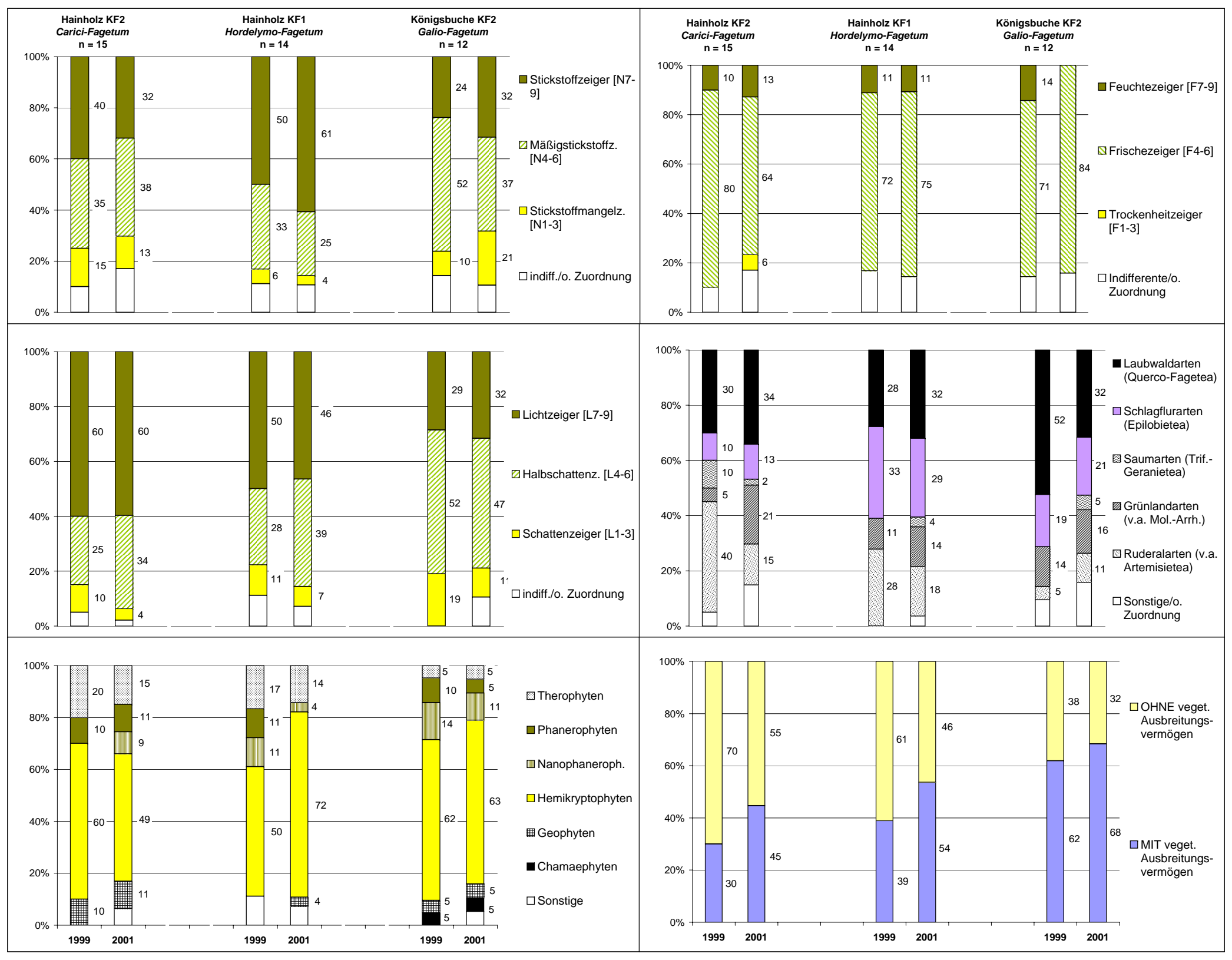

Abb. 60: Qualitative Artengruppenspektren derjenigen Arten, die auf drei Buchen-Windwurfflächen des südwestlichen

Harzvorlandes teilweise nur auf Wurzeltellern vorkamen (entsprechend Tab. A32); Strauch- und Feldschicht zusammengefasst;

Werte unter $3 \%$ nicht angezeigt. 


\section{DISKUSSION}

\subsection{Einleitung und Definitionen}

\subsubsection{Grundsätzliches zur Herangehensweise}

Die Eingangsthesen besagen, dass die Windwurfsukzession in mitteleuropäischen BuchenWäldern der Optimalphase in erster Linie stark standortsabhängig verläuft und sich Störungsflächengröße und forstliche Räumungsmaßnahmen besonders auf basenreichen, gut wasser- und nährstoffversorgten Standorten nur wenig bemerkbar machen (Kap. 1.4). Trotzdem sollte man jedes Windwurfgebiet als Einzelfall betrachten und dabei berücksichtigen, dass Faktoren wie die Zusammensetzung des Vorbestandes und der Vorausverjüngung, das Ausmaß an Bodenstörung, der Umfang und die Qualität der Samenbank, die langfristige Präsenz von Störzeigern in der Krautschicht auch schon vor dem Sturm, der Wildverbiss sowie die Erreichbarkeit der Flächen für Diasporen (Entfernung entsprechender Samenbäume) einen maßgeblichen Einfluss auf die Windwurfsukzession haben (KoOP 1982; KENK et al. 1991; FISCHER 1996a; HETZEL \& REIF 1997; SCHMIDT-SCHÜTZ \& HuSs 1998; SCHMIDT-SCHÜTZ 1999). Die nun folgende Diskussion will die einzelnen Einflussfaktoren zunächst getrennt beleuchten und differenzierende Untersuchungsparameter ausscheiden. Dabei erfolgt eine kapitelweise Unterteilung in Standort (5.3), Nutzungsaspekte (5.4), Störungsflächengröße (5.5) und sonstige, meist nicht mit eigenen Untersuchungsergebnissen belegte Einflussfaktoren (5.6). Pflanzensoziologische Aspekte der Windwurfsukzession (5.2) und Sonderstandorte (5.7) finden ebenfalls Berücksichtigung. Anschließend sollen allgemeine Sukzessionsmodelle (5.8) diskutiert und Folgerungen für Naturschutz und forstliche Praxis (5.9) abgeleitet werden.

Das Hauptaugenmerk der vorliegenden Untersuchungen lag auf der Waldbodenvegetation. Deren Kenngrößen wie z.B. Artenzahl und Artenzusammensetzung eignen sich ganz hervorragend als Indikatoren für gerichtete oder ungerichtete Veränderungen und als Diversitätsmaß in Wäldern, was durch mehrere Studien schon länger belegt ist (DIERSCHKE 1988; SCHMIDT 1991, 1999a+b; BRUNET et al. 1996; FISCHER 1998; SCHMIDT \& WECKESSER 2001; ОHEIMB 2003). Bei den hier u.a. verwendeten Artengruppenspektren (Kap. 4.3) erwies es sich als sinnvoll, jeweils vier Betrachtungsebenen (qualitativ und quantitativ sowie absolut und relativ) einzubeziehen. Nur so können Dominanzwechsel und Artenwechsel sicher voneinander unterschieden werden, was aber den etwas aufwändigeren Blick in die Vegetationstabellen zum Zwecke der weiteren Differenzierung nicht ersetzen kann und soll.

In Anlehnung an ScHÖLCH (1998) werden unter dem auch in der forstlichen Literatur unscharf verwendeten Begriff „Pionierbaumarten“ hier die Birke (Betula pendula), die Zitterpappel (Populus tremula), die Salweide (Salix caprea), die Schwarzerle (Alnus glutinosa) und die Vogelbeere (Sorbus aucuparia) verstanden. Die übrigen werden als „Schlusswald-, Klimax- oder Wirtschaftsbaumarten“ bezeichnet, ohne Berücksichtigung 
zeitweiliger Pioniereigenschaften innerhalb der letztgenannten Gruppe, z.B. bei der Esche (FISCHER et al. 1998).

\subsubsection{Fluktuation, Regeneration, Sukzession - Begrifflichkeiten der Vegetationsdynamik}

Während Fluktuationen eine kurzfristig-ungerichtete Änderung der Dominanzverhältnisse oder der Populationsstruktur ohne eintretenden Artenwechsel kennzeichnen, versteht man unter Sukzessionen die meist längerfristig-gerichtete Veränderung der Vegtationsstruktur, welche mit der Abfolge unterschiedlich zusammengesetzter Pflanzenbestände an einem Wuchsort einhergeht (SCHMIDT 1991; DiERSCHKE 1994). Kurzfristige, z.B. witterungsbedingte Fluktuationen von langfristig-gerichteten Sukzessionen zu unterscheiden, stellt sich als ein Problem dar, das durch den weitverbreiteten Mangel an Langzeitstudien mit lückenlosem, d.h. jährlich erfolgenden Aufnahmen bedingt ist. Ersatzweise angewandte „Raum-für-Zeit-Modelle“ (PICKETT 1989), bei denen aus dem räumlichen Nebeneinander von Sukzessionsstadien auf deren zeitliche Folge geschlossen wird, sind mit großen Unsicherheiten behaftet (PLACHTER 1992; TOPP 1998). Dies dürfte ganz besonders auf die stark menschlich beeinflussten mitteleuropäischen Wälder zutreffen und die Notwendigkeit von Langzeitstudien eher noch unterstreichen (SCHMIDT 1991, 1998a). Besonders für perennierende Arten mit klonalem Wachstum sind diese unabdingbar (BRUNET \& TYLOR 2000). Als Gründe für interannuelle Fluktuationen von Feldschichtarten in Wäldern werden häufig Niederschlags- und Temperaturschwankungen, zeitweilig geändertes Strahlungsangebot, die Nettoprimärproduktion des Vorjahres, Konkurrenzeffekte (z.B. Beschattung durch höherwüchsige Nachbarn), Pilzinfektionen und der Wildverbiss genannt (RUNGE 1981; SCHMIDT 1988, 1991; BRUnet \& TYLER 2000; BÖHLING 2003). Demgegenüber führen zunehmende Bodenversauerung, atmogene Stickstoffdeposition, globale und lokale Klimaveränderung und Nutzungseinstellung bzw. -umstellung eher zu langfristig-gerichteten Veränderungen in Waldbeständen (BÜRGER 1991; BÖHLING 1992, 2003; BRUNET et al. 1996; GRABIETZ \& FIEDLER 1996; AHRNS \& HOFMANN 1998; HAKES 1999).

Der Begriff Sukzession wird in der Literatur häufig generalisierend verwendet, was sicher kein Problem darstellt, so lange er im Einzelfall hinreichend genau erklärt wird und sich im Rahmen anerkannter Definitionen bewegt (siehe dazu die Übersicht in FISCHER \& KLOTZ 1999). Die vegetationsdynamischen Prozesse nach Windwurf in den hier untersuchten Buchen-Wäldern können entweder als sekundär-progressive Sukzession oder besser noch als zyklische Regeneration aufgefasst werden (vgl. DIERSCHKE 1988; SCHMIDT 1991; FisCHER \& KLOTZ 1999). Ein relativ reifes und naturnahes Ökosystem war Ausgangspunkt der Entwicklungen, nicht ein waldartenarmes oder naturfernes. Die Klimaxgesellschaft ist nach derzeitigem Erkenntnisstand ein relativ schnell erreichbares Ziel (zumindest keines, das erst in 100 Jahren realistisch sein wird) und pflanzensoziologisch gesehen wird der von der 
Ausgangsgesellschaft vorgegebene Rahmen kaum verlassen. Die alten Waldbedingungen werden sich - wenn auch nicht zu 100\% identisch - früher oder später wieder einstellen bzw. haben sich unter rasant aufkommender Naturverjüngung teilweise schon wieder eingestellt (SCHMIDT 2002a, 2003; KOMPA \& SCHMIDT 2003). Im Gegensatz zur Bewaldung bisher waldfreier Standorte bleibt ein großer Grundstock der im Altbestand vorhandenen und überwiegend den Querco-Fagetea zugehörigen Arten erhalten (vgl. Gegenüberstellung von Brachflächensukzession und Kahlschlagregeneration in SCHMIDT 1991).

\subsection{Zur Soziologie und Phänologie von Schlagflur- und Ruderalgesellschaften}

Die pflanzensoziologische Einordnung von Schlagflurvegetation gilt als problematisch (DIERSCHKE 1988). Während sich die Ausgangsbestände der hiesigen UG relativ klar als für den nordwestdeutschen Raum standortstypische Buchen-Waldgesellschaften klassifizieren lassen (Carici-, Hordelymo-, Galio odorati- und Luzulo-Fagetum), wurde der Pflanzensoziologie der Schlagflur- und Ruderalgesellschaften in der vorliegenden Arbeit kein eigener Platz eingeräumt. Im Zusammenhang mit seinen Untersuchungen zur Kahlschlagvegetation südniedersächsischer Buchen-Wälder spricht DIERSCHKE (1988) von einer Überlagerung der Waldarten durch eine Schlagflur, die sich nicht durch eine Abfolge floristisch-qualitativ unterscheidbarer Stadien auszeichnet, sondern eher durch einen Dominanzwechsel. Die pflanzensoziologische Abgrenzung solcher einander überlagernder „Zwillingsgesellschaften“ (DIERSCHKE 1988) ist in Anbetracht der rasanten Vegetationsentwicklung auf Kahlflächen, seien sie natürlich oder anthropogen entstanden, schwer möglich. Die Frage nach dem Sinn pflanzensoziologischer Zuordnungen ließe sich zukünftig nicht nur bei großflächiger, „katastrophaler“ Störung mit ausgeprägteren Schlagflur- und Ruderalstadien stellen, sondern auch bei kleinflächiger Lückendynamik in älteren Naturwäldern, welche in Deutschland derzeit aber noch selten sind. Momentan stammen ja fast alle Beschreibungen zur Wald-Syntaxonomie Mitteleuropas aus Wirtschafts-, nicht aus Natur- oder gar Urwäldern (ABS et al. 1999). Sie stellen gewissermaßen durch die Bewirtschaftungsweise jahrhundertelang fixierte Sukzessionstadien dar, die aber bei Nutzungseinstellung keinen Bestand haben werden (KoOP 1982). Dennoch werden auch in Naturwäldern solche zu durchlaufende Sukzessionsstadien häufig noch als eigene Assoziationen beschrieben, die auf der Klassifizierung der Kulturlandschaft basieren. Deshalb schlägt KооP (1982) vor, Wälder mit parallel anzutreffenden Sukzessionsstadien nach dem flächenmäßig dominanten Stadium zu benennen und nicht nach dem potenziell möglichen Endzustand. Diese Diskussion soll hier aber nicht weiter verfolgt werden. Bei den vorliegenden Untersuchungen im großflächigen Windwurf des Harzvorlandes ließen sich Anklänge an die Verbände Epilobion (Buntsandstein) und Atropion (Zechstein) finden. Besonders auf Buntsandstein ist die Überprägung der alten Waldbodenflora durch eine Himbeer-Flur (Rubetum idaei bei OBERDORFER 1973 bzw. Fragario-Rubetum idaei bei 
PASSARGE 1998) und ein Holunder-Gebüsch (Sambucetum racemosae bei OBERDORFER 1973 bzw. Impatieni-Sambucetum racemosae bei PASSARgE 1998) charakteristisch. Auf der flachgründigen und bislang Strauchschicht-armen Gips/Dolomit-Kuppe des Bollerkopfes ließe sich ein temporäres Epilobio-Atropetum belladonnae bzw. ein Epilobio angustifoliiSenecionetum fuchsii (bei SCHUBERT et al. 2001) beschreiben. Unklar ist, in welchem Umfang sich zukünftig auch hier die Himbeere etablieren kann. Man beachte die steigenden, aber relativ geringen Himbeer-Deckungen in der Strauch- und Feldschicht bis zum vierten Sukzessionsjahr (Tab. A22). Dagegen dürften in den beiden Varianten auf Dolomit (Hainholz-KF1, Nüllberg) mit ihrer rasanten Eschen- und Buchen-Naturverjüngung bzw. Pflanzung momentan bestehende Rubus-Gesellschaften schon bald keine Chance mehr haben. Die eingangs erwähnten Dominanzwechsel machen sich natürlich auch phänologisch bemerkbar. Erwähnenswert sind auf dem Nüllberg die zeitweiligen Massenbestände von Alliaria petiolata und Stachys sylvatica (zweites Jahr), Euphorbia amygdaloides (zweites und drittes Jahr), Hypericum perforatum (drittes Jahr) und Cirsium arvense (viertes Jahr), die unter schnellwüchsiger Esche und Himbeere zunehmend verschwanden. Sehr schön anzuschauen war auch der jährlich wechselnde Blühaspekt auf dem Bollerkopf (1998: Convallaria majalis - 1999: Atropa bella-donna, Mycelis muralis - 2000: Atropa belladonna, Hypericum perforatum - 2001: Carduus crispus, Cirsium arvense, Senecio ovatus), der die ganze Zeit vom Gelb der Impatiens parviflora-Herden begleitet wurde. In der Königsbuchen-KF2 kam es zum massenhaften Auflaufen von Impatiens parviflora (zweites und drittes Jahr), welches anschließend von der Himbeere überdeckt wurde. Der Krücker leuchtete in den Farben von Digitalis purpurea, Epilobium angustifolium und Impatiens parviflora (zweites Jahr), bevor auch hier Himbeer-Holunder-Gebüsche vordrangen. Weniger bunt erschienen die durch Himbeere, Brombeere, Holunder und Brennessel dominierte Rotenberg-Abt. 1228 und die stark Eschen-beherrschte Hainholz-KF1. Letztere fiel lediglich im Mai durch ihre dichten, schneeweißen Allium ursinum-Blütenteppiche auf. Einen recht abwechslungsreichen Einblick in die Phänologie der Hainholz-KF1 (Hordelymo-Fagetum) gibt die Fotoserie in Abb. 61 a+b. Gut erkennbar ist die im Sukzessionsverlauf immer dichter schließende Strauchschicht (Esche, ferner Himbeere und Holunder) und die immer weniger sichtbaren Allium-Blütenteppiche auf den Frühjahrsfotografien (Mai 2000, 2001).

\subsection{Zum Einfluss des Standortes auf die Windwurfsukzession}

\subsection{1 Übersicht über differenzierende Untersuchungsparameter}

Zunächst sollen Untersuchungsparameter zusammenfassend diskutiert werden, die im Rahmen der vorliegenden Arbeit standörtlich differenzierenden Charakter haben. Vielfältige und nicht näher quantifizierbare Überlagerungen z.B. mit Nutzungsaspekten sind dabei möglich und wahrscheinlich. 


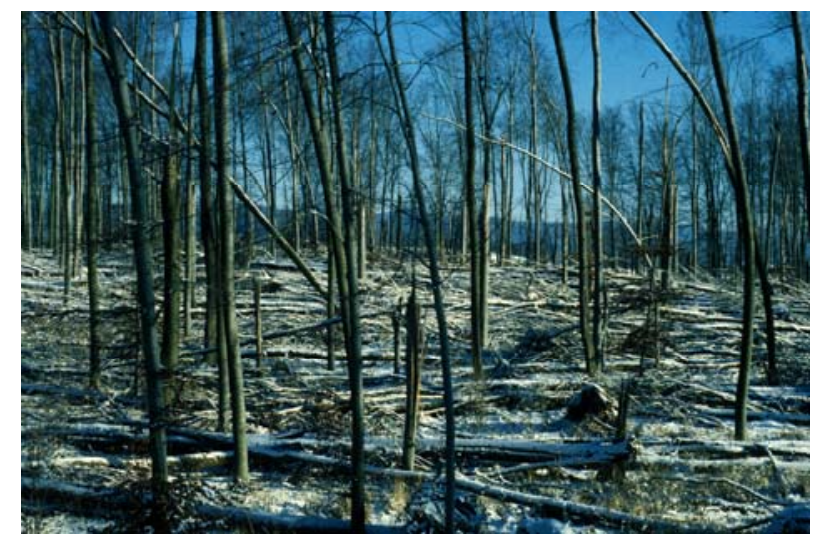

Abb. 61 a:

Fotoserie:

Hainholz-KF1

von Nordwesten

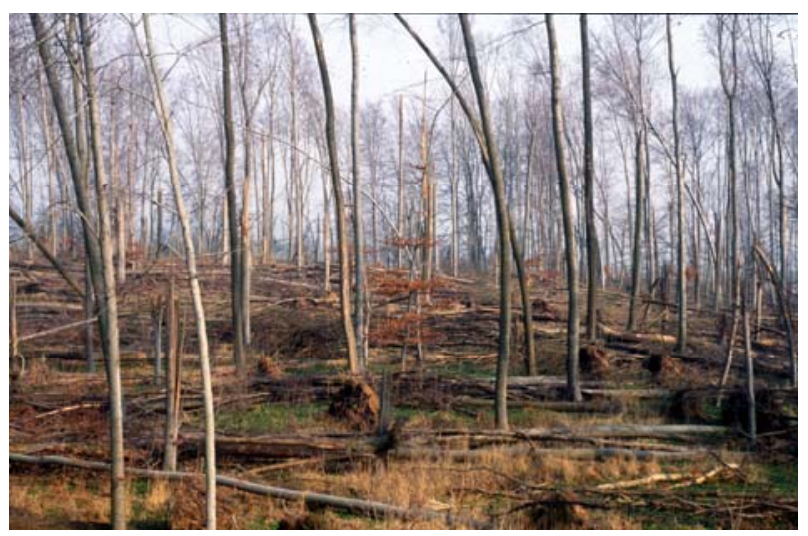

Januar 2000

März 2000

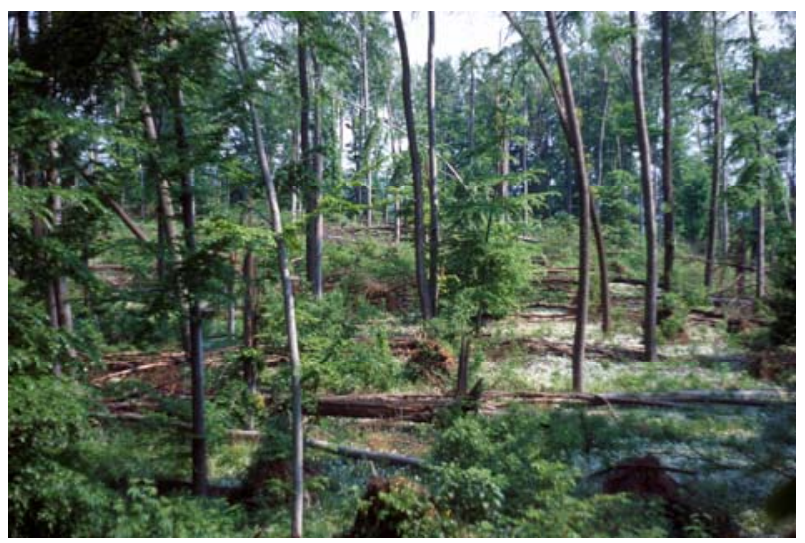

Mai 2000

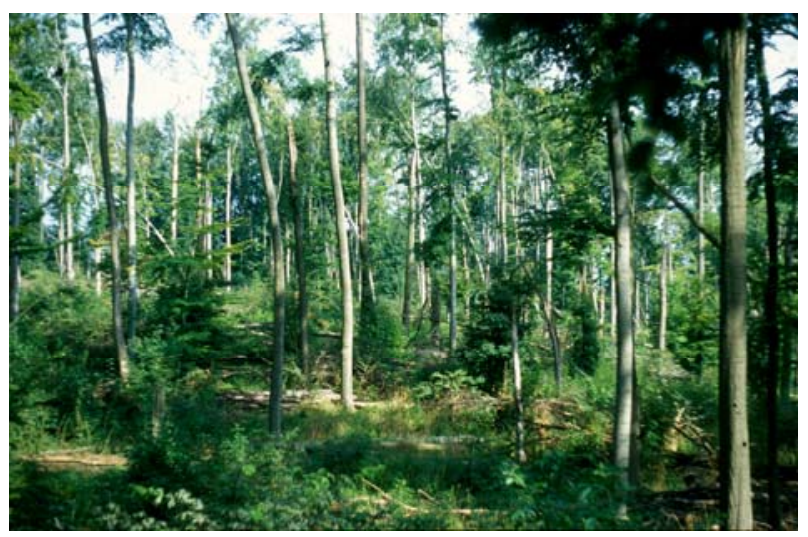

August 2000

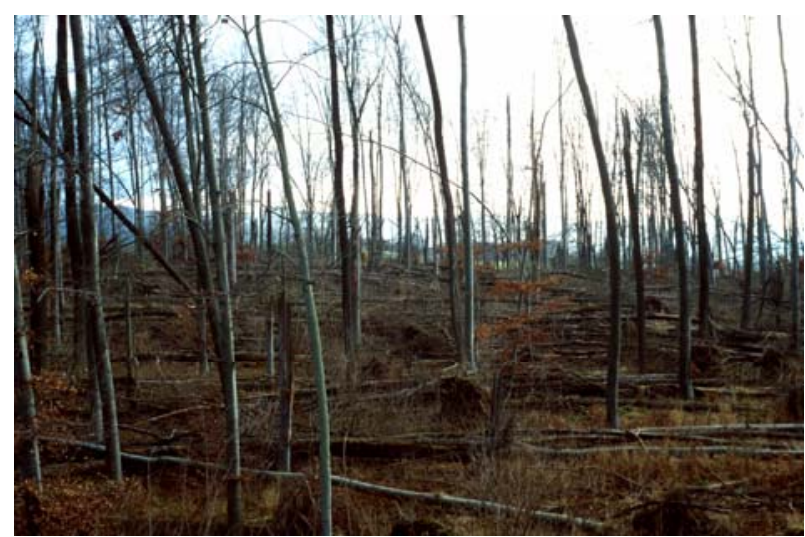




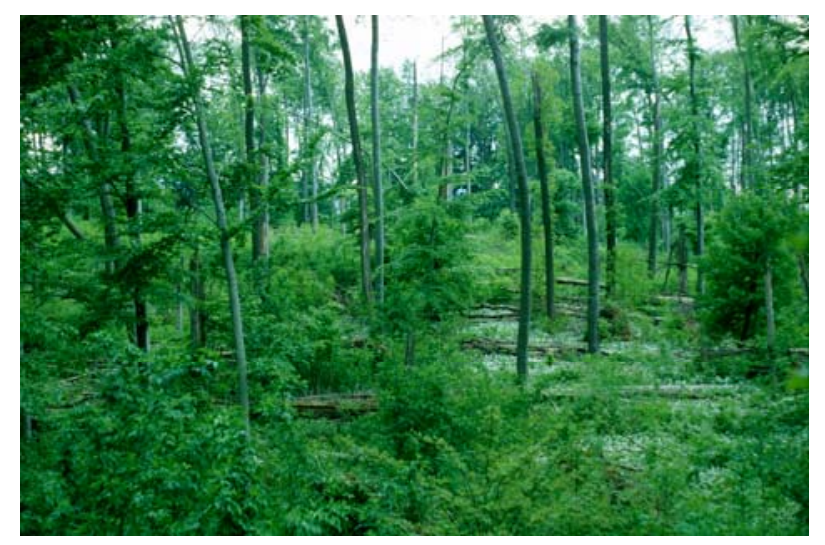

Abb. 61 b:

Fotoserie:

Hainholz-KF1

von Nordwesten

Mai 2001

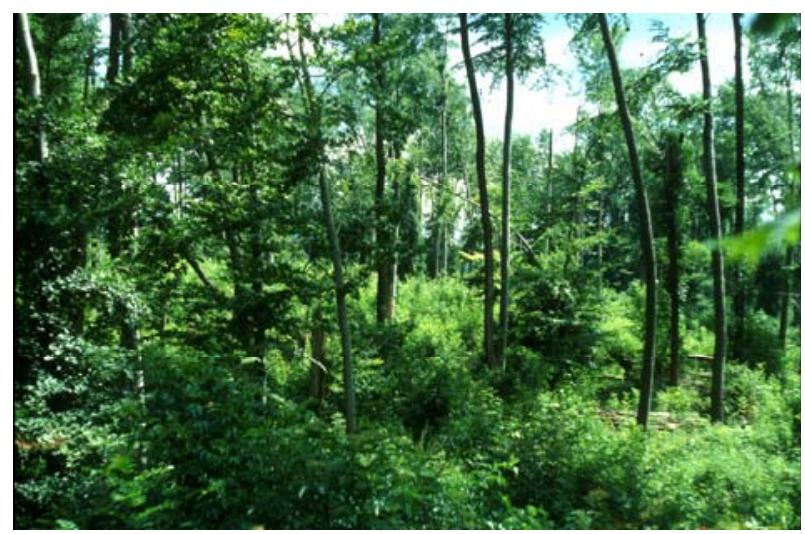

Juli 2001

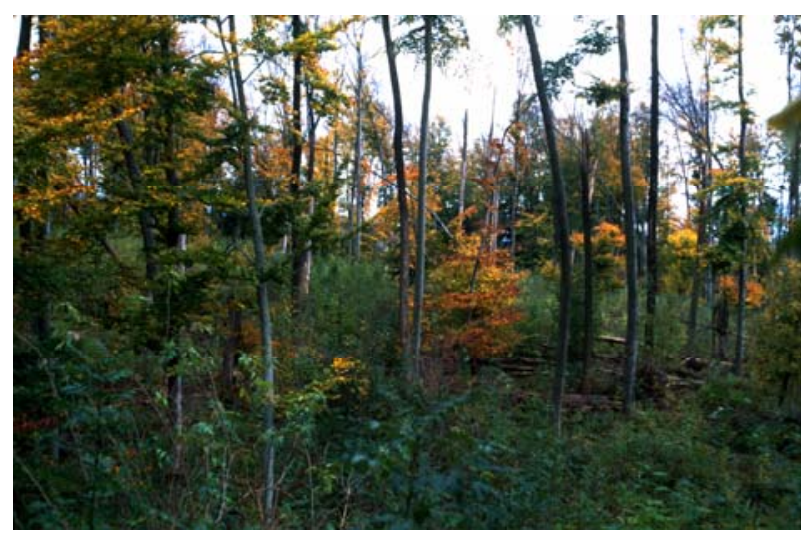

Oktober 2001

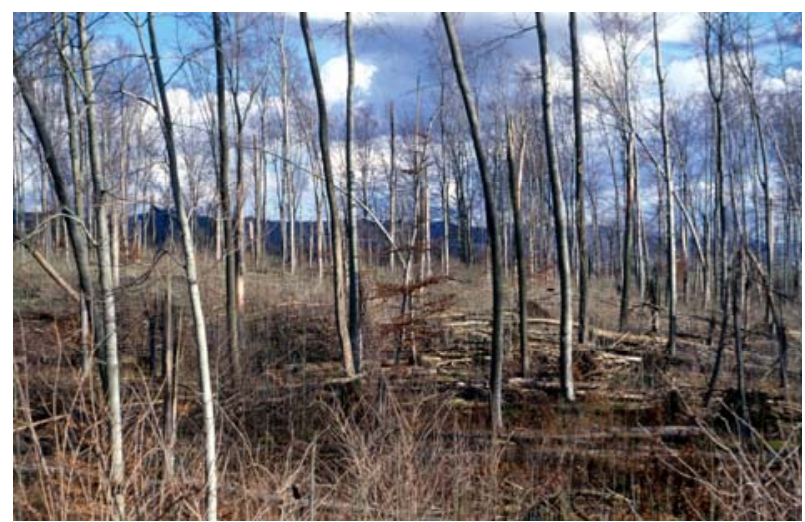

Februar 2002

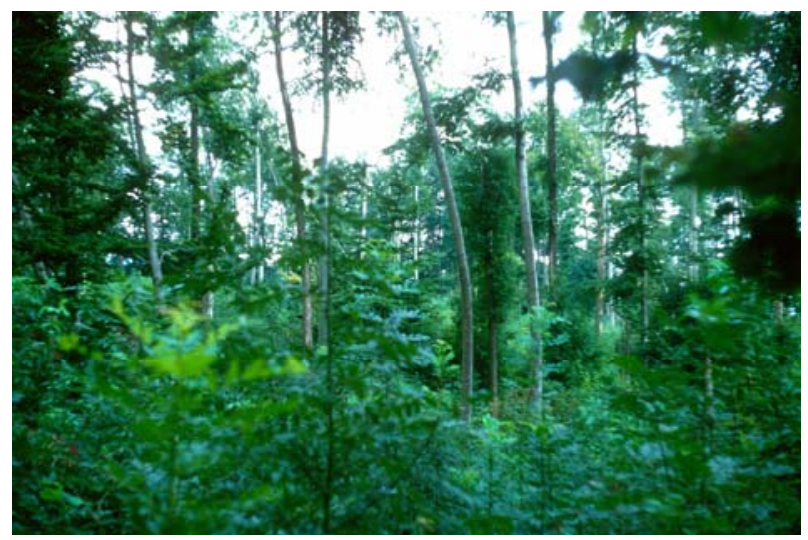


Von den strukturellen Parametern differenzieren vor allem die Artenzahlen und die Deckungssummen nach dem Standort (Tab. 16; Kap. 4.2). Bei den vegetationsökologischen Parametern gehören bei qualitativer Betrachtung nach den Zeigerwerten der Stickstoffhaushalt, die Lichtverhältnisse sowie die Bodenreaktion zu den entscheidenden Standortsfaktoren, nicht aber der Wasserhaushalt. Mit den Wuchsformen, dem vegetativen Ausbreitungsvermögen und der Zusammensetzung der Baumartenverjüngung (Kap. 4.5) sind weitere Faktoren zu nennen, die funktionale Aspekte der untersuchten Wälder und deren Standorte im Sukzessionsverlauf kennzeichnen. Hingegen tragen Hemerobiestufe, RAUNKIAER'scher Lebensformtyp, Samenbank- und Ausbreitungstyp nicht, die Einteilung nach pflanzensoziologischen Klassen nur schwach zu einer Standortsdifferenzierung bei (Kap. 4.3). Bei quantitativer Betrachtung dagegen erlauben mehr oder weniger alle in Tab. 16 bzw. in Kap. 4.3 aufgeführten Parameter eine Standortsdifferenzierung. Dabei sind die einzelnen Gebiete unterschiedlich stark betroffen, so dass in einigen Fällen jedes UG ein fast individuelles Bild aufweist. Meistens kann aber relativ klar zwischen basenreichen und basenarmen Standorten unterschieden werden. Teilweise heben sich auch nur die beiden tiefgründigen Dolomit-Standorte oder der flachgründige Gips/Dolomit-Standort von den übrigen Varianten ab. Eine genauere Gegenüberstellung ist Tab. 16 zu entnehmen.

Tab. 16: Beitrag einzelner struktureller und vegetationsökologischer Parameter zur Standortsdifferenzierung nach großflächigem Sturmwurf; quantitative Betrachtung unter Berücksichtigung der Deckungsgrade; alle verbalen Angaben (z.B. „gering“ oder „häufig“) sind immer relativ, d.h. in Bezug zum Vergleichsstandort zu verstehen.

\begin{tabular}{|c|c|c|}
\hline & basenreiche Standorte & basenarme Standorte \\
\hline Artenzahl & $\begin{array}{l}\text { artenreich (gilt nicht für die relativ } \\
\text { carbonatarme Hainholz-KF1) }\end{array}$ & artenarm \\
\hline $\begin{array}{l}\text { Deckungssummen } \\
\text { Strauchschicht }\end{array}$ & $\begin{array}{l}\text { Strauchschicht deckt im vierten } \\
\text { Sukzessionsjahr noch geringer als } \\
\text { Feldschicht (auf flachgründigem Gips- } \\
\text { Standort Strauchschicht wesentlich } \\
\text { geringer deckend als auf tiefgründigem } \\
\text { Dolomit-Standort) }\end{array}$ & $\begin{array}{l}\text { Strauchschicht deckt im vierten } \\
\text { Sukzessionsjahr genauso stark oder stärker } \\
\text { als Feldschicht }\end{array}$ \\
\hline $\begin{array}{l}\text { Deckungssummen } \\
\text { Feldschicht }\end{array}$ & $\begin{array}{l}\text { hochdeckend (gilt nicht für flachgründigen } \\
\text { Gips-Standort) }\end{array}$ & geringdeckend \\
\hline $\begin{array}{l}\text { maximale } \\
\text { Wuchshöhen der } \\
\text { Vegetations- } \\
\text { schichten }\end{array}$ & $\begin{array}{l}\text { Strauchschicht: kein Beitrag (Ausnahme: } \\
\text { geringe Wuchshöhen auf flachgründigem } \\
\text { Gips-Standort); } \\
\text { Feldschicht: (tendenziell) hoch }\end{array}$ & $\begin{array}{l}\text { Strauchschicht: kein Beitrag } \\
\text { Feldschicht: (tendenziell) gering }\end{array}$ \\
\hline $\begin{array}{l}\text { \%-Anteil von } \\
\text { Feldschichtarten mit } \\
>1 \% \text { mittlerem DG } \\
\text { an Gesamtartenzahl }\end{array}$ & kein Beitrag & kein Beitrag \\
\hline $\begin{array}{l}\text { Strauchschicht- } \\
\text { Evenness }\end{array}$ & kein Beitrag & $\begin{array}{l}\text { kein Beitrag } \\
\text { (Ausnahme: Stagnation in der vermutlich } \\
\text { schon länger sehr Strauchschicht-reichen } \\
\text { Rotenberg-Abt. 1228) }\end{array}$ \\
\hline
\end{tabular}


Fortsetzung von Tab. 16.

\begin{tabular}{|c|c|c|}
\hline & basenreiche Standorte & basenarme Standorte \\
\hline $\begin{array}{l}\text { Feldschicht- } \\
\text { Evenness }\end{array}$ & $\begin{array}{l}\text { kein Beitrag } \\
\text { (Ausnahme: fortlaufende Erhöhung auf } \\
\text { ehemals dünn besiedeltem Gips-Standort) }\end{array}$ & kein Beitrag \\
\hline $\begin{array}{l}\text { qualitative } \\
\text { floristische } \\
\text { Ähnlichkeit } \\
\text { (zwischen zweitem } \\
\text { und viertem } \\
\text { Sukzessionsjahr) }\end{array}$ & $\begin{array}{l}\text { standortabhängig; } \\
\text { (innerhalb der belassenen Varianten } \\
\text { Carici-Fagetum mit geringerer Ähnlichkeit } \\
\text { als Hordelymo-Fagetum) }\end{array}$ & nicht standortabhängig \\
\hline $\begin{array}{l}\text { quantitative } \\
\text { floristische } \\
\text { Ähnlichkeit } \\
\text { (zwischen zweitem } \\
\text { und viertem } \\
\text { Sukzessionsjahr) } \\
\end{array}$ & $\begin{array}{l}\text { kein Beitrag } \\
\text { (innerhalb der belassenen Varianten } \\
\text { Carici-Fagetum mit geringerer Ähnlichkeit } \\
\text { als Hordelymo-Fagetum) }\end{array}$ & $\begin{array}{l}\text { kein Beitrag } \\
\text { (im Luzulo-Fagetum geringere Ähnlichkeit } \\
\text { als im Galio odorati-Fagetum) }\end{array}$ \\
\hline Stickstoffzeigerwert & $\begin{array}{l}\text { Stickstoffzeiger stark vertreten (tw. } \\
\text { dominierend), Mäßigstickstoffzeiger } \\
\text { subdominant }\end{array}$ & $\begin{array}{l}\text { Stickstoffzeiger schwach vertreten, } \\
\text { Mäßigstickstoffzeiger dominant }\end{array}$ \\
\hline Feuchtezeigerwert & $\begin{array}{l}\text { Frischezeiger dominieren konstant, } \\
\text { Feuchtezeiger schwach vertreten (auf } \\
\text { Dolomit abnehmend) }\end{array}$ & $\begin{array}{l}\text { Frischezeiger dominieren nur zu } \\
\text { Sukzessionsbeginn, Feuchtezeiger stark } \\
\text { vertreten (konstant bis zunehmend) }\end{array}$ \\
\hline Reaktionszeigerwert & $\begin{array}{l}\text { auf Dolomit: Basenzeiger dominieren (auf } \\
\text { Gips indifferente Arten), Mäßigsäurezeiger } \\
\text { schwach vertreten, kaum Säurezeiger }\end{array}$ & $\begin{array}{l}\text { Basenzeiger sehr schwach vertreten, } \\
\text { Mäßigsäurezeiger stark vertreten, aber } \\
\text { nicht dominant, Säurezeiger } \\
\text { unterschiedlich stark vertreten }\end{array}$ \\
\hline Lichtzeigerwert & $\begin{array}{l}\text { Lichtzeiger schwach oder subdominant } \\
\text { vertreten }\end{array}$ & $\begin{array}{l}\text { Lichtzeiger stark vertreten und ab dem } \\
\text { vierten Sukzessionsjahr dominierend }\end{array}$ \\
\hline $\begin{array}{l}\text { Pflanzen- } \\
\text { soziologische } \\
\text { Zuordnung }\end{array}$ & $\begin{array}{l}\text { Waldarten dominant (gilt nicht für Gips- } \\
\text { Standort: dort sehr starke Ruderalstadien), } \\
\text { Schlagflurarten untergeordnet, } \\
\text { Grünlandarten schwach vertreten }\end{array}$ & $\begin{array}{l}\text { Waldarten untergeordnet, Schlagflurarten } \\
\text { dominant, Grünlandarten stark vertreten }\end{array}$ \\
\hline Wuchsformen & $\begin{array}{l}\text { hoher Anteil an Krautigen (dominierend) } \\
\text { und Baumartenverjüngung, niedriger } \\
\text { Anteil an Sträuchern und Grasartigen, } \\
\text { kaum Farne }\end{array}$ & $\begin{array}{l}\text { niedriger Anteil an Krautigen und } \\
\text { Baumartenverjüngung, hoher Anteil an } \\
\text { Sträuchern (dominierend), Grasartigen und } \\
\text { Farnen }\end{array}$ \\
\hline $\begin{array}{l}\text { Lebensformtyp } \\
\text { (RAUNKIAER) }\end{array}$ & $\begin{array}{l}\text { auf Dolomit: hoher Anteil an Geophyten } \\
\text { und Chamaephyten, aber kaum } \\
\text { Therophyten (auf Gips: geringer } \\
\text { Geophyten- und Chamaephyten-, aber } \\
\text { hoher Therophytenanteil), baumartenreich } \\
\text { und strauchartenarm }\end{array}$ & $\begin{array}{l}\text { geringer Anteil an Geophyten (gilt nicht } \\
\text { für Königsbuche), kaum Chamaephyten, } \\
\text { hoher (aber sinkender) Anteil an } \\
\text { Therophyten, baumartenarm und } \\
\text { strauchartenreich (Sträucher dominierend) }\end{array}$ \\
\hline Ausbreitungstyp & $\begin{array}{l}\text { geringer Anteil an endozoochoren und } \\
\text { bsds. auf Dolomit hoher Anteil an } \\
\text { anemochoren Arten, Myrmekochorie stark } \\
\text { vertreten }\end{array}$ & $\begin{array}{l}\text { hoher Anteil an endozoochoren und } \\
\text { geringer Anteil an anemochoren Arten, } \\
\text { Myrmekochorie nur schwach und nur im } \\
\text { Luzulo-Fagetum vertreten }\end{array}$ \\
\hline $\begin{array}{l}\text { vegetatives } \\
\text { Ausbreitungs- } \\
\text { vermögen }\end{array}$ & $\begin{array}{l}\text { Arten mit vegetativem } \\
\text { Ausbreitungsvermögen schwach vertreten, } \\
\text { auf Dolomit noch dominierend; auf Gips } \\
\text { untergeordnet, aber zunehmend }\end{array}$ & $\begin{array}{l}\text { Arten mit vegetativem } \\
\text { Ausbreitungsvermögen generell } \\
\text { dominierend und weiter zunehmend }\end{array}$ \\
\hline Samenbanktyp & $\begin{array}{l}\text { geringer und schwach zunehmender Anteil } \\
\text { von Arten mit langfristiger Samenbank (>5 } \\
\text { J.); hoher und fast konstanter Anteil von } \\
\text { Arten mit kürzerfristiger (bsds. temporärer) } \\
\text { Samenbank }\end{array}$ & $\begin{array}{l}\text { hoher und meist zunehmenderAnteil von } \\
\text { Arten mit langfristiger Samenbank (>5 J.); } \\
\text { geringer und weiter abnehmender Anteil } \\
\text { von Arten mit kürzerfristiger (bsds. } \\
\text { temporärer) Samenbank }\end{array}$ \\
\hline
\end{tabular}


Fortsetzung von Tab. 16.

\begin{tabular}{|c|c|c|}
\hline & basenreiche Standorte & basenarme Standorte \\
\hline Hemerobietyp & $\begin{array}{l}\text { auf Dolomit: Dominanz oligohemerober } \\
\text { Arten; auf Gips: in etwa Parität zwischen } \\
\text { oligo- und mesohemeroben Arten }\end{array}$ & $\begin{array}{l}\text { generell Dominanz der oligohemeroben } \\
\text { Arten (im Galio-Fagetum stärker als im } \\
\text { Luzulo-Fagetum) }\end{array}$ \\
\hline $\begin{array}{l}\text { Baumarten- } \\
\text { verjüngung }\end{array}$ & $\begin{array}{l}\text { verjüngungsreich; } \\
\text { auf Dolomit: Eschen-Dominanz mit } \\
\text { unterständiger Buche; auf Gips: mehrere } \\
\text { Baumarten in etwa gleichen Anteilen; } \\
\text { Pionierbaumarten generell wenig präsent }\end{array}$ & $\begin{array}{l}\text { verjüngungsarm; } \\
\text { Buchen-Dominanz mit unterschiedlich } \\
\text { starker Beimischung anderer Baumarten; } \\
\text { nur im Luzulo-Fagetum stärkere, aber nicht } \\
\text { dominante Präsenz von Pionierbaumarten }\end{array}$ \\
\hline
\end{tabular}

Die recht deutliche Standortsdifferenzierung, die durch die Mehrzahl der hier aufgeführten Parameter ermöglicht wird, spricht für deren Eignung als Indikatoren und beschreibende Instrumente von Sukzessionsprozessen. Allerdings handelt es sich bei ihnen um strukturelle oder ökologisch-funktionale Ableitungen, die den Standort nur indirekt widerspiegeln. Deshalb sollen in den nachfolgenden Kapiteln (5.3.2, 5.3.3, 5.3.4, 5.3.5) diejenigen Standortsfaktoren diskutiert werden, die in der Windwurfsukzession als wesentlich erachtet werden und bei denen man die größten Veränderungen nach der Störung vermuten sollte (Licht, Nährstoff- und Wasserhaushalt, Bodenreaktion - siehe Kap. 1.2 und 1.3). Neben den abgeleiteten ELLENBERG-Zeigerwerten stehen hierfür teilweise auch direkt erhobene standortsökologische Messungen zur Verfügung (Kap. 4.1; Tab. 1).

$\mathrm{Zu}$ den in Tab. 16 aufgeführten strukturellen Parametern sei erwähnt, dass die Deckung der Bodenvegetation auf bestimmten Standorten im Harzvorland (Königsbuche-KF2, HainholzKF2) nicht schon im ersten Jahr nach der Auflichtung zunahm, sondern relativ sprunghaft erst ab dem zweiten (SCHMIDT 2002a; KOMPA \& SCHMIDT 2003). Dieser Effekt war auch aus anderen Windwurfgebieten bereits beschrieben worden (z.B. mehrere Untersuchungen zitiert in ScHÖLCH 1998). Nach diesen Studien kann innerhalb von etwa vier Jahren ein fast vollständiger Vegetationsschluss auf dem Waldboden erreicht werden. Ganz ähnlich stellte LUGINBÜHL (2000) für das Hainholz fest, dass nicht alle Untersuchungsparameter gleichschnell eine Reaktion auf die Auflichtung zeigten. Im ersten Jahr nach dem Sturm (d.h. 1998) reagierten Artenzahl und quantitative floristische Ähnlichkeit, dagegen erst im zweiten Jahr die Feld- und Strauchschichtdeckungen sowie die qualitative floristische Ähnlichkeit.

\subsubsection{Lichtverhältnisse}

Unbestritten ist das Licht ein entscheidender Faktor für die Entwicklung vieler Waldbodenpflanzen (z.B. EBER 1972; SchMidT 1988; MrotzeK et al. 1996). Es gibt aber auch Studien, die den Einfluss der Lichtverhältnisse auf einzelne Artengruppen oder Vegetationsschichten relativieren. So erwies sich der Lichtfaktor bei einem Vergleich ungestörter Buchen-Waldgesellschaften Süddeutschlands (LEUSCHNER 1999) von eher untergeordneter Bedeutung speziell für die Krautschichtdeckung. BRUNET et al. (1996) argumentieren, dass die typische Waldbodenflora an das gelegentliche Auftreten von 
Kronenlücken adaptiert ist und deshalb zumindest eine naturnahe Waldbewirtschaftung trotz unvermeidlicher Auflichtung nur wenig Einfluss auf diese Artengruppe hat. In experimentellen Lochhieben in einem Hordelymo-Fagetum auf Kalk fand ScHMIDT (1997, 2002c) eine geringe Abhängigkeit der Bodenvegetation von den Lichtverhältnissen (kaum Unterschiede zwischen Lücke und Bestand), bei der Baumartenverjüngung dagegen eine sehr große. Allerdings herrschten in der Krautschicht der dortigen Schlaglücken schon nach wenigen Sukzessionsjahren wieder ähnliche Lichtverhältnisse wie im geschlossenen Bestand. Durch die vorliegende Arbeit wird diese Beobachtung auch für die untersuchten Windwurfgebiete gut nachvollziehbar. Eines der bemerkenswertesten Ergebnisse ist ja, dass auf den im vierten Sukzessionsjahr von Himbeere oder Esche beherrschten Windwurfflächen waldtypische Schattenzeiger (L1-3) im Deckungsgrad nur relativ und teilweise sehr undeutlich abnehmen (Abb. 29), absolut gesehen aber stagnieren oder sogar zunehmen (Tab. A22, A23). Der Beschattungseffekt der üppigen Strauchschicht bedingt zwar eine Stagnation in der Feldschicht, die Schattenzeiger sind davon aber offensichtlich kaum betroffen (Abb. 20, Tab. A22, A23). Selbst auf dem Strauchschicht-armen Bollerkopf blieben die auch anfangs relativ geringen Schattenzeiger-Anteile bislang stabil (Abb. 29).

Die aus Kap. 4.3.1 ersichtliche Standortsabhängigkeit der Lichtzeiger (L7-9) in der Windwurfsukzession, nach der die basenarmen Flächen stärker von dieser Artengruppe beherrscht werden als die basenreichen, ist auf die Zusammenfassung von Feld- und Strauchschicht in der Auswertung zurückzuführen. Dadurch machen sich die hohen Deckungsanteile der Himbeere (L7) bemerkbar. Nach LUGINBÜHL (2000) nahm im Hainholz nach dem 1997er Sturm die Anzahl der Lichtzeiger in der Krautschicht überpropotional zur allgemeinen Artenzahlerhöhung zu, wobei das Carici-Fagetum eine höhere Anzahl an Lichtzeigern als das Hordelymo-Fagetum aufwies. Die vorliegenden Untersuchungen bestätigen diese Artenzahlunterschiede nur beim Vergleich belassener Flächen. Auf dem geräumten Nüllberg (ebenfalls Hordelymo-Fagetum) lassen sich etwa gleichviele Lichtzeiger mit ähnlichen Abundanzanteilen finden wie im Carici-Fagetum auf dem Bollerkopf (Abb. 29; Tab. 3, A22, A23).

\subsubsection{Wasserhaushalt}

Der windwurf- oder kahlschlagbedingte Wegfall der Transpiration und Interzeption der ehemaligen Baumschicht bedingt normalerweise eine zunehmende Bodenvernässung und vermehrtes Auftreten von Feuchte- und Nässezeigern. Dies kommt besonders auf stauenden, weniger auf gut drainierten Böden zum Tragen (FISCHER 1992; FISCHER et al. 1998). So stieg z.B. nach den Untersuchungen von POLOMSKI \& KUHN (2000) der Bodenwassergehalt in Buchen-Wäldern auf basenreichen Böden nach Kahlschlag deutlich an, ging durch die anschließend einsetzende Krautschichtentwicklung langsam zurück und blieb dennoch oberhalb der des Bestandes. Auf Windwurfflächen in Rheinland-Pfalz wurden vergleyte 
Böden häufig überstaut, da die Pionierstrauchvegetation anfangs nicht alles überschüssige Bodenwasser aufnehmen konnte (SCHÜLER 1999). Daneben entwickelten sich dort dichte Grasdecken, die v.a. im Sommer eine enorme Wasserkonkurrenz bedingten und die Wiederbewaldung verzögerten. Auch im Harzvorland haben die Grasartigen und die Pioniersträucher mit ihren relativ hohen Abundanzanteilen v.a. im Buntsandstein offensichtlich eine ganz ähnliche Wirkung (Kap. 4.3.6).

Ebenfalls auf den stauenden Buntsandstein-Böden zeigten erwartungsgemäß die Feuchtezeiger (F7-9) ihren Verbreitungsschwerpunkt (Kap. 4.3.2; Tab. A22, A23). Erstaunlich war jedoch das kurzzeitige und geringdeckende Auftreten von Feuchtezeigern selbst auf den relativ trockenen und gut drainierten Gips-Standorten wie der Hainholz-KF2 (betraf Cirsium palustre, Stachys sylvatica, Alopecurus geniculatus, Deschampsia cespitosa, Epilobium hirsutum, E. parviflorum, Eupatorium cannabinum, Ranunculus repens, Impatiens noli-tangere, Poa trivialis).

Die große Bedeutung des Wasserfaktors speziell für den Keimerfolg vieler Baumarten wird durch eine Vielzahl von Studien belegt. Mit Untersuchungen nach einer Buchen-Mast ermittelte z.B. BurSCHEL (1961), dass die Trockenheit in den Monaten Februar bis Mai der hauptsächliche Mortalitätsfaktor für auflaufende Buchen-Keimlinge sein kann. Ebenso ist bekannt, dass lückige Bodenvegetation und Moos aufgrund des abgemilderten Mikroklimas hervorragende Etablierungsplätze darstellen (BURSCHEL et al. 1964; HOMANN \& ENGELS 1991; LÄSSIG et al. 1995a; HETZEL \& REIF 1998; WILMANNS et al. 1998).

Auch nach der Keimung bleiben besonders Buchen-Jungpflanzen u.a. für Wasserstress sehr anfällig. Auf größeren Kahlflächen mittlerer Standorte fanden z.B. BOLTE \& ROLOFF (1992) im Umkreis von Buchen-Überhältern eine erhebliche Wasser- und Nährstoffkonkurrenz für die nachwachsende Verjüngung (gekoppelt mit erhöhter Bodenacidität), was zu einer beträchtlichen Reduktion des Höhenwachstums der jungen Buchen führte. Solche wachstumsreduzierenden Effekte ließen sich im Harzvorland auch sehr gut an der Laubbaumverjüngung im Umfeld einzelner Eschen-Überhälter in der Hainholz-KF1 beobachten. Mittels Blattwasserpotenzialmessungen an Eschen- und Buchen-Naturverjüngung in Bestandeslücken konnte HoRN (2002) den Austrocknungseffekt von Altbäumen auf den Boden nachweisen. Dabei ergaben sich speziell bei jungen Buchen zunehmend negativer werdende pre-dawn-Potenziale entlang eines Gradienten von der Lückenmitte in den Bestand hinein. Möglicherweise verursachen die ausgedehnten Pionierstrauchfluren auf den windgeworfenen Buntsandstein-Standorten im Harzvorland einen ähnlichen oder sogar noch größeren Konkurrenzeffekt um die Ressource Wasser wie stehende Altbäume. Neben der geringeren Anzahl lebender Samenbäume auf Windwurfflächen könnte dies ein weiterer Erklärungsbeitrag für die abnehmende Buchen-Verjüngungsdichte mit zunehmender Störungsintensität entlang des Gradienten Bestand - Lücke - Flächenwurf sein (siehe Kap. 4.5.2). 


\subsubsection{Bodenreaktion}

Nach LeUSCHNER (1999) sind bodensaure Waldstandorte in Deutschland etwa 3-4 mal so häufig anzutreffen wie basenreich-alkalische. Basensättigung, pH-Wert und austauschbare Kationen (Ca, K, Mg) differenzieren besser zwischen nährstoffreichen und -armen Waldgesellschaften als der Stickstoff. Wenn man den Basenreichtum des Standortes als einen wesentlichen Faktor der Artenzahlentwicklung in Buchen-Wäldern annimmt (u.a. BRUNET et al. 1996; LEUSCHNER 1999; SCHMIDT 1999a+b), dann lässt sich die im Harzvorland gefundene standörtliche Abstufung im Artenzahlniveau zum Teil bereits damit erklären. Die beiden carbonatreicheren Zechstein-Standorte (Hainholz-KF2, Nüllberg) waren ja deutlich artenreicher als die übrigen, carbonatärmeren bzw. -freien Varianten (Tab. 1). Ebenso verzeichneten diese beiden UG die rapidesten Artenzahlanstiege von 1999 zu 2001, welche sich hauptsächlich in der Krautschicht bemerkbar machten, während die Strauchschicht diesbezüglich zwischen allen sieben Varianten nur wenig differierte (Abb. 17). Vermutlich auch deshalb liegt innerhalb der Buntsandstein-Standorte der 1991 gekalkte Krücker trotz seines natürlicherweise artenärmeren Luzulo-Fagetum leicht über dem Artenzahlniveau des Rotenberges (Galio odorati-Fagetum). Nach den Untersuchungen von ScHMIDT (2002b) führen die bundesweit üblichen Kompensationskalkungen zu erhöhten Artenzahlen vor allem durch basen- und stickstoffzeigende Nichtwaldarten. In verschiedenen süddeutschen Fageten stiegen Artenzahl und Deckungsgrad der Feldschicht mit zunehmender Basensättigung und pH-Wert an, nahmen aber u.a. mit zunehmender Tiefgründigkeit des Bodens ab (LEUSCHNER 1999). Auch im belassenen Windwurf des Harzvorlandes ist z.B. das flachgründige und sehr carbonatreiche Carici-Fagetum (mittlerer $\mathrm{pH}\left[\mathrm{H}_{2} \mathrm{O}\right]-$ Wert: 7,3) artenreicher als das tiefergründige und carbonatarme Hordelymo-Fagetum ( $\mathrm{pH}\left[\mathrm{H}_{2} \mathrm{O}\right]$-Wert: 6,2 - siehe Tab. 1).

Desweiteren existieren große Unterschiede in der Verjüngung der SchlusswaldLaubbaumarten zwischen basenarmen, basenreich-flachgründigen und basenreichtiefgründigen Standorten, die sich in einer starken Zunahme der Verjüngungsdichte in eben dieser Reihenfolge ausdrücken (Abb. 43, 44). Eine ähnliche Verjüngungssituation wie im Hordelymo-Fagetum auf Dolomit im Hainholz wurde auch auf Kalk auf der Schwäbischen Alb (Hetzel \& ReIF 1996, 1998) oder auf Basalt im Vogelsberg (Willig 1994, 2002b; KEITEL 1998, 1999) gefunden, worauf auch in Kap. 5.8.1 noch eingegangen wird. In gut wasser- und nährstoffversorgten Geländepartien dominieren dort Esche, Buche und BergAhorn in der Naturverjüngung. In der Regel ist auf basenreichen Buchen-Waldstandorten deutscher Mittelgebirge und Hügelländer mit einer sehr schnellen Wiederbewaldung nach Windwurf im Sinne der Klimaxgesellschaft zu rechnen, während basenarme Standorte zunächst von Pionierstrauchfluren oder Pionierwäldern (bei entsprechender Vorbestockung auch von Fichte oder Kiefer) dominiert werden. Bei einem Vergleich von basenarmen, mittleren und basenreichen Standorten in Fichten-Sturmwurfgebieten Baden-Württembergs (SAYER \& REIF 1998) zeigten sich trotz der andersartigen Vorbestockung viele Parallelen zum Harzvorland. Dabei ließ sich ebenso wie hier eine deutliche Gliederung entlang des 
Gradienten der Basenversorgung finden, die sich in Artenzahlniveau, Deckungsgradentwicklung, Wuchshöhe der Strauch- und Baumschicht sowie im floristischen Arteninventar ausdrückte (basenreiche Standorte am artenreichsten, am schnellsten und stärksten deckend, mit den wüchsigsten Gehölzen, mit vielen überdauernden Waldarten des Vorbestandes). Die Naturverjüngung wurde dort innerhalb der ersten 5 Jahre nach dem Sturm zwar überall von Fichte dominiert, aber zusätzlich kamen auf sauer-armen Standorten noch Tanne, Kiefer und Vogelbeere hinzu, auf mittleren Birke und Salweide und auf basenreichen Buche, Esche und Berg-Ahorn (Tab. 21). Auf basenreichen Standorten mit nicht standortsgerechter Vorbestockung (z.B. Fichte) existiert also nach Windwurf eine Tendenz, nach der sich die zahlreich auflaufende Laubbaumverjüngung Terrain (zurück)erobert und eine Entwicklung hin zu Laubmischwäldern (z.B. potentiell natürliches Hordelymo-Fagetum) auch ohne forstliche Eingriffe sicher ist. Die zu schnelle Zersetzung der sauren Fichten-Streu auf Kalkböden und die zu schwache Mykorrhizierung der Fichten-Keimlinge werden als Begründung dafür genannt. Auf basenarmen Standorten trifft dies nicht zu (SCHMIDT-SCHÜTZ \& HuSS 1996; HETZEL \& REIF 1998; SAYER \& REIF 1998).

\subsubsection{Stickstoffversorgung}

Der nach Windwurf normalerweise zu erwartende Mineralisierungsschub (z.B. MELLERT et al. 1998) ist hier nicht untersucht worden. Da kein Anstieg der Artenzahl- oder Abundanzanteile von stärkeren Stickstoffzeigern (N7-9) beobachtet wurde (Kap. 4.3.4), war eine verstärkte N-Mineralisation mittels dieses Parameters auch nicht nachweisbar. Auch LUGINBÜHL (2000) ermittelte nach dem 1997er Sturm im Hainholz, dass die Mineralstickstoffkonzentration des Bodens nicht mit der Vegetationsentwicklung oder der Störungsintensität korreliert und ein Mineralisierungsschub im Jahr 1999 bereits nicht mehr nachweisbar war. Dennoch ist bekannt, dass die sich nach Sturmwurf entwickelnde Vegetation eine wichtige Zwischenspeicherfunktion für Nährstoffe erfüllt. Bei Lochhiebsexperimenten in einem bodensauren Buchen-Wald im Solling (BAUHUS 1994; BARTSCH et al. 1999) reduzierten sich anfangs hohe Stoffausträge u.a. an Nitrat, nachdem sich eine hochdeckende Feldschicht entwickelte. Nach MELLERT et al. (1998) und BARTSCH et al. (1999) ist schon ab 40-50\% Deckungsgrad der Waldbodenvegetation mit diesem Effekt zu rechnen. Etwa 10 Jahre nach der Anlage experimenteller Lochhiebe in einem HordelymoFagetum auf Kalk (ScHMIDT 1997, 2002c) ließ sich nur noch wenig freies Nitrat in der Bodenlösung feststellen, wobei der Stickstoffkreislauf viel stärker durch die rasch emporwachsende Baumartenverjüngung als durch die Bodenvegetation beeinflusst wurde. In den hiesigen Windwurfgebieten spielt es offenbar keine Rolle, ob die Bodenvegetation von Baumartenverjüngung, Sträuchern oder Krautigen geprägt ist bzw. ob es sich dabei um ausgesprochene Stickstoffzeiger handelt. Sowohl die Mäßigstickstoff- als auch die Stickstoffzeiger erhöhten ihre absoluten Deckungssummen in der Strauch- und Feldschicht in 
etwa demselben Maße, was sich an den annähernd stabil bleibenden Abundanzspektren ablesen lässt (Kap. 4.3.4). So ist z.B. Rubus idaeus als die dominanteste Art im Sukzessionsgeschehen vieler Untersuchungsflächen im Harzvorland nach ELLENBERG et al. (1992) mit einem N-Zeigerwert von 6 auch eher als Mäßigstickstoffzeiger einzuschätzen. Ähnliches gilt für die allermeisten Brombeer-Unterarten (Rubus fruticosus agg.) mit NZeigerwerten zwischen 4 und 6. Auffällig ist jedoch, dass auf basenarmen und basenreichen Standorten jeweils unterschiedliche Wuchsformengruppen von dem Nährstoffüberschuss profitieren (Kap. 4.3.6). Während im Buntsandstein hauptsächlich Pioniersträucher an Stoffproduktion und -kreislauf beteiligt sind, sind es im Zechstein vor allem die Baumartenverjüngung und/oder krautige Arten. Ob die in allen Untersuchungsvarianten leicht ansteigenden Artenzahlanteile von Stickstoffmangelzeigern (Tab. 6) bereits jetzt schon als erster (vager) Hinweis auf die Abschöpfung des Nährstoffüberschusses interpretiert werden können, ist fraglich.

Nach LuginBüHL (2000) nahm im Hainholz die Anzahl der Stickstoffzeiger nach dem Sturm etwa proportional zur allgemeinen Artenzahlerhöhung zu, was sich in den vorliegenden Untersuchungen ganz ähnlich durch die stabilen qualitativen Artengruppenspektren ausdrückt (Tab. 3). Allerdings fand LuginBÜHL (2000) im Carici-Fagetum höhere $\mathrm{N}_{\min }$-Gehalte des Bodens und eine höhere Anzahl an Stickstoffzeigern als im Hordelymo-Fagetum. Mit den hier gewonnenen Ergebnissen (Kap. 4.3.4, Tab. A22, A23) kann dies nur bezüglich der absoluten Artenzahlen, nicht jedoch bezüglich der absoluten und relativen Abundanzen bestätigt werden. Demnach sind Stickstoffzeiger im Hordelymo-Fagetum (Hainholz-KF1) quantitativ deutlich stärker verbreitet als im Carici-Fagetum (Hainholz-KF2). Das stimmt auch mit den Untersuchungen von LEUSCHNER (1999) in ungestörten Buchen-Waldgesellschaften überein, denen zufolge im trockenheitsbeeinflussten Carici-Fagetum signifikant niedrigere Nettomineralisationsraten zu finden waren als im Luzulo-, Galio odorati- und HordelymoFagetum, welche sich in der Höhe ihrer N-Nachlieferung kaum unterschieden.

Auch der Übergang von der annuellen zur perennen Phase der Vegetationsbesiedlung erfolgt offenbar standorts- bzw. nährstoffabhängig. Ein Primärsukzessions-Experiment (BORNKAMM 1987) durchlief auf nährstoffreicherem Lehm die verschiedenen Phasen schneller als auf nährstoffärmerem Sand. Die mit etwa 30\% über mindestens vier Sukzessionsjahre ungewöhnlich lange stabilen Abundanzanteile der Therophyten (Abb. 35) in der HainholzKF2 könnte man dementsprechend als Hinweis auf die allgemeine standörtliche Ungunst (nicht unbedingt nur der relativen Nährstoffarmut) dieser flachgründigen, mäßig trockenen Rendzinen bewerten. 


\subsection{Zum Einfluss der forstlichen Bewirtschaftung auf die Windwurfsukzession}

\subsection{1 Übersicht über differenzierende Untersuchungsparameter}

Im Folgenden sollen Untersuchungsparameter zusammenfassend diskutiert werden, die differenzierenden Charakter bezüglich der Bewirtschaftungsart vor und nach dem Sturmwurf haben. Vielfältige und nicht näher quantifizierbare Überlagerungen z.B. mit dem Standorteinfluss sind auch hier möglich und wahrscheinlich. In den Zechstein-Gebieten empfiehlt sich der Vergleich zwischen den standörtlich ähnlichen Flächen von Hainholz-KF1 (belassen, Naturwald) und Nüllberg (geräumt, Wirtschaftswald). Für die belassene HainholzKF2 (Carici-Fagetum) gibt es kein geräumtes standörtliches Pendant. In den BuntsandsteinGebieten werden Unterschiede besonders beim Vergleich der seit 30 Jahren unbewirtschafteten Königsbuche und den durchgehend bewirtschafteten Wäldern deutlich. Ein Vergleich innerhalb des Luzulo-Fagetum (belassener Krücker-Osthang vs. geräumtes Krücker-Plateau) lässt dagegen kaum Unterschiede zutage treten. Von einem nutzungsgeschichtlichen Vergleich der Zechstein-Standorte (Nüllberg, Hainholz) wird abgesehen, da der Zeitraum der Null-Nutzung im Hainholz noch sehr gering ist (Tab. 1).

Von den strukturellen Parametern tragen am ehesten die Artenzahlen, der Anteil von Arten mit $>1 \%$ mittlerer Deckung an der Gesamtartenzahl und teilweise auch die floristische Ähnlichkeit zu einer Differenzierung nach der Bewirtschaftungsart bei (Kap. 4.2). Was die vegetationsökologischen Parameter betrifft, so gibt es bei qualitativer, ungewichteter Betrachtung keine bewirtschaftungsdifferenzierenden Parameter. Bei quantitativer, gewichteter Betrachtung dagegen tragen die Baumartenverjüngung, der Licht-, Reaktionsund Stickstoffzeigerwert, die Einteilung nach pflanzensoziologischen Klassen, Wuchsformen, RAUNKIAER'schem Lebensformtyp sowie nach Samenbank-, Ausbreitungs-, und Hemerobietyp zu einer mehr oder weniger deutlichen Differenzierung nach aktueller Bewirtschaftungsart (Räumung/Belassung) bzw. langjähriger Nutzungsgeschichte bei (Kap. 4.3, 4.5). Eine vollständigere Gegenüberstellung dazu gibt Tab. 17.

Tab. 17: Beitrag einzelner struktureller und vegetationsökologischer Parameter zur Differenzierung entsprechend der Bewirtschaftungsgeschichte und/oder der aktuellen Behandlung nach großflächigem Sturmwurf; quantitative Betrachtung unter Berücksichtigung der Deckungsgrade; alle verbalen Angaben (z.B. „gering“ oder „häufig“) sind immer relativ, d.h. in Bezug zum Vergleichsstandort zu verstehen; $\mathrm{NW}=$ Naturwald, $\mathrm{WW}=$ Wirtschaftswald, Dol. = Dolomit, BS = Buntsandstein, ger. = geräumt, bel. = belassen.

\begin{tabular}{|c|c|c|}
\hline & $\begin{array}{l}\text { langjährige Bewirtschaftungsgeschichte } \\
\text { nur auf Buntsandstein: } \\
\text { Vergleich des Naturwaldes Königsbuche } \\
\text { (KF2) mit Wirtschaftswaldflächen }\end{array}$ & $\begin{array}{c}\text { Behandlung nach dem Sturm: } \\
\text { Belassung/Räumung } \\
\text { alle Standorte auf Buntsandstein und auf } \\
\text { Zechstein }\end{array}$ \\
\hline Artenzahl & NW: artenarm; WW: artenreich & bel.: artenarm; ger.: artenreich \\
\hline Deckungssummen & $\begin{array}{l}\text { kein Beitrag } \\
\text { (Ausnahme: in der Rotenberg-Abt. } 1228 \\
\text { kaum Anstieg der Strauchschicht- } \\
\text { Deckung) }\end{array}$ & kein Beitrag \\
\hline
\end{tabular}


Fortsetzung von Tab. 17

\begin{tabular}{|c|c|c|}
\hline & langjährige Bewirtschaftungsgeschichte & $\begin{array}{l}\text { Behandlung nach dem Sturm: } \\
\text { Belassung/Räumung }\end{array}$ \\
\hline $\begin{array}{l}\text { \%-Anteil von } \\
\text { Feldschichtarten mit } \\
>1 \% \text { mittlerem DG } \\
\text { an Gesamtartenzahl }\end{array}$ & kein Beitrag & $\begin{array}{l}\text { bel.: niedrig und gleichbleibend bis } \\
\text { ansteigend; ger.: hoch und gleichbleibend } \\
\text { bis sinkend }\end{array}$ \\
\hline Evenness & kein Beitrag & kein Beitrag \\
\hline $\begin{array}{l}\text { qualitative } \\
\text { floristische } \\
\text { Ähnlichkeit } \\
\text { (zwischen 2. u. } 4 . \\
\text { Sukzessionsjahr) } \\
\end{array}$ & kein Beitrag & $\begin{array}{l}\text { Dol. bel.: große Ähnlichkeit; Dol. ger.: } \\
\text { geringe Ähnlichkeit; } \\
\text { BS: kein Beitrag }\end{array}$ \\
\hline $\begin{array}{l}\text { quantitative } \\
\text { floristische } \\
\text { Ähnlichkeit } \\
\text { (zwischen 2.u.4. } \\
\text { Sukzessionsjahr) } \\
\end{array}$ & kein (deutlicher) Beitrag & $\begin{array}{l}\text { Dol. bel.: große Ähnlichkeit; Dol. ger.: } \\
\text { geringe Ähnlichkeit; BS: im Galio- } \\
\text { Fagetum geräumte Flächen mit größerer } \\
\text { Ähnlichkeit als belassene, im Luzulo- } \\
\text { Fagetum kein Beitrag } \\
\end{array}$ \\
\hline Stickstoffzeigerwert & $\begin{array}{l}\text { NW: Stickstoffzeiger schwach vertreten; } \\
\text { WW: Stickstoffzeiger stark vertreten }\end{array}$ & kein Beitrag \\
\hline Feuchtezeigerwert & kein Beitrag & kein Beitrag \\
\hline Reaktionszeigerwert & $\begin{array}{l}\text { NW: sehr geringe Anteile an Basenzeigern } \\
\text { WW: hohe Anteile an Basenzeigern } \\
\end{array}$ & kein Beitrag \\
\hline Lichtzeigerwert & $\begin{array}{l}\text { NW: Lichtzeiger anfangs noch schwach } \\
\text { und Schattenzeiger stark vertreten; } \\
\text { WW: Lichtzeiger von Anfang an stark und } \\
\text { Schattenzeiger schwach vertreten }\end{array}$ & $\begin{array}{l}\text { Dol. bel.: Lichtzeiger schwach und } \\
\text { Schattenzeiger stark vertreten; } \\
\text { Dol. ger.: Lichtzeiger stark und } \\
\text { Schattenzeiger schwach vertreten } \\
\end{array}$ \\
\hline $\begin{array}{l}\text { Pflanzen- } \\
\text { soziologische } \\
\text { Zuordnung }\end{array}$ & $\begin{array}{l}\text { NW: anfangs noch Schlagflurarten } \\
\text { schwach und Waldarten stark vertreten; } \\
\text { WW: Schlagflurarten von Beginn an stark } \\
\text { und Waldarten schwach vertreten }\end{array}$ & $\begin{array}{l}\text { Dol. bel.: geringer Anteil an Schlagflur- } \\
\text { und Ruderalarten und hoher Anteil an } \\
\text { Waldarten; Dol. ger.: hoher Anteil an } \\
\text { Schlagflur- und Ruderalarten und geringer } \\
\text { Anteil an Waldarten } \\
\end{array}$ \\
\hline Wuchsformen & 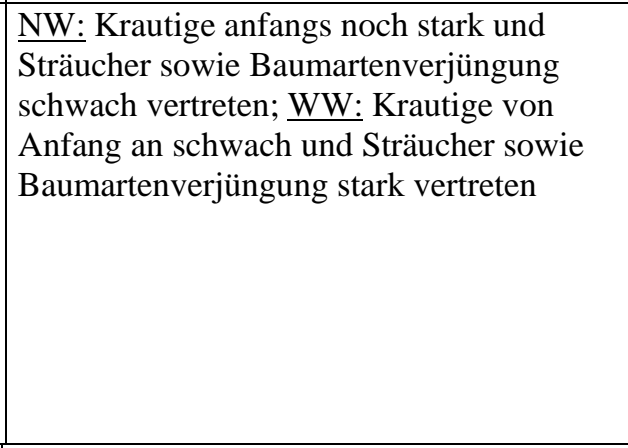 & $\begin{array}{l}\text { Dol. bel.: schwacher Rückgang von } \\
\text { Krautigen und schwache Expansion von } \\
\text { Sträuchern; Dol. ger.: starker Rückgang } \\
\text { von Krautigen und starke Expansion von } \\
\text { Sträuchern; BS bel.: starker Rückgang von } \\
\text { Krautigen und starke Expansion von } \\
\text { Sträuchern; wenig Baumartenverjüngung; } \\
\text { BS ger.: schwacher Rückgang von } \\
\text { Krautigen und schwache Expansion von } \\
\text { Sträuchern; starke Baumartenverjüngung } \\
\text { bzw. Pflanzung }\end{array}$ \\
\hline $\begin{array}{l}\text { Lebensformtyp } \\
\text { (RAUNKIAER) }\end{array}$ & $\begin{array}{l}\text { NW: starke Expansion der } \\
\text { Nanophanerophyten von geringem } \\
\text { Ausgangsniveau; starker Rückgang von } \\
\text { Geophyten; kaum Phanerophyten; WW: } \\
\text { von Anfang an starke Präsenz von } \\
\text { Nanophanerophyten (wenig } \\
\text { expandierend); geringe und konstante } \\
\text { Geophytenanteile; hohe } \\
\text { Phanerophytenanteile }\end{array}$ & $\begin{array}{l}\text { Dol. bel.: schwache Expansion der } \\
\text { Nanophanerophyten; geringe und } \\
\text { konstante Hemikryptophytenanteile; hohe, } \\
\text { aber zurückgehende Geophytenanteile; } \\
\text { konstante Chamaephytenanteile; Dol. ger.: } \\
\text { starke Expansion der Nanophanerophyten; } \\
\text { hohe, aber zurückgehende } \\
\text { Hemikryptophytenanteile; geringe, aber } \\
\text { konstante Geophytenanteile; starker } \\
\text { Rückgang an Chamaephyten }\end{array}$ \\
\hline
\end{tabular}


Fortsetzung von Tab. 17

\begin{tabular}{|c|c|c|}
\hline & langjährige Bewirtschaftungsgeschichte & $\begin{array}{c}\text { Behandlung nach dem Sturm: } \\
\text { Belassung/Räumung }\end{array}$ \\
\hline Ausbreitungstyp & $\begin{array}{l}\text { NW: sehr starker Rückgang autochorer } \\
\text { Arten von hohem Ausgangsniveau } \\
\text { zugunsten endozoochorer; } \\
\text { WW: unterschiedlich starker Rückgang } \\
\text { autochorer Arten von niedrigem } \\
\text { Ausgangsniveau zugunsten endozoochorer }\end{array}$ & $\begin{array}{l}\text { Dol. bel.: schwacher Rückgang autochorer } \\
\text { Arten zugunsten endozoochorer, hoher } \\
\text { Anteil anemochorer Arten; Dol. ger.: } \\
\text { starker Rückgang autochorer Arten } \\
\text { zugunsten endozoochorer; niedriger Anteil } \\
\text { anemochorer Arten; BS bel.: starker } \\
\text { Rückgang autochorer Arten von hohem } \\
\text { Ausgangsniveau zugunsten endozoochorer; } \\
\text { BS ger.: schwacher Rückgang autochorer } \\
\text { Arten von geringem Ausgangsniveau }\end{array}$ \\
\hline \begin{tabular}{|l|} 
vegetatives \\
Ausbreitungs- \\
vermögen
\end{tabular} & kein Beitrag & kein Beitrag \\
\hline Hemerobietyp & $\begin{array}{l}\text { NW: starker und expandierender Anteil } \\
\text { von oligohemeroben Arten zu Lasten } \\
\text { mesohemerober; eu-hemerobe Arten } \\
\text { bedeutungslos; WW: geringer und nur } \\
\text { schwach expandierender Anteil an } \\
\text { oligohemeroben Arten; mesohemerobe } \\
\text { Arten stark, eu-hemerobe Arten von } \\
\text { geringer Bedeutung (ihre absoluten DGS } \\
\text { aber deutlich stärker als im NW) }\end{array}$ & $\begin{array}{l}\text { Dol. bel.: oligohemerobe Arten dominieren } \\
\text { sehr stark, sehr geringer und stagnierender } \\
\text { Anteil an mesohemeroben Arten; eu- } \\
\text { hemerobe Arten bedeutungslos; Dol. ger.: } \\
\text { oligohemerobe Arten dominieren weniger } \\
\text { stark, hoher und steigender Anteil an } \\
\text { mesohemeroben Arten; geringer Anteil an } \\
\text { eu-hemeroben Arten (ihre absoluten DGS } \\
\text { deutlich stärker als im belassenen } \\
\text { Flächenwurf); BS bel.: hoher und } \\
\text { unterschiedlich stark zurückgehender } \\
\text { Anteil an mesohemeroben Arten zugunsten } \\
\text { oligohemerober, eu-hemerobe Arten } \\
\text { bedeutungslos; BS ger.: hoher und nur } \\
\text { wenig zurückgehender Anteil } \\
\text { mesohemerober Arten zu Gunsten } \\
\text { oligohmerober, eu-hemerobe Arten von } \\
\text { geringer Bedeutung (ihre absoluten DGS } \\
\text { deutlich stärker als im belassenen } \\
\text { Flächenwurf - gilt aber nur im Rotenberg!) }\end{array}$ \\
\hline Samenbanktyp & $\begin{array}{l}\text { NW: starke Expansion von Arten mit } \\
\text { langfristiger Samenbank >5 J. von } \\
\text { geringem Ausgangsniveau zu Lasten von } \\
\text { Arten mit kürzerfristiger (bsds. } \\
\text { temporärer) Samenbank; WW: schwache } \\
\text { Expansion von Arten mit langfristiger } \\
\text { Samenbank >5J. von hohem } \\
\text { Ausgangsniveau }\end{array}$ & $\begin{array}{l}\text { Dol. bel.: geringer und wenig } \\
\text { expandierender Anteil an Arten mit } \\
\text { langfristiger Samenbank >5J.; Dol. ger.: } \\
\text { hoher, aber wenig expandierender Anteil } \\
\text { an Arten mit langfristiger Samenbank; BS } \\
\text { bel.: starke Expansion von Arten mit } \\
\text { langfristiger Samenbank > 5J. von } \\
\text { geringem Ausgangsniveau zu Lasten von } \\
\text { Arten mit kürzerfristiger Samenbank; BS } \\
\text { ger.: geringe Expansion von Arten mit } \\
\text { langfristiger Samenbank >5J. von sehr } \\
\text { hohem Ausgangsniveau }\end{array}$ \\
\hline $\begin{array}{l}\text { Baumarten- } \\
\text { verjüngung }\end{array}$ & $\begin{array}{l}\text { NW: sehr geringe Naturverjüngung; } \\
\text { WW: stärkere Naturverjüngung bzw. } \\
\text { Pflanzung }\end{array}$ & \begin{tabular}{|l} 
Dol. bel.: starke Naturverjüngung; Dol. \\
ger.: trotz Pflanzung kaum Unterschiede \\
zur belassenen Variante; BS bel.: geringe \\
Naturverjüngung; BS ger.: starke \\
Naturverjüngung bzw. Pflanzung
\end{tabular} \\
\hline
\end{tabular}




\subsubsection{Einfluss forstlicher Maßnahmen nach dem Sturm: belassene vs. geräumte Windwurfflächen}

Belassene Windwurfflächen besitzen eine deutlich größere strukturelle und kleinstandörtliche Vielfalt als geräumte. Letztere wiederum weisen eine hohe Ähnlichkeit zu geräumten Kahlschlagflächen auf (HETZEL \& REIF 1996; FischeR et al. 1998; WiLMANNS et al. 1998). Geräumte Windwurfflächen sind gegenüber belassenen durch vergleichsweise weniger Beschattung, niedrigere relative Luftfeuchte, mehr Luftbewegung und Evapotranspiration sowie häufigeren Frostwechsel charakterisiert. Auf edaphisch entsprechenden Standorten dient der freigelegte Rohboden in den Fahr- und Rückespuren der Keimung und Etablierung v.a. von Bodenverdichtungs- und Vernässungszeigern. Die Beeinflussung geräumter Windwurfflächen durch schwere Forsttechnik, der damit verbundene Diasporeneintrag und die Neuschaffung von als Keimplätzen dienenden Bodenverwundungen sind hauptverantwortlich für den meist größeren Artenreichtum geräumter gegenüber belassenen Windwurfflächen. So sprechen FISCHER \& JEHL (1999) bei ihren Untersuchungen im Bayerischen Wald von „management pathways“ (hoher Störungsgrad) vs. „untouched pathways“ (geringer Störungsgrad). Auch nach den vorliegenden Untersuchungen sind geräumte/aufgeforstete Flächen generell artenreicher als belassene (Abb. 17). Besonders deutlich zeigt sich das im Hordelymo-Fagetum, bei dem der Artenzahlanstieg in der belassenen Hainholz-KF1 moderat und auf niedrigem Niveau erfolgte, auf dem geräumten Nüllberg dagegen rapide und auf dem höchsten Niveau aller UG. Da die qualitativen Artengruppenspektren aller UG in den ersten vier Sukzessionsjahren stabil bleiben (Kap. 4.3), kann dieser Zuwachs an floristischer Vielfalt keiner einzelnen Artengruppe zugerechnet werden, sondern allen in etwa demselben Maße. Die Artenzahlentwicklung spiegelt sich u.a. in der Entwicklung der floristischen Ähnlichkeit wider (Kap. 4.2.8). Die geräumten NüllbergFlächen haben sich floristisch sehr stark verändert, die belassene Hainholz-KF1 dagegen nur wenig (betrifft beide verwendete Indizes, Abb. 26). In den Buntsandstein-Gebieten mit ihrer relativ moderaten Artenzahlerhöhung liegen solch deutliche Unterschiede zumindest bei ungewichteter Betrachtung (Jaccard-Index) nicht vor. Das Bemerkenswerte im Harzvorland ist, dass dieser Artenzahlanstieg vier Jahre lang anhält (Tab. A22, A23). Im badenwürttembergischen Fichten-Windwurf wurde das Artenzahlmaximum standortsunabhängig bereits nach zwei Jahren erreicht (SAYER \& REIF 1998).

Präferenzen bestimmter Arten für geräumte oder belassene Flächen drücken sich meist in höheren Deckungsgraden, weniger in exklusivem Vorkommen aus (Tab. 18, A22, A23). Es gibt auch Arten, die sich in den einzelnen UG gegenläufig verhalten oder die nur in einem Gebiet eine Rolle spielen. Neben der Räumung kommt hier auch der Standorteinfluss einschließlich der Unterschiede bei der pflanzensoziologischen Ausgangssituation zum Tragen. Auffällig ist jedoch, dass besonders in den Zechstein-Gebieten viele typische Laubwaldarten mit einer Wuchsortpräferenz auf belassenen Flächen zu nennen sind, im Buntsandstein dagegen kaum. Auf den geräumten Flächen aller Standorte lassen sich 
hauptsächlich Nichtwaldarten ausscheiden, besonders deutlich im Buntsandstein (Unterteilung entsprechend ELLENBERG et al. 1992; OBERDORFER 2001). Rote-Liste-Arten spielen nirgendwo eine Rolle (Tab. 18, A22, A23).

Tab. 18: Beispiele für die Bevorzugung geräumter oder belassener Flächen durch einige Arten in großflächig geworfenen Buchen-Wäldern des Harzvorlandes (entspr. Tab. A22, A23).

\begin{tabular}{|l|l|l|}
\hline belassen & \multicolumn{1}{|c|}{ Zechstein } & \multicolumn{1}{c|}{ Buntsandstein } \\
\hline geräumt & $\begin{array}{l}\text { Alium ursinum, Atropa bella-donna, Circaea } \\
\text { Fraxinus excelsior, Hordelymus europaeus, } \\
\text { Galeobdolon luteum, Mercurialis perennis }\end{array}$ & $\begin{array}{l}\text { Digitalis purpurea, Epilobium angustifolium } \\
\text { (Luzulo-Fagetum), Oxalis acetosella (Galio } \\
\text { odorati-Fagetum) }\end{array}$ \\
\hline $\begin{array}{l}\text { Alliaria petiolata, Brachypodium sylvaticum, } \\
\text { Carex muricata, Cirsium arvense, Euphorbia } \\
\text { amygdaloides, Galium odoratum, Hypericum } \\
\text { perforatum, Rubus idaeus, Rumex obtusifolius, } \\
\text { Viola reichenbachiana }\end{array}$ & $\begin{array}{l}\text { Rubus fruticosus agg., Galeopsis tetrahit, } \\
\text { Rumex obtusifolius, Poa angustifolia, } \\
\text { Sambucus nigra, Urtica dioica }\end{array}$ \\
\hline
\end{tabular}

In der Literatur ist belegt, dass überlebende Waldarten auf belassenen Windwurfflächen oftmals höhere Deckungen behalten als auf geräumten (z.B. FISCHER et al. 1998). Zusätzlich ist im belassenen Windwurf eine enorme Artenvielfalt an holzzersetzenden Pilzen und bestimmten Tierartengruppen vertreten (z.B. Arthropoden, Vögel). Im Gegensatz zu den Gefäßpflanzen sind darunter auch viele seltene und geschützte Arten zu finden (FISCHER et al. 1998).

Hinzuweisen ist ferner auf die recht hohen Brombeer-Deckungen, die im vierten Jahr nach dem Sturm in den beiden geräumten Buntsandstein-Varianten erreicht werden (RotenbergAbt. 1228: $11 \%$ in der Strauch- und 1\% in der Feldschicht; Krücker-Plateau: 6\% in der Strauch- und 5\% in der Feldschicht - siehe Tab. A23). Somit lässt sich die Brombeere v.a. im Buntsandstein als Profiteur räumungsbedingter Bodenverwundungen benennen (Tab. 18; Kap. 5.4.2). Daneben spielen aber vermutlich auch das schon längerfristige Persistieren dieser Art im Vorbestand, die langjährige Durchforstung und speziell in der Rotenberg-Abt. 1228 der hohe Eichen-Anteil des dortigen Altbestandes (lichteres Kronendach als in BuchenReinbeständen) eine Rolle. Dadurch sind eine relativ starke Mineralisation und günstige Humusformen angezeigt. Auch nach den Untersuchungen von WALTER et al. (1998) in Tannen-Wäldern auf Buntsandstein trat die Brombeere nach Auflichtung hauptsächlich auf durchforstungsbedingt besseren Humusformen mit stärkeren Deckungen auf, auf weniger günstigen Humusformen reagierte sie verhaltener. Auch die Himbeere zeigt nach den vorliegenden Untersuchungen einen Ausbreitungsschwerpunkt auf geräumten Flächen, was sich aber nur im Zechstein beobachten lässt (Tab. A22, A23).

Akzeptiert man die 1\% mittlere Deckung als Untergrenze für „höherdeckende“ Feldschichtarten (Abb. 18; Tab. A22, A23), so findet bezüglich dieses Parameters eine langsame Angleichung von belassenen Flächen (geringe Anteile an Gesamtartenzahl, eher ansteigend) und geräumten Flächen (hohe Anteile an Gesamtartenzahl, eher sinkend) statt. Diese Feststellung spiegelt sich zumindest teilweise auch in den Evenness-Werten wider 
(Abb. 19), denn die belassenen Flächen mit Hordelymo- und Galio odorati-Fagetum sind geringer durchmischt als die geräumten. Insgesamt bestätigt sich die von WILMANNS et al. (1998) getroffene Feststellung, dass es ganz allgemein nur wenige Arten sind, die die Syndynamik von Freiflächenbiozönosen nach Kahlschlag oder Windwurf bestimmen.

Weiterhin ergeben sich nach Tab. 17 größere Unterschiede in den Dominanzen ökologischfunktionaler Artengruppen. Demnach sind geräumte Windwurfflächen im Vergleich zu belassenen durch besonders hohe Abundanzanteile folgender Artengruppen charakterisiert: Arten mit langfristiger Samenbank $>5$ Jahre (alle Standorte), Schlagflurarten (nicht im Luzulo-Fagetum), Ruderalarten (nur im Hordelymo-Fagetum), Lichtzeiger (nur im Hordelymo-Fagetum), Sträucher (nicht im Luzulo-Fagetum), Baumartenverjüngung (nur im Buntsandstein: hier wahrscheinlich Aufforstungseffekt), Grasartige (nur im Luzulo-Fagetum), endozoochor verbreitete Arten (nicht im Luzulo-Fagetum) sowie meso- und euhemerobe Arten (nicht im Luzulo-Fagetum). Die in Klammern angegebenen Einschränkungen weisen schon darauf hin, dass man - abgesehen vielleicht von der tendenziell stärkeren Verbreitung von Arten mit langfristiger Samenbank auf geräumten Flächen - nur wenig generalisieren kann und sich ein stärkerer Standorteinfluss auch hier interferierend bemerkbar macht.

Die Bodenfreilegung durch schwere Räumtechnik im Zuge der Windwurf-Aufarbeitung besitzt auch für die Verjüngung große Bedeutung. Sie schafft einerseits günstige Keimungsbedingungen für Gehölze und fördert deren Ansamung (Rohbodenkeimplätze). Andererseits kann sie gleichzeitig die Wiederbewaldung stark verzögern, indem natürliche Verjüngungsvorräte und Mykorrhiza vernichtet, Holz als potenzielles Keimsubstrat (v.a für Fichte) entfernt und zu Staunässe bzw. raschem Austrocknen des Bodens führende Bodenverdichtungen hervorgerufen werden (LÄssIG et al. 1995a+b; WOHLGEMUTH et al. 1995; KÖBerle 1997; SAYER \& ReIF 1998; SCHMIDT-SchÜTZ \& HuSS 1998; BROGGi 2000; LÄSSIG 2000; LÄSSIG \& SCHÖNENBERGER 2000; ALDINGER et al. 2001; MÄRKL \& EGGLSEER 2001; LÄSSIG \& MotsCHALOW 2002; SCHÖNENBERGER et al. 2002, 2003). Beispielsweise führten erhöhter Trockenstress und Spätfrosthäufigkeit auf nach dem 1972er „NiedersachsenOrkan“ maschinell geräumten Windwurfflächen zu starken Pflanzausfällen (HuSs 1991). Zunehmende Räumungsintensität kann sich u.a. in immer stärker reduzierter Verjüngung der Schlusswaldbaumarten zeigen (SCHMIDT-SchÜTZ \& HUSS 1997, 1998; LÄSSIG \& Motschalow 2002). Dagegen sind es vor allem Pionierbaumarten (Birke, Aspe, Salweide), Pioniersträucher (Himbeere u.a.) sowie krautige Schlagflur- und Ruderalarten, die von der Räumung profitieren (FISCHER et al. 1990, 2002; ALDINGER et al. 2001; MÄRKL \& EGGLSEER 2001).

Die Differenzierung zwischen geräumten und belassenen Windwurfflächen kann regional und standörtlich unterschiedlich stark ausgeprägt sein (FISCHER et al. 1998; siehe auch Kap. 5.8.1). Dass sich Schlusswaldbaumarten auf belassenen Flächen besser verjüngen als auf geräumten, unterstreichen z.B. für die Fichte FISCHER et al. (1990, 2002) sowie SCHMIDT-SchÜTZ \& Huss (1997, 1998), für Laubbaumarten HETZEL \& REIF (1996, 
1998) sowie ALDINGER \& KENK (2000) und für Nadelgehölze LÄSSIG \& MotSCHALOW (1999, 2000a, 2000b, 2002). In diesen Studien waren Pionierwälder und andere Pionierstadien tendenziell stärker auf geräumten Flächen zu finden. Im Bayerischen Wald z.B. etablierte sich Schlagflurvegetation auf belassenen Flächen nur im Bereich der Wurzelteller, auf geräumten dagegen flächig (FISCHER et al. 1990, 2002). In Baden-Württemberg waren wichtige Schlagflurarten qualitativ zwar ähnlich verbreitet, aber auf geräumten Flächen signifikant häufiger als auf belassenen (SAYER \& REIF 1998; HETZEL \& REIF 1998). Die auffällige Dominanz der Himbeere im geräumten Windwurf der Schwäbischen Alb wird dort auf die verstärkte Mineralisation infolge räumungsbedingter Bodenverwundung zurückgeführt. Das lässt sich im Buchen-Windwurf des Harzvorlandes nur bei einem Vergleich innerhalb der Zechstein-Standorte feststellen, während sich im Buntsandstein die Himbeer-Fluren bis zum vierten Jahr nach dem Sturm überall etwa gleichstark ausprägten (Tab. A22, A23). Auch auf Windwurfflächen bayerischer Wirtschaftswälder und der Schweizer Alpen trat Schlagflurvegetation in allen Nutzungsvarianten etwa gleichstark auf (FISCHER 1996; LÄSSIG \& SCHÖNENBERGER 1994). In basenarmen Windwurfgebieten Baden-Württembergs fanden SAYER \& REIF (1998) auf belassenen Flächen anfangs sogar eine stärker ausgebildete Schlagflurvegetation als auf geräumten (ähnliche Situation wie im Luzulo-Fagetum des Krückers, Kap. 4.3.5), bei der Baumartenverjüngung dagegen kaum Unterschiede. In einem baden-württembergischen Fichten-Sturmwurfbannwald konstatierten HANKE \& PISOKE (1999): „Der Einfluss der Räumung auf die Verjüngungszahlen ist nicht eindeutig“. Etwa 10 Jahre nach „Vivian/Wiebke“ argumentieren WoHLGEMUTH et al. (2002) für Fichtendominierte Schweizer Bergwälder sogar, dass räumungsbedingte Bodenstörungen zu sehr hohen Sämlingsdichten und zur Kompensation der räumungsbedingen Verluste in der Naturverjüngung führten (geräumte Standorte dort verjüngungsreicher als belassene). Die UG im Harzvorland müssen in diesem Punkt standörtlich getrennt eingeordnet werden. Auffällig sind hier im Buntsandstein die (aufforstungsbedingt) höheren Deckungssummen und -anteile des Baumartenjungwuchses auf geräumten Flächen. Im Zechstein dagegen verhält es sich bei den absoluten Deckungssummen genau umgekehrt (Tab. A22), bei den Deckungssummenanteilen herrscht etwa Gleichstand (Abb. 34, 35).

\subsubsection{Einfluss der Nutzungsgeschichte: Naturwälder vs. Wirtschaftswälder}

Die Nutzungsgeschichte des Bestandes vor dem Sturm ist wichtig für die Interpretation der Unterschiede in der Windwurfsukzession, hier am Beispiel der vier Buntsandstein-Varianten (Tab. 17 links; Kap. 4.2 und 4.3). Der Flächenwurf des Naturwaldes Königsbuche setzte sich anfangs (d.h. 1999) bei den meisten Parametern von den Varianten mit langjähriger forstlicher Nutzung ab, bevor im weiteren Verlauf eine Angleichung erfolgte (KOMPA \& SCHMIDT 2003). 30 Jahre Null-Nutzung in der Königsbuche zeigten sich demnach in relativ geringen Artenzahlen sowie vergleichsweise geringen Abundanzanteilen an Epilobietea-Arten, 
Sträuchern, endozoochoren Arten, Arten mit langfristiger Samenbank (>5 J.) sowie Licht(L7-9), Basen- (R7-9) und Stickstoffzeigern (N7-9). Entsprechend hoch waren dort die Abundanzanteile an Querco-Fagetea-Arten, krautigen Arten, Geophyten, autochoren Arten, Arten mit temporärer bis kurzfristiger Samenbank ( $<5$ J.) sowie an Schatten- (L1-3), Frische(F4-6), Mäßigstickstoff- (N4-6) und Mäßigsäurezeigern (R4-6). Der Abundanzanteil oligohemerober Arten nahm im Naturwald nach dem Sturm wieder stärker zu als im Wirtschaftswald. Die relativ hohen Abundanzanteile der Basenzeiger im Wirtschaftswald weisen auf einen möglichen Einfluss forstlicher Kalkungsmaßnahmen hin (Tab. 1). Die anfängliche Artenarmut des Naturwaldes Königsbuche erklärt sich aus der Tatsache, dass in

Buchen-Wäldern der Optimalphase nach Nutzungseinstellung ein Artenrückgang insbesondere bei Schlagflur- und Ruderalarten einsetzt, die Krautschicht ausgeschattet, die Buche begünstigt und langfristig andere Baumarten verdrängt werden (MEYER 1995; BRUNET et al. 1996; ElLENBerg 1996; LeUSCHNER 1998; SCHMidT 1999a+b, 2002a). Solche Tendenzen sind auf basenreichen Standorten weniger stark ausgeprägt als auf basenarmen (SCHMIDT et al. 1996; SCHMIDT 1999a+b). So ist z.B. das seit 1993 forstwirtschaftlich wenig genutzte Hainholz mit durchschnittlich 92 Gefäßpflanzenarten/ha einer der artenreichsten niedersächsischen Naturwälder (SCHMIDT 2002a, 2003) - vielleicht sogar der artenreichste. Das lässt sich nicht allein mit dem Artenzahlanstieg nach Sturmwurf begründen, sondern auch mit dem geologischen Ausgangssubstrat (Zechsteingips und -dolomit) und den dadurch bedingten heterogenen Standortsbedingungen.

Aus Vergleichsuntersuchungen zwischen Natur- und Wirtschaftswäldern z.B. in Niedersachsen und Hessen (SCHMIDT 1999a, 2003; SCHMIDT \& WECKESSER 2001) ist bereits bekannt, dass auch in Wirtschaftswäldern ohne Windwurf die Strauchschicht einschließlich der Naturverjüngung stärker ausgebildet ist als in Naturwäldern, was auf das höhere Lichtangebot in durchforsteten Beständen und die enorme Konkurrenzkraft der Buche (v.a. nach Nutzungseinstellung) zurückgeführt wird. Damit korrespondiert die anfangs sehr unterschiedliche Deckung der Pionier-Strauchschicht und der Lichtzeiger (L7-9) in den fast benachbart liegenden Nutzungsvarianten des Galio-Fagetum im Rotenberg. In der RotenbergAbt. 1228 könnten die hohen Strauchschicht-Deckungssummen (91\% in 2001) zusätzlich noch durch den hohen Eichen-Anteil des Vorbestandes (Kap. 2.1.4) verursacht worden sein. Ihre relativ geringe Zunahme in der Zeitreihe schlägt sich auch in einer relativ hohen quantitativen floristischen Ähnlichkeit (Percentage Similarity) zwischen 1999 und 2001 im Gegensatz zum benachbart liegenden Naturwald Königsbuche nieder.

Neben der bereits erwähnten Kronenauflichtung spielt in Wirtschaftswäldern das langfristige oberirdische Persistieren von waldfremden Störzeigern bzw. deren Aktivierung aus der Samenbank infolge durchforstungsbedingter Bodenverwundungen eine Rolle. Deshalb haben Wirtschaftswälder bei ausschließlicher Betrachtung der Gefäßpflanzenflora auch eine vergleichsweise hohe $\alpha$-Diversität (Artenzahl je Flächeneinheit). FISCHER (1996b) spricht von regelrechter „Durchseuchung“ langjähriger Wirtschaftswälder mit Störzeigern. In der 
Startphase von Windwurf- oder Kahlschlagsukzessionen nutzen diese Arten ihren Konkurrenzvorteil aus, während sie in Naturwäldern oder wenig durchforsteten Wäldern eine geringere Rolle spielen und ein Neueintrag etwas länger dauert bzw. erst forstliche Räumungsmaßnahmen wieder dafür sorgen (FISCHER 1987; FISCHER et al. 1998). Nach einer Reihe von Untersuchungen (BRUNET et al. 1996; SCHMIDT 1999a; HÄRDTLE et al. 2001; VON OнЕIмв 2003) sind speziell Schlagflur-, Ruderal-, Grünland-, Heide- und Saumarten in Wirtschaftswäldern typischerweise stärker vertreten als in Naturwäldern, wo wiederum Laubwaldarten stärker dominieren. Hinsichtlich Rote-Liste-Arten ergeben sich jedoch häufig keine Unterschiede (SCHMIDT 1999a; SchmidT \& WECKESSER 2001). Bei den Windwurfgebieten im Harzvorland können diese Aussagen für die Abundanzanteile der Schlagflur- und Laubwaldarten (im Zechstein zusätzlich für die der Saum- und Ruderalarten) bestätigt werden. Es ist weiterhin auffällig, dass starke N-Zeiger (z.B. die beiden HolunderArten) im Naturwald Königsbuche schwächer vertreten sind als in den drei anderen Varianten mit langjähriger forstlicher Vornutzung. Jedoch lassen sich im Harzvorland aus der rein qualitativen Artenzusammensetzung keine nutzungsgeschichtlichen Unterschiede zwischen den Vergleichsstandorten ableiten (Kap. 4.3). Das steht in Übereinstimmung mit den Aussagen von SCHMIDT \& WECKESSER (2001), wonach die Waldbodenflora naturnah bewirtschafteter Wälder durch forstliche Maßnahmen nicht nennenswert beeinträchtigt wird. In südschwedischen Buchen- und Eichen-Wäldern fanden BRUNET et al. (1996) ebenfalls keine Beeinflussung der typischen Waldbodenflora durch naturnahen Waldbau, da die Waldarten an das gelegentliche Auftreten von Kronenlücken und Bodenverwundungen angepasst und entsprechend persistent sind. Anders verhält es sich dagegen mit solchen auf Totholz angewiesenen Artengruppen wie Moose, Flechten und Pilze (GroßE-BraUCKMAnN 1994; SCHMIDT \& WeCKESSER 2001; SCHLECHTE 2002). In anderen NaturwaldWirtschaftswald-Vergleichen (z.B. ABs et al. 1999 in Bialowiesa/Ostpolen) ergaben sich wiederum nur geringfügige floristische Unterschiede (im Urwald geringfügig weniger Störzeiger), aber viel stärkere in der Bestandesstruktur (im Urwald geklumpteres Auftreten der Gehölze, größerer Totholzreichtum und heterogenere, aber geringere Verjüngung). Eine Zunahme der Textur-Evenness mit länger andauernder Null-Nutzung ermittelten HÄRDTLE et al. (2001) in Naturwäldern. Beim Vergleich von windgeworfenen Bergwäldern des Ural und der Schweizer Alpen kamen LÄssig \& Motschalow (1999, 2000a, 2000b, 2002) zu dem Schluss, dass Windwurf in naturnahen Gebirgswäldern weniger strukturverändernd zu wirken scheint als in bewirtschafteten. Die häufig aus ökonomischen Gründen naturverjüngten und relativ naturnahen Ural-Wälder waren nach dem Sturm v.a. bei Pionierbäumen arten- und verjüngungsreicher als die Schweizer Wirtschaftswälder. Innerhalb der Ural-Standorte waren wiederum naturverjüngte Wälder im Vergleich zu gepflanzten sturmresistenter und stufiger (mehr innere Waldränder), allerdings auch vorratsärmer.

Auch die historische Nutzungsform ist ein sehr wichtiger Faktor für bestimmte Verbreitungsmuster von Arten (ZACHARIAs 1993, 1994). So gelten typische Arten historisch 
alter Wälder oftmals als Niederwaldzeiger (BONN \& PosCHLOD 1998). Sie erscheinen heute als häufig in den Roten Listen zu findende historische Nutzungsrelikte mit hohem Isolationsund Auslöschungsrisiko, aber möglicherwiese auch mit sehr langer Persistenz v.a. in sehr großen Waldgebieten (ZACAHRIAS 1994; BONN \& POSCHLOD 1998). Für ihre Fernausbreitung waren und sind Zufallsprozesse sowie die direkte anthropogene Verschleppung sehr bedeutend (z.B. Menschen, Tiere, Fahrzeuge, Sturmverdriftung). Möglicherweise ist es heute so, dass natürliche und anthropogene Störungen (also z.B. Sturmwurf und Räumung) in gewissem Umfang eine Art Ersatzfunktion für früher weit verbreitete dynamische Prozesse wie z.B. Waldweide und Niederwaldwirtschaft darstellen, welche in den Wäldern der historischen Kulturlandschaft maßgeblich zur Neuetablierung von Diasporen und zu ihrem Austausch zwischen isolierten Populationen beitrugen. Allerdings ist dieser „Ersatz“ eher funktionaler Natur und betrifft heute teilweise völlig andere Arten als damals. Die heute ausbreitungsrelevanten Prozesse in Wäldern führen oftmals zur Förderung von Neophyten oder Ruderalarten. Beispielsweise wird die relativ schnelle Ausbreitung des auch im Harzvorland-Windwurf sehr dominanten Wald-Störzeigers Impatiens parviflora mit Wegebau und Holztransport in Verbindung gebracht (LOHMEYER \& SUKOPP 1992). Trotzdem kann ein „Mehr“ an natürlicher Dynamik in unseren Wäldern die Wahrscheinlichkeit von „ausbreitungsrelevanten Zufallsprozessen“ (BONN \& POSCHLOD 1998) sicher nicht nur für Störzeiger, sondern auch für Waldarten erhöhen.

Zur Nutzungsgeschichte im weiteren Sinne gehört auch der langfristige, teils nutzungs-, teils immissionsbedingte Vegetationswandel in deutschen Buchen-Wäldern, der in den letzten Jahren belegt wurde (z.B. BÜRGER 1991 für den Schwarzwald; BöHLING 1992, 2003 für den Stadtwald Hannover bzw. südwestdeutsche Buchen-Wälder; GRABIETZ \& FIEDLER 1996 sowie AHRNs \& HOFMANN 1998 für den Hainich; HAKEs 1999 für nordhessische Basalt- und KalkBuchenwälder; WECKESSER \& SCHMIDT 2004 für den Solling). Obwohl für die untersuchten Waldgebiete mitunter regional verschiedene Ursachen zutrafen, kam es überall zu Artenzahlerhöhungen und stärkerer Verbreitung vor allem von Stickstoffzeigern, während mesophile Arten sowie Säure- und Nährstoffarmutszeiger zurückgingen. Als Gründe werden die atmogene Deposition von Stickstoff und alkalischen Stäuben, Kalkungsmaßnahmen, die verbesserte Bodenmineralisation in Verbindung mit Kronenverlichtung und die Regeneration der Waldböden nach Aufgabe historischer Nutzungsformen genannt. Andererseits führte die Einstellung bzw. Verminderung der forstlichen Bewirtschaftung zu einem dichter schließenden Kronendach und damit zu einem subatlantischer getönten Waldinnenklima. Zu den Gewinnern der genannten Entwicklungen zählten einerseits Ruderalarten und Störzeiger, andererseits aber auch anspruchsvollere Laubwaldarten (z.B. Allium ursinum) und die Klimaxbaumart Buche. 


\subsection{Zum Einfluss der Störungsflächengröße auf die Windwurfsukzession}

Der Einfluss der Störungsflächengröße auf die Windwurfsukzession wurde in der vorliegenden Arbeit überwiegend mittels Verjüngungsaufnahmen auf den BuntsandsteinStandorten geprüft (Kap. 4.5). Königsbuche (Galio odorati-Fagetum) und Krücker (LuzuloFagetum) können in den Varianten „Bestand“ und „Lücken“ im vierten Sukzessionsjahr (2001) als relativ verjüngungsreich eingeschätzt werden, wenn man die von BURSCHEL \& HuSs (1997) als ausreichende Sämlingsdichte angegebenen 5.000-10.000/ha (gültig für Buche) zu Grunde legt. Mit zunehmender Störungsflächengröße nehmen aber in beiden UG die Sämlingszahlen stark ab, wobei die Buche als Klimaxbaumart Anteile am Artenspektrum der Naturverjüngung verliert. Neben der entlang des Störungsgradienten sinkenden Anzahl vorhandener Alt-Buchen (Samenbäume) dürften hierbei auch Konkurrenzeffekte eine entscheidende Rolle spielen. Im Flächenwurf führt die Konkurrenz der Sträucher, vermutlich auch der Grasartigen im Moment noch zu einer Hemmung der jungen Bäume, so dass man gemessen am oben genannten Richtwert - in der Königsbuche nicht mehr und im Krücker gerade noch von einer gesicherten Naturverjüngung sprechen kann. Während bei lückiger Störung nur eine geringe Beeinflussung der Baumartenverjüngung durch konkurrierende Vegetation zu bestehen scheint, trifft dies für den Flächenwurf nicht mehr zu. Einen ähnlichen Stückzahlgradienten vom Bestand hin zum Flächenwurf fand bereits LEMKE (2001) im September 1999 an insgesamt 28 im gesamten Reservat systematisch verteilten Gitternetzpunkten in der Königsbuche. Damals wurden deutlich höhere Stückzahlen gefunden als zwei Jahre später in KF2 (vorliegende Untersuchungen). Der Bestand war dabei mit 80.500/ha (96\% Buchen-Anteil), die Lücken mit 33.000-43.000/ha (85 bzw. 79\% BuchenAnteil) und der Flächenwurf mit 19.500/ha (97\% Buchen-Anteil) notiert worden. Bei ähnlichen Untersuchungen in Bergmischwäldern bayerischer Mittelgebirge und der Alpen fanden BURSCHEL \& BINDER (1993) etwa 10 Jahren nach unterschiedlich starker Auflichtung ebensolche abnehmenden Buchen-Verjüngungsdichten vom Bestand (27.000/ha) über Schirmhiebsflächen (16.000-23.000/ha) zur Kahlfläche (2.000/ha).

Die Frage ist, ob sich auch auf basenreichen Buchen-Standorten solche Gradienten ergeben. Mit hoher Wahrscheinlichkeit zeigt sich hier ein anderes Muster. Im Beispiel des BasaltBuchenwaldes im hessischen Naturwaldreservat Weiherskopf (WILLIG 1994, 2002b; KEITEL 1998, 1999) war der benachbart stehende Buchen-Altbestand mit rund 25.000 Sämlingen/ha ähnlich verjüngungsreich wie der großflächige Windwurf und wies auch eine ähnliche Baumartenzusammensetzung beim Jungwuchs auf (85\% Edellaubbäume und 13\% Buche, siehe Tab. 21). Auch im Flächenwurf des Harzvorlandes ist auf basenreich-tiefgründigen Standorten schnellwüchsige Eschen-Verjüngung mit zahlreicher, unterständiger Buche typisch (Kap. 4.5.1), für die kleineren Windwurflücken (z.B. im Hainholz) kann dies nach eigenen Beobachtungen zumindest angenommen werden.

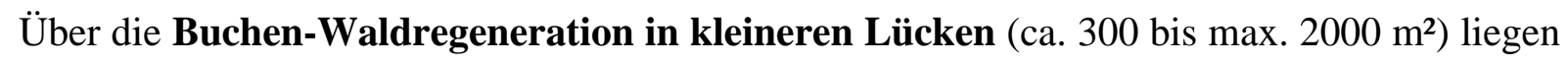
bereits umfangreiche Studien vor. So ist bekannt, dass dieser Prozess unter maßgeblicher 
Beteiligung der Buche oder völlig Buchen-dominiert abläuft (ELLENBERG et al. 1986; KNAPP \& JesChKe 1991; Griese 1995; KorPel 1995; MEYer 1995; SCHMIDT 1996, 1997, 1998b; SCHMIDT et al. 1996; MEYER et al. 2000). Dabei liegen in natürlichen Buchen-Wäldern der gemäßigten Zone die Lückengrößen ohne großflächige Störung um die $100 \mathrm{~m}^{2}$ bei Gesamtflächenanteilen von weit unter 10\% (TABAKU 1999). Das Ausbleiben von Pionierwaldstadien in Lücken begründen KENK et al. (1991) mit dem ausreichenden Vorhandensein von Samenbäumen der Schlusswaldbaumarten im angrenzenden Bestand, deren relativ schwere Samen (im Gegensatz zu den leichteren der Pionierbaumarten) nicht fernverbreitet werden. Hinzu kommt, dass in kleineren Lücken ein ausgeglicheneres, waldähnliches Mikroklima die Verjüngung der Schlußwaldbaumarten begünstigt, während in größeren Lücken die Einwanderung von Pionierarten durch zunehmendes Freiflächenklima gefördert wird (KoOP 1982 für nordwestdeutsche und ostpolnische Naturwälder). In neuseeländischen Buchen- (Nothofagus) Wäldern stellten STEWART et al. (1991) fest, dass die Naturverjüngung der eher lichtbedürftigen Nothofagus fusca um so stärker auftrat, je größer und lichter die Lücke war, während in kleineren Lücken (unter $400 \mathrm{~m}^{2}$ ) die schattentolerantere Nothofagus menziesii dominierte. Auch hier war das Geschehen von den Schlusswaldbaumarten bestimmt. Zwischenzeitliche Pionierwälder wurden dennoch z.B. aus den Karpaten beschrieben, allerdings nur in größeren Windwurflücken (KORPEL 1995). In der dortigen Buchen- und Buchenmischwaldstufe können Salweide und Zitterpappel (basenreiche Standorte) sowie Birke und Eberesche (saure Standorte) nach 10-15 Jahren vorübergehend geschlossene Vorwaldstadien ausbilden, die aber spätestens nach 25-30 Jahren von den nachwachsenden Klimaxbaumarten verdrängt werden.

Eine Vergleichsmöglichkeit bieten weiterhin die Untersuchungsergebnisse aus experimentellen Lochhieben. So beobachteten BARTSCH et al. (1999) und BARTSCH \& VOR (2000) in unbehandelten Lochhieben des bodensauren Buchen-Waldes (Luzulo-Fagetum) im Solling eine zeitverzögerte Besiedlung mit Buchen-Naturverjüngung, welche etwa ab dem fünften Jahr nach der Auflichtung dominierende Krautschicht-Deckungsgrade und Sämlingszahlen von etwa 50.000-120.000/ha erreichte. Krautige Vegetation entwickelte sich dagegen kaum. Verglichen damit sind die Windwurflücken des Krückers und der Königsbuche mit rund 35.000 bzw. 9.000/ha relativ arm an Sämligen, ausserdem ist hier die Buche weniger dominant. Der hohe Eschen-Anteil in den belassenen Lücken am KrückerOsthang ist standörtlich zu erklären, da hier bereits eine Übergangszone zum benachbarten Zechstein-Gebiet des Hainholzes verläuft (Abb. 46). Experimentelle Lochhiebe auf basenreichen Standorten unterscheiden sich bezüglich der Verjüngungszahlen kaum von denen auf Buntsandstein, wohl aber bezüglich der Baumartenzusammensetzung. LAMBERTz \& SchMidT (1999) fanden im Göttinger Wald auf Muschelkalk (Hordelymo-Fagetum) in Lücken 120.000 Sämlinge/ha (30\% Buchen-Anteil) und im angrenzenden Bestand sogar 180.000/ha (12\% Buchen-Anteil). Im Göttinger Wald und am Ossenberg (Dransfelder Hochfläche) ermittelte HORN (2002) ebenfalls auf Muschelkalk etwa 60.000-120.000 
Sämlinge/ha (20-64\% Buchen-Anteil). Edellaubhölzer (v.a. Esche) erreichten in beiden Untersuchungen sehr hohe Anteile. Sämlingsdichte und Baumartenverhältnis zwischen Buche und Edellaubhölzern gestalteten sich ähnlich wie im Harzvorland. Ein weiteres Charakteristikum solcher Lochhiebe auf basenreichen Standorten besteht darin, dass die Buche an den etwas schattigeren Lückenrändern häufig höhere Verjüngungsdichten und höhere Zuwächse erreicht als im Lückenzentrum, wo wiederum die Esche eindeutig überlegen ist. Allerdings läuft der Selbstausdünnungsprozess bei beiden Baumarten im Lückenzentrum am intensivsten ab (z.B. WAGNER 1999).

Nicht nur die Baumartenverjüngung, sondern auch die krautige Vegetation und Sträucher müssen in diese Betrachtung einbezogen werden. In den Buntsandstein-Windwurfgebieten des Harzvorlandes wurde mit zunehmender Störungsflächengröße eine zunehmende floristische Veränderung hin zu waldfremden Sukzessionsstadien festgestellt, die sich in abnehmenden Anteilen von Waldarten bei konstanten Absolutwerten ausdrückt (KoMPA \& SCHMIDT 2003), d.h. die alte Waldvegetation persistiert hier unter störungsbedingt unterschiedlich stark ausgeprägten Pionierstadien. Für die basenreichen Windwurfstandorte des Hainholzes ermittelte LugINBÜHL (2000), dass mit zunehmender Störungsintensität Artenzahl, Krautschichtdeckung sowie Evenness immer stärker zu- und die floristische Ähnlichkeit abnahmen. Ab 25\% relativem Lichtgenuss ließ sich allerdings kein stärker förderlicher Einfluss der Auflichtung auf die Krautschichtentwicklung mehr feststellen. Auf den basenarmen Buntsandstein-Standorten (z.B. Naturwald Königsbuche) können solche Aussagen zumindest bezüglich Artenzahl, floristischer Ähnlichkeit und Krautschichtdeckung bestätigt werden (KOMPA \& SCHMIDT 2003). Ein weiteres Beispiel für die Störungsflächenabhängigkeit beschrieb z.B. STÜDELI (1998) in Buchen-Mischwäldern der Schweizer Jura. Mit zunehmender Größe der Bestandeslücke fand er eine steigende Zahl von Sambucus nigra-Büschen und eine zunehmende (endozoochore) Samenverbreitung durch Vögel. Eine ganz ähnliche Ausbreitung von Sambucus racemosa entlang des Störungsgradienten liegt auch im Naturwald Königsbuche vor, was dort ebenso zu steigenden Abundanzanteilen endozoochor verbreiteter Arten beiträgt (KOMPA \& ScHMIDT 2003). Für das Wuchsverhalten der Himbeere als dem dominantesten Pionierstrauch des HarzvorlandWindwurfes ist der Störungsgradient ebenfalls bedeutsam. In den Lücken eines tiefgründigen Dolomit-Standortes (Hainholz) entwickelte sich Rubus idaeus zahlreicher und fruchtbarer als im benachbarten Flächenwurf, zeigte in den Lücken aber ab dem vierten Jahr bereits Selbstausdünnungstendenzen, während die Triebzahl im Flächenwurf weiter anstieg (Kap. 4.4.2).

Auch für das Ausmaß von Fichten-Verjüngung haben Größe und Ausformung der Störungsfläche sowie der Abstand vom nächsten fruktifizierenden Bestand große Bedeutung, was mehrere Autoren aus Süddeutschland und der Schweiz betonen. So nennen MößNANG \& KÜHNEL (1999) für bayerische Fichten-Windwurfgebiete maximal ein bis zwei Stammlängen Entfernung vom Bestandesrand bzw. 1-2 ha maximale Flächengröße als Eckdaten für eine 
ausschließlich auf Naturverjüngung basierende Bestandesneugründung. Auf Kalk-Standorten der Schwäbischen Alb fanden HETzEL \& REIF (1998) bei geschlossenem oder nur mäßig aufgelockertem Kronendach eine relativ geringe Fichten-Verjüngung, im Flächenwurf dagegen etwa das 6-fache der Pflanzenzahlen. Ähnliches berichten REIF \& PRZYBILLA (1995) aus den Hochlagen des Bayerischen Waldes.

Aus anderen Literaturauswertungen (SCHÖLCH et al. 1994; SCHÖLCH 1998) geht hervor, dass Samendichte und Naturverjüngung vieler Baumarten mit zunehmender Entfernung vom Waldrand bzw. Samenbaum exponentiell abnehmen. In der Schweiz ermittelten LÄSSIG et al. (1995a) im Windwurf etwa 2-3 Millionen Fichten-Samen/ha, im stehenden Bestand dagegen 20-30 Millionen. In kleinen und mittleren Windwürfen unter 2,6 ha erreichten dort immerhin noch 20-30\% der Samenmenge des Bestandes den Boden. Die Autoren geben etwa $100 \mathrm{~m}$ als Grenzentfernung für eine ausreichende Ansamung von Fichte und auch von BergAhorn an. Nach den Untersuchungen von WAGNER (1999) in experimentellen Lochhieben von Buchen-Eschen-Mischbeständen betrug die mittlere Ausbreitungsdistanz der Bucheckern etwa $8 \mathrm{~m}$, die der Eschen-Früchte dagegen $40 \mathrm{~m}$. Für die Esche wurde dabei eine Windrichtungsabhängigkeit ihrer verdrifteten Fruchtmenge festgestellt.

Folgendes Fazit lässt sich ableiten: In windgeworfenen Buchen-Wäldern auf basenarmem Buntsandstein spielt die Störungsflächengröße eine bedeutende Rolle, was sich bei abnehmender Kronenüberschirmung in ausgedehnten Pionierstrauchfluren, in sinkenden Verjüngungszahlen und sinkenden, aber weiterhin dominierenden Anteilen der Klimaxbaumart Buche ausdrückt. Für die basenreicheren Zechstein-Standorte wurde dieser Gradient nicht überprüft, jedoch lassen die wesentlich höheren Verjüngungszahlen aus dem Flächenwurf sowie der Vergleich mit anderen Buchen-Windwurfgebieten und experimentellen Lochhieben die Schlussfolgerung zu, dass zumindest auf den tiefgründigen, gut wasser- und nährstoffversorgten Dolomit-Standorten die Störungsflächengröße für eine gesicherte Naturverjüngung der Schlusswaldbaumarten keine allzu große Bedeutung haben dürfte. Hier erfolgt die Waldregeneration im Sinne der Klimaxgesellschaft ohne Pionierstadien und mit hoher Geschwindigkeit. Auf den flachgründigen Gips-Standorten läuft diese Entwicklung vermutlich stark verlangsamt ab. Unklar bleibt der Einfluss gradueller Unterschiede in der Störungsintensität, die laut Definition (Kap. 3.1.1) nicht in das grobe Schema Bestand - Lücke - Flächenwurf passen. So ist im Flächenwurf die Restüberschirmung doch recht unterschiedlich (Carici-Fagetum 1\%, Hordelymo-Fagetum 6-8\%, Galio odoratiFagetum 6-10\%, Luzulo-Fagetum 1-2\%). Eventuell trägt auch dies in unbekanntem Umfang zu Unterschieden in der Vegetationsentwicklung bei.

\subsection{Zu sonstigen Einflussfaktoren}

Eine ganze Reihe weiterer Einflussfaktoren, die größtenteils nicht mit eigenen Untersuchungen belegt werden konnten, sollen hier zusammenfassend diskutiert werden. 


\subsubsection{Vorteilsausnutzung am Sukzessionsbeginn: der Vorbestand an Bodenvegetation und Verjüngung}

Als räumlich nahes Beispiel liegt hierfür der von LugINBÜHL (2000) für das Hainholz durchgeführte Vorher-Nachher-Vergleich (1996 zu 1998/1999) vor. Demzufolge hatten z.B. Artenzahl und Krautschichtdeckung vor dem Sturm maßgeblichen Einfluss auf die Entwicklung in den Jahren danach. Im vor dem Sturm geringdeckenden Carici-Fagetum waren später höhere Artenzahlen, geringere floristische Ähnlichkeit und höhere Krautschichtdeckungen $\mathrm{zu}$ finden als im Hordelymo-Fagetum. Bezüglich der Krautschichtdeckung kommen die vorliegenden Untersuchungen zu einem anderen Ergebnis, da hier der Krautschicht-reiche Frühjahrsaspekt, insbesondere die Allium-Fazies des Hordelymo-Fagetum mit einbezogen wurde (Kap. 4.2.3). Die flachgründigen Gips/DolomitStandorte (z.B. Hainholz-KF2) waren kurz nach dem Sturmwurf zwar nicht unbedingt artenreicher (siehe 1998er Daten in Tab. A22), jedoch lichter und stärker mit Offenlandarten durchsetzt als die tiefgründigen Dolomit-Standorte (z.B. Hainholz-KF1). Nach dem Sturm nahm das Carici-Fagetum dann eine Entwicklung, bei der es sich bezüglich einiger Parameter vom Ausgangszustand weiter entfernte als die meisten anderen Bestände (betrifft vor allem Artenzahlen/Kap. 4.2.1 und qualitative floristische Ähnlichkeit/Kap. 4.2.8).

Die Nach-Windwurf-Entwicklung vieler Arten ist häufig nur mit bereits längerfristigem, wenn auch quantitativ geringfügigem Persistieren in den Vorbeständen zu erklären (FISCHER et al. 1998). So sprechen WiLMANNS et al. (1998) vom Durchhalten bzw. Neukeimen bereits vor dem Sturm vorhandener Arten als einem syndynamisch bedeutsamen Prozess auf Kahlflächen im Wald. Arten mit entsprechend angepassten Besiedlungs- und Nährstoffverwertungsstrategien sind nach der Auflichtung im Vorteil. Solche Mechanismen wurden z.B. für Calamagrostis epigejos in Kiefern-Wäldern untersucht (BRÜNN et al. 1996), nach denen die Art gleichermaßen auf Lichtstellung und erhöhtes N-Angebot mit enormen Wuchsraten reagierte. Im geworfenen Luzulo-Fagetum des Krückers machen vermutlich ebendiese Faktoren das relativ starke Wachstum von C. epigejos erklärbar. Für Rubus idaeus, die bei dichtem Kronenschluss kaum Existenzchancen hat, wird langfristiges Persistieren in kleineren Lichtlücken oder an Baumstümpfen beschrieben (z.B. UlANOVA 2000b). Ebenso untersuchten WALTER et al. (1998) das latente Vorhandensein lichtbedürftiger Brombeeren in ungestörten Beständen vor allem auf (durchforstungsbedingt) besseren Humusformen. Carex brizoides und Arrhenatherum elatius sind weitere Beispiele solcher nach Windwurf „dominanzfähigen“, bereits im Vorbestand existierenden Störzeiger (HETZEL \& REIF 1998 sowie MÄRKL \& EGGLSEER 2001 für den baden-württembergischen Fichten-Windwurf).

Auch für die Verjüngungsentwicklung und die einsetzende Wiederbewaldung nach dem Sturm ist der Vorbestand enorm wichtig. Es liegen Untersuchungen vor, bei denen der Hauptteil der Verjüngung bereits vor dem Sturm „in Warteposition“ vorhanden war (FISCHER et al. 1990; WiLlig 1994, 2002b; HetZel \& ReIF 1997, 1998; KeITEL 1998, 1999; MäRKL \& EGGLSEER 2001), bei anderen keimte der Hauptteil der jungen Bäume aber erst wesentlich 
später (SCHÖNENBERGER \& WASEM 1999; SCHÖNENBERGER 2002; WOHLGEMUTH et al. 2002). Speziell für eine Neuansamung von Baumarten scheint dabei nur ein kleines Zeitfenster zu existieren (MÄRKL \& EGGLSEER 2001). So sprechen z.B. LÄSSIG et al. (1995a+b) angesichts sehr geringer Fichten-Naturverjüngungsvorräte in den 1990 geworfenen Schweizer Bergwäldern von einem im Vergleich zu geworfenen Tieflagenwäldern ungünstigen Start. SCHÖNENBERGER (2002) führt zu denselben Untersuchungsgebieten aus, dass der Hauptteil der 10 Jahre nach dem Sturm gefundenen Sämlinge nicht aus der Vorverjüngung stammte und v.a. auf basenarmen Standorten gerade mal ein Fünftel der auf Bergwald-Standorten geforderten Verjüngungsdichte erreicht wurde. SAYER \& REIF (1998) sowie SCHMIDT-SCHÜTZ \& HuSs (1998) beobachteten in Süddeutschland diesbezüglich eher ein „Sowohl-als-auch“. Auf basenarmen oder vernässenden Fichten-Standorten setzte sich die Naturverjüngung nach Windwurf hauptsächlich aus den bereits vorhandenen Sämlingen der Fichte und neuanfliegenden Pionierbaumarten zusammen. Bei den hiesigen Buchen-Wäldern wird dagegen von hohen Verjüngungszahlen der Klimax-Baumarten vor dem Sturm ausgegangen, was auf den Buntsandstein-Standorten durch den direkten Vergleich des stehenden Bestandes mit benachbarten Lücken und Flächenwürfen deutlich hervortritt (Abb. 46 bis 48). Auch für die nicht mittels eines derartigen Gradienten untersuchten Zechstein-Standorte im Harzvorland kann diese Aussage gelten.

\subsubsection{Synergien mit anderen Störungsarten}

Für Synergie-Effekte zwischen verschiedenen natürlichen Störungsarten lassen sich in der Literatur viele Beispiele finden. Im osthessischen Bergland ließ ein Eisregen die Sukzession auf einem über 50jährigen Eichen-Kahlschlag (Luzulo-Fagetum-Standort) noch einmal von vorn ablaufen (GREGOR \& SEIDLING 1997, 1998, 1999). Borkenkäferbefall verursachte im belassenen Fichten-Windwurf des Bayerischen Waldes nach dem 1984er Sturm zunächst eine Lückenaufweitung, kam im Jahr 1989 vollständig zum Erliegen und trat mit einer erneuten Gradation erst 1996 wieder in Erscheinung (JEHL 2001). Derartige Schädlingskalamitäten spielen bei verrottendem Buchen-Holz in Mitteleuropa aber keine Rolle. Beschrieben wurde desweiteren die Lückenaufweitung in Buchen-Beständen durch Sonnenbrand (z.B. REMMERT 1987; JENSSEN \& HOFMANN 1996), was im Harzvorland bisher ebenfalls nicht beobachtet wurde. Interessant ist auch die etwa 400 Jahre zurückreichende Betrachtung in KulaKowsKi \& VeBLen (2002): Teilweise vor Jahrhunderten durch Waldbrand beeinflusste Bestände eines subalpinen Nadelwaldes in Colorado/USA waren deutlich weniger von Sturmwurf niederer bis mittlerer Intensität betroffen als Bestände, die in ihrer Geschichte nicht abgebrannt waren. Nicht zuletzt müssen bei den hiesigen Untersuchungsgebieten die Trittspuren erwähnt werden, die der Bearbeiter (aber auch das Wild) gewissermaßen als „Störung in der Störung“ hinterließ und die zur Einschleppung von Diasporen der Offenlandarten beitrugen. 


\subsubsection{Samenbank}

Die Samenbank kann die Nutzungsgeschichte eines Bestandes bis zu einem Jahrhundert lang dokumentieren (HÄRDTLE et al. 2001). Sie wird deshalb häufig auch als das „Gedächtnis des Bodens“ bezeichnet (z.B. WILMANNS et al. 1998). Die Samenbankaktivierung nach Windwurf wurde bereits von einer Vielzahl von Autoren untersucht. Samenbank und aktuelle Vegetation befanden sich dabei generell kaum in Übereinstimmung, da Waldarten nur selten eine langfristige Samenbank aufbauen. Während Waldarten als K-Strategen mehr in die Überlebensrate weniger Keimlinge investieren, verwenden Störzeiger als R-Strategen ihre Ressourcen für eine riesige Samenproduktion. Letztere sind deshalb in der Samenbank häufig überrepräsentiert (FISCHER 1987; APFFELSTAEDT \& BERNHARDT 1996; WILMANNS et al. 1998; HYATT \& CASPER 2000). Bei ihren Untersuchungen in süddeutschen Windwurfgebieten stellten WiLMANNS et al. (1998) fest, dass die meisten neuetablierten Arten aus der Samenbank aufliefen, während Einwanderung eine nachgeordnete Bedeutung besaß. Im Buchen-Windwurf auf Kalk in den Stemwehder Bergen (APFFELsTAEDT \& BERNHARDT 1996) war die Samenbank etwa 20 Jahre nach dem Sturm sowohl im Bestand als auch im Windwurf qualitativ von Waldarten und quantitativ von Schlagflurarten dominiert, wobei die Samenbank der Windwurffläche größer war. Während im Bestand vegetative Vermehrung überwog, vermehrten sich manche Waldarten (Stachys sylvatica, Milium effusum) fakultativ generativ und bildeten eine persistente Samenbank geringeren Umfangs aus. Die Autoren stellten fest, dass während langer Perioden ohne Störung der Diasporenvorrat der Störzeiger im Wald abnimmt. Zu ähnlichen Ergebnissen kam auch WESTPHAL (2001) bei Samenbankuntersuchungen wiederbewaldeter Ackerflächen in der Lüneburger Heide. Dort wurde mit zunehmendem Alter des Waldbestandes ein abnehmender Anteil von Nichtwaldarten in der Samenbank bei gleichzeitig ansteigendem Waldartenanteil sowie eine sinkende prozentuale Übereinstimmung zwischen Samenbank und aktueller Vegetation gefunden. Desweiteren fanden ABS et al. (1999) bei einem Urwald-WirtschaftswaldVergleich im ostpolnischen Bialowiesa (Tilio-Carpinetum) nur geringfügige floristische Unterschiede, dafür aber umso größere in der Samenbank (im Urwald Samenbank insgesamt kleiner und Störzeiger viel seltener). In Windwurflücken eines Buchen-Eichen-Waldes in Pennsylvania (USA) untersuchten HyATT \& CASPER (2000) Sameneintrag, Keimung und Samenmortalität. Danach beeinflussen diese Faktoren bereits im ersten Jahr die räumliche Verteilung von Artendichte und -diversität in der langfristigen Samenbank. Rubus allegheniensis war dort ein Beispiel, bei dem stark dominantes Auftreten in der Samenbank und auch in der Lückenvegetation übereinstimmten. Wollte man bei den vorliegenden Untersuchungen die Abundanzanteile der Ruderal- und Schlagflurarten als indirektes Maß für den Umfang der Samenbankaktivierung nach dem Sturm nehmen (Kap. 4.3.5), so erscheinen besonders die langjährig bewirtschafteten Bestände auf Buntsandstein (Krücker, RotenbergAbt. 1228), aber auch die Gips/Dolomit-Kuppe des Bollerkopfes (Hainholz-KF2) als stark mit 
Störzeigern durchsetzt. Dies ist aber mit Vorbehalt zu betrachten, da weder der Sameneintrag von außen noch die Samenbank des Bodens untersucht wurden.

\subsubsection{Verbissbelastung}

Einerseits ist es unstrittig, dass der Wildverbiss entscheidend für die Verjüngungsentwicklung sein kann und bei entsprechendem Angebot auch ein „Gradient der Verbissattraktivität“ bestimmter Baumarten existiert. So fanden MAYER et al. (1980) in kroatischen Urwäldern einen geringer werdenden Verbiss in der Reihenfolge Tanne $>$ Fichte $>$ Buche. Auch im Vergleich zu vielen Edellaubhölzern ist die Attraktivität der Buche eher gering, so dass selektiver Wildverbiss die Baumartenzusammensetzung in der Verjüngung entmischen und den Trend zu Buchen-dominierten Beständen fördern kann (STRIEPEN 1999 für nordrheinwestfälische Buchen-Naturwaldreservate).

Andererseits wird die Bedeutung der Verbissbelastung speziell in Windwurf- und sonstigen Waldschadensgebieten in der Literatur sehr unterschiedlich eingeschätzt. So bezeichnen BURSCHEL \& Binder (1993) den Wildverbiss als einziges Problem bei der natürlichen Wiederbewaldung in Bergmischwäldern bayerischer Mittelgebirge und der Alpen. Ähnlich äussern sich LÄssıG et al. (1995a+b) für die Schweizer Bergwälder nach „Vivian/Wiebke“. Auf belassenen Windwurfflächen scheint die Verbissbelastung tendenziell geringer zu sein als auf geräumten, was teilweise mit einem Zauneffekt der Verhaue begründet wird. Derartige Unterschiede fanden z.B. HANKE \& PISOKE (1999) im Sturmwurfbannwald Teufelsries/Baden-Württemberg, HeTZEL \& REIF (1996) auf der Schwäbischen Alb sowie FISCHER \& JEHL (1999) im Nationalpark Bayerischer Wald.

Es liegen aber auch Untersuchungen vor, die zu teilweise anderslautenden Schlüssen kommen. Im Bayerischen Wald wurde von JeHL (2001) ein abnehmender Verbiss bei Fichte und Vogelbeere vom 8. zum 13. Jahr nach dem 1984er Sturmwurf festgestellt. Etwa 10 Jahre nach den 1990er Stürmen bezeichnen auch SENN et al. (2002) den Einfluss des Verbisses auf Naturverjüngung und Pflanzung in ehemals Fichten-dominierten Windwurfgebieten der Schweizer Nordalpen als vernachlässigbar gering und fanden auch - abgesehen vom etwas stärkeren Verbiss von Vogelbeere und Hochstauden wie Epilobium angustifolium - kaum Unterschiede zwischen gezäunten und ungezäunten Plots. Die Geschwindigkeit, mit der die jungen Bäume aus der Verbisszone herauswachsen, hat somit eine große Bedeutung. Ebenso kam eine weitere Untersuchung aus dem Schweizer Nationalpark zu dem Ergebnis, dass der Verbiss insbesondere durch das Rotwild den Sukzessionsverlauf in einem durch Schneedruck gestörten Pinus montana-Bestand kaum beeinflusste (KRÜSI \& MOSER 2000). Auch bei Zäunungsexperimenten auf kleineren Waldlichtungen in der dortigen subalpinen Stufe (CAMENISCH \& SCHÜTZ 2000) liessen sich weder bezüglich der Artenvielfalt noch der Bodenvegetation oder der Baumverjüngung unterschiedliche Entwicklungen inner- und ausserhalb des Zaunes feststellen. 
Neben der Verbissattraktivität einzelner Baumarten, der Wuchsleistung der Verjüngung und der Räumung bzw. Belassung von Windwurfflächen spielt auch die Art der herbivoren Großsäuger eine Rolle. In den hiesigen Untersuchungsgebieten existiert nur Reh-, kein Rotwild. Ob die Verhaue im belassenen Windwurf tatsächlich einen Zauneffekt ausbilden, mag dahingestellt sein. Es ist ebenso gut möglich, dass speziell das Rehwild bei ausreichendem Nahrungsangebot gerade diese Bereiche aufsucht, sobald das unübersichtliche Terrain erkundet und die Fluchtwege sicher sind. Ein verjüngungsschädigender Wildeinfluss kann zumindest für die Bestände auf tiefgründigen Dolomit-Standorten nahezu ausgeschlossen werden, was aus den hohen Verjüngungszahlen (Kap. 4.5) und der großen Wuchsleistung der Eschen-dominierten Strauchschicht im vierten Jahr nach dem Sturm geschlossen werden kann (im Mittel $4 \mathrm{~m}$ hoch, Abb. 25). Die KF2 im Naturwald Königsbuche auf Buntsandstein ist zudem gezäunt (Tab. 1).

Abschließend soll erwähnt werden, dass auch der Verbiss durch Kleinsäuger einen nicht unbeträchtlichen Einfluss auf Naturverjüngung oder Pflanzung haben kann (CAMENISCH \& SCHÜTZ 2000; ОTт 2002).

\subsubsection{Mykorrhiza}

Die Mykorrhizierung ist für die Etablierung junger Bäume in Windwurfgebieten sehr wichtig. So ist z.B. bekannt, dass dieser Prozess bei jungen Fichten etwa 3 Monate nach der Keimung beginnt. Während die Fichte mehrere Mykorrhizapartner nutzen kann, benötigt die Buche ganz spezielle (HeTzEL \& REIF 1998; ReXer et al. 1999). Der Artenvielfalt der MykorrhizaPilze war nach den Untersuchungen von REXER et al. (1999) auf geräumten Windwurfflächen geringer als auf belassenen bzw. im stehenden Bestand. Während natürlich mykorrhizierte Bäumchen aus der Naturverjüngung vergleichsweise resistent gegenüber fakultativ pathogenen Pilzen sind, stellten GÖRKE \& OBERWINKLER (1999) bei gepflanzten Baumexemplaren einen relativ starken Befall mit diesen Pilzen fest. Dies kann vor allem unter klimatischen Stressbedingungen zum Selektionsfaktor werden. Somit existieren für die Naturverjüngung (besonders der Buche) nach Windwurf wesentlich bessere Startchancen als für gepflanzte Exemplare (FISCHER et al. 1998). Dies ist ein wichtiger Grund, bereits vorhandenen Jungwuchs in die Wiederbewaldung von Sturmwurfflächen einzubeziehen und bodenschonende Räumverfahren zu benutzen. Da hierzu keine Untersuchungen aus dem Harzvorland vorliegen, können solche Einflüsse nur vermutet werden. Es ist z.B. auffällig, dass innerhalb der Zechstein-Standorte die geräumten Nüllberg-Flächen trotz nachfolgender Bepflanzung eher noch geringere Baumarten-Deckungen erreichen als die standörtlich vergleichbare, aber belassene Hainholz-KF1. Im Buntsandstein trifft allerdings genau das Gegenteil zu: die bepflanzten Flächen sind bezüglich der Baumarten deckungsstärker als die belassenen (Kap. 4.3.6 und Tab. A22, A23). 


\subsubsection{Alter des Waldstandortes und Mechanismen der Artenausbreitung nach Störung}

Mehr als 30 der in BonN \& PosCHLOD (1998) zusammengestellten Zeigerarten historisch alter Wälder kommen auch in den UG des Harzvorlandes häufiger vor (Tab. A22 bis A25). Deshalb lassen sich die hiesigen Windwurfgebiete nach ZACHARIAS (1994) als historisch alte Wälder („ancient forests“) einordnen. Typische Waldarten haben in der Regel eine artspezifisch eingeschränkte Ausbreitungsfähigkeit und eine langsame Kolonisationsrate, keine langfristige Samenbank, überwiegend myrmeko- und autochore Nahausbreitungsmechanismen, zeigen eine Bindung an feucht-schattige Standorte und gehören überwiegend zu den Querco-Fagetea. Demgegenüber finden sich in historisch jungen Sekundärwäldern („recent forests“) weniger Querco-Fagetea-Arten, verstärkt Arten mit zoound anemochoren Fernausbreitungsmechanismen, die $\mathrm{zu}$ den Pioniergehölzen, Offenlandrelikten, nitrophilen Saumarten und Verlichtungszeigern gerechnet werden können (Hermy et al. 1993; Zacharias 1993, 1994; Bonn \& Poschlod 1998; Heinken 1998). Letzteres gilt in den hier untersuchten Windwurfgebieten ja eher für die störungsbedingten Vegetationsmuster (Artengruppenspektren in Kap. 4.3; Tab. 16, 17). Nach HeRMY et al. (1993) ergibt sich nach einer europaweiten Zusammenstellung von Arten (ungestörter) historisch alter Wälder die in Tab. 19 dargestellte Aufteilung bezüglich der Ausbreitungstypen. In den damit verglichenen Windwurfgebieten fällt der relativ hohe Anteil der Anemo- und der Epizoochoren auf, welches offenbar die bevorzugten Verbreitungsmuster der Störungszeiger sind. Auch WilmanNs et al. (1998) nennen besonders Anemo- und Zoochorie als Ausbreitungsstrategien neueinwandernder Arten auf Kahlflächen im Wald.

Tab. 19: Vergleich der Ausbreitungstypenanteile von Arten historisch alter Wälder in Europa und den hier untersuchten Windwurfgebieten (Angaben aus dem Harzvorland entsprechend dem qualitativen Spektrum in Tab. 11, basierend auf Tab. A22, A23).

\begin{tabular}{|l|c|c|c|c|}
\hline & $\begin{array}{c}\text { typische Arten } \\
\text { historisch alter } \\
\text { Wälder in Europa } \\
\text { (Liste in HERMY et } \\
\text { al. 1993) }\end{array}$ & $\begin{array}{c}\text { Anteil an der } \\
\text { Gesamtartenzahl der } \\
\text { einbezogenen } \\
\text { Waldgebiete } \\
\text { (HERMY et al. 1993) }\end{array}$ & $\begin{array}{c}\text { Buchen-Wälder im } \\
\text { Harzvorland } \\
\text { 2 Jahre nach } \\
\text { Windwurf }\end{array}$ & $\begin{array}{c}\text { Buchen-Wälder im } \\
\text { Harzvorland } \\
\text { 4 Jahre nach } \\
\text { Windwurf }\end{array}$ \\
\hline Myrmekochore & $40 \%$ & $12 \%$ & $2-10 \%$ & $2-8 \%$ \\
\hline Anemochore & $20 \%$ & $35 \%$ & $\mathbf{3 7 - 4 7 \%}$ & $\mathbf{4 0 - 4 8 \%}$ \\
\hline Endozoochore & $22 \%$ & $30 \%$ & $14-19 \%$ & $13-20 \%$ \\
\hline Epizoochore & $9 \%$ & $5 \%$ & $\mathbf{1 5 - 2 1 \%}$ & $\mathbf{1 7 - 2 2 \%}$ \\
\hline Hydrochore & $4 \%$ & $12 \%$ & $0-1 \%$ & $0-1 \%$ \\
\hline Baro- und Autochore & $5 \%$ & $4 \%$ & $11-22 \%$ & $10-17 \%$ \\
\hline
\end{tabular}

Durch die oben genannten Eigenschaften typischer Waldarten stellte sich das biotische Potenzial historisch alter Wälder nacheiszeitlich erst in einem langen Prozess ein (nach Angaben verschiedener Autoren in ZACHARIAs 1994 etwa 350-800 Jahre). Aktuell wird dadurch sowohl die Besiedlung jüngerer Wälder als auch die Wiedererlangung der alten Populationsgröße nach einem Wechsel der Standortsbedingungen (z.B. Durchforstung, Einbringung fremder Baumarten, völlige Zerstörung) erschwert, wobei dieser Nachteil durch 
Großflächigkeit des Waldstandortes und durch Restpopulationen der entsprechenden Art in benachbarten Refugien relativiert wird (HERMY et al. 1993). Da es sich aber bei Windwurf nicht um einen die Vegetation und den Standort völlig zerstörenden Prozess handelt, das Waldarteninventar in der Sukzession nachweislich erhalten bleibt (Kap. 4.3.5) und der nächste intakte Bestand kaum mehr als ein paar hundert Meter entfernt steht, ist hier nicht mit extrem langen Regenerationszeiträumen zu rechnen. Ein Blick nach Nordamerika zeigt, dass Störungsarten wie Feuer oder Vulkanausbruch den Standort viel stärker beeinträchtigen als ein Windwurf und zu wesentlich länger andauernden Regenerationsprozessen führen. Aber auch dort ist die Richtung der ablaufenden Sukzessionen stark von der Überlebensrate der alten Waldvegetation abhängig (HALPERN 1988; HALPERN et al. 1990; DE GRANPRE et al. 2000).

\subsection{Windwurfbedingte Kleinstandorte (microsites)}

\subsubsection{Kronen- und Stammverhaue geworfener Laubbäume}

Mit 20-35\% mittlerer Totholzdeckung und 0-14\% lebenden Kronenteilen wurden im belassenen Windwurf des Harzvorlandes im vierten Jahr nach dem Sturm ähnlich hohe Verhaudeckungen ermittelt wie z.B. von HeTzEL \& REIF (1998) im Fichten-Windwurf der Schwäbischen Alb (dort etwa 33\% Verhaudeckung, aufsummiert aus Stammholz, Gipfel und Reisighaufen). Im Harzvorland lässt sich im Untersuchungszeitraum eine teils rapide (Königsbuche-KF2, Hainholz-KF2), teils sehr langsame Abnahme (Hainholz-KF1) des Deckungsgrades liegend-lebender Baumkronen feststellen (siehe auch ScHMIDT 2002a), worin sich neben der standörtlichen Situation vermutlich auch noch weitere individuelle Faktoren widerspiegeln. Unklar ist beispielsweise, warum gerade der flachgründige Gips/Dolomit-Standort des Bollerkopfes (Hainholz-KF2) und der Buntsandstein-Standort am Krücker-Osthang noch die höchsten Deckungen lebender Kronen aufweisen (9 bzw. 14\% in 2001, siehe Tab. A22, A23). Wahrscheinlich werden viele Stämme noch eine ganze Zeit lang austreiben und fruktifizieren. Zur Überlebensdauer geworfener Laubbaumkronen gibt es in der Literatur kaum Angaben. Häufig werden „mehrere Jahre“ als Zeitraum angegeben (FALINSKI 1976; KoOP 1982; KeITEL 1998, 1999). Aus dem norddeutschen Naturwald Kienmoor berichtete UNKRIG (mündliche Mitteilung) von einer seit 30 Jahren am Boden liegenden Eiche, die immer noch austreibt. Aus südwestdeutschen „Lothar“Windwurfgebieten nach 1999 nennt ALDINGER (unveröffentlicht) speziell für die Buche stark differierende Überlebensraten von 9\% nach 4 Jahren (Bannwald Burghard), 21\% nach knapp 2 Jahren (Bannwald Ofenberg) und aber auch 0\% nach 9 Monaten (Bannwald Rötteler Wald). Ein weiteres Phänomen ist in diesem Zusammenhang das vegetative Ausbreitungsvermögen vieler Waldbaumarten, das von der Forstwirtschaft lange unterschätzt wurde. So beschreibt KоOP (1987) u.a. aus nordwestdeutschen Naturwäldern das vollständige Überleben und die vegetative Vermehrung geworfener Bäume bei weiterem Wurzelkontakt. An geworfenen 
Stämmen z.B. von Carpinus betulus und Fagus sylvatica, die auf Wurzeltellerbuckeln benachbarter Bäume liegen, beobachtete er die Bildung von stammbürtigen Adventivwurzeln sowie von stammbürtigen Ausläufern. Letztere bewurzeln sich und führen zur Etablierung neuer Individuen, obwohl der geworfene Baum stirbt. Weiterhin kann die Bewurzelung nach unten gebogener Äste oder Stammteile erfolgen. Für Fraxius excelsior wird die vegetative Vermehrung durch senkrecht wachsende, stammbürtige Sprosse beschrieben (KoOP 1987). Dabei dichtet sich der geworfene Baum hinter dem letzten dieser Sprosse mit einem Wundkallus gegen den weiterfaulenden Stammteil ab und überlebt auf diese Art zumindest teilweise. Solche senkrecht wachsenden, stammbürtigen Sprosse an geworfenen EschenStämmen waren nach dem 1997er Sturm auch im Hainholz öfters zu beobachten.

Zur Zersetzungsgeschwindigkeit der Baumleichen lassen sich verschiedene Angaben finden. Beipielsweise gehen HoMANN \& ENGELS (1991) bei den Bergfichtenwäldern vernässter Hanglagen im Nationalpark Bayerischer Wald von etwa 100-200 Jahren aus. Dagegen nennt KORPEL (1995) etwa 20-30 Jahre für Laubbäume und bis zu 50 Jahre für Nadelgehölze in den Karpaten. Ebenfalls etwa 20 bis 30 Jahre (auf sehr feuchten Standorten kürzer) beziffert KoOP (1982) für ehemalige norddeutsche Hudewälder (Neuenburg, Hasbruch) und einen ostpolnischen Urwald (Bialowiesa). Auf Kleinstandorten mit vermodernden Baumleichen wuchsen in den letztgenannten Wäldern typischerweise Arten wie z.B. Athyrium filix-femina, Dryopteris dilatata, Milium effusum, Oxalis acetosella, Geranium robertianum und eine Phegopteris-Art. Hingegen verhindern solche Strukturen die Ansiedlung z.B. von Allium ursinum etwa noch 5-10 Jahre nach ihrem vollständigen Verschwinden (FALINSKI 1976, 1978). In den vorliegenden Untersuchungen wurden keine gesonderten Vegetationsaufnahmen für stamm- oder kronennahe Kleinstandorte gemacht. Jedoch erscheint es auf den ersten Blick einleuchtend, dass die Vegetation in den stark schattigen und klimatisch ausgeglicheneren Verhauen eine andere Entwicklung nimmt als auf deren Umgebungsflächen. Auf diesen wiederum nehmen aber Verjüngung oder Pionierstrauchfluren zu, so dass es mit zunehmender Strauchschichtdeckung zu einer Nivellierung der anfangs vorhandenen kleinstandörtlichen Unterschiede kommen dürfte. Nach den Untersuchungen von HeTZEL \& REIF (1998) in Fichten-Windwurfgebieten erwiesen sich besonders Rubus idaeus, Sambucus racemosa, Arrhenatherum elatius und Impatiens noli-tangere als Besiedlungsspezialisten der Stamm- und Kronenbereiche. In der Literatur vielfach belegt ist auch die Moderholz- bzw. Rannenverjüngung der Fichte (LÄSSIG \& SCHÖNENBERGER 1993, 2002; ReIF \& PRZYBILla 1995; UlANOVA 2000b; JEHL 2001), die aber für die im Harzvorland vorherrschenden Laubbaumarten keine Bedeutung hat.

\subsubsection{Wurzelteller}

Windwurfbedingte Bodenstörungen durch aufgeklappte Wurzelteller sind ein wesentliches und teilweise noch sehr lange Zeit sichtbares Merkmal von natürlich gestörten 
Waldökosystemen. Für den Artenreichtum naturnaher Wälder hat die Bodenstörung durch Wurzelteller möglicherweise eine größere Bedeutung als Lücken im Kronendach (NAKASHIZUKA 1989 für temperate Mischwälder Westjapans). Darüber hinaus begünstigt sie die Etablierung neuer und stabiler Baumgenerationen (UlANOva 2000b für boreale FichtenWälder Zentralrusslands). Einen Überblick zu kleinstandörtlichen Parametern - insbesondere zu Wurzeltellern - gibt Tab. 20.

Tab. 20: Verschiedene kleinstandörtliche Parameter in Windwurfgebieten, getrennt nach Autor und Region.

\begin{tabular}{|c|c|c|c|}
\hline Autor und Gebiet & Wurzeltellerdichte & $\begin{array}{c}\text { Bodendeckung } \\
\text { durch Wurzelteller }\end{array}$ & sonstige Angaben \\
\hline $\begin{array}{l}\text { FALINSKI (1976, 1978): Bialowiesa- } \\
\text { Urwald (Ostpolen), „Normalfall“ eines in } \\
\text { der Alterungsphase befindlichen Tilio- } \\
\text { Carpinetum ohne katastrophalen } \\
\text { Windwurf }\end{array}$ & $\begin{array}{l}\text { bei durchschnitt- } \\
\text { lichem jährlichem } \\
\text { Windwurf: } \\
\text { 2-4,5 Stämme/ha }\end{array}$ & $\begin{array}{l}\text { bei durchschnitt- } \\
\text { lichem jährlichem } \\
\text { Windwurf: } \\
2 \%\end{array}$ & $\begin{array}{l}\text { Wurzeltellerfläche } \\
\text { (Fichte): } 2-40 \mathrm{~m}^{2}\end{array}$ \\
\hline $\begin{array}{l}\text { LYFORD \& MCLEAN (1966), zitiert in } \\
\text { ELLENBERG et al. (1986): häufig durch } \\
\text { Tornados geworfene kanadische } \\
\text { Küstenwälder }\end{array}$ & $\begin{array}{l}\text { 1000/ha } \\
\text { (etwa 100/ha müssen } \\
\text { in Nordamerika als } \\
\text { „normal“ gelten) }\end{array}$ & k.A. & k.A. \\
\hline $\begin{array}{l}\text { ULANOVA (2000): boreale Fichten- } \\
\text { Wälder des zentralrussischen Tieflands }\end{array}$ & k.A. & $\begin{array}{l}\text { Flächenwurf: } \\
\text { 15-25\% }\end{array}$ & k.A. \\
\hline $\begin{array}{l}\text { HETZEL \& REIF (1998): Fichten-Wälder } \\
\text { der Schwäbischen Alb }\end{array}$ & k.A. & $\begin{array}{l}\text { Flächenwurf: } \\
6 \%\end{array}$ & k.A. \\
\hline $\begin{array}{l}\text { JEHL (2001): Berg-Fichtenwälder des NP } \\
\text { Bayerischer Wald }\end{array}$ & $\begin{array}{l}\text { Flächenwurf: } \\
\text { 100-600/ha }\end{array}$ & k.A. & $\begin{array}{l}\text { mittlere } \\
\text { Wurzeltellerfläche } \\
\text { (Fichte): } 5 \mathrm{~m}^{2}\end{array}$ \\
\hline $\begin{array}{l}\text { KOMPA (vorliegende Untersuchungen): } \\
\text { Buchen-Wälder im südwestlichen } \\
\text { Harzvorland }\end{array}$ & $\begin{array}{l}\text { Flächenwurf: } \\
\text { 200-400/ha }\end{array}$ & $\begin{array}{l}\text { Flächenwurf: } \\
4-8 \%\end{array}$ & $\begin{array}{l}\text { mittlere } \\
\text { Wurzeltellerfläche } \\
\text { (Buche): } 2-4 \mathrm{~m}^{2}\end{array}$ \\
\hline
\end{tabular}

Das langfristig durch eingeebnete Wurzelteller entstandene Kleinrelief (Wurfböden, moundand-pit topography) wird von ELLENBERG et al. (1986) als Indiz eines historisch alten Waldstandortes gewertet, der niemals unter den Pflug kam. Nach den Untersuchungen aus dem Solling (Niedersachsen) stammten besonders große Wurzeltellerbuckel aus der Zeitepoche der Waldweide, da durch die Auflichtung einzelne Buchen besonders ausladende Kronen- und Wurzelsysteme ausbilden konnten. Bei Böden mit Staunässeneigung sind die Wurzelteller flach und bleiben lange stabil, während sie auf flachgründigen Rendzinen schneller zerfallen und die Physiognomie der Windwurfflächen nur relativ kurze Zeit prägen (ELLENBERG et al. 1986). Dies ist im Harzvorland auch heute schon in der relativ bodenfeuchten Königsbuche (pseudovergleyte Parabraunerde) im Gegensatz zum relativ trockenen Bollerkopf (Rendzina) zu beobachten oder mindestens zu erahnen. Die Einebnungsdauer einer Wurzeltellermulde wird von FALINSKI $(1976,1978)$ im ostpolnischen Bialowiesa-Urwald mit etwa 8 Jahren angegeben. Dort werden auch ohne großflächigen Windwurf etwa zwei Drittel der Bestandesfläche innerhalb von 10 Jahren in die kleinstandörtliche Dynamik gefallener Bäume einbezogen und durch sie nachhaltig verändert. Dagegen geht UlANOVA (2000) beim borealen Fichten-Wald Zentralrusslands von einer 
(vollständigen) Rekonstruktionsdauer der alten Bodenverhältnisse im Bereich der Wurzelteller von etwa 200-300 Jahren aus, bei flachgründigen Böden von etwa 100-200 Jahren.

Die frisch aufgeklappten Wurzelteller in Windwurfgebieten müssen als Extremstandorte gelten. Die Niederschlagsmenge pro Grundfläche ist relativ gering, der Grundwasseranschluss nicht mehr vorhanden und die Austrocknungsneigung relativ hoch (FISCHER et al. 1990). Die Wurzeltellerböden unterscheiden sich nach ELLENBERG et al. (1986) gegenüber dem nicht aufgebrochenen Boden der umgebenden Fläche durch größeres Porenvolumen, größere Luftkapazität, schnellere Wasserinfiltration, höhere pH-Werte, höhere Basensättigung und höhere Schwermetallgehalte.

Unter diesen Voraussetzungen stellt sich natürlich die Frage nach der Bedeutung der Wurzelteller für eine pflanzliche Besiedlung. In der Literatur werden Wurzelteller auffällig häufig als bevorzugte Keim- und Etablierungsplätze für anfliegende Samen von Fichte und/oder Pionierbäumen genannt (NAKASHIZUKA 1989; HoMANN \& ENGELS 1991; REIF \& Przybilla 1995; HetZel \& Reif 1998; Ulanova 2000b; Jehl 2001; Wohlgemuth et al. 2002). Im baden-württembergischen Fichten-Windwurf kam es trotz dieses häufigen Anfluges von Fichte und Pionierbäumen langfristig nicht zur Etablierung der Bäume (FISCHER et al. 1998). Auch in den Hochlagen des Bayerischen Waldes fanden REIF \& PrzYBILla (1995) auf sehr alten Wurzeltellern keine Fichten-Verjüngung mehr. Demgegenüber konnten KULAKOWSKI \& VEBLEN (2003) noch 65 Jahre nach dem Windwurf in einem subalpinen Nadelwald Colorados/USA Wurzelteller als bevorzugte Keim- und Siedlungsplätze für Abies lasiocarpa und Picea engelmannii ausmachen.

Unter den einzelnen Wurzeltellerabschnitten gelten die Mulden (pits) als besonders verjüngungsfeindlich, da sie mit Laub zugeweht werden oder stark vernässen. Dort wachsen Feuchtezeiger, teilweise sogar initiale Wasser- und Sumpfpflanzenvegetation (FALINSKI 1976, 1978; KoOP 1982). Dagegen werden die Wurzeltellerbuckel oder deren Randbereiche häufig als Besiedlungsorte für Baumartenverjüngung angegeben (LYFORD \& MCLEAN 1966 zitiert in ELLENEBERG et al. 1986; LÄSSIG \& SCHÖNENBERGER 1993; BATTAGLIA et al. 2000). Weiterhin wurden v.a. Moose als wichtige (Erst-)Besiedler beobachtet (KoOP 1982; ELLENBERG et al. 1986). In anderen Untersuchungen werden Wurzelteller allgemein als Etablierungsort für Pionierstadien genannt, wobei Rubus idaeus als Spezialist dieser Kleinstandorte gilt (KoOP 1982; FISCHER et al. 1990; HETZEL \& REIF 1998; MCALISTER et al. 2000).

Im Gegensatz dazu lassen sich kaum Beispiele finden, in denen aufgeklappte Wurzelteller als bevorzugte Etablierungsorte für die Verjüngung von Schlusswald-Laubbaumarten fungieren. Nach den Untersuchungen von HetzeL \& ReIF (1998) in windgeworfenen FichtenReinbeständen auf Kalk-Standorten der Schwäbischen Alb dominierte die Fichte zwar die Verjüngung auf Wurzeltellern (Anflug und Keimung auf Rohboden), Laubbäume jedoch die der Umgebungsflächen. In ehemaligen Buchen-Reinbeständen waren die Wurzelteller dort 
deutlich verjüngungsärmer (Buche und Esche) als die Umgebungsflächen. Offenbar kann die Buche (möglicherweise alle zoochoren Baumarten mit großen Samen?) wegen mangelndem Eintrag den Rohbden kaum zur Keimung nutzen. Auch bezüglich der Lebens- und Ausbreitungsstrategien ließen sich nach Literaturangaben Wuchsortpräferenzen finden. Auf Wurzeltellern eines Quercus-Pinus-Bestandes auf sandig-trockenem Standort in Minnesota/USA dominierten annuelle und bienne Arten (PALMER et al. 2000). In temperaten westjapanischen Mischwäldern bevorzugten anemochore Arten mit kleinen Samen den gestörten Waldboden zur Etablierung, während zoochore Arten mit großen Samen keine Verbreitungspräferenzen besaßen (NAKASHIZUKA 1989). An gestörten Stellen kann generative Vermehrung dominieren, auf ungestörtem Waldboden dagegen vegetative (ULANOVA 2000b für boreale Fichten-Wälder Russlands). Im Gegensatz dazu stellten PALMER et al. (2000) bei ihren Wurzeltelleruntersuchungen in Minnesota/USA fest, dass sich - ganz ähnlich wie im Harzvorland - die qualitativen Wuchsformenspektren der Wurzelteller und die ihrer Umgebungsfläche sehr ähnlich waren und sich im mehrjährigen Sukzessionsverlauf kaum veränderten. Das galt dort ebenso für die Wuchsformenspektren der verschwundenen und der neu auftretenden Arten.

Im Harzvorland lässt sich mit den vorliegenden Untersuchungen bestätigen, dass die Wurzelteller deutlich stärker mit Kryptogamen (v.a. Moosen) besiedelt waren als die Umgebungsfläche (Tab. 14). Das (qualitative) Arteninventar der Wurzelteller und der sie umgebenden Flächen war sich innerhalb der ersten vier Jahre nach dem Sturm erstaunlich ähnlich und blieb konstant. Dagegen trat bei den Dominanzverhältnissen sowohl auf den Wurzeltellern als auch auf den $100 \mathrm{~m}^{2}$-Plots eine teilweise beträchtliche, standörtlich differenzierte Dynamik innerhalb des Untersuchungszeitraumes auf (Abb. 54 bis 59). Während sich auf der flachgründigen Gipskuppe des Bollerkopfes (Hainholz-KF2) Wurzelteller und umgebende Fläche bezüglich der Abundanzanteile verschiedener Artengruppen fast identisch entwickelten, waren die Wurzelteller auf den tiefergründigen Buntsandstein- und Dolomit-Standorten bevorzugte Ausbreitungsorte für Schlagflurarten, Sträucher, Hemikryptophyten und Lichtzeiger, auf Buntsandstein zusätzlich noch für Feuchteund Stickstoffzeiger sowie für Arten ohne vegetatives Ausbreitungsvermögen. Dagegen waren typische Waldarten, Geophyten und Baumjungwuchs auf diesen Wurzeltellern wesentlich schwächer verbreitet als auf der Umgebungsfläche. Die Wurzelteller sind also im vorliegenden Fall keineswegs als Verjüngungsschwerpunkt der Schlusswaldbaumarten aufzufassen, was die o.g. Beobachtungen von der Schwäbischen Alb (HETZEL \& REIF 1998) und die Vermutung einer generellen „Etablierungsungunst“ u.a. für Buche auf Wurzeltellern zu bestätigen scheint. Insgesamt kristallisierten sich im Untersuchungszeitraum nachfolgend aufgeführte „Besiedlungsspezialisten“ der Wurzelteller heraus, wobei die Himbeere auffälligerweise immer an erster Stelle genannt werden muss (vergleiche Tab. A26 bis A31; mit Wuchsortpräferenz nach Deckungsgrad): 
1. Carici-Fagetum auf flachgründiger Rendzina über Gips/Dolomit (Hainholz-KF2): Rubus idaeus (Mulde), Impatiens parviflora (Mulde), Atropa bella-donna (Oberseite, Mulde), Mycelis muralis (Mulde) und Senecio ovatus (Mulde).

2. Hordelymo-Fagetum auf tiefgründigem Dolomit-Verwitterunsglehm (Hainholz-KF1): Rubus idaeus (Oberseite, Mulde), Galeobdolon luteum (Mulde) und Atropa bella-donna (Oberseite).

3. Galio odorati-Fagetum auf Löss über Buntsandstein (Königsbuche-KF2): Rubus idaeus (Mulde), Sambucus racemosa (Oberseite), Athyrium filix-femina (Mulde) und Juncus effusus (Mulde).

Nach nordamerikanischen Studien finden die größten Veränderungen in den ersten Jahren nach dem Windwurf statt. Danach verlangsamt sich dieser Prozess, kommt aber durch den zunehmenden Zerfall der Wurzelteller auch nicht völlig zum Stillstand. Teilweise findet diese „sukzessionale Annäherung“ der Kleinstandorte innerhalb weniger Jahre statt (7-8 Jahre bei PEterson \& PiCKetT 1995), während andere Autoren (z.B. BEATTY 1984) über jahrzehntelang anhaltende floristische Unterschiede zwischen alten Wurzeltellerbuckeln und deren Umgebungsflächen berichten. Nach dem Beispiel eines geworfenen Pinus strobusBestandes auf sandigem Standort in Minnesota (MCALISTER et al. 2000) wurden die einzelnen Wurzeltellerabschnitte der umgebenden Fläche im Verlauf von 14 Jahren immer ähnlicher. Scheinbar sind die Bodenbedingungen (lehmig-feucht oder sandig-trocken) für diese Angleichungsgeschwindigkeit ausschlaggebend. Auch im Harzvorland ist mit dieser Angleichung zu rechnen, was auf den tiefgründigeren Dolomit- und Buntsandstein-Standorten langsamer von statten geht als auf dem flachgründigen Gips/Dolomit-Standort. Insgesamt veränderten sich die Wurzelteller floristisch stärker als die Umgebungsflächen, was sowohl anhand qualitativer als auch quantitativer Indizes festgestellt werden konnte (Kap. 4.6.5). Obwohl keine generell nur auf Wurzeltellern vorkommende Art registriert wurde, ergab sich ein standortsabhängig unterschiedlich hoher Prozentsatz von Arten, für die der Wurzelteller in manchen Untersuchungsflächen der alleinige Wuchsort war (betraf 0-50\% der Strauchschichtund 18-41\% der Feldschichtarten). Auf den tiefgründigen, Strauchschicht-reichen Standorten (Dolomit, Buntsandstein) spielten die Wurzelteller als „Ausweichlebensraum“ für bestimmte Arten eine tendenziell größere Rolle als auf dem flachgründigen, Strauchschicht-armen Gips/Dolomit-Standort (Kap. 4.6.8). Für 4 Strauch- und 54 Feldschichtarten konnte eine Bedeutungszunahme der Wurzelteller als (gelegentlich) alleiniger Wuchsort festgestellt werden. Dabei handelte es sich meist um Licht-, Halbschatten- und Mäßigstickstoff-zeigende Arten unterschiedlicher soziologischer Zuordnung, bei denen man vermuten könnte, dass sie konkurrenzbedingt zunehmend auf die Wurzelteller „ausweichen“. 


\subsection{Zusammenfassende Diskussion: Windwurfdynamik in Wäldern}

\subsubsection{Typische Sukzessionsmuster im überregionalen Vergleich}

Die Übersicht über die Naturverjüngung und die Bodenvegetation in Tab. 21 konzentriert sich auf großflächige Windwurf- und Kahlschlagflächen in Deutschland (Harzvorland, Vogelsberg, Schwarzwald, Schwäbische Alb, Neckarland, Bayerischer Wald) und in den Schweizer Alpen.

Aus der tabellarischen Zusammenstellung kann abgeleitet werden, dass sich überregional ähnliche Sukzessionsmuster in erster Linie entlang des Gradienten der Basenversorgung ergeben und andere Faktoren, wie z.B. die Vorbestockung, die Räumung/Belassung und die Störungsart (Windwurf/Kahlschlag) einen wichtigen, aber nachgeordneten Einfluss haben.

Tab.: 21: Verjüngungszahlen je ha und dominierende Bodenvegetation in Windwurfgebieten im überregionalen Vergleich (nach Autor, Gebiet, Standort und Sukzessionsdauer).

\begin{tabular}{|c|c|c|c|c|}
\hline Autor & $\begin{array}{l}\text { Untersuchungs- } \\
\text { gebiet, Waldtyp } \\
\text { und Standort }\end{array}$ & $\begin{array}{c}\text { Alter der } \\
\text { Bestände nach } \\
\text { Sturmwurf oder } \\
\text { Kahlschlag }\end{array}$ & $\begin{array}{c}\text { Verjüngungszahlen und } \\
\text { dominierende } \\
\text { Baumarten }\end{array}$ & Bodenvegetation \\
\hline $\begin{array}{l}\text { KOMPA \& } \\
\text { SCHMIDT } \\
\text { (2003) } \\
\text { bzw. } \\
\text { vorliegende } \\
\text { Arbeit }\end{array}$ & $\begin{array}{l}\text { südwestliches } \\
\text { Harzvorland } \\
\text { (Niedersachsen), } \\
\text { Buchen-Wälder } \\
\text { auf Buntsandstein } \\
\text { und Zechstein }\end{array}$ & $\begin{array}{l}\text { 4 Jahre nach } \\
\text { 1997er Sturm; } \\
\text { belassen }\end{array}$ & $\begin{array}{l}\text { Buntsandstein: } \\
\text { 1.500-4.700/ha (Buche, } \\
\text { vereinzelt Pionierbäume) } \\
\text { Gips/Dolomit: } \\
\text { 9.000/ha (Berg-Ahorn, } \\
\text { Esche, Buche, Spitz- } \\
\text { Ahorn, Salweide); } \\
\text { Dolomit: } \\
\text { 52.000/ha (75\% Esche, } \\
\text { 23\% Buche) }\end{array}$ & $\begin{array}{l}\text { Buntsandstein: } \\
\text { Himbeer-Holunder- } \\
\text { Gebüsch; } \\
\text { Gips/Dolomit: } \\
\text { gehölzarme Schlagflur- } \\
\text { und Ruderalstadien; } \\
\text { Dolomit: } \\
\text { Waldarten dominant, } \\
\text { kaum Schlagflora }\end{array}$ \\
\hline $\begin{array}{l}\text { WILLIG } \\
(1994,2002 b) \\
\text { KEITEL } \\
(1998,1999)\end{array}$ & $\begin{array}{l}\text { Vogelsberg } \\
\text { (Hessen), NWR } \\
\text { Weiherskopf, } \\
\text { Buchen-Wald auf } \\
\text { Basalt } \\
\end{array}$ & $\begin{array}{l}4 \text { Jahre nach } \\
\text { 1990er Sturm; } \\
\text { belassen }\end{array}$ & $\begin{array}{l}\text { frische Standorte: } \\
\text { im Mittel 29.000/ha ( } 85 \% \\
\text { Edellaubhölzer, davon } \\
48 \% \text { Esche; ferner } 12 \% \\
\text { Buche) }\end{array}$ & $\begin{array}{l}\text { Mischflora aus Wald- } \\
\text { (60\% Anteil) sowie } \\
\text { Schlagflur- und } \\
\text { Ruderalarten }\end{array}$ \\
\hline $\begin{array}{l}\text { SCHÖLCH et } \\
\text { al. (1994) }\end{array}$ & $\begin{array}{l}\text { Schwarzwald und } \\
\text { Neckarland } \\
\text { (Baden- } \\
\text { Württemberg), } \\
\text { Nadelmischwälder } \\
\text { auf Buntsandstein } \\
\text { und Buchen- } \\
\text { Mischwälder auf } \\
\text { Muschelkalk }\end{array}$ & $\begin{array}{l}\text { 3- bis max. } \\
\text { 11jährige Bestände } \\
\text { nach Kahlschlag } \\
\text { oder 1986er } \\
\text { Windwurf, } \\
\text { geräumt, aber nicht } \\
\text { aufgeforstet }\end{array}$ & $\begin{array}{l}\text { Buntsandstein: } \\
\text { 4.000-12.000/ha (Fichte, } \\
\text { Tanne, Kiefer, Buche, } \\
\text { Pionierbäume) } \\
\text { Muschelkalk: } \\
\text { 9.000-42.000/ha (Esche, } \\
\text { Buche, Eiche, } \\
\text { Pionierbäume), } \\
\text { Schlusswaldbaumarten } \\
\text { dominieren fast überall } \\
\end{array}$ & k.A. \\
\hline $\begin{array}{l}\text { HANKE \& } \\
\text { PISOKE } \\
(1999)\end{array}$ & $\begin{array}{l}\text { Schwarzwald, } \\
\text { Fichten-Tannen- } \\
\text { Wald auf } \\
\text { vernässtem } \\
\text { Buntsandstein }\end{array}$ & $\begin{array}{l}5 \text { Jahre nach } \\
\text { 1990er Sturm; } \\
\text { belassen }\end{array}$ & $\begin{array}{l}\text { 15.000/ha (Fichte, Tanne, } \\
\text { kaum Pionierbäume) }\end{array}$ & k.A. \\
\hline
\end{tabular}


Fortsetzung von Tab. 21.

\begin{tabular}{|c|c|c|c|c|}
\hline Autor & $\begin{array}{l}\text { Untersuchungs- } \\
\text { gebiet, Waldtyp } \\
\text { und Standort }\end{array}$ & $\begin{array}{c}\text { Alter der } \\
\text { Bestände nach } \\
\text { Sturmwurf oder } \\
\text { Kahlschlag }\end{array}$ & $\begin{array}{l}\text { Verjüngungszahlen und } \\
\text { dominierende } \\
\text { Baumarten }\end{array}$ & Bodenvegetation \\
\hline $\begin{array}{l}\text { SAYER \& } \\
\text { REIF (1998) }\end{array}$ & $\begin{array}{l}\text { Baden- } \\
\text { Württemberg; } \\
\text { ehemals Fichten- } \\
\text { dominierte Wälder } \\
\text { auf sauren, } \\
\text { mittleren und } \\
\text { basenreichen } \\
\text { Standorten }\end{array}$ & $\begin{array}{l}5 \text { Jahre nach dem } \\
\text { 1990er Sturm }\end{array}$ & $\begin{array}{l}\text { saure Standorte: } \\
\text { max. 40.000/ha (Fichte) } \\
\text { bzw. 2.000/ha (Tanne, } \\
\text { Kiefer, Pionierbäume); } \\
\text { mittlere Standorte: } \\
\text { max. 20.000/ha (Fichte) } \\
\text { bzw. 5.000/ha (Salweide, } \\
\text { Birke) } \\
\text { basenreiche Standorte: } \\
\text { max. 20.000/ha (Fichte) } \\
\text { bzw. 2.000/ha (Buche, } \\
\text { Esche, Berg-Ahorn) }\end{array}$ & $\begin{array}{l}\text { saure Standorte: } \\
\text { Vergrasung (Avenella } \\
\text { flexuosa), Heidelbeere, } \\
\text { später Schlagflur } \\
\text { (Digitalis purpurea, } \\
\text { Pteridium aquilinum) } \\
\text { mittlere Standorte: } \\
\text { Epilobion- oder Atropion- } \\
\text { Schlagflur, Himbeer- } \\
\text { Holunder-Gebüsch } \\
\text { basenreiche Standorte: } \\
\text { Atropion-Schlagflur, } \\
\text { Himb.-Holunder-Gebüsch }\end{array}$ \\
\hline $\begin{array}{l}\text { HETZEL \& } \\
\text { REIF (1998) }\end{array}$ & $\begin{array}{l}\text { Schwäbische Alb } \\
\text { (Baden- } \\
\text { Württemberg), } \\
\text { Fichten- und } \\
\text { Buchen-Wälder } \\
\text { auf Kalk }\end{array}$ & $\begin{array}{l}5 \text { Jahre nach } \\
\text { 1990er Sturm, } \\
\text { belassen (Angaben } \\
\text { nur zu Verjüngung } \\
\text { über } 30 \mathrm{~cm} !)\end{array}$ & $\begin{array}{l}\text { Fichten-Wälder: } \\
\text { ca. 4.000/ha } \\
\text { (Fichte, ferner Buche, } \\
\text { Berg-Ahorn, Esche), } \\
\text { Buchen-Wälder: } \\
\text { ca. 45.000/ha } \\
\text { (75\% Buche, 22\% Esche); } \\
\text { Pionierbäume überall } \\
\text { unbedeutend }\end{array}$ & $\begin{array}{l}\text { Himbeer-Holunder- } \\
\text { Gebüsch }\end{array}$ \\
\hline $\begin{array}{l}\text { HOMANN \& } \\
\text { ENGELS } \\
(1991)\end{array}$ & $\begin{array}{l}\text { NP Bayerischer } \\
\text { Wald, } \\
\text { Bergfichtenwälder } \\
\text { auf vernässtem } \\
\text { bodensauren } \\
\text { Standort } \\
\end{array}$ & $\begin{array}{l}3 \text { Jahre nach } \\
\text { 1983er Sturm; } \\
\text { belassen }\end{array}$ & $\begin{array}{l}\text { im Mittel 17.300/ha (74\% } \\
\text { Fichte, ferner Buche, } \\
\text { Vogelbeere, Salweide) }\end{array}$ & $\begin{array}{l}\text { Waldbodenflora erhalten; } \\
\text { Vergrasung } \\
\text { (Calamagrostis villosa), } \\
\text { ferner Rubus idaeus, } \\
\text { Oxalis acetosella }\end{array}$ \\
\hline JEHL (2001): & $\begin{array}{l}\text { NP Bayerischer } \\
\text { Wald, } \\
\text { Bergfichtenwälder } \\
\text { auf vernässtem } \\
\text { bodensauren } \\
\text { Standort } \\
\end{array}$ & $\begin{array}{l}\text { 13 Jahre nach } \\
\text { 1984er Sturm; } \\
\text { belassen }\end{array}$ & $\begin{array}{l}\text { im Mittel 6.700/ha } \\
\text { (Fichte) und 500/ha } \\
\text { (Vogelbeere), } \\
\text { Pionierbäume } \\
\text { bedeutungslos }\end{array}$ & $\begin{array}{l}\text { Waldbodenflora erhalten, } \\
\text { starke Vergrasung } \\
\text { (Calamagrostis villosa), } \\
\text { ferner Rubus idaeus und } \\
\text { Epilobium angustifolium }\end{array}$ \\
\hline $\begin{array}{l}\text { FISCHER et al. } \\
\text { (2002) }\end{array}$ & $\begin{array}{l}\text { NP Bayerischer } \\
\text { Wald; bodensaure } \\
\text { Aufichtenwälder }\end{array}$ & $\begin{array}{l}\text { 15 Jahre nach } \\
\text { 1983er Sturm; } \\
\text { belassen und } \\
\text { geräumt }\end{array}$ & $\begin{array}{l}\text { 6.000-16.900/ha; } \\
\text { belassen: } \\
\text { Fichte } \\
\text { geräumt: } \\
\text { Birken-Vorwald }\end{array}$ & $\begin{array}{l}\text { belassen: } \\
\text { Schlagflur nur auf } \\
\text { Wurzeltellern; } \\
\text { geräumt: } \\
\text { Schlagflur flächig }\end{array}$ \\
\hline $\begin{array}{l}\text { SCHÖNEN- } \\
\text { BERGER } \\
(2002) \\
\text { flächen- } \\
\text { basierte } \\
\text { Aufnahme, } \\
\text { ohne } \\
\text { Keimlinge }\end{array}$ & $\begin{array}{l}\text { Schweizer Alpen, } \\
\text { Fichten-dominierte } \\
\text { subalpine } \\
\text { Bergwälder auf } \\
\text { basenarmen und } \\
\text { basenreichen } \\
\text { Standorten }\end{array}$ & $\begin{array}{l}\text { 10 Jahre nach } \\
\text { 1990er Sturm; } \\
\text { belassen und } \\
\text { geräumt }\end{array}$ & $\begin{array}{l}\text { basenarm: } \\
900-1.500 / \text { ha } \\
\text { basenreich: } \\
\text { ca. } 2.500 / \text { ha } \\
\text { (Fichte, Berg-Ahorn, } \\
\text { Vogelbeere, Weide) }\end{array}$ & $\begin{array}{l}\text { anfangs Galeopsis- } \\
\text { Epilobium-Stadium, dann } \\
\text { Vergrasung } \\
\text { (Calamagrostis villosa, } \\
\text { Agrostis capillaris, } \\
\text { Avenella flexuosa) oder } \\
\text { Schlagflur (Rubus idaeus, } \\
\text { Epilobium angustifolium) }\end{array}$ \\
\hline $\begin{array}{l}\text { WOHLGE- } \\
\text { MUTH et al. } \\
\text { (2002) } \\
\text { Aufnahme auf } \\
\text { Kleinstand- } \\
\text { orten incl. } \\
\text { Keimlinge }\end{array}$ & $\begin{array}{l}\text { S. } \\
\text { SCHÖNENBERGER } \\
(2002)\end{array}$ & $\begin{array}{l}\text { S. } \\
\text { SCHÖNENBERGER } \\
(2002)\end{array}$ & $\begin{array}{l}\text { basenarm: } \\
\text { 2.000-5.000/ha } \\
\text { basenreich: } \\
\text { 20.000-45.000/ha } \\
\text { (Fichte, Berg-Ahorn, } \\
\text { Vogelbeere, Weide) } \\
\text { stärkste Verjüngung auf } \\
\text { gestörten Kleinstandorten! }\end{array}$ & $\begin{array}{l}\text { S. } \\
\text { SCHÖNENBERGER } \\
(2002)\end{array}$ \\
\hline
\end{tabular}


Für die Therophyten-reichen Schlagflur- und Ruderalstadien der sturmgeworfenen CariciFageten auf Gipskarst (z.B. Hainholz-KF2) ließen sich deutschlandweit keine vergleichbaren Beispiele finden. Die größten Parallelen zu den übrigen basenreichen Windwurfflächen des Harzvorlandes (Hordelymo-Fagetum) haben demzufolge der Buchen-Windwurf auf frischen Basalt-Standorten im Vogelsberg (WILLIG 1994, 2002b; KeITEL 1998, 1999) und der auf KalkStandorten in Baden-Württemberg (SchöLCH et al. 1994; HetzeL \& REIF 1998). Die Sukzession ist dort überall gekennzeichnet durch eine dominante und auch ohne forstliche Maßnahmen ausreichende Naturverjüngung der Schlusswaldbaumarten, wobei im Vergleich zum Altbestand eine vorübergehende Baumarteninversion von Buche zu Edellaubhölzern (v.a. Esche) in der Verjüngung auftreten kann (nicht z.B. in Kalk-Buchenwäldern der Schwäbischen Alb). Die alte Waldbodenflora dominiert entweder weiterhin oder persistiert unter einer unterschiedlich stark und ausdauernd ausgeprägten Schlagflur, von der aber keine generell verdrängende Wirkung ausgeht. Am Beispiel des Vogelsberg-Windwurfes (BasaltBuchenwald) zeigt sich auf engem Raum die Standortsabhängigkeit der Sukzession in Gestalt einer stark unterschiedlichen Vegetationsentwicklung in frischen und wechselfeuchten Geländepartien (WILLIG 2002b). Während der frische Standortsbereich die in Tab. 21 gezeigte Entwicklung nahm, wurde auf den wechselfeuchten Standorten nur ein Bruchteil dieser Sämlingsdichte bei stark abweichender Baumartenzusammensetzung erreicht (Buche etwa 50\%, weiterhin Fichte und Pionierbäume stark vertreten). Langfristig kam es in den letztgenannten Beständen zur Dominanz von Störzeigern in der Bodenvegetation (besonders Brombeere, Himbeere, Deschampsia cespitosa), während unter den Waldarten lediglich Athyrium filix-femina und Luzula luzuloides von den Windwurfbedingungen profitierten.

Die im Harzvorland ablaufenden Sukzessionen erinnern weiterhin - mit einiger Verkürzung im zeitlichen Verlauf und Abwandlung in der Artenzusammensetzung - an die Vegetationsentwicklung auf geräumten Buchenwald-Kahlschlägen, wie sie von DIERSCHKE (1988) in einem Hordelymo-Fagetum des Göttinger Waldes bzw. von RUNGE (1993) in einem Galio odorati-Fagetum in der Nähe von Münster (Westfalen) beobachtet wurden. Dort wechselten jeweils mehrjährige Pionierkraut-, Rubus-Gebüsch- und Birken-Vorwaldstadien einander ab, bei denen aber ähnlich wie im Harzvorland ein Grundstock typischer Waldarten unter Himbeere und Birke überdauerte und die Buchen-Verjüngung langfristig zunahm. Im Harzvorland wurden die überwiegend krautigen und zu den Artemisietea gehörenden Ruderalarten spätestens nach vier Jahren zugunsten von meist strauchartigen EpilobieteaArten wie Himbeere, Holunder und Brombeere (Buntsandstein) oder durch EschenVerjüngung (Zechstein) verdrängt (Kap. 4.3.5; Tab. A22, A23). Im Carici-Fagetum auf flachgründigem Gipskarst werden die krautigen Zwischenstadien etwas länger andauern, aber auch hier etablierte sich ja mit etwa 9.000 Sämlingen/ha bereits eine im mittleren Bereich liegende Naturverjüngung mit stark dominierenden Anteilen der Schlusswaldbaumarten.

Die tiefgründigen Dolomit-Standorte das Harzvorlandes erreichen bei den Verjüngungszahlen ähnliche Größenordnungen wie die Kalk-Standorte der Schwäbischen Alb und liegen über 
denen der Basalt-Standorte im Vogelsberg (Kap. 4.5; Tab. 21). Die Gehölze erscheinen im Hordelymo-Fagetum des Harzvorlandes recht schnellwüchsig. Die Strauchschicht erreichte z.B. in der Hainholz-KF1 nach vier Sukzessionsjahren bereits durchschnittlich $4 \mathrm{~m}$ (siehe Abb. 25). Knapp 30\% des gesamten Baumjungwuchses war hier zu diesem Zeitpunkt höher als $150 \mathrm{~cm}$ (Abb. 44), etwa 5\% der jungen Eschen erreichten Wuchshöhen über $3 \mathrm{~m}$ (Maximalhöhe $490 \mathrm{~cm}$ - nach Vermessung, hier nicht dargestellt). Im Hordelymo-Fagetum des Vogelberg-Windwurfes betraf dies nach vier Jahren etwa 10\% der Eschen (WILLIG 1994, 2002b; KeITEL 1998, 1999). Maximale Jahreszuwächse der Esche von 1 m und mehr sind auf basenreichen Windwurfstandorten offensichtlich normal. Die Eschen-Verjüngung im zitierten Galio-Fagetum bei Münster brauchte etwa 7 Jahre, um die 4 m-Grenze zu erreichen (RUNGE 1993). Die Deckungssummen der Feldschicht waren vier Jahre nach dem Sturm bei bis dahin steigender Tendenz mit durchschnittlich 150\% im Harzvorland ähnlich hoch wie im Vogelsberg (130\%). Im selben Zeitraum zeigten sich im Harzvorland im Vergleich zum Vogelsberg auch ähnliche Abundanzanteile an Lichtzeigern (v.a. Hainholz-KF2 bzw. Nüllberg) und an Stickstoffzeigern (v.a. Hainholz-KF1).

Wie in Kap. 5.8.1 bereits erwähnt, verzeichnen die Buche und andere Laubbaumarten in Baden-Württemberg gute Verjüngungserfolge auf basenreichen Windwurfflächen mit künstlicher Fichten-Vorbestockung und leiten dort trotz dominanter Fichten-Verjüngung einen natürlichen Baumartenwechsel hin zu Laubmischwäldern ein (HETZEL \& REIF 1998; SAYER \& REIF 1998; MÄRKL \& EGGLSEER 2001). Auf basenarmen Standorten dagegen bleibt die Fichte die einzige erfolgreiche Schlusswaldbaumart mit sehr hohen Verjüngungszahlen (Tab. 21). Für Standorte mittlerer Basenversorgung kann weiterhin eine Pionierbaum-reiche Verjüngung (z.B. Birke, Salweide) mit unterschiedlich ausgeprägter Schlagflur charakteristisch sein (Tab. 21; SCHÖLCH et al. 1994; SAYER \& REIF 1998), auf sehr sauren oder vernässten Standorten aber teilweise auch das Ausbleiben von Pionierwäldern (Tab. 21; SAYER \& REIF 1998; HANKE \& PISOKE 1999). Im sauren Bereich scheint Vergrasung (Calamagrostis, Avenella, Deschampsia) generell eine größere Rolle zu spielen. Die basenarmen Buchen-Standorte des Harzvorlandes (Galio odorati-, Luzulo-Fagetum) lassen sich hier nur bedingt einordnen. Einerseits sind die Verjüngungszahlen unter den ausgedehnten Pionierstrauchfluren relativ gering. Andererseits dominiert die Klimaxbaumart Buche bereits in der Verjüngung, so dass Pionierwälder weitgehend fehlen. Auch die alte Waldbodenflora bleibt fast vollständig erhalten.

Die Fichten-Windwürfe auf Bergwald-Standorten des Bayerischen Waldes (FISCHER et al. 1990, 2002; JeHL 2001) und der Schweizer Alpen (SCHÖNENBERGER et al. 2002, 2003) nehmen eine ganz eigene und von den Hügelländern abweichende Entwicklung. Die Fichte gehört dort - im Gegensatz zu den meisten anderen in Tab. 21 aufgeführten Beispielen - zur natürlichen Waldgesellschaft und die klimatischen Bedingungen sind im Vergleich zu tiefergelegenen Standorten um einiges ungünstiger. Aber auch hier zeichnet sich kein 
genereller Artenwechsel ab und die in der Verjüngung dominante Klimaxbaumart Fichte wird sich langfristig wieder durchsetzen. Allerdings wird die Waldregeneration nach dem 1990er Windwurf besonders in den Schweizer Alpen aufgrund sehr geringer Verjüngungszahlen und -raten (auf basenreichen und basenarmen Standorten gleichermaßen) länger dauern als in den Tieflagen. In der montanen Stufe veranschlagen ScHÖNENBERGER et al. (2003) etwa 20 weitere Jahre, in der alpinen sogar 30. Dort muss sich die Wiederbewaldung aus Neuansamung vollziehen, in den Tieflagen dagegen meist aus Vorverjüngung (SCHÖNENBERGER \& WASEM 1999; SchÖNENBERger 2002). Am Anfang der Windwurfsukzession in Schweizer Bergwäldern beschreiben SCHÖNENBERGER et al. (2002, 2003) einheitlich zunächst ein Hohlzahn- (Galeopsis) Stadium (in Anklängen auch auf geräumten Buntsandstein-Flächen des Harzvorlandes zu finden, siehe Tab. A23) und anschließend ein Gras-Stadium (Calamagrostis villosa, Agrostis capillaris, Avenella flexuosa) oder eine Himbeer-Hochstauden-Flur. Vergrasung und Schlagflur sind ebenso für die bodensauren Windwurfgebiete des Bayerischen Waldes charakteristisch (Tab. 21). Für Calamagrostis villosa sowie für bestimmte Hochstaudenarten und Farne wird häufig eine verjüngungsverdämmende Wirkung in den Bergwäldern beschrieben (REIF \& PRZYBILLA 1995; Wohlgmuth et al. 2002). Desweiteren spielt die Vogelbeere im Gebirge als Pionierbaumart eine wichtige Rolle (Tab. 21). Eine besondere Bedeutung haben hier offenbar auch die Wurzelteller. Im Bayerischen Wald trat Schlagflurvegetation auf belassenen Flächen nur im Bereich der Wurzelteller auf, auf geräumten dagegen flächig. In den Schweizer Bergwäldern sind auch 10 Jahre nach dem Sturm Wurzelteller und andere gestörte Kleinstandorte bevorzugte Wuchsorte der Verjüngung, wobei nach Extrapolation der Werte auf die Gesamtfläche auf basenarmen Standorten das Doppelte bis Dreifache der Verjüngungszahlen der Umgebungsflächen erreicht wurde, auf basenreichen sogar das Zehnbis Zwanzigfache (WohlgEMuth et al. 2002, Tab. 21). Im Gegensatz dazu fallen die Wurzelteller im Buchen-Windwurf des Harzvorlandes gegenüber ihren Umgebungsflächen nicht so stark auf. Sie waren zwar in den ersten vier Sukzessionsjahren besonders auf Buntsandstein- und auf tiefgründigen Dolomit-Standorten durch besonders hohe Abundanzanteile an Stör- und Lichtzeigern sowie Sträuchern und durch besonders niedrige an Baumartenjungwuchs, Geophyten und typischen Laubwaldarten charakterisiert. Bezüglich des (qualitativen) Arteninventars waren sie ihrer Umgebungsfläche aber erstaunlich ähnlich. Auf den flachgründigen Gips/Dolomit-Rendzinen galt dies sogar für die Mengenverhältnisse der untersuchten Artengruppen (Kap. 4.6.7 und 5.7.2).

Nicht zuletzt soll erwähnt werden, dass in allen verglichenen Windwurfstudien eine generelle Artenzahlerhöhung infolge der natürlichen Störung festgestellt wurde. Übereinstimmend sind dabei basenreiche Standorte artenreicher als basenarme, geräumte Windwurfflächen sind artenreicher als belassene. Die sich meist sehr dynamisch entwickelnde Strauchschicht dunkelt die anfangs oft üppig entwickelte Feldschicht zunehmend aus. Die Ergebnisse der 
vorliegenden Arbeit aus Buchen-Windwurfgebieten des Harzvorlandes bestätigen dies ausnahmslos.

Fazit: Überregional feststellbare Trends nach Windwurf sind demzufolge: 1. (bezüglich floristischer Veränderungen): kein kompletter Artenwechsel, aber in Artenzahl und Abundanz unterschiedlich starkes Überdauern der alten Waldbodenflora unter unterschiedlich lang andauernden baum- oder strauchdominierten Pionierstadien, bevorzugt auf sauren oder Bergwald-Standorten auch Gras- und Farn-Dominanzen. 2. (bezüglich der Waldregeneration): auf basenreichen Standorten der Mittelgebirge und Hügelländer rasante Wiederbewaldung mit Klimaxbaumarten (in Buchen-Wäldern teilweise Baumarteninversion von Buche zu Edellaubhölzern möglich; bei künstlicher Fichten-Vorbestockung langsame Rückeroberung des Standortes durch Laubbaumarten); auf basenarmen Standorten verlangsamte Regeneration infolge stärker überlagernder Pionierstadien (bei künstlicher Fichten-Vorbestockung weiterhin Fichten-Dominanz); in Hochlagen-Wäldern - mit oder ohne Pionierstadien - generell stark verlangsamte Wiederbewaldung.

\subsubsection{Konkurrenzfähigkeit der Buche (Fagus sylvatica) nach Windwurf}

Die Buche wäre von Natur aus fast überall in Nordwestdeutschland vorherrschend. Ihre Konkurrenzüberlegenheit resultiert aus ihrer Langlebigkeit und Beschattungsfähigkeit (geringe Lichtdurchlässigkeit der Kronen), der hohen Überlebensfähigkeit ihres Jungwuchses im Schatten und ihrer weiten Standortsamplitude. Buchen-Feinwurzeln erscheinen mit hoher Abundanz besonders im Kronenzwischenraum und sind sehr erfolgreich bei der Eroberung nährstoffreicherer Bodenhorizonte. Buchen-Wälder der Optimalphase besitzen eine unterwuchsarme Hallenstruktur und ein gleichmäßig hohes Kronendach (JAHN 1979, 1983; VON LOCHOW 1990; LEUSCHNER 1998). Ohne großflächige Störung erfolgt die Verjüngung in diesen Wäldern überwiegend durch die schattentolerante Klimaxbaumart Buche selbst (SCHMIDT 2002a, 2003). Eine Trockenheits- und Nährstoffmangelgrenze der Buche lässt sich im atlantischen Nordwestdeutschland nicht erkennen (LEUSCHNER et al. 1993; HEINKEN 1995; LEUSCHNER 1998), die Nässegrenze ist weniger gut geklärt. Es lassen sich sowohl Beispiele finden, bei denen die Buche auf vernässten Böden abstirbt, als auch andere, wo sie selbst auf grund- und stauwasserbeeinflussten Böden weiter zunimmt (MEYER et al. 2000; SCHMIDT 2000). Typisch für die Buche ist die schubweise Verjüngung nach Mastjahren und die relativ hohe Mortalität der Jungpflanzen. Konkurrenzbedingt entwickelt sich Buchen-Jungwuchs optimal im strahlungsarmen Bereich mit unter 12\% relativem Lichtgenuss. Die BuchenVerjüngung ist auffällig an den Kronenradius der Altbäume gebunden, konkurrierende Bodenvegetation existiert im Altbestand normalerweise kaum (UNKRIG 1997b). Nach TOPOLIANTZ \& PONGE (2000) fördern flache Sandsteinböden sowohl die Mast als auch die Keimlingsmortalität der Buche, Bestandeslücken (Auflichtung) fördern die Verjüngung. 
Somit ist Licht der Hauptfaktor für eine erfolgreiche Keimlingsetablierung, Trockenheit dagegen für eine hohe Keimlingsmortalität. In guten Samenjahren der Buche können Verjüngungsdichten von 200.000-300.000 Keimlingen/ha erzeugt werden (BROGGI 2000).

In vorangegangenen Jahrhunderten bedingte die Einführung der Niederwaldwirtschaft einen Rückgang der Buche, da diese ein standorts- und alterslimitiertes Ausschlagvermögen besitzt (JAHN 1983, 1990; POTT 1988). Durch den Übergang von der Mittel- oder Niederwaldzur Hochwaldwirtschaft vor etwa anderthalb Jahrhunderten, den Rückgang der Waldweide, die daraus resultierende Regeneration der Waldböden und die anthropogene Entwässerung wurden viele Waldstandorte wieder „Buchen-fähig“. Die Buche nahm in Nordwestdeutschland zu, bevor sie aus waldbaulichen Gründen wieder Flächenanteile an die Nadelhölzer verlor (JAHN 1983). Mittlerweile lässt sich in nordwestdeutschen, aber auch in bayerischen Naturwäldern eine Tendenz erkennen, dass die Buche nach Nutzungseinstellung auf Kosten anderer Baumarten deutlich zunimmt oder gar zur Dominanz gelangt. Dies betrifft Buchen-Waldgesellschaften in der Wachstums- und Optimalphase, aber auch derzeit nicht Buchen-dominierte Bestände. Der Fichten-Anteil in diesen Naturwäldern reduziert sich ständig durch Windwurf und Borkenkäferbefall (MEYER 1997; KÖLBEL 1999; MEYER et al. 2000). Somit sind es in erster Linie gezielte waldbauliche Eingriffe oder großflächige natürliche Störungen, die diese Entwicklung unterbrechen könnten.

Allgemein sind Freiflächensituationen für Buchen-Verjüngung als ungünstig einzuschätzen, was aber in Windwurfgebieten durch aufwachsende und beschattende Vegetation standörtlich unterschiedlich schnell kompensiert wird. Auf tonigen Keuper-Böden Baden-Württembergs fand z.B. KÖBERLE (1997) im großflächigen Fichten-Windwurf zwar verjüngungsverdämmende Vegetation (Gräser, Holunder, Brombeere). Jedoch wuchs BuchenNaturverjüngung am besten dort, wo Wurzeln stehengebliebener Bäume die Gras- und Krautkonkurrenz behinderten. Dichte Brombeer-Fluren stellten in diesem Beispiel für Fichten- und Buchen-Verjüngung langfristig kein Problem dar, dagegen aber für Tanne und Ahorn. Nach KeITEL (1998) ist die Buche in der Lage, mit nur 2.000 Sämlingen/ha langfristig stabile (Dominanz-)Bestände aufzubauen, nach BURSCHEL \& HUSS (1997) müssen 5.00010.000/ha für eine gesicherte Verjüngung vorhanden sein. Sollte die niedrigere Grenze generell zutreffend sein, so ist in den Windwurfgebieten des Harzvorlandes selbst auf den Pionierstrauch-dominierten Buntsandstein-Standorten von keiner langfristigen Ablösung des Buchen-Waldes auszugehen. Sollte die obere Grenze zutreffen, so bleibt diese Aussage wenigstens für die Zechstein-Standorte bestehen.

In den vorangegangenen Kapiteln wurde bereits mehrfach eine in Buchen-Wäldern basenreicher Standorte nach Auflichtung auftretende Baumarteninversion in der Verjüngung von Buche zu Edellaubhölzern erwähnt, die eine vorrübergehe Pionierrolle v.a. der Esche kennzeichnet. Die meist unterständige, aber reichlich vorhandene und gut entwickelte Buche dürfte nach etwa 20-35 Jahren wieder vorherrschen (ZÜGE 1986; DIERSCHKE 1988). Der Verjüngungsvorteil der Edellaubbäume gegenüber der Buche lässt sich 
nicht nur durch die Zusammensetzung der Vorausverjüngung, sondern teilweise auch durch das Fruktifizierungsalter erklären. Esche und Berg-Ahorn fruktifizieren mit 40-50 Jahren, die Buche erst mit 60-80 Jahren, nach anderen Angaben allerdings bereits mit 50 Jahren (BuRSCHEL \& Huss 1997; KeITEL 1999). Das in der Forstwirtschaft häufig so bezeichnete Phänomen der „Vereschung“ bei gleichzeitigem Absterben von Buchen-Verjüngung wurde von RUST \& SAVILL (2000) in Gewächshausexperimenten untersucht. Demnach führte v.a. die Wasserkonkurrenz zwischen Buchen- und Eschen-Sämlingen zu deutlich reduziertem Wachstum und Überleben von Buchen-Sämlingen. Zu teilweise abweichenden Resultaten kam HORN (2002) bei Untersuchungen zur Eschen- und Buchen-Naturverjüngung in Bestandeslücken südniedersächsischer Laubmischwälder. Trotz unterschiedlicher ökophysiologischer Strategien bei der Konkurrenz um die Ressource Wasser, die besonders in sommerlichen Trockenphasen eine Unterlegenheit der Buche gegenüber der Esche erkennen lassen, kam es dort nicht zum Ausfall der Buche in den meist Eschen-dominierten Verjüngungshorsten. Auch bezüglich der Feinwurzelkonkurrenz konnte kein Nachweis erbracht werden, dass die meist oberflächennah wurzelnden jungen Eschen eine Verdrängung der Buchen-Feinwurzeln in tiefere Bodenhorizonte bewirken. Allerdings zeigte die Verjüngung der Buche eine größere Variabilität im vertikalen Durchwurzelungsverhalten als die der Esche.

Dass der beschriebene Dominanzwechsel in windgeworfenen Buchen-Wäldern nur zeitweise anhalten wird, deutet sich heute schon z.B. im hessischen Naturwaldreservat Weiherskopf an. Nach WILLIG (2002b) vergrößerte die Buche im dortigen Flächenwurf ihren Verjüngungsanteil von 11 (1990) auf 16\% (1998). Solche Entwicklungen sind - bei jetzt bereits höherem Verjüngungsanteil der Buche - in Zukunft auch in den Zechstein-Gebieten des Harzvorlandes zu erwarten.

\subsubsection{Sukzessionsmodelle in natürlich gestörten Wäldern unter besonderer Berücksichtigung des Mosaik-Zyklus-Konzeptes}

Nach Untersuchungen aus verschiedenen europäischen Urwäldern, deutschen Naturwaldreservaten und Lochhiebsexperimenten ist davon auszugehen, dass in BuchenWäldern die Waldregeneration in kleineren Lücken (max. $2000 \mathrm{~m}^{2}$ ) unter maßgeblicher Beteiligung der Buche oder völlig Buchen-dominiert abläuft, langfristige krautige Zwischenstadien bzw. Pionierwälder weitestgehend oder völlig ausbleiben und sich auch die Artendiversität nur geringfügig erhöht (LEIBUNDGUT 1978; KNAPP \& JESCHKE 1991; GRIESE 1995; KORPEL 1995; MEYER 1995; SCHMIDT 1996, 1997, 1998b; SCHMIDT et al. 1996; TABAKU 1999; MEYer et al. 2000). Der von Remmert (1985, 1987, 1991) postulierte Mosaikzyklus mit Beispielen aus den Tropen (völlige Vernichtung der Schattenzeiger in treefall gaps) bzw. aus dem Bialowiesa-Urwald in Ostpolen (10 Jahre langen Brennessel-Dominanz in Windwurflücken) konnte für Buchen-Wälder in Deutschland nicht bestätigt werden, 
weswegen seine Gültigkeit speziell für dieses Waldökosystem relativiert oder in Frage gestellt werden muss (SCHMIDT 1998b, 2002a, 2003; KOMPA \& SCHMIDT 2003). Komplette Baumartenwechsel und zeitaufwändige Zyklen (langjährige Gräser/Kräuter- und Pionierwaldstadien) sind bislang nicht zu beobachten. Es erfolgt scheinbar auch nur selten ein Absterben der Buchen infolge Sonnenbrandes an den Lückenrändern, wie es z.B. von JENSSEN \& HOFMANN (1996) aus dem mecklenburgischen NSG „Serrahn“ berichtet wurde. Jedoch mahnt MEYER (1997) im Falle der deutschen Naturwaldreservate zur Vorsicht, da wegen ihrer langjährigen forstlichen Überprägung und des weitgehenden Fehlens natürlicher Altersstadien (Terminal- bzw. Zerfallsphasen) alle Aussagen mit großer Unsicherheit behaftet sind und sich bisherige Erkenntnisse der deutschen (Buchen-)Naturwaldforschung überwiegend auf die Optimalphase beschränken. Allerdings beschreibt LEIBUNDGUT (1978), dass auch in „richtigen“ Buchen-Urwäldern die Optimalphase lange andauert und großflächige, rein altersbedingte Zusammenbrüche eher selten sind. Stattdessen wird die einförmige Hallenstruktur dieser Wälder aus einem Mosaik stark ungleichaltriger Baumexemplare gebildet. Bei einem Vergleich deutscher und albanischer Buchen-Wälder (TABAKU 1999) wurden mittlere Lückengrößen von 60-74 $\mathrm{m}^{2}$ (Albanien) bzw. 97-183 $\mathrm{m}^{2}$ (Deutschland) gefunden, die den Normalfall einer Lückendynamik ohne großflächige Störung darstellen. Wegen der erst seit kurzem zurückliegenden forstlichen Nutzung waren die Lücken in Deutschland noch größer als die in den albanischen Urwäldern.

Eine andere mögliche Betrachtungsebene fokussiert nicht auf die einzelne Lücke und die darin ablaufenden Regenerationsprozesse, sondern auf die Gesamtheit von Entwicklungsstadien in einem Waldgebiet. Für Buchen-dominierte Urwälder der Westkarpaten nennt KORPEL (1995) etwa 30-40 ha als Flächeneinheit, innerhalb derer ein Waldökosystem als „dynamisch ausgewogen“ gelten kann, d.h. in dem alle Waldentwicklungsstadien (Heranwachsens-, Optimal- und Zerfallsstadium) dauerhaft parallel vertreten sind. Ähnliche Minimum-Strukturarealgrenzen, die auch als Richtwerte für die Größe von Naturwaldreservaten dienen können, konstruiert KoOP (1982) nach Erfahrungen aus - in der Vergangenheit forstlich überprägten - Naturwäldern in Polen und Deutschland (z.B. 10 ha für das Stellario- und Tilio-Carpinetum, 25 ha für das Milio-Fagetum und 40 ha für das Fago-Quercetum). Nur ein kleiner Teil der von Koop (1982) untersuchten Waldfläche war stabil im Sinne der Klimaxgesellschaft. Im Wirtschaftswald dagegen dürfte es schwierig sein, solche „Mosaiksteine“ oder „Parallelstadien“ überhaupt zu finden. Im Plenterwald z.B. erfolgt die Ausschaltung der natürlichen Alters- und Zerfallsphase des Waldes (THOMAsIUS 1988). Bezüglich der Regenerationsprozesse kommt die femel- oder plenterartige Waldnutzung den Verhältnissen im Naturwald jedoch am nächsten (TABAKU 1999).

Im Gegensatz zur eingangs geführten Argumentation sieht KoOP (1982) solche parallel auftretenden Stadien als Teil eines Mosaikzyklus im REMMERT'schen Sinne und konstatiert am Beispiel von drei seit etwa 100 Jahren unbewirtschafteten Wäldern (Bialowiesa/Ostpolen, Hasbruch und Neuenburg/Niedersachsen) mit unterschiedlichen Waldgesellschaften (Fago- 
Quercetum, Stellario- und Tilio-Carpinetum, Milio-Fagetum) die Abfolge von: 1. einem mehrere Jahrzehnte andauernden Kräuterstadium (Pteridium, Rubus, Hochstauden) 2. einem Vorwaldstadium (Betula, Populus, Sorbus) 3. einem Hauptwaldstadium (Fagus, Carpinus, Tilia) und 4. einem Endstadium (Taxus, Ilex). Ein Baumartenwechsel ließ sich dort etwa in Form von Fagus- und Carpinus- bzw. von Ilex- und Taxus-Verjüngung unter Schirm des jeweiligen Hauptwaldstadiums beobachten. Angesichts dieses Beispiels könnte man fragen, ob die Gültigkeit des REMMERT'schen Mosaik-Zyklus-Konzeptes nicht doch eher von der Definition oder vom betrachteten Zeitraum abhängt?!

So argumentieren z.B. AHRns \& HofMAnN (1998), dass dies alles eine Frage der syntaxonomischen Feingliederung ist und sich am Beispiel des plenterartig genutzten KalkBuchenwaldes im Hainich (Thüringen) bei entsprechend ausdifferenzierter pflanzensoziologischer Betrachtung mindestens vier zeitlich aufeinanderfolgende und mosaikartig miteinander verzahnte Entwicklungsstadien (Mosaikzyklen) unterscheiden lassen. Dem wird hier aber nicht gefolgt, denn in der bisherigen Argumentation (siehe dazu auch Kap. 5.2 und 5.8.1) wird von einer sukzessionsbedingten Überlagerung der ursprünglichen Waldvegetation, nicht von floristisch streng abgrenzbaren Einheiten ausgegangen. Sowohl unter den speziellen Bedingungen einer Windwurfsukzession als auch unter den Bedingungen einer gelegentlichen, anthropogen verursachten Lückendynamik (z.B. Plenterhieb) werden solche wie auch immer gearteten Mosaikzyklen mit hoher Geschwindigkeit durchlaufen. Eine pflanzensoziologische Differenzierung hat somit jeweils nur kurze Zeit Bestand und würde teilweise auch nur sehr undeutlich sichtbar werden.

Zum Schluss dieser Betrachtung soll als Resüme' festgehalten werden: Bei kleinflächiger (lückiger) Störung in mitteleuropäischen Buchen-Wäldern der Optimalphase liegen bisher keine Hinweise auf einen generellen Artenwechsel vor, der das Mosaik-Zyklus-Konzept stützen könnte.

Wie verhält es sich nun aber mit großflächigen Störungen? Möglicherweise führen erst großflächige und häufige Störungen zu einem entsprechenden Artenwechsel mit ausgeprägten Pionierstadien. Nach den vorliegenden Untersuchungen aus dem Harzvorland kommt es nach Buchen-Windwurf auf Buntsandstein einerseits zu einer Erweiterung des Arteninventars sowie zu teilweise massiven Dominanzverschiebungen. Diese Veränderung ist umso stärker, je größer die gestörte Fläche ist (KOMPA \& SCHMIDT 2003). Somit könnte man vermuten, dass die Windwurfsukzession auf Buntsandstein zumindest bei großflächiger Störung dem Modell des Mosaik-Zyklus-Konzeptes schon näherkommt. Andererseits bleibt aber die Dominanz der Klimaxbaumart Buche in der Verjüngung prinzipiell bestehen, behalten typische Waldarten konstante Deckungssummen unter den üppig entwickelten Himbeer-Fluren und findet ein Artenwechsel nicht statt. Noch weniger lässt sich das Mosaik-Zyklus-Konzept mit der Windwurfsukzession auf basenreichen Standorten stützen, wo die Waldregeneration besonders auf tiefgründigen, gut wasser- und nährstoffversorgten Böden selbst bei 
flächenhafter, mehrere Hektar großer Störung ohne Zwischenstadien aus der Naturverjüngung der Klimaxbaumarten heraus erfolgt und ein Baumartenwechsel nicht stattfindet (WILLIG 1994, 2002b; Hetzel \& ReIF 1998; KeItel 1998, 1999; KomPA \& SCHMidT 2001, 2002; SCHMIDT 2002a, 2003). Etwas ungünstiger gestaltet sich die Situation auf den flachgründigen und für eine Gehölzentwicklung eher suboptimalen Gipskarst-Standorten (z.B. HainholzKF2). Aber obwohl hier in den ersten vier Jahren überwiegend Schlagflur- und Ruderalarten sowie Therophyten vorherrschten, deutet der hohe Anteil an Schlusswaldbaumarten in einer mittelstark ausgeprägten Verjüngung die künftige Entwicklungsrichtung zum BuchenMischwald an. Ein 20jähriges Stauden- und Grasstadium und ein daran anschließendes ca. 200jähriges Pionierbaumstadium (REMMERT 1991) sind im Harzvorland nirgendwo zu erwarten. Lichte Pionierwälder könnten sich am ehesten noch im Luzulo-Fagetum des Krückers auf Buntsandstein entwickeln und dürften dort aber für die nachwachsenden Buchen eher förderlich und für die derzeitigen Pionierstrauchfluren eher nachteilig sein. Angesichts der geringen Verjüngungsanteile von Birke und Salweide im Krücker ist es aber fraglich, wie stark und wie langfristig sich dieser Pionierwald ausprägen wird. Insgesamt lassen die Ergebnisse v.a. aus dem Hainholz den Schluss zu, dass auch bei großflächiger Störung die Regenerationsprozesse in mitteleuropäischen Buchen-Wäldern rascher verlaufen als bisher vielfach angenommen wird (SCHMIDT 2002a, 2003). Auch die vorübergehende Pionierfunktion von Edellaubhölzern (v.a. der Esche) nach Windwurf auf basenreichen Standorten bringt keine eigene, Buchen-freie Waldgesellschaft hervor und verhindert die langfristige Kontinuität des Buchen-Waldes nicht (FISCHER et al. 1998; LAMBERTZ \& SCHMIDT 1999; SCHMIDT 2002a, 2003).

In der Windwurfsukzession liegt also eine Parallelität von Entwicklungsstadien vor. Ein wesentliches Untersuchungsergebnis aus dem Harzvorland waren ja die stabil bleibenden qualitativen Artengruppenspektren und somit das Überdauern typischer Waldarten unter verschiedenartigen Pionierstadien oder verschiedenartiger Baumartenverjüngung, was sich u.a. auch in weitestgehend stabilen absoluten Deckungssummen der meist krautigen, oligohemeroben, frische- und schattenzeigenden Querco-Fagetea-Arten ausdrückt (Kap. 4.3 und Tab. A22, A23). Es lassen sich einige Beispiele dafür finden, dass dies nicht nur in der Startphase der Windwurfsukzession so ist und eine räumliche und zeitliche Parallelität solcher Stadien auch langfristig der Normalfall bleibt. Auf dem Kahlschlag eines HordelymoFagetum im Göttinger Wald überdauerten nach DIERSCHKE (1988) die Waldarten alle aufeinanderfolgenden Sukzessionsstadien (Krautiges Pionier-, Rubus-Gebüsch- und Vorwaldstadium) recht gut und nahmen langfristig wieder zu. Während des gesamten Untersuchungszeitraumes (16 Jahre) wurden etwa 50\% der Gesamtartenzahl aus den Waldarten des Altbestandes gebildet, wodurch kein genereller Artenwechsel abgeleitet werden kann. Dieses „Ausharren“ der alten Waldbodenflora und somit eine hohe Resilienz des Ökosystems Buchen-Wald stellte auch ScHMIDT (1997) bei experimentellen Lochhieben 
in Kalk-Buchenwäldern fest. Obwohl innerhalb der ersten 7 Jahre nach der Auflichtung starke Verluste z.B. bei Allium ursinum und Mercurialis perennis auftraten (beides im Harzvorland bislang nicht zu beobachten - Tab. A22, A23), kam es dort insgesamt nur zu minimalen qualitativen Artenverschiebungen. Aus dem osthessischen Bergland liegt eine mit etwa 50 Beobachtungsjahren ungewöhnlich lange Zeitreihe vor, mit der die Sukzession auf einem Eichen-Kahlschlag (Luzulo-Fagetum-Standort) studiert wurde (GREGOR \& SEIDLING 1997, 1998, 1999). Einer mehrjährigen krautigen Schlagflur folgte dort ein Himbeer-Gebüsch (bis 9. Jahr), ein Sambucus-Frangula-Gebüsch (bis 13. Jahr) und anschließend ein räumliches und zeitliches Nebeneinander von Vor- (Betula, Populus, Salix), Zwischen- (Quercus, Carpinus, Prunus, Acer) und Schlusswaldstadien (Fagus sylvatica). In einem nordwestdeutschen Naturwaldreservat in den Stemwehder Bergen mit Galio odorati-Fagetum auf KreideKalkmergel (APFFELSTAEDT \& BERNHARDT 1996) verfügte der dort belassene lückige Windwurf etwa 20 Jahre nach den Stürmen von 1972/76 noch immer über das komplette Arteninventar der Ausgangsgesellschaft mit mäßig starker Buchen-Verjüngung und wurde von höherdeckenden Schlagflur- und Ruderalarten lediglich überlagert. Ebensowenig wurde im baden-württembergischen Windwurf nach 1990 eine strikte zeitliche Vegetationsabfolge ersichtlich, sondern vielmehr die parallele Etablierung von Gehölzen früher, mittlerer und späterer Stadien (FISCHER et al. 1998). Die Sukzession windgeworfener mitteleuropäischer Buchen-Wälder sollte man vor diesem Hintergrund also weniger als zyklische Abfolge von unterschiedlichen Artenkombinationen interpretieren, sondern eher als zwischenzeitliche, standörtlich unterschiedlich ausgeprägte Überlagerung des ursprünglichen Artenspektrums durch Schlagflur- und Ruderalarten.

\subsubsection{Eingangshypothesen: Standortsgradient und standörtliche Gunst}

Der eingangs (Kap. 1.4) postulierte Standortsgradient beinhaltet eine Abnahme der Wiederbewaldungsgeschwindigkeit im Sinne der Klimaxgesellschaft von frischen, basen- und nährstoffreichen Standortsverhältnissen hin zu (trockenen?) sauer-armen Bedingungen. Dabei sollte in derselben Richtung die Bedeutung der Störungsflächengröße zunehmen. Die Hypothesen 1-3 werden durch die Ergebnisse der vorliegenden Arbeit bestätigt (Standortsabhängigkeit der Windwurfsukzession, verlangsamte Wiederbewaldung mit zunehmender standörtlicher Ungunst, kein genereller Artenwechsel, insgesamt große Resilienz des Ökosystems Buchen-Wald). Zur Stützung dieser Hypothesen können nach den vorliegenden Untersuchungen, die ja nur einen Teil der Standortsamplitude von BuchenWäldern repräsentieren, vor allem die Artengruppenspektren (Kap. 4.3) und die Verjüngungszahlen (Kap. 4.5) herangezogen werden. Laubwaldtypische Artengruppen überdauern die Störung auf basenreich-tiefgründigen Standorten besser (d.h. mit höheren absoluten und relativen Abundanzen) als auf basenarmen. Die Verjüngungsdichte der Schlusswald-Laubbaumarten ist besonders auf basenreich-tiefgründigen Standorten 
wesentlich höher als auf basenarmen und verspricht eine rasante Wiederbewaldung des geworfenen Hordelymo-Fagetum. Sie erscheint auf basenreichen Windwurfstandorten weniger abhängig von der Störungsflächengröße als auf basenarmen (Literaturvergleich bzw. eigene Erhebungen, siehe Kap. 5.5 und 5.8.1).

Die Bestätigung oder Verwerfung von Hypothese Nr. 4, welche auf basenreichen Standorten einen relativ geringen Einfluss forstlicher Räumungsmaßnahmen auf die Windwurfsukzession postuliert und auf basenarmen einen relativ großen (Kap. 1.4), ist eine Frage des betrachteten Parameters. Die quantitativen Artengruppenspektren (Kap. 4.3) scheinen bei einen Vergleich von Hordelymo-Fagetum und Galio odorati-Fagetum diese Vermutung zu stützen. Trotz Räumung/Befahrung wirken die tiefgründigen Dolomit-Standorte (Nüllberg) besonders bei Laubwald-typischen Artengruppen „stabil“, d.h. sowohl gegenüber dem Ausgangszustand als auch gegenüber der belassenen Variante (Hainholz-KF1) relativ wenig verändert. Im Vergleich dazu haben sich die Bestände im Rotenberg besonders in der geräumten Variante (Abt. 1228) in „schlusswaldferne“, Pionierstrauch-dominierte Zwischenstadien verwandelt. In der belassenen Königsbuche dagegen sind auch nach vier Sukzessionsjahren die typischen Waldarten vergleichsweise stärker vertreten. Dementsprechend profitieren die im Flächenwurf (!) verjüngungsarmen Buntsandstein-Standorte von einer Aufforstung mit Klimaxbaumarten mehr als die verjüngungsreichen Dolomit-Standorte, bei denen eine solche Aufforstung eher sinnlos erscheint. Weiterhin könnte man anführen, dass auf geräumten Buntsandstein-Flächen ganz besonders viele Beispiele von stark etablierten Waldstörzeigern zu finden sind, während im Zechstein sowohl auf belassenen, in geringerem Umfang auch auf geräumten Flächen viele Waldarten durch den Windwurf begünstigt werden (Tab. 18, A22, A23). Gegen die generelle Gültigkeit dieser Hypothese spricht aber, dass sich im Krücker (Luzulo-Fagetum) geräumte und belassene Bestände weitestgehend identisch, wenn auch ähnlich wie im Galio odorati-Fagetum relativ „schlusswaldfern“ entwickelten. Auch die Artenzahlentwicklung und die damit verbundene floristische Ähnlichkeit eignen sich kaum für eine Argumentation zugunsten Hypothese 4, denn besonders auf basenreich-geräumten Standorten (z.B. Nüllberg) sorgte ja der drastische Artenzahlanstieg für sehr starke floristische Veränderungen, während bei geringerer Basenversorgung des Bodens (z.B. Krücker, Rotenberg) die Artenzahlunterschiede zwischen geräumten und belassenen Flächen eher gering ausfallen und zumindest die qualitative floristische Ähnlichkeit zum Ausgangszustand in allen Behandlungsvarianten vergleichsweise hoch ist (Kap. 4.2.1 und 4.2.8). Die Vielzahl möglicher Einflussfaktoren (Kap. 5.3, 5.4, 5.5, 5.6) bzw. ihre Überschneidung machen es schwierig, bezüglich Hypothese 4 zu einer verallgemeinerbaren Aussage zu kommen.

Zur Untermauerung der postulierten Erstrangigkeit des Standortes als Einflussfaktor in der Sukzession nach Störung sollen auch die nun zitierten Erkenntnisse anderer Autoren dienen: 
So betont FALINSKI (1986) am Beispiel des ostpolnischen Bialowiesa-Urwaldes die Rolle der Nährstoff- und Wasserversorgung für die Geschwindigkeit der Waldregeneration. Die dort auf ärmeren Böden stockenden Subassoziationen des Tilio-Carpinetum reagierten nach Störung (z.B. Wild, Windwurf) mit größerer Dynamik (lang andauernde Artenzahlanstiege, längere Regenerationsdauer) als die Subassoziationen auf besser versorgten Böden (geringe Artenzahlanstiege, relativ stabile Struktur der Krautschicht, eher Fluktuation statt Sukzession). Extrem nährstoffarme Standorte gleichen KoOP (1982) zufolge fast dauerhaften Pionierstadien, besser versorgte Standorte dagegen entwickeln sich schneller in Richtung Klimax. Das Fazit des Autors: je extremer Boden, Klima und Wasserhaushalt, umso größer die innere Dynamik und das zeitliche Ausmaß des Verjüngungsprozesses. Nach LEUSCHNER (1994, 1997) verläuft die Waldregeneration nach Störung auf basenarmen Standorten und in stark fragmentierten Landschaften langsamer und es können durchaus Baumartenwechsel auftreten. Länger andauernde Schlagflur- und Vorwaldstadien mit z.T. verjüngungshemmender Vegetation wurden in den süddeutschen Fichten-Windwurfgebieten ja eher auf mittleren und sauren Standorten beschrieben, nicht auf basenreichen (HETZEL \& REIF 1998; BAUER 2001; MÄRKL \& EGLSEER 2001). Die relativ langsame Regeneration des montanen, bodensauren Fichten-Waldes im Bayerischen Wald (FISCHER \& JEHL 1999; FISCHER et al. 2002) und die verzögerte Wiederbewaldung der Schweizer Bergfichtenwälder (SCHÖNENBERger \& WASEM 1999; SCHÖNENBERGER 2002) sind weitere Beispiele für den Einfluss der standörtlichen Ungunst.

Bezogen auf die großflächigen Windwurfgebiete des Harzvorlandes und ausgehend von den im vierten Sukzessionsjahr vorgefundenen Verjüngungsdichten (Kap. 4.5) müsste man folgenden Gradienten der standörtlichen Gunst aufstellen, der entsprechende Rückschlüsse auf die Regenerationsgeschwindigkeit der unterschiedlichen Buchen-Waldgesellschaften zulässt:

tiefgründiger Dolomit-Standort (Hordelymo-Fagetum, sehr günstig) > flachgründiger Gips- oder Gips/Dolomit-Standort (Carici-Fagetum, mäßig günstig) > Buntsandstein (Luzulo- bzw. Galio odorati-Fagetum, relativ ungünstig).

Im europäischen Vergleich (Kap. 5.8.1) werden die Buchen-Windwurfgebiete des Harzvorlandes insgesamt eine relativ schnelle Entwicklung hin zur potenziellen Schlusswaldgesellschaft nehmen, die um einiges zügiger abläuft als z.B. die Windwurfsukzession auf vernässten oder hochmontanen oder Heidestandorten mit nicht standortsgerechter Vorbestockung. Die von KENK et al. (1991) als Erfahrungswert einer durchschnittlichen Wiederbewaldungsdauer für viele Waldökosysteme genannten 10 Jahre oder weniger dürften im großflächigen Windwurf des Harzvorlandes auf den tiefgründigen Dolomit-Standorten bereits erfüllt sein, auf den flachgründigen Gips-Standorten könnte dies 
mit einiger Zeitverzögerung ebenso zutreffen. Im Buntsandstein bleibt die Entwicklung abzuwarten, von einer generellen Ablösung des Buchen-Waldes ist aber auch auf diesen Standorten nicht auszugehen.

\subsection{Konsequenzen für Naturschutz und Waldbau}

\subsubsection{Biodiversität und Naturschutz}

Bei Fokussierung auf die Parameter „Gefäßpflanzenarten“ und „ $\alpha$-Diversität“ (Artenzahl pro Flächeneinheit) läßt sich im hier untersuchten Buchen-Windwurf in den ersten Sukzessionsjahren durchaus eine ansteigende Organismenvielfalt feststellen. Es sind aber zumeist waldfremde „Allerweltsarten“ und nicht etwa Rote-Liste-Arten, die zu diesem Zuwachs an Biodiversität beitragen. Besonders geräumte Windwurfflächen mit ihren artenreichen Schlag- und Ruderalfluren sind zwar hochdivers, aber im Vergleich zum Ausgangsbestand bzw. zum belassenen Windwurf häufig auch relativ naturfern bzw. waldfremd. Sie erscheinen „doppelt gestört“ (erst Windwurf als natürliche und danach Räumung/Bepflanzung als anthropogene Störgröße). Hohe Diversität und Naturnähe stellen sich unter diesem Gesichtspunkt als gegensätzliche Pole dar (KOMPA \& ScHMIDT 2002). Deshalb sollten weitere Diversitätsniveaus in die Betrachtung einbezogen werden, etwa die $\beta$ Diversität als Vielfalt von Gesellschaften entlang ökologischer Gradienten (z.B. Bestand Lücke - Flächenwurf oder basenreich - mittel - basenarm) oder die $\chi$-Diversität als Gesamtvielfalt einer Landschaft (WHITTAKER 1973). Da der konventionell bewirtschaftete Wald eine Vielzahl von Waldentwicklungsstadien überhaupt nicht zulässt, können v.a. belassene Windwurfflächen zu einer Erhöhung der natürlichen Biodiversität in unseren Wäldern beitragen. Für geräumte Windwurfflächen trifft diese Aussage wesentlich schwächer zu, da sie eine vergleichsweise geringe strukturelle und kleinklimatische Heterogenität besitzen (WILMANNS et al. 1998). Der naturschutzfachliche Wert belassener Windwurfflächen liegt also nicht in der (oftmals gar nicht vorhandenen) Seltenheit einzelner Phanerogamenarten, sondern eher in der strukturellen Diversität und der Seltenheit der Biozönose an sich (FISCHER et al. 1998). Bei holzbewohnenden Tieren und Pilzen kommt neben dem störungsbedingten Zugewinn an struktureller Diversität noch ein weiteres wichtiges Naturschutzargument hinzu, nämlich die hohe Anzahl von Rote-Liste-Arten oder seltenen Zönosen. In den Schweizer Bergwäldern ließen sich z.B. nach den 1990er Stürmen auf Windwurfflächen bis zu 60\% mehr Arthropoden-Arten gegenüber ungestörten FichtenBeständen finden, allerdings keine Unterschiede zwischen geräumten und belassenen Flächen hinsichtlich Individuenzahl und Rote-Liste-Arten (LÄSSIG et al. 1995a; DuELLI \& OBRIST 1999). Im Buchen-Windwurf des hessischen Naturwaldreservates Weiherskopf fand SCHLECHTE (2002) eine äußerst artenreiche und dynamische Pilzflora und besonders ab dem sechsten Sukzessionsjahr auch viele Rote-Liste-Arten, die auf dem liegenden Sturmholz ein Refugium fanden, dass ihnen im Wirtschaftswald normalerweise entzogen ist. Für 
Windwurfgebiete der Zukunft empfehlen LÄSSIG \& SCHÖNENBERGER (2000) ein Nebeneinander von geräumten und belassenen Flächen zur Erhöhung der floristischen und faunistischen Diversität. Auch KUHN (1995) sieht das vorhandene „Sturmholz als Kapital, auf dessen Zinsen sowohl Naturverjüngung als auch Biodiversität angewiesen sind“.

In ungestörten Wäldern lassen sich zum Thema „Natürlichkeit vs. Diversität“ ähnliche Feststellungen treffen wie im Windwurf. So argumentieren SCHMIDT \& WECKESSER (2002) bzw. WECKESSER (2003) im Hinblick auf bodensaure Luzulo-Fagetum-Standorte des Sollings, dass Artenzahlen allein nicht geeignet sind, die Diversität eines Bestandes zu beschreiben. Vielmehr sollten funktionale ökologische und naturschutzfachliche Aspekte mit einbezogen und der Natürlichkeitsgrad eines Bestandes höher bewertet werden als seine Diversität. Steigende Gefäßpflanzen-Artenzahlen bedeuteten im genannten Beispiel gleichzeitig sinkende Naturnähe, weil sie in erster Linie mit zunehmenden Anteilen an standortsfremder Fichte und waldfremden Störzeigern korreliert waren. Somit wäre die Einbringung der Fichte in ehemals naturnahe Buchen-Wälder als anthropogene Störung zu begreifen, die zwar mit der Störungsart „Räumung von Windwurfflächen“ nur sehr bedingt vergleichbar ist, aber eine ähnliche ökologische Funktion übernimmt und ähnliche Resultate zeigt.

Nach WECKESSER (2003) ist der Hemerobiegrad als reziprokes Maß der Natürlichkeit ein geeignetes Mittel zur Bewertung der Naturnähe von Waldbeständen. Überträgt man das auf die hiesigen Windwurfgebiete (Kap. 4.3.11), so sind die Bestände im Harzvorland auch nach dem Sturm sehr naturnah geblieben. Euhemerobe Arten spielen insgesamt kaum eine Rolle, fast überall dominieren oligohemerobe Arten. Dennoch sollte man die tendenziell größere Bedeutung meso- bis euhemerober Arten im langjährigen Wirtschaftswald bzw. auf geräumten Windwurfflächen gegenüber der teilweise zunehmende Dominanz oligohemerober Arten im langjährigen Naturwald bzw. im belassenen Windwurf beachten (Abb. 39). Darin spiegelt sich der Einfluss der Bewirtschaftungsgeschichte bzw. der Räumung - wenn auch nicht besonders prägnant - wider.

Diese Beispiele sollen verdeutlichen, dass Arten- und Strukturvielfalt nur bedingt zahlenmäßig bewertbar sind, die Betrachtungsebene eine entscheidende Rolle spielt und die Natürlichkeit der gesamten Waldbiozönose an erster Stelle aller ökologischen, aber auch waldbaulichen Überlegungen stehen sollte. Speziell für den Erhalt naturnaher Buchen-Wälder hat die Bundesrepublik Deutschland - völlig unabhängig von der Anzahl vorzufindender Rote-Liste-Arten - eine hohe Verantwortung, da ein Großteil des natürlichen Areals dieser Ökosysteme auf deutschem Territorium liegt (SsYMANK et al. 1998).

\subsubsection{Waldbauliche Praxis}

Geschwindigkeit und Qualität der natürlichen Wiederbewaldung nach Windwurf sind entscheidende Faktoren, auf deren Kenntnis die Entwicklung möglichst naturnaher Waldbaukonzepte in Zukunft beruhen muss. Die in letzter Zeit immer stärker erhobene 
Forderung nach natürlicher Wiederbewaldung oder gar völliger Belassung von Windwurfflächen ist ökologisch und ökonomisch sinnvoll, um teure Aufforstungen auf ein notwendiges Maß zu beschränken (z.B. BROGGI 2000; LÄSSIG 2000; WILLIG 2001). Noch Anfang der 1990er Jahre kritisierten KENK et al. (1991) die oft recht enge Auslegung des gesetzlichen Wiederaufforstungsgebotes nach natürlichen Störungen und dem daraus resultierenden geringen Stellenwert der natürlichen Wiederbewaldung in der forstlichen Theorie und Praxis. Sie wiesen aber schon damals auf einen nach den 1990er „Vivian/Wiebke“-Würfen einsetzenden Gesinnungswandel hin. In Baden-Württemberg sollten demzufolge Sturmwurfflächen bis 0,3 ha regulär aus Naturverjüngung wiederbewaldet werden. Nach den 1999er „Lothar“-Würfen gibt es im selben Bundesland nunmehr die Absicht, auf zwei Dritteln dieser neueren Sturmflächen die Bestandesbegründung überwiegend oder ausschließlich aus natürlichen Verjüngungsvorräten erfolgen zu lassen (Aldinger et al. 2001; STEINFATH 2001). Auch aus der Schweiz wird von einem derartigen Richtungswechsel berichtet, der vor dem Hintergrund sehr großer Windwurfflächen auch durch ökonomische Überlegungen herbeigeführt wurde. So blieben dort immerhin etwa 20\% des „Lothar“-Sturmholzes im Wald liegen (LÄSSIG 2002). Neben den hohen Aufforstungskosten spielt dabei meistens auch der zwangsläufige Preiseinbruch auf dem Holzmarkt eine Rolle (z.B. in der Schweiz: bei Nadelholz minus 35\%, bei Laubholz minus 15-40\% nach BRÄKER \& BÜRGI 2000). Wenn man dagegen bedenkt, dass nur 4\% der Schweizer Waldfläche vom 1999er Orkan betroffen waren, so ergibt sich eine starke Relativierung solch anthropozentrisch geprägter Begriffe wie „Sturmkatastrophe“. Wieviel kleiner erscheint dagegen erst der Windwurf im Harzvorland (Kap. 1.1 und 2.1)! Dennoch darf man nicht aus den Augen verlieren, dass für den einzelnen betroffenen Privatwaldbesitzer solch ein Sturm oft tatsächlich katastrophale wirtschaftliche Folgen hat. Zu Recht weist KuHN (1995) darauf hin, dass das Liegenlassen von Sturmholz in der Bevölkerung oft nicht akzeptiert ist. Aus dem Harzvorland liegen ebensolche persönlichen Erfahrungen des Autors vor. Es wird vermutlich noch eine ganze Zeit vergehen, bis allgemein anerkannt ist, dass die natürliche Dynamik in Wäldern ihren Platz haben soll. Wegen der offensichtlich hohen Resilienz mitteleuropäischer Buchen-Wälder empfiehlt ScHMIDT (2003) eine gelassene Herangehensweise an die Sturmwurfproblematik, was durch die vorliegenden Ergebnisse aus Zechstein-Windwurfgebieten im südwestlichen Harzvorland gestützt wird. Doch Windwurf geschieht nicht nur in naturnahen Wäldern und auf günstigen Standorten. So sehen ANGST et al. (2000) Stürme auch als Chance für einen Waldumbau, der das zukünftige Landschaftsbild nachhaltig verändern wird. Sie sind gewissermaßen Katalysatoren für ohnehin schon geplante Waldumbaumaßnahmen (z.B. von naturferneren Nadel- zu naturnäheren Laubholz- oder Mischbeständen). Für die waldbauliche Praxis nach Sturmwurf sind deshalb differenzierte Einzelfallentscheidungen unter der Gesamtschau aller Einflussfaktoren anstrebenswert. Konkret mündete dies in folgende waldbauliche Empfehlungen, die sich mittlerweile auf mehr als ein Jahrzehnt Praxiserfahrung stützen: 
1. Eine genaue Analyse der Verjüngungsvorräte direkt nach dem Sturm sollte die Grundlage für weiteres forstliches Tun oder Lassen sein (KENK et al. 1991; FISCHER et al. 1998; WILLIG 2001). Solch eine Bewertung sollte im Spätwinter der 2. bis 3. (maximal 5.) Vegetationsperiode nach der Störung erfolgen (SCHÖLCH 1998; ALDINGER \& KENK 2000; BROGGI 2000; LÄSSIG 2000). Der Verjüngung unter $30 \mathrm{~cm}$ Wuchshöhe ist dabei in Zukunft mehr Beachtung zu schenken (HETZEL \& REIF 1998). In 50-100 m breiten Randzonen der Windwurffläche zu angrenzend stehenden Beständen ist häufig mit ausreichender Verjüngung bzw. ausreichendem Sameneintrag zu rechnen und ein forstlicher Eingriff nicht erforderlich (KENK et al. 1991; Aldinger \& KENK 2000). Für kleinere Lücken unter $200 \mathrm{~m}^{2}$ empfiehlt Huss (1991) sogar, die Forstleute sollten „20 Jahre nicht hinschauen“ und die Selbstheilungskräfte des Bestandes staunend zur Kenntnis nehmen.

2. Bei der Jungbestandspflege sind punktuelle Eingriffe zugunsten der Wirtschaftsbaumarten flächigen Durchforstungen vorzuziehen (SCHÖLCH 1998; EHRING 2001; FLÖß 2001). Ergänzungspflanzungen sind nur bei nicht ausreichender Verjüngung und bei angestrebtem Baumartenwechsel nötig. Sie sollten frühestens nach 3 bis 5 Beobachtungsjahren vorgenommen werden - es sei denn, es zeichnen sich Dominanzen verjüngungshemmender Bodenvegetation wie Adlerfarn, Reitgras oder Brombeere ab (ALDINGER \& KENK 2000; Aldinger et al. 2001; Aldinger \& Michiels 2001). In Gebieten mit entsprechend hoher Verbissbelastung sollte die Bejagung intensiviert werden (Huss 1991; BROGGI 2000; ALDINGER et al. 2001). Die Naturverjüngung gilt als gesichert, wenn 2.000 Laubbäume/ha bzw. 1.000 Nadelbäume/ha mindestens eine Vegetationsperiode ohne nennenswerten Verbiss und mit unbeschädigtem Spross überlebt haben (ALDINGER \& KENK 2000).

3. Pionierbaum-reiche Vorwaldstadien sollten ganz bewusst in die Wiederbewaldung einbezogen werden. Vielfach belegt ist deren Schutzeffekt auf Kahlflächen für darunter nachwachsende Schlusswaldbaumarten in Hinsicht auf Ausgeglichenheit des Bestandesklimas (Wind-, Frost- und Dürreschutz), Verbissschutz durch Ablenkung, Minderung des Konkurrenzdruckes durch die Bodenvegetation, Abschöpfung überschüssigen Bodenwassers auf Pseudogley-Standorten und Verbesserung der Wuchsform der künftigen Baumgeneration (Huss 1991; KenK et al. 1991; GRUNDMANN 1997; KÖBERLE 1997; SCHMIDT-SCHÜTZ \& HuSS 1998; SCHMIDT-SCHÜTZ 1999; FLÖß 2001).

4. Möglichst bodenschonende Räumverfahren sollten bevorzugt werden, um Vorausverjüngung und Mykorrhiza so wenig wie möglich zu zerstören (BROGGI 2000; ALDINGER et al. 2001; FISCHER et al. 2002).

5. Auf großen Kahlflächen sollten einzelne überlebende Bäume geschont werden. Ihre Bedeutung beruht auf ihrer Wirkung als Wasserpumpe (wichtig auf vernässenden Pseudogley-Standorten), Schattenspender, Frostschutz, Samenquelle, Wurzelkonkurrenten zu krautiger und grasartiger Vegetation, als Anzeiger für Schädlingsbefall und auch zur Marktentlastung (KÖBERLE 1997; SCHÖNENBERGER et al. 2002, 2003). 
6. Leistungsschwache und schwer zugängliche sowie für den Arten- und Biotopschutz relevante Sturmwurfflächen sollten bevorzugt der Sukzession überlassen werden (HuSS 1991). Auf sehr steilen Gebirgswald-Standorten sollte das Sturmholz aus Gründen des Lawinenschutzes ebenfalls belassen werden (LÄSSIG et al. 1995a; LÄSSIG \& SCHÖNENBERGER 2000).

Bezogen auf die untersuchten Buchen-Waldstandorte im Harzvorland soll abschließend folgender Bewirtschaftungsvorschlag gemacht werden, der nach zukünftigen Windwürfen zur Anwendung kommen könnte:

1. Carici-Fagetum auf flachgründiger Rendzina über Gips oder Gips/Dolomit: generell belassen (geringe Produktivität und Seltenheit des Standortes).

2. Hordelymo-Fagetum auf tiefgründigem Dolomit-Verwitterungslehm: räumen und anschließend belassen (Wiederbewaldung unabhängig von der Störungsflächengröße gewährleistet).

3. Galio odorati- und Luzulo-Fagetum auf Löss über Buntsandstein: in Lücken räumen und anschließend belassen (Wiederbewaldung gewährleistet); im Flächenwurf: räumen und bei Bedarf pflanzen (häufig zu schwache Naturverjüngung). 


\section{ZUSAMMENFASSUNG}

In den Jahren 1998-2001 wurden im südwestlichen Harzvorland in Windwurfgebieten auf Zechstein und auf Unterem Buntsandstein vegetationsökologische und gehölzkundliche Erhebungen auf Dauerflächen durchgeführt, um die Sukzessionsdynamik und Regeneration natürlich gestörter Buchen-Wälder in Abhängigkeit von Standort, ehemaliger Nutzungsgeschichte, aktueller forstlicher (Nicht-)Behandlung und Störungsflächengröße zu studieren. In den ausgewählten Untersuchungsgebieten stocken für den nordwestdeutschen Raum typische Buchen-Waldgesellschaften mit relativ großer Naturnähe (teilweise Status Naturwald oder Naturschutzgebiet). Nach dem lokalen Orkan vom Juni 1997 wurde ein Teil der geworfenen Waldbestände (Hainholz, Krücker, Königsbuche) sich selbst überlassen und somit einer besonderen Form der ökologischen Langzeitforschung zugänglich gemacht. Dabei kann deutschlandweit erstmalig Buchen-Windwurf auf Gipskarst studiert werden. Die Standortsamplitude der untersuchten Bestände bewegt sich im Bereich mäßig-trocken bis frisch, es lässt sich grob eine Dreiteilung in basenreich-flachgründige (Gips/Dolomit, Rendzinen, Carici-Fagetum), basenreich-tiefgründige (Dolomit, Rendzina-Braunerden, Hordelymo-Fagetum) und basenarme Standorte (Buntsandstein, lössüberdeckte Braun- und Parabraunerden, Galio odorati-/Luzulo-Fagetum) vornehmen. Der Schwerpunkt der vorliegenden Untersuchungen lag auf sieben Varianten mit großflächigem Windwurf, wobei für die einbezogenen Waldgesellschaften jeweils ein belassenes und ein geräumtes/aufgeforstetes Pendant existiert (nicht im Carici-Fagetum: dort nur belassener Flächenwurf). Es wurden Vegetationsaufnahmen (Grundfläche 100 m²; Zeitraum 1998-2001 oder kürzer), Wurzeltelleraufnahmen (Zeitraum 1999-2001) und Verjüngungsaufnahmen (Grundfläche $20 \mathrm{~m}^{2}$; Sommer 2001) durchgeführt sowie bodenchemische Parameter und der relative Lichtgenuss ermittelt. Ferner wurden populationsbiologische Daten zu Rubus idaeus erhoben. Speziell im Buntsandstein wurden bei der Verjüngungsinventur zusätzlich die beiden Straten „Lücken“ (lückiger Windwurf) und „Bestand“ (intakter oder nur randlich beeinflusster Wald) einbezogen. Die Untersuchungsflächen waren im belassenen Windwurf geklumpt in Form lückenlos gerasterter Kernflächen angeordnet, in den geräumten Varianten dagegen unsystematisch verteilt.

\section{ERGEBNISSE:}

In allen Varianten mit Flächenwurf erfolgte im vierjährigen Untersuchungszeitraum ein kontinuierlicher Anstieg der mittleren Artenzahlen auf unterschiedlichem Niveau, in der belassenen Variante des Hordelymo-Fagetum nahmen sie ab dem vierten Sukzessionsjahr (2001) bereits wieder ab. Die basenreichen Standorte waren artenreicher als die basenarmen, geräumte Flächen waren artenreicher als belassene. Das höchste Niveau erreichten nach vier Jahren mit 50 bzw. 57 Feldschichtarten/100 $\mathrm{m}^{2}$ die beiden carbonatreichsten Standorte 
(belassenes Carici-Fagetum bzw. geräumtes Hordelymo-Fagetum), während ansonsten 20-30 Feldschichtarten/100 $\mathrm{m}^{2}$ notiert wurden. In der Regel blieben die Feldschicharten etwa gleichstark durchmischt (stagnierende Evenness, im Carici-Fagetum jedoch steigende). Dagegen kam es in der Strauchschicht zur dominanten Entwicklung einzelner Gehölzarten (teilweise massiv abnehmende Evenness). Nur relativ wenige Arten vor allem der Feldschicht erreichten mittlere Deckungsgrade von über 1\%. Ihr Anteil an der Gesamtartenzahl betrug im vierten Jahr nach dem Sturm variantenabhängig etwa 10-18\% (Feldschicht) bzw. 18-68\% (Strauchschicht). Somit wurden die floristischen Veränderungen häufig durch neuauflaufende Arten im unteren Deckungsgradbereich verursacht. Quantitativ betrachtet (Index Percentage Similarity) veränderten sich die belassene Variante des Hordelymo-Fagetum und die geräumte Variante des Galio odorati-Fagetum am wenigsten, die geräumte Variante des Hordelymo-Fagetum dagegen am stärksten. Die Strauchschicht-Deckungen stiegen auf allen Standorten in unterschiedlichem Maße kontinuierlich an. Der flachgründige Gips/DolomitStandort (Carici-Fagetum) wies nach vier Sukzessionsjahren die schwächste Gehölzentwicklung auf (12\% mittlere Strauchschicht-Deckungssumme gegenüber $60-90 \%$ auf den übrigen Standorten). Durch die Gehölzexpansion sank der relative Lichtgenusses über der Feldschicht z.B. in der belassenen Variante des Hordelymo-Fagetum von 54\% (1998) auf 12\% (2001). Aufgrund dieses Ausschattungseffektes stagnierten die KrautschichtDeckungen oder gingen zurück. Im Zechstein war die Feldschicht bislang dichter ausgeprägt als die Strauchschicht, im Buntsandstein übertrifft eine zunehmend dichter schließende Strauchschicht die Feldschichtwerte oder nähert sich ihnen an. Die Kryptogamendeckung nahm auf allen Standorten zu, wobei sich das Luzulo-Fagetum mit maximal 37\% Deckungsgrad im vierten Jahr als der Kryptogamen-reichste Standort erwies. Die mittlere Zahl aufgeklappter Wurzelteller/100 $\mathrm{m}^{2}$ variierte zwischen 1 und 4, die der liegenden Stämme zwischen 6 und 10 (belassener Flächenwurf). Die mittlere Totholzdeckung lag bei 22-36\% (belassene Varianten) bzw. bei 5-13\% (geräumte). Die Deckung liegend-lebender Baumkronen nahm kontinuierlich ab und ging nach vier Jahren teilweise gegen Null, teilweise trieben vor allem geworfene Buchen und Eschen immer noch neu aus (in 2001: 514\% mittlere Deckung). Bezüglich der mittleren Maximalhöhe der Strauchschicht ergab sich im vierten Sukzessionsjahr die Reihenfolge Hordelymo-Fagetum (belassen: 3,80 m) > Galio odorati-/ Hordelymo-Fagetum (beide geräumt: jeweils 3,20 m) > Galio odorati-Fagetum (belassen: 2,70 m) > Carici-Fagetum (2,30 m).

In der bisherigen Sukzessionsreihe war in allen Varianten eine Konstanz der qualitativen Artengruppenspektren festzustellen. Die einzelnen ökologisch-funktionalen Artengruppen erhöhten ihre Artenzahlen in etwa demselben Maß, so dass sich ihr prozentuales Verhältnis zueinander kaum oder gar nicht veränderte. Dies traf auf alle elf untersuchten Parameter zu (ökologisches Verhalten nach vier verschiedenen ELLENBERG-Zeigerwerten, Einteilung nach 
pflanzensoziologischen Klassen, Wuchsformen, Lebensform-, Samenbank-, Ausbreitungsund Hemerobietypen sowie nach dem Vorhandensein vegetativer Ausbreitungsmechanismen).

Unter Einbeziehung der Deckungsgrade (quantitative Artengruppenspektren) zeigten sich teilweise massive Verschiebungen in den Dominanzverhältnissen. Im Carici-Fagetum bestimmten innerhalb der ersten vier Sukzessionsjahre meist krautige Schlagflur- und Ruderalarten das Bild. Diese Bestände waren charakterisert durch vergleichsweise hohe Abundanzanteile an Stickstoff-, Frische-, Halbschatten- und Lichtzeigern, gegenüber der Bodenreaktion indifferenten Arten, Epilobietea- und Artemisietea-Arten, Krautigen, Therophyten, Arten mit kurzfristiger bis temporärer Samenbank ( $<5$ Jahre) und Arten mit generativem Ausbreitungsvermögen, ferner durch etwa gleichgroße Anteile an auto-, anemound endozoochoren bzw. oliogo- und mesohemeroben Arten. Im Hordelymo-Fagetum bestimmten hohe Anteile der alten Waldbodenflora, vor allem aber eine starke Baumartenverjüngung (v.a. Esche) die Physiognomie der Windwurfflächen. Diese Bestände waren charakterisiert durch vergleichsweise hohe Abundanzanteile an Stickstoff-, Frische-, Basen-, Schatten- und Halbschattenzeigern, Querco-Fagetea-Arten, Baumartenverjüngung, Krautigen, Geophyten, Chamaephyten, anemo- und myrmekochoren sowie oligohemeroben Arten, Arten mit vegetativem Ausbreitungsvermögen und Arten mit kurzfristiger bis temporärer Samenbank ( $<5$ Jahre). Im Galio odorati- und Luzulo-Fagetum wurde das Bild durch ausgedehnte Pionierstrauchfluren bestimmt (hauptsächlich Rubus idaeus, ferner auch Sambucus racemosa, S. nigra und Rubus fruticosus agg.). Diese Bestände waren charakterisiert durch vergleichsweise hohe Abundanzanteile an Mäßigstickstoff-, Feuchte-, Mäßigsäure- und Lichtzeigern, Epilobietea- und Molinio-Arrhenatheretea-Arten, Sträuchern, Grasartigen und Farnen, an oligohemeroben und endozoochoren Arten, Arten mit langfristiger Samenbank (>5 Jahre) und Arten mit vegetativem Ausbreitungsvermögen. Insgesamt ist unter der meist üppig entwickelten Strauchschicht ein Überdauern Laubwald-typischer Artengruppen $\mathrm{zu}$ beobachten und auch in Zukunft wahrscheinlich, was sich trotz unterschiedlich stark abnehmender Abundanzanteile dieser Gruppen in ihren konstanten oder teilweise noch zunehmenden absoluten Deckungssummen zeigte. Diese Tendenz war im Zechstein stärker ausgeprägt als im Buntsandstein. Krautige Ruderalfluren stellten auf den meisten Standorten nur ein kurzfristiges Zwischenstadium dar (Ausnahme: Carici-Fagetum). Sie wurden spätestens ab dem vierten Sukzessionsjahr von Pionierstrauchfluren (Buntsandstein) oder starker Baumartenverjüngung (tiefgründige Dolomit-Standorte) verdrängt. Eine Hauptkomponentenanalyse (PCA) zeigte auf den belassenen ZechsteinFlächen die relativ geringen Veränderungen bei den jeweils dominantesten Arten (im Hordelymo-Fagetum: Allium ursium, Galeobdolon luteum, Fraxinus excelsior, in der geräumten Variante zusätzlich Rubus idaeus; im Carici-Fagetum: Impatiens parviflora). Demgegenüber entwickelten sich die Buntsandstein-Flächen meist gleichgerichtet von Impatiens parviflora-Dominanzstadien hin zu ausgedehnten Himbeer-Fluren. 
Standortsübergreifende Unterschiede zwischen geräumten und belassenen Flächen ließen sich neben den bereits genannten Artenzahlunterschieden lediglich anhand der vergleichsweise stärkeren Überprägung geräumter Flächen durch Arten mit langfristiger Samenbank (>5 Jahre) feststellen. Darüber hinaus ergaben sich standortsabhängig unterschiedlich starke Präferenzen einzelner ökologisch-funktionaler Artengruppen in einer der beiden Nutzungsvarianten. Beispielsweise traten auf geräumten/bepflanzten Flächen folgende Gruppen stärker auf: Schlagflurarten (nicht im Luzulo-Fagetum), Ruderalarten (nur im Hordelymo-Fagetum), Lichtzeiger (nur im Hordelymo-Fagetum), Baumartenverjüngung (nur im Buntsandstein: hier wahrscheinlich Aufforstungseffekt), Sträucher (nicht im LuzuloFagetum), Grasartige (nur im Luzulo-Fagetum), endozoochore Arten (nicht im LuzuloFagetum) sowie meso- und euhemerobe Arten (nicht im Luzulo-Fagetum). Es lassen sich sowohl für die basenreichen als auch die basenarmen Standorte jeweils unterschiedliche Arten benennen, die entweder die geräumte oder die belassene Variante „bevorzugen“. Dies drückt sich meist in höheren Deckungsgraden, seltener in exklusivem Vorkommen aus. Im Buntsandstein (beide Nutzungsvarianten) gehören hierzu hauptsächlich Stör- und Verlichtungszeiger, im Zechstein betrifft dies auf den belassenen Flächen v.a. Laubwaldarten (Querco-Fagetea), auf den geräumten Flächen eine Mischung aus verschiedenen Gruppen. Ein Teil der Pioniergehölze hatte auf geräumten Flächen einen Ausbreitungsschwerpunkt (z.B. Rubus fruticosus agg. v.a. im Buntsandstein, Rubus idaeus und Sambucus racemosa v.a. im Hordelymo-Fagetum, Sambucus nigra v.a. im Galio odorati-Fagetum).

Am Beispiel der Buntsandstein-Varianten ließ sich die unterschiedliche Entwicklung in langjährigen Natur- bzw. Wirtschaftswäldern studieren. Obwohl sich die Plots im bisherigen Sukzessionsverlauf anglichen, zeichnete sich der etwa 30 Jahre ungenutzte Naturwald Königsbuche anfangs (d.h. 1999) noch durch relativ geringe Artenzahlen sowie geringe Abundanzanteile an Licht-, Basen- und Stickstoffzeigern, Epilobietea-Arten, Sträuchern, endozoochoren sowie meso- bis euhemeroben Arten und Arten mit langfristiger Samenbank (>5 Jahre) aus. Entsprechend hoch waren dort anfangs die Abundanzanteile an Schatten-, Frische-, Mäßigstickstoff- und Mäßigsäurezeigern, Querco-Fagetea-Arten, Krautigen, Geophyten, autochoren und oligohemeroben Arten sowie Arten mit temporärer bis kurzfristiger Samenbank $(<5$ Jahre). Damit bestätigen sich Erkenntnisse aus Vergleichsuntersuchungen von nicht geworfenen Natur- und Wirtschaftswäldern in der Optimalphase.

Entsprechend der Deckungsgradentwicklung einzelner Arten wurden fünf verschiedene Sukzessionsmuster ausgeschieden (keine Änderung, Deckungsmaximum im ersten, zweiten/dritten und vierten Jahr nach dem Sturm, Deckungseinbruch nach drei Jahren). Dabei zeigte sich, dass es nur wenige Laubwaldarten gibt, die tatsächlich quantitativ zurückgedrängt werden (z.B. Stachys sylvatica, Hordelymus europaeus). Unter den Arten, die zumindest 
zeitweise von der Windwurfsituation profitieren, sind auch viele Waldarten der Ausgangsgesellschaft vertreten (z.B. Carex sylvatica, Galeobdolon luteum, Athyrium filixfemina).

Die Naturverjüngung der Baumarten wurde im Flächenwurf des Hordelymo-Fagetum bei insgesamt sehr hohen Verjüngungszahlen (im vierten Sukzessionsjahr: etwa 52.000/ha) durch hohe Eschen-Anteile mit unterständiger Buche dominiert. Das Carici-Fagetum erreicht mit etwa 9.000 Sämlingen/ha trotz der Flachgründigkeit des Standortes eine im mittleren Bereich liegende Verjüngungsdichte. Esche, Buche, Berg- und Spitz-Ahorn sowie Sal-Weide teilten sich hier das Artenspektrum. Im Buntsandstein lagen die Verjüngungsdichten weit hinter denen im Zechstein zurück, wobei im Luzulo-Fagetum mit etwa 4.700 Sämlingen/ha gerade noch von einer ausreichenden Naturverjüngung gesprochen werden kann, im Galio odoratiFagetum mit nur 1.500/ha dagegen nicht mehr. Auf den basenarmen Standorten dominierte die Buche in der Verjüngung. Pionierbaumarten fehlten oder waren nur schwach vertreten. Lediglich im Luzulo-Fagetum erreichten Betula pendula und Salix caprea subdominante Anteile (etwa ein Drittel der Sämlingszahl), die in den nächsten Jahren zu lichten, Pionierwald-artigen Stadien führen könnten.

Der Einfluss der Störungsflächengröße auf die Waldregeneration wurde im vierten Sukzessionsjahr u.a. am Beispiel der Verjüngungsdichte im Buntsandstein (Galio odorati-/ Luzulo-Fagetum) geprüft. Mit zunehmender Ausdehnung der gestörten Fläche und abnehmender Überschirmung erhöhte sich der relative Lichtgenuss über der jeweils höchsten Vegetationsschicht vom Bestand (7-15\%) über die Lücken (41-44\%) zum Flächenwurf (9195\%). In ebendieser Reihenfolge sanken die Sämlingszahlen beträchtlich und verlor die Klimaxbaumart Buche Anteile am Baumartenspektrum der Naturverjüngung, behielt aber weiterhin die Dominanz. Während in den Windwurflücken der basenarmen Standorte die Waldregeneration hin zur standortstypischen Buchen-Waldgesellschaft unmittelbar gewährleistet ist, wird sie im Flächenwurf durch die Konkurrenz der Pionierstrauchfluren und Grasartigen längere Zeit in Anspruch nehmen. Hierin besteht ein wesentlicher Unterschied zu den basenreichen Standorten, wo die Waldregeneration hin zur Klimaxgesellschaft weitestgehend unabhängig von der Störungsflächengröße und ohne Pionierstadien aus der Verjüngung der standortstypischen Laubbaumarten sehr schnell voranschreitet, wobei ein vorübergehender Dominanzwechsel (Baumarteninversion) von der Buche zur Esche zu beobachten ist. Auch die Himbeere (Rubus idaeus) als der dominanteste Pionierstrauch im Windwurf des Harzvorlandes zeigte eine Störungsflächenabhängigkeit. Auf einem DolomitStandort (Hordelymo-Fagetum) entwickelte sich die Art bei ansteigender Tendenz in Windwurflücken zahlreicher und fertiler als im benachbarten Flächenwurf, zeigte aber in den Lücken ab dem vierten Sukzessionsjahr bereits Selbstausdünnungstendenzen. 
Die aufgeklappten Wurzelteller als windwurfbedingte Klein- und Sonderstandorte wurden auf belassenen Flächen im Carici-, Hordelymo- und Galio odorati-Fagetum untersucht. Standortsunabhängig kam etwa ein Drittel des Gesamtarteninventars der Untersuchungsflächen nicht auf den Wurzeltellern vor. Andererseits wurde keine Art ermittelt, die - bezogen auf ein UG als Gesamtstratum - ausschließlich auf Wurzeltellern zu finden war. Das Carici-Fagetum war im Gegensatz zu den anderen Beständen ganz besonders stark durch Arten gekennzeichnet, die sowohl auf Wurzeltellern als auch auf ihren Umgebungsflächen an Stetigkeit und Deckungsgrad zunahmen (sehr hohe Besiedlungsdynamik). Das Arteninventar der Wurzelteller und das ihrer Umgebungsfläche war auf allen Standorten erstaunlich ähnlich und blieb innerhalb der ersten vier Sukzessionsjahre konstant. Dagegen trat bei den Dominanzverhältnissen eine standörtlich differenzierte Dynamik auf. Während sich im Carici-Fagetum die betrachteten ökologischfunktionalen Artengruppen auf Wurzeltellern und ihrer Umgebungsfläche quantitativ fast identisch entwickelten, waren die Wurzelteller im Hordelymo- und im Galio odorati-Fagetum bevorzugte Ausbreitungsorte für Lichtzeiger, Epilobietea-Arten, Sträucher und Hemikryptophyten (auf Buntsandstein auch für Feuchte- und Stickstoffzeiger sowie für Arten ohne vegetatives Ausbreitungsvermögen). Typische Laubwaldarten, Geophyten und Baumjungwuchs waren dort dagegen nur relativ schwach verbreitet. Es lassen sich „Besiedlungsspezialisten“ der Wurzelteller benennen, wobei die Himbeere, aber auch Moose standortsübergreifend an erster Stelle stehen. Insgesamt veränderten sich die Wurzelteller floristisch stärker als ihre Umgebungsflächen, woraus eine zunehmende Angleichung resultiert. Diese erfolgt auf dem flachgründigen Gips/Dolomit-Standort schneller als auf den produktiveren Dolomit- bzw. Buntsandstein-Standorten. Es zeigte sich ein standortsabhängig unterschiedlich hoher Prozentsatz von Arten, für die der Wurzelteller in manchen $100 \mathrm{~m}^{2}$ Plots der alleinige Wuchsort war (betraf 0-50\% der Strauchschicht- und 18-41\% der Feldschichtarten). Im Strauchschicht-reichen Hordelymo- bzw. Galio odorati-Fagetum spielten die Wurzelteller als konkurrenzbedingter „Ausweichlebensraum“ für bestimmte Arten vorerst eine tendenziell größere Rolle als im Strauchschicht-armen Carici-Fagetum.

\section{DISKUSSION:}

Die hier beschriebene Vegetationsentwicklung nach Windwurf liefert bisher keine Hinweise auf einen generellen Artenwechsel, wie er z.B. von REMMERT (1985, 1987, 1991) im MosaikZyklus-Konzept postuliert wurde. Eher sollte von einer zwischenzeitlichen Überlagerung der alten Waldbodenflora gesprochen werden, die hauptsächlich vom Standort, mit zunehmender standörtlicher Ungunst auch von der Störungsflächengröße abhängig ist und eine teilweise massive Verschiebung in den Dominanzverhältnissen der Arten einschliesst. Günstige Standorte mit guter Wasser-, Basen- und Nährstoffversorgung und tiefgründigen Böden (hier: Hordelymo-Fagetum) werden sich sehr schnell in Richtung Klimaxgesellschaft entwickeln, relativ ungünstige Standorte mit geringerer Basenversorgung oder flachgründigen Böden 
(hier: Carici-, Galio odorati- und Luzulo-Fagetum) werden dafür etwas länger brauchen. Von einer generellen Ablösung des standortstypischen Buchen-Waldes ist im Harzvorland nirgendwo auszugehen. Entsprechend formulierte Hypothesen konnten durch die eigenen Ergebnisse und anhand eines Literaturvergleiches bestätigt werden. Der Einfluss forstlicher Maßnahmen auf die Windwurfsukzession (Bodenstörung, Diasporeneintrag, Aufforstung) variiert je nach betrachtetem Parameter und ist häufig ebenfalls standortsabhängig.

Im europaweiten Vergleich sind die hier untersuchten Buchen-Windwurfgebiete insgesamt als naturnah und sehr resilient einzuschätzen. Die Waldregeneration im Zechstein des Harzvorlandes weist große Parallelen zu basenreichen Buchen-Windwurfgebieten der Schwäbischen Alb (Kalk) und des Vogelsberges (Basalt) auf.

Der naturschutzfachliche Wert insbesondere belassener Windwurfflächen kann in der Seltenheit und im Strukturreichtum dieser Zönosen gesehen werden, was sich nicht unbedingt in messbaren Diversitätsparametern (z.B. sind geräumte Flächen artenreicher als belassene) oder in - bei den Phanerogamen kaum vorhandenen - Rote-Liste-Arten ausdrücken lässt. Vor diesem Hintergrund wurden Diversität und Naturnähe als teilweise gegensätzliche Pole diskutiert. Für vergleichbare Windwurfsituationen der Zukunft wurde die Empfehlung gegeben, die seltenen und wenig produktiven Gipskarst-Standorte (Carici-Fagetum) generell zu belassen, bei den verjüngungsreichen Dolomit-Standorten (Hordelymo-Fagetum) dagegen nach der Räumung auf die natürliche Wiederbewaldung zu vertrauen. Im Buntsandstein (Galio odorati-/Luzulo-Fagetum) sollten nach einer Räumung nur die Flächenwürfe, nicht jedoch die Windwurflücken bepflanzt werden. 


\section{LITERATURVERZEICHNIS}

ABS, C.; FisCHER, A. \& FALINSKI, J.B. (1999): Vegetationsökologischer Vergleich von Naturwald und Wirtschaftswald, dargestellt am Beispiel des Tilio-Carpinetum im Waldgebiet von Bialowieza/Nordost-Polen. - Forstw. Cbl. 118: 181-196.

AD HOC-ARBEITSGRUPPE BODEN DER GEOLOGISCHEN LANDESÄMTER UND DER BUNDESANSTALT FÜR GEOWISSENSCHAFTEN UND ROHSTOFFE (Hrsg.)(1994): Bodenkundliche Kartieranleitung. 4. Aufl. - Göttingen, 392 S.

AHrns, C. \& Hofmann, G. (1998): Vegetationsdynamik und Florenwandel im ehemaligen mitteldeutschen Waldschutzgebiet „Hainich“ im Intervall 1963-1995. - Hercynia N.F. 31: 33-64.

ALAND (1994): Pflege- und Entwicklungsplan Gipskarstlandschaft Hainholz. - Hannover.

ALDINGER, E.; BROCKAMP, U.; HAUSCHILD, R. \& UlRICH, T. (2001): Sukzession nach Sturmwurf - Ergebnisse nach Wibke und Lothar. - Schriftenr. Freib. Forstl. Forschung 18: 31-39.

Aldinger, E. \& KENK, G. (2000): Natürliche Wiederbewaldung von Sturmflächen. Merkblätter der Forstlichen Versuchs- und Forschungsanstalt Baden-Württemberg 51, 11 S., Freiburg/Brsg.

ALDINGER, E. \& MiCHIELS, H.-G. (2001): Schlussfolgerungen zur natürlichen Wiederbewaldung in Baden-Württemberg aus standortskundlicher Sicht - Kriterien zur Beurteilung der Naturverjüngung auf Sturmwurfflächen. - Ber. Freib. Forstl. Forschung 25: 123-131.

ANGST, C.; LÄSSIG, R. \& BRANG, P. (2000): Nach Windwürfen stabile Bestände schaffen. Wald und Holz 8: 27-30.

APFFELSTAEDT, F. \& BERNHARDT, K.-G. (1996): Vegetations- und populationsbiologische Untersuchungen zur Dynamik von Naturwaldzellen und Windwurfflächen in Nordrhein-Westfalen. - Tuexenia 16: 43-63.

ARBEITSKREIS STANDORTSKARTIERUNG IN DER ARBEITSGEMEINSCHAFT FORSTEINRICHTUNG (Hrsg.)(1985): Forstliche Wuchsgebiete und Wuchsbezirke in der Bundesrepublik Deutschland. - Landwirtschaftsverlag Münster-Hiltrup, $170 \mathrm{~S}$.

ARBEITSKREIS STANDORTSKARTIERUNG IN DER ARBEITSGEMEINSCHAFT FORSTEINRICHTUNG (Hrsg.)(1996): Forstliche Standortsaufnahme. 5. Aufl. - IHW-Verlag Eching, 352 S.

Arevalo, J.R.; DeCoster, J.K.; MCAlister, S.D. \& PAlmer, M.W. (2000): Changes in two Minnesota forests during 14 years following catastrophic windthrow. - J. Veg. Sci. 11(6): 833-840.

Bachofen, H.; BÜrgi, A.; Commarmot, B.; ERni, V.; Frutig, F.; LemM, R.; Oswald, K.; Thees, O.; WASEM, U. \& ZINGG, A. (2000): Waldmanagement nach „Lothar“ Anregungen zum weiteren Vorgehen. - Inf.bl. Forsch.bereich Wald (WSL) 2: 2-5.

BARDEN, L.S. (1979): Tree replacement in small canopy gaps of a Tsuga canadensis forest in the southern Appalachians, Tennessee. - Oecologia 44: 141-142.

BARTELS, H. (1993): Gehölzkunde. - Ulmer, Stuttgart, 336 S.

BARTSCH, N.; BAUHUS, J. \& VOR, T. (1999): Auswirkungen von Auflichtung und Kalkung auf das Sickerwasser in einem Buchenbestand (Fagus sylvatica L.) im Solling. Forstarchiv 70: 218-223.

BARTSCH, N. \& VOR, T. (2000): Gap processes in an Acid Mor Beech Forest Ecosystem in the Solling. - unveröff. Exkursionsführer Univ. Göttingen, Inst. f. Waldbau I, 17 S.

BATTAGLIA, L.L.; FORE', S.A. \& SHARITZ, R.R. (2000): Seedling emergence, survival and size relation to light and water availability in two bottomland hardwood species. - J. Ecol. 88: 1041-1050. 
BAuHus, J. (1994): Stoffumsätze in Lochhieben. - Ber. Forsch.zentr. Waldökosysteme Göttingen A/113: 1-181.

BAUER, E.-M. (2001): Populationsbiologische und pflanzensoziologische Untersuchungen im Bereich des Sturmwurf-Bannwaldes „Silbersandgrube“. - Ber. Freib. Forstl. Forschung 32: 5-53.

BEATTY, S.W. (1984): Influence of microtopography and canopy species on spatial patterns of forest understorey plants. - Ecology 65: 1406-1419.

Bernd, T.; SCHLicht, R.; SCHLimme, H. \& Vladi, F. (1994): Errichtung und Sicherung schutzwürdiger Teile von Natur und Landschaft mit gesamtstaatlich repräsentativer Bedeutung. Projekt: Gipskarstlandschaft Hainholz, Niedersachsen. - Natur und Landschaft 69(7/8): 337-342.

BEZIRKSREGIERUNG BRAUNSCHWEIG (2000): Verordnung über das Naturschutzgebiet „Gipskarstlandschaft Hainholz“ im Landkreis Osterode am Harz vom 06.04.2000.

BöHLING, N. (1992): Floristischer Wandel von Waldgesellschaften. Mögliche Auswirkungen von Bodenversauerung und Nährstoffanreicherung in der südlichen Eilenriede (Stadtwald Hannover). - Naturschutz und Landschaftplanung 1/92: 16-19.

BÖHLING, N. (2003): Dauerflächen-Untersuchungen in einem Eichen-Hainbuchenwald im Vorland der Schwäbischen Alb (Südwestdeutschland), 1978-2001: Der Niedergang von Scilla bifolia und die Invasion von Allium ursinum. - Tuexenia 23: 131-161.

Bolte, A. \& Roloff, A. (1992): Einfluss von Buchenüberhältern auf Bodenvegetation und Naturverjüngung. - Allg. Forst- u. Jagdztg. 164(6): 97-102.

Bonn, S. \& PoschloD, P. (1998): Ausbreitungsbiologie der Pflanzen Mitteleuropas. - Quelle \& Meyer, Wiesbaden, $404 \mathrm{~S}$.

BoRnKAMM, R. (1987): Veränderungen der Phytomasse in den ersten zwei Jahren einer Sukzession auf unterschiedlichen Böden. - Flora 179: 179-192.

BRÄKER, O.U. \& BÜRGI, A. (2000): Lothar: Der Orkan, der Wald und das Holz. Kompetenz-Zentrum Holz (WSL) 1: 17-19.

BrandT, A.; KemPE, S.; SEEgER, M. \& Vladi, F. (1976): Geochemie, Hydrographie und Morphologie des Gipskarstgebietes von Düna/Südharz. - Geol. Jb. C15: 3-55.

BREWER, R. \& MERRITT, P.G. (1978): Windthrow and tree replacement in a climax beechmaple forest. - Oikos 30: 149-152.

BRogGI, M.F. (2000): Wird die Bedeutung der Forschung nach „Vivian“ und „Lothar“ unterschätzt? - Inf.bl. Forsch.ber. Wald (WSL) 2: 1-5.

BRÜNIG, E.F. (1973): Sturmschäden als Risikofaktor bei der Holzproduktion in den wichtigsten Holzerzeugungsgebieten der Erde. - Forstarchiv 44: 137-140.

BRÜNN, S.; GRIES, D. \& SCHMIDT, W. (1996): Reaktion von Calamagrostis epigejos (L.) ROTH auf Unterschiede im Licht- und Stickstoffangebot. - Verh. Ges. f. Ökol. 26: 775780.

Brunet, J.; FALKENGREN-GreRUP, U. \& TYLER, G. (1996): Herb layer vegetation of south Swedish beech and oak forests - effects of management and soil acidity during one decade. - For. Ecol. a. Managem. 88: 259-272.

BRUNET, J. \& TYLER, G. (2000): Interannual variability in abundance of field layer species in a south Swedish deciduous wood. - Flora 195: 97-103.

BÜCKING, W. (1997): Naturwald, Naturwaldreservate, Wildnis in Deutschland und Europa. Forst und Holz 52: 515-522.

BÜRGER, R. (1991): Immissionen und Kronenverlichtung als Ursache für Veränderungen der Waldbodenvegetation im Schwarzwald. - Tuexenia 11: 407-424.

BuRsCHEL, P. (1961): Untersuchungen über die natürliche Verjüngung der Buche. - Allg. Forstzeitschr. 16(6): 108-113.

Burschel, P. \& BindeR, F. (1993): Bodenvegetation - Verjüngung - Waldschäden. - Allg. 
Forstzeitschr. 5: 216-223.

Burschel, P. \& Huss, J. (1997): Grundriß des Waldbaus. 2. Auflage - Pareys Studientexte 49: 1-487.

Burschel, P.; Huss, J. \& KalBhenN, R. (1964): Die natürliche Verjüngung der Buche. Schriftenr. d. Forstl. Fak. d. Univ. Göttingen, Bd. 34.

CAMENISCH, M. \& SCHÜTZ, M. (2000): Zeitliche und räumliche Variabilität der Vegetation auf vierjährigen Zäunungen in der Val Trupchun (Schweizerischer Nationalpark). Nat.-park-Forsch. Schweiz 89: 165-188.

CONRAD, T. (1997): Vergleich von Vegetation und Kationengehalten der Böden der Gipsbuchenwälder im NSG Hainholz (Osterode am Harz) und des NSG OchsenburgZiegelhüttental (Kyffhäuser). - unveröff. Dipl. Univ. Göttingen, Albrecht-von-HallerInstitut für Pflanzenwissenschaften, $108 \mathrm{~S}$.

DEGRANDPRE', L.; MORISSETTE, J. \& GAUTHIER, S. (2000): Long-term post-fire changes in the northeastern boreal forest of Quebec. - J. Veg. Sci. 11: 791-800.

DEUTSCHER WETTERDIENST (1964): Klimaatlas von Niedersachsen. - Selbstverlag des Deutschen Wetterdienstes, Offenbach/Main.

DiERsCHKE, H. (1985): Pflanzensoziologische und ökologische Untersuchungen in Wäldern Süd-Niedersachsens. II. Syntaxonomische Übersicht der Laubwaldgesellschaften und Gliederung der Buchenwälder. - Tuexenia 5: 491-521.

DiERSCHKE, H. (1988): Pflanzensoziologische und ökologische Untersuchungen in Wäldern Süd-Niedersachsens. IV. Vegetationsentwicklung auf langfristigen Dauerflächen von Buchenwald-Kahlschlägen. - Tuexenia 8: 307-326.

DiERSCHKE, H. (1989): Artenreiche Buchenwaldgesellschaften Nordwest-Deutschlands. - Ber. Reinh.-Tüxen-Ges. 1: 107-148.

DierschKe, H. (1994): Pflanzensoziologie. Grundlagen und Methoden. - Ulmer, Stuttgart, $683 \mathrm{~S}$.

DoROW, W.H.O. (2002): Zoologische Untersuchungen auf der Sturmwurffläche Tierordnungen, Heteroptera (Wanzen), Hymenoptera (Hautflügler). - Mitt. Hess. Landesforstverwaltung 38: 79-118.

Duelli, P. \& OBRIst, M.K. (1999): Räumen oder Belassen? Die Entwicklung der faunistischen Biodiversität auf Windwurfflächen im schweizerischen Alpenraum. Verh. Ges. f. Ökol. 29: 193-200.

EBER, W. (1972): Über das Lichtklima von Wäldern bei Göttingen und seinen Einfluß auf die Bodenvegetation. - Scripta Geobotanica 3: 1-150.

EHRING, A. (2001): Sukzessionsbedingte Wiederbewaldung im Stromberg. - Schriftenr. Freib. Forstl. Forschung 18: 40-45.

ELLENBERG, H. (1996): Vegetation Mitteleuropas mit den Alpen in ökologischer, dynamischer und historischer Sicht. 5. Aufl. - Ulmer, Stuttgart, 1096 S.

Ellenberg, H.; MAYer R. \& SchauermanN, J. (Hrsg.)(1986): Ökosystemforschung Ergebnisse des Sollingprojektes 1966-1986. - Ulmer, Stuttgart, 507 S.

EllenberG, H.; WeBer, H.; DÜll, R.; WIRTH, V.; WERnER, W. \& PAUlisSEN, D. (1992): Zeigerwerte von Pflanzen in Mitteleuropa. 2. Aufl. - Scripta Geobotanica 18: 1-258.

ELLWANGER, G. (1999): Zur Bedeutung des vorgeschlagenen FFH-Gebietes „Gipskarstgebiet bei Osterode“ für das europäische Schutzgebietssystem NATURA 2000. - Göttinger Naturkundl. Schriften 5: 169-178.

FALINSKI, J.B. (1976): Windwürfe als Faktor der Differenzierung und der Veränderung des Urwaldbiotopes im Licht der Forschungen auf Dauerflächen. - Phytocoenosis 5.2: 85107.

FALINSKI, J.B. (1978): Uprooted trees, their distribution and influence in the primeval forest biotope. - Vegetatio 38(3): 175-183. 
FALINSKI, J.B. (1986): Vegetation dynamics in temperate lowland primeval forests. Ecological studies in Bialowiesa Forest. - Geobotany 8: 1-537.

FisCHER, A. (1987): Untersuchungen zur Populationsdynamik am Beginn von Sekundärsukzessionen. Die Bedeutung von Samenbank und Samenniederschlag für die Wiederbesiedlung vegetationsfreier Flächen in Wald- und Grünlandgesellschaften. - Diss. Bot. 110: 1-234.

FISCHER, A. (1992): Long term vegetation development in Bavarian Mountain Forest ecosystems following natural destruction. - Vegetatio 103: 93-104.

FISCHER, A. (1996a): Die Entwicklung der Biozönosen auf Sturmwurfflächen. Ein Vorwort zu den Berichten aus dem PAÖ-Sturmwurfflächenprojekt. - Veröff. PAÖ 16: 285-287.

FISCHER, A. (1996b): Forschung auf Dauerbeobachtungsflächen im Wald - Ziele, Methoden, Analysen, Beispiele. - Arch. für Nat.-Lands.- 35: 87-106.

FISCHER, A. (Hrsg.)(1998): Die Entwicklung von Wald-Biozönosen nach Sturmwurf. Umweltforschung in Baden-Württemberg. - ecomed, Langsberg, $427 \mathrm{~S}$.

FISCHER, A.; ABS, G. \& LENZ, F. (1990): Natürliche Entwicklung von Waldbeständen nach Windwurf. Ansätze einer „Urwaldforschung“ in der Bundesrepublik. - Forstw. Cbl. 109: 309-326.

FISCHER, A.; Honold, A.; REIF, A.; FunKE, W. \& BÜCKING, W. (1998): Die SturmwurfBiozönosen in der Startphase der Bestandesentwicklung. - in: FISCHER, A. (Hrsg.): Die Entwicklung von Wald-Biozönosen nach Sturmwurf. - ecomed, Landsberg: 357-374.

FISCHER, A. \& JEHL, H. (1999): Vegetationsentwicklung auf Sturmwurfflächen im Nationalpark Bayerischer Wald aus dem Jahre 1983. - Forstl. Forschungsber. München 176: 93-101.

FISCHER, A. \& KLOTZ, S. (1999): Zusammenstellung von Begriffen, die in der VegetationsDauerbeobachtung eine zentrale Rolle spielen. - Tuexenia 19: 3-11.

FISCHER, A.; LINDNER, M.; ABS, C. \& LASCH, P. (2002): Vegetation dynamics in Central European forest ecosystems (near-natural as well as managed) after storm events. Folia Geobotanica 37: 17-32.

FISCHER, A. \& MÖßMER, R. (Hrsg.)(1999): Forschung in Sturmwurf-Ökosystemen Mitteleuropas. Seminar des Fachgebietes Geobotanik und der Bayer. Landesanstalt für Wald und Forstwirtschaft. - Forstl. Forschungsber. München 176: 1-150.

FlechtNeR, G. (2002): Die Rolle der Käfer beim Abbau von Buchen-Totholz auf der Sturmfläche. - Mitt. d. Hess. Landesforstverwaltung 38: 123-146.

FLÖß, M. (2001): Struktur und Dynamik der sukzessionsgestützten Wiederbewaldung im Baar-Schwarzwald. - Schriftenr. Freib. Forstl. Forschung 18: 46-52.

FRANK, D.; KLOTZ, S. \& WeSTHUS, W. (1990): Botanisch-ökologische Daten zur Flora der DDR. 2. Aufl. - Wiss. Beiträge Martin-Luther-Univ. Halle-Wittenberg 32(P41): 1167.

FuCHS, H. \& KunTH, S. (1999): Erfassung der Bestandesstrukturen und Störungsarten in Beziehung zu den Standorts- und Vegetationseinheiten im Naturschutzgebiet Hainholz mit Hilfe von Luftbildauswertung und GIS. - Abschlußbericht Inst. f. Forsteinrichtung u. Ertragskunde Univ. Göttingen, 21 S.

FULL, J. (1999): Die Verjüngung im Naturwaldreservat „Königsbuche“ im ersten Jahr nach dem Sturm von 1997. - unveröff. Dipl. Univ. Göttingen, Institut für Waldbau, 82 S.

GEOLOGISCHE SPECIALKARTE VON PREUSSEN UND DEN THÜRINGSCHEN STAATEN (1884): Blätter Duderstadt (4427), Gieboldehausen (4327) und Weissenborn-Lüderode (4428), Maßstab 1 : 25.000, mit Erläuterungstext, Berlin.

Glavac, V. (1996): Vegetationsökologie: Grundfragen, Aufgaben, Methoden. - Fischer, Jena-Stuttgart-Lübeck-Ulm, 358 S.

GLÄSSER, R. (1994): Das Klima des Harzes. - Kovac, Hamburg, 341 S. 
GÖRKE, C. \& OBERWINKLER, F. (1999): Pilze - ein biotischer Stressfaktor für juvenile Bäume bei der Wiederbewaldung? - Forstl. Forschungsber. München 176: 53-60.

GRABIETZ, S. \& FIEDLER, H.J. (1996): Umweltbedingte Vegetationsveränderungen nach 3 Jahrzehnten in einem Buchen-Plenterwald des Hainich. - Arch. f. Nat.- Lands.- 34: 245-255.

GravenhORST, G.; Giesemann, J.; KREILEIN, H. \& RASCHENDORFER, M. (1991): Die Zunahme von Sturmschäden in den Wäldern des Vorderen Hunsrück und ihre mögliche atmosphärische Ursache. - Mitt. Forstl. Versuchsanst. Rheinl.-Pfalz 19: 4068.

GREGOR, T. \& SEIDLING, W. (1997): 50 Jahre Vegetationsentwicklung auf einer Schlagfläche im osthessischen Bergland. - Forstw. Cbl. 116: 218-231.

GrEgOR, T. \& SEIDLING, W. (1998): 50jährige Sukzession auf einer Waldschlagfläche im osthessischen Bergland - Folgerungen für den Naturschutz. - Schriftenr. f. Landschaftspl. u. Naturschutz 58: 291-301.

Gregor, T. \& SEIDLING, W. (1999): 50 Jahre Vegetationsentwicklung eines EichenAltbestandes im osthessischen Bergland. - Tuexenia 19: 193-205.

GRIESE, F. (1995): Zur Konkurrenz von Buche, Eiche und Fichte im Naturwald Totenberg. Forstarchiv 66: 159-166.

Grime, J.P.; HodGson, J.G. \& HunT, R. (1989): Comparative plant ecology: a functional approach to common British species. - Unwin Hyman, London, 742 S.

GroßE-BraucKMANN, H. (1994): Holzzersetzende Pilze - Aphyllophorales und Heterobasidiomycetes - des Naturwaldreservates Karlswörth: Eine Dokumentation und Vergleiche mit verwandten Waldgesellschaften. - Naturwaldreservate in Hessen 4: 1-119.

GRUNDMANN, V. (1997): Verjüngung auf Windwurfflächen. - Allg. Forstzeitschr. 52: 974976.

HAASE, H. (1954): Das neue Bild der Niederschlagsverteilung im Harz. - Roemeriana 1: 145154.

HÄRDTLE, W.; OHEIMB, G. VON \& WestPhAL, C. (2001): Vergleichende Untersuchungen zur Struktur und Vegetation von Natur- und Wirtschaftswäldern des Tieflandes auf der Grundlage räumlich expliziter Vegetationsmodelle. - Ber. d. Reinh.-Tüxen-Ges. 13: 183-196.

HAEUPLER, H. (1982): Evenness als Ausdruck der Vielfalt in der Vegetation. Untersuchungen zum Diversitätsbegriff. - Diss. Bot. 65: 1-268.

HAKES, W. (1991): Das Galio odorati-Fagenion im Habichtswald bei Kassel Untersuchungen zur ökologischen Feingliederung. - Tuexenia 11: 381-406.

HAKES, W. (1999): Jüngere Veränderungen der Vegetationsstruktur in nordhessischen Buchenwäldern und ihre standörtlichen Ursachen - Ergebnisse einer neunjährigen Monitoringstudie. - NUA-Seminarber. 4: 301-303.

HALPERN, C.B. (1988): Early successional pathways and the resistance and resilience of forest communities. - Ecology 69(6): 1703-1715.

HalPeRn, C.B.; Frenzen, P.M.; MEANS, J.E. \& FrankLin, J.F. (1990): Plant succession in areas of scorched and blown-down forest after the 1980 eruptions of Mount St. Helens, Washington. - J. Veg. Sci. 1(2): 181-194.

HANKE, U. \& PiSOKE, T. (1999): Der Sturmwurfbannwald „Teufelsries“ Waldstrukturerhebung mit forstlicher Grundaufnahme und Luftbilddokumentation. Ber. Freib. Forstl. Forschung 6: 1-80.

Harcombe, P.A.; Bill, C.J.; Fulton, M.; Glitzenstein, J.S.; MARKs, P.L. \& Elsik, I.S. (2002): Stand dynamics over 18 years in a southern mixed hardwood forest, Texas, USA. - J. Ecol. 90: 947-957. 
HEINKEN, T. (1995): Naturnahe Laub- und Nadelwälder grundwasserferner Standorte im niedersächsischen Tiefland: Gliederung, Standortsbedingungen, Dynamik. - Diss. Bot. 239: 1-313.

HeINKEN, T. (1998): Zum Einfluss des Alters von Waldstandorten auf die Vegetation in bodensauren Laubwäldern des niedersächsischen Tieflandes. - Arch. für Nat.- Lands.37: 201-232.

HERMY, M.; BREMT, P. VAN DEN \& TACK, G. (1993): Effects of site history on woodland vegetation. - in: BroeKMEYer, M.E.A.; Vos, W. \& KoOP, H. (eds.): European Forest Reserves. - Pudoc Scientific Publishers, Wageningen: 219-231.

Hetzel, G. \& ReIf, A. (1996): Die Vegetation der Kahlflächen im Wald und ihre Beziehung zu Standort, vorangegangener Nutzung und Wildverbiß, mit besonderer Berücksichtigung der Bannwaldgebiete bei Langenau, Bebehausen und Bad Waldsee. - Veröff. PAÖ 16: 289-305.

Hetzel, G. \& ReIF, A. (1997): Der Einfluß von Bestandesgeschichte und forstlichen Maßnahmen auf die Entwicklung der Sturmwurfflächen auf Kalkverwitterungslehm der Schwäbischen Alb. - Veröff. PAÖ 22: 129-135.

Hetzel, G. \& REIF, A. (1998): Vegetationsdifferenzierung und Baumartenverjüngung von Sturmwurfflächen auf Kalkverwitterungslehmen der Schwäbischen Alb. - in: FischeR, A. (Hrsg.): Die Entwicklung von Wald-Biozönosen nach Sturmwurf. ecomed, Landsberg: 169-187.

Heupel, M. \& BlOCK, J. (1991): Untersuchungen zum Einfluss des Standortes und der Bestandesstruktur auf das Ausmaß von Sturmschäden im vorderen Hunsrück. - Mitt. Forstl. Versuchsanst. Rheinl.-Pf. 19: 57-68.

HoFmANN, W. (1960): Beobachtungen über die Windwurfgefährdungen der Rotbuche auf der Fränkischen Platte. - Forstw. Cbl. 79: 202-206.

Homann, M. \& Engels, F. (1991): Was kommt nach dem Sturm? - Allg. Forstzeitschr. 12: 630-633.

Honold, A. \& OBerwinkLeR, F. (1998): Pilze im Totholz. - in: Fischer, A. (Hrsg.): Die Entwicklung von Wald-Biozönosen nach Sturmwurf. - ecomed, Landsberg: 213-226.

HORN, A. (2002): Konkurrenz zwischen natürlich verjüngten Eschen und Buchen in Bestandeslücken: Wachstum, Feinwurzelverteilung und ökophysiologische Reaktion auf Austrocknung. - Ber. Forsch.zentr. Waldökosysteme Göttingen A/177: 1-203.

HuBRIG, M. (1999): Dokumentation der Sturmschäden vom 29.6.1997 in Niedersachsens Wäldern, verursacht durch „Schwere lokale Stürme“. - Aus dem Walde 52: 4-139.

Huss, J. (1991): Konzeptionen zur Wiederbewaldung von Sturmschadensflächen. - Allg. Forstzeitschr. 1: 25-30.

Huss, J. \& HEHN, M. (Hrsg.)(2001): Wiederbewaldung von Sturmschadensflächen. Waldbauliche Strategien in Forschung und Praxis: Erfahrungen und Empfehlungen. Ber. Freib. Forstl. Forschung 25: 1-148.

HyATT, L.A. \& CASPER, B.B. (2000): Seed bank formation during early secondary succession in a temperate deciduous forest. - J. Ecol. 88: 516-527.

JAHN, G. (1979): Zur Frage der Buche im nordwestdeutschen Flachland. - Forstarchiv 50: 8595.

JAHN, G. (1983): Die Buche auf dem Vormarsch im Flachland des westlichen Mitteleuropa. Forst- u. Holzwirt 38: 142-145.

JAHN, G. (1990): Landschaft und Wald im Wandel der Zeiten. - Forst und Holz 3: 53-58.

JEHL, H. (2001): Die Waldentwicklung nach Windwurf in den Hochlagen des Nationalparks

Bayerischer Wald. - Nationalpark Bayerischer Wald, Wissenschaftl. Reihe 14: 49-98.

Jenssen, M. \& Hofmann, G. (1996): Der natürliche Entwicklungszyklus des baltischen 
Perlgras-Buchenwaldes (Melico-Fagetum). - Beiträge f. Forstwirtschaft u. Landschaftsökologie 30: 114-124.

KEITEL, W. (1998): Verjüngungsentwicklung einer Windwurffläche. Naturwaldreservat Weiherskopf. - Allg. Forstzeitschr. 11: 587-590.

KeITEL, W. (1999): Sukzession nach Sturmwurf in einem Waldgersten-Buchenwald. - NUASeminarber. 4: 286-289.

KENK, G.; MENGES, U. \& BÜRGER, R. (1991): Natürliche Wiederbewaldung von Sturmwurfflächen? - Allg. Forstzeitschr. 2: 96-100.

KLEYER, M. (1995): Biological traits of vascular plants. A database. - Arbeitsberichte Inst. f. Landschaftsplanung und Ökologie Univ. Stuttgart, N.F. 2, 23 S. + Diskette.

KNAPP, H.D. \& JESCHKE, L. (1991): Naturwaldreservate und Naturwaldforschung in den ostdeutschen Bundesländern. - Schr.-R. f. Vegetationskde. 21: 21-54.

KÖBERLE, F. (1997): Wiederbewaldung von Sturmflächen. - Allg. Forstzeitschr. 24: 13001301.

KöLBEL, M. (1999): 25 Jahre Naturwaldreservate in Deutschland - eine Standortbestimmung. - NUA-Seminarber. 4: 32-39.

KÖNIG, N. \& FORTMANN, H. (1996): Probenvorbereitungs-, Untersuchungs- und Elementbestimmungsmethoden des Umweltanalytik-Labors der Niedersächsischen Forstlichen Versuchsanstalt und des Zentrallabors II des Forschungszentrums Waldökosysteme. Teil 4. - Ber. Forsch.zentr. Waldökosysteme Göttingen B/49: 1435.

KOMPA, T. \& SCHMIDT, W. (2001): Vegetationsentwicklung nach Windwurf in Buchenwäldern des südwestlichen Harzvorlandes. - Ber. d. Reinh.-Tüxen-Ges. 13: 251-255.

KOMPA, T. \& SCHMIDT, W. (2002): Vegetationsentwicklung nach Windwurf in Buchenwäldern des südwestlichen Harzvorlandes. - in: BUNDESAMT FÜR NATURSCHUTZ (Hrsg.): Treffpunkt Biologische Vielfalt II: 125-131.

KOMPA, T. \& SCHMIDT, W. (2003): Buchenwald-Sukzession nach Windwurf auf Buntsandstein im südwestlichen Harzvorland. - Tuexenia 23: 95-130.

KoOP, H. (1982): Waldverjüngung, Sukzessionsmosaik und kleinstandörtliche Differenzierung infolge spontaner Waldentwicklung. - in: Struktur und Dynamik von Wäldern. Ber. d. Int. Symposien d. Int. Ver. f. Vegetationsk.: 235-267, Rinteln.

KoOP, H. (1987): Vegetative reproduction of trees in some European natural forests. Vegetatio 72: 103-110.

KoRPEL, S. (1995): Die Urwälder der Westkarpaten. - Fischer, Stuttgart, 310 S.

KRÜSI, B.O. \& Moser, B. (2000): Der Einfluss von Schnee und Huftieren auf den Sukzessionsverlauf in einem Bergföhrenwald im Schweizerischen Nationalpark (Munt La Schera). - Nat.park-Forsch. Schweiz 89: 131-164.

KuHN, N. (1995): Die standörtliche Abhängigkeit der Vivian-Windwürfe. - Inf.bl. Forsch.bereich Landsch.ökol. (WSL) 28: 1-3.

KULAKOWSKI, D. \& VEBLEN, T.T. (2002): Influences of fire history and topography on the pattern of a severe wind blowdown in a Colorado subalpine forest. - J. Ecol. 90: 806819.

KulaKowsKi, D. \& VeBlen, T.T. (2003): Subalpine forest development following a blowdown in the Mount Zirkel Wilderness, Colorado. - J. Veg. Sci. 14(5): 653-660.

LÄssIG, R. (2000): Die natürliche Dynamik nach Sturmwürfen nutzen. - Allg. Forstzeitschr. 3: $112-115$.

LÄSSIG, R. (2002): Lothar-Forschung eine lohnende Investition. Naturnahen Waldbau und Krisenstrategie verbessern. - Inf.bl. Forsch.bereich Wald (WSL) 10: 1-3.

LÄSSIG, R.; BLEISTEIN, U. \& SCHÖNENBERGER, W. (1995a): Windwurf im Bergwald - Risiko 
und Chance zugleich. - Wald und Holz 76(15): 40-45.

LÄSSIG, R.; EGLI, S.; ODERMATT, O.; SCHÖNENBERGER, W.; STÖCKLI, B. \& WOHLGEMUTH, T. (1995b): Beginn der Wiederbewaldung auf Windwurfflächen. - Schweiz. Zeitschr. Forstwes. 146(11): 893-911.

LÄSsIG, R. \& MotSCHALOW, S.A. (1999): Die Häufigkeit starker Stürme und ihre Auswirkungen auf die Wälder der Ural-Region. - Forstl. Forschungsber. München 176: $30-41$.

LÄSSIG, R. \& MoTSCHALOW, S.A. (2000a): Frequency and characteristics of severe storms in the Urals and their influence on the development, structure and management of the boreal forests. - Forest Ecol. a. Managem. 135: 179-194.

LÄSSIG, R. \& MotSCHALOW, S.A. (2000b): Waldforschung - Folgen von Windwürfen. OstWest-Partnerschaft am Beispiel von Stürmen auf Wälder im Ural. - Forum für Wissen (WSL): 37-45.

LÄSSIG, R. \& MOTSCHALOW, S.A. (2002): Wiederbewaldung von belassenen und geräumten Sturmwurfflächen im Mittel-Ural. - Mitt. d. Hess. Landesforstverwaltung 38: 23-34.

LÄSSIG, R. \& SCHÖNENBERGER, W. (1993): Forschung auf Sturmschadenflächen in der Schweiz. - Forst und Holz 9(48): 244-249.

LÄSSIG, R. \& SCHÖNENBERGER, W. (1994): Untersuchungen auf Windwurfflächen am Beispiel der Forschungsfläche Uaul Cavorgia-Funtauna bei Disentis. - Bündnerwald 47(5): 23-31.

LÄSSIG, R. \& SCHÖNENBERGER, W. (2000): Nach „Lothar“ von „Vivian“-Erfahrungen profitieren. - Wald und Holz 2: 31-35.

LAMBERTZ, B. \& SCHMIDT, W. (1999): Auflichtungen in Buchen- und Buchenmischbeständen auf Muschelkalk und Buntsandstein. - Untersuchungen zur Verjüngungs- und Vegetationsstruktur. - Verh. Ges. f. Ökol. 29: 81-88.

LEIBUNDGUT, H. (1978): Über die Dynamik europäischer Urwälder. - Allg. Forstzeitschr. 33(24): 686-690.

LEMKE, M. (2001): Flora und Vegetation im Naturwald Königsbuche (Forstamt Reinhausen). - unveröff. Dipl. Univ. Göttingen, Institut für Waldbau, 72 S.

LEUSCHNER, C. (1994): Walddynamik auf Sandböden in der Lüneburger Heide, NWDeutschland. - Phytocoenologia 22: 289-324.

LEUSCHNER, C. (1998): Mechanismen der Konkurrenzüberlegenheit der Rotbuche. - Ber. Reinh.-Tüxen-Ges. 10: 5-18.

LEUSCHNER, C. (1999): Zur Abhängigkeit der Baum- und Krautschicht mitteleuropäischer Waldgesellschaften von der Nährstoffversorgung des Bodens. - Ber. Reinh.-TüxenGes. 11: 109-131.

Leuschner, C.; Rode, M. \& Heinken, T. (1993): Gibt es eine Nährstoffmangel-Grenze der Buche im nordwestdeutschen Flachland? - Flora 188: 239-249.

LOCHOW, A. vON (1990): Die Buche in den Waldgesellschaften Nordwestdeutschlands. Ergebnisse aus Strukturanalysen in den niedersächsischen Naturwaldreservaten. Natur und Landschaft 65(6): 319-325.

LOHMEYER, W. \& SuKOPP, H. (1992): Agriophyten in der Vegetation Mitteleuropas. - Schr.R. f. Vegetationskde. 25: 1-185.

LORIMER, C.G. (1980): Age structure and disturbance history of a southern Appalachian virgin forest. - Ecology 61: 1169-1184.

LugINBÜHL, U. (2000): Einfluß des Ausgangszustandes und der Störungsintensität auf die sekundäre Sukzession nach Windwurf im Gips-Buchenwald NSG Hainholz (Osterode) nach zwei Jahren. - unveröff. Dipl. Univ. Göttingen, Albrecht-von-Haller-Institut für Pflanzenwissenschaften, $105 \mathrm{~S}$.

LYFORD, W.H. \& MAC LEAN, D.W. (1966): Mound and pit microrelief in relation to soil 
disturbance and tree distribution in New Brunswick, Canada. - Harvard Forest Papers $15,13 \mathrm{~S}$.

MÄRKL, G. \& EGLSEER, C. (2001): Verjüngungs- und Vegetationsentwicklung in SturmwurfBannwäldern (1993-1998/1999). - Ber. Freib. Forstl. Forschung 32: 61-205.

MAYER, H. (1985): Baumschwingungen und Sturmgefährdungen des Waldes. Wissenschaftl. Mitt. Meteorolog. Inst. Univ. München 51: 1-247.

Mayer, H.; NeumanN, M. \& Sommer, H.-G. (1980): Bestandesaufbau und Verjüngungsdynamik unter dem Einfluss natürlicher Wilddichten im kroatischen Urwaldreservat Corkova Uvala/Plitvicer Seen. - Schweiz. Zeitschr. f. Forstwesen 131: 45-70.

McAlister, S.D.; Palmer, M.W.; Arevalo, J.R. \& DeCoster, J.K. (2000): Species composition on tipup mounds and pits created by catastrophic windthrow in a Minnesota forest. - Proceedings $41^{\text {st }}$ IAVS symposium: 104-107.

MELlERT, K.-H.; KÖLlinG, C. \& ReHFuESS, K.E. (1998): Vegetationsentwicklung und Nitrataustrag auf 13 Sturmkahlflächen in Bayern. - Forstarchiv 69: 3-11.

Meusel, H. (1939): Die Vegetationsverhältnisse der Gipsberge im Kyffhäuser und im südlichen Harzvorland. - Hercynia 2: 1-372.

MEYER, P. (1995): Untersuchungen waldkundlicher Entwicklungstendenzen und methodischer Fragestellungen in Buchen- und Buchenmischbeständen niedersächsischer Naturwaldreservate. - Diss. Univ. Göttingen, Fak. Forstwiss. u. Waldökolgie, 239 S.

MeYer, P. (1997): Zur Populationsdynamik in nordwestdeutschen Naturwäldern.

Erkenntniswert der Naturwaldforschung für Forstwirtschaft und Naturschutz. - Forst u. Holz 52(18): 532-538.

MEYER, P. (2000): Waldkundliche Dauerbeobachtung von Naturwaldflächen in den Niedersächsischen Landesforsten. - unveröff. methodischer Leitfaden, Niedersächsische Forstliche Versuchsanstalt (NFV), Göttingen, 44 S.

MeYer, P.; UnKRiG, W. \& Griese, F. (2000): Dynamik der Buche (Fagus sylvatica L.) in nordwestdeutschen Naturwäldern. - Forst u. Holz 55: 470-477.

MÖßNANG, M. \& KÜHNEL, S. (1999): Natürliche Verjüngung auf Sturmwurfflächen vom Februar 1990 in Bayern. Ergebnisse von Dauerbeobachtungsflächen und Folgerungen für die Praxis. - Forstl. Forschungsber. München 176: 61-69.

MrotzeK, R.; Perona, L. \& SchmidT, W. (1996): Einfluß von Licht und ausgewählten Bodenfaktoren auf die Verteilung von Urtica dioica L. und Mercurialis perennis L. in der Bodenvegetation des Buchenwaldökosystems der „Fallstudie Zierenberg“. - Verh. Ges. f. Ökol. 26: 559-564.

NAKASHIZUKA, T. (1989): Role of uprooting in composition and dynamics of an old-growth forest in Japan. - Ecology 70: 1273-1278.

OBERDORFER, E. (2001): Pflanzensoziologische Exkursionsflora. 8. Aufl. - Ulmer, Stuttgart, $1051 \mathrm{~S}$.

OBERDORFER, E. (1973): Die Gliederung der Epilobietea angustifolii-Gesellschaften am Beispiel süddeutscher Vegetationsaufnahmen. - Acta Bot. Sc. Hung. 19: 235-253.

OHEIMB, G. VON (2003): Einfluss forstlicher Nutzung auf die Artenvielfalt und Artenzusammensetzung der Gefäßpflanzen in norddeutschen Laubwäldern. - Kovac, Hamburg, $261 \mathrm{~S}$.

Oтт, B. (2002): Analyse der erfolgsbestimmenden Faktoren buchendominierter

Freiflächenkulturen nach Sturmwurf im hessischen Vogelsberg. - Ber. Forsch.zentr. Waldökosysteme Göttingen A/182: 1-180.

OтTо, H.-J. (1994): Waldökologie. - Ulmer, Stuttgart, 391 S.

Palmer, M.W.; McAlister, S.D.; Arevalo, J.R. \& DeCoster, J.K. (2000): Changes in the 
understorey during 14 years following catastrophic windthrow in two Minnesota forests. - J. Veg. Sci. 11(6): 841-854.

PASSARGE, H. (1998): Gehölz-Gesellschaften auf Waldschlägen Nordostdeutschlands. Tuexenia 18: 63-84.

Peterson, C.J. \& ReBertus, A.J. (1997): Tornado damage and initial recovery in three adjacent, lowland temperate forests in Missouri. - J. Veg. Sci. 8: 559-564.

Peterson, C.J. \& PiCKETT, S.T.A. (1995): Forest reorganization: a case study in an oldgrowth catastrophic blowdown. - Ecology 76: 763-774.

PICKETT, S.T.A. (1989): Space-for-time substitution as an alternative to long-term studies. in: LiKENS, G.E. (ed.): Long-term studies in ecology. - Springer, New York: 110-135.

PIELOU, E.C. (1984): The interpretation of ecological data. - Wiley-Interscience, New York, $263 \mathrm{~S}$.

PLACHTER, H. (1992): Ökologische Langzeitforschung und Naturschutz. - Veröff. PAÖ 1: 5996.

PolOMSKI, J. \& KUHN, N. (2000): Kahlschlagbedingte Veränderunge im Wurzelraum eines Buchenniederwaldes auf Rendzina. - in: MERBACH, W.; WitTenMayer, L. \& Augustin, B.G. (Hrsg.): Rhizodeposition und Stoffverwertung. 10. Borkheider Seminar zur Ökophysiologie des Wurzelraumes: 65-71.

PoTT, R. (1988): Entstehung von Vegetationstypen und Pflanzengesellschaften unter dem Einfluß des Menschen. - Düsseldorfer Geobot. Kolloq. 5: 27-54.

ReIf, A. \& Przybilla, M. (1995): Zur Regeneration der Fichte (Picea abies) in den Hochlagen des Nationalparks Bayerischer Wald. - Hoppea 56: 467-514.

REMMERT, H. (1985): Was geschieht im Klimax-Stadium? Ökologisches Gleichgewicht durch Mosaik aus desynchronen Zyklen. - Naturwissenschaften 72: 505-512.

REMmert, H. (1987): Sukzessionen im Klimax-System. - Verh. Ges. f. Ökol. 16: 27-34.

REMMERT, H. (1991): Das Mosaik-Zyklus-Konzept und seine Bedeutung für den Naturschutz. Eine Übersicht. - Laufener Seminarbeitr. (ANL) 5: 5-15.

ReXeR, K.H.; HonOlD, A. \& OBeRwinkLeR, F. (1999): Mykorrhiza, Holz- und Streuabbau: Pilze in ökologischen Schlüsselrollen bei der Wiederbewaldung nach Sturmwurf. Forstl. Forschungsber. München 176: 42-52.

RothmaleR, W. (2002): Exkursionsflora von Deutschland. Band 4: Gefäßpflanzen. Kritischer Band. 9. Aufl. - Spektrum, Heidelberg-Berlin, 948 S.

RungE, F. (1981): Änderungen der Krautschicht in einem Eichen-Hainbuchenwald im Lauf von 21 Jahren. - Natur und Heimat 41: 89-93.

RungE, F. (1993): Änderung der Flora eines Buchenwaldkahlschlages im Laufe von sieben Jahren. - Tuexenia 13: 71-73.

Rust, S. \& SAVILL, P.S. (2000): The root systems of Fraxinus excelsior and Fagus sylvatica and their competitive relationships. - Forestry 73: 499-508.

SAYER, U. \& REIF, A. (1998): Die Entwicklung der Vegetation im überregionalen Vergleich. in: FISCHER, A. (Hrsg.): Die Entwicklung von Wald-Biozönosen nach Sturmwurf. ecomed, Landsberg: 146-168.

SCHÄFER, M. (2002): Zersetzung der sturmgeworfenen Buchenstämme. - Mitt. d. Hess. Landesforstverwaltung 38: 49-60.

SCHERZINGER, W. (1996): Naturschutz im Wald - Qualitätsziele einer dynamischen Waldentwicklung. - Ulmer, Stuttgart, $447 \mathrm{~S}$.

Schlechte, G.B. (2002): Sukzession der holzzerstörenden Pilze auf der Sturmwurffläche. Mitt. d. Hess. Landesforstverwaltung 38: 61-78.

ScHMIDT, W. (1983): Experimentelle Syndynamik - Neuere Wege zu einer exakten Sukzessionsforschung, dargestellt am Beispiel der Gehölzentwicklung auf Ackerbrachen. - Ber. Deutsch. Bot. Ges. 96: 511-533. 
SCHMIDT, W. (1988): Langjährige Veränderungen der Krautschicht eines Kalkbuchenwaldes (Dauerflächenuntersuchungen). - Tuexenia 8: 327-338.

SCHMiDT, W. (1991): Die Veränderung der Krautschicht in Wäldern und ihre Eignung als pflanzlicher Bioindikator. - Schr.-R. f. Vegetationskde. 21: 77-96.

SCHMIDT, W. (1996): Zur Entwicklung der Verjüngung in zwei Femellücken eines Kalkbuchenwaldes. - Forst u. Holz 51(7): 201-205.

ScHMidT, W. (1997): Zur Vegetationsdynamik von Lochhieben in einem Kalkbuchenwald. Forstw. Cbl. 116: 207-217.

SCHMIDT, W. (1998a): Vegetationskundliche Langzeitforschung auf Dauerflächen Erfahrungen und Perspektiven für den Naturschutz. - Schr.-R. f. Landschaftspfl. u. Natursch. 58: 353-375.

ScHMiDT, W. (1998b): Dynamik mitteleuropäischer Buchenwälder: Kritische Anmerkungen zum Mosaik-Zyklus-Konzept. - Naturschutz u. Landschaftsplanung 30(8/9): 242-249.

SCHMIDT, W. (1999a): Die Bodenvegetation als Indikator für die biotische Mannigfaltigkeit von Wäldern - Beispiele aus Naturwaldreservaten und Wirtschaftswäldern. - Verh. Ges. f. Ökol. 29: 133-143.

SCHMIDT, W. (1999b): Biotische Mannigfaltigkeit - Indikator für intakte Wälder? Beispiele aus Studien über die Bodenvegetation in Naturwaldreservaten. - NUA-Seminarber. 4: 108-127.

SCHMIDT, W. (2000): Eiche, Hainbuche oder Rotbuche? - Zur Vegetation und

Baumartenzusammensetzung von stau- und grundwasserbeeinflussten Wäldern des nordwestdeutschen Tieflandes. Ergebnisse aus den Naturwäldern Hasbruch und Pretzetzer Landwehr. - Tuexenia 20: 21-43.

SCHMIDT, W. (2002a): Die Naturschutzgebiete Hainholz und Staufenberg am Harzrand Sukzessionsforschung in Buchenwäldern ohne Bewirtschaftung (Exkursion E). Tuexenia 22: 151-214.

SCHMIDT, W. (2002b): Einfluss der Bodenschutzkalkungen auf die Waldvegetation. Forstarchiv 73: 43-54.

SCHMIDT, W. (2002c): Stickstoffkreislauf in Schlaglücken eines Kalkbuchenwaldes. - Allg. Forst- und Jagdzeitung 173(4): 67-76.

SCHMIDT, W. (2003): Vielfalt im Urwald - Einfalt im Wirtschaftswald? Untersuchungen zur Gefäßpflanzendiversität in Naturwaldreservaten. - Kleine Senckenberg-Reihe 45: 185204.

SCHMIDT, W. \& WECKESSER, M. (2001): Struktur und Diversität der Waldvegetation als Indikatoren für eine nachhaltige Waldnutzung. - Forst und Holz 15: 493-498.

SCHMIDT, W. \& WECKESSER, M. (2002): Structure and species diversity of forest vegetation as indicators of forest sustainability. - in: SPELLMANN, H. (ed.): Presentations of the $5^{\text {th }}$ International Workshop of the EU-LIFE-Project: „Demonstration of Methodes to Monitor Sustainable Forestry“ 2001-05-05 - 2001-05-08. Germany. - Cuvillier, Göttingen: 68-78.

SCHMIDT, W.; WeITEMEIER, M. \& HolZAPFEL, C. (1996): Vegetation dynamics in canopy gaps of a beech forest on limestone - The influence of the light gradient on species richness. - Verh. Ges. f. Ökol. 25: 253-260.

SCHMIDT-SCHÜTZ, A. (1999): Wiederbewaldung von Fichten-Sturmwurfflächen auf vernässenden Standorten mit Hilfe von Pioniergehölzen. - Forstl. Forschungsber. München 176: 120-130.

SchMIDT-SchÜTZ, A. \& HuSs, J. (1996): Gelenkte Sukzessionen mit Hilfe von Pioniergehölzen als Alternativen zu Kahlflächenaufforstungen. - Veröff. PAÖ 16: 307-324.

SCHMIDT-SCHÜTZ, A. \& HUSS, J. (1997): Wiederbewaldung von Sturmschadensflächen mit 
Hilfe von Pioniergehölzen. - Veröff. PAÖ 22: 137-152.

SCHMIDT-SCHÜTZ, A. \& HUSS, J. (1998): Wiederbewaldung von Fichten-Sturmwurfflächen auf vernässenden Standorten mit Hilfe von Pioniergehölzen. - in: FISCHER, A. (Hrsg.): Die Entwicklung von Wald-Biozönosen nach Sturmwurf. - ecomed, Landsberg: 188211.

SCHÖLCH, M. (1998): Zur natürlichen Wiederbewaldung ohne forstliche Steuerung: dargestellt an Beispielen aus Baden-Württemberg. - Schriftenr. Freib. Forstl. Forschung 1: 1-245.

SCHÖLCH, M.; EH, M. \& KENK, G. (1994): Natürliche Wiederbewaldung von Sturmflächen. Allg. Forstzeitschr. 2: 92-95.

SCHÖNENBERGER, W. (2002): Post windthrow stand regeneration in Swiss mountain forests: the first ten years after the 1990 storm Vivian. - For. Snow Landsc. Res. 77: 61-80.

SchÖnenberger, W.; Angst, C.; BrÜndL, M.; Dobbertin, M.; Duelli, P.; EgLi, S.; Frey, W.; Gerber, W.; Kupferschmid-AlbisetTI, A.D.; LÜSCher, P.; SENN, J.; WeRMELINGER, B. \& WoHLGEMUTH, T. (2003): Vivians Erbe - Waldentwicklung nach Windwurf im Gebirge. - Merkblätter für die Praxis (WSL) 36: 1-12.

SCHÖNENBERGER, W.; FISCHER, A. \& InNES, J. (eds.)(2002): Vivian's Legacy in Switzerland - impact of windthrow on forest dynamics. - For. Snow Landsc. Res. 77(1/2): 1-224.

SCHÖNENBERGER, W. \& WASEM, U. (1999): Der Beginn der Wiederbewaldung von Sturmwurfflächen im Gebirge - Ein Zwischenbericht. - Forstl. Forschungsber. München 176: 102-110.

SCHÖNFELDER, P. (1978): Vegetationsverhältnisse auf Gips im südwestlichen Harzvorland Eine vergleichende Untersuchung unter besonderer Berücksichtigung der Naturschutzprobleme. - Natursch. u. Landschaftspfl. Nieders. 8: 1-110.

SCHUBERT, R.; HiLBIG, W. \& KLOTZ, S. (2001): Bestimmungsbuch der Pflanzengesellschaften Deutschlands. - Spektrum, Heidelberg-Berlin, 472 S.

SCHÜLER, G. (1999): Wasserhaushalt auf Sturmwurfflächen in Rheinland-Pfalz. - Forstl. Forschungsber. München 176: 4-17.

SEITZ, M. (1998): Aufnahme einer Sturmschadensfläche im Naturwald Königsbuche. unveröff. Dipl. FH Hildesheim-Holzminden, FB Forstwirtschaft und Umweltmanagement (Göttingen), $51 \mathrm{~S}$.

SENN, J.; WASEM, U. \& ODERMATT, O. (2002): Impact of browsing ungulates on plant cover and tree regeneration in windthrow areas. - For. Snow Landsc. Res. 77: 161-170.

SmiRnova, O.V.; Zaugolnova, L.B.; IstOMinA, I.I. \& KhANinA, L.G. (2000): Population mosaic cycles in forest ecosystems. - Proceedings $41^{\text {st }}$ IAVS symposium: 108-112.

SSYMANK, A.; HAUKE, U.; RÜCKRIEM, C. \& SCHRÖDER, E. (1998): Das europäische Schutzgebietssystem NATURA 2000. - Schr.-R. f. Landschaftspfl. u. Natursch. 53: 1558.

STEWART, G.H.; Rose, A.B. \& VeBlen, T.T. (1991): Forest development in canopy gaps in old-growth beech (Nothofagus) forests, New Zealand. - J. Veg. Sci. 2(5): 679-690.

STEINFATH, M. (2001): 40000 ha Kahlflächen wiederbewalden. Den Aufräumarbeiten nach „Lothar“ folgt die Wiederbewaldung. - Allg. Forstzeitschr. 20: 1064-1065.

STRIEPEN, K. (1999): Einfluss des Wildverbisses auf die Verjüngungsentwicklung von Buchen- und Buchenmischwäldern in Nordrhein-Westfalen. - NUA-Seminarber. 4: 91-107.

STÜDELI, T. (1999): Factors controlling seed dispersal of Sambucus nigra in forest gaps. Bull. Geobot. Inst. ETH 65: 92-93.

TABAKU, V. (1999): Struktur von Buchen-Urwäldern in Albanien im Vergleich mit deutschen Buchen-Naturwaldreservaten und -Wirtschaftswäldern. - Cuvillier, Göttingen, 206 S.

Thomas, A.; MrotzeK, R. \& SCHMidT, W. (1995): Biomonitoring in Buchenwäldern. 
Aufgaben, Methoden und Organisation eines koordinierten Biomonitoringsystems in naturnahen Waldökosystemen der Bundesrepublik Deutschland. - Angewandte Landschaftsökologie 6: 1-150.

THOMAsIUS, H. (1988): Sukzession, Produktivität und Stabilität natürlicher und künstlicher Waldökosysteme. - Arch. Nat.schutz Landsch.forsch. 28: 3-21.

THOMPSON, K.; BAKKER, J. \& BEKKER, R. (1997): The soil seed bank of North West Europe: methodology, density and longevity. - Cambridge University Press, 176 S.

TILMAN, D. (1989): Ecological experimentation: Strengths and conceptual problems. - in: LIKENS, G.E. (ed.): Long-term studies in ecology - Approaches and alternatives. Springer, New York-Berlin-Heidelberg-London-Paris-Tokio: 136-157.

TOPOLIANTZ, S. \& PONGE, J.-F. (2000): Influence of site conditions on the survival of Fagus sylvatica seedlings in an old-growth beech forest. - J. Veg. Sci. 11(3): 369-374.

Topp, W. (1998): Raum-für-Zeit-Ansätze als Alternativen zu ökologischen Langzeitforschungen. - Schr.-R. f. Landschaftspfl. u. Natursch. 58: 21-45.

UlAnOvA, N.G. (2000a): Plant age stages during succession in woodland clearings in Central Russia. - Proceedings $41^{\text {st }}$ IAVS symposium: $80-83$.

Ulanova, N.G. (2000b): The effects of windthrow on forests at different spatial scales: a review. - Forest Ecol. a. Management 135: 155-167.

UNKRIG, W. (1997a): Sturmfolgenforschung im Naturschutzgebiet Hainholz und im Naturwald Königsbuche. - unveröff. Projektskizze, Niedersächsische Forstliche Versuchsanstalt (NFV), Göttingen, 12 S.

UNKRIG, W. (1997b): Zur Verjüngung von Fichte und Buche im Naturwald Sonnenkopf. Forst und Holz 52(18): 538-543.

UnKRIG, W.; MENCH, A. \& KAHLE, M. (1999): Waldkundliche Auswertung terrestrisch aufgenommener Probekreise im Rahmen des Untersuchungsauftrages „Sukzession auf Windwurfflächen im Naturschutzgroßprojekt Gipskarstlandschaft Hainholz“. Abschlußbericht Inst. f. Forsteinrichtung u. Ertragskunde Univ. Göttingen, 15 S.

VAN DEN Berge, K.; MAdDELEIN, D. \& MuYs, B. (1993): Recent structural changes in the beech forest reserve of Groenendaal (Belgium). - in: BROEKMEYER, M.E.A.; Vos, W. \& Koop, H. (eds.): European Forest Reserves. - Pudoc Scientific Publishers, Wageningen: 195-198.

VLADI, F. (1976): Quartärgeologische Untersuchungen zu den Terrassen der Sieber am Südwestrande des Harzes. - unveröff. Dipl. Univ. Hamburg, Geol.-Paläont. Inst., 109 S.

VLADI, F. (1998): Flußversinkungen am südwestlichen Harzrand - jüngere Beobachtungen und Deutungen. - NNA-Ber. 11(2): 82-87.

WAGNER, S. (1999): Ökologische Untersuchungen zur Initialphase der Naturverjüngung in Eschen-Buchen-Mischbeständen. - Schriften aus der Forstl. Fak. d. Univ. Göttingen und der Niedersächs. Forstl. Versuchsanstalt 129: 1-262.

WALTER, H. \& LIETH, H. (1967): Klimadiagramm - Weltatlas.

WAlter, M.; KRUG, M.; SCHREINER, M. \& REIF, A. (1998): Brombeeren auf oberem Buntsandstein. - Allg. Forstzeitschr. 11: 600-603.

WeCKESSER, M. (2003): Die Bodenvegetation von Buchen-Fichten-Mischbeständen im Solling - Struktur, Diversität und Stoffhaushalt. - Cuvillier, Göttingen, 157 S.

WECKESSER, M. \& SCHMIDT, W. (2004): Gehen dem Luzulo-Fagetum die Trennarten verloren? Veränderungen der Bodenvegetation in bodensauren Buchen- und Fichtenwäldern des Sollings in mehr als drei Jahrzehnten. - Tuexenia 24: 191-206.

WESTPHAL, C. (2001): Theoretische Gedanken und beispielhafte Untersuchungen zur Naturnähe von Wäldern im Staatlichen Forstamt Sellhorn (Lüneburger Heide). - Ber. Forsch.zentr. Waldökosysteme Göttingen A/174: 1-189. 
WHITTAKER, R.H. (ed.)(1973): Ordination and classification of communities. - Handbook of Vegetation Science 5, Den Haag.

WILD, S. (2000): Vegetationsentwicklung auf Sturmwurfflächen auf Buntsandstein (Krücker, Großschutzgebiet Hainholz). - unveröff. Dipl. Univ. Göttingen, Institut für Waldbau, $74 \mathrm{~S}$.

WILLIG, J. (1994): Naturwaldforschung auf Windwurfflächen. Untersuchungen im hessischen Naturwaldreservat Weiherskopf. - Allg. Forstzeitschr. 11: 583-585.

WILLIG, J. (2001): Natürliche Entwicklung von Wäldern nach Sturmwurf. - Allg. Forstzeitschr. 20: 1066-1067.

WILLIG, J. (Hrsg.)(2002a): Natürliche Entwicklung von Wäldern nach Sturmwurf. 10 Jahre Forschung im Naturwaldreservat Weiherskopf. - Mitt. d. Hess. Landesforstverwaltung 38: $1-165$.

WILLIG, J. (2002b): Sturmwurf im Naturwaldreservat Weiherskopf - Forschungskonzept, Vegetations- und Verjüngungsentwicklung. - Mitt. d. Hess. Landesforstverwaltung 38: 35-48.

Wilmanns, O.; Bauer, E.-M.; Goetze, D.; Hermann-Nitritz, B.; Kollmann, J.; Staub, F. \& WotKe, S.A. (1998): Populationsbiologische Studien auf Sturmwurf- und Kahlschlagflächen. - in: FISCHER, A. (Hrsg.): Die Entwicklung von Wald-Biozönosen nach Sturmwurf. - ecomed, Landsberg: 130-145.

Wohlgemuth, T.; Kunn, N.; LÜSCher, P.; KulL, P. \& WÜThrich, H. (1995): Vegetationsund Bodendynamik auf rezenten Windwurfflächen in den Schweizer Nordalpen. Schweiz. Zeitschr. Forstwes. 146(11): 873-891.

WoHLGEMUTH, T.; KUlL, P. \& WÜTHRICH, H. (2002): Disturbance of microsites and early tree regeneration after windthrow in Swiss mountain forests due to the winter storm Vivian 1990. - For. Snow Landsc. Res. 77: 17-47.

ZACHARIAS, D. (1993): Flora und Vegetation von Wäldern der Querco-Fagetea im nördlichen Harzvorland Niedersachsens unter besonderer Berücksichtigung der EichenHainbuchen-Mittelwälder. - Diss. Univ. Braunschweig, 293 S.

ZACHARIAS, D. (1994): Bindung von Gefäßpflanzen an Wälder alter Waldstandorte im nördlichen Harzvorland Niedersachsens - ein Beispiel für die Bedeutung des Alters von Biotopen für den Pflanzenartenschutz. - NNA-Ber. 3: 76-88.

ZIERL, H. \& SIEGRIST, J. (1999): Sturmwürfe 1990 im Nationalpark Berchtesgaden und Folgeentwicklungen. - Forstl. Forschungsber. München 176: 82-92.

ZÜGE, J. (1986): Wachstumsdynamik eines Buchenwaldes auf Kalkgestein - mit besonderer Berücksichtigung der interspezifischen Konkurrenzverhältnisse. - Diss. Univ. Göttingen, Fak. Forstwiss. u. Waldökologie, 213 S. 


\section{ANHANG}

Inhalt:

Tab. A22: Entwicklung der mittleren Deckungsgrade im Flächenwurf der Windwurfgebiete auf Zechstein im südwestlichen Harzvorland in den Jahren 1998 - 2001.

Tab. A23: Entwicklung der mittleren Deckungsgrade im Flächenwurf der Windwurfgebiete auf Buntsandstein im südwestlichen Harzvorland in den Jahren 1998 - 2001.

Tab. A24: Pflanzensoziologische Zuordnung nach DiERSCHKE (1989) der Untersuchungsflächen mit Flächenwurf in den Zechstein-Gebieten im ersten (bzw. zweiten) sowie im vierten Jahr nach dem Sturm.

Tab. A25: Pflanzensoziologische Zuordnung nach DiERSCHKE $(1985,1989)$ der Untersuchungsflächen mit Flächenwurf in den Buntsandstein-Gebieten im ersten (bzw. zweiten) sowie im vierten Jahr nach dem Sturm.

Tab. A26: Hainholz-KF2: Deckungsgradvergleich Wurzelteller/Untersuchungsfläche $1999+2001$.

Tab. A27: Hainholz-KF2: Stetigkeitsvergleich Wurzelteller/Untersuchungsfläche $1999+2001$.

Tab. A28: Hainholz-KF1: Deckungsgradvergleich Wurzelteller/Untersuchungsfläche $1999+2001$.

Tab. A29: Hainholz-KF1: Stetigkeitsvergleich Wurzelteller/Untersuchungsfläche $1999+2001$.

Tab. A30: Königsbuche-KF2 (Flächenwurf): Deckungsgradvergleich Wurzelteller/Untersuchungsfläche $1999+2001$.

Tab. A31: Königsbuche-KF2 (Flächenwurf): Stetigkeitsvergleich Wurzelteller/Untersuchungsfläche $1999+2001$.

Tab. A32: Prozentuale Häufigkeit, mit der manche Arten nur auf Wurzeltellern vorkamen. 
Tab. A22: Entwicklung der mittleren Deckungsgrade im Flächenwurf der Windwurfgebiete auf Zechstein im südwestlichen Harzvorland in den Jahren 1998-2001; grau unterlegt = mindestens

Verdoppelung/Halbierung des mittleren Deckungsgrades; "+" = <0,1\% mittlere Deckung; "\#" = enthält neben der Naturverjüngung auch gepflanzte Exemplare; DGS = Deckungsgradsumme; k.A. = keine Angabe.

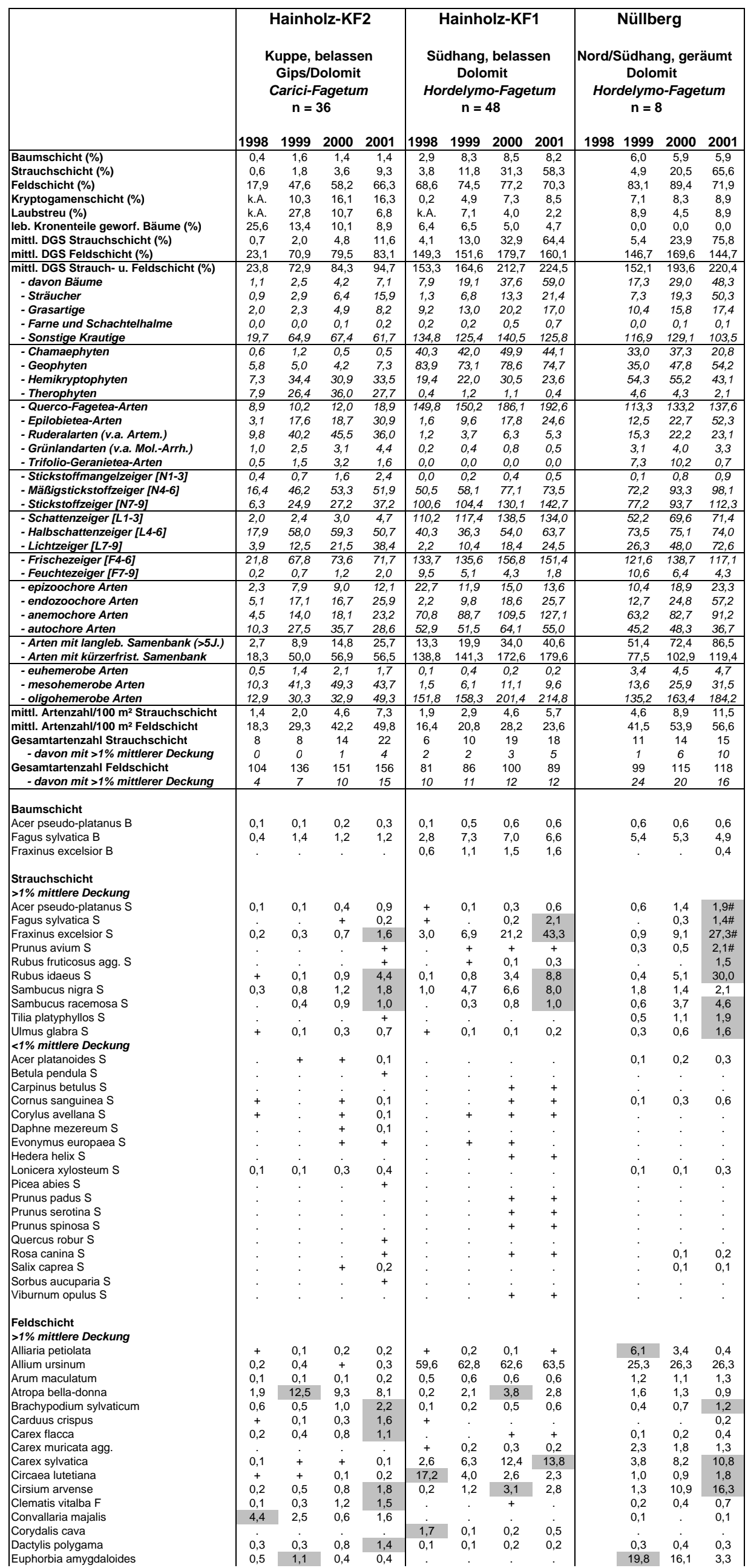




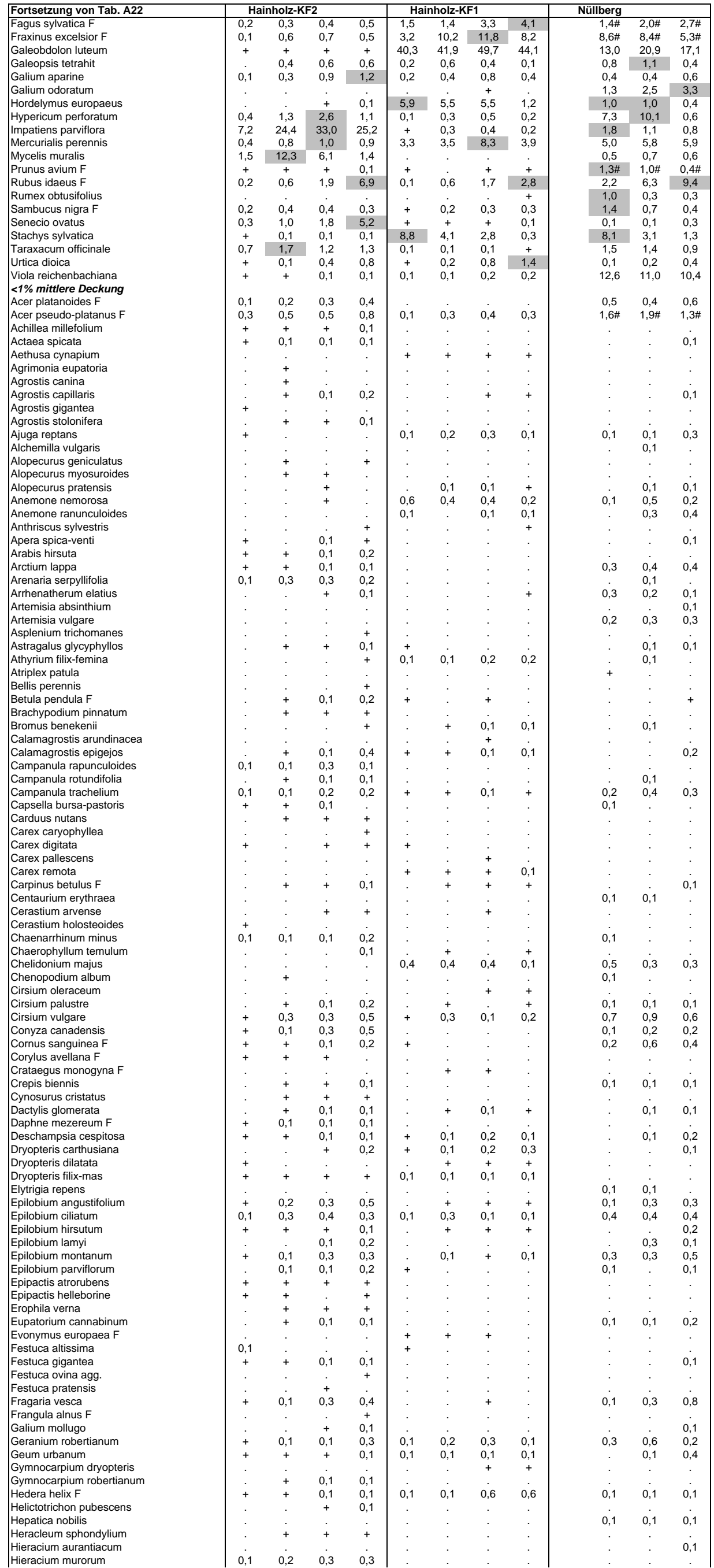




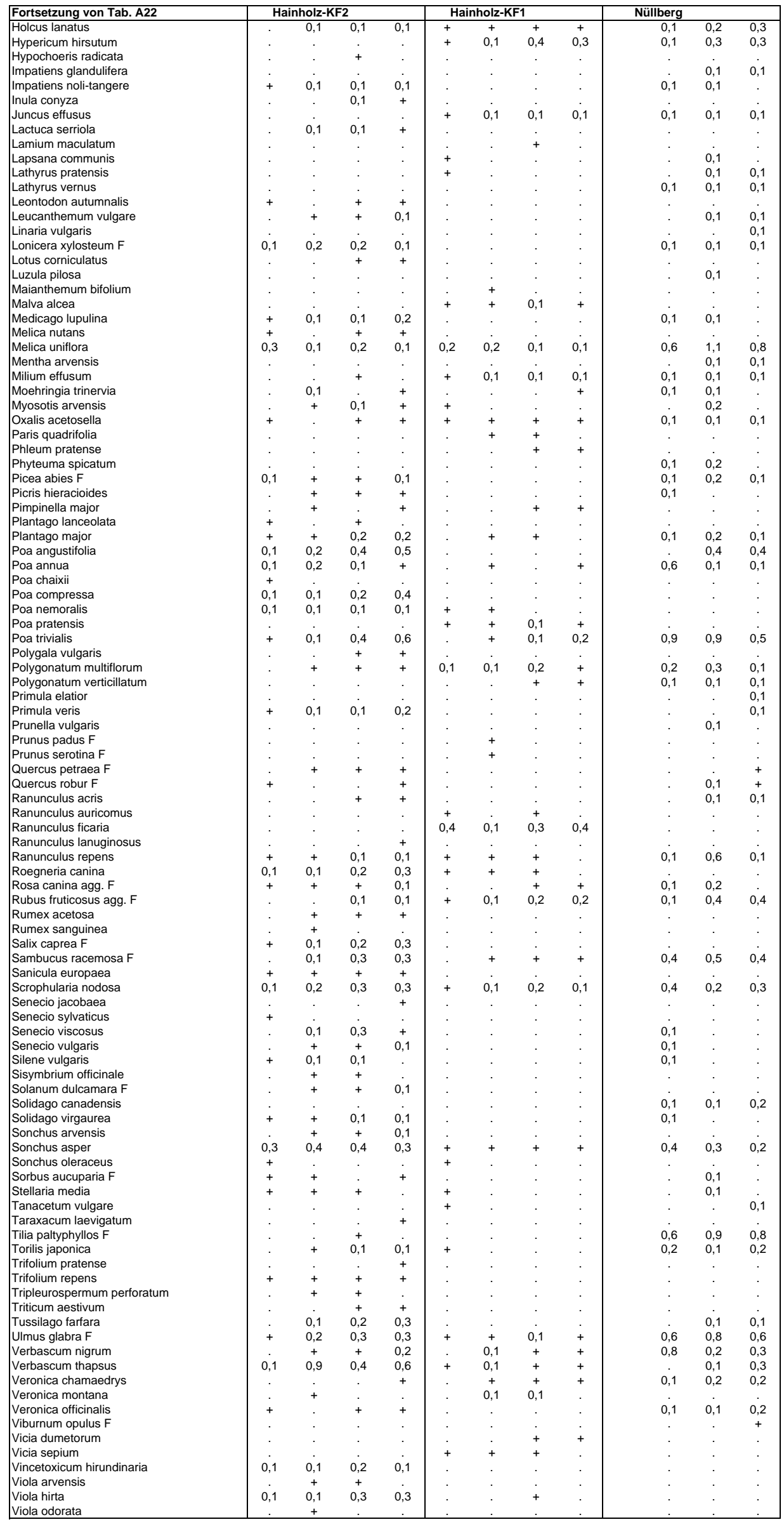


Tab. A23: Entwicklung der mittleren Deckungsgrade im Flächenwurf der Windwurfgebiete auf Buntsandstein im südwestlichen Harzvorland in den Jahren 1998-2001; grau unterlegt = mindestens Verdoppelung/Halbierung des mittleren Deckungsgrades;

"+" = <0,1\% mittlere Deckung; "\#" = enthält neben der Naturverjüngung auch gepflanzte Exemplare; DGS = Deckungsgradsumme; k.A. = keine Angabe

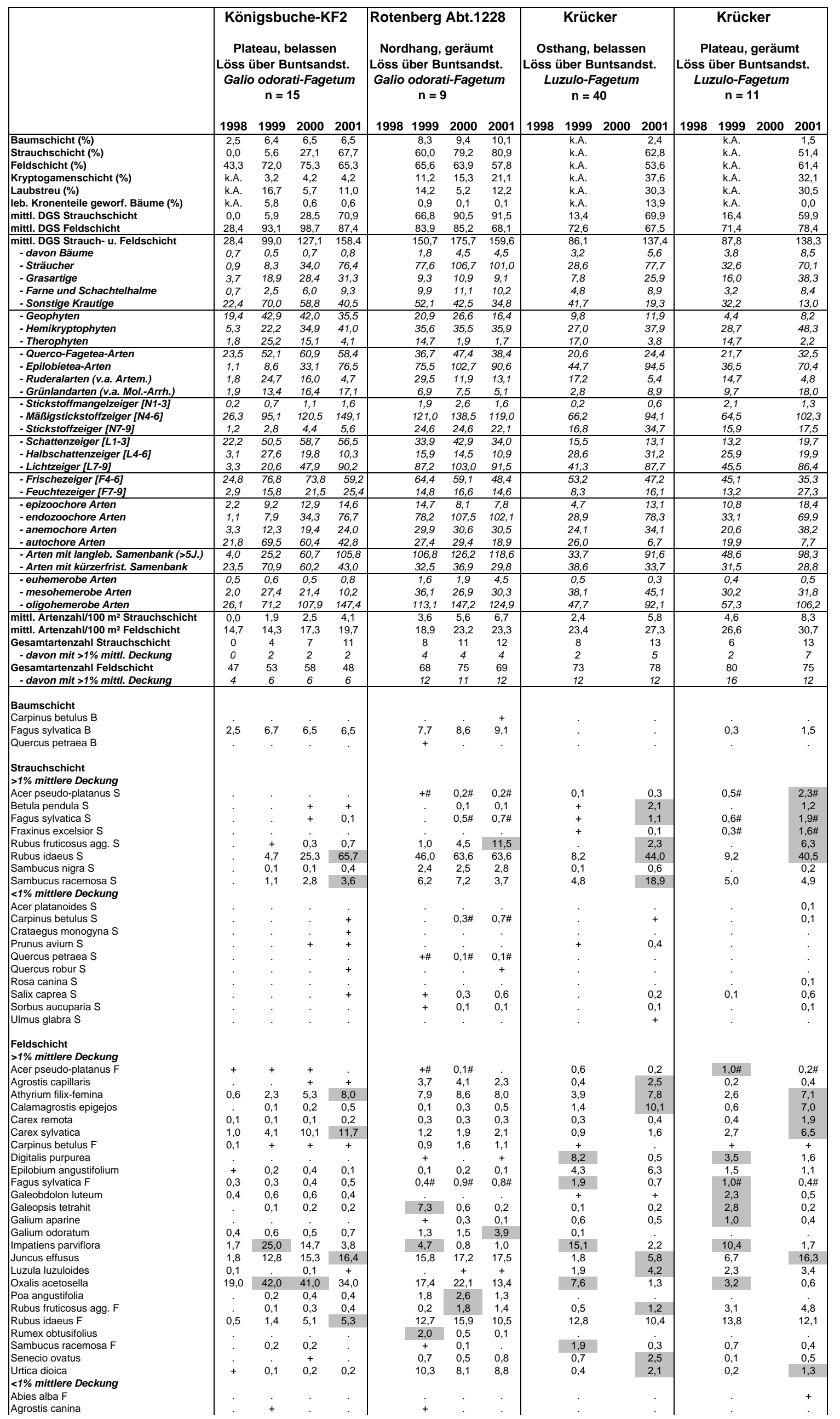




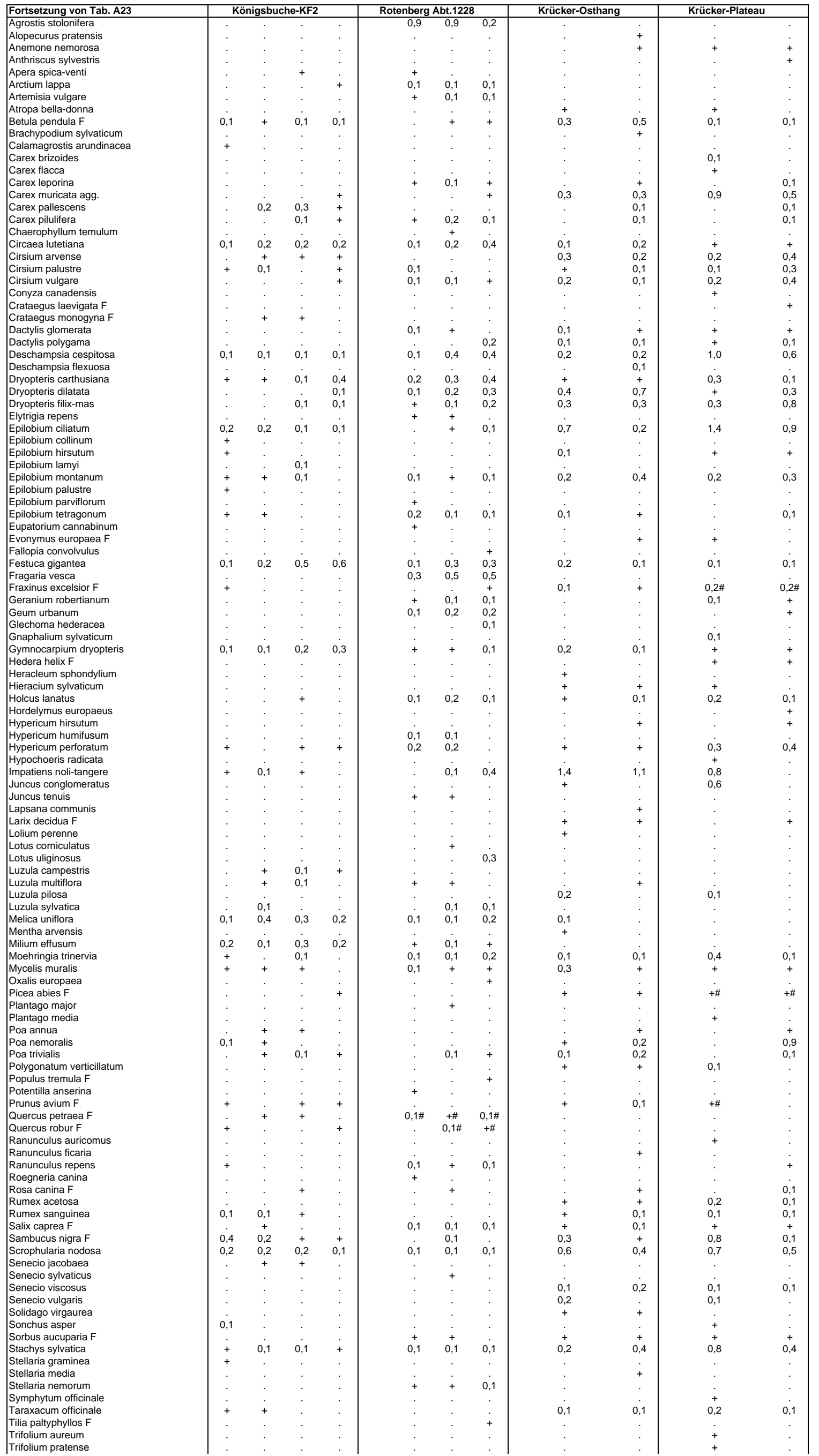




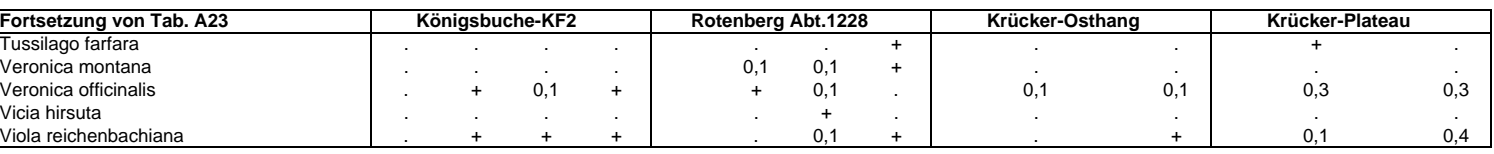


Tab. A24: Pflanzensoziologische Zuordnung nach DIERSCHKE (1989) der Untersuchungsflächen mit Flächenwurf in den Zechstein-Gebieten im ersten (bzw. zweiten) sowie im vierten Jahr nach dem Sturm (1998/1999 sowie 2001); (Artname)* = Arten mit differenzierendem Charakter, die von den Angaben von DIERSCHKE (1989) abweichen;

(wegen besserer Übersichtlichkeit veränderte Anordnung der Untersuchungsvarianten).

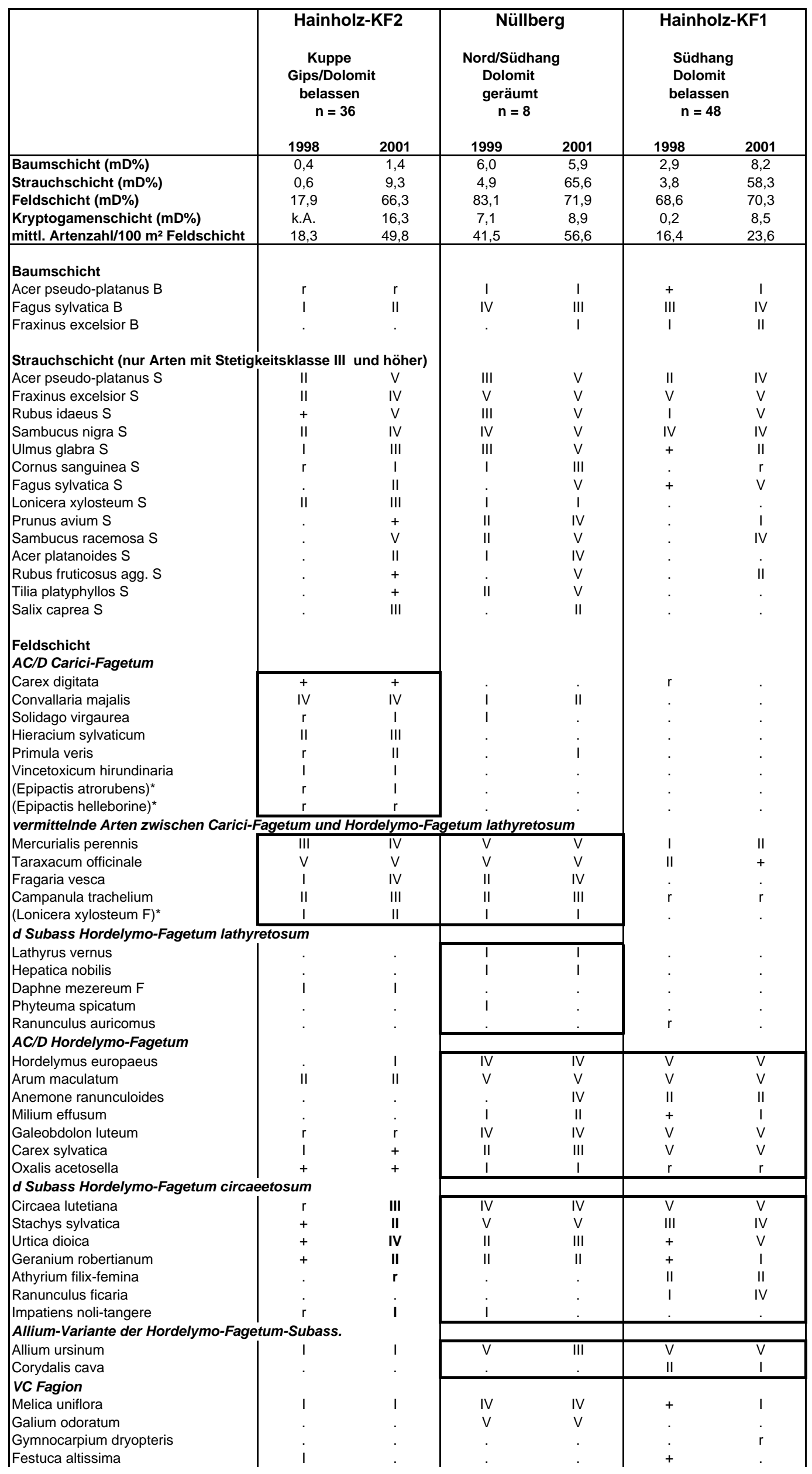




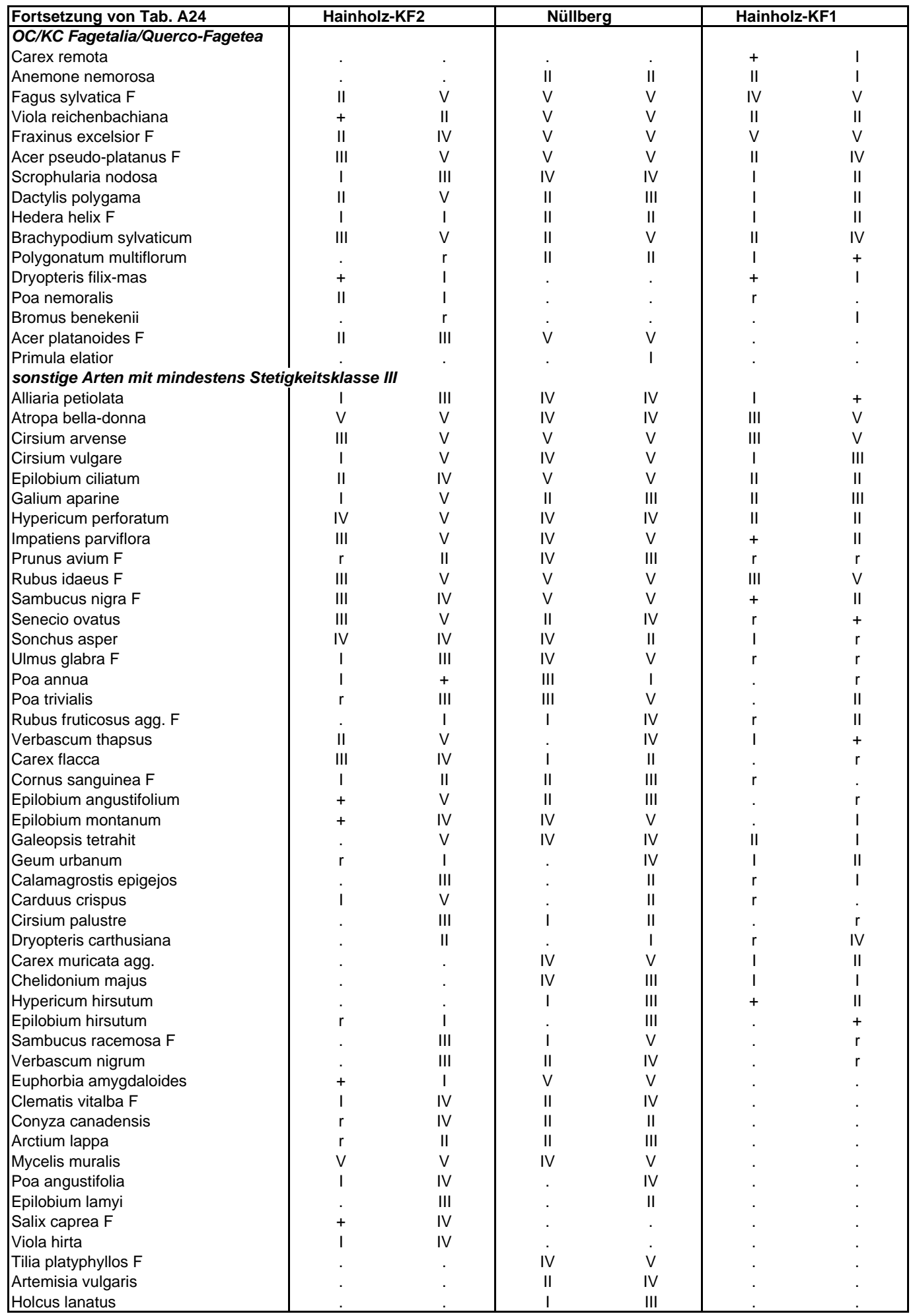


Tab. A25: Pflanzensoziologische Zuordnung nach DIERSCHKE $(1985,1989)$ der Untersuchungsflächen mit Flächenwurf in den Buntsandstein-Gebieten im ersten (bzw. zweiten) sowie im vierten Jahr nach dem Sturm (1998/1999 sowie 2001); $(\text { Artname })^{*}=$ Arten mit differenzierendem Charakter, die von den Angaben von DIERSCHKE $(1985,1989)$ abweichen.

\begin{tabular}{|c|c|c|c|c|c|c|c|c|}
\hline & \multicolumn{2}{|c|}{$\begin{array}{l}\text { Königsbuche KF2 } \\
\text { Plateau } \\
\text { Löss über Buntsandst. } \\
\text { belassen } \\
n=15\end{array}$} & \multicolumn{2}{|c|}{\begin{tabular}{|} 
Rotenberg Abt. 1228 \\
Nordhang \\
Löss über Buntsandst. \\
geräumt \\
n=9
\end{tabular}} & \multicolumn{2}{|c|}{$\begin{array}{c}\text { Krücker } \\
\text { Osthang } \\
\text { Löss über Buntsandst. } \\
\text { belassen } \\
n=40\end{array}$} & \multicolumn{2}{|c|}{$\begin{array}{c}\text { Krücker } \\
\text { Plateau } \\
\text { Löss über Buntsandst. } \\
\text { geräumt } \\
n=11\end{array}$} \\
\hline & 1998 & 2001 & 1999 & 2001 & 1999 & 2001 & 1999 & 2001 \\
\hline Baumschicht (mD\%) & 2,5 & 6,5 & 8,3 & 10,1 & k.A. & 2,4 & k.A. & 1,5 \\
\hline Strauchschicht (mD\%) & 0,0 & 67,7 & 60,0 & 80,9 & k.A. & 62,8 & k.A. & 51,4 \\
\hline Feldschicht (mD\%) & 43,3 & 65,3 & 65,6 & 57,8 & k.A. & 53,6 & k.A. & 61,4 \\
\hline Kryptogamenschicht (mD\%) & k.A. & 4,2 & 11,2 & 21,1 & k.A. & 37,6 & k.A. & 32,1 \\
\hline mittl. Artenzahl/100 $\mathrm{m}^{2}$ Feldschicht & 14,7 & 19,7 & 18,9 & 23,3 & 23,4 & 27,3 & 26,6 & 30,7 \\
\hline \multicolumn{9}{|l|}{ Baumschicht } \\
\hline Carpinus betulus & . & . & . & 1 & . & . & . & . \\
\hline Fagus sylvatica & IV & IV & III & III & . & + & + & II \\
\hline Quercus petraea B & . & . & + & . & . & . & . & . \\
\hline \multicolumn{9}{|c|}{ Strauchschicht (nur Arten mit Stetigkeitsklasse III oder höher) } \\
\hline Rubus idaeus S & . & $\mathrm{V}$ & $\mathrm{V}$ & $\mathrm{V}$ & $\mathrm{V}$ & $\mathrm{V}$ & $\mathrm{V}$ & $\mathrm{V}$ \\
\hline Sambucus racemosa S & . & V & V & V & V & V & V & V \\
\hline Acer pseudo-platanus S & . & . & + & 1 & + & III & V & $\mathrm{V}$ \\
\hline Fagus sylvatica S & . & II & . & V & $r$ & IV & V & V \\
\hline Salix capraea S & . & + & + & III & . & I & II & IV \\
\hline Sambucus nigra S & . & III & II & IV & I & I & . & II \\
\hline Betula pendula S & . & I & . & II & + & $\mathrm{V}$ & . & IV \\
\hline Rubus fruticosus agg. $\mathrm{S}$ & . & III & IV & V & . & IV & . & $\vee$ \\
\hline Carpinus betulus $\mathrm{S}$ & . & II & . & V & . & $r$ & . & + \\
\hline Fraxinus excelsior S & . & . & . & . & $r$ & I & III & V \\
\hline \multicolumn{9}{|l|}{$\begin{array}{l}\text { Feldschicht } \\
\text { AC Galio odorati-Fagetum }\end{array}$} \\
\hline Galium odoratum & IV & $\mathrm{V}$ & $\bar{V}$ & $\mathrm{~V}$ & r & . & . & . \\
\hline Melica uniflora & $\mathrm{I}$ & II & + & II & + & . & . & . \\
\hline \multicolumn{9}{|c|}{ d Subass Galio odorati-Fagetum circaeetosum } \\
\hline Circaea lutetiana & $\mathrm{I}$ & III & $\mathrm{I}$ & IV & I & III & + & + \\
\hline Festuca gigantea & I & III & 1 & III & + & + & + & II \\
\hline (Milium effusum)* & I & III & + & I & . & . & . & . \\
\hline Veronica montana & . & . & 1 & I & . & . & . & . \\
\hline Gymnocarpium-Variante des Galio o & orati-Fage & ircaeetos & & & & & & \\
\hline Gymnocarpium dryopteris & $\mathrm{I}$ & IV & + & II & II & + & + & + \\
\hline (Dryopteris carthusiana)* & + & $\mathrm{V}$ & II & $\mathrm{V}$ & + & + & + & I \\
\hline vermittelnde Arten zwischen verschi & denen Su & iationen & Jes Galio o & und des & uzulo-Fag & & & \\
\hline Athyrium filix-femina & $\mathrm{V}$ & $\mathrm{V}$ & $\mathrm{V}$ & $\mathrm{V}$ & $\mathrm{V}$ & $\mathrm{V}$ & $\bar{V}$ & $\mathrm{~V}$ \\
\hline Dryopteris dilatata & & II & I & IV & III & III & + & III \\
\hline Juncus effusus & V & V & V & IV & $\mathrm{V}$ & V & $\mathrm{V}$ & V \\
\hline Carex remota & 1 & II & III & III & 1 & II & 1 & III \\
\hline Carex pilulifera & . & III & + & ॥ & . & I & . & I \\
\hline Impatiens noli-tangere & 1 & . & . & IV & IV & $\mathrm{V}$ & II & \\
\hline AC Luzulo-Fagetum & & & & & & & & \\
\hline Luzula luzuloides & I & 1 & . & . & $\mathrm{V}$ & $\mathrm{V}$ & $\bar{V}$ & $\mathrm{~V}$ \\
\hline Agrostis capillaris & . & + & il & II & 1 & III & II & IV \\
\hline Deschampsia flexuosa & . & . & . & . & . & + & . & . \\
\hline d Subass Luzulo-Fagetum galietosu & & & & & & & & \\
\hline Fraxinus excelsior F & + & . & . & 1 & + & $r$ & II & III \\
\hline Acer pseudo-platanus F & II & . & + & . & III & III & V & III \\
\hline (Stachys sylvatica)* & + & + & 1 & . & II & IV & IV & IV \\
\hline Luzula pilosa & . & . & . & . & I & . & I & . \\
\hline Galeobdolon luteum & I & 1 & . & . & $\mathrm{r}$ & $r$ & + & + \\
\hline Hedera helix F & . & . & . & . & . & . & + & + \\
\hline Anemone nemorosa & . & . & . & . & . & r & + & + \\
\hline VC/OC/KC Fagion/Fagetalia/Querco- & agetea & & & & & & & \\
\hline Fagus sylvatica $\mathrm{F}$ & IV & $\mathrm{V}$ & IV & V & $\mathrm{V}$ & $\mathrm{V}$ & 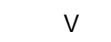 & IV \\
\hline Carex sylvatica & $\mathrm{V}$ & $\mathrm{V}$ & III & III & IV & $\mathrm{V}$ & V & $\mathrm{V}$ \\
\hline Scrophularia nodosa & II & I & II & II & $\mathrm{V}$ & IV & V & $\mathrm{V}$ \\
\hline Dryopteris filix-mas & . & III & + & III & II & II & + & III \\
\hline Carpinus betulus $F$ & III & + & $\mathrm{V}$ & $\mathrm{V}$ & $r$ & . & 1 & + \\
\hline Dactylis glomerata & . & . & + & . & + & + & + & + \\
\hline Dactylis polygama & . & . & . & il & 1 & II & + & I \\
\hline Viola reichenbachiana & . & + & . & . & . & + & + & IV \\
\hline Poa nemoralis & il & . & . & . & r & II & . & III \\
\hline Quercus robur F & I & + & . & II & . & . & . & . \\
\hline Brachypodium sylvaticum & . & . & . & . & . & $r$ & . & . \\
\hline Sonstige Arten mit mindestens Ste & keitsklas & & & & & & & \\
\hline Urtica dioica & + & III & V & V & III & V & II & III \\
\hline Oxalis acetosella & V & V & V & V & IV & IV & IV & $\mathrm{V}$ \\
\hline Epilobium angustifolium & 1 & III & II & II & $\mathrm{V}$ & V & $\mathrm{V}$ & $\mathrm{V}$ \\
\hline Impatiens parviflora & $\mathrm{V}$ & $\mathrm{V}$ & $\mathrm{V}$ & $\mathrm{V}$ & $\mathrm{V}$ & $\mathrm{V}$ & V & $\mathrm{V}$ \\
\hline Rubus idaeus $\mathrm{F}$ & $\mathrm{V}$ & $\mathrm{V}$ & V & V & V & $\vee$ & $\vee$ & V \\
\hline Betula pendula $\mathrm{F}$ & III & I & . & I & III & IV & II & ॥ \\
\hline Calamagrostis epigejos & . & IV & i & III & IV & $\mathrm{V}$ & III & $\mathrm{V}$ \\
\hline Cirsium palustre & + & + & + & . & + & I & II & III \\
\hline
\end{tabular}




\begin{tabular}{|c|c|c|c|c|c|c|c|c|}
\hline Fortsetzung von Tab. A 25 & Köni & & Rot & t. 1228 & & eau & & hang \\
\hline Cirsium vulgare & & $\mathrm{I}$ & $\mathrm{I}$ & $\mathrm{I}$ & II & II & II & IV \\
\hline Epilobium ciliatum & III & 1 & . & II & IV & III & IV & $\mathrm{V}$ \\
\hline Epilobium montanum & + & . & I & I & I & IV & 1 & IV \\
\hline Galeopsis tetrahit & . & III & $\mathrm{V}$ & III & 1 & III & IV & II \\
\hline Moehringia trinervia & + & . & II & III & + & 1 & II & I \\
\hline Rubus fruticosus agg. $F$ & . & III & III & V & III & IV & V & V \\
\hline Senecio ovatus & . & . & III & IV & III & V & + & $\mathrm{V}$ \\
\hline Digitalis purpurea & . & . & I & I & V & V & V & V \\
\hline Galium aparine & . & . & + & II & III & V & V & V \\
\hline Sambucus nigra $F$ & IV & + & . & . & II & + & II & II \\
\hline Veronica officinalis & . & II & + & . & I & 1 & III & IV \\
\hline Sambucus racemosa F & . & . & + & . & v & III & IV & IV \\
\hline Cirsium arvense & . & I & . & . & II & II & II & IV \\
\hline Taraxacum officinale & 1 & . & r. & . & + & II & III & II \\
\hline Senecio viscosus & . & . & . & 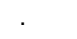 & II & III & 1 & I \\
\hline Carex pallescens & . & IV & . & 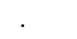 & . & + & . & I \\
\hline Poa angustifolia & . & IV & III & III & . & . & . & . \\
\hline Fragaria vesca & $\dot{\square}$ & . & III & III & & . & & \\
\hline
\end{tabular}


Tab. A26: Hainholz-KF 2: Deckungsgradvergleich Wurzelteller (WT)/Untersuchungsfläche (Ufl) $1999+2001$; alle Angaben in \%; grau unterlegt = mindestens Verdoppelung/Halbierung des mittleren Deckungsgrades; "+" = <0,1\% mittl. Deckung.

\begin{tabular}{|c|c|c|c|}
\hline & $\begin{aligned} \text { Ufl } \\
n\end{aligned}$ & $\left.\mathrm{~m}^{2}\right)$ & $\begin{array}{c}\text { WT } 9 \\
n=\end{array}$ \\
\hline & 1999 & 2001 & 1999 \\
\hline mittl. Anzahl Wurzelteller je 100 m² $^{2}$ & 3,7 & 3,7 & \\
\hline mittl. Wurzeltellerfläche je $100 \mathrm{~m}^{2}(\%)$ & 6,2 & 8,1 & \\
\hline Gesamtartenzahl Strauchschicht & 8 & 18 & 0 \\
\hline Gesamtartenzahl Feldschicht & 112 & 140 & 50 \\
\hline $\begin{array}{l}\text { mittl. Artenzahl Strauchschicht } \\
\text { davon nur auf WT vorkommend }\end{array}$ & $\begin{array}{l}2,0 \\
0,0\end{array}$ & 7,5 & 0,0 \\
\hline mittl. Artenzahl Felschicht & 31,5 & 56,1 & 7,1 \\
\hline davon nur auf WT vorkommend & 1,8 & 4,4 & \\
\hline mittl. Fläche des WT-Abschnitts ( $\left.\mathbf{m}^{2}\right)$ & & & \\
\hline mittl. vegetationsfreie Fläche (\%) & 17,8 & 7,4 & 60,8 \\
\hline mittl. DGS Strauchschicht (\%) & 2,3 & 14,0 & 0,0 \\
\hline mittl. DGS Feldschicht (\%) & 78,3 & 85,8 & 24,7 \\
\hline mittl. Deckung Kryptogamen (\%) & 11,1 & 16,7 & 12,6 \\
\hline mittl. Deckung Laubstreu + Totholz (\%) & 50,0 & 34,9 & 1,5 \\
\hline Baumschicht (Sommeraufnahme) & & & \\
\hline Fagus sylvatica $B$ & 1,7 & 1,8 & \\
\hline Strauchschicht (Gesamtartenzahl: 18) & & & \\
\hline 1. auf Ufl und auf WT zunehmend (5 Arte & entsp & $27,8 \%$ & \\
\hline Acer pseudo-platanus $S$ & 0,2 & 1,1 & . \\
\hline Lonicera xylosteum S & 0,1 & 0,2 & . \\
\hline Rubus idaeus S & 0,1 & 4,3 & . \\
\hline Sambucus nigra $\mathrm{S}$ & 1,4 & 3,5 & . \\
\hline Sambucus racemosa S & 0,2 & 0,8 & . \\
\hline 6. nicht auf $W T$ vorkommend (13 Arten $\mathrm{e}$ & tsprich & 3\%) & \\
\hline Acer platanoides S & . & 0,2 & . \\
\hline Betula pendula S & . & + & . \\
\hline Prunus avium $\mathrm{S}$ & . & + & . \\
\hline Cornus sanguinea $\mathrm{S}$ & . & + & . \\
\hline Corylus avellana S & . & 0,1 & . \\
\hline Daphne mezereum S & . & 0,1 & . \\
\hline Evonymus europaea S & . & + & . \\
\hline Fagus sylvatica S & 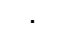 & 0,2 & . \\
\hline Fraxinus excelsior $\mathrm{S}$ & 0,2 & 1,8 & . \\
\hline Rubus fruticosus agg. $\mathrm{S}$ & . & + & . \\
\hline Salix caprea S & . & 0,3 & . \\
\hline Tilia platyphyllos S & . & + & . \\
\hline Ulmus glabra S & 0,2 & 1,1 & . \\
\hline Feldschicht (Gesamtartenzahl: 150) & & & \\
\hline 1. auf Ufl und auf WT zunehmend (62 Ar & $n$ ents & t 41,1 & \\
\hline Acer platanoides $\mathrm{F}$ & 0,1 & 0,4 & . \\
\hline Acer pseudo-platanus F & 0,5 & 0,9 & + \\
\hline Agrostis capillaris & ${ }^{\circ}$ & 0,2 & . \\
\hline Agrostis stolonifera & & 0,1 & . \\
\hline Allium ursinum & 0,9 & 0,5 & + \\
\hline Arabis hirsuta & + & 0,2 & . \\
\hline Brachypodium sylvaticum & 0,3 & 1,0 & + \\
\hline Bromus benekenii & . & + & . \\
\hline Calamagrostis epigejos & . & 0,6 & . \\
\hline Campanula rotundifolia & + & 0,1 & + \\
\hline Carduus crispus & 0,2 & 1,4 & + \\
\hline Carex flacca & 0,5 & 1,5 & + \\
\hline Carpinus betulus $\mathrm{F}$ & + & 0,2 & . \\
\hline Prunus avium $\mathrm{F}$ & + & 0,1 & . \\
\hline Cirsium arvense & 0,5 & 1,1 & 0,1 \\
\hline Cirsium palustre & + & 0,3 & . \\
\hline Cirsium vulgare & 0,2 & 0,5 & + \\
\hline Clematis vitalba & + & 0,5 & . \\
\hline Conyza canadensis & 0,3 & 1,3 & + \\
\hline Cornus sanguinea $\mathrm{F}$ & + & 0,1 & . \\
\hline Crepis biennis & + & 0,1 & . \\
\hline Dactylis polygama & + & 0,6 & . \\
\hline Dryopteris carthusiana & & 0,2 & . \\
\hline Epilobium angustifolium & 0,1 & 0,4 & + \\
\hline Epilobium ciliatum & 0,3 & 0,4 & + \\
\hline Epilobium hirsutum & . & 0,1 & . \\
\hline Epilobium lamyi & . & 0,3 & . \\
\hline Epilobium montanum & 0,1 & 0,4 & + \\
\hline Epilobium parviflorum & 0,1 & 0,3 & \\
\hline Fagus sylvatica F & 0,4 & 0,5 & 0,1 \\
\hline Festuca ovina agg. & & 0,1 & \\
\hline Fragaria vesca & + & 0,5 & . \\
\hline Galeopsis tetrahit & 0,5 & 0,6 & 0,1 \\
\hline Galium aparine & 0,1 & 0,9 & . \\
\hline Galium mollugo agg. & & 0,1 & . \\
\hline Geranium robertianum & 0,1 & 0,3 & + \\
\hline Gymnocarpium robertianum & & 0,1 & . \\
\hline Helictotrichon pubescens & & 0,1 & . \\
\hline Holcus lanatus & 0,1 & 0,2 & 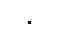 \\
\hline Hypericum perforatum & 1,0 & 1,1 & 0,2 \\
\hline Leontodon autumnalis & . & 0,1 & . \\
\hline Leucanthemum vulgare & + & 0,2 & . \\
\hline Lotus corniculatus & & + & 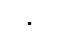 \\
\hline Medicago lupulina & 0,1 & 0,2 & + \\
\hline Pimpinella major & + & 0,2 & . \\
\hline Poa angustifolia & 0,3 & 0,7 & + \\
\hline Poa trivialis & 0,1 & 0,9 & . \\
\hline Quercus robur F & 乎 & 0,1 & . \\
\hline Roegneria canina & 0,2 & 0,4 & + \\
\hline Rosa canina agg. $\mathrm{F}$ & + & 0,1 & \\
\hline Rubus idaeus $\mathrm{F}$ & 0,5 & 7,3 & 0,1 \\
\hline Salix caprea F & 0,1 & 0,3 & . \\
\hline Sambucus racemosa F & 0,1 & 0,3 & 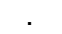 \\
\hline Scrophularia nodosa & 0,1 & 0,3 & + \\
\hline Senecio ovatus & 0,7 & 5,9 & 0,3 \\
\hline
\end{tabular}




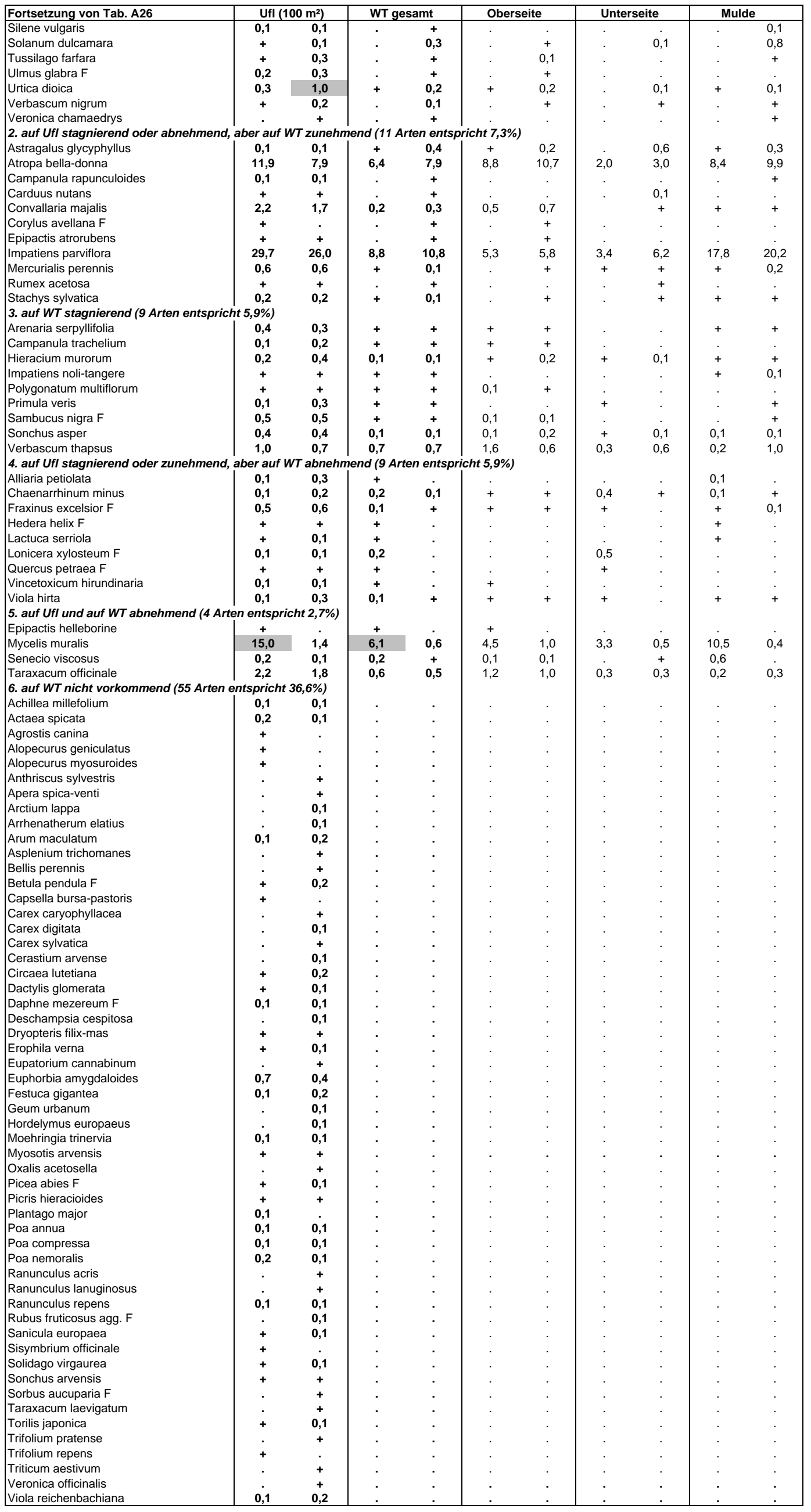


Tab. A27: Hainholz-KF2: Stetigkeitsvergleich Wurzelteller (WT)/Untersuchungsfläche (Ufl) $1999+2001$; alle Angaben in \%; grau unterlegt = mindestens Verdoppelung/Halbierung der Stetigkeit.

\begin{tabular}{|c|c|c|c|c|c|}
\hline & & $\begin{array}{l}\left.0 \mathrm{~m}^{2}\right) \\
5\end{array}$ & $\begin{array}{c}\text { WT } 9 \\
n\end{array}$ & & \\
\hline & 1999 & 2001 & 1999 & 2001 & 1999 \\
\hline mittl. Anzahl Wurzelteller je 100 m²$^{2}$ & 3,7 & 3,7 & & & \\
\hline mittl. Wurzeltellerfläche je $100 \mathrm{~m}^{2}(\%)$ & 6,2 & 8,1 & & & \\
\hline Gesamtartenzahl Strauchschicht & 8 & 18 & 0 & 5 & 0 \\
\hline Gesamtartenzahl Feldschicht & 112 & 140 & 50 & 87 & 32 \\
\hline mittl. Artenzahl Strauchschicht & 2,0 & 7,5 & 0,0 & 0,7 & 0,0 \\
\hline davon nur auf $W T$ vorkommend & 0,0 & 0,1 & & & \\
\hline $\begin{array}{l}\text { mittl. Artenzahl Felschicht } \\
\text { davon nur auf WT vorkommend }\end{array}$ & $\begin{array}{l}31,5 \\
1,8\end{array}$ & $\begin{array}{l}56,1 \\
4,4\end{array}$ & 7,1 & 16,5 & 3,9 \\
\hline mittl. Fläche des WT-Abschnitts $\left(\mathrm{m}^{2}\right)$ & & & & & 1,6 \\
\hline mittl. vegetationsfreie Fläche (\%) & 17,8 & 7,4 & 60,8 & 42,5 & 71,0 \\
\hline mittl. DGS Strauchschicht (\%) & 2,3 & 14,0 & 0,0 & 4,3 & 0,0 \\
\hline mittl. DGS Feldschicht (\%) & 78,3 & 85,8 & 24,7 & 35,9 & 23,5 \\
\hline mittl. Deckung Kryptogamen (\%) & 11,1 & 16,7 & 12,6 & 28,0 & 10,1 \\
\hline mittl. Deckung Laubstreu + Totholz (\%) & 50,0 & 34,9 & 1,5 & 3,3 & 0,0 \\
\hline Baumschicht (Sommeraufnahme) & & & & & \\
\hline Fagus sylvatica $\mathrm{B}$ & 20,0 & 20,0 & . & . & . \\
\hline Strauchschicht (Gesamtartenzahl: 18) & & & & & \\
\hline 1. Auf Ufl und auf WT zunehmend (5 Arte & entsp & t $27,7 \%$ & & & \\
\hline Acer pseudo-platanus S & 33,3 & 80,0 & . & 9,7 & . \\
\hline Lonicera xylosteum S & 13,3 & 40,0 & . & 3,2 & . \\
\hline Rubus idaeus S & 13,3 & 100,0 & . & 54,8 & . \\
\hline Sambucus nigra S & 46,7 & 80,0 & . & 6,5 & . \\
\hline Sambucus racemosa S & 20,0 & 73,3 & . & 3,2 & . \\
\hline 6. auf WT nicht vorkommend (13 Arten e & tsprich & ,3\%) & & & \\
\hline Acer platanoides S & 13,3 & 33,3 & . & . & . \\
\hline Betula pendula S & . & 6,7 & . & . & . \\
\hline Prunus avium S & . & 20,0 & . & . & . \\
\hline Cornus sanguinea $\mathrm{S}$ & . & 13,3 & . & . & . \\
\hline Corylus avellana S & . & 26,7 & . & . & . \\
\hline Daphne mezereum S & . & 20,0 & . & . & . \\
\hline Evonymus europaea S & . & 6,7 & . & . & . \\
\hline Fagus sylvatica S & 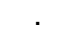 & 46,7 & . & . & . \\
\hline Fraxinus excelsior $\mathrm{S}$ & 40,0 & 66,7 & . & . & . \\
\hline Rubus fruticosus agg. $\mathrm{S}$ & . & 6,7 & . & . & . \\
\hline Salix caprea S & . & 53,3 & . & . & . \\
\hline Tilia platyphyllos S & 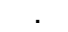 & 13,3 & . & . & . \\
\hline Ulmus glabra S & 20,0 & 60,0 & . & . & . \\
\hline Feldschicht (Gesamtartenzahl: 150) & & & & & \\
\hline 1. auf Ufl und auf WT zunehmend (68 Art & $n$ entsp & ht $45,3 \%$ & & & \\
\hline Acer platanoides $\mathrm{F}$ & 26,7 & 46,7 & & 3,2 & \\
\hline Acer pseudo-platanus F & 86,7 & 93,3 & 12,9 & 25,8 & 9,7 \\
\hline Agrostis capillaris & . & 40,0 & . & 3,2 & . \\
\hline Agrostis stolonifera & . & 20,0 & . & 3,2 & . \\
\hline Arabis hirsuta & 6,7 & 33,3 & . & 3,2 & 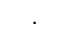 \\
\hline Astragalus glycyphyllus & 13,3 & 20,0 & 6,5 & 9,7 & 3,2 \\
\hline Brachypodium sylvaticum & 40,0 & 93,3 & 3,2 & 25,8 & 3,2 \\
\hline Bromus benekenii & . & 6,7 & . & 3,2 & . \\
\hline Calamagrostis epigejos & & 80,0 & 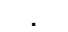 & 6,5 & . \\
\hline Carduus crispus & 46,7 & 93,3 & 6,5 & 41,9 & . \\
\hline Carex flacca & 66,7 & 80,0 & 9,7 & 48,4 & . \\
\hline Carpinus betulus $\mathrm{F}$ & 13,3 & 46,7 & . & 3,2 & . \\
\hline Prunus avium $\mathrm{F}$ & 6,7 & 40,0 & . & 3,2 & \\
\hline Cirsium arvense & 93,3 & 100,0 & 19,4 & 32,3 & 3,2 \\
\hline Cirsium palustre & 20,0 & 66,7 & . & 12,9 & . \\
\hline Cirsium vulgare & 60,0 & 100,0 & 3,2 & 25,8 & . \\
\hline Clematis vitalba & 6,7 & 73,3 & . & 12,9 & . \\
\hline Conyza canadensis & 6,7 & 73,3 & . & 29,0 & . \\
\hline Cornus sanguinea $\mathrm{F}$ & 13,3 & 26,7 & . & 3,2 & . \\
\hline Crepis biennis & 6,7 & 26,7 & . & 3,2 & . \\
\hline Dactylis polygama & 26,7 & 80,0 & . & 19,4 & . \\
\hline Dryopteris carthusiana & & 46,7 & & 9,7 & \\
\hline Epilobium angustifolium & 20,0 & 86,7 & 3,2 & 25,8 & 3,2 \\
\hline Epilobium ciliatum & 60,0 & 80,0 & 6,5 & 38,7 & . \\
\hline Epilobium hirsutum & . & 13,3 & . & 3,2 & . \\
\hline Epilobium lamyi & . & 66,7 & . & 32,3 & . \\
\hline Epilobium montanum & 26,7 & 86,7 & 3,2 & 67,7 & . \\
\hline Epilobium parviflorum & 20,0 & 60,0 & . & 25,8 & . \\
\hline Epipactis atrorubens & 6,7 & 20,0 & . & 6,5 & . \\
\hline Festuca ovina agg. & & 13,3 & . & 3,2 & . \\
\hline Fragaria vesca & 6,7 & 80,0 & 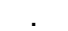 & 22,6 & \\
\hline Galeopsis tetrahit & 93,3 & 100,0 & 25,8 & 61,3 & 3,2 \\
\hline Galium aparine & 53,3 & 100,0 & 6,5 & 35,5 & 3,2 \\
\hline Galium mollugo agg. & & 26,7 & $r_{0}$ & 3,2 & . \\
\hline Geranium robertianum & 13,3 & 26,7 & 3,2 & 6,5 & . \\
\hline Gymnocarpium robertianum & 6,7 & 13,3 & . & 3,2 & 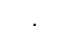 \\
\hline Helictotrichon pubescens & & 26,7 & 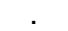 & 3,2 & \\
\hline Hieracium murorum & 46,7 & 73,3 & 9,7 & 25,8 & 6,5 \\
\hline Holcus lanatus & 20,0 & 33,3 & $x_{0}$ & 3,2 & \\
\hline Hypericum perforatum & 93,3 & 100,0 & 16,1 & 54,8 & 6,5 \\
\hline Lactuca serriola & 6,7 & 13,3 & 3,2 & & 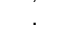 \\
\hline Leontodon autumnalis & $\cdot$ & 20,0 & . & 6,5 & . \\
\hline Leucanthemum vulgare & 6,7 & 40,0 & . & 6,5 & . \\
\hline Lotus corniculatus & & 6,7 & . & 3,2 & \\
\hline Medicago lupulina & 20,0 & 33,3 & 9,7 & 12,9 & 9,7 \\
\hline Plantago major & 20,0 & 40,0 & & 3,2 & \\
\hline Poa angustifolia & 46,7 & 73,3 & 3,2 & 9,7 & . \\
\hline Poa trivialis & 13,3 & 66,7 & . & 22,6 & . \\
\hline Primula veris & 33,3 & 53,3 & 3,2 & 6,5 & r. \\
\hline Quercus robur F & & 33,3 & - & 6,5 & 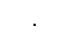 \\
\hline Roegneria canina & 6,7 & 20,0 & . & 6,5 & . \\
\hline Rosa canina agg. $\mathrm{F}$ & 6,7 & 33,3 & & 3,2 & \\
\hline Rubus idaeus $\mathrm{F}$ & 80,0 & 100,0 & 16,1 & 58,1 & 3,2 \\
\hline
\end{tabular}




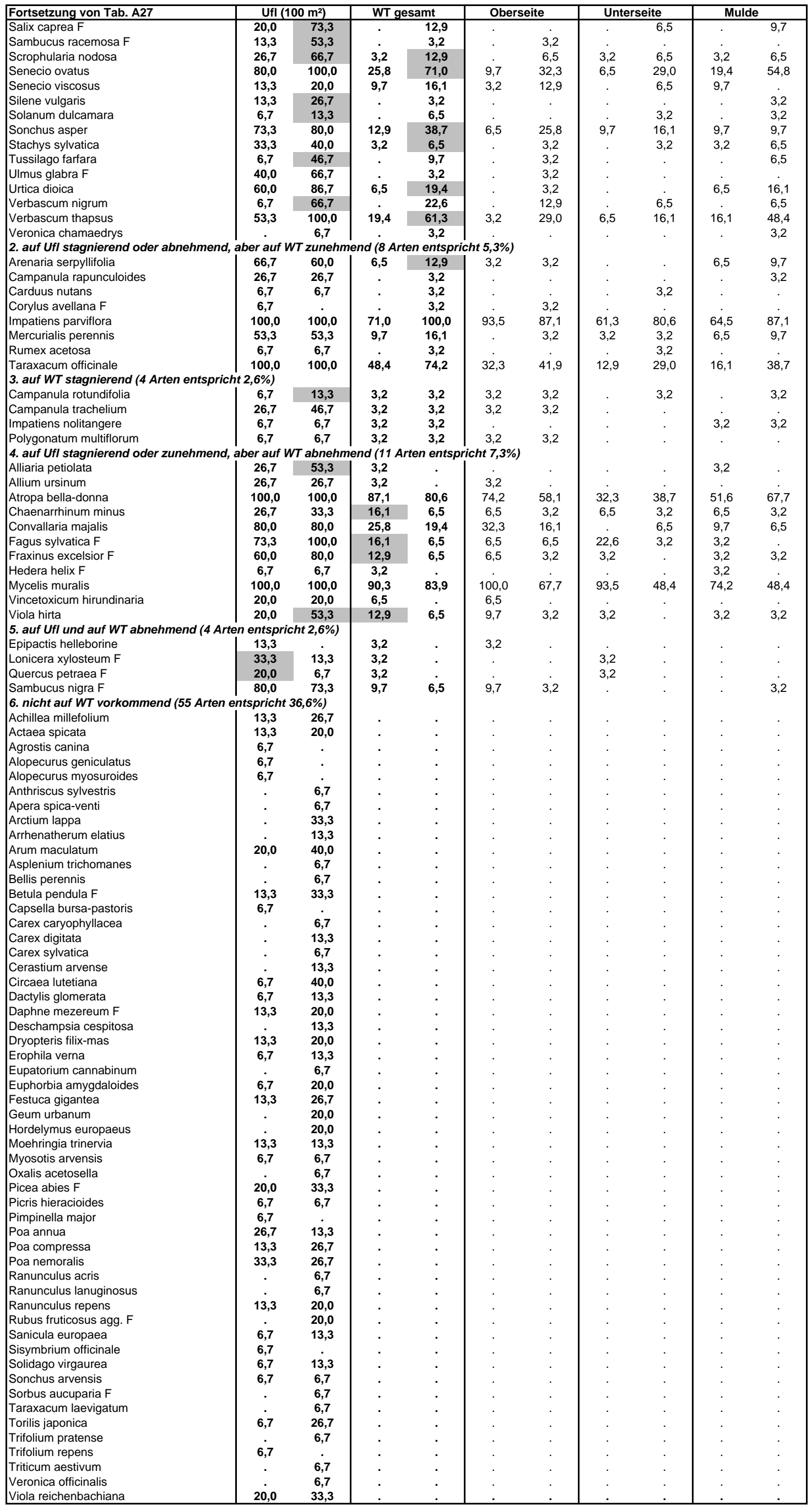


Tab. A28: Hainholz-KF1: Deckungsgradvergleich Wurzelteller (WT)/Untersuchungsfläche (Ufl) $1999+2001$ alle Angaben in \%; grau unterlegt $=$ mindestens Verdoppelung/Halbierung des mittleren Deckungsgrades; $+"=<0,1 \%$ mittl. Deckung.

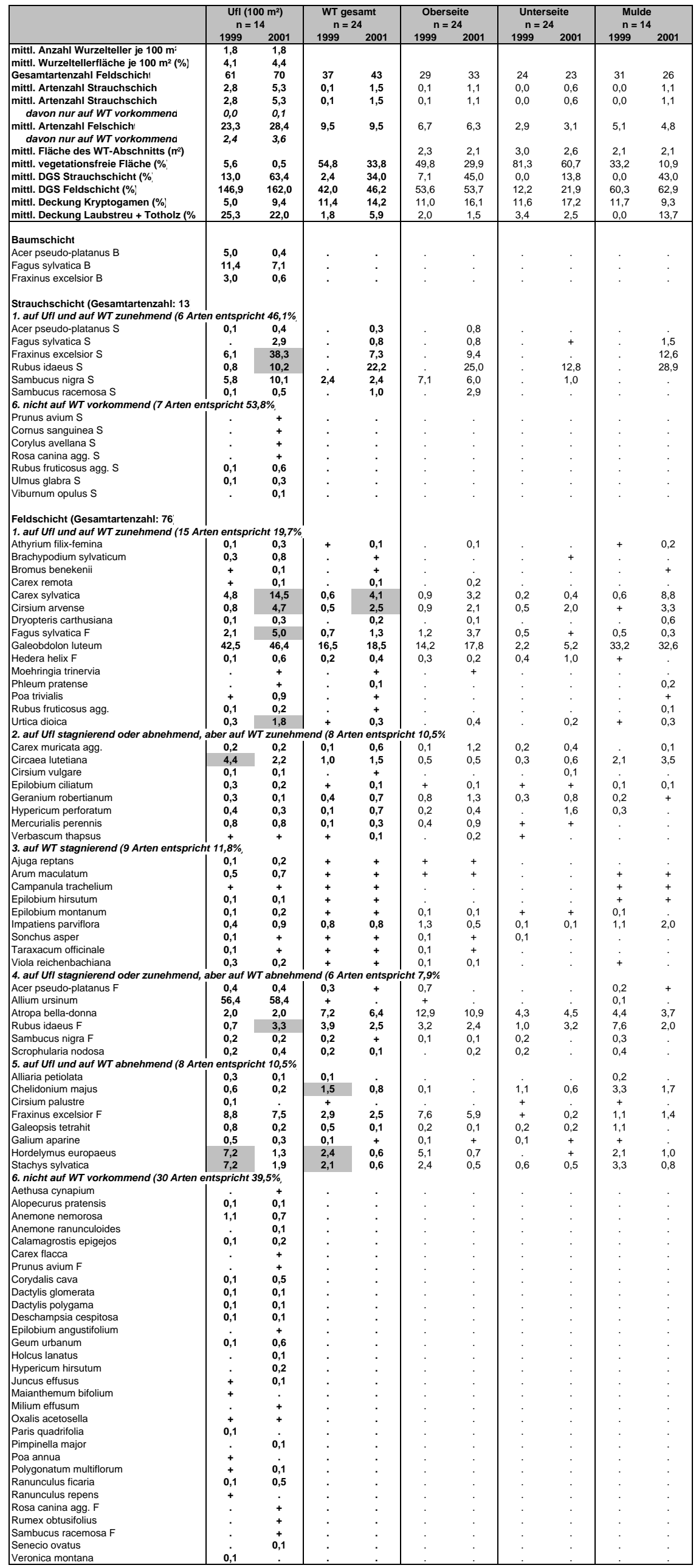


Tab. A29: Hainholz-KF1: Stetigkeitsvergleich Wurzelteller (WT)/Untersuchungsfläche (Ufl) 1999 + 2001; alle Angaben in \%; grau unterlegt $=$ mindestens Verdoppelung/Halbierung der Stetigkeit.

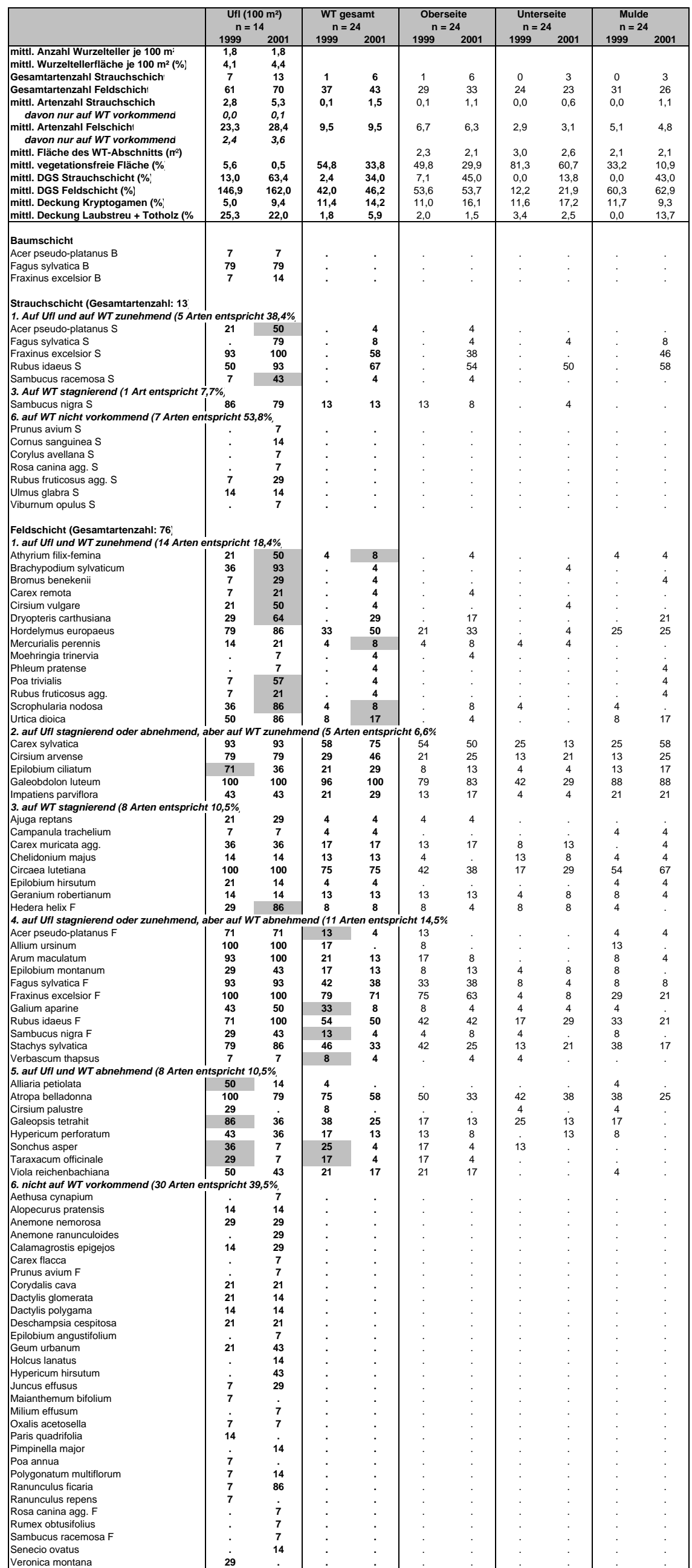


Tab. A30: Königsbuche-KF 2 (Flächenwurf): Deckungsgradvergleich Wurzelteller (WT)/Untersuchungsfläche (Ufl) 1999 + 2001; alle Angaben in \%; grau unterlegt = mindestens Verdoppelung/Halbierung des mittleren

Deckungsgrades;"+" = <0,1\% mittl. Deckung.

\begin{tabular}{|c|}
\hline \\
\hline \\
\hline mittl. Anzahl Wurzelteller je 100 m² $^{2}$ \\
\hline mittl. Wurzeltellerfläche je $100 \mathrm{~m}^{2}(\%)$ \\
\hline Gesamtartenzahl Strauchschicht \\
\hline Gesamtartenzahl Feldschicht \\
\hline mittl. Artenzahl Strauchschicht \\
\hline davon nur auf $W T$ vorkommend \\
\hline $\begin{array}{l}\text { mittl. Artenzahl Felschicht } \\
\text { davon nur auf WT vorkommend }\end{array}$ \\
\hline mittl. Fläche des WT-Abschnitts $\left(\mathrm{m}^{2}\right)$ \\
\hline mittl. vegetationsfreie Fläche (\%) \\
\hline mittl. DGS Strauchschicht (\%) \\
\hline mittl. DGS Feldschicht (\%) \\
\hline mittl. Deckung Kryptogamen (\%) \\
\hline mittl. Deckung Laubstreu + Totholz (\%) \\
\hline Baumschicht \\
\hline Fagus sylvatica B \\
\hline Strauchschicht (Gesamtartenzahl: 9) \\
\hline 1. Auf Ufl und auf WT zunehmend (4 Art \\
\hline Fagus sylvatica $\mathrm{S}$ \\
\hline Rubus fruticosus agg. $\mathrm{S}$ \\
\hline Rubus idaeus S \\
\hline Sambucus racemosa S \\
\hline 3. Auf WT stagnierend (1 Art $=11,1 \%$ ) \\
\hline Sambucus nigra S \\
\hline 6. Nicht auf $W T$ vorkommend (4 Arten $=$ \\
\hline Betula pendula S \\
\hline Carpinus betulus $\mathrm{S}$ \\
\hline Prunus avium $\mathrm{S}$ \\
\hline Salix caprea S \\
\hline Feldschicht (Gesamtartenzahl: 61) \\
\hline 1. Auf Ufl und auf WT zunehmend (14 Ar \\
\hline Betula pendula F \\
\hline Calamagrostis epigejos \\
\hline Carex muricata agg. \\
\hline Carex pilulifera \\
\hline Carex sylvatica \\
\hline Dryopteris carthusiana \\
\hline Dryopteris dilatata \\
\hline Dryopteris filix-mas \\
\hline Galium odoratum \\
\hline Gymnocarpium dryopteris \\
\hline Hypericum perforatum \\
\hline Milium effusum \\
\hline Picea abies $\mathrm{F}$ \\
\hline Urtica dioica \\
\hline 2. Auf Ufl stagnierend oder abnehmend, \\
\hline Epilobium angustifolium \\
\hline Impatiens parviflora \\
\hline Veronica officinalis \\
\hline 3. Auf WT stagnierend (3 Arten $=4,9 \%$ ) \\
\hline Galeopsis tetrahit \\
\hline Luzula luzuloides \\
\hline Poa angustifolia \\
\hline 4. Auf Ufl stagnierend oder zunehmend, \\
\hline Agrostis capillaris \\
\hline Athyrium filix-femina \\
\hline Carex pallescens \\
\hline Carex remota \\
\hline Circaea lutetiana \\
\hline Fagus sylvatica F \\
\hline Juncus effusus \\
\hline Rubus fruticosus agg. $\mathrm{F}$ \\
\hline Rubus idaeus $\mathrm{F}$ \\
\hline Viola reichenbachiana \\
\hline 5. Auf Ufl und auf WT abnehmend (10 Ar \\
\hline Epilobium ciliatum \\
\hline Epilobium montanum \\
\hline Oxalis acetosella \\
\hline Poa nemoralis \\
\hline Quercus petraea F \\
\hline Rumex sanguinea \\
\hline Sambucus nigra F \\
\hline Sambucus racemosa $\mathrm{F}$ \\
\hline Scrophularia nodosa \\
\hline Stachys sylvatica \\
\hline 6. Nicht auf WT vorkommend (21 Arten = \\
\hline Acer pseudo-platanus F \\
\hline Agrostis canina \\
\hline Carpinus betulus $\mathrm{F}$ \\
\hline Cirsium arvense \\
\hline Cirsium palustre \\
\hline Cirsium vulgare \\
\hline Deschampsia cespitosa \\
\hline Epilobium tetragonum \\
\hline Festuca gigantea \\
\hline Galeobdolon luteum \\
\hline Impatiens noli-tangere \\
\hline Luzula campestris \\
\hline Luzula multiflora \\
\hline Luzula sylvatica \\
\hline Melica uniflora \\
\hline Mycelis muralis \\
\hline Poa annua \\
\hline Poa trivialis \\
\hline Salix caprea F \\
\hline Senecio jacobaea \\
\hline Taraxacum officinal \\
\hline
\end{tabular}


Tab. A31: Königsbuche-KF2 (Flächenwurf): Stetigkeitsvergleich Wurzelteller (WT)/Untersuchungsfläche (Ufl) 1999 + 2001; alle Angaben in \%; grau unterlegt = mindestens Verdoppelung/Halbierung der Stetigkeit.

\begin{tabular}{|c|}
\hline \\
\hline \\
\hline mittl. Anzahl Wurzelteller je $100 \mathrm{~m}^{2}$ \\
\hline mittl. Wurzeltellerfläche je $100 \mathrm{~m}^{2}(\%)$ \\
\hline Gesamtartenzahl Strauchschicht \\
\hline Gesamtartenzahl Feldschicht \\
\hline mittl. Artenzahl Strauchschicht \\
\hline davon nur auf $W T$ vorkommend \\
\hline mittl. Artenzahl Felschicht \\
\hline $\begin{array}{l}\text { davon nur auf WT vorkommend } \\
\text { mittl. Fläche des WT-Abschnitts ( }\left(^{2}\right)\end{array}$ \\
\hline mittl. vegetationsfreie Fläche (\%) \\
\hline mittl. DGS Strauchschicht (\%) \\
\hline mittl. DGS Feldschicht (\%) \\
\hline mittl. Deckung Kryptogamen (\%) \\
\hline mittl. Deckung Laubstreu + Totholz (\%) \\
\hline Baumschicht \\
\hline Fagus sylvatica $B$ \\
\hline Strauchschicht (Gesamtartenzahl: 9) \\
\hline 1. Auf Ufl und WT zunehmend ( 3 Arten = \\
\hline Fagus sylvatica S \\
\hline Rubus fruticosus agg. $\mathrm{S}$ \\
\hline Sambucus racemosa S \\
\hline 2. Auf Ufl stagnierend oder abnehmend, \\
\hline $\begin{array}{l}\text { Rubus idaeus } \mathrm{S} \\
\text { 5. Auf Ufl und } W T \text { abnehmend (1 Art }=1\end{array}$ \\
\hline Sambucus nigra $S$ \\
\hline 6. Nicht auf WT vorkommend (4 Arten $=$ \\
\hline Betula pendula S \\
\hline Carpinus betulus $\mathrm{S}$ \\
\hline Prunus avium $\mathrm{S}$ \\
\hline Salix caprea S \\
\hline Feldschicht (Gesamtartenzahl: 61) \\
\hline 1. Auf Ufl und auf WT zunehmend (16 A \\
\hline Betula pendula F \\
\hline Calamagrostis epigejos \\
\hline Carex muricata agg. \\
\hline Carex pallescens \\
\hline Carex pilulifera \\
\hline Dryopteris carthusiana \\
\hline Dryopteris dilatata \\
\hline Dryopteris filix-mas \\
\hline Galeopsis tetrahit \\
\hline Galium odoratum \\
\hline Gymnocarpium dryopteris \\
\hline Hypericum perforatum \\
\hline Milium effusum \\
\hline Picea abies F \\
\hline Urtica dioica \\
\hline Veronica officinalis \\
\hline 2. Auf Ufl stagnierend oder abnehmend, \\
\hline Carex sylvatica \\
\hline Impatiens parviflora \\
\hline 3. Auf WT stagnierend ( 5 Arten $=8,2 \%$ ) \\
\hline Athyrium filix-femina \\
\hline Circaea lutetiana \\
\hline Epilobium angustifolium \\
\hline Juncus effusus \\
\hline Oxalis acetosella \\
\hline 4. Auf Ufl stagnierend oder zunehmend, \\
\hline Agrostis capillaris \\
\hline Carex remota \\
\hline Fagus sylvatica F \\
\hline Poa angustifolia \\
\hline Rubus fruticosus agg. $\mathrm{F}$ \\
\hline Rubus idaeus $\mathrm{F}$ \\
\hline Viola reichenbachiana \\
\hline 5. Auf Ufl und auf WT abnehmend (10 A \\
\hline Epilobium ciliatum \\
\hline Epilobium montanum \\
\hline Luzula luzuloides \\
\hline Luzula multiflora \\
\hline Quercus petraea F \\
\hline Rumex sanguinea \\
\hline Sambucus nigra F \\
\hline Sambucus racemosa F \\
\hline Scrophularia nodosa \\
\hline Stachys sylvatica \\
\hline 6. Nicht auf WT vorkommend (21 Arten = \\
\hline Acer pseudo-platanus $\mathrm{F}$ \\
\hline Agrostis canina \\
\hline Carpinus betulus $\mathrm{F}$ \\
\hline Cirsium arvense \\
\hline Cirsium palustre \\
\hline Cirsium vulgare \\
\hline Deschampsia cespitosa \\
\hline Epilobium tetragonum \\
\hline Festuca gigantea \\
\hline Galeobdolon luteum \\
\hline Impatiens noli-tangere \\
\hline Luzula campestris \\
\hline Luzula sylvatica \\
\hline Melica uniflora \\
\hline Mycelis muralis \\
\hline Poa annua \\
\hline Poa nemoralis \\
\hline Poa trivialis \\
\hline Salix caprea F \\
\hline Senecio jacobaea \\
\hline Taraxacum officinale \\
\hline
\end{tabular}


Tab. A32: Prozentuale Häufigkeit, mit der manche Arten nur auf Wurzeltellern vorkamen; UG mit Flächenwurf; nur Ufl mit einem Ufl-WT-Vergleich (entsprechend Tab. 14); grau unterlegt $=$ mindestens Verdoppelung/Halbierung der prozentualen Häufigkeit.

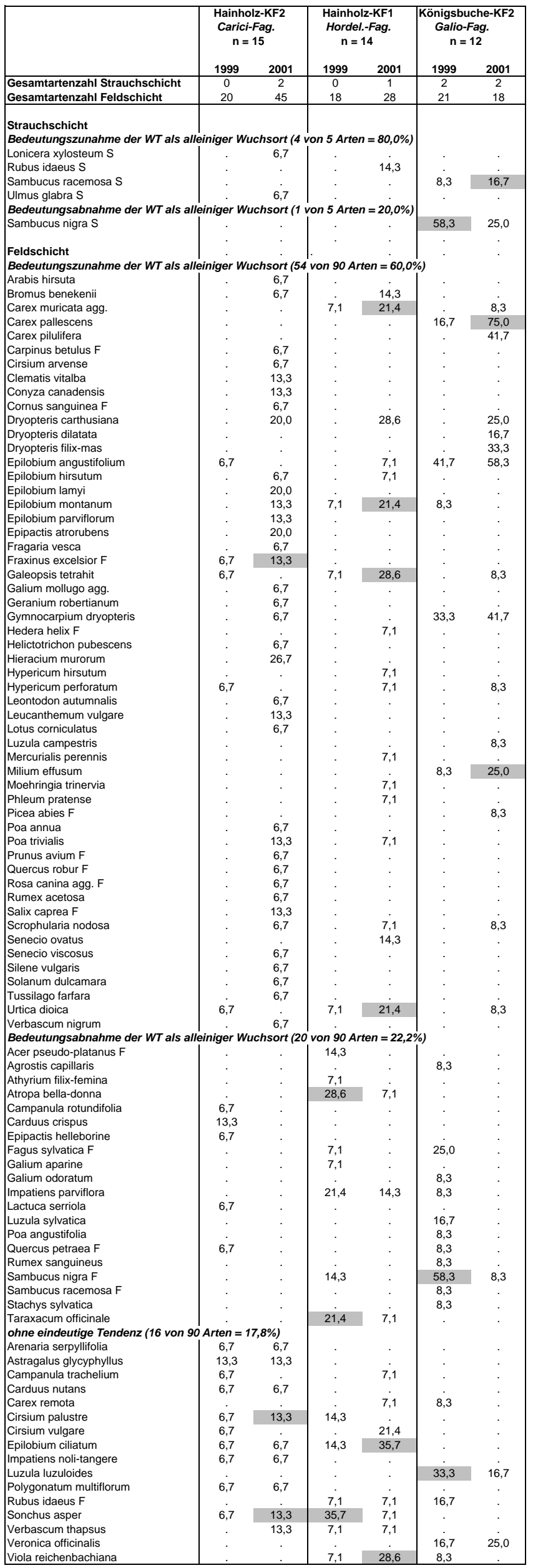




\section{LEBENSLAUF}

\section{Thomas Kompa}

geb. am 15.09.1969 in Frankenberg (Sachsen)

1 Tochter

Staatsbürgerschaft: Bundesrepublik Deutschland

\section{Schulbildung:}

1976 bis 1986:

Polytechnische Oberschule in Karl-Marx-Stadt (Chemnitz)

1988:

Abitur in Karl-Marx-Stadt (Chemnitz)

\section{Grundwehrdienst:}

$11 / 1988$ bis $01 / 1990$

\section{Studium:}

09/1990 bis 06/1997: Studium der Biologie in Halle/Saale; Hauptfach Geobotanik, Nebenfächer Tierökologie, Geographie und Naturschutz.

Abschluss:

Diplom-Biologe; Diplomarbeitsthema „Grünlandvegetation in der Saale-Aue bei Holleben (Saalkreis) in Abhängigkeit von unterschiedlicher Nutzungsgeschichte und -intensität" unter Betreuung von Prof. Ernst-Gerhard Mahn.

\section{Beruflicher Werdegang:}

07/1997 bis 09/1998: Wiss. Mitarbeiter in einem BMBF-Forschungsprojekt am Institut für Geobotanik der Universität Halle (Einfluss von Managementmaßnahmen auf spezifische Sukzessionsstadien in der mitteldeutschen Bergbaufolgelandschaft).

seit 03/1999:

Wiss. Mitarbeiter an der Universität Göttingen (Institut für Waldbau) und Anfertigung einer Doktorarbeit bei Prof. Wolfgang Schmidt zum Thema „Die Initialphase der Vegetationsentwicklung nach Windwurf in BuchenWäldern auf Zechstein- und Buntsandstein-Standorten des südwestlichen Harzvorlandes."

\section{Bisherige Veröffentlichungen:}

KOMPA, T. (1998): Fledermäuse (Chiroptera). - in: LANDESAMT FÜR UMWELTSCHUTZ (Hrsg.): Arten- und Biotopschutzprogramm Stadt Halle (Saale). - Ber. LA Umweltsch. Sachs.-Anh. 4: 295-303, Halle (Saale).

KOMPA, T.; GRÜTTNER, A. \& MAHN, E.G. (1999): Zum Einfluß von Standort und Nutzungsgeschichte auf die Grünlandvegetation in der Saale-Aue bei Holleben (Saalkreis). - Hercynia N.F. 32: 191-230, Halle (Saale).

KOMPA, T. \& SCHMIDT, W. (2001): Vegetationsentwicklung nach Windwurf in Buchenwäldern des südwestlichen Harzvorlandes. - Ber. d. Reinh.-Tüxen-Ges. 13: 251-255, Hannover.

KOMPA, T. \& SCHMIDT, W. (2002): Vegetationsentwicklung nach Windwurf in Buchenwäldern des südwestlichen Harzvorlandes. - in: Bundesamt für Naturschutz: Treffpunkt Biologische Vielfalt II: 125131, Bonn-Bad Godesberg.

KOMPA, T. \& SCHMIDT, W. (2003): Buchenwald-Sukzession nach Windwurf auf Buntsandstein. Tuexenia 23: 95-130, Göttingen. 